Michael Szeliga

Push und Pull in der Markenpolitik

Ein Beitrag zur modellgestützten

Marketingplanung am Beispiel des Reifenmarktes 


\section{Michael Szeliga}

\section{Push und Pull in der Markenpolitik}

Angesichts der hohen Komplexität von Entscheidungen in der Markenpolitik sehen sich Markenartikelhersteller vor der Herausforderung, Planungsinstrumente zu konzipieren, die sie bei der Entscheidungsfindung unterstützen und eine quantifizierbare Bewertung von Handlungsalternativen ermöglichen. Diese Arbeit hat sich daher zum Ziel gesetzt, einen Beitrag zur Entwicklung dieser Instrumente zu liefern. Hierbei wird die Tradition der bereits in den 70er Jahren intensiv diskutierten modelltheoretischen Ansätze fortgeführt und ein Simulationsmodell zur Planung von Markendurchsetzungsstrategien des Push and Pull entwickelt. Im Vordergrund steht die Frage des aufeinander abgestimmten Einsatzes des handels- und endabnehmerorientierten Marketinginstrumentariums. Den situativen Kontext zur Veranschaulichung der einzelnen Prozeßstufen bei der Entwicklung eines solchen Modells bildet der Privatkundenbereich des deutschen Reifenmarktes.

Michael Szeliga wurde 1964 in Steinheim/Westf. geboren. Er studierte Betriebswirtschaftslehre an der Universität Bielefeld und der University of Georgia/ USA. Während des Studiums war er als Entwickler von PC-Software sowie als Berater von Unternehmen bei der Einführung von EDV-Systemen selbständig tätig. Nach Abschluß als Diplom-Kaufmann sowie als Master of Business Administration (MBA) arbeitete er während der Zeit seiner Promotion als wissenschaftlicher Mitarbeiter am Institut für Marketing der Universität Münster. 
Push und Pull in der Markenpolitik

Ein Beitrag zur modellgestützten Marketingplanung am Beispiel des Reifenmarktes 


\section{SCHRIFTEN ZU \\ MARKETING UND MANAGEMENT \\ Herausgegeben von Prof. Dr. Dr. h.c. Heribert Meffert}

Band 29

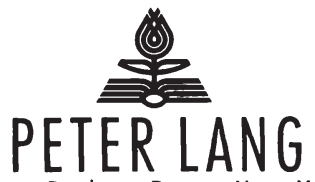

Frankfurt am Main - Berlin - Bern - New York - Paris - Wien 


\section{Michael Szeliga}

\section{Push und Pull \\ in der Markenpolitik}

Ein Beitrag zur modellgestützten Marketingplanung

am Beispiel des Reifenmarktes

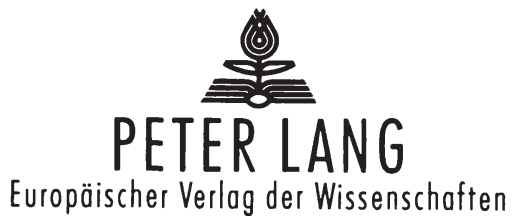

Michael Szeliga - 978-3-631-75081-0

Downloaded from PubFactory at 01/11/2019 08:32:20AM

via free access 
Die Deutsche Bibliothek - CIP-Einheitsaufnahme

Szeliga, Michael:

Push und Pull in der Markenpolitik : ein Beitrag zur modellgestützten Marketingplanung am Beispiel des Reifenmarktes / Michael Szeliga. - Frankfurt am Main ; Berlin ; Bern ; New York ; Paris ; Wien : Lang, 1996

(Schriften zu Marketing und Management ; Bd. 29)

Zugl.: Münster (Westfalen), Univ., Diss., 1995

ISBN 3-631-49759-8

NE: GT

Open Access: The online version of this publication is published on www.peterlang.com and www.econstor.eu under the international Creative Commons License CC-BY 4.0. Learn more on how you can use and share this work: http://creativecommons. org/licenses/by/4.0.

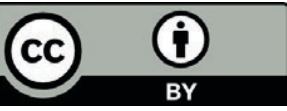

This book is available Open Access thanks to the kind support of ZBW - Leibniz-Informationszentrum Wirtschaft.

\author{
D 6 \\ ISSN 0176-2729 \\ ISBN 3-631-49759-8 \\ ISBN 978-3-631-75081-0 (eBook) \\ (C) Peter Lang GmbH \\ Europäischer Verlag der Wissenschaften \\ Frankfurt am Main 1996 \\ Alle Rechte vorbehalten.
}

Das Werk einschließlich aller seiner Teile ist urheberrechtlich geschützt. Jede Verwertung außerhalb der engen Grenzen des

Urheberrechtsgesetzes ist ohne Zustimmung des Verlages unzulässig und strafbar. Das gilt insbesondere für

Vervielfältigungen, Übersetzungen, Mikroverfilmungen und die Einspeicherung und Verarbeitung in elektronischen Systemen.

Printed in Germany 123467 


\section{Vorwort des Herausgebers}

Markenartikelhersteller sehen sich heute in einem zuvor nicht gekannten Ausmaß vor der Herausforderung, sowohl die externe Wirkungseffizienz und Effektivität ihrer markenpolitischen Maßnahmen zu erhöhen als auch gleichzeitig ihre innere Planungseffizienz zu steigern. Angesichts der hohen Komplexität von Entscheidungen im Spannungsfeld zwischen Hersteller, Handel und Endabnehmer wird damit der Einsatz von Planungsinstrumenten notwendig, die eine Strukturierung des Planungsproblems und die quantifizierbare Bewertung der Handlungsalternativen ermöglichen.

Bereits in den 70er Jahren hat sich die Forschung intensiv damit auseinandergesetzt, solche Instrumente auf Basis modellgestützter Ansätze zu entwickeln. Obgleich für eine gewisse Zeit etwas in den Hintergrund getreten, zeigen die gegenwärtigen Publikationen wieder ein deutliches Interesse, diese Tradition modelltheoretischer Arbeiten zur Fundierung betriebswirtschaftlicher Entscheidungen fortzuführen.

Vor diesem Hintergrund wird in der vorliegenden Arbeit das Ziel verfolgt, ein computergestütztes Modell zur Unterstützung markenpolitischer Entscheidungen zu entwickeln. Aus der Vielzahl möglicher Fragestellungen der Markenführung greift der Verfasser dabei die Aufgabe der Planung von Strategien der Markendurchsetzung auf, also solchen Strategien, die einen Orientierungsrahmen für den integrierten handels- und endabnehmergerichteten Instrumenteeinsatz liefern. In der Literatur wird in diesem Zusammenhang häufig auch von Push- und Pull-Strategien gesprochen. Letztlich führt diese Aufgabe zur Problemstellung der Ressourcenallokation, bei der das Marketingbudget $\mathrm{zu}$ bestimmen und möglichst optimal auf die vorgeschaltete Beeinflussung des Handels und die direkte Ansprache der Endabnehmer aufzuteilen ist.

Den Ausgangspunkt der Modellentwicklung stellt die inhaltliche Analyse des Planungsgegenstandes dar. Hierbei werden der Reifenmarkt als beispielhaft gewählter situativer Kontext vorgestellt, markenpolitische Ziele systematisiert und ausgewählt sowie der durch vorgelagerte Entscheidungen über Grundsatzstrategien der Markierung und Positionierung gegebene strategische Rahmen beschrieben. Des weiteren werden das im Reifenmarkt zur Verfügung stehende Push- und Pull-Instrumentarium gekennzeichnet sowie 
die zwischen den Instrumentebereichen bestehenden Wirkungsbeziehungen und Interdependenzen charakterisiert.

Das Modell selbst setzt an der Vorstellung des Entscheidungsträgers über die realen Beziehungen innerhalb des zugrundeliegenden Marktes an. Ziel ist es, das "interne Modell" des Entscheidungsträgers explizit darzulegen, um so ein Planungsinstrument mit "intelligenzverstärkender" Funktion zu schaffen. Der zentrale Grundgedanke dieses Ansatzes ist darin zu sehen, daß die Einschätzungen und Erfahrungen des Entscheidungsträgers bei der Modellbildung berücksichtigt werden und von der Fähigkeit des Manager Gebrauch gemacht wird, Informationen zu verdichten und damit Wirkungsbeziehungen subjektiv schätzen zu können. Besondere Aufmerksamkeit wird vom Verfasser in diesem Zusammenhang der Problematik gewidmet, daß die Ermittlung von Variablenwerten und funktionalen Zusammenhängen stets mit Unsicherheiten behaftet ist. An die Stelle deterministischer Variablen treten in dem Modell daher subjektiv geschätzte, stochastische Größen und Wirkungsbeziehungen.

Das auf Basis dieser grundlegenden Gestaltungsprinzipien entwickelte globalanalytische Makromodell erlaubt die Simulation der Wirkungen unterschiedlicher Kombinationen von Aktivitätsniveaus des Push- und PullInstrumentariums unter Annahme zuvor festgelegter, situativer Rahmenbedingungen. Mit Hilfe der Methode der Risikosimulation werden Enwartungswerte und Risikoprofile für verschiedene markenpolitische Ziele berechnet und damit die Grundlage geschaffen, eine der Planungsunsicherheit adäquate Bewertung von Strategiealternativen vornehmen zu können.

Über die theoretische Konzeption hinaus wird das Modell als computergestütztes Programm implementiert. Neben der Simulation mit diskreten Werten für die Aktivitätsniveaus der Entscheidungsvariablen bietet das Programm im Rahmen der Sensitivitätsanalyse die Möglichkeit, diese Niveaus innerhalb eines anzugebenden Bereiches automatisch variieren zu lassen. In Verbindung mit dem Prinzip der Einzelschrittmethode läßt sich hiermit der Erreichungsgrad markenpolitischer Ziele stufenweise erhöhen und ein zumindest lokales Optimum erzielen. 
Zum Abschluß der Arbeit verdeutlicht der Verfasser schließlich den praktischen Einsatz des Modells beispielhaft anhand einer fiktiven Reifenmarke. Ausgehend von den bisher für die Marke angenommenen Aktivitätsniveaus des Push- und Pull-Instrumentariums gelangt er dabei mit der beschriebenen Vorgehensweise zu einer erheblichen Verbesserung des als Oberziel gewählten Markenwertes.

Insgesamt stellt die Arbeit eine wertvolle Bereicherung der wissenschaftlichen Diskussion auf dem Gebiet modelltheoretischer Ansätze dar und gibt aufschlußreiche Hinweise für die Planung markenpolitischer Strategien. Es ist zu wünschen, daß dieser Beitrag in Wissenschaft und Praxis auf breite Resonanz stößt.

Münster, im August 1995

Prof. Dr. Dr. h.c. H. Meffert 
Michael Szeliga - 978-3-631-75081-0

Downloaded from PubFactory at 01/11/2019 08:32:20AM

via free access 


\section{Vorwort des Verfassers}

Markenartikelhersteller stehen heute vor der Herausforderung, sowohl die externe Wirkungseffizienz und Effektivität markenpolitischer Maßnahmen zu erhöhen, als auch die interne Planungseffizienz zu steigern. Dies gilt im besonderen Maße für die Planung von Markendurchsetzungsstrategien, bei der eine integrierte Betrachtung der Beziehungen zwischen Hersteller, Handel und Endabnehmer notwendig ist. Um so wichtiger ist es für die Markenartikelhersteller, über geeignete Planungsinstrumente zu verfügen, die eine Strukturierung des Planungsproblems und die Bewertung von Handlungsalternativen ermöglichen. Vor diesem Hintergrund war es das Ziel, die Tradition modelltheoretischer Arbeiten fortzusetzen und ein entscheidungsunterstützendes System für die Planung von Markendurchsetzungsstrategien zu entwickeln. Den situativen Kontext bildet der Privatkundenbereich des deutschen Reifenmarktes.

Als Grundlage der Arbeit werden zunächst der markenpolitische Planungsprozeß strukturiert und Strategien der Markendurchsetzung als Instrumentalstrategien charakterisiert, die mit ihren Ausprägungsformen der Push- und Pull-Orientierung zur Durchsetzung markenpolitischer Grundsatzstrategien der Markierung und Positionierung dienen. Des weiteren werden die für eine Entscheidung über die Art der Markendurchsetzung relevanten Faktoren des Verhaltens von Handel und Endabnehmer analysiert und der Zusammenhang von Erstausrüstungs- und Ersatzmarkt kritisch überprüft. Die Auswahl und Einordnung der relevanten markenpolitischen Ziele, die Untersuchung der Rahmenbedingungen durch vorgegebene Entscheidungen über die Markierung und Positionierung sowie die Systematisierung und Darstellung von Einsatzmöglichkeiten für die Marketinginstrumente vervollständigen die inhaltliche Analyse der Planung von Markendurchsetzungsstrategien.

Aufbauend hierauf wird ein entscheidungsunterstützendes Modell entwickelt, das dem Prinzip des benutzerorientiert-subjektiven Ansatzes folgt. Vor dem Hintergrund der zu beachtenden Strukturdefekte, den in der Literatur beschriebenen Gestaltungsanforderungen sowie der Diskussion ausgewählter Modellansätze wird das Modell als globalanalytisches Makromodell mit verhaltensbezogenen Variablen konzipiert, das stochastische Variablen und Beziehungen enthält, periodenübergreifende Wirkungen berücksichtigt und 
als Lösungsmethode die Risikosimulation verwendet. Über die theoretische Konzeption hinaus wird das Modell als computergestütztes Simulationsprogramm implementiert und dessen Anwendung anhand eines Beispiels verdeutlicht.

Bei der Abfassung der Arbeit erfuhr ich umfassende Unterstützung durch verschiedene Personen. Mein besonderer Dank gilt an dieser Stelle meinem akademischen Lehrer, Herrn Professor Dr. Dr. h.c. Heribert Meffert, der die Themenstellung anregte und die Fertigstellung der Arbeit umfassend förderte. Herrn Professor Dr. Heinz-Lothar Grob sei für die Übernahme des Zweitgutachtens ebenfalls herzlich gedankt.

Mein Dank gilt ferner allen Kollegen und Kolleginnen am Institut für Marketing, die mich während der Erstellung der Arbeit entlastet und vielfältig unterstützt haben. Hervorheben möchte ich Herrn Dr. Manfred Kirchgeorg, der mir während meiner Assistentenzeit in persönlicher wie auch fachlicher Form stets als Ansprechpartner und Berater zur Verfügung stand und mir eine Vielzahl wichtiger Impulse gab. Meinen Dank möchte ich insbesondere auch den Herren Dipl.-Kfm. Jörg Meurer, Dr. Nicolaus Müller und Dr. Stefan Wöllenstein aussprechen. Ihre ständige Bereitschaft zur Diskussion und ihre konstruktiv-kritischen Hinweise haben die Abfassung der Arbeit begleitet und maßgeblich zum Gelingen beigetragen. Frau Dipl.-Kffr. Karin Hillebrand sowie Herr Dipl.-Kfm. Frithjof Netzer waren mir nicht nur bei der Durchsicht der Manuskripte behilflich sondern haben mich während der gesamten Erstellungszeit in vorbildlich kollegialer Form unterstützt. Dank gebührt in diesem Zusammenhang auch Herrn Dipl.-Kfm. Jesko Perrey, der mich ebenfalls sehr engagiert unterstützte. Für die exzellente Anfertigung der Grafiken und die hervorragende Abwicklung technischer Aufgaben bei der Manuskripterstellung danke ich Frau Sabine Voß und Herrn Oliver Voß.

Darüber hinaus möchte ich meinen Eltern danken. Ohne ihre Bereitschaft, häufig zu verzichten und statt dessen in meine Ausbildung zu investieren, wäre diese Arbeit wahrscheinlich niemals entstanden. Schließlich gebührt ein ganz besonderer Dank meiner Christiane. Sie hat in den letzten Jahren nicht nur viel Geduld und Verständnis gezeigt, sondern mir stets mit tatkräftiger Unterstützung und wertvollem Rat zur Seite gestanden. 


\section{Inhaltsverzeichnis}

A. Modellgestützte Planung von Markendurchsetzungsstrategien als Herausforderung für die Markenpolitik

1. Gegenstand und Komplexität markenpolitischer Entscheidungen im Zeichen veränderter Rahmenbedingungen.

2. Push und Pull als Ausprägungsformen von Markendurchsetzungsstrategien

3. Gegenstand und Anforderungen einer modellgestützten Planung in der Markenpolitik

4. Ziel und Gang der Untersuchung

B. Analyse des Planungsgegenstandes von Markendurchsetzungsstrategien

1. Der Reifenmarkt als situativer Kontext für die Planung von Markendurchsetzungsstrategien

1.1 Teilmärkte des Reifenmarktes und Absatzmarktstruktur 35

1.2 Wettbewerbssituation und Marktentwicklung 40

1.3 Endabnehmerbezogene Situation 45

1.31 Bedarfsverhalten 45

1.32 Einkaufsstättenwahlverhalten

1.33 Informationsquellennutzung und Markenwahlverhalten 50

1.4 Handelsbezogene Situation 53

1.41 Rollenverständnis des Handels 53 
1.42 Machtbeziehungen zwischen Hersteller und Handel 57

1.43 Warenpräsentation und Bedienungsform im Handel. 59

1.5 Bedeutung der Erstausrüstung im Reifenmarkt 61

2. Markenpolitische Ziele und Grundsatzstrategien der Reifenhersteller als Ausgangspunkt der Planung von Markendurchsetzungsstrategien ..67

2.1 Markenpolitische Ziele als anzustrebende Vorzugszustände 67

2.11 Systematisierung des Zielsystems 67

2.12 Ziele der Nachfragestärke 71

2.13 Ziele der Angebotsstärke 78

2.2 Grundsatzstrategien der Reifenhersteller als strategische Rahmenbedingungen für Markendurchsetzungsstrategien 80

2.21 Grundsatzstrategien der Markierung 80

2.22 Grundsatzstrategien der Positionierung 83

3. Einsatz des Push- und Pull-Instrumentariums als Ausdruck von Markendurchsetzungsstrategien

3.1 Darstellung des Push- und Pull-Instrumentariums im Reifenmarkt .87

3.11 Instrumente im Produktbereich 88

3.12 Instrumente im Kommunikationsbereich 92

3.13 Instrumente im Kontrahierungsbereich 95

3.14 Instrumente im Distributionsbereich .99

3.2 Aktivitătsniveau des Push- und Pull-Instrumentariums. 101 
3.3 Wirkungsbeziehungen und Interdependenzen des Pushund Pull-Instrumentariums

3.31 Wirkungsbeziehungen des Push-Instrumentariums 105

3.32 Wirkungsbeziehungen des Pull-Instrumentariums 108

3.33 Zeitlich-horizontale Interdependenzen zwischen Push- und Pull-Instrumentarium

3.34 Zeitlich-vertikale Interdependenzen zwischen

Push- und Pull-Instrumentarium

C. Modellgestützte Planung von Markendurchsetzungsstrategien

1. Grundlagen der Gestaltung eines Modells zur Planung von Markendurchsetzungsstrategien.....

1.1 Strukturdefekte und Anforderungen an das Modell als Ausgangspunkt der Entwicklung.... 118

1.2 Darstellung von Ansätzen zur Gestaltung von Modellen 126

1.3 Implikationen für die eigene Modellentwicklung. 131

2. Darstellung und Würdigung ausgewählter Modelle der Literatur 132

2.1 CIM-Devellopement-Reifenmarktmodell 133

2.2 BRANDAID-Modell 141

2.3 Modell von Farris, Olver und De Kluyver 147

2.4 Würdigung der Modelle und Implikationen für die eigene Modellentwicklung 
3. Entwicklung eines Reifenmarktsimulationsmodells zur Planung von Markendurchsetzungsstrategien

3.1 Kennzeichnung der Struktur der Gesamtmodells und Darstellung der Teilmodelle

3.11 Herstellerentscheidungsmodell ......................................161

3.12 Endabnehmermodell ......................................................163

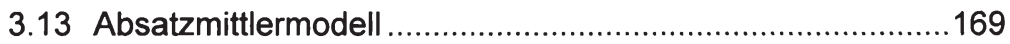

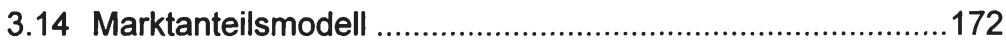

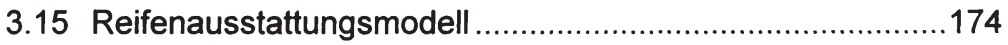

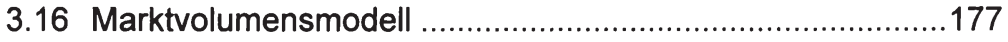

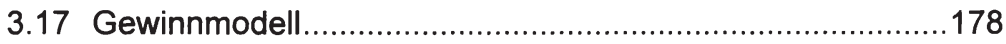

3.2 Eingangsvariablen und Erklärungsgleichungen .........................182

3.21 Ermittlung von Werten der Eingangsvariablen ...................182

3.22 Bestimmung von Parametern der Erklärungs-

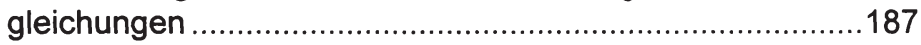

3.3 Berechnung und Interpretation der Modellergebnisse .................198

3.31 Berechnung der Modellergebnisse durch Risikosimulation

3.32 Interpretation der Modellergebnisse und

Entscheidung für eine Handlungsalternative .....................208

3.4 Sensitivitätsanalyse und Optimierung ....................................211

3.5 Computergestützte Implementierung des Modells .....................215 


\section{XV}

4. Planung von Markendurchsetzungsstrategien mit dem

Reifenmarktsimulationsmodell anhand eines fiktiven Beispiels

4.1 Datensituation des Beispiels

4.2 Ausgangssimulation und Optimierung

225

D. Zusammenfassung und Ausblick

1. Zusammenfassende Würdigung der modellgestützten

Planung von Markendurchsetzungsstrategien

2. Implikationen für die Unternehmenspraxis

238

3. Implikationen für die weitere Forschung

239

Anhang

Literaturverzeichnis 261 
Michael Szeliga - 978-3-631-75081-0

Downloaded from PubFactory at 01/11/2019 08:32:20AM

via free access 


\section{Abbildungsverzeichnis}

Abb. 1: Prozeß der Planung in der Markenpolitik

Abb. 2: Push und Pull als Konzepte zur Beschreibung mehrstufiger Zusammenhänge im vertikalen Martktsystem von Hersteller, Handel und Endabnehmer

Abb. 3: Begriffsverwendung von Push und Pull in der Literatur 21

Abb. 4: Push- und Pull-Orientierung in der Markendurchsetzungsstrategie

Abb. 5: Stufen der Entwicklung eines Modells zur Unterstützung der Planung in der Markenpolitik

Abb. 6: Struktur des Reifenmarktes 37

Abb. 7: Anteil der Vertriebswege auf dem deutschen Reifenmarkt im PKW-Privatkundenbereich (in 1993).

Abb. 8: Herstellermarktanteile auf dem deutschen Reifenmarkt im PKW-Privatkundenbereich (in 1993)

Abb. 9: Absatzvolumen PKW-Reifen im Ersatzgeschäft 42

Abb. 10: Gründe für die Wahl der Einkaufsstätte 48

Abb. 11: Einfluß von Informationsquellen auf die Kaufentscheidung beim Reifenkauf

Abb. 12: Kriterien zur Beurteilung von Marken durch Reifenfachhändler

Abb. 13: Vom Händler bevorzugt im Verkaufsgespräch empfohlene Marke

Abb. 14: Einfluß der Erstausrüstung auf das Reifenersatzgeschäft (Berechnung Teil 1). 
Abb. 15: Einfluß der Erstausrüstung auf das Reifenersatzgeschäft (Berechnung Teil 2)

Abb. 16: Hierarchisch strukturiertes Zielsystem für die Planung von Markendurchsetzungsstrategien.

Abb.17: Markenpolitische Ziele zur Beschreibung der Nachfragestärke einer Marke

Abb. 18: Markierungsstrategien im Reifenmarkt 81

Abb. 19: Ausprägungen verschiedener Positionierungen im Reifenmarkt 84

Abb. 20: Systematisierung des Push- und Pull-Instrumentariums 88

Abb. 21: Handelsgerichtete Leistungen der Continental AG 92

Abb. 22a: Entwicklung des Werbeaufkommens im deutschen Reifenmarkt

Abb. 22b:Aufteilung des Werbeaufkommens auf Medien und Reifenmarken in 1993 96

Abb. 23: Schematische Darstellung der Wirkungsbeziehungen des Push-und Pull-Instrumentariums.

Abb. 24: Zusammenhang von POS-Stärke und Aktivitätsniveau des Push-Instrumentariums

Abb. 25: Zeitlich-horizontale Interdependenzen zwischen Push- und Pull-Instrumentarium im Hinblick auf das Marktanteilziel

Abb. 26: Vereinfachte Darstellung der zeitlich-horizontalen Interdependenzen des Push- und Pull-Instrumentariums.

Abb. 27: Idealtypischer Verlauf einer Marken- bzw. Produkteinführung mit Wechsel von einer Push- zur Pull-Orientierung 
Abb. 28: Anforderungen an die Modellgestaltung .............................. 124

Abb. 29: Dimensionen zur Systematisierung von Modelltypen ............... 126

Abb. 30: Struktur des CIM-DEVELOPPEMENT-Modells ....................... 134

Abb. 31: Grundlegende Beziehungen des im

CIM-DEVELOPPEMENT abgebildeten Reifenmarktes

Abb. 32: Struktur des BRANDAID-Modells .......................................... 141

Abb. 33: Struktur des Modells von Farris, Olver und De Kluyver ............ 148

Abb. 34: Struktur des Reifenmarktsimulationsmodells .......................... 158

Abb. 35: Grundstruktur der Variablenbeziehungen des Modells............. 160

Abb. 36: Prozeß des Reifenkaufes innerhalb der Einkaufsstätte ............ 164

Abb. 37: Präferenz- und Akzeptanzstruktur der Reifenkäufer .................. 168

Abb. 38: Berechnung des Marktanteils .............................................. 174

Abb. 39: Ermittlung der zum Kaufzeitpunkt montierten Reifenmarke...... 176

Abb. 40: Stichprobenverteilung der jährlichen Fahrleistung.................. 184

Abb. 41: Trapezvariablen der Modellvariablen ....................................... 186

Abb. 42: Responsefunktion des ADBUDG-Modells ............................. 189

Abb. 43: Verfallsfunktion des ADBUDG-Modells.................................... 190

Abb. 44: Konkaver und s-förmiger Funktionsverlauf der

modifizierten ADBUDG-Funktion

Abb. 45: Zusammenhang von Aktivitätsniveau des Marketinginstruments und der Erwartungsvariablen unter Berücksichtigung des unsicheren Wirkungszusammenhanges ........................... 195 


\section{$X X$}

Abb. 46: Ermittlung der stochastischen Beziehung zwischen Aktivitätsniveau des Marketinginstruments und der Erwartungsvariablen bei trapezförmiger Wahrscheinlichkeitsverteilung

Abb. 47: Ablauf der Monte-Carlo-Simulation.............................................204

Abb. 48: Risikoprofile zweier Strategien der Markendurchsetzung...........209

Abb. 49: Risikoprofile des Markenwertes bei Ausgangs- und optimiertem Budget. 


\section{Abkürzungsverzeichnis}

a.a.O.

Abb.

AG

asw

Aufl.

Bd.

BFuP

BRV

bspw.

bzw.

ca.

DBW

Dekra

d.h.

Diss.

DM

Ed.

EDV

etc.

e.V.

f., ff.

FAZ

FN

GDI

$\mathrm{GmbH}$

H.

Hrsg.

i.d.R.

LZ am angegebenen Ort

Abbildung

Aktiengesellschaft

Absatzwirtschaft

Auflage

Band

Betriebswirtschaftliche Forschung und Praxis

Bundesverband Reifenhandel und Vulkaniseurhandwerk

e.V.

beispielsweise

beziehungsweise

circa

Die Betriebswirtschaft

Deutscher Kraftfahrzeug-Überwachungs-Verein e.V.

das heißt

Dissertation

Deutsche Mark

Edition

Elektronische Datenverarbeitung

et cetera

eingetragener Verein

folgende, fortfolgende

Frankfurter Allgemeine Zeitung

Fußnote

Gottlieb Duttweiler Institut

Gesellschaft mit beschränkter Haftung

Heft

Herausgeber

in der Regel

Lebensmittel Zeitung 
$\mathrm{km}$

Mio.

n

No.

$\mathrm{Nr}$.

O.J.

o.V.

Pkw

POS

S.

sog.

Sp.

TÜV

u.a.

usw.

vgl.

WiSt

WISU

Z.B.

ZfB

$\mathrm{ZfbF}$

ZFP

z.T.
Kilometer

Millionen

Stichprobengröße

Number

Nummer

ohne Jahr

ohne Verfasser

Personenkraftwagen

Point of Sale

Seite

sogenannte

Spalte

Technischer Überwachungsverein e.V.

und andere, unter anderem

und so weiter

vergleiche

Wirtschaftswissenschaftliches Studium

Das Wirtschaftsstudium

zum Beispiel

Zeitschrift für Betriebswirtschaft

Zeitschrift für betriebswirtschaftliche Forschung Zeitschrift für Forschung und Praxis zum Teil 


\section{A. Modellgestützte Planung von Markendurchsetzungsstrategien als Herausforderung für die Markenpolitik}

\section{Gegenstand und Komplexität markenpolitischer Entscheidungen im Zeichen veränderter Rahmenbedingungen}

Markenartikelhersteller sehen sich heute einer Umwelt gegenüber, die sowohl durch die Dynamik als auch durch die Vielfalt der Entwicklungen wichtiger unternehmerischer Rahmenbedingungen zu einer bislang nicht gekannten Komplexität markenpolitischer Entscheidungen führt. Im Spannungsfeld zwischen verändertem Konsumentenverhalten, gestiegenem Selbstbewußtsein des Handels und einer zunehmenden Wettbewerbsintensität stehen die Hersteller vor der Herausforderung, ihre Effizienz und Effektivität ${ }^{1}$ in der Markenpolitik zu verbessern, um den Erfolg der Marken ${ }^{2}$ langfristig zu sichern. ${ }^{3}$

1 In der Effizienz kommt das Verhältnis zwischen Zielerreichung und eingesetzten Ressourcen zum Ausdruck, während mit dem Begriff der Effektivität der Zielerreichungsgrad beschrieben wird. Vgl. dazu Welge, M. K., Unternehmungsführung, Band 3, Controlling, Stuttgart 1983, S. 450 sowie die dort angegebene Literatur.

2 Unter dem Begriff der Marke kann die Kennzeichnung von Produkten mit einem über die Zeit weitgehend konstanten Marktauftritt verstanden werden, von denen erwartet wird, daß sie eine stets gleichbleibende oder verbesserte Qualität aufweisen und in einem breiteren Absatzraum erhältlich sind. Da die Marke damit nicht losgelöst von den durch sie gekennzeichneten Produkten betrachtet werden kann, wird der Begriff Marke hier im Sinne von "Produkt(e) einer Marke" verwendet. Eine begriffliche Trennung von Marken- und Produktebene wird lediglich in solchen Fällen vorgenommen, wo sie aus inhaltlichen Gründen notwendig wird. Vgl. zum Begriff der Marke Mellerowicz, K., Markenartikel - die ökonomischen Gesetze ihrer Preisbildung und Preisbindung, München, Wien 1963, S. 39; Kühn, R., Der Markenartikel, in: Marketing Journal, H. 5, 1983, S. 374 ff.; Größer, H., Der klassische Markenartikel - Versuch einer Wesensbestimmung, in: Markenartikel, $H$. 5, 1991, S. $200 \mathrm{ff}$. Zur Entwicklung des Markenbegriffes vgl. Leitherer, E., Die Entwicklung des Markenwesens - Von den Ursprüngen bis zum Beginn der fünfziger Jahre. Veröffentlichung der Gesellschaft zur Erforschung des Markenwesens e.V., Hrsg: Berekoven, L., München 1988 sowie Berekoven, L., Von der Markierung zur Marke, in: Marke und Markenartikel als Instrumente des Wettbewerbs, Hrsg.: Dichtl, E., Eggers, W., München 1992, S. 25 ff.

3 Vgl. Meffert, H., Herausforderungen an die Markenführung in den 90er Jahren, in: Markenartikel, H. 6, 1991, S. 264 f.; Meffert, H., Markenführung in der Bewährungsprobe, in: Markenartikel, H. 10, 1994, S. $478 \mathrm{ff}$. 
Das Konsumentenverhalten ist durch einen umfassenden Wertewandel gekennzeichnet, bei dem die Vielschichtigkeit der Bedürnnisse in den Vordergrund getreten ist. ${ }^{4}$ Der Verbraucher tendiert dazu, die Ausgaben für Basisbedürfnisse ("Needs") zu minimieren und das Einkaufsbudget auf Käufe mit Erlebniswert ("Wants") zu konzentrieren. Dabei verhält sich der Konsument heute zunehmend kritischer beim Kauf, mit der Folge, daß von inm nur Marken mit einer hohen Eigenständigkeit durch eine entsprechende Preisbereitschaft honoriert werden. ${ }^{5}$ Auch scheint der Preis als Qualitätsindikator an Bedeutung verloren zu haben, da aufgeklärte Verbraucher nicht zuletzt durch eine fortschreitende Homogenisierung des Angebotes in der Lage sind, die tatsächliche Leistung einer Marke beurteilen zu können. Parallel zu dieser Entwicklung wird es schwieriger, die Marke im Wettbewerbsumfeld kommunikativ zu profilieren. Die auf den Konsumenten einwirkende Informationsflut bei gleichzeitiger Fraktionierung der Zielgruppen durch eine ständig steigende Medienvielfalt erschwert es zusehends, den Endabnehmer mit der eigenen Botschaft zu erreichen. 6

Die Beziehungen zwischen Hersteller und Handel sind traditionell durch unterschiedliche Zielvorstellungen geprägt, bei denen sich die Markenorientierung des Herstellers und das Sortimentsdenken ${ }^{7}$ des Handels gegen-

Vgl. Windhorst, K-G., Wertewandel und Konsumentenverhalten, Münster 1985, S. 85 ff.; Meffert, H., Perspektiven des Marketing im Spannungsfeld zwischen Standardisierung und Differenzierung, Thesen zu den Herausforderungen der 90er Jahre, in: Markenartikel, H. 10, 1986, S. 442 ff.; derselbe, Erfolgreiches Marketing in den neunziger Jahren, in: Marketing im Umbruch - Chancen und Gefahren für Unternehmer, Zürich 1991, S. $11 \mathrm{ff}$.

5 Vgl. die Ergebnisse der Verbraucherstudie der BBDO, BBDO (Hrsg.): Der neue Realismus - Verbraucherverhalten in der Rezession, Düsseldorf 1994; Meffert, H., Markenführung in der Bewährungsprobe, a.a.O., S. 478 ff.; o. V., Der gute Ruf entscheidet, in: asw, H. 9, 1990, S. 36; Westbrook, G., Der Preisabstand schwindet dahin. Trends auf dem europäischen Eigenmarkenmarkt, in: LZ, Nr. 40, 8. Oktober 1993, S. 158 f.; Köhler, R., Marke und Verbraucher - Zu den Grundlagen lebenslanger Bindung, in: Markenartikel, H. 7, 1993, S. $340 \mathrm{ff}$.

6 Vgl. Unger, F., Markenpolitik und -strategie angesichts geänderten Verbraucherverhaltens, in: VM International, H. 2, 1990, S. 4 ff.; o. V., Gefahr im Marketing, in: w\&v, Nr. 27, 6. Juli 1990, S. 12; Merkle, H., Globale Trends - Globale Chance für die Werbung, in: Markenartikel, H. 7, 1994, S. 338; Mann, G., Entwicklungstendenzen des Markenartikels - Herstellerperspektive, in: Markenartikel, H. 10, 1994, S. 487. 
überstehen. ${ }^{8}$ Hierbei hat sich in den letzten Jahren das Machtgefüge zwischen den beiden Marktteilnehmern zugunsten des Handels verschoben. ${ }^{9}$ Die Konzentration von Handelsunternehmen, verbesserte Informationssysteme zur Überprüfung der Profitabilität von Produkten und das Angebot einer Vielzahl gleichwertiger und damit austauschbarer Alternativen haben die Machtposition des Handels in seiner Rolle als "Gatekeeper" zum Konsumenten gestärkt.10 Darüber hinaus befindet sich der Hersteller in einer Reihe von Branchen in direktem Wettbewerb mit den Eigenmarken ${ }^{11}$ des Handels, die nicht mehr ausschließlich im Niedrigpreissegment positioniert sind, sondern sich auch immer häufiger im mittleren und höheren Preissegment finden.12 Der Handel bedient sich hierbei zusehends eines Marke-

Sachleistungen zum Zweck der Verwertung im Absatzmarkt unter Einschluß der durch handelsübliche Manipulationen im Betrieb neu entstandenen Sachleistungen" zu verstehen. Vgl. Gümbel, R., Die Sortimentspolitik in den einzelnen Betrieben des Wareneinzelhandels, Köln, Opladen 1963, S. 59.

Vgl. Fritz, W. u.a., Unternehmensziele in Industrie und Handel, in: DBW, H. 4, 1985, S. 375 ff.; Pepels, W., Die Beziehungen von Marketing-Interessen auf Herstellerund Handelsstufe, in: Markenartikel, H. 10, 1988, S. 494 ff.; Dichtl, E., Müller, S., Herausforderungen für die Markenartikelindustrie an der Schwelle zu einem neuen Jahrzehnt, Ergebnisse einer Delphi-Befragung, Arbeitspapier Nr. 94 des Instituts für Marketing der Universität Mannheim, Mannheim 1992, Anhang I, S. 28; Bielefeld, K. W., Marken II: Kampf um die Wertschöpfung, in: Harvardmanager, H. 3, 1992, S. $34 \mathrm{ff}$.

Vgl. Bellino, H., Strategische Verkaufsförderung ist Push-Marketing, in: Thexis, H. 2, 1993, S. $6 \mathrm{ff}$.

Vgl. Ahlert, D., Distributionspolitik, Das Management des Absatzkanals, Stuttgart, New York 1985, S. 97; Hansen, U., Absatz- und Beschaffungsmarketing des Einzelhandels. Eine Aktionsanalyse, Göttingen 1990, S. 44 ff.; Meffert, H., MarketingManagement - Analyse, Strategie, Implementierung, Wiesbaden 1994, S. $164 \mathrm{ff}$.

Bei Eigenmarken des Handels bzw. Handelsmarken handelt es sich um markierte Produkte, für die sich die jeweilige Handelsorganisation verantwortlich zeigt und die ausschließlich in den zur Handelsorganisation zugehörigen Einkaufsstätten erhältlich sind. Neben den "klassischen Handelsmarken" zählen hierzu auch Gattungsmarken, bei denen nicht der Markenname, sondern die Warenbezeichnung im Vordergrund der Markierung steht. Vgl. Meffert, H., Markenpolitik im Wettbewerb (I), in: WISU, H. 9, 1983, S. 407 ff. Für eine ausführliche Diskussion der Formen von Handelsmarken vgl. Huber, W. R., Markenpolitische Strategien des Konsumgüterherstellers, Frankfurt/Main 1988, S. 21 ff. Zum Verbraucherverhalten gegenüber Eigenmarken vgl. Gordon, W., Handelsmarken, Das Äquivalent für Erfolg in den Neunzigern, in: Planung und Analyse, H. 3, 1994, S. 52 ff.

12 Für eine Übersicht der Marktanteile von Hersteller- und Handelsmarken in ausgewählten Branchen und Produktbereichen siehe Batzer, E., Greipl, E., Die Bedeutung von Hersteller- und Handelsmarke für den Handel, in: Marke und Markenarti- 
tinginstrumentariums, dessen Einsatz in der Vergangenheit als Domäne der Markenartikelhersteller galt. In Verbindung mit der handelsspezifischen Nähe zum Konsumenten wird er damit zu einem ernsthaften Konkurrenten bei der Gestaltung und Führung von Marken. ${ }^{13}$

Auch zwischen den Markenartikelherstellern ist eine kontinuierlich steigende Wettbewerbsintensität zu beobachten. Ansteigende Konditionenforderungen des Handels, die Erhöhung der Anzahl gleichzeitig geführter, miteinander konkurrierender Marken sowie eine verstärkte Vorwärtsintegration zur Kontrolle von Absatzwegen führen zu verringerten Deckungsbeiträgen und höheren Fixkostenbelastungen. Um dem entstehenden Renditedruck auszuweichen, werden vielfach aggressive Maßnahmen zur Umsatzsteigerung ergriffen, die oftmals mit einer Preisreduzierung einhergehen und damit zur weiteren Verschärfung der Wettbewerbssituation beitragen. ${ }^{14}$ Zudem ist auf Herstellerseite ebenfalls eine Konzentration festzustellen, die durch eine zunehmende Zahl von Markenakquisitionen ausgelöst wurde. ${ }^{15}$ Der damit verbundene Trend zu einer Oligopolisierung birgt gleichermaßen das Risiko einer noch zunehmenden Intensivierung der Konkurrenzauseinandersetzungen wie auch die Chance zur Stabilisierung der Wettbewerbssituation. ${ }^{16}$

kel als Instrumente des Wettbewerbs, Hrsg.: Dichtl, E., Eggers, W., München 1992, S. 201 sowie Mei-Folter, A., Barber, F., Händler als Markengestalter, Aufbau vertikaler Handelsmarken als Zukunftschance, in: LZ, Nr. 41, 15. Oktober 1993, S. J 14.

13 Vgl. Michael, B. M., Die Marke ist tot, Es lebe die Marke!, in: Markenartikel, H. 1, 1994, S. 22 ff.; GDI-Handelstrendletter 1/94, Gottlieb Duttweiler Institut, Rüschlikon 1994.

14 Vgl. Riesenbeck, H., Voss, W.-D., Marke oder Masse, in: Manager Magazin, H. 6, 1989 , S. $223 \mathrm{ff}$.

15 Vgl. Meffert, H., Herausforderungen an die Markenführung in den 90er Jahren, a.a.O., S. 265. Konzentrationstendenzen auf Herstellerseite werden vom Handel als wesentliche Bedrohung empfunden. Vgl. hierzu Dichtl, E., Müller, S., Herausforderungen für die Markenartikelindustrie an der Schwelle zu einem neuen Jahrzehnt, a.a.O., S. 24. Eine Übersicht ausgewählter Unternehmensakquisitionen im Markenartikelbereich findet sich bei Sander, M., Die Bestimmung und Steuerung des Wertes von Marken. Eine Analyse aus Sicht des Markeninhabers, Heidelberg 1994, S. 51. $\mathrm{Zu}$ einer Untersuchung der Erfolgsquoten von Akquisitionen vgl. Blex, W., Marchal, G., Risiken im Akquisitionsprozeß - Ein Überblick, in: BFuP, H. 2, 1990, S. $85 \mathrm{ff}$.

16 Zu Eigenschaften oligopolistischer Märkte vgl. Meffert, H., Marketing, Grundlagen der Absatzpolitik, 7. Aufl., Wiesbaden 1986, S. $315 \mathrm{ff}$. 
In der Literatur finden sich vielfältige Vorschläge, auf die skizzierten Entwicklungen mit einer Neuorientierung in der Markenpolitik zu reagieren. Diese reichen von einer "identitätsbezogenen Markenführung" über die "Stärkung der Markenkompetenz" bis hin zur "Gestaltung fraktaler Marken". Dabei werden mitunter bisherige Grundprinzipien der Markenpolitik, wie die Vermittlung eines zentralen Nutzenversprechens, eine eindeutige Positionierung oder die Kontinuität im Auftritt der Marke in Frage gestellt. ${ }^{17}$

Ohne Zweifel verdeutlichen solche Konzepte einerseits die Notwendigkeit, daß sich in einer Marke Ideen und Visionen widerspiegeln müssen, um den bestehenden Herausforderungen begegnen zu können. Andererseits tragen solche Vorschläge durch z. T. ungenaue und "diffuse" Begrifflichkeiten wenig zu einer systematischen und effizienten Planung in der Markenpolitik bei. Zur langfristigen Sicherung des Markenerfolges ist jedoch nicht nur die externe Wirkungseffizienz und Effektivität markenpolitischer Maßnahmen zu erhöhen, sondern ebenso die unternehmensinterne Planungseffizienz zu steigern. Um so wichtiger ist es für die Markenartikelhersteller, über Planungsinstrumente zu verfügen, die dazu beitragen, daß

- durch die Strukturierung der zugrundeliegenden Situation, der Ziele und Handlungsalternativen die Komplexität markenpolitischer Entscheidungen reduziert wird,

- die Handlungsalternativen unter Einsatz möglichst objektiver und interpersonal nachvollziehbarer Methoden bewertet werden können und sich damit das Risiko vermeidbarer Handlungsfehler verringert,

- durch die frühzeitige Antizipation von Entwicklungen rechtzeitig Maßnahmen ergriffen werden können, um auf Chancen und Risiken in geeigneter Form und mit einer ausreichenden Handlungsflexibilität ${ }^{18}$ zu reagieren.

Die Problembereiche der markenpolitischen Planung, verstanden als die Vorbereitung und das Treffen von Entscheidungen über die Führung von

17 Vgl. Meffert, H., Markenführung in der Bewährungsprobe, a.a.O., S. 479 sowie die dort angegebene Literatur.

18 Zum Begriff der Handlungsflexibilität vgl. Meffert, $H$., Größere Flexibilität als Unternehmungskonzept, in: ZfbF, H. 2, 1985, S. $121 \mathrm{ff}$. 
Marken unter der gedanklichen Vorwegnahme zukünftiger Ereignisse, ${ }^{19}$ lassen sich dem entscheidungsorientierten Ansatz folgend dabei in einem $\mathrm{Pa}$ radigma mit sechs zentralen Fragestellungen zusammenfassen:20

- Unter Berücksichtigung welcher markenpolitischen Ziele

- und Beachtung welcher situativen Faktoren

- sollen welche Markierungsstrategie

- und welche Markenpositionierungsstrategie gewählt,

- mit welcher Strategie gegenüber den Marktteilnehmern durchgesetzt und

- durch welche konkreten Maßnahmen umgesetzt werden?

Markenpolitische Ziele sind als Vorzugszustände zu kennzeichnen, die für die Führung einer Marke als erstrebenswert angesehen werden. Abgeleitet aus übergeordneten Unternehmens- und Marketingzielen ${ }^{21}$ stellen sie Sollgrößen dar, die es durch die Wahl geeigneter Handlungsalternativen über eine Abfolge von Zustandsänderungen (Trajektoriekonzept)22 zu erreichen gilt. Darüber hinaus ermöglicht erst die Festlegung von Inhalt, Ausmaß und Zeitbezug der Ziele, die Konsequenzen von Handlungsalternativen zu bewerten und in eine Reihenfolge der Vorziehenswürdigkeit zu bringen. ${ }^{23}$

19 Zum Begriff der Planung vgl. Koch, H., Integrierte Unternehmensplanung, Wiesbaden 1982, S. 3 ff. Eine ausführliche Begriffsdiskussion findet sich bei Voigt, K. I., Strategische Planung und Unsicherheit, Wiesbaden 1992, S. $6 \mathrm{ff}$.

20 Zum entscheidungsorientierten Ansatz siehe insbesondere Heinen, E., Zum Wissenschaftsprogramm der entscheidungsorientierten Betriebswirtschaftslehre, in: ZfB, H. 4, 1969, S. 207 ff.; Meffert, H., Marketing und strategische Unternehmensführung - ein wettbewerbsorientierter Kontingenzansatz, in: Strategische Unternehmensplanung, Hrsg.: Hahn, D., Taylor, B., 4. Aufl., Heidelberg, Wien 1986, S. 660 ff.; Rühli, E., Entscheidungsorientierter Ansatz und Allgemeine Betriebswirtschaftslehre, in: Die Betriebswirtschaftslehre im Spannungsfeld zwischen Generalisierung und Spezialisierung, Hrsg.: Kirsch, W.; Picot, A., Wiesbaden 1989, S. 99 ff.

21 Zu Marketingzielen vgl. Haedrich, G., Tomczak, T., Strategische Markenführung, Planung und Realisierung von Marketingstrategien für eingeführte Produkte, Bern, Stuttgart 1990, S. 84 ff.; Tolle, E., Steffenhagen, H., Kategorien des Markenerfolges und einschlägige Meßmethoden, in: Markenartikel, H. 8, 1994, S. $378 \mathrm{ff}$.

Zum Trajektoriekonzept vgl. Köhler, R., Beiträge zum Marketing-Management, 2. Aufl., München 1991, S. 35 f.

23 Vgl. Meffert, H., Unternehmensziele, in: Jahrbuch des Marketing, Hrsg.: Schöttle, K. M., Essen 1971, S. 23; Heinen, E., Grundlagen betriebswirtschaftlicher Entscheidungen, Das Zielsystem der Unternehmung, 3. Aufl., Wiesbaden 1976, S. 89 ff. 
Welche Handlungsalternative den höchsten Zielerreichungsgrad verspricht, ist abhängig von den jeweils geltenden situativen Faktoren. Diese beschreiben den für die Planung zugrundeliegenden Kontext, bei dem im allgemeinen zwischen einer internen und einer externen Unternehmenssituation differenziert wird. Während zur internen Situation Faktoren zählen, die unter der alleinigen Kontrolle des jeweiligen Unternehmens stehen, werden Faktoren der externen Situation vorrangig durch unternehmensfremde Umstände beeinflußt. ${ }^{24}$

Bei den Handlungsalternativen wird in aller Regel zwischen strategischen und operativen Entscheidungstatbeständen differenziert. Von einer strategischen Handlungsalternative bzw. Strategie kann gesprochen werden, wenn mit dieser ein bedingter, langfristig orientierter Verhaltensplan festgelegt wird. ${ }^{25}$ Es handelt sich also um zeitablaufbezogene Entscheidungen, die unter der Annahme einer bestimmten Entwicklung für das zukünftige Verhalten getroffen werden. Dabei soll hier eine Mehrebenenbetrachtung zugrunde gelegt werden. Demnach ist der Begriff der Strategie nicht auf eine übergeordnete Betrachtungs- und Entscheidungsebene beschränkt, bei der als Objekt ein Unternehmen oder Geschäftsfeld angenommen wird, sondern kann auch auf hierarchisch untergeordneten Ebenen, d. h. in Verbindung mit einer Marke oder einem einzelnen Produkt Anwendung finden. ${ }^{26}$

24 Der aus der Organisationstheorie stammende Kontingenz- bzw. situative Ansatz verfolgt in diesem Zusammenhang explizit das Forschungsziel, für die Planung relevante Kontextvariablen zu identifizieren und durch Klassifikation und Typologisierung geeignete "Situationscluster" zu bilden, um auf deren Basis zu situationsgerechten Empfehlungen für die Wahl der Handlungsalternative zu gelangen. Zum situativen Ansatz vgl. Meffert, $\mathrm{H}$., Marketingstrategien in unterschiedlichen Marktsituationen, in: Handbuch des Marketing, Anforderungen an Marketingkonzeptionen aus Wissenschaft und Praxis, Hrsg.: Bruhn, M., München 1989, S. 277 ff.; Kieser, A., Kubicek, H., Organisation, 3. Aufl., Berlin, New York 1992, S. 45 ff. Zu den Begriffen der Klassifikation und Typologisierung vgl. Knoblich, $H$., Die typologische Methode in der Betriebswirtschaftslehre, in: WiSt, H. 4, 1972, S. $141 \mathrm{ff}$.

25 Für eine ausführliche Diskussion des Strategiebegriffes vgl. Steffenhagen, $H_{\text {., }}$ Der Strategiebegriff in der Marketingplanung, Arbeitspapier des Instituts für Marketing der Universität Münster, Nr. 29, Münster 1982. Vgl. auch Müller, N., Marketingstrategien in High-Tech-Märkten, Typologisierung, Ausgestaltungsformen und Einflußfaktoren auf der Grundlage strategischer Gruppen, Diss., Münster 1994, S. 11 ff. Eine Übersicht der Elemente unterschiedlicher Definitionen von Strategien findet sich bei Voigt, K. I., Strategische Planung und Unsicherheit, a.a.O., S. $216 \mathrm{ff}$.

Wenngleich Strategien mit dem Übergang auf höhere Betrachtungsebenen an Komplexität und Relevanz für das Gesamtunternehmen gewinnen, so ist das 
In der Literatur zur Marketingplanung, d. h. der marktbezogenen Entscheidungsfindung, wird eine Vielzahl unterschiedlicher Strategien und Ansätze zu deren Systematisierung aufgeführt. ${ }^{27}$ Im Hinblick auf markenpolitische Entscheidungstatbestände bietet es sich an, zwischen Strategien der Markierung, der Markenpositionierung und der Markendurchsetzung zu unterscheiden.

Strategische Entscheidungstatbestände der Markierung beziehen sich auf zwei verschiedene Dimensionen. ${ }^{28}$ Zum einen ist festzulegen, wie viele Produkte unter einer Marke geführt werden sollen. Abhängig davon, ob jeweils nur eine, mehrere oder alle Produkte des Herstellers unter einer Marke gefaßt werden, können die Produkt-, Familien- und Dachmarkenstrategie voneinander unterschieden werden. ${ }^{29}$ Zum anderen ist die Anzahl der Marken pro Markt zu bestimmen. Wird vom Hersteller auf jedem Markt lediglich eine Marke geführt, handelt es sich um eine Einmarkenstrategie. Bei gleichzeitigem Angebot mehrerer Marken auf demselben Markt kann von einer Mehrmarkenstrategie gesprochen werden. In diesem Fall ist ferner die Beziehung der Marken untereinander näher zu beschreiben, die in einem substitutiven oder komplementären Verhältnis zueinander stehen können. Während substitutive Marken miteinander

Grundprinzip der Strategieformulierung auf jeder Ebene gleich. Vgl. dazu Steffenhagen, H., Marketing, Eine Einführung, 2. Aufl., Stuttgart u.a. 1991, S. 119 f. Zum Begriff des strategischen Geschäftsfeldes vgl. Kreilkamp, E., Strategisches Management und Marketing, Berlin, New York 1987, S. 316 ff.; Hinterhuber, H. H., Strategische Unternehmensführung, II. Strategisches Handeln, 5. Aufl., Berlin, New York 1992, S. 123 ff.; Meffert, H., Marketing-Management, a.a.O., S. $41 \mathrm{ff}$.

Bei der Systematisierung von Strategien lassen sich Partial- und integrative Ansätze voneinander unterscheiden. Während Partialansätze jeweils nur einen Ausschnitt des strategischen Entscheidungsproblems erfassen, sind integrative Ansätze bemüht, das gesamte Entscheidungsspektrum der Planung von Strategien abzudecken. Zu einer zusammenfassenden Darstellung von Partial- und integrativen Ansätzen vgl. Meffert, H., Marketing-Management, a.a.O., S. 109 ff.

Vgl. Kapferer, J.-N., Die Marke - Kapital des Unternehmens, Landsberg/Lech 1992 , S. $157 \mathrm{ff}$.

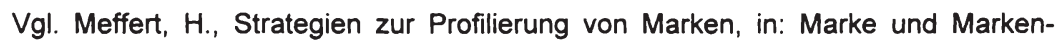
artikel als Instrumente des Wettbewerbs, Hrsg.: Dichtl, E., Eggers, W., München 1992, S. 137 ff.; Müller, G.-M., Dachmarkenstrategie, in: Markenartikel, H. 4, 1994, S. $142 \mathrm{ff}$. 
konkurrieren, ergänzen sich komplementäre Marken im Hinblick auf die verschiedenen Anforderungen der Konsumenten. ${ }^{30}$

Die Strategien der Markenpositionierung beziehen sich auf die Gestaltung der Marke und ihrer wesensbestimmenden Eigenschaften. Diese drücken sich zum einen in der physischen Beschaffenheit des Produktes aus. Zum anderen zeigen sie sich in immateriellen, nur durch die Kommunikation vermittelbaren Merkmalen. Ziel der Markenpositionierungsstrategie ist es, durch die Herausstellung bestimmter Markeneigenschaften sowohl eine dominierende Stellung in der Psyche des Konsumenten als auch eine Differenzierung gegenüber den Wettbewerbern zu erreichen. ${ }^{31}$ Dazu wird in einem mehrdimensionalen Raum, dessen Dimensionen vom Konsumenten wahrgenommene und als wichtig erachtete Eigenschaften darstellen, eine Position zu besetzen versucht, die möglichst gut dessen Idealvorstellung entspricht und zugleich in mindestens einer Dimension eine dauerhafte Überlegenheit gegenüber der Konkurrenz bietet (Wettbewerbsvorteil). ${ }^{32}$

Abhängig vom jeweiligen Aggregationsgrad reichen die Positionierungsentscheidungen von der Einordnung einer Marke auf strategische Grunddimensionen bis hin zur Festlegung differenzierter Eigenschaftsausprägun-

30 So kann beispielsweise aus Unternehmens- und Markenakquisitionen heraus die Situation entstehen, daß zwei ähnlich positionierte und vom Konsumenten als Substitute betrachtete Marken parallel auf dem gleichen Markt geführt werden und damit in unmittelbarer Konkurrenzbeziehung zueinander stehen. Ebenso ist jedoch möglich, daß ein Unternehmen zur besseren Entsprechung unterschiedlicher Endabnehmerbedürfnisse oder zur Absicherung der Preisposition seiner Erstmarke mit weiteren, sogenannten Zweitmarken auf dem Markt vertreten ist. Vgl. hierzu ausführlich Höhl, J., Zweitmarken - Ein entscheidungsorientierter Ansatz aus der Sicht des Markenartikelherstellers bei Gütern des täglichen Bedarfs, Gelsenkirchen 1982.

31 Vgl. Rieger, B., Die Kunst sich zwischen die Stühle zu plazieren, Plazieren - Positionieren - Segmentieren, in: asw, H. 10, 1984, S. 156 ff.; Meffert, H., Markenstrategien als Waffe im Wettbewerb, in: Handbuch strategische Führung, Hrsg.: Henzler, H. A., Wiesbaden 1988, S. 585 ff.; Aaker, D. A., Management des Markenwertes, Frankfurt/Main, New York 1992, S. $140 \mathrm{ff}$.

32 Vgl. Freter, M., Marktsegmentierung, Stuttgart 1983, S. 34 ff.; Pepels, W., Positionierungsstrategien, in: Markenartikel, H. 4, 1994, S. 423. Zum Wettbewerbsvorteil

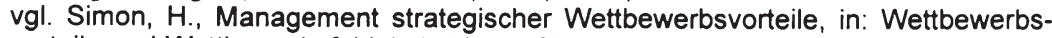
vorteile und Wettbewerbsfähigkeit, Hrsg.: Simon, H., Stuttgart 1988, S. $1 \mathrm{ff}$;; Porter, M., Wettbewerbsvorteile, Frankfurt/Main 1992, S. 177 ff. 
gen. ${ }^{33}$ Während letztere nur in Abhängigkeit von der konkreten Produktart gekennzeichnet werden können, haben sich in der Literatur als zentrale, allgemeingültige Grunddimensionen ${ }^{34}$ die Merkmale der Innovation, der Qualität, der Produktprogrammabdeckung, des emotionalen Zusatznutzens sowie des Preises herauskristallisiert. ${ }^{35}$ Zur vereinfachten Beschreibung der gewählten Position konzentriert man sich dabei häufig auf diejenige Dimension, in der ein Wettbewerbsvorteil angestrebt wird. Steht der Preis im Vordergrund, so kann von einer preisorientierten Markenpositionierungsstrategie gesprochen werden. Die Fokussierung auf eine oder die Kombination

33 Zur Ermittlung von Eigenschaftsdimensionen und wahrgenommenen Markenpositionen sowie zur Unterstützung der Positionierungsentscheidung ist eine Reihe methodischer Hilfsmittel entwickelt worden. Vgl. hierzu z. B. Brockhoff, K., Produktpolitik, 3. Aufl., Stuttgart, Jena 1993, S. 124 ff.; Reutterer, T., Analytische Modelle zur Unterstützung von Positionierungsentscheidungen - Versuch einer Bestandsaufnahme, in: der markt, H. 2, 1994, S. $88 \mathrm{ff}$.

34 Vgl. hierzu insbesondere die empirische Untersuchung von Bolz, J., Wettbewerbsorientierte Standardisierung der internationalen Marktbearbeitung, Eine empirische Analyse in europäischen Schlüsselmärkten, Darmstadt 1992, S. 35 ff. In den letzten Jahren hat zudem die ökologische Dimension als Wettbewerbsfaktor an Bedeutung gewonnen. Vgl. Kirchgeorg, M., OKologieorientiertes Unternehmensverhalten, Wiesbaden 1990, S. 109; o. V., Pull-Effekt à la Öko, in: asw, H. 11, 1991, S. 60 ff.; Wicke, L., Der Umweltschutz als Herausforderung und Chance für den Markenartikel, in: Marke und Markenartikel als Instrumente des Wettbewerbs, Hrsg.: Dichtl, E., Eggers, W., München 1992, S. 157 ff.

Eine innovationsorientierte Positionierung ist durch eine ständige Weiterentwicklung der Marke zur Aufrechterhaltung einer Pionierposition im Markt gekennzeichnet. Die Qualitätsorientierung einer Marke zeigt sich in einem hohen Erfüllungsgrad der vom Endabnehmer an die Leistung gestellten Erwartungen. Hierbei werden Eigenschaften wie Gebrauchsnutzen, Haltbarkeit oder Zuverlässigkeit vom Endabnehmer zur Beurteilung der Qualität herangezogen. Unter der Programmabdeckung ist die Anzahl der Produktvarianten einer Marke zu verstehen, mit der differenzierten Wünschen des Endabnehmers entsprochen wird. Der emotionale Zusatznutzen einer Marke tritt z. B. in Form der Vermittlung eines besonderen Prestiges, Lebensstils oder bestimmter Erlebniswelten auf. Zu den Grunddimensionen vgl. Meffert, $H$., Marketing-Management, a.a.O., S. 126 ff. Zum emotionalen Zusatznutzen vgl. insbesondere auch Konert, F.-J., Vermittlung emotionaler Erlebniswerte - Eine Positionierungs- und Profilierungsstrategie für Markenartikel auf gesättigten Märkten, in: Markenartikel, H. 12, 1986, S. 576 ff.; Kroeber-Riel, W., Konsumentenverhalten, 5. Aufl., München 1992, S. 112 ff.; Meffert, H., Entscheidungsorientierter Ansatz der Markenpolitik, in: Handbuch Markenartikel, Hrsg.: Bruhn, M., Stuttgart, 1994, S. 173 ff.; Zur Vermittlung von Erlebniswelten vgl. Weinberg, P., Erlebnismarketing, München 1992. 
mehrerer anderer Dimensionen kann als leistungsorientierte Markenpositionierungsstrategie bezeichnet werden. ${ }^{36}$

Markierungs- und Markenpositionierungsstrategien stellen Grundsatzstrategien dar. Indem sie die Komplexität der Planung in der Markenpolitik auf Basisentscheidungen zu reduzieren versuchen, tragen sie zur Systematisierung und Beschreibung der Handlungsalternativen sowie der mit diesen verbundenen Vor- und Nachteile bei. Gleichzeitig wird mit Strategien aber auch eine "Lenkleistung" verbunden. Strategien sollen den grundsätzlichen Weg zur Erreichung der gesetzten Ziele aufzeigen und damit rahmengebend für hierarchisch untergeordnete, operative Entscheidungen auf der Ebene der Marketinginstrumente ${ }^{37}$ wirken. ${ }^{38}$

Vergegenwärtigt man sich jedoch den hohen Komplexitätsgrad, der sich aus der nahezu unbegrenzten Anzahl an Kombinationsmöglichkeiten ${ }^{39}$ von Marketinginstrumenten ergibt, so wird deutlich, daß mit der Vorgabe von Grundsatzstrategien die Breite des Entscheidungskanals nur unzureichend eingeschränkt wird. Eine weitere Komplexitätsreduktion läßt sich durch die Einführung von Instrumentalstrategien erreichen. Diese sind als Bündel zusammengehöriger Marketinginstrumente zu verstehen, die im Sinne der grundsatzstrategischen Entscheidungen den Schwerpunkt für den Instrumenteeinsatz definieren. Sie bilden damit einen gedanklichen Rahmen, der die auf

36 Häufig wird anstelle des Begriffs der leistungsorientierten Strategie auch von einer Qualitătsführerschaft, Differenzierungsstrategie oder Präferenzstrategie gesprochen. Vgl. Porter, M. E., Wettbewerbsstrategie, Frankurt/Main 1992, S. 62 ff; Becker, J., Marketing-Konzeption, Grundlagen des strategischen MarketingManagements, 5. Aufl., München 1993, S. $154 \mathrm{ff}$.

37 In den Marketinginstrumenten kommen die marktgerichteten Aktivitäten des Herstellers zum Ausdruck. Zum Begriff des Marketinginstrumentes vgl. Meffert, H., Marketing, a.a.O., S. $114 \mathrm{ff}$.

38 Vgl. Haedrich, G., Tomczak, T., Strategische Markenführung, a.a.O., S. 145; Becker, J., Marketing-Konzeption, a.a.O., S. $113 \mathrm{ff}$.

39 So resultieren z. B. allein aus 5 Instrumenten mit jeweils nur 6 diskreten Ausprägungen bereits $5^{6}=15625$ Kombinationsmöglichkeiten. Berücksichtigt man zudem, daß mit denselben Instrumenten eine Vielzahl qualitativ variierender Varianten realisierbar ist, erstrecken sich die Kombinationsmöglichkeiten ins Unendliche. 
operativer Ebene erfolgende Planung des Marketinginstrumenteeinsatzes und der daraus abzuleitenden konkreten Maßnahmen erleichtert. 40

Vor dem Hintergrund der skizzierten Marktentwicklungen wird deutlich, daß sich insbesondere das Spannungsfeld von Hersteller, Handel und Endabnehmer intensiviert hat. Aus Sicht des Herstellers ist daher vor allem die Frage des aufeinander abgestimmten Einsatzes von Marketinginstrumenten bei der Bearbeitung von Handel und Konsument ${ }^{41}$ zur Durchsetzung einer Marke von Bedeutung. Die erfolgreiche Markendurchsetzung, verstanden als die Schaffung und Aufrechterhaltung der Nachfrage des Endabnehmers sowie der angestrebten Präsenz und Förderung der Marke im Handel, setzt die gleichzeitige Berücksichtigung der Interessen beider Marktteilnehmer und der zwischen innen bestehenden Beziehungen voraus.

Aus dieser Perspektive bietet es sich an, solche Instrumentalstrategien zu formulieren, die einen Orientierungsrahmen für den integrierten handelsund endabnehmerorientierten Instrumenteeinsatz liefern. Diese werden im folgenden als Markendurchsetzungsstrategien bezeichnet, deren Ausprägungen in Abhängigkeit von der gewünschten Schwerpunktsetzung des Marketinginstrumentariums zu beschreiben sind. Bei einer eher handelsorientierten Ausrichtung wird von einer Push-Orientierung gesprochen, während der stärker endabnehmerorientierte Instrumenteeinsatz einer PullOrientierung entspricht.

Die besondere Relevanz der Frage nach einer geeigneten Markendurchsetzungsstrategie ergibt sich insbesondere auch aus den weitreichenden Konsequenzen, die mit dieser Entscheidung verbunden sein können. Je stärker ein Hersteller bei der Durchsetzung seiner Marke die Unterstützung des Handels in Anspruch nimmt, um so größer sind die von den Absatzmitt-

40 Vgl. Haedrich, G., Gussek, F., Tomczak, T., Instrumentelle Strategiemodelle als Komponenten im Marketingplanungsprozeß, in: DBW, H. 2, S. 208 f. Der hier gewählte Begriff der Instrumentalstrategie entspricht dem von diesen Autoren verwendeten Begriff des instrumentellen Strategiemodells. Vgl. hierzu auch Becker, J., Marketing-Konzeption, a.a.O., S. 468; Meffert, H., Marketing-Management, a.a.O., S. $24 \mathrm{ff}$.

41 Als Endabnehmer werden in der vorliegenden Arbeit nur Konsumenten und keine Organisationen betrachtet, so daß die Begriffe Endabnehmer und Konsument synonym verwendet werden können. 
lern 42 geforderten Zugeständnisse und der handelsorientierte Ressourceneinsatz. Aufgrund von Beschränkungen der verfügbaren Ressourcen verringern sich damit gleichzeitig die Handlungsalternativen des Herstellers zur direkten Einflußnahme auf den Endabnehmer. Mit zunehmender Dauer gerät der Hersteller infolgedessen in eine wachsende Abhängigkeit vom Handel, die in der Literatur auch als "destruktiver Zyklus distributiver Phänomene" bezeichnet wird. 43

Trotz dieser Erkenntnis kommen Tomczak und Gussek in einer Untersuchung der deutschen Konsumgüterindustrie zu dem Ergebnis, daß bei $46 \%$ der von innen befragten Unternehmen eine deutliche Dominanz einer pushorientierten Markendurchsetzung festzustellen ist. ${ }^{44}$ Auch die Organisationsformen der Markenartikelhersteller spiegeln eine wachsende Push-Orientierung wider. Während das Produkt-Management ${ }^{45}$ mit seinem primär auf den Endabnehmer ausgerichteten Marketing an Bedeutung verliert, wird die Stellung des handelsorientierten Vertriebsbereiches innerhalb der Unternehmen ausgebaut. 46

Als Absatzmittler werden im allgemeinen rechtlich und wirtschaftlich selbständige Unternehmen bezeichnet, die Ware auf eigenes Risiko kaufen, um sie weiter zu veräußern. Vgl. Ahlert, D., Distributionspolitik, a.a.O., S. 47. Die Begriffe Handel, Absatzmittler (Plural) und Händler (Plural) werden hier synonym verwendet.

Vgl. Tomczak, T., Gussek, F., Handelsorientierte Anreizsysteme der Konsumgüterindustrie, in: ZfB, H. 7, 1992, S. 785 f. sowie die dort angegebene Literatur; vgl. auch Achenbaum, A. A., Mitchel, F. K., Pulling away from push marketing, in: Harvard Business Review, No. 3, 1987, S. $38 \mathrm{ff}$.

Vgl. Tomczak, T., Gussek, F., Handelsorientierte Anreizsysteme der Konsumgüterindustrie, a.a.O., S. $797 \mathrm{ff}$. Der Untersuchung lag eine empirische Befragung von 206 Marken der deutschen Konsumgüterindustrie zugrunde. Vgl. auch Gussek, F., Erfolg in der strategischen Markenführung, Wiesbaden 1992, S. 308. Dabei wurde eine im folgenden noch zu erläuternde zielgruppenbezogene Abgrenzung der Marketinginstrumente vorgenommen.

Zu den Aufgaben des Produktmanagement vgl. Meffert, $\mathrm{H}$., Produktmanagement: Funktionen und organisatorische Eingliederung, in: WISU, 1979, S. 68 ff. u. $120 \mathrm{ff}$; Haedrich, G., Tomczak, T., Strategische Markenführung, a.a.O., S. $31 \mathrm{ff}$.

Vgl. Zentes, J., Trade-Marketing, Eine neue Dimension in den Hersteller-HändlerBeziehungen, in: Marketing ZFP, H. 4, 1989, S. 226. Vgl. auch Kemmna, H., Das Key Account Management der 90er Jahre, in: Thexis, H. 1, 1990, S. 32 f.; Voß, W.D., Marketing in der Sackgasse? - Ansatzpunkte zur Weiterentwicklung des Marketing in der Konsumgüterindustrie, in: Marktorientierte Unternehmensführung im Umbruch, Effizienz und Flexibilität als Herausforderungen des Marketing, Hrsg. Bruhn, M., Meffert, H., Wehrle, F., Stuttgart 1994, S. 122. 
Diese starke Ausrichtung der Markenartikelhersteller auf den Handel ist sicherlich zum einen auf dessen zunehmende Machtposition zurückzuführen. Zum anderen kann aber auch vermutet werden, daß bei steigender Konkurrenzintensität und abnehmender Wirkung endabnehmergerichteter Kommunikationsaktivitäten die Push-Orientierung zu einem höheren finanziellen Markenerfolg führt als eine pull-orientierte Markendurchsetzung. Festzuhalten bleibt in jedem Fall, daß die Frage des Push und Pull bei der Markendurchsetzung von zentraler Bedeutung für die Planung im Rahmen der Markenpolitik ist.

Abbildung 1 zeigt den skizzierten Planungsprozeß zusammenfassend auf. Dabei sind die bisher beschriebenen Planungsstufen um die Phasen der Informationsbeschaffung, der Implementierung von Maßnahmen und der sich anschließenden Kontrolle ergänzt. Einem erweiterten Verständnis des Planungsbegriffes zufolge sind diese Phasen insofern im Gesamtzusammenhang zu berücksichtigen, als es sich bei der Planung im Rahmen der Markenpolitik um einen kontinuierlichen Prozeß der Führung einer Marke handelt. Die Planungsschritte sind über Rückkopplungsprozesse miteinander verbunden, so daß spätere Informationen, Entwicklungen und Erfahrungen wiederum in die verschiedenen Prozeßstufen der Planung einfließen können. 47

\section{Push und Pull als Ausprägungsformen von Markendurchsetzungs- strategien}

Die Begriffe des Push und Pull werden in der Literatur zur Markenpolitik unterschiedlich weit interpretiert und mit verschiedener Akzentsetzung verwendet. Voneinander unterscheiden lassen sich die

- systembezogene,

- zielbezogene,

- objektbezogene,

- strategische und

- instrumentelle Begriffsfassung.

47 Vgl. zum Prozeß der Planung Haedrich, G., Tomczak, T., Strategische Markenführung, a.a.O., S. 32; Meffert, H., Marketing-Management, a.a.O., S. 25 ff. 


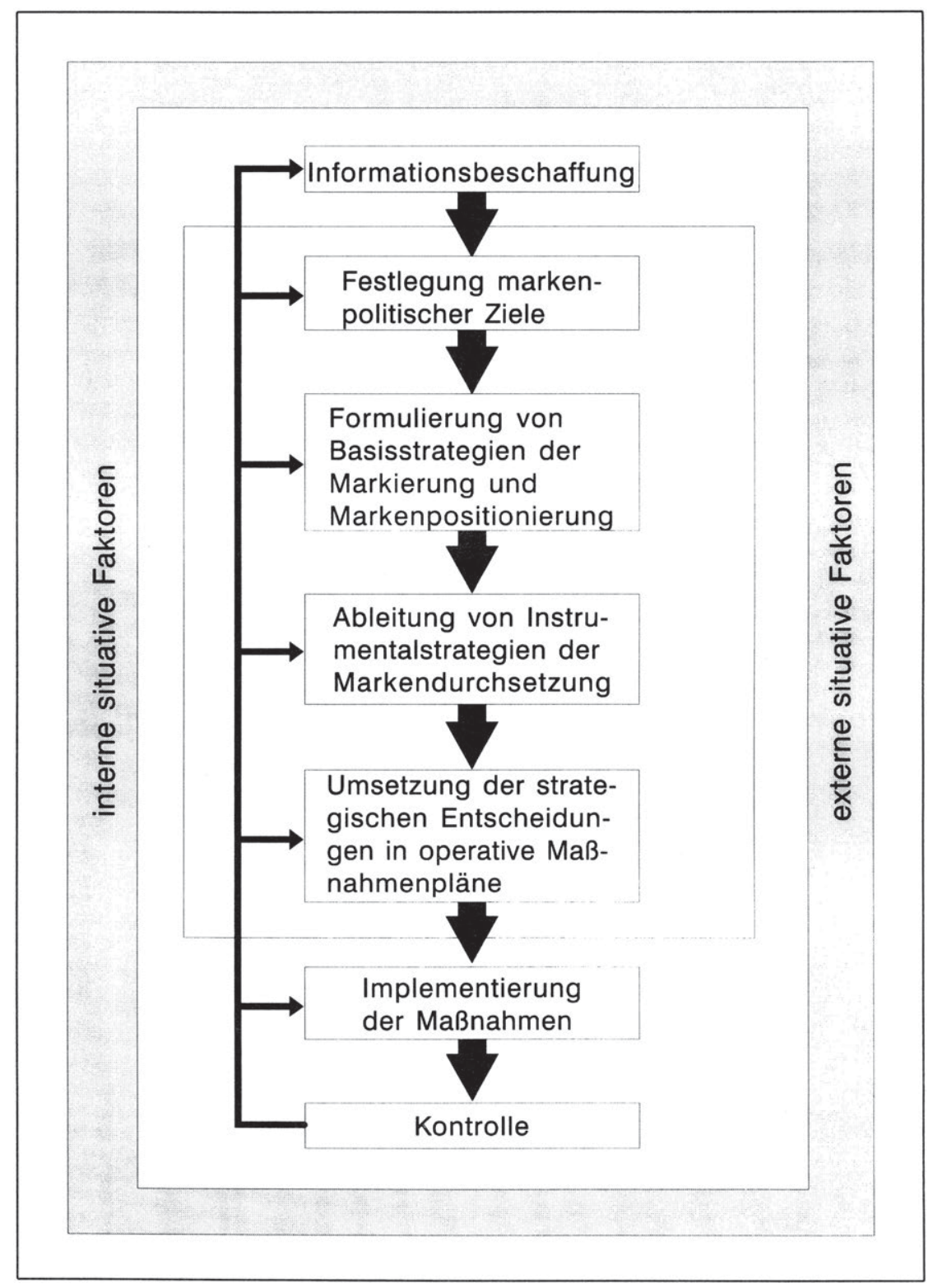

Abb. 1: Prozeß der Planung in der Markenpolitik 
Im Sinne eines systembezogenen Begriffsverständnisses erfolgt unter der Bezeichnung des Push und Pull zumeist eine Beschreibung mehrstufiger Wirkungszusammenhänge im vertikalen Marktsystem ${ }^{48}$ von Hersteller, Handel und Endabnehmer, die durch entsprechende Stimuli und Reaktionen gekennzeichnet sind (vgl. Abbildung 2). 49

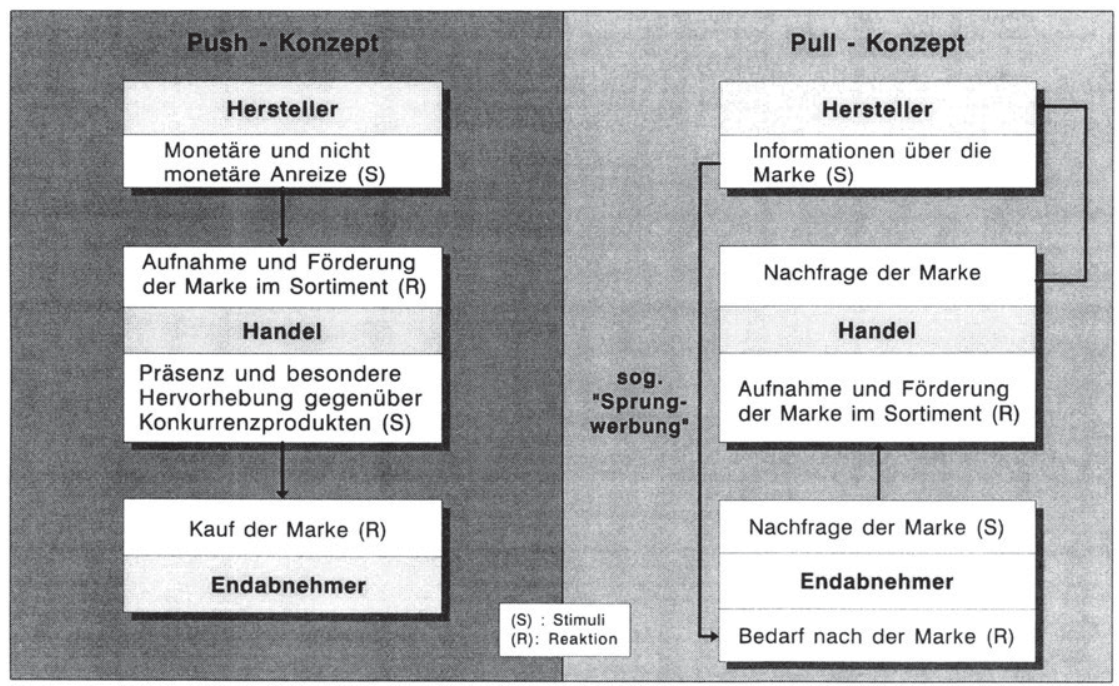

Abb. 2: Push und Pull als Konzepte zur Beschreibung mehrstufiger Zusammenhänge im vertikalen Marktsystem von Hersteller, Handel und Endabnehmer

Beim Push-Konzept werden dem Handel vom Hersteller bestimmte Anreize geboten, die diesen dazu veranlassen sollen, die Marke in das Sortiment

Als vertikales Marktsystem können allgemein solche Marktkonstellationen bezeichnet werden, bei denen der Absatz einer Leistung nicht direkt vom Hersteller an den Endabnehmer erfolgt, sondern mindestens eine eigenverantwortliche Marktstufe zwischengeschaltet ist. Vgl. Kunkel, R., Vertikales Marketing im Herstellerbereich, Bestimmungsfaktoren und Gestaltungselemente stufenübergreifender MarketingKonzeptionen, München 1977, S. 20 sowie Florenz, der nach einer ausführlichen Diskussion zu dem Ergebnis kommt, daß nicht die rechtliche Eigenständigkeit, sondern lediglich die Eigenverantwortlichkeit als Eigenschaft dieser Marktstufe erforderlich ist. Vgl. Florenz, P. J., Konzept des vertikalen Marketing, Entwicklung und Darstellung am Beispiel der deutschen Automobilwirtschaft, Diss., Köln 1991, S. 27. 
aufzunehmen und zu fördern. Durch die Präsenz der Marke im Handel und inre besondere Hervorhebung im Vergleich zu Konkurrenzprodukten kommt es zum Kauf durch den Endabnehmer. Im Rahmen des Pull-Konzeptes wird der Konsument dagegen direkt durch den Hersteller angesprochen. Der hierdurch angeregte Bedarf führt zur aktiven Nachfrage des Endabnehmers beim Handel, der sich seinerseits dadurch veranlaßt sieht, die Marke im Sortiment zu führen. 50

In einer zielbezogenen Interpretation finden sich die Begriffe des Push und Pull zur Kennzeichnung der Angebotsstärke einer Marke im Handel (Push-Stärke) sowie der Stärke ihrer Nachfrage durch den Endabnehmer (Pull-Stärke). Dieses Begriffsverständnis geht im wesentlichen auf eine jüngere Arbeit von Olver und Farris zurück, die darüber hinaus vorschlagen, hierbei jeweils zwei Dimensionen zu unterscheiden, die sie mit Breite (breadth) und Tiefe (depth) des Push bzw. Pull bezeichnen. ${ }^{51}$ Die Breite des Push entspricht dem Distributionsgrad52 der Marke, während sich dessen Tiefe in der "in-store-attractiveness" ausdrückt. Letztere zeigt die relative Stärke, mit der eine Marke im Vergleich zu Konkurrenzprodukten innerhalb der Einkaufsstätte präsentiert wird (z. B. Anteil am Regalplatz oder Umfang der Werbung am Point of Sale). ${ }^{53}$ Die Breite des Pull drückt sich in der "unmodified preference" aus, d. h. dem vom Handelseinfluß bereinigten Umfang der Nachfrage. 54 Die Tiefe wird anhand der "wahren Markentreue"

50 Diese Konzepte lassen sich grundsätzlich auch auf vertikale Systeme der Konstellation "Zulieferer-Weiterverarbeiter-Handel-Endabnehmer" übertragen. Dabei findet in der Literatur vor allem die Frage nach der Realisierbarkeit eines Pull-Konzeptes für Zulieferer besonderes Interesse. Vgl. Engelhardt, W. H., Mehrstufige Absatzstrategien, in: ZfbF-Kontaktstudium, 1976, S. 175 ff.; Kunkel, R., Vertikales Marketing im Herstellerbereich, a.a.O.; Rudolph, M., Mehrstufiges Marketing für Einsatzstoffe, Anwendungsvoraussetzungen und Strategietypen, Frankfurt/Main u. a. 1989.

51 Vgl. hierzu und im folgenden Olver, J. M, Farris, P. W., Push and Pull: A One-Two Punch for Packaged Products, in: Sloan Management Review, Fall 1989, S. $53 \mathrm{ff}$.

52 Zum Distributionsgrad vgl. Kap. B. 2.13 dieser Arbeit.

53 Zur Operationalisierung der "in-store-attractiveness" wird ein Index empfohlen, dessen Variablen die für die Marke relevanten Aktivitäten des Handels in der Verkaufsstätte umfassen. Vgl. auch Farris, P., Olver, J., De Kluyver, C., The Relationship between Distribution and Market Share, in: Marketing Science, No. 2, Spring 1989, S. $110 \mathrm{f}$.

54 Olver und Farris beschreiben dieses Vorgehen wie folgt: "Breadth of Pull has been measured as share of preference in a fair choice situation - one in which all brands 
beschrieben, die sich nach Olver und Farris darin äußert, daß Endabnehmer bei Nichtverfügbarkeit einer Marke den Kauf zeitlich verschieben oder zu einer anderen Einkaufsstätte wechseln. 55

Aus der Angebots- und Nachfragestärke läßt sich unmittelbar ein objektbezogenes Begriffsverständnis ableiten, bei dem mit Push und Pull bestimmte Absatzeigenschaften der Marke ausgedrückt werden. Als PullMarken können solche beschrieben werden, die sich durch eine hohe Nachfragestärke seitens der Endabnehmer auszeichnen. Diese Marken werden vom Konsumenten im Handel verlangt, d. h. der Absatz erfolgt aufgrund einer starken Präferenz der Endabnehmer gegenüber der Marke. Im Gegensatz dazu sind Push-Marken dadurch gekennzeichnet, daß eine solche Nachfragestärke fehlt. Der Absatz einer solchen Marke wird im wesentlichen durch den Angebotsdruck geschaffen und aufrechterhalten, der im Handel auf den Endabnehmer wirkt.

Bei der strategiebezogenen Verwendung dienen die Begriffe des Push und Pull in der Literatur im allgemeinen zur Kennzeichnung von Strategien der Absatzmittlerakquisition und Regalplatzsicherung. ${ }^{56}$ Diese stark auf den Absatzmittler bezogene Sichtweise des Push und Pull erklärt sich aus dem

enjoy equal distribution breadth and depth". Vgl. Olver, J. M, Farris, P. W., Push and Pull, a.a.O., S. 55.

55 Diese Größe wird als "resistance to compromise" bezeichnet. Olver und Farris versuchen hiermit, die "wahre Markentreue" vom gewohnheitsmäßigen Kaufverhalten abzugrenzen. Bei letzterem würde der Endabnehmer sofort ein alternatives Produkt wählen, falls die ursprünglich zu kaufen beabsichtigte Marke nicht verfügbar ist. Vgl. Olver, J. M, Farris, P. W., Push and Pull, a.a.O., S. 55. Vgl. auch Day, G. S., Buyer Attitudes and Brand Choice Behavior, New York, London 1970, S. 66 f. Für eine ausführliche Diskussion der Ausprägungsformen von Markentreue vgl. Nolte, $\mathrm{H}$., Die Markentreue im Konsumgüterbereich, Bochum 1976, S. 10 ff.

56 Zu den Strategien der Absatzmittlerakquisition und Regalplatzsicherung vgl. Ahlert, D., Distributionspolitik, a.a.O., S. 58 ff. Der Begriff des Regalplatzes ist als Ausdruck für den Umfang jener Leistungen des Handels zu verstehen, durch die potentielle Käufer mit dem Produkt konfrontiert werden. Vgl. Cairns, J. P., Suppliers, Retailers and Shelf Space, in: Journal of Marketing, No. 3, 1962, S. $34 \mathrm{ff}$. In der Literatur besteht allerdings keine einheitliche Meinung darüber, welche Aktivitäten des Handels genau dem Regalplatz zuzurechnen sind. Hansen nimmt hier z. B. eine Erweiterung von der "Unterbringung der Produkte auf händlerischen Verkaufsflächen" zur "Präsentation des Laden-Werbematerials" vor. Vgl. Hansen, P., Die handelsgerichtete Absatzpolitik der Hersteller im Wettbewerb um den Regalplatz, Berlin 1972, S. 93 f. sowie S. 186 ff. 
handelsorientierten Fokus des Marketing in den 70er Jahren.57 Die wachsende Nachfragemacht des Handels durch seine Gate-Keeper-Funktion und die Knappheit des Regalplatzes lenkten in dieser Entwicklungsperiode des Marketing die Interessen verstärkt auf Aspekte der Beeinflussung von Absatzmittlern. Mit Push und Pull werden in diesem Zusammenhang die beiden Alternativen des Herstellers ausgedrückt, seine Produkte entweder durch einen direkten Druck auf den Handel in die Regale "hineinzupressen" oder aber einen auf den Handel wirkenden "Sog" durch die Nachfrage der Endabnehmer zu erzeugen.

In der instrumentellen Begriffsfassung wird mit Push und Pull die Ausrichtung des eingesetzten Marketinginstrumentariums beschrieben. Dabei lassen sich grundsätzlich zwei Ansätze voneinander unterscheiden, nach denen die Einordnung eines Marketinginstrumentes als Push- oder PullInstrument vorgenommen werden kann. Die Abgrenzung kann sowohl zielgruppen- als auch wirkungsbezogen erfolgen.

Bei einer zielgruppenbezogenen Abgrenzung erfolgt die Unterscheidung von Push- und Pull-Instrumenten danach, ob es sich um handels- oder endabnehmergerichtete Aktivitäten des Herstellers handelt. ${ }^{8}$ Während mit

$57 \mathrm{Zu}$ den Entwicklungsstufen des Marketing vgl. Meffert, H., Entwicklungslinien des Marketing - Akzente der marktorientierten Führung in den 90er Jahren, in: Jahrbuch des Marketing, Hrsg.: Schöttle, K. M., 5. Aufl., Essen 1990, S. 12 ff. Zu grundlegenden Arbeiten dieser Zeit, die sich mit der Beeinflussung des Handels auseinandersetzen, vgl. Hansen, P., Die handelsgerichtete Absatzpolitik der Hersteller im Wettbewerb um den Regalplatz, a.a.O.; Dingeldey, K., Herstellermarketing im Wettbewerb um den Handel, Berlin 1975. Eine weitere Fundierung der Hersteller-Handelsbeziehungen lieferten in dieser Phase vor allem Arbeiten, die durch Ziele, Rollen, Macht und Kommunikation diese Beziehung zu erklären versuchen. Hier sind insbesondere zu nennen: Steffenhagen, H., Konflikt und Kooperation in Absatzkanälen, Ein Beitrag zur verhaltensorientierten Marketingtheorie, in: Schriftenreihe Unternehmensführung und Marketing, Bd. 5, Hrsg.: Meffert, H., Wiesbaden 1975; Kümpers, U. A., Marketingführerschaft, Eine Verhaltenswissenschaftliche Analyse des vertikalen Marketing, Diss., Münster 1976; Thies, G., Vertikales Marketing, Marktstrategische Partnerschaft zwischen Industrie und Handel, Berlin, New York 1976.

58 Eine solche Abgrenzung wird z. B. von Gussek vorgenommen. Dieser bezeichnet ein Marketing-Mix, das "quasi beim Handel stehenbleibt" als handelsorientiertes Teilmix mit Push-Bestrebungen, während alle anderen Instrumente dem Endabnehmer-Mix und damit dem Pull-Konzept angehören. Vgl. Gussek, F., Erfolg in der strategischen Markenführung, a.a.O, S. $306 \mathrm{ff}$. 
ersteren eine unmittelbare Reaktion des Handels herbeigeführt werden soll, sind letztere auf die direkte Beeinflussung des Endabnehmers ausgerichtet.

Die Problematik einer solchen Abgrenzung läßt sich am Beispiel des Instrumentes der Verkaufsförderung verdeutlichen. ${ }^{59}$ Diese kann zum einen handelsgerichtete Maßnahmen, wie z. B. die Gewährung von Sonderrabatten, umfassen, die den Handel zu einer stärkeren Förderung der Marke veranlassen sollen. Zum anderen kann die Verkaufsförderung aber auch endabnehmergerichtet eingesetzt werden. Ein Beispiel hierfür sind vom Hersteller am Point of Sale durchgeführte Werbeaktionen. Diese zwei Formen der Verkaufsförderung richten sich in ihrem unmittelbaren Bezug also zunächst auf unterschiedliche Zielgruppen und wären somit nach einer zielgruppenbezogenen Abgrenzung im ersten Fall als Push- und im zweiten Fall als Pull-Instrument zu charakterisieren.

Einer solchen Einordnung stehen jedoch zwei Argumente gegenüber. Erstens wird mit beiden Formen der beispielhaft genannten Verkaufsförderung die Angebotsstärke erhöht, d. h. der Angebotsdruck der Marke auf den Endabnehmer im Handel steigt. Somit entsprechen beide Fälle eher dem Gedanken des Push- denn dem des Pull-Konzeptes. Zweitens erfolgt die Werbeaktion zwar durch den Hersteller, aber in der Einfluß- und Entscheidungssphäre des Absatzmittlers und mit Bindung an den Ort der Einkaufsstätte. Eine Zuordnung dieser Maßnahme zu den Instrumenten des Pull würde der Zielsetzung des Pull-Konzeptes widersprechen, endabnehmergerichtete Aktivitäten möglichst autonom und unabhängig vom Handel gestalten zu können, um damit einen Gegenpol zur Handelsmacht zu schaffen.

Eine geeignetere Form der Unterscheidung scheint deshalb die wirkungsbezogene Abgrenzung von Push- und Pull-Instrumenten. So lassen sich alle Marketinginstrumente, die eine unmittelbare Erhöhung der Angebotsstärke der Marke im Handel bewirken, als Push-Instrumente bezeichnen. Pull-Instrumente sind demnach solche, die direkt auf die Steigerung der Nachfragestärke einer Marke bei den Endabnehmern abzielen. Um zusätzlich den Zielgruppenbezug zum Ausdruck zu bringen, wird ergänzend zwischen Push-Instrumenten differenziert, die zum Hineinverkauf der Marke in den Handel (Push-Instrumente des Hineinverkaufs) und zum Herausver- 
kauf der Marke aus dem Handel an den Endabnehmer (Push-Instrumente des Herausverkaufs) dienen.

Für die vorliegende Arbeit soll eine Begriffsfassung des Push und Pull gewählt werden, die sich im Sinne des entscheidungsorientierten Ansatzes auf Handlungsalternativen des Unternehmens bezieht. Wie bereits aufgezeigt, bietet es sich dabei an, Push und Pull zur Kennzeichnung von Instrumentalstrategien der Markendurchsetzung zu verwenden, mit denen die grundsätzliche Ausrichtung des Marketinginstrumentariums auf Handel und Endabnehmer beschrieben wird. Diese läßt sich nunmehr mit Hilfe der Instrumentezuordnung genauer definieren. Konzentriert sich die Markendurchsetzungsstrategie auf Marketinginstrumente, die dem Push-Instrumentarium zuzuordnen sind, wird diese als push-orientierte Markendurchsetzungsstrategie bezeichnet. Liegt der geplante Schwerpunkt bei PullInstrumenten, wird analog von einer pull-orientierten Markendurchsetzungsstrategie gesprochen.

Obgleich die zuvor dargestellten und in Abbildung 3 zusammengefaßten Begriffsverwendungen in der Literatur mitunter den Eindruck entstehen lassen, daß es sich bei Push und Pull um zwei alternative Ansätze der Markenführung handelt, ist festzustellen, daß in der Praxis nahezu jede Markendurchsetzungsstrategie beide Elemente umfaßt.

\begin{tabular}{|l|l|l|}
\hline Ansatz & Begriff & Inhalt \\
\hline Systembezogen & $\begin{array}{l}\text { Push-/Pull- } \\
\text { Konzept }\end{array}$ & $\begin{array}{l}\text { Mehrstufige Wirkungszusammenhänge im vertikalen Markt- } \\
\text { system, die ihren Ausgang in der Stimulierung des Handels } \\
\text { (Push) oder des Endabnehmers (Pull) durch den Hersteller } \\
\text { haben }\end{array}$ \\
\hline Zielbezogen & $\begin{array}{l}\text { Push-/Pull- } \\
\text { Brelte u. Tiefe }\end{array}$ & $\begin{array}{l}\text { Stärke des Angebots einer Marke im Handel (Push) und der } \\
\text { Nachfrage durch den Endabnehmer (Pull) }\end{array}$ \\
\hline Objektbezogen & $\begin{array}{l}\text { Push-/Pull- } \\
\text { Marke }\end{array}$ & $\begin{array}{l}\text { Eigenschaft der Marke, den Absatz durch Angebotsdruck } \\
\text { im Handel (Push) oder die Nachfrage des Endabnehmers } \\
\text { (Pull) zu erreichen }\end{array}$ \\
\hline $\begin{array}{l}\text { Strategle- } \\
\text { bezogen }\end{array}$ & $\begin{array}{l}\text { Push-/Pull- } \\
\text { Strategie }\end{array}$ & $\begin{array}{l}\text { Handelsbeeinflussende Strategien der Absatzmittlerakqui- } \\
\text { sition und Regalplatzsicherung }\end{array}$ \\
\hline $\begin{array}{l}\text { Instrumente- } \\
\text { bezogen }\end{array}$ & $\begin{array}{l}\text { Push-/Pull- } \\
\text { Instrumente }\end{array}$ & $\begin{array}{l}\text { Ausrichtung der Marketinginstrumente, Einordnung als } \\
\text { Push- oder Pull-Instrumente (zielgruppen-/wirkungsbe- } \\
\text { zogene Abgrenzung) }\end{array}$ \\
\hline
\end{tabular}

Abb. 3: Begriffsverwendung von Push und Pull in der Literatur 
Push und Pull in ihrer Reinform stellen daher nur zwei Extrempunkte eines Kontinuums möglicher Markendurchsetzungsstrategien dar.60 Die Stärke der Push- bzw. der Pull-Orientierung zeigt sich in den Anteilen der jeweils eingesetzten Marketinginstrumente und steigt mit dem relativen Gewicht des Push- bzw. Pull-Instrumentariums. Kommen Push- und Pull-Instrumente in gleichem Maße zum Tragen, liegt eine "ausgeglichene" Markendurchsetzungsstrategie vor. Abbildung 4 zeigt diesen Zusammenhang graphisch auf.

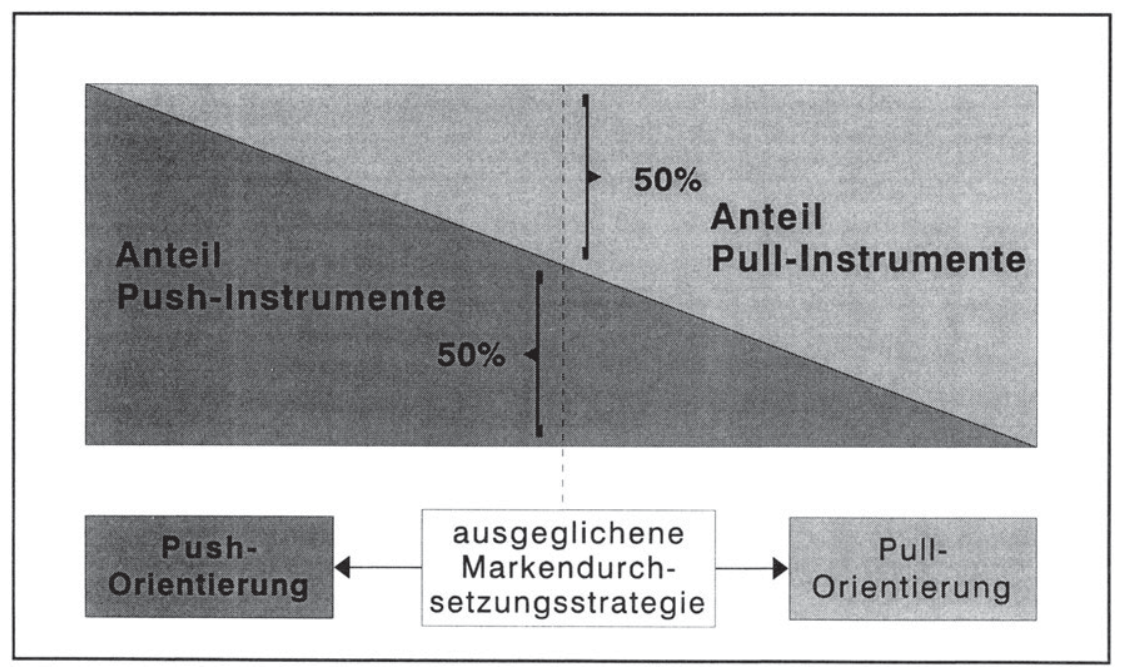

Abb. 4: Push- und Pull-Orientierung in der Markendurchsetzungsstrategie

\section{Gegenstand und Anforderungen einer modellgestützten Planung in der Markenpolitik}

Die Komplexität markenpolitischer Entscheidungen läßt den Einsatz unterstützender Planungsinstrumente sinnvoll erscheinen. Deren grundsätzliche Aufgabe besteht darin, durch Informationsverdichtung auf der einen und die Berücksichtigung von Interdependenzen innerhalb des Planungsproblems auf der anderen Seite die Entscheidungsfindung zu vereinfachen und

60 Vgl. Träger, W., Push und Pull: Oder was soll der Hersteller beim Einzelhandel tun?, in: Marketing-Journal, H. 4, 1969, S. 262 f.; Gussek, F., Erfolg in der strategischen Markenführung, a.a.O., S. 305. 
gleichzeitig zur besseren Fundierung markenpolitischer Entscheidungen beizutragen. 61 Die betriebswirtschaftliche Theorie bedient sich hierzu der modellhaften Abbildung des dem Planungsproblem zugrundeliegenden realen Systems. ${ }^{62}$ In aller Regel wird dabei eine Quantifizierung der Zusammenhänge angestrebt, indem die Beziehungen in Form mathematischer Funktionen abgebildet werden.

Diese quantitativen Modelle besitzen den Vorteil, auch bei mehrstufigen Beziehungen die Auswirkungen der Veränderung einer Modellgröße auf das Modellergebnis ermitteln zu können, ohne daß Zwischenschritte jeweils inhaltlich zu interpretieren bzw. zu verbalisieren sind.63 Grundsätzlich bietet die Verwendung quantitativer Modelle darüber hinaus folgende Vorzüge:64

61 Vgl. Voß, W.-D., Modellgestützte Markenpolitik, Planung und Kontrolle markenpolitischer Entscheidungen auf der Grundlage computergestützter Informationssysteme, Wiesbaden 1983, S. 7 f.

62 Als System wird in der Literatur die Gesamtheit von geordneten Elementen verstanden, die über Eigenschaften verfügen und über Relationen miteinander in Verbindung stehen. Vgl. Hünerberg, R., Marketingsysteme und -modelle, Zur Entscheidungslehre im Marketing, Opladen 1974, S. 25. Dieses Grundverständnis von Systemen wurde in der Forschungsrichtung der Systemtheorie dahingehend ausgebaut, daß stark formalisierte und möglichst vollständige Analysen der Beziehungen innerhalb eines Systems angestrebt werden. Zum systemtheoretischen Ansatz vgl. Meffert, H., Systemtheorie aus betriebswirtschaftlicher Sicht, in: Systemanalyse in den Wirtschafts- und Sozialwissenschaften, Hrsg.: Schenk, K. E., Berlin 1971, S. 174 ff.; Baetge, J., Betriebswirtschaftliche Systemtheorie, Opladen 1974. Zu Systemanalysen in der Marketingplanung vgl. z. B. die Arbeiten von Rütschi, K., Marketing-Modelle, Systemanalyse, Formalisierung und Aufbau, Winterthur 1972, und Zantov, R., Systemorientierte Marketinganalyse der Banken, Die Systemforschung als Grundlage generell anwendbarer Analysemodelle, dargestellt am Beispiel der Bankmarketinganalyse, Florenz 1978.

63 Vgl. Meffert, H., Steffenhagen, H., Marketing-Prognosemodelle, Stuttgart 1977 , S. 29 f. Zum Modellbegriff vgl. Hünerberg, R., Marketingsysteme und -modelle, a.a.O., S. 36 ff.; Gaul, W., Both, M., Computergestütztes Marketing, Berlin u. a. 1990, S. $68 \mathrm{ff}$.

64 Vgl. Dannenberg, J., Mikrocomputergestützte Instrumente der strategischen Unternehmensplanung, Wiesbaden 1990, S. 96 ff.; Hanssmann, F., Systemforschung am Beispiel der strategischen Unternehmensmodelle, in: WiSt, H. 8, S. 357 f; Ritzerfeld, U., Marketing-Mix-Strategien in Investitionsgütermärkten - Marktstrukturspezifische Strategieempfehlungen aufgrund einer Analyse von Lieferanten-KundenBeziehungen und einer Simulationsstudie, Diss., Aachen 1993, S. 108 ff. Im folgenden werden unter dem Begriff des Modells stets quantitative Modelle verstanden. 
- Die Formalisierung der Beziehungen erfordert eine intensive Analyse des zugrundeliegenden realen Systems, gibt Hinweise auf fehlende Informationen und zeigt logische Inkonsistenzen im Verständnis der Systemzusammenhänge auf.

- Die Darstellung der Beziehungen sowie die Offenlegung der getroffenen Annahmen systematisiert die Diskussion über die Entscheidungsfindung und erhöht deren interpersonale Nachvollziehbarkeit.

- Die mathematische Modellformulierung ermöglicht eine computerbasierte Implementierung des Modells und damit eine Erhöhung von Vollständigkeit, Genauigkeit und Geschwindigkeit der Informationsverarbeitung des Entscheidungsträgers ("Intelligenzverstärkung"). Die Konsequenzen verschiedener Handlungsoptionen und alternativer Situationen sind entsprechend schnell zu ermitteln.

In der Literatur findet sich eine Vielzahl von Modellen, die in generischer Form Teilaspekte markenpolitischer Planungsprobleme abbilden. Hierzu zählen vor allem Modelle zur Abbildung von Markenwahlentscheidungen des Endabnehmers, zur Positionierung von Marken, zur Budgetierung endabnehmergerichteter Kommunikationsausgaben sowie zur Gestaltung von Distributionssystemen. ${ }^{65}$ Vergleichsweise wenige Modelle setzen sich jedoch mit einer integrierten Betrachtung der Beziehungen zwischen Hersteller, Handel und Endabnehmer auseinander.66 Liegt dem Planungsproblem obendrein ein realer Markt zugrunde, existiert häufig kein der Problemstellung adäquates Modell, da oftmals marktspezifische Besonderheiten zu berücksichtigen sind.

Insofern ist die Aufgabe der modellgestützten Marketingplanung vielfach gleichzusetzen mit der Entwicklung eines neuen entscheidungsunter-

65 Auf eine nähere Beschreibung dieser Modelle sei an dieser Stelle verzichtet. Ausführliche Darstellungen sowie zusammenfassende Übersichten finden sich Z. B. bei Funke, U. H., Mathematical Models in Marketing, A collection of Abstracts, Berlin u.a. 1976; Topritzhofer, E., Absatzwirtschaftliche Modelle des Kaufentscheidungsprozesses unter besonderer Berücksichtigung des Markenwahlaspektes, Wien 1974, S. 58 ff.; Maas, R.-M., Absatzwege, Konzeptionen und Modelle, Wiesbaden 1980, S. 240 ff.; Lilien, G. L., Kotler, P., Moorthy, K. S., Marketing Models, Englewood Cliffs 1992, S. 19 ff.; Ritzerfeld, U., Marketing-Mix-Strategien in Investitionsgütermärkten, a.a.O., S. $115 \mathrm{ff}$.

Vgl. zur Diskussion einiger ausgewählter Modelle Kap. C. 2.3 dieser Arbeit. 
stützenden Modells. ${ }^{67}$ Hierbei hat der für die Markenpolitik verantwortliche Entscheidungsträger die Zielsetzungen und Anforderungen an ein solches Modell zu formulieren und auf Basis der inhaltlichen Analyse des Planungsgegenstandes die konzeptionelle Modellstruktur festzulegen. Die programmtechnische Umsetzung im Rahmen der sich anschließenden computergestützten Implementierung wird dagegen, zumindest bei komplexen Modellen, auf EDV-Spezialisten zu übertragen sein (vgl. Abbildung 5). 68

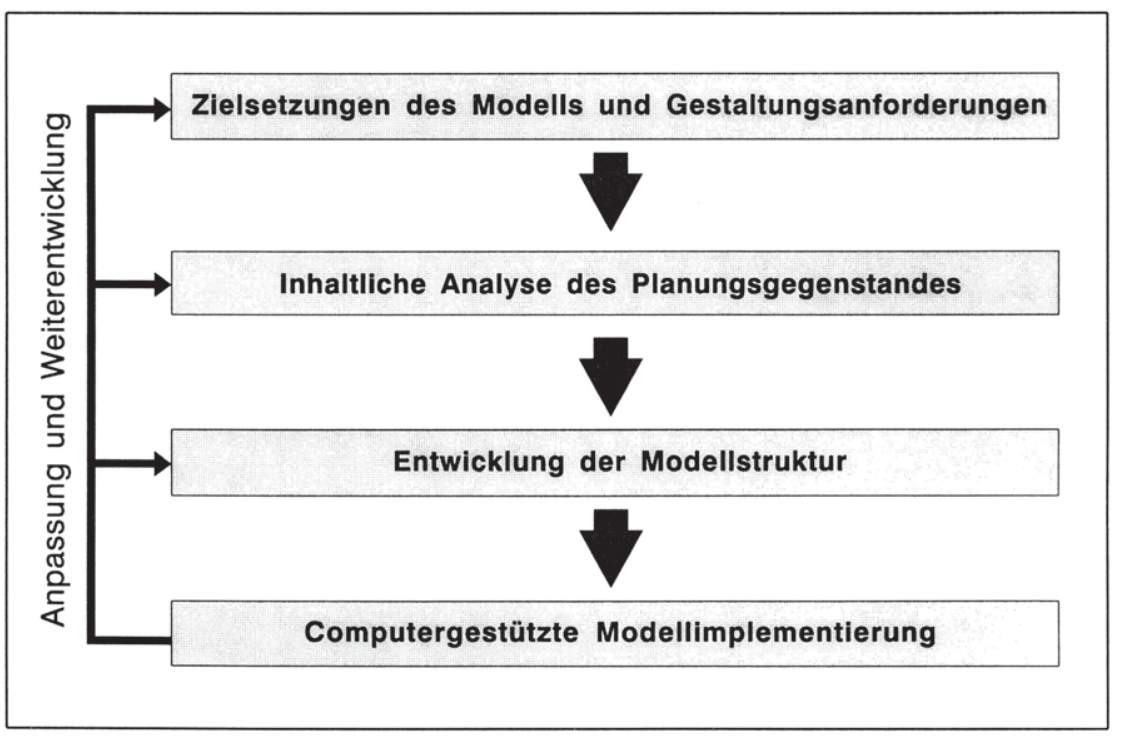

Abb. 5: Stufen der Entwicklung eines Modells zur Unterstützung der Planung in der Markenpolitik

67 Aufgrund der gewählten Definition des Planungsbegriffes sind die Bezeichnungen "entscheidungsunterstützendes Modell" und "Modell zur Unterstützung der Planung" synonym zu verstehen.

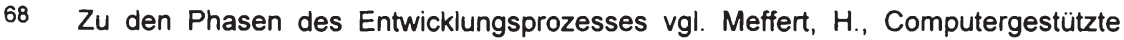
Marketing-Informationssysteme und Marketing-Modelle, in: Computergestützte Marketingplanung, Hrsg.: Hansen, H. R., München 1974, S. 13 ff. In der Literatur existieren vielfältige Beschreibungen möglicher Phasenkonzepte zur Entwicklung von Modellen. Je nach Betrachtungsschwerpunkt sind diese stärker auf konzeptionelle Analysen des Planungsgegenstandes, der konzeptionellen oder technischorientierten, computergestützten Implementierung oder der organisatorischen Verankerung solcher Modelle in den Unternehmen ausgerichtet. Für die vorliegende Arbeit erscheint die hier gewählte Strukturierung der innerhalb des Entwicklungsprozesses zu berücksichtigenden Stufen zweckmäßig. 
Im ersten Schritt ist hinsichtlich der Modellzielsetzung zwischen Modellen zur Beschreibung und Erklärung des zugrundeliegenden realen Systems, zur Prognose zukünftiger Entwicklungen oder zur unmittelbaren Entscheidungsfindung zu unterscheiden. 69 Beschreibungsmodellen kommt die Aufgabe zu, das der Planung zugrundeliegende reale System von seiner grundsätzlichen Struktur her oder konkret-rechnerisch darzustellen. Dieser Modellansatz beschränkt sich in aller Regel auf die Verwendung von Definitionsgleichungen, bei denen auf Hypothesen über den Zusammenhang zwischen den Modellvariablen verzichtet wird.

Erklärungsmodelle formulieren dagegen solche Hypothesen explizit in sogenannten Erklärungsgleichungen. Diese beschreiben die Beziehung zwischen den Handlungsalternativen (Entscheidungsvariablen) und den zu erwartenden Handlungsfolgen (Erwartungsvariablen) unter Berücksichtigung der bestehenden situativen Faktoren (Kontextvariablen). Erklärungsgleichungen werden ferner vielfach durch ein System von Nebenbedingungen ergänzt, die den zulässigen Bereich der Handlungsvariablen einschränken. ${ }^{70}$ Als eine spezielle Art von Erklärungsmodellen werden in der Literatur häufig Prognosemodelle betrachtet. Sie dienen dazu, Aussagen über zukünftige Entwicklungen bzw. den Eintritt von Ereignissen zu ermöglichen. In diesem Sinne können sie als Erklärungsmodelle interpretiert werden, die auf eine Abbildung der in Zukunft geltenden Zusammenhänge ausgerichtet sind. Allerdings existiert auch eine Reihe von Prognosemodellen, bei denen die unabhängigen Variablen entweder nicht im Entscheidungsbereich des Unternehmens liegen oder aber nicht explizit formuliert werden. Dies gilt beispielsweise für Trendprognosen der allgemeinen Nachfrageentwicklung auf einem Markt. ${ }^{71}$

69 Vgl. hierzu und im folgenden Meffert, H., Computergestützte Marketing-Informationssysteme, Konzeptionen, Modellanwendungen, Entwicklungsstrategien, Wiesbaden 1975, S. 57 ff.; Biethahn, J., Simulation - eine Methode zur Findung betriebswirtschaftlicher Entscheidungen?, in: Biethahn, J., Schmidt, B., Simulation als betriebliche Entscheidungshilfe, Methoden, Werkzeuge, Anwendungen, Berlin u.a. 1987, S. 82; Homburg, C., Modellgestützte Unternehmensplanung, Wiesbaden 1991, S. $267 \mathrm{ff}$.

70 Vgl. Heinen, E., Einführung in die Betriebswirtschaftslehre, 9. Aufl., Wiesbaden 1985, S. 158.

71 Zur Trendprognose vgl. Meffert, H., Steffenhagen, H., Marketing-Prognosemodelle, a.a.O., S. $63 \mathrm{ff}$. 
Die Erweiterung eines Erklärungsmodells um die mathematische Formulierung einer Zielfunktion führt schließlich zum Entscheidungsmodell. ${ }^{72}$ Charakteristisch für diese Klasse von Modellen ist die Zielsetzung, optimale oder zumindest befriedigende Kombinationen von Entscheidungsvariablen zu finden. Entscheidungsmodelle werden daher häufig unmittelbar im Hinblick auf eine bestimmte Lösungsmethode konzipiert, wodurch Art und Umfang des Modells zumeist von vornherein eingeschränkt sind..$^{73}$

Im Vorgriff auf spätere Ergebnisse kann hier bereits festgehalten werden, daß der Einsatz von Entscheidungsmodellen zur Unterstützung der Planung im Rahmen der Markenpolitik nicht geeignet ist. Die Komplexität der Problemstellung sowie die noch aufzuzeigenden Strukturdefekte ${ }^{74}$ des Planungsgegenstandes lassen eine unmittelbare Optimierung bei der Entscheidungsfindung nicht zu. Vielmehr bietet es sich an, ein Erklärungsmodell zu konzipieren, dessen Erwartungsvariablen mit den markenpolitischen Zielen übereinstimmen, so daß die Auswirkungen verschiedener Ausprägungen der Entscheidungsvariablen unter alternativen Annahmen über die Datenkonstellation direkt bewertet werden können.

Daß ein solches Erklärungsmodell einen prognostischen Charakter besitzen und zudem auch auf Prognosen über zukünftige, nicht unmittelbar vom Unternehmen beeinflußbare Entwicklungen aufbauen muß, versteht sich bereits aus der Zukunftsorientierung der Planung. Erst im Anschluß an die Erfüllung dieser Zielsetzung ist zu untersuchen, inwieweit z. B. durch den Einsatz der Simulationsmethode verbesserte oder optimale Lösungen des Planungsproblems gefunden werden können. 75

72 Vgl. auch Heinen, E., Einführung in die Betriebswirtschaftslehre, a.a.O., S. 215 f.

73 Die Auseinandersetzung mit Entscheidungsmodellen findet insbesondere im Rahmen der betriebswirtschaftlichen Teildisziplin des Operations Research statt. Dabei werden zumeist kleine, wohl-strukturierte Problemstellungen zugrunde gelegt. Vgl. hierzu Homburg, C., Modellgestützte Unternehmensplanung, a.a.O., S. $274 \mathrm{ff}$.

Vgl. Kap. C. 1.1 dieser Arbeit.

Vgl. Kap. C. 3.4 dieser Arbeit. 
Zur Festlegung der Gestaltungsanforderungen sind zwei grundsätzlich verschiedene Ansätze der Entwicklung und des Einsatzes von entscheidungsunterstützenden Modellen 76 zu unterscheiden, die als realsystemorientiert-objektiv und benutzerorientiert-subjektiv bezeichnet werden können.

Der realsystemorientiert-objektive Ansatz entspricht der Vorstellung, daß Modelle isomorphe oder zumindest homomorphe Abbildungen der Realität sein müssen. ${ }^{77}$ Entsprechend dieser Auffassung sind Modelle um so eher für die Entscheidungsunterstützung geeignet, je wirklichkeitsgetreuer und genauer sie die Realität widerspiegeln. In ihrer Anwendung sind solchen Modellen jedoch enge Grenzen gesetzt, die sich aus der Komplexität des realen Systems sowie den unzulänglichen Möglichkeiten der Formalisierung und empirischen Überprüfung von Zusammenhängen ergeben.78 Der realsystemorientiert-objektive Ansatz ist damit eher zur Unterstützung von Entscheidungen geeignet, die auf Grundlage weniger, gut beobachtbarer und in ihren Wirkungen verläßlich prognostizierbarer Beziehungen zu treffen sind.

Der benutzerorientiert-subjektive Ansatz setzt bei der Modellbildung an der Vorstellung des Entscheidungsträgers über das reale System an. Ziel ist es, dessen sogenanntes "interne Modell" in expliziter Form zu formulieren und damit ein Instrument der "Intelligenzverstärkung" zu schaffen. ${ }^{79}$ Der zentrale Grundgedanke besteht darin, die Einschätzungen und Erfahrungen des Entscheidungsträgers bei der Modellbildung zu berücksichtigen. Dabei soll insbesondere von der Fähigkeit des Managers Gebrauch gemacht wer-

76 Vgl. hierzu und im folgenden Meffert, H., Computergestützte Marketing-Informationssysteme, a.a.O., S. $53 \mathrm{ff}$.

77 Mit der Forderung nach Homomorphie wird verlangt, daß ein Modell eine strukturidentische Abbildung des zugrundeliegenden realen Systems darstellt. Das Kriterium der Isomorphie fordert darüber hinaus, daß zwischen Modell und realem System eine eineindeutige, d. $h$. in beide Richtungen eindeutige Beziehung zwischen den Elementen besteht. Vgl. Zentes, J., Die Optimalkomplexion von Entscheidungsmodellen, Köln u. a. 1976, S. 33 f.

78 Vgl. Haedrich, G., Informationssystem und Marketingplanung, in: Operationale Entscheidungshilfen für die Marketingplanung, Hrsg.: Haedrich, G., Berlin, New York 1977, S. 26 f.; Bäuerle, P., Zur Problematik der Konstruktion praktikabler Entscheidungsmodelle, in: ZfB, H. 2, 1989, S. $175 \mathrm{ff}$. 
den, verschiedenste Informationen zu verdichten und damit Wirkungszusammenhänge subjektiv schätzen zu können. Objektiv gemessene Zusammenhänge dienen dabei durchaus als Informationsgrundlage, werden aber vor dem Hintergrund weiterer Aspekte, Erkenntnisse und Erfahrungen ergänzt und interpretiert. 80 Auf Little zurückgehend wird in der Literatur ein solches Instrument als Decision Calculus bezeichnet. 81

Hinsichtlich der an ein entscheidungsunterstützendes Modell zu stellenden Anforderungen dürfen der realsystemorientiert-objektive und benutzerorientiert-subjektive Ansatz nicht als sich ausschließende Alternativen verstanden werden. Es kann zum einen nicht das Ziel sein, durch das Streben nach einer detailgetreuen Abbildung der Realität zu einem Modell zu gelangen, das die angesprochenen Grenzen überschreitet und damit zu einer Scheingenauigkeit der Ergebnisse führt. ${ }^{82}$ Zum anderen ist es nicht sinnvoll, ein Modell zu entwickeln, das zwar die subjektiven Vorstellungen des Entscheidungsträgers wiedergibt, nicht aber mit dem realen System übereinstimmt. Vielmehr müssen sich beide Ansätze ergänzen. Im Sinne eines evolutionären Entwicklungsprozesses erscheint es dabei vorteilhaft, von einem benutzerorientiert-subjektiv gestalteten Modell auszugehen, das anschließend, soweit möglich, schrittweise zu objektivieren ist, indem die vermuteten Beziehungen überprüft und angepaßt werden. 83

Der zweite Schritt in der Entwicklung eines entscheidungsunterstützenden Modells besteht in der inhaltlichen Analyse des Planungsgegenstandes. Hierbei sind zunächst die markenpolitischen Ziele des Herstellers zu erfassen und zu systematisieren. Im Rahmen dieser Analyse sind insbeson-

80 Vgl. Haedrich, G., Informationssystem und Marketingplanung, a.a.O., S. 27.

81 Zum Konzept des Decision Calculus vgl. Little, J. D. C., Models and Managers: The Concept of a Decision Calculus, in: Management Science, No. 8, 1970, S. $466 \mathrm{ff}$. Deutsche Übersetzung: Little, J. D. C., Modelle und Manager, das Konzept eines Decision Calculus, in: Operationale Entscheidungshilfen für die Marketingplanung, Hrsg.: Haedrich, G., Berlin, New York 1977, S. $201 \mathrm{ff}$.

82 Zum Problem der optimalen Komplexität von Modellen vgl. Zentes, J., Die Optimalkomplexion von Entscheidungsmodellen, a.a.O., S. 33 ff.; Bäuerle, P., Zur Problematik der Konstruktion praktikabler Entscheidungsmodelle, a.a.O., S. $177 \mathrm{ff}$.; Heinhold, M. Simultane Unternehmensplanungsmodelle - ein Irrweg?, in: DBW, H. 6 , 1989, S. $689 \mathrm{ff}$.

Vgl. Meffert, H., Computergestützte Marketing-Informationssysteme, a.a.O., S. 55. 
dere die Zielinhalte zu präzisieren, um im Ergebnis zu einer operationalen Zielformulierung zu gelangen. ${ }^{84}$ Gleiches gilt für die Handlungsalternativen, die dem Unternehmen im Rahmen seiner Markenpolitik zur Verfügung stehen. ${ }^{85}$ Sofern nicht schon als Ziele formuliert, sind ferner Größen zur Beschreibung von Marktteilnehmerreaktionen darzustellen und zu operationalisieren. Gerade in der Markenpolitik, bei der es wesentlich um die Beeinflussung des Marktteilnehmerverhaltens geht, ist jedoch davon auszugehen, daß diese Reaktionsgrößen bereits weitgehend Bestandteil des Zielsystems sind. Des weiteren zählt zur Analyse des Planungsgegenstandes die Erfassung des situativen Kontextes. Hier sind vor allem das reale Marktsystem zu kennzeichnen und dabei im besonderen die Faktoren zu identifizieren, die Einfluß auf den Wirkungszusammenhang zwischen Handlungsalternativen und Marktteilnehmerreaktionen bzw. markenpolitischen Zielen nehmen.

Im dritten Schritt erfolgt die Entwicklung der Modellstruktur. Hierbei sind die gewonnenen Erkenntnisse aus der inhaltlichen Problemanalyse in eine adäquate Modellstruktur zu überführen. In diesem Zusammenhang sind zunächst weitere Entscheidungen hinsichtlich des Aggregations- und Abstraktionsgrades des Modells, der Berücksichtigung von Unsicherheit in den Variablen und Beziehungen, der Beachtung dynamischer Zusammenhänge, des Modellumfangs sowie der zu verwendenden Lösungsmethode zu treffen. 86

Im Anschluß daran sind die in das Modell einzubeziehenden Handlungsalternativen und Ziele sowie die darüber hinausgehenden Größen der Marktteilnehmerreaktion als Variablen zu definieren und über Erklärungsgleichungen in Beziehung zueinander zu setzen. Inwieweit die situativen Faktoren in Form von Kontextvariablen ebenfalls Bestandteil des Modells

84 Ein Ziel ist operational, wenn hierfür eine Meßvorschrift vorliegt. Vgl. Heinen, E., Einführung in die Betriebswirtschaftslehre, a.a.O., S. 99 f. Allgemein ist unter dem Begriff der Operationalisierung die Übersetzung eines theoretischen Begriffes oder Sachverhaltes in eine Sprachebene mit direktem Bezug zu beobachtbaren Größen zu verstehen. Vgl. hierzu Andritzky, K., Die Operationalisierbarkeit von Theorien zum Konsumentenverhalten, Berlin 1976, S. 20 ff.

Vgl. Heinen, E., Einführung in die Betriebswirtschaftslehre, a.a.O., S. 125.

Vgl. zu diesen Entscheidungen Kap. C. 1.2 dieser Arbeit. 
sind, ist abhängig vom grundsätzlich gewählten Konzept. Während diese beim realsystemorientiert-objektiven Ansatz zwingend aufgenommen werden müssen, um Veränderungen der Datensituation im Modell berücksichtigen zu können, kann dies beim benutzerorientiert-subjektiven Ansatz implizit, d. h. über die Wirkungsabschätzungen des Entscheidungsträgers, erfolgen.

In der letzten Stufe wird schließlich die computergestützte Implementierung vorgenommen. In dieser Phase ist das bislang konzeptionell entwickelte Modell in Programmbefehle umzusetzen, die dessen computergestützte Berechnung ermöglichen. Ferner sind in diesem Zusammenhang entsprechende Ein- und Ausgabeschnittstellen zu implementieren, die den Anforderungen der Benutzerfreundlichkeit eines Modells genügen. 87

Die eigentlich zur vorherigen, konzeptionellen Stufe der Entwicklung zu zählende Kalibrierung des Modells, ${ }^{88}$ d. h. die Schätzung von Funktionsparametern zur Festlegung des genauen Verlaufs von Erklärungsfunktionen, kann beim subjektiv-benutzerorientierten Ansatz sinnvollerweise erst nach bzw. während der Implementierungsphase erfolgen. Die vom Entscheidungsträger vorzunehmenden Wirkungsabschätzungen erfordern eine unmittelbare Kontrolle ihrer Auswirkungen auf die Erwartungsvariablen, damit von der Form und Stärke ihres Zusammenhangs falsch eingeschätzte Beziehungen in einem iterativen Prozeß korrigiert werden können. Diese Möglichkeit setzt allerdings voraus, daß zumindest die Grundstruktur des Modells implementiert ist. ${ }^{89}$

87 Zur Gestaltung von Ein- und Ausgabeschnittstellen vgl. Turban, E., Decision Support and Expert Systems, Managerial Perspectives, New York 1988, S. 86 ff.; Herrmann, H.-J., Modellgestützte Planung im Unternehmen, Entwicklung eines Rahmenkonzeptes, Wiesbaden 1992, S. $348 \mathrm{ff}$.

88 Zur Kalibrierung von Modellen vgl. Lilien, G. L., Kotler, P., Moorthy, K. S., Marketing Models, a.a.O., S. $676 \mathrm{ff}$.

89 Zum Vorgehen bei der Implementierung von entscheidungsunterstützenden Modellen vgl. Turban, E., Decision Support and Expert Systems, a.a.O., S. 122 ff.; Brombacher, R., Entscheidungsunterstützungssysteme für das MarketingManagement, Gestaltungs- und Implementierungsansatz für die Konsumgüterindustrie, Berlin, Heidelberg 1988, S. $182 \mathrm{ff}$. 


\section{Ziel und Gang der Untersuchung}

Mit der vorliegenden Arbeit wird die generelle Zielsetzung verfolgt, einen Beitrag zur modellgestützten Planung von Strategien der Markendurchsetzung zu leisten. Hierbei soll insbesondere das Vorgehen bei der inhaltlichen Analyse des Planungsgegenstandes sowie bei der Entwicklung eines geeigneten Modells aufgezeigt werden.

Aus dieser generellen Zielsetzung leiten sich mehrere Teilziele ab. Als erstes Teilziel ist eine konzeptionelle Analyse der markenpolitischen Ziele sowie der Handlungsalternativen des Unternehmens vorzunehmen. Zudem sind die Wirkungs- und Interdependenzbeziehungen zwischen den Entscheidungstatbeständen und den Zielen zu erfassen und die sie beeinflussenden Faktoren zu identifizieren.

Eine solche Untersuchung hat dabei immer vor dem Hintergrund eines bestimmten Marktsystems und der sich daraus für das Unternehmen ergebenden Umweltsituation zu erfolgen. Erst dieser situative Kontext erlaubt die Konkretisierung der Ziele, Handlungsalternativen und Beziehungen. Daher wird dieser Arbeit der deutsche Reifenmarkt als Anschauungsobjekt zugrunde gelegt. Der Reifenmarkt, und hier insbesondere der Privatkundenbereich, gestatten aus mehreren Gründen eine prägnante Analyse:

- Im Gegensatz zu vielen Gütern des täglichen Bedarfs erfolgt der Reifenkauf zumeist nicht im Verbund mehrerer Produkte, so daß die Wahl der Einkaufsstätte und die Kaufentscheidung allein im Hinblick auf ein Produkt getroffen werden. Damit können sich überlagernde, durch den gleichzeitigen Kauf verschiedener Produkte entstehende Effekte bei der Untersuchung weitgehend ausgeschlossen werden.

- Am Point of Sale besteht eine sehr hohe Möglichkeit der Einflußnahme seitens des Händlers auf die Kaufentscheidung des Endabnehmers. Damit läßt sich deutlich die Wirkung einer Variation der veränderten Angebotsstärke einer Marke aufzeigen.

- Das Distributionssystem im Reifenmarkt ist durch eine Vielzahl eigenständiger Absatzmittler gekennzeichnet. Daraus ergeben sich Entscheidungsspielräume für die Markendurchsetzungsstrategie, wie sie bei der überaus hohen Machtkonzentration auf einigen Märkten, wie beispielsweise im Lebensmitteleinzelhandel, nicht bestehen. 
Als zentrales zweites Teilziel wird die Entwicklung eines eigenen Modellansatzes angestrebt. Dabei soll zunächst eine Modellstruktur konzipiert werden, die im Sinne des benutzerorientiert-subjektiven Ansatzes alle relevanten Entscheidungsvariablen und Wirkungsbeziehungen umfaßt. Zudem sollen die ebenfalls noch zu kennzeichnenden Strukturdefekte des Planungsproblems durch die Einbeziehung stochastischer Wirkungsbeziehungen und den Einsatz des Instrumentes der Risikosimulation Berücksichtigung finden. Damit werden vor allem die Unsicherheiten in der Planung von Markendurchsetzungsstrategien erfaßt und bei der Entscheidungsfindung explizit beachtet.

Im Rahmen des dritten Teilziels soll schließlich die modellgestützte Planung der Markendurchsetzungsstrategie für eine konkrete Situation durchgeführt werden. Hierzu wird eine zwar fiktive, jedoch von ihren Ausprägungen realistische Reifenmarke zugrunde gelegt.

Mit den beschriebenen Zielen ist bereits der Gang der Untersuchung vorgezeichnet. Im Hauptkapitel B wird zunächst der Planungsgegenstand näher gekennzeichnet. Dazu werden im ersten Schritt die grundlegende Struktur des Reifenmarktes aufgezeigt und die zu beachtenden Einflußfaktoren herausgearbeitet. 90 Vor dem Hintergrund dieses situativen Kontextes werden anschließend als Ausgangspunkt der Planung die markenpolitischen Ziele definiert und die durch Grundsatzstrategien der Reifenhersteller vorgegebenen strategischen Rahmenbedingungen vorgestellt. Die Darstellung des Push- und Pull-Instrumentariums sowie die Untersuchung der Wirkungsund Interdependenzbeziehungen zwischen den Instrumenten und den markenpolitischen Zielen ist Gegenstand des dritten Teils dieses Kapitels.

90 Die Analyse des Reifenmarktes stützt sich auf folgende Informationsgrundlagen: 1. Offene, nicht standardisierte Gespräche mit Experten eines Reifenherstellers.

2. Expertengespräche, die mit Reifenfachhändlern im Rahmen einer explorativen Studie geführt wurden, bei denen in offener Fragestellung die Themenbereiche der Bedeutung von Marken im Reifengeschäft, des Käuferverhaltens sowie Kriterien der Sortimentsbildung behandelt wurden. 3. Marktforschungsergebnisse verschiedener Studien eines Reifenherstellers. Da die Gesprächsinhalte und Studien z. T. als "sensibel" einzustufen sind, muß aus Gründen der Vertraulichkeit auf deren explizite Darstellung sowie auf die konkrete Nennung der betroffenen Reifenmarken verzichtet werden. 
Im Hauptkapitel C erfolgt die Entwicklung eines eigenen entscheidungsunterstützenden Reifenmarktsimulationsmodells. Dazu wird das Planungsproblem zunächst im Hinblick auf seine Strukturdefekte untersucht, um daraufhin die Gestaltungsanforderungen an das Modell konkretisieren zu können. Weitere Erkenntnisse und Ansatzpunkte für eine geeignete Modellierung des Planungsproblems werden im Anschluß daran durch die Diskussion ausgewählter Modelle der Literatur gewonnen. Nach der Entwicklung der Modellstruktur wird auf Grundlage trapezförmiger Wahrscheinlichkeitsverteilungen auf die Bestimmung stochastischer Variablen eingegangen. Um die bestehenden Unsicherheiten des Planungsproblems auch im Zusammenhang zwischen den Variablen abbilden zu können, wird zudem die sogenannte ADBUDG-Funktion zu einer stochastischen Beziehung erweitert. Diese Art der Modellkonstruktion erfordert den Einsatz der Risikosimulation als Lösungsmethode. Deren Ablaufschritte werden für das konkrete Modell näher beschrieben, da sie sich in Haupt- und Untersimulation aufteilen.

Im weiteren ist aufzuzeigen, wie die mit dem Modell berechneten Handlungsalternativen zu bewerten und auszuwählen sind. Darüber hinaus wird eine Möglichkeit vorgestellt, mit Hilfe der Sensitivitätsanalyse eine Verbesserung oder sogar Optimierung der Ausgangslösung zu erreichen. Um den Einsatz des Modells zu demonstrieren, wird schließlich dessen computergestützte Implementierung beschrieben und anhand eines konkreten Beispiels die Planung von Markendurchsetzungsstrategien vorgenommen.

Im Hauptkapitel D erfahren die gewonnenen Erkenntnisse bei der Entwicklung eines entscheidungsunterstützenden Modells zur Planung von Markendurchsetzungsstrategien sowohl unter praxisrelevanten als auch forschungsspezifischen Aspekten eine abschließende Würdigung. 


\section{B. Analyse des Planungsgegenstandes von Markendurchsetzungs- strategien}

\section{Der Reifenmarkt als situativer Kontext für die Planung von Mar- kendurchsetzungsstrategien}

Die Analyse des Reifenmarktes als situativen Kontext für die Planung setzt zunächst die Unterscheidung verschiedener Teilmärkte sowie die Kennzeichnung der grundlegenden Absatzmarktstruktur voraus. Dabei ist die aus Sicht des Reifenherstellers relevante Umweltsituation und hier vor allem die Aufgabenumwelt näher zu beschreiben. 91 Neben der Darstellung von Wettbewerbssituation und Marktentwicklung sind in diesem Zusammenhang die Besonderheiten im Verhalten der verschiedenen Marktteilnehmer zu untersuchen. Im Hinblick auf die Konsumenten sind das Bedarfsverhalten, das Verhalten bei der Einkaufsstättenwahl sowie das Vorgehen bei der Informationsbeschaffung und der Markenwahlentscheidung zu betrachten. Bezüglich der handelsbezogenen Situation sind das Rollenverständnis des Handels und dessen Machtposition gegenüber dem Hersteller zu analysieren. Ferner ist die Bedeutung der Art der Warenpräsentation und der Bedienungsform im Reifenmarkt herauszustellen.

\subsection{Teilmärkte des Reifenmarktes und Absatzmarktstruktur}

Der Reifenmarkt gliedert sich in mehrere Teilmärkte. Auf dem Erstausrüstungsmarkt befindet sich der Reifenanbieter in der Rolle des Zulieferers

91 Zur Systematisierung der externen Umweltsituationen von Unternehmen werden im allgemeinen die Kategorien der globalen und aufgabenspezifischen Umwelt voneinander unterschieden. Unter dem Begriff des Aufgabenumfeldes werden alle Interaktionspartner subsumiert, zu denen das Unternehmen im Rahmen seiner Tätigkeit Beziehungen unterhält. Als solche sind Abnehmer (Handel, Weiterverarbeiter und Endabnehmer), Zulieferer, Wettbewerber sowie sonstige Anspruchsgruppen zu verstehen. Die globale Umwelt umfaßt alle Umweltfaktoren, die nur mittelbar über eine Veränderung der Aufgabenumwelt auf das Unternehmen einwirken. Hierzu zählen u. a. gesamtwirtschaftliche, politisch-rechtliche, sozio-kulturelle sowie ökologische Einflüsse. Vgl. Bourgeois III, L. J., Strategy and Environment - A conceptual integration, in: Academy of Management Review, No. 1, 1980, S. 33; Meffert, H., Marktanalyse, in: Handwörterbuch der Planung, Hrsg.: Szyperski, N., Stuttgart 1989, Sp. 1022; Heß, G., Marktsignale und Wettbewerbsstrategie, Theoretische Fundierung und Fälle aus der Unternehmenspraxis, Stuttgart 1991. S. $65 \mathrm{ff}$; Steinmann, H., Schreyögg, G., Management, Stuttgart 1990, S. $137 \mathrm{ff}$. 
für Hersteller (= Erstausrüster) von Pkws und Nutzfahrzeugen. Für sich allein betrachtet ist das Erstausrüstungsgeschäft eher unattraktiv. Die Qualitätsanforderungen und Anspruchshaltungen der Erstausrüster erfordern einen außerordentlich hohen Ressourceneinsatz im Bereich der Forschung und Entwicklung. Gleichzeitig sehen sich die Reifenhersteller einem erheblichen Preisdruck ausgesetzt, der aus dem Zwang der Erstausrüster resultiert, eigene Kostensenkungspotentiale auszuschöpfen. Trotz niedriger Deckungsspannen und einem teilweise sogar defizitären Geschäft ist der Erstausrüstungsmarkt dennoch hart umkämpft. 92

Ein Grund hierfür ist in dem hohen Absatzvolumen zu sehen, das mit wenigen Kunden realisiert und auf mehrere Jahre hinaus sichergestellt werden kann. Damit wird eine Grundauslastung der Produktion erreicht, die trotz niedriger Margen einen Beitrag zur Deckung bestehender Fixkosten leistet. Dies gilt insbesondere, da seitens der Automobilindustrie eine Tendenz zur stärkeren Konzentration der Beschaffungsaktivitäten auf eine geringe Anzahl von Zulieferern besteht. Der zweite Grund findet sich in der Verbindung zum Ersatzgeschäft. Die Tätigkeit eines Reifenherstellers in der Erstausrüstung beeinflußt direkt oder indirekt das Empfehlungsverhalten des Handels und die Kaufentscheidung des Endabnehmers.

Das damit bereits angesprochene Ersatzgeschäft teilt sich in die weiteren Märkte des Flotten- und Nutzfahrzeug- sowie des Privatkundenbereichs auf. Im Flotten- und Nutzfahrzeugbereich handelt es sich bei den Endabnehmern um gewerbliche Kunden, wie Unternehmen mit Fuhrparks oder Transportunternehmen. Diese betrachten den Reifenkauf als Investitionsentscheidung, die ihre eigene Kostenstruktur beeinflußt. Insbesondere für Transportunternehmen ist die Wirtschaftlichkeit des Reifens im Sinne der Kilometerleistung und des Einflusses auf die Kraftstoffkosten von hoher Relevanz für die eigene Wettbewerbsfähigkeit.

Der Privatkundenbereich umfaßt den PKW-Reifenabsatz an private Endkunden. Dieser Markt ist durch Endabnehmer geprägt, die Reifen überwiegend als problemlose Produkte betrachten, an denen sie zumeist wenig

92 Vgl. o. V., Wir liefern nicht an Hinz und Kunz, Interview mit Bridgestone Geschäftsführer Freitag, in: asw, H. 7, 1990, S. 15; Sasseen, J., Tyre Makers on the Rack, in: International Management, March 1993, S. 40 f. 
Interesse zeigen.93 Die Mehrheit der Reifenkäufer ist nicht in der Lage, die Technologie und Leistung eines Reifens differenziert zu beurteilen. Für den unbedarften Käufer besteht die einzig wahrnehmbare Eigenschaft darin, daß ein Reifen "rund und schwarz" ist.94

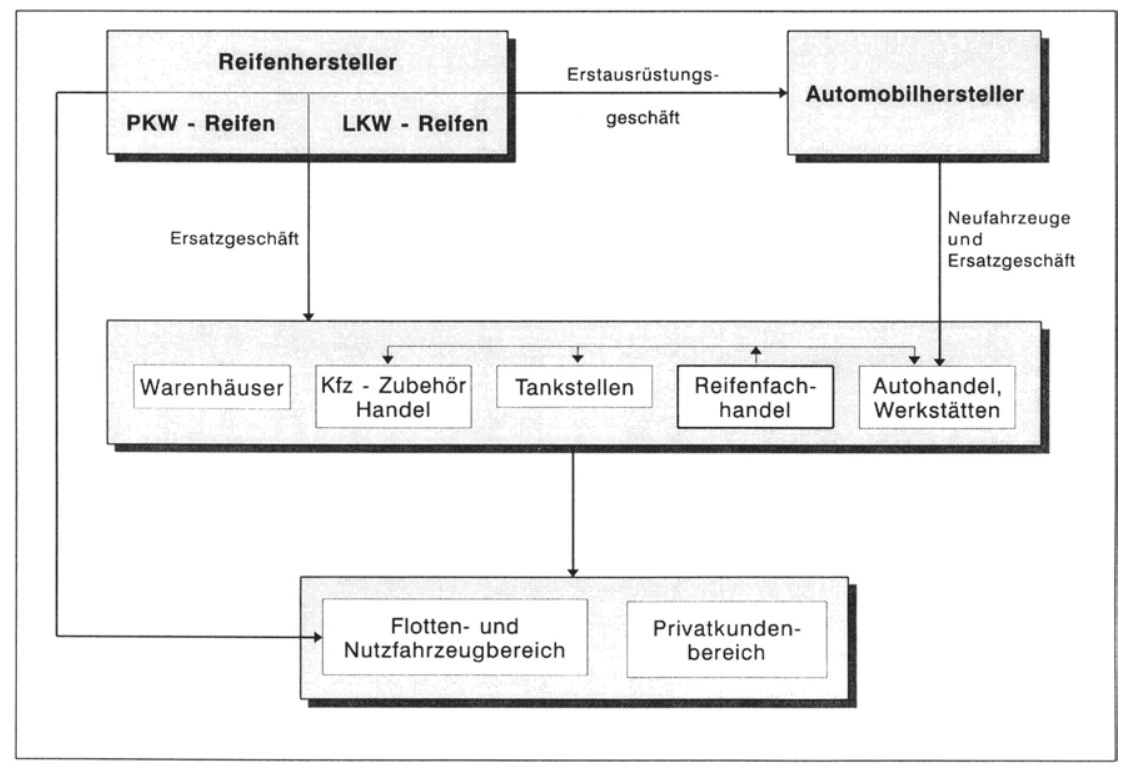

Abb. 6: Struktur des Reifenmarktes

Abbildung 6 zeigt die grundlegende Absatzmarktstruktur des Reifenmarktes auf. Über die direkte Beziehung zu den Automobilherstellern erfolgt zum einen der Absatz im Rahmen der Erstausrüstung. Zum anderen gelangen über diesen Absatzweg auch Reifen in das Ersatzgeschäft, wenn Automobilhersteller überschüssige Bestände ihren Vertragshändlern zuleiten. Als

93 Vgl. Simon, R. S., Marketing-Service als Mittel zur Differenzierung im stagnierenden Reifenmarkt, in: Thexis, H. 1, 1986, S. 28.

94 Vgl. Belz, Chr., Konstruktives Marketing in gesättigten und schrumpfenden Märkten, Band 2, Habil., St. Gallen 1987, S. 155. Wie im Zusammenhang mit der Beschreibung des Aufbaus der Untersuchung bereits abgegrenzt, steht dieser Teilmarkt im Vordergrund und wird im weiteren Verlauf der Arbeit differenzierter zu analysieren sein. Der Flotten- und Nutzfahrzeugbereich wird demnach im folgenden aus der Betrachtung ausgeschlossen. 
weitere Absatzmittler sind Reifenfachhändler, Kfz-Werkstätten, der KfzZubehör-Handel sowie Warenhäuser, SB-Märkte etc. auf dem Ersatzmarkt tätig. Zwischen den Reifenherstellern und diesen Absatzmittlern bestehen einerseits direkte Lieferbeziehungen. Andererseits nimmt ein Teil der Reifenfachhändler, neben seinem Einzelhandelsgeschäft, auch Großhandelsfunktionen wahr. Von diesen Großhändlern werden im wesentlichen kleinere Reifenhändler sowie Automobilhäuser beliefert. 95

Zu Beginn der 90er Jahre existierten in den alten Bundesländern zwischen 3200 und 3400 Reifenhandelsunternehmen. ${ }^{96}$ Nimmt man die 2500 kleineren Reifenhändler mit einem Umsatz von weniger als 0,5 Million DM/Jahr aus, verbleiben ca. 800 anerkannte Reifenfachhandelsunternehmen. 97 Über den Reifenfachhandel erfolgt mit einem Anteil von 55,2\% auch der Großteil des Reifenabsatzes an die Endabnehmer. Etwa $23 \%$ dieses Marktanteils sind unter Kontrolle der Reifenhersteller. Im Zuge einer verstärkten Vorwärtsintegration wurden selbständige Händler von Reifenherstellern aufgekauft und in Händlerketten integriert. ${ }^{98} \mathrm{Da}$ im Rahmen dieser sogenannten "controlled distribution" nicht nur herstellereigene, sondern ebenfalls Konkurrenzmarken im Sortiment geführt werden, ist die enge Verbindung zwischen Hersteller und Handel für den Endabnehmer nicht in allen Fällen ersichtlich. Weitere $17 \%$ des Marktanteils des Reifenfachhandels entfallen auf freie, nationale Großketten.

95 Vgl. Bundesverband Reifenhandel und Vulkaniseurhandwerk e. V. (Hrsg.): Erfolgskonzept 2000, Der deutsche Reifenfachhandel und sein Wettbewerbsumfeld Chancen einer zukünftig profitablen Marktbehauptung, Köln 1990, S. 81 ff. Im folgenden zitiert als BRV, Erfolgskonzepte 2000. Aus Gründen der Vollständigkeit ist in Abbildung 6 eine direkte Absatzbeziehung zwischen Reifenherstellern und gewerblichen Kunden eingetragen, die bei einzelnen Großkunden besteht.

In den neuen Bundesländern bestanden $1990 \mathrm{ca} .400$ Reifenhandelsunternehmen. Vgl. BRV, Erfolgskonzepte 2000, a.a.O., S. 106.

$97 \mathrm{Zu}$ einem Anforderungsprofil, das der BRV zur Qualitätssicherung des nicht geschützten Begriffes des Reifenfachhandels bzw. -fachbetriebs entwickelt hat, vgl. Bundesverband Reifenhandel und Vulkaniseurhandwerk e.V. (Hrsg.): "TÜV-geprüfter Reifen-Fachbetrieb - freiwillige Prüfung gemäß Anforderungsprofil des BRV und des TÜV Bayern/Sachsen", Köln 1994. Im folgenden werden die Begriffe des Reifenhändlers und Reifenfachhändlers synonym verwendet.

98 So werden von Reifenherstellern die folgenden Handelsketten kontrolliert: A3 (Bridgestone), EUROMASTER (Michelin), Holert (Dunlop), Pneumobil (Pirelli), Vergölst (Continental), Goodyear Franchise (Goodyear). 
Neben dem Reifenfachhandel stellen Automobilhandel und Kfz-Werkstätten mit 29 \% Marktanteil den zweitstärksten Vertriebsweg auf dem Reifenmarkt dar. Die verbleibende Absatzmenge teilen sich die schon zuvor genannten Vertriebswege des Kfz-Zubehör-Handels (6,0 \%), Tankstellen $(5,1 \%)$ sowie sonstige Absatzstellen (SB-Märkte, Warenhäuser etc. 4,7\%) (vgl. Abbildung 7). .99

\section{Reifenfachhandel \\ Automobilhandel/ Werkstätten}

Kfz/ZubehörHandel

Tankstellen

Sonstige

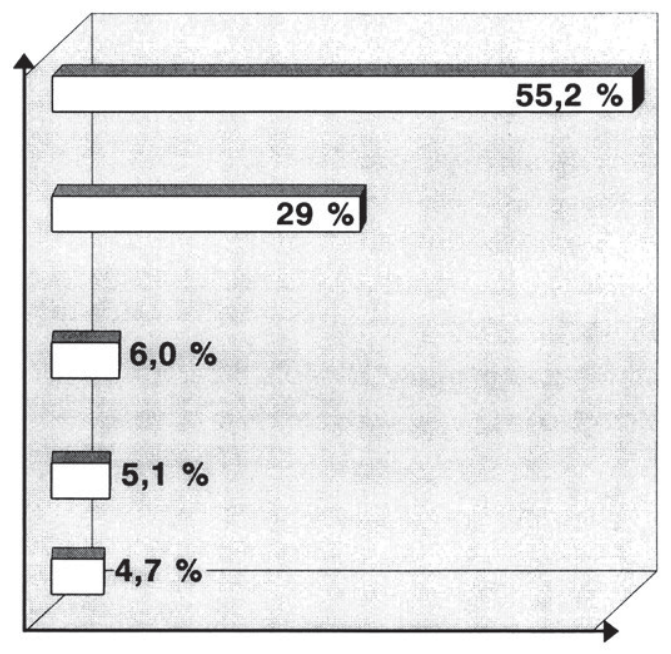

Abb. 7: Anteil der Vertriebswege auf dem deutschen Reifenmarkt im PKWPrivatkundenbereich (in 1993)

(Quelle: BRV, Reifenersatzgeschäft 1993/94)

Die Angaben zu den Marktanteilen entstammen einer unveröffentlichten Statistik eines Reifenherstellers, zu den Vertriebswegen vgl. Bundesverband Reifenhandel und Vulkaniseurhandwerk e.V. (Hrsg.): Reifenersatzgeschäft Deutschland 1993/94 Marktdaten. Im folgenden zitiert als BRV, Reifenersatzgeschäft 1993/94. Die Zahlen beziehen sich auf abverkaufte Reifen. Bezüglich der Lieferbeziehung zwischen Hersteller und den Vertriebswegen ergibt sich aufgrund der Großhandelsfunktion des Reifenfachhandels und der direkten Belieferung von Vertragshändlern durch die Automobilhersteller eine andere prozentuale Aufteilung. Vgl. hierzu BRV, Erfolgskonzepte 2000, a.a.O., S. 24, S. 69 und S. 83. 
Im Vergleich der Vertriebswege steht besonders der selbständige Reifenfachhändler unter einem hohen Wettbewerbsdruck. So sind nationale Ketten durch zentral entwickelte Unternehmenskonzepte und hohe Absatzvolumina in der Lage, umfassende Kostenvorteile zu realisieren. Der Automobilhandel verfügt über den Vorteil einer starken, fahrzeugbezogenen Bindung seiner Kunden sowie einer Spezialisierung auf bestimmte Fahrzeugmarken. Für Absatzmittler der weiteren Vertriebswege stellt der Reifenabsatz nur einen Teil des Gesamtgeschäftes dar. Im Vergleich hierzu befindet sich der Reifenfachhandel in einer relativ ungünstigen Position. Er sieht sich gefordert, bei vergleichsweise geringen Absatzmengen ein breites Sortiment an Reifenmarken, -größen und -typen für verschiedene Fahrzeuge vorrätig zu halten und ist nahezu ausschließlich vom Reifengeschäft abhängig. ${ }^{100}$

\subsection{Wettbewerbssituation und Marktentwicklung}

Die Wettbewerbssituation auf dem Reifenmarkt war in den letzten Jahren durch starke Konzentrationsprozesse geprägt. Zum Ausbau ihrer Marktanteile und zur Stärkung der Machtposition gegenüber Handel und Automobilherstellern akquirierten die weltweit operierenden Reifenhersteller in größerem Umfang Konkurrenzunternehmen. Teilten sich 1985 noch 14 Anbieter etwa $75 \%$ des Weltmarktes, waren es 1993 nur noch 6 Unternehmen. ${ }^{101}$ Auch auf dem deutschen Markt besitzen durch Unternehmenszusammenschlüsse die sechs größten Reifenhersteller einen Marktanteil von ca. $85 \%$ (vgl. Abbildung 8).

Ausgelöst durch erhebliche Überkapazitäten in der Produktion wurde der Konzentrationsprozeß von einem intensiven Preiswettbewerb begleitet. ${ }^{102}$

100 Zur Einschätzung der Situation des Reifenfachhandels vgl. auch BRV, Erfolgskonzepte 2000, a.a.O., S. 108 ff. Die der Arbeit zugrundeliegenden Informationen über die Vertriebswege beziehen sich wesentlich auf die Einschätzungen von Reifenfachhändlern (vgl. auch Kap. A. 4, FN 90 dieser Arbeit). Dementsprechend gelten die folgenden Ausführungen primär für den Absatz von Reifen über den Vertriebsweg des Reifenfachhandels.

101 Vgl. Sasseen, J., Tyre Makers on the Rack, a.a.O., S. 40.

102 Vgl. o. V., Weltgrößter Reifenhersteller erwartet schwere Zeiten, in: Handelsblatt vom 27.6 .90$, S. 23. 


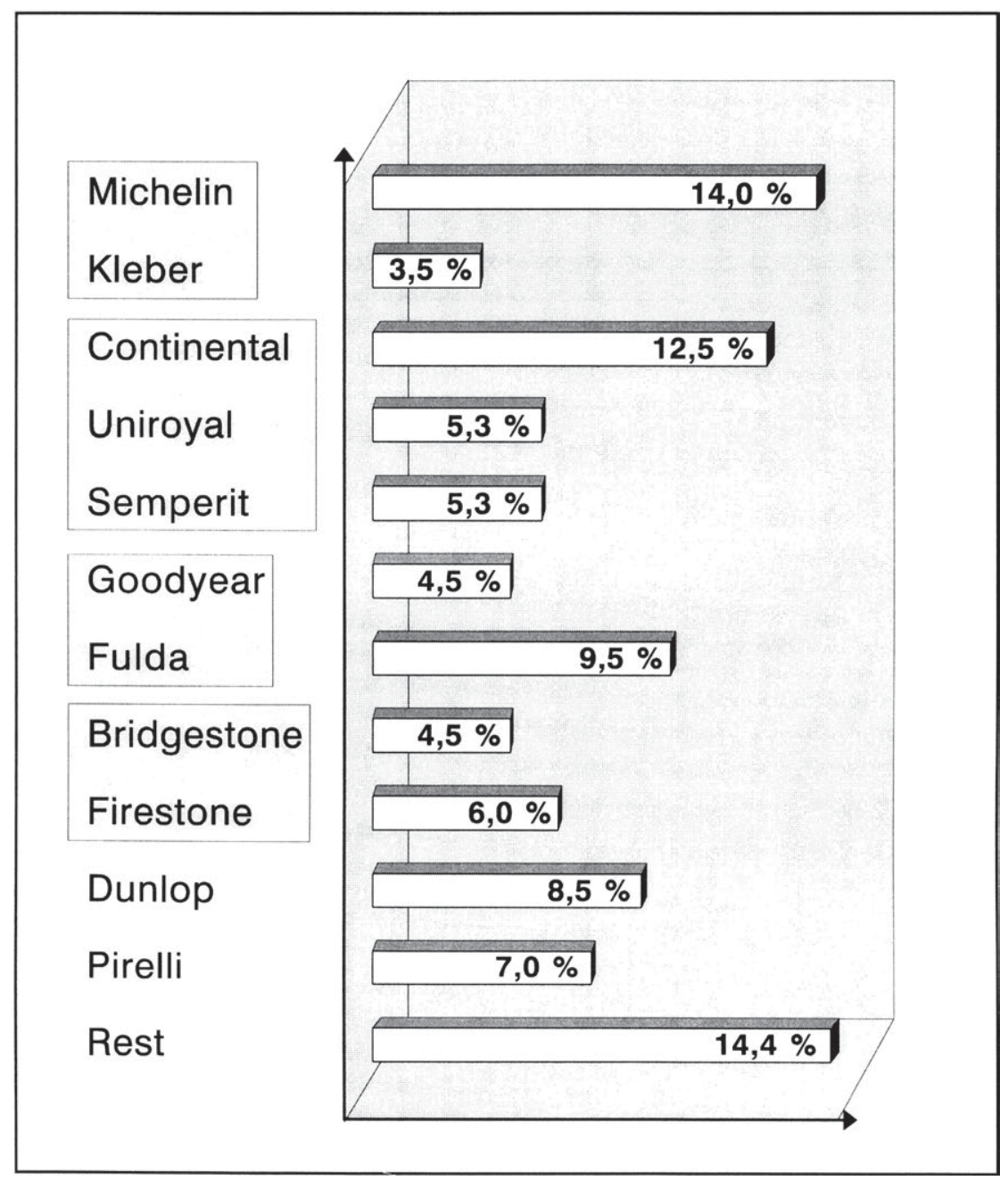

Abb. 8: Herstellermarktanteile auf dem deutschen Reifenmarkt im PKWPrivatkundenbereich (in 1993)

(Quelle: Unveroffentlichte Statistik eines Reifenherstellers)

Die Verringerung der Deckungsspannen und die Folgekosten aus den Akquisitionen führten dazu, daß zu Beginn der 90er Jahre alle bedeutenden Anbieter erhebliche Verluste verzeichneten. Wenngleich in der Folgezeit 
eine leichte Erholung des Preisniveaus eingetreten ist und tiefgreifende Rationalisierungsmaßnahmen zu einer Verbesserung der Ertragssituation beigetragen haben, ${ }^{103}$ bleibt der hohe Preis- und Ertragsdruck auf dem Reifenmarkt bestehen. ${ }^{104}$

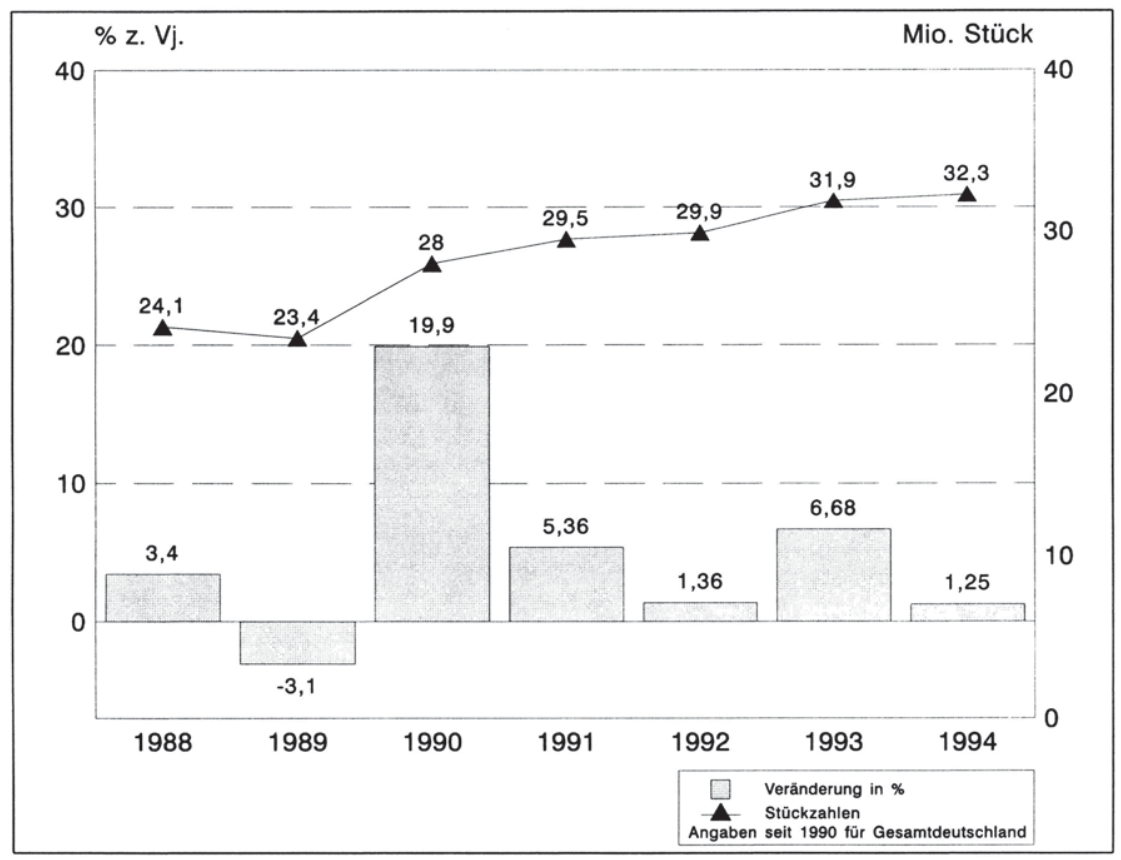

Abb. 9: Absatzvolumen PKW-Reifen im Ersatzgeschäft (Quelle: BRV, Reifengeschaft 1993/94)

103 Vgl. o. V., Continental enwartet ein schwieriges Jahr, in: FAZ vom 27.2.93, S. 16; o. V., Der Wintereinbruch hilft dem Handel mehr als den Herstellern, in: FAZ vom 27.2.1993, S. 16.

104 Vgl. o. V., Continental ist der Reifenmarkt eingebrochen, in: FAZ vom 22.10.93, S. 22 ; o. V., Reifenkonzern spürt die Wende zum Besseren, in: Handelsblatt vom 26./27.8.1994, S. 21; Wirtschaftsverband der deutschen Kautschukindustrie e. V. (Hrsg.), Kautschukindustrie: Probleme trotz leichter Konjunkturerholung, Pressenotiz vom 4.10.1994; o. V., Höherer Gewinn bei Continental, in: FAZ vom 14.3.1995, S. 19. 
Die Marktentwicklung war im Hinblick auf das Absatzvolumen in der jüngeren Vergangenheit durch ein stagnierendes, allenfalls moderat wachsendes Volumen gekennzeichnet (vgl. Abbildung 9). ${ }^{105}$ Die wesentlichen Ursachen sind in den abnehmenden Steigerungsraten des Fahrzeugbestandes, den sich nicht weiter erhöhenden, durchschnittlichen Jahreskilometerleistungen der Fahrzeuge sowie in der durch technische Verbesserungen gestiegenen Reifenlebensdauer (Reifenlaufleistung) zu sehen. Darüber hinaus zeigten die Endabnehmer rezessionsbedingt in den letzten Jahren eine starke Kaufzurückhaltung. Von einer ähnlichen Situation wird ebenfalls in der Zukunft auszugehen sein. Für die Entwicklung des Fahrzeugbestandes wird ein begrenztes Wachstum vorhergesagt, und hinsichtlich der durchschnittlichen Jahreskilometerleistung ist nach pessimistischen Einschätzungen ein Rückgang zu erwarten. ${ }^{106}$

Mit Blick auf die Technologiesituation ist die Marktentwicklung vor allem durch eine kontinuierliche Verbesserung der Fahreigenschaften sowie durch eine genaue Abstimmung der Reifen auf die Anforderungen der Automobilhersteller bestimmt. Obgleich die grundsätzlich verwendete Reifentechnologie bereits seit Beginn der 70er Jahre auf dem Konzept des Stahlgürtelreifens beruht, erneuern die Hersteller Schätzungen zufolge jährlich rund $30 \%$ ihres Reifensortimentes. Zumindest Verlautbarungen der Hersteller nach werden dabei durch veränderte Reifenprofile und Mischungsverhältnisse der Reifenbestandteile stetig verbesserte Haftungs- und Lenkeigenschaften, geringere Geräuschentwicklungen und eine Verminderung des Kraftstoffverbrauches erreicht. Mit Niederquerschnitts- und Breitreifen wurden zudem Weiterentwicklungen in den Markt eingeführt, die nicht nur technisch, sondern auch von der äußeren Form her verändert wurden. ${ }^{107}$

105 Die Absatzzahlen beziehen sich auf das Ersatzgeschäft. Der in der Statistik ausgewiesene Absatzzuwachs von 19,9 \% in 1990 beruht auf der erstmaligen Berücksichtigung der neuen Bundesländer.

106 Vgl. Belz, Chr., Konstruktives Marketing in gesättigten und schrumpfenden Märkten, a.a.O., S. 142 ff.; Deutsche Shell Aktiengesellschaft (Hrsg.): Motorisierung in Deutschland: Mehr Senioren fahren länger Auto, Shell Szenarien des PKWBestands und der Neuzulassungen bis zum Jahre 2010 mit einem Ausblick auf 2020, Hamburg 1993, S. 11 ff.; Sasseen, J., Tyre Makers on the Rack, a.a.O., S. 40 f.; BRV, Erfolgskonzepte 2000, S. 8 ff.

107 Vgl. Belz, Chr., Konstruktives Marketing in gesättigten und schrumpfenden Märkten, a.a.O., S. $141 \mathrm{ff}$.; o. V., Wir liefern nicht an Hinz und Kunz, a.a.O., S. $15 \mathrm{ff}$. 
Eine geringe Bedeutung bei PKW-Reifen besitzt nach Expertenmeinungen die anfänglich als sehr erfolgreich prognostizierte Technologie der Runderneuerung, bei der abgefahrene Reifen durch das Auftragen einer neuen äußeren Schicht wieder gebrauchsfähig werden. Obgleich in bezug auf Sicherheit und Lebensdauer einem Neureifen gleichwertig und unter Umweltgesichtspunkten vorteilhaft, konnten sich runderneuerte PKW-Reifen bislang nicht am Markt durchsetzen. Ein wesentlicher Grund dafür besteht in dem unmittelbaren Wettbewerb mit preislich ähnlich positionierten, fabrikneuen Reifen, die vom Endabnehmer im direkten Vergleich vielfach vorgezogen werden. ${ }^{108}$

Ebenso wenig erfolgreich waren bisher Versuche der Hersteller, innovative Produkttechnologien durchzusetzen, bei denen Reifen und Felgen miteinander verbunden sind und damit nicht nur verbesserte Fahreigenschaften, sondern auch eine höhere Pannensicherheit garantieren. So scheiterte zu Beginn der 80er Jahre die Einführung des sogenannten TRX-Reifensystems von Michelin, wie sich in ähnlicher Weise auch das von Continental 1987 eingeführte Conti Tire System (CTS) bislang nicht erfolgreich etablieren konnte. Trotz der verbesserten Eigenschaften zeigten weder Handel noch Erstausrüster genügend Bereitschaft, auf eine Technologie umzusteigen, für die zusätzliche Montagesysteme und spezielle Felgen notwendig sind. 109

108 Quelle: Unveröffentlichte Protokolle von Gesprächen mit Experten eines Reifenherstellers. Der prozentuale Anteil von runderneuerten PKW-Reifen am gesamten Ersatzgeschäft ist kontiniuerlich von $12 \%$ in 1988 bis auf $7 \%$ in 1993 gesunken. Bei den hier nicht weiter betrachteten LKW-Reifen lag dieser Anteil dagegen in 1993 bei $44 \%$. Vgl. BRV, Reifenersatzgeschäft 1993/94.

109 Das CTS-System ist nach Herstellerauskunft der herkömmlichen Reifentechnologie nicht nur in allen Laufeigenschaften überlegen, sondern garantiert nach einem Defekt überdies einen Pannenlauf von $400 \mathrm{~km}$ und ermöglicht damit einen Verzicht auf das Reserverad. Vgl. Belz, Chr., Konstruktives Marketing in gesättigten und schrumpfenden Märkten, a.a.O., S. 198 ff.; o. V., Wir liefern nicht an Hinz und Kunz, a.a.O., S. $15 \mathrm{ff}$. 


\subsection{Endabnehmerbezogene Situation}

\subsection{Bedarfsverhalten}

Im Bedarf des Endabnehmers kommt der auf eine Produktart oder Marke konkretisierte Wunsch zur Befriedigung eines Bedürnnisses zum Ausdruck. ${ }^{110}$ Als auslösendes Moment des Kaufprozesses stellt die Wahrnehmung eines Bedarfs den Zeitpunkt dar, zu dem der Endabnehmer bereit ist, gedanklich den Kauf zu planen, Informationssuch- und Bewertungsprozesse einzuleiten und schließlich den Kaufakt zu vollziehen. ${ }^{111}$ Ausschlaggebend für den Hersteller sind die Beeinflußbarkeit des Zeitpunktes der Bedarfswahrnehmung sowie die Frage nach der Art und der Intensität des Bedarfs.

Die Reifenbranche kann als typisches Beispiel für einen Markt gelten, bei dem der Zeitpunkt der Bedarfswahrnehmung weitgehend außerhalb des Einflußbereiches des Herstellers liegt. 112 Obwohl der Reifen als Verbindungselement zwischen Fahrzeug und Straße ein wichtiger Sicherheitsfaktor ist und inm überdies eine durchaus anspruchsvolle Technologie zugrunde liegt, ist nahezu der gesamte Bedarf durch technische Faktoren bestimmt.

Der Reifenkauf wird als verschleißbedingter Ersatzkauf betrachtet, der bei Defekt oder bei Unterschreiten einer gesetzlich vorgeschriebenen Mindestprofiltiefe notwendig wird. Insofern handelt es sich um einen "Mußkauf", der dem Kunden keinen eigenen Nutzen stiftet, sondern im Gegenteil mit z. T.

110 Bedürfnisse werden in der Literatur sowohl als empfundene Mangelzustände als auch als autonom entstehende oder durch Sozialisation gelernte Antriebskräfte im Inneren des Menschen beschrieben. Vgl. Nieschlag, R., Dichtl, E., Hörschgen, H., Marketing, 15. Aufl., Berlin 1988, S. $144 \mathrm{ff}$.

111 Vgl. Amstutz, A. E., Computer Simulation of Competitive Market Response, Cambridge (Mass.), London 1976, S. 185.

112 Vgl. Belz, Chr., Konstruktives Marketing in gesättigten und schrumpfenden Märkten, a.a.O., S. 155. 
recht hohen Kosten verbunden ist. ${ }^{113}$ So zeigt auch die Befragung von Reifenkäufern nach den Gründen für ihren Kauf folgendes Ergebnis:114

- Reifen abgefahren (70,3\%)

- Reifen beanstandet von TÜVIDEKRA (15,1\%)

- Reifen beschädigt $(9,8 \%)$

- Reifenpanne (4,2\%)

- Reifen gefallen nicht mehr $(0,6 \%)$

Von den Herstellern könnte grundsätzlich versucht werden, den Endabnehmer hinsichtlich des technischen Zustandes seines Reifens und der damit verbundenen Sicherheitsproblematik zu sensibilisieren. Es wäre auf die Risiken einer Unterschreitung der Mindestprofiltiefe hinzuweisen und zudem eine größere Mindestprofiltiefe als die gesetzlich vorgeschriebene anzuraten. Hierbei besteht allerdings die Gefahr, daß die Art des ausgelösten Bedarfs sich eher auf die Produktart des Reifens denn auf eine bestimmte Marke bezieht. Da aus Sicht des Endabnehmers keine ausreichende Differenzierung zwischen den angebotenen Reifen besteht, ${ }^{115}$ werden Maßnahmen des Herstellers zu einem großen Teil markenunspezifische Stimulierungswirkungen auslösen, die nicht nur der eigenen, sondern ebenso konkurrierenden Marken zugute kommen. Zudem wäre damit zu rechnen, daß die Bedarfsintensität, d. h. die Stärke des Kaufwunsches, gering ist. Der Endabnehmer wird trotz eines stimulierenden Impulses vom Kauf absehen, wenn inm hierdurch größerer Beschaffungsaufwand im Sinne von Such- und Wegezeit entsteht. Die Einflußnahme auf das Bedarfsverhalten seitens des Herstellers setzt damit voraus, daß der Bedarf von vornherein im Hinblick auf die eigene Marke kanalisiert und ohne weiteren Beschaffungsaufwand für den Endabnehmer in einen Kauf umgesetzt werden kann.

113 Der Durchschnittspreis für PKW-Sommerreifen betrug 1993135 DM pro Reifen. Hinzu kommen Kosten für die Montage in Höhe von ca. 10 DM. Vgl. BRV, Erfolgskonzepte 2000 , S. 84.

114 Die Ergebnisse stammen aus einer unveröffentlichten Studie eines Reifenherstellers (Stichprobengröße $n=1005$ ).

Vgl. Kap. B. 1.33 dieser Arbeit. 


\subsection{Einkaufsstättenwahlverhalten}

Der Entscheidung des Endabnehmers für die Wahl einer bestimmten Einkaufsstätte liegt ein Beurteilungsprozeß zugrunde, bei dem die bestehenden Alternativen im Hinblick auf unterschiedliche Kriterien bewertet werden. Die Vielzahl der in der Literatur in diesem Zusammenhang aufgeführten Beurteilungskriterien läßt sich dabei wie folgt systematisieren:116

- Leistungskriterien stellen auf Merkmale ab, die unmittelbar zur Charakterisierung der Leistung von Einkaufsstätten herangezogen werden. Hierzu zählen u. a. die Tiefe und Breite des Sortiments ${ }^{117}$, das Preisniveau und die Preisgünstigkeit ${ }^{118}$ sowie die Servicequalität. ${ }^{119}$

- Erreichbarkeitskriterien berücksichtigen den Zeit- und Kostenaufwand, der mit dem Weg zur Einkaufsstätte verbunden ist. Dazu gehören Kriterien wie Entfernung zur Einkaufsstätte, Verkehrsanbindung zu deren Standort etc.

- Verfügbarkeitskriterien beziehen sich schließlich auf das konkrete Vorhandensein einer bestimmten, zum Kauf beabsichtigten Marke, wobei zwischen ständiger und temporärer Verfügbarkeit unterschieden werden kann.

116 Für eine Übersicht von Beurteilungskriterien zur Einkaufsstättenwahl und Möglichkeiten zu deren Systematisierung vgl. Heinemann, M., Einkaufsstättenwahl und Firmentreue des Konsumenten, Wiesbaden 1976, S. 153 ff. sowie die dort angegebene Literatur.

117 Mit der Sortimentstiefe wird die Zahl alternativer Kaufmöglichkeiten gekennzeichnet, während mit der Sortimentsbreite das Angebot additiver Kaufmöglichkeiten beschrieben wird. Vgl. Gümbel, R., Die Sortimentspolitik in den einzelnen Betrieben des Wareneinzelhandels, a.a.O., S. 62.

118 Während das Preisniveau kennzeichnend für die grundsätzliche "Leistungsklasse" ist, bezieht sich die Preisgünstigkeit auf die Position des Handelsunternehmens innerhalb einer solchen Leistungsklasse. Vgl. Diller, H., Preisimage und Preiswerbung im Reifenhandel, Arbeitspapier Nr. 18 des Lehrstuhls für Marketing an der Universität Erlangen-Nürnberg, Nürnberg 1993.

119 Die Servicequalität umfaßt Eigenschaften wie Kompetenz und Vertrauenswürdigkeit des Verkaufspersonals, sachliche und personelle Ausstattung, Verläßlichkeit in der Ausführung von Serviceleistungen etc. Vgl. Berry, L. L., Zeithaml, V. A., Parasuraman, A., Five Imperatives for Improving Service Quality, in: Sloan Management Review, No. 4, 1990, S. 29 ff. 
Die relative Gewichtung dieser drei Kriteriengruppen gibt Aufschluß darüber, welche Bedeutung die Wahl der Einkaufsstätte für den Endabnehmer beim Reifenkauf besitzt und zeigt darüber hinaus auf, welchen Aufwand dieser generell für den Weg zur Einkaufsstätte zu tragen bereit ist. Für den Reifenmarkt stellt Abbildung 10 die wichtigsten Gründe dar, die von Reifenkäufern für die Wahl einer Einkaufsstätte genannt werden. ${ }^{120}$

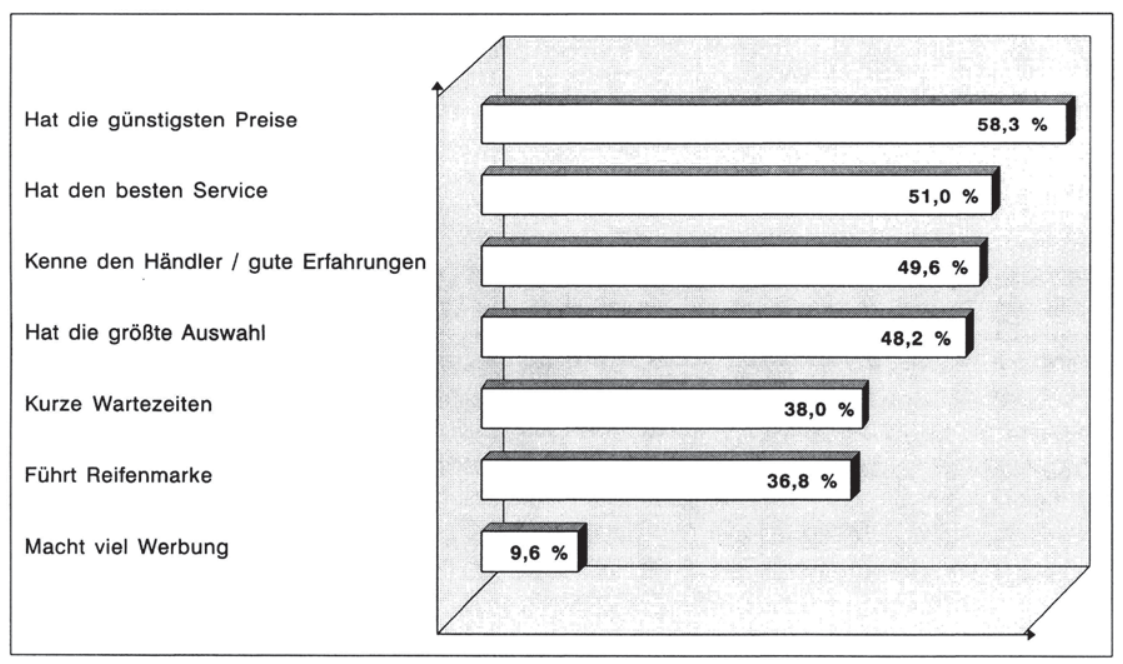

Abb. 10: Gründe für die Wahl der Einkaufsstätte (Quelle: Unveröffentlichte Studie eines Reifenherstellers)

Nahezu die Hälfte der befragten Personen gibt als Grund ihrer Einkaufsstättenwahl an, gute Erfahrungen mit einem bestimmten Reifenhändler in der Vergangenheit gemacht zu haben. Da es beim Reifenkauf zu einem persönlichen Kontakt zwischen Verkaufspersonal und Endabnehmer kommt, der zudem häufig die Form eines beratenden Gespräches annimmt, drückt sich in den positiven Erfahrungen nicht nur die Zufriedenheit mit der Leistung des Händlers aus, sondern auch ein Vertrauen in dessen Empfehlungsverhalten. In diesem Zusammenhang ist zu berücksichtigen, daß die recht hohen

120 Befragt wurden Kunden des Reifenfachhandels. Mehrfachnennungen waren möglich. Die Ergebnisse entstammen einer unveröffentlichten Studie eines Reifenherstellers $(n=1005)$. 
Kosten des Reifenkaufs, die lange Nutzungsdauer eines Reifens sowie die geringen Produktkenntnisse des Endabnehmers zu einem relativ hohen Kaufrisiko führen. ${ }^{121}$ Daher ist davon auszugehen, daß der Endabnehmer eine hohe Bindung an den Händler seines Vertrauens zeigt und ein Wechsel allein aufgrund einer bestimmten Reifenmarke eher unwahrscheinlich ist. Ein hoher Distributionsgrad ist demnach Voraussetzung für den Absatzerfolg.

Fast die Hälfte der Reifenkäufer nannte die Auswahl an angebotenen Reifen als Kriterium für die Wahl des Reifenhändlers. Die Sortimentsleistung der Einkaufsstätte nimmt hier deutlich mehr Einfluß auf das Kaufverhalten des Endabnehmers als die einzelne Marke. Im Hinblick auf diese Käufergruppe ist der Vertrieb über Absatzmittler, die aufgrund ihres geringen Sortimentsumfanges eine starke Förderung der Marke des Herstellers ermöglichen, wenig erfolgversprechend. Geeignet ist vielmehr ein Konkurrenzumfeld am Point of Sale, bei dem eine hohe Sortimentstiefe besteht, die eigene Marke jedoch eine herausragende Stellung einnimmt.

Bezüglich der Anforderungen an die Erreichbarkeit der Einkaufsstätte sind zwei Aspekte grundlegend. Zum einen dürfte die geringe Wertschätzung des Produktes "Reifen" dazu führen, daß der Endabnehmer eine möglichst unkomplizierte und schnelle Abwicklung seines Kaufvorganges anstrebt und damit auf möglichst nahegelegene Einkaufsstätten zurückgreift. Zum anderen sind durch die Motorisierung auch mittlere Entfernungen innerhalb einer Stadt schnell überbrückbar, so daß der Endabnehmer nicht auf die unmittelbar nächstgelegene Einkaufsstätte angewiesen ist. Prinzipiell kann somit angenommen werden, daß zumindest ein Distributionsgrad bestehen muß, bei dem die Einkaufsstätte innerhalb weniger Autominuten erreicht werden kann.

Die Verfügbarkeit einer bestimmten Reifenmarke wird von $36,8 \%$ der Befragten als Grund für die Wahl der Einkaufsstätte genannt. Bei diesen Personen ist anzunehmen, daß sie vor dem Kauf Informationen darüber einholen, wo die Marke geführt wird. Sie werden sich bei ihrer Auswahl auf

$121 \mathrm{Zu}$ einer ausführlichen Diskussion von Kaufrisiken und deren Bedeutung für das Informationsverhalten vgl. Katz, R., Informationsquellen der Konsumenten, Eine Analyse der Divergenzen zwischen Beurteilung und Nutzung. Wiesbaden 1983, S. $77 \mathrm{ff}$. 
die entsprechenden Einkaufsstätten beschränken oder bei Nichtverfügbarkeit der Marke in eine andere Einkaufsstätte wechseln. Damit zeigt sich bei einer nicht unerheblichen Zahl von Käufern, daß die Absicht, eine bestimmte Marke zu kaufen, ausreichend stark ist, um einen gewissen Such- und Wegeaufwand zu akzeptieren.

\subsection{Informationsquellennutzung und Markenwahlverhalten}

Die Bildung der einer Markenwahl zugrundeliegenden Präferenzen ${ }^{122}$ setzt Informationen über die Eigenschaften der angebotenen Alternativen und die Möglichkeit zu deren Bewertung voraus. Daher wird der Endabnehmer nach Wahrnehmung eines produktartbezogenen Bedarfs einen Informationssuchprozeß einleiten, dessen Intensität von bereits bestehenden Präferenzen, dem bisherigen Informationsstand, der Bedeutung des Kaufes sowie dem wahrgenommenen Kaufrisiko abhängig ist. ${ }^{123}$

Grundsätzliche Informationsquellen, die bereits in der Vergangenheit zum gegenwärtigen Informationsstand beigetragen haben oder aber zu dessen Erweiterung genutzt werden können, sind die eigenen Erfahrungen, die Kommunikation des Herstellers, das Produkt selbst, die Beratung im Handel, das soziale Umfeld (Freunde, Bekannte) und schließlich neutrale Organisationen sowie deren Publikationen (Verbraucherberatung, Fachzeitschriften). ${ }^{124}$

122 Zum Begriff der Präferenz vgl. Kap. B. 2.212 dieser Arbeit.

123 Das Ausmaß und die Intensität, mit der ein Endabnehmer neue Informationen aktiv sucht oder passiv aufnimmt, werden häufig anhand der Typen der extensiven, limitierten und habitualisierten sowie der impulsiven Kaufentscheidung erklärt. Vgl. hierzu ausführlich Weinberg, P., Das Entscheidungsverhalten von Konsumenten, Paderborn 1981.

124 Zur Systematisierung von Informationsquellen vgl. Meffert, $H$., Die Beurteilung und Nutzung von Informationsquellen beim Kauf von Konsumgütern, in: Konsumentenverhalten und Informationen, Hrsg.: Meffert, H., Steffenhagen, H., Freter, H., Wiesbaden 1979, S. 42 ff. Aus den Informationsquellen kann der Endabnehmer zum einen objektive Angaben über die Markeneigenschaften und die relevanten Beurteilungskriterien gewinnen, zum anderen aber auch bestehende Meinungen und Bewertungen übernehmen, die von einem differenzierten Markenbild bis hin zu einer eindimensionalen Empfehlung für oder gegen eine Marke reichen können. Vgl. Kroeber-Riel, W., Konsumentenverhalten, a.a.O, S. 264. 
Die Art der genutzten Informationsquellen sowie deren Einfluß auf die Präferenzbildung zeichnet nicht nur die relevanten Wege der Beeinflussung des Endabnehmers vor, sondern zeigt darüber hinaus das zu erwartende Markenwahlverhalten auf. Dabei geht es nicht darum, die Frage der Markenwahl in Abhängigkeit von den Eigenschaften der Marke zu beantworten. Hier ist vielmehr festzustellen, über welche Informationskanäle und in welchem Maße der Endabnehmer in seiner Wahlentscheidung beeinflußbar ist.

Für den Reifenmarkt liegt eine Studie vor, in der Reifenkäufer nach den Gründen für ihre Kaufentscheidung befragt wurden. Hieraus läßt sich unmittelbar das Nutzungsverhalten der Endabnehmer im Hinblick auf die verschiedenen Informationskanäle ableiten (vgl. Abbildung 11). ${ }^{125}$

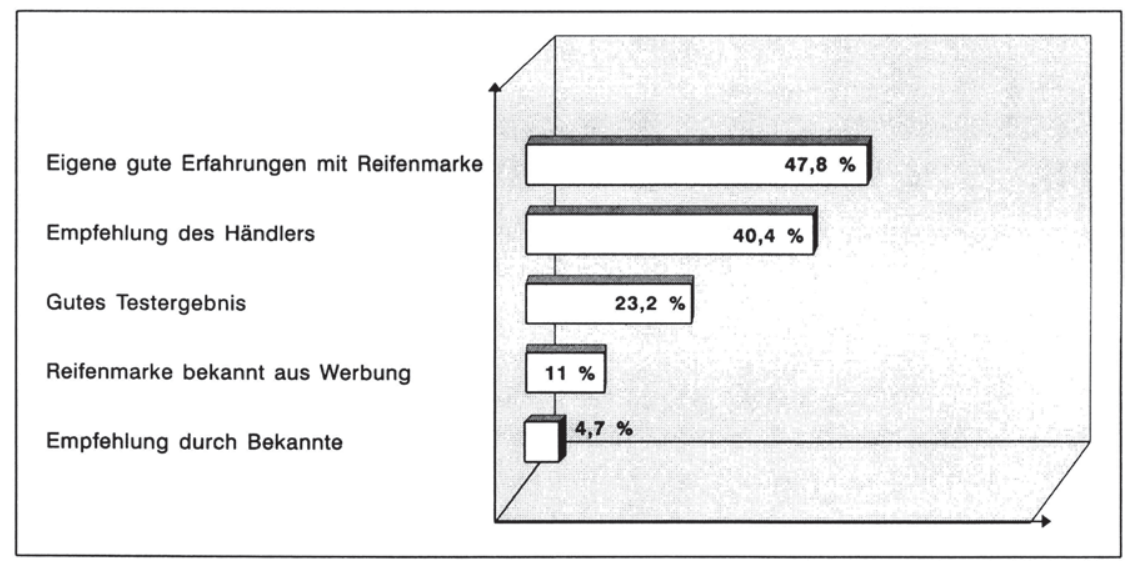

Abb. 11: Einfluß von Informationsquellen auf die Kaufentscheidung beim Reifenkauf

(Quelle: Unveroffentlichte Studie eines Reifenherstellers)

Der eigenen positiven Erfahrung mit der Reifenmarke kommt offensichtlich die höchste Bedeutung für die Präferenzbildung zu. Grundsätzlich ist für Endabnehmer, die sich wesentlich auf eigene Erfahrungen stützen, anzu-

125 Es wurden Kunden des Reifenfachhandels nach den jeweils drei wichtigsten Gründen für ihre Kaufentscheidung befragt. Mehrfachnennungen waren möglich. Die Ergebnisse stammen aus einer unveröffentlichten Studie eines Reifenherstellers $(n=1005)$. 
nehmen, daß sie bereits mit einer bestimmten Kaufabsicht für eine Marke die Einkaufsstätte betreten. Der Käufer rekurriert auf den Eindruck, den er bei der Nutzung des Reifens gewonnen hat und entscheidet sich bei Zufriedenheit für dieselbe Marke. Besonders ausgeprägt ist dieses Verhalten beim Wechsel nur eines Reifens, bei dem quasi nur eine Ergänzung der vorhandenen Reifenausstattung vorgenommen wird und keine eigenständige Markenwahlentscheidung stattfindet. Beachtenswert ist dabei in diesem Markt, daß die am Fahrzeug montierte Reifenmarke nicht zwingend auf eine frühere Wahlentscheidung des Reifenkäufers zurückzuführen ist, sondern die Reifen häufig im Rahmen des Fahrzeugneu- und -gebrauchtkaufes übernommen werden.

Eine ebenfalls sehr hohe Bedeutung kommt der persönlichen Beratung in der Einkaufsstätte zu. Dabei ist davon auszugehen, daß neben den 40,4\% der befragten Personen, die die Empfehlung des Händlers als einen der wesentlichen Gründe für ihre Markenwahl nennen, noch ein wesentlich größerer Personenkreis diese Informationsquelle zur Beschaffung vermeintlich objektiver Informationen nutzt, um auf deren Grundlage zu einer eigenständigen Bewertung der Alternativen zu gelangen. Hiermit wird der hohe Einfluß des Händlers auf die Präferenzbildung und Kaufentscheidung des Endabnehmers unterstrichen.

Die Empfehlung neutraler Quellen, z. B. in Form von Testergebnissen, wird von fast einem Viertel aller Befragten als kaufentscheidend betrachtet. Die Pflege von Kontakten des Herstellers zu Organisationen, die vom Endabnehmer als objektiv angesehen werden und entsprechende Beratungen durchführen bzw. Publikationen herausgeben, scheint somit wesentlich zum Absatzerfolg einer Marke beizutragen. Allerdings bleibt zu hinterfragen, ob der Endabnehmer sich vor dem Kauf über entsprechende Testergebnisse informiert oder hiervon erst in der Einkaufsstätte durch entsprechende Werbematerialien des Herstellers oder die Beratung des Händlers erfährt. ${ }^{126}$

Auffallend ist, daß die Herstellerkommunikation nur von wenigen Befragten als Informationsquelle genannt wird. Dies kann durch die geringe Wahrnehmung von Herstellerinformationen seitens der Endabnehmer erklärt wer-

126 Zum Einfluß von Warentestergebnissen auf das Kaufverhalten siehe ausführlich Fritz, W., Warentest und Konsumgütermarketing, Wiesbaden 1984. 
den. Aufgrund des geringen Interesses, das dem Reifen als Produkt entgegengebracht wird, ist der Endabnehmer im wesentlichen nur in der Phase des aktuellen Bedarfs bereit, entsprechende Informationen aufzunehmen. ${ }^{127}$ Diese Phase dürfte vergleichsweise kurz sein, da es nach Auftreten des Bedarfs, aufgrund der dann bestehenden sicherheitstechnischen und gesetzlichen Notwendigkeiten des Reifenwechsels, relativ schnell zum Kauf kommen wird.

Eine weitere Erklärung beruht darauf, daß die Inhalte der Kommunikation des Herstellers als ungeeignet zur Bewertung von Reifen gesehen werden, da sie keinen informativen Charakter besitzen. ${ }^{128}$ Schließlich ist als dritte Möglichkeit eine relativ geringe Glaubwürdigkeit zu nennen, die herstellerkontrollierten Informationen seitens der Endabnehmer zugemessen wird. ${ }^{129}$

Von untergeordneter Bedeutung für den Reifenkauf sind Informationen aus dem sozialen Umfeld. Lediglich 4,7\% der Reifenkäufer nennen die daraus resultierenden Empfehlungen als wichtigen Grund für ihre Kaufentscheidung.

\subsection{Handelsbezogene Situation}

\subsection{Rollenverständnis des Handels}

Das Verhalten des Handels bei der Entscheidung über die Aufnahme und Förderung einer Marke wird wesentlich durch dessen grundsätzliches Rollenverständnis geprägt. Damit sind die Aufgaben und Funktionen angesprochen, die der Handel im Rahmen des Distributionssystems über-

127 Das situationsabhängige Interesse an einem Produkt wird in der Literatur auch als situatives Involvement beschrieben. Vgl. Trommsdorff, V., Konsumentenverhalten, Stuttgart u. a. 1989, S. 47; Kroeber-Riel, W., Konsumentenverhalten, a.a.O, S. 376. Zur Aktivierung der Informationsaufnahmebereitschaft vgl. Kroeber-Riel, W., Strategie und Technik der Werbung, Verhaltenswissenschaftliche Ansätze, 3. Aufl., Stuttgart u.a. 1991, S. $98 \mathrm{ff}$.

128 Zu Beispielen der Reifenwerbung vgl. auch Kroeber-Riel, W., Strategie und Technik der Werbung, a.a.O., S. $84 \mathrm{ff}$.

129 Zur Glaubwürdigkeit unterschiedlicher Informationsquellen vgl. die Ergebnisse von Katz, R., Informationsquellen der Konsumenten, a.a.O., S. 40. 
nimmt. ${ }^{130}$ Der Handel kann sich einmal als Marktvermittler verstehen, womit seine primäre Aufgabe in der Bereitstellung der Marke und entsprechender Informationen des Herstellers an einem für den Endabnehmer zugänglichen Ort besteht. ${ }^{131}$ In einer solchen Rolle ist der Handel an der Aufnahme von Marken interessiert, deren Abverkauf keine besonderen eigenen Anstrengungen erfordert und deren Umsatz durch die Nachfrage des Endabnehmers mit einem vergleichsweise hohen Grad an Verläßlichkeit gesichert ist. Demgegenüber beinhaltet die Rolle des Marktgestalters die aktive Stimulierung des Endabnehmerbedarfs und Beeinflussung der Kaufentscheidung durch den Handel. Um Gestaltungsfreiräume zu erhalten, werden Marken bevorzugt, für die zwar eine Akzeptanz seitens der Endabnehmer besteht, bei denen der Handel über Art und Umfang des Angebotes jedoch möglichst eigenständig entscheiden kann. Zudem werden primär Marken geführt, deren Hersteller bereit sind, die Schaffung und Aufrechterhaltung der Endabnehmernachfrage durch den Handel in Form materieller oder immaterieller Leistungen zu honorieren. ${ }^{132}$

Abbildung 12 zeigt Kriterien sowie deren Bedeutung, die vom Reifenfachhandel zur Beurteilung von Marken herangezogen werden. Diese stellen die Grundlage für Entscheidungen des Händlers über die Aufnahme und Förderung einer Marke dar. ${ }^{133}$ Auffallend ist zunächst, daß der Lieferservice und die Qualität des Reifens für wichtiger erachtet werden als die Ertragsmöglichkeiten. Der besondere Stellenwert des Lieferservice erklärt sich daraus, daß aufgrund der Vielzahl an Produktvarianten die Lagerreichweite der ein-

130 Zur Analyse des Rollenverständnisses von Teilnehmern des Distributionssystems vgl. Steffenhagen, H., Konflikt und Kooperation in Absatzkanälen, a.a.O., S. 85 ff.; Gümbel, R., Handel, Markt und Ökonomik, Wiesbaden 1985, S. 59 ff.

131 In der Literatur werden die Funktionen der Markterschließung, -beeinflussung und Marktforschung auch als Vermittleraufgaben des Handels beschrieben. Diese Begriffsverwendung scheint jedoch wenig zutreffend, soweit der Handel eigenständig agiert und nicht etwa nur Informationen vom Hersteller zum Endabnehmer und umgekehrt übermittelt. Insofern soll hierfür der Begriff des Marktgestalters verwendet werden. Vgl. Oehme, W., Handelsmarketing, 2. Aufl., München 1992, S. 141; Barth, K., Betriebswirtschaftslehre des Handels, Wiesbaden 1988, S. 447; Hansen, U., Absatz- und Beschaffungsmarketing des Einzelhandels, a.a.O., S. 15.

132 Die Rollen des Marktvermittlers und -gestalters sind als Extreme eines Kontinuums möglicher Rollenverständnisse des Händlers zu verstehen.

133 Befragt wurden Reifenfachhändler. Die Ergebnisse stammen aus einer unveröffentlichten Studie eines Reifenherstellers $(n=436)$. 
zelnen Variante beschränkt ist und der Handel somit auf eine zuverlässige und schnelle Belieferung zur Aufrechterhaltung seiner eigenen Lieferfähigkeit angewiesen ist. Die Anforderung an die Qualität des Reifens beruht auf der Befürchtung, daß schlechte Erfahrungen des Endabnehmers mit einer Marke in unmittelbaren Zusammenhang mit der Beratungsleistung des Handels gebracht werden und außerdem zusätzlicher Aufwand durch Reklamationen entsteht.

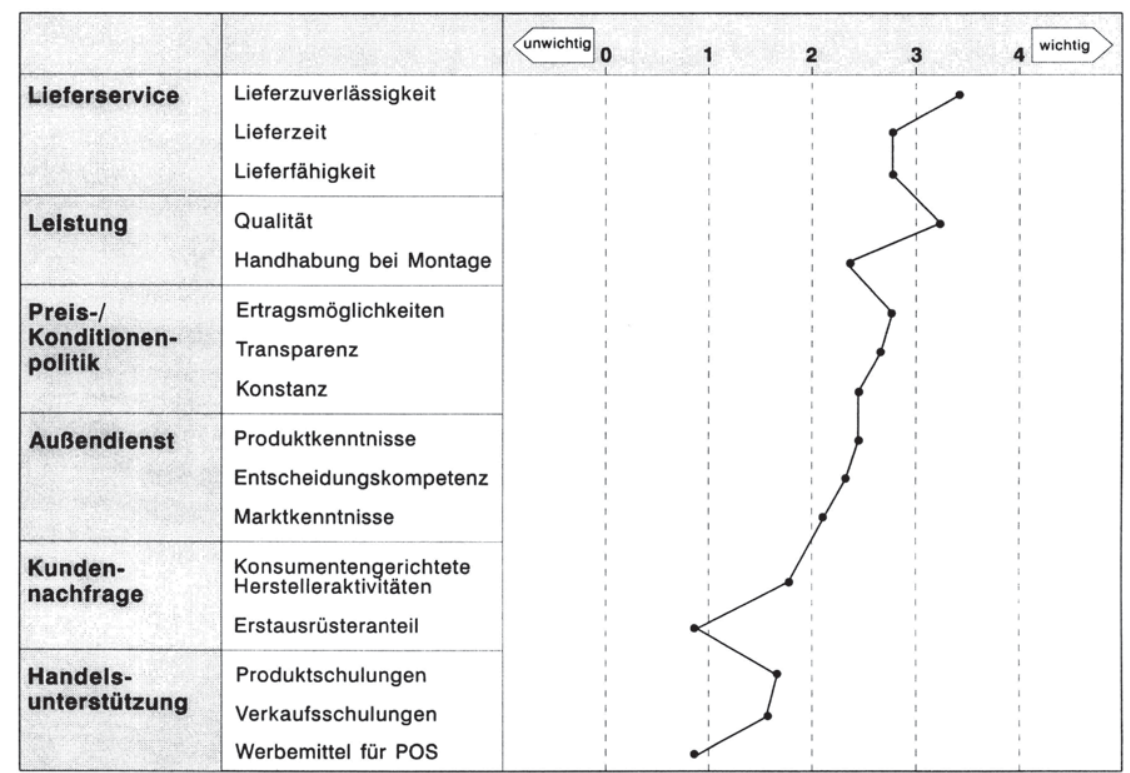

Abb. 12: Kriterien zur Beurteilung von Marken durch Reifenfachhändler (Quelle: Unveroffentlichte Studie eines Reifenherstellers)

Insgesamt zeigt sich bei den weiteren Kriterien, daß eher solche Leistungen des Herstellers bevorzugt werden, die zu den Instrumenten des Hineinverkaufs zählen. Hierzu gehören gute Ertragsmöglichkeiten des Handels, die Erleichterung der Kalkulation durch Transparenz und Konstanz der Preis- und Konditionenpolitik sowie die kompetente Beratung durch den Außendienst. Demgegenüber werden die Unterstützung beim Herausverkauf oder gar die Schaffung einer eigenständigen Kundennachfrage als weniger wichtig erachtet. Dies läßt den Schluß zu, daß der Reifenfachhandel sich eher als Marktgestalter versteht. 
Diese Erkenntnisse spiegeln sich auch im Empfehlungsverhalten des Handels wider. Aus Expertengesprächen mit Reifenfachhändlern wird deutlich, daß der Händler nicht nur bei Käufern ohne konkrete Kaufabsicht die Markenwahl steuert, sondern auch Gegenempfehlungen bei solchen Kunden ausspricht, die bereits eine Vorauswahl für eine Marke getroffen haben. ${ }^{134}$ Damit wird in erheblichem Maße Einfluß auf die Kaufentscheidung des Endabnehmers genommen, wobei der Handel gezielt ausgewählte Marken in den Vordergrund stellt. Hinsichtlich der Anteile, zu denen die einzelnen Marken vom Handel bevorzugt empfohlen werden, zeigen sich recht deutliche Unterschiede (vgl. Abbildung 13). ${ }^{135}$

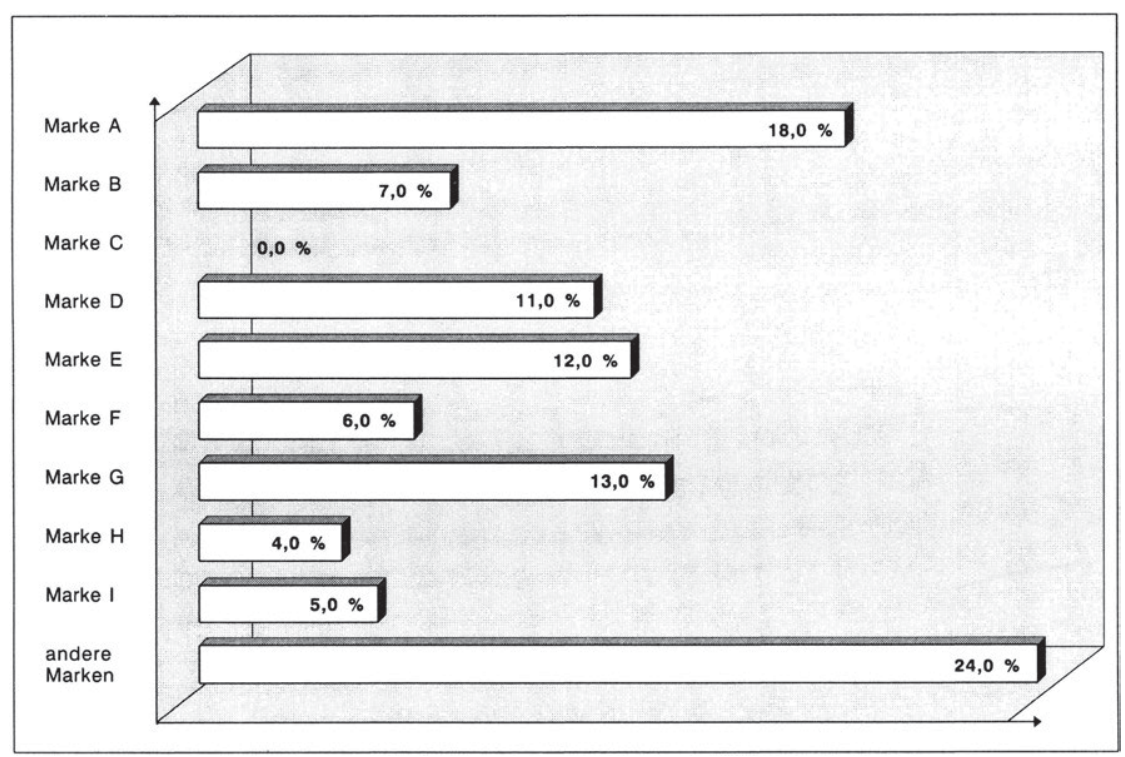

Abb. 13: Vom Händler bevorzugt im Verkaufsgespräch empfohlene Marke (Quelle: Unveroffentlichte Studie eines Reifenherstellers)

134 Quelle: Unveröffentlichte Protokolle von Expertengesprächen mit Reifenfachhändlern.

135 Befragt wurden Reifenfachhändler. Die Ergebnisse stammen aus einer unveröffentlichten Studie eines Reifenherstellers $(n=200)$. 
Allerdings läßt sich aus Expertengesprächen auch entnehmen, daß sich die Empfehlungen des Handels z. T. an der aktuell am Fahrzeug montierten Reifenmarke orientieren. Hier schränkt sich die Rolle des Marktgestalters dahingehend ein, daß bei der Beratung der Weg des geringsten Widerstandes gesucht wird. Dieser wird in der Empfehlung einer Marke vermutet, mit der vom Reifenkäufer bereits zufriedenstellende Erfahrungen gemacht wurden.

\subsection{Machtbeziehungen zwischen Hersteller und Handel}

Die Beziehung von Hersteller und Handel wird wesentlich durch die Machtpotentiale der beiden Marktteilnehmer bestimmt. Diese kommen in den Möglichkeiten zum Ausdruck, das Verhalten des jeweils anderen positiv oder negativ zu sanktionieren. In der Literatur wird daher auch von Belohnungs- und Bestrafungsmacht gesprochen. ${ }^{136}$ Das Ausmaß des Machtpotentials ergibt sich aus der Abhängigkeit eines Marktteilnehmers von den Entscheidungen des jeweils anderen. Dabei kann die Abhängigkeit als potentielle, relative Veränderung des Zielerreichungsgrades verstanden werden, die mit der Einflußnahme verbunden ist.

Für das Verhalten von Hersteller und Handel ist allerdings weniger die isolierte Betrachtung der Höhe des Machtpotentials als vielmehr die Machtverteilung von Bedeutung. In einem symmetrischen Machtverhältnis ist die Abhängigkeit beider Marktteilnehmer voneinander gleich hoch ausgeprägt. Weder Handel noch Hersteller können Forderungen gegenüber dem anderen durchsetzen, ohne Gegenforderungen in gleicher Höhe erfüllen zu müssen. Demgegenüber ist beim asymmetrischen Machtverhältnis die Abhängigkeit eines der Marktteilnehmer geringer. Entsprechend dem Grad der

136 Eine weit verbreitete, auf French und Raven zurückgehende Klassifikation von Machtgrundlagen nennt darüber hinaus als weitere Arten der Macht die legitimierte Macht, die Identifikationsmacht sowie die Expertenmacht. Diese können jedoch nicht der Begriffsebene der Belohnungs- und Bestrafungsmacht zugeordnet werden. Vielmehr handelt es sich um eine Beschreibung der Machtbasis und der damit zusammenhängenden Einflußmöglichkeiten, die sich letztlich stets in Form einer materiellen oder immateriellen Belohnung oder Bestrafung niederschlagen. Vgl. French, J. R. P., Raven, B., The Bases of Social Power, in: Study in Social Power, Hrsg.: Cartwright, D., 3. Aufl., Ann Arbor 1959, S. 150 ff.; Kümpers, U. A., Marketingführerschaft, a.a.O., S. 109. Zu einer Übersicht von Sanktionsgrundlagen vgl. auch Ahlert, D., Distributionspolitik, a.a.O., S. $104 \mathrm{ff}$. 
Überlegenheit steht diesem die Möglichkeit offen, Einfluß auf die Entscheidungen des anderen Marktteilnehmers zu nehmen, ohne seinerseits mit entsprechenden Gegenreaktionen rechnen zu müssen. ${ }^{137}$

Die zu einem großen Teil durch kleine und mittelständische Reifenfachhändler geprägte Handelsstruktur scheint zunächst auf ein geringes Machtpotential im Handel hinzuweisen. Die relativ niedrigen Umsätze bieten dem einzelnen Reifenfachhändler keine ausreichende Bestrafungsmacht. Seine Auslistung der Marke hat nur wenig Einfluß auf die Ertragssituation des Reifenherstellers, zumal sich diesem durch die große Anzahl an Reifenhändlern dem Anschein nach genügend Alternativen bieten. Eine Bestrafungsmacht mit Bedeutung besitzen demnach nur nationale Großketten, die jeweils einen entsprechend hohen Umsatz auf sich vereinen. Für die von Wettbewerbern kontrollierte Distribution ist in diesem Zusammenhang von vornherein davon auszugehen, daß Fremdfabrikate nur geführt werden, um eine gewisse Sortimentsbreite präsentieren zu können, die Nachfrage aber auf die eigenen Marken gelenkt wird.

Wenngleich hiermit eine relativ schwache Abhängigkeit des Reifenherstellers vom Handel zu bestehen scheint, so ist im Hinblick auf die Machtverteilung zwischen den beiden Marktteilnehmern festzustellen, daß umgekehrt die Abhängigkeit des Handels vom Hersteller noch wesentlich geringer ist. Der Grund hierfür ist in der hohen Austauschbarkeit der Marken untereinander zu suchen. Expertenmeinungen zufolge finden sich auf dem Reifenmarkt nur eine, allenfalls zwei Reifenmarken, die geführt werden müssen, da sie vom Endabnehmer verlangt werden und für dessen Wahl der Einkaufsstätte von Bedeutung sind. Alle weiteren Marken werden in ihren jeweiligen Preisklassen vom Handel als substituierbar betrachtet. ${ }^{138}$

Allerdings macht der Handel von den Substitutionsmöglichkeiten im Rahmen der Beschaffung nur für einen Teil seines Sortimentes Gebrauch. Im allge-

137 Vgl. Steffenhagen, H., Konflikt und Kooperation in Absatzkanälen, a.a.O., S. $107 \mathrm{ff}$.

138 Quelle: Unveröffentlichte Protokolle von Expertengesprächen mit Reifenfachhändlern. 
meinen werden bestimmte Kernmarken ${ }^{139}$ geführt, von denen der Händler auch bei kurzfristig attraktiveren Konkurrenzangeboten nicht abweicht. Langjährige Erfahrungen mit dem Hersteller sowie gute Beziehung zu dessen Außendienstmitarbeitern tragen zu einer gewissen Herstellertreue des Reifenhändlers bei. ${ }^{140}$ Günstige Angebote werden dagegen für das Randund Zusatzsortiment genutzt. Hier sucht der Handel die Möglichkeit, seine Gesamtrentabilität durch möglichst attraktive, wenn auch nur für einen begrenzten Zeitraum oder eine beschränkte Menge gültige Preiskonditionen zu erhöhen. ${ }^{141}$

\subsection{Warenpräsentation und Bedienungsform im Handel}

Warenpräsentation und Bedienungsform beschreiben die Art der Konfrontation des Käufers mit den Produkten der Marke im Handel. Die Warenpräsentation kann durch das physische Produkt selbst, anhand von Produktmustern oder durch Abbildungen und mündliche Beschreibungen erfolgen. Mit der Bedienungsform wird der Umfang konkretisiert, in dem sich das Verkaufspersonal in den Auswahl- und Entscheidungsprozeß des Endabnehmers einschaltet. Während bei der Fremdbedienung der Verkäufer als vermittelnde Person zwischen das Produkt und den Käufer tritt, ist die Selbstbedienung dadurch gekennzeichnet, daß der Endabnehmer ohne Unterstützung des Verkaufspersonals seine beabsichtigte Kaufentscheidung weiter konkretisiert und die Warenentnahme selbständig tätigt. ${ }^{142}$

139 Kernmarken bilden als ständig geführte Marken das Zentrum des Gesamtsortimentes, das durch Rand- und Zusatzmarken ergänzt wird. Vgl. Oehme, W., Handelsmarketing, a.a.O., S. 141.

140 Die Herstellertreue führt jedoch ebenso dazu, daß die Akquisition von Händlern, die bereits von Wettbewerbern beliefert werden, erschwert ist. Insofern ist das Machtpotential des einzelnen Händlers im Hinblick auf eine Auslistung höher als zunächst anzunehmen, da sich damit für den Hersteller die Anzahl und Attraktivität möglicher Händleralternativen verringert.

141 Quelle: Unveröffentlichte Protokolle von Expertengesprächen mit Reifenfachhändlern.

142 Neben den reinen Ausprägungsformen der Fremd- und Selbstbedienung sind Mischtypen möglich, bei denen der Endabnehmer entweder zunächst eine Vorauswahl aus den angebotenen Produktalternativen trifft und sich erst im fortgeschrittenen Stadium der Kaufentscheidung an das Verkaufspersonal wendet oder aber dieses ausschließlich bei auftretendem Beratungsbedarf in Anspruch nimmt. Vgl. 
Im Reifenmarkt erfolgen etwa $95 \%$ des Absatzes über Distributionswege mit Fremdbedienung. Hieraus ergeben sich Implikationen für die Bedarfsfeststellung seitens des Handels. Im persönlichen Verkaufsgespräch wird der Endabnehmer seine Präferenz für eine bestimmte Marke artikulieren, und der Reifenhändler hat zudem die Möglichkeit, die Akzeptanz weiterer Marken direkt zu prüfen. Das Absatzpotential einer Marke wird damit unmittelbar offensichtlich. Bei der Selbstbedienung bestünde demgegenüber die Notwendigkeit, entsprechende Bedarfsanalysen im Rahmen von Marktforschungsstudien durchzuführen. In der Einkaufsstätte selbst würde der Reifenkäufer zudem bei Nichtverfügbarkeit der Marke entweder stillschweigend auf eine alternative Reifenmarke ausweichen oder die Einkaufsstätte verlassen, ohne daß dem Händler der Grund für den Kaufverzicht offensichtlich würde.

\section{Auch bezüglich der Einflußmöglichkeiten auf die Kaufentscheidung} unterscheiden sich Eigen- und Fremdbedienung erheblich. Neben der Wirkung des Produktes selbst bzw. entsprechender Werbematerialien geht bei der Eigenbedienung die Beeinflussungswirkung allein von der Größe und Qualität der sichtbaren Präsentationsfläche aus. ${ }^{143}$ Gerade die physische Präsentation der Produkte ist im Reifenmarkt jedoch wenig aussagekräftig, da Unterschiede in der Leistung durch Anschauung kaum zu beurteilen sind. ${ }^{144}$ Bei der Fremdbedienung bestehen dagegen nicht nur wesentlich mehr Möglichkeiten der Vermittlung von Informationen über die Marke, sondern der Handel kann durch eine individuell auf den Käufer abgestimmte Beratung und Argumentation erheblichen Einfluß auf die Kaufentscheidung nehmen.

Bidlingmaier, J., Geschäftsprinzipien und Betriebsformen des Einzelhandels, in: ZfbF, H. 12, 1963, S. 590; Oehme, W., Handelsmarketing, a.a.O., S. 179. Zu Grundlagen des persönlichen Verkaufs vgl. Domman, D., Erfolgreicher Persönlicher Verkaufen, in: Handbuch Marketing-Kommunikation, Hrsg.: Berndt, R., Hermanns, A., Wiesbaden 1993, S. $751 \mathrm{ff}$.

143 Daß häufig die Wirkung einer Verkaufsflächenvariation weit überschätzt wird, zeigt eine Untersuchung von Curhan. Vgl. Curhan, R. C., The Relationship Between Shelf Space and Unit Sales in Supermarkets, in: Journal of Marketing Research, November 1972, S. 406 ff. Für ein Beispiel zur Flächenoptimierung auf Basis von Artikelgruppen siehe Schwärzler, A., Rentabel variieren, in: asw, H. 7, 1991, S. $64 \mathrm{ff}$.

144 Ausnahmen bestehen z. B. bei einem Vergleich von "Standardreifen" und Breitreifen. 
So kann der Reifenhändler durch die Verfügbarkeit mehrerer Marken dem Wunsch nach Angebotsvielfalt entgegenkommen, gleichzeitig im Verkaufsgespräch die Interessen des Endabnehmers jedoch bewußt auf eine bestimmte Marke lenken. Ein solches Vorgehen zeigt sich besonders deutlich bei herstellerkontrollierten Distributionskanälen. Hier konnte in einem Fall festgestellt werden, daß fast $80 \%$ des Gesamtumsatzes einer Reifenkette auf die eigenen Marken des Herstellers entfallen, obgleich auch Marken der Wettbewerber geführt werden. ${ }^{145}$ Selbst für den Fall, daß der Endabnehmer mit einem konkreten, markenbezogenen Kaufwunsch die Einkaufsstätte betritt, kann durch das Empfehlungsverhalten die Kaufentscheidung somit vergleichsweise gut beeinflußt werden, indem eine vom Käufer akzeptierte Alternative angeboten wird.

\subsection{Bedeutung der Erstausrüstung im Reifenmarkt}

Im Rahmen der Untersuchung des Stellenwertes der Erstausrüstung im Reifenmarkt ist zunächst festzuhalten, daß zwischen Reifenhersteller, Automobilhersteller und Endabnehmer eigenständige, vom Ersatzgeschäft losgelöste Beziehungen bestehen, auf die prinzipiell die Konzepte des Push und Pull übertragbar sind. In diesem Fall werden bei einer pushorientierten Markendurchsetzungsstrategie die Aktivitäten auf den Automobilhersteller ausgerichtet. Mit einer pull-orientierten Strategie würde der Reifenhersteller wiederum eine direkte Beeinflussung des Endabnehmers zu erreichen versuchen, um durch den ausgelösten Nachfragesog den Automobilhersteller zur Ausrüstung seiner Fahrzeuge mit der entsprechenden Reifenmarke zu bewegen. ${ }^{146}$ Das Zitat eines Vorstandsmitgliedes des Reifenherstellers Pirelli verdeutlicht diese Strategie in anschaulicher Form: "Wenn etwa der BMW-Kunde schon bei der Neuanschaffung darauf besteht, daß sein Wagen mit einem Pirelli-Reifen ausgerüstet ist, haben wir ... uns der Willkür der Autoindustrie entzogen."147

145 Quelle: Unveröffentlichte Protokolle von Expertengesprächen mit Reifenfachhändlern.

146 Vgl. Rudolph, M., Mehrstufiges Marketing für Einsatzstoffe, a.a.O., S. 35 ff.; Backhaus, K., Investitionsgütermarketing, 2. Aufl., München 1990, S. 312 ff.

147 Vgl. o. V., Unwucht im Ersatzgeschäft, in: Industriemagazin, H. 2, 1987, S. 143. 
In der vorliegenden Arbeit soll jedoch weniger diese eigenständige Beziehung von Interesse sein als vielmehr der Einfluß des Erstausrüstungsgeschäftes auf den Ersatzmarkt. Seitens der Reifenhersteller wird der Erstausrüstung eine überaus hohe Bedeutung zugemessen. ${ }^{148}$ Als Begründung wird insbesondere angeführt, daß die Ausstattung eines Neufahrzeuges mit einer bestimmten Reifenmarke zu einer Kette von markengleichen Folgekäufen führt. Zuzustimmen ist der Annahme, daß der Endabnehmer unabhängig davon, ob er den aktuell montierten Reifen selbständig gekauft oder mit dem Fahrzeug übernommen hat, beim Ersatzkauf vielfach dieselbe Marke erwirbt. Zudem wirkt sich die auf guten Erfahrungen begründete hohe Akzeptanz für die gefahrene Reifenmarke positiv auf das Verhalten des Handels aus und führt damit gleichzeitig zu einem verstärkten Angebot der Marke. Insofern ist diese Begründung für die Bedeutung der Erstausrüstung grundsätzlich richtig. Allerdings läßt sich anhand eines vereinfachten Beispiels demonstrieren, daß der unterstellte, direkte Zusammenhang zum Ersatzkauf wesentlich schwächer ist als zunächst zu vermuten wäre.

Ausgangspunkt des Beispiels ist die Annahme, daß ein Hersteller der Marke A zum Zeitpunkt $t=0$ (Beginn des ersten Planungsjahres) im Erstausrüstungsgeschäft einen Marktanteil von $10 \%$ erreicht. Bei einer unterstellten (konstanten) Anzahl von 2 Mio. Neufahrzeugen pro Jahr wären demnach 0,2 Mio. neue Fahrzeuge in $t=0$ mit der Marke $A$ bereift. ${ }^{149}$ Ferner sei angenommen, daß die Lebensdauer eines Fahrzeuges 12,5 Jahre und die eines Reifens 3 Jahre beträgt. ${ }^{150}$ Damit kommt es 3, 6, 9 und 12 Jahre nach der Erstausrüstung eines Fahrzeuges zu einem Reifenwechsel. Zu diesen Zeitpunkten sind jeweils insgesamt 8 Mio. Fahrzeuge im Ersatzgeschäft zu bereifen. So wird beispielsweise in $t=3$ ein Reifenwechsel für alle Fahrzeuge erforderlich, die in $t=0, t=-3, t=-6$ und $t=-9$ als Neufahrzeuge erstmalig ausgestattet wurden. ${ }^{151}$ Für den Reifenwechsel soll unterstellt werden, daß für

148 Vgl. Sasseen, J., Tyre Makers on the Rack, a.a.O., S. 40; Unveröffentlichte Protokolle von Gesprächen mit Experten eines Reifenherstellers.

149 Die Darstellung des Beispiels anhand absoluter Fahrzeugzahlen dient lediglich der Veranschaulichung. Das Ergebnis ist unabhängig von der gewählten Anzahl jährlicher Neufahrzeuge.

Vgl. BRV, Reifenersatzgeschäft 1993/94.

151 Hierbei sei vereinfachend angenommen, daß die Intervalle des Reifenwechsels für alle Fahrzeuge gleich sind. 
Marke A eine Wiederkaufwahrscheinlichkeit von $50 \%$ besteht. Empirisch ermittelte Ergebnisse zeigen, daß es sich hierbei um einen Wert handelt, dessen Größe für bestimmte Marken durchaus realistisch ist. ${ }^{152}$

\begin{tabular}{|l|c|c|c|c|c|}
\hline Zeitpunkt t & $\mathbf{0}$ & $\mathbf{3}$ & $\mathbf{6}$ & $\mathbf{9}$ & $\mathbf{1 2}$ \\
\hline $\begin{array}{l}\text { Anzahl mit Marke A bereifter } \\
\text { Fahrzeuge vor Reifenwechsel } \\
\text { in t }\end{array}$ & $\begin{array}{c}\text { Erst- } \\
\text { aus- } \\
\text { rüstung }\end{array}$ & 0,2 Mio. & 0,1 Mio & 0,05 Mio. & 0,025 Mio. \\
\hline $\begin{array}{l}\text { Wiederkaufwahrscheinlich- } \\
\text { keit der Marke A }\end{array}$ & $50 \%$ & $50 \%$ & $50 \%$ & $50 \%$ \\
\hline $\begin{array}{l}\text { Anzahl mit Marke A bereifter } \\
\text { Fahrzeuge nach Reifen- } \\
\text { wechsel in t }\end{array}$ & 0,1 Mio. & 0,05 Mio & 0,025 Mio. & $0,0125 \mathrm{Mio}$ \\
\hline $\begin{array}{l}\text { Gesamtzahl der in t bereiften } \\
\text { Fahrzeuge im Ersatzgeschäft }\end{array}$ & 8 Mio. & 8 Mio & 8 Mio. & 8 Mio. \\
\hline $\begin{array}{l}\text { Marktanteil Marke A bezogen } \\
\text { auf Gesamtzahl der in t bereif- } \\
\text { ten Fahrzeuge im Ersatzge- } \\
\text { schäft }\end{array}$ & & $1,25 \%$ & $0,625 \%$ & $0,3125 \%$ & $0,15625 \%$ \\
\hline
\end{tabular}

Abb. 14: Einfluß der Erstausrüstung auf das Reifenersatzgeschäft (Berechnung Teil 1)

Abbildung 14 stellt die Entwicklung der sich augenscheinlich aus der Erstausrüstung ergebenden Marktanteile im Ersatzgeschäft dar. Vor dem ersten Wechsel sind die im Rahmen der Erstausrüstung ausgestatteten 0,2 Mio. Fahrzeuge mit der Marke A bereift. Bei jedem Reifenwechsel entscheiden sich $50 \%$ der Reifenkäufer für die bereits montierte Marke. Nach dem ersten Wechsel verbleiben somit 0,1 Mio. Fahrzeuge, bis zum Zeitpunkt $t=12$ noch 12.500 Fahrzeuge mit Bereifung der Marke A. Bezogen auf die Gesamtzahl der jeweils in $\mathrm{t} z \mathrm{u}$ bereifenden Fahrzeuge beträgt der erreichte

152 Die Ergebnisse stammen aus einer unveröffentlichten Studie eines Reifenherstellers. 
Marktanteil zwischen $1,25 \%$ in $t=3$ und $0,16 \%$ in $t=12 .{ }^{153}$ Gelingt es dem Hersteller, in jedem Jahr einen Erstausrüstungsanteil von $10 \%$ zu realisieren, addieren sich die zuvor ermittelten Marktanteile wie in Abbildung 15 dargestellt. Der aufgrund des Erstausrüstungsanteils maximal zu erreichende Marktanteil im Ersatzgeschäft berechnet sich demnach auf 2,34 \%.

Das Ergebnis dieses durchaus auf realistischen Annahmen beruhenden Beispiels zeigt deutlich auf, daß die Bedeutung der Erstausrüstung für den unmittelbar durch den Wiederkauf zu erzielenden Marktanteil nicht überschätzt werden darf. Vor allem, da die empirisch ermittelte Wiederkaufwahrscheinlichkeit nicht als "automatischer" Folgekauf interpretierbar ist. Vielmehr kann die Tatsache, daß sich nach einem Reifenwechsel die gleiche Marke am Fahrzeug befindet wie zuvor, auf mehrere Ursachen zurückzuführen sein. Sowohl die eigene Entscheidung des Käufers als auch die Empfehlung des Händlers für die betreffende Marke kann sich vollkommen unabhängig von der Reifenausstattung des Fahrzeuges eingestellt haben. In diesen Fällen ist der beobachtete "Wiederkauf" nicht mehr ursächlich auf die Erstausrüstung zurückzuführen. Ein Kauf dieser Marke hätte auch unabhängig von der Ausstattung des Fahrzeuges stattgefunden. Damit liegt in dem Beispiel der tatsächlich durch die Erstausrüstung induzierte Marktanteil im Ersatzgeschäft unterhalb des berechneten Ergebnisses.

Für den seitens der Reifenhersteller proklamierten Erfolg von Marken, die auch in der Erstausrüstung verwendet werden, scheinen vielmehr andere Ursachen wesentlich zu sein. Reifenhersteller, die sich dauerhaft als Zulieferer qualifizieren wollen, sind aufgrund der Anforderungen der Automobilindustrie gezwungen, stets technologisch fortschrittliche und qualitativ hochwertige Leistungen zu erbringen. Selbst wenn die für das Erstausrüstungsund Ersatzgeschäft entwickelten Reifen nicht zwingend identisch sind, ist dennoch in einem hohen Maße von einem Know-how-Transfer zwischen diesen beiden Bereichen auszugehen. Zudem ist anzunehmen, daß der Hersteller auch im Ersatzgeschäft nicht seine Kompetenz gefährden wird, da hiervon negative Auswirkungen auf die Stellung als Erstausrüster ausgehen könnten.

153 Dabei sei angenommen, daß jeweils alle Reifen eines Fahrzeuges zu diesen Zeitpunkten gewechselt werden. 


\begin{tabular}{|c|c|c|c|c|c|c|c|c|c|c|c|c|c|c|c|c|}
\hline Zeitpunkt (Jahr) & 0 & 1 & 2 & 3 & 4 & 5 & 6 & 7 & 8 & 9 & 10 & 11 & 12 & 13 & 14 & 15 \\
\hline \multirow{8}{*}{$\begin{array}{l}\text { Marktanteil Marke A be- } \\
\text { zogen auf Gesamtzahl } \\
\text { der in } t \text { bereiften Fahr- } \\
\text { zeuge (in \%) }\end{array}$} & $E$ & & & 1,25 & & & 0,625 & & & 0,3125 & & & 0.15625 & & & \\
\hline & & $E$ & & & 1,25 & & & 0,625 & & & 0,3125 & & & 0,15625 & & \\
\hline & & & E & & & 1,25 & & & 0,625 & & & 0,3125 & & & 0,15625 & \\
\hline & & & & $E$ & & & 1,25 & & & \begin{tabular}{|l|} 
\\
\end{tabular} & & & 0,3125 & & & 0,15625 \\
\hline & & & & & $E$ & & & 1,25 & & & 0,625 & & & 0,3125 & & \\
\hline & & & & & & $E$ & & & 1,25 & & & \begin{tabular}{|l|l|} 
\\
, 625
\end{tabular} & & & 0,3125 & \\
\hline & & & & & & & $E$ & & & 1,25 & & & 0,625 & & & 0,3125 \\
\hline & & & & & & & & $E$ & & & 1,25 & & & 0,625 & & \\
\hline \multirow{5}{*}{$\begin{array}{c}\mathrm{E}=\underset{\text { Zeitpunkt der }}{\text { Erstausrüstung }} \\
\text { Zrtus }\end{array}$} & & & & & & & & & $E$ & & & 1,25 & & & 0 & \\
\hline & & & & & & & & & & $E$ & & & 1,25 & & & 0,625 \\
\hline & & & & & & & & & & & $\mathrm{E}$ & & & 1,25 & & \\
\hline & & & & & & & & & & & & $E$ & & & 1,25 & \\
\hline & & & & & & & & & & & & & $E$ & & & 1,25 \\
\hline $\begin{array}{l}\text { Summe der Marktanteile } \\
\text { (in \%, gerundet) }\end{array}$ & & & & 1,25 & 1,25 & 1,25 & 1,88 & 1,88 & 1,88 & 2,19 & 2,19 & 2,19 & 2,34 & 2,34 & 2,34 & 2,34 \\
\hline
\end{tabular}

Abb. 15: Einfluß der Erstausrüstung auf das Reifenersatzgeschäft (Berechnung Teil 2) 
Damit entspricht die Qualität der Produkte tatsächlich einem hohen, stets aktuellen Niveau, das bei Herstellern ohne Erstausrüstungsgeschäft aufgrund des fehlenden Leistungsdrucks seitens der Automobilindustrie möglicherweise nicht in der gleichen Konstanz erbracht wird. Zum anderen signalisiert die Ausstattung eines Neufahrzeuges mit einer bestimmten Marke deutlich ein gewisses Vertrauen, das der Automobilhersteller in den Reifen setzt. Schließlich wird dieser kein Produkt wählen, durch das die Zufriedenheit der Kunden bezüglich seiner Fahrzeuge beeinträchtigt werden könnte. Für Handel und Endabnehmer wird somit indirekt eine Empfehlung ausgesprochen, welche Marke grundsätzlich, d. h. nicht nur in bezug auf das entsprechend erstausgerüstete Fahrzeug, ohne Risiko zu empfehlen bzw. zu kaufen ist.

Neben der Qualität kann durch die Erstausrüstung ein emotionaler Zusatznutzen der Reifenmarke zum Ausdruck kommen. So geht von bestimmten Automobilherstellern (z. B. Porsche, BMW, Mercedes) das Image der Sportlichkeit oder Exklusivität aus, das sich auch auf den Reifen übertragen kann. Beispielsweise gelang es dem Unternehmen Bridgestone durch die Alleinausrüstung eines bestimmten Porschemodells, einen seiner Reifen als "Porschereifen" zu vermarkten und sich damit als Marke mit sportlichem Image zu positionieren. ${ }^{154}$

Automobilhersteller beeinflussen das Ersatzgeschäft darüber hinaus durch offizielle Freigabelisten, auf denen bestimmte Reifen als geeignet für den betreffenden Fahrzeugtyp ausgewiesen werden. ${ }^{155} \mathrm{Um}$ in diese Listen rechtzeitig aufgenommen $\mathrm{zu}$ werden und damit möglichst frühzeitig im Lebenszyklus 156 eines PKW-Modells in das Ersatzgeschäft eintreten zu können, werden von Experten gute Beziehungen zu den Automobilherstellern als besonders wichtig erachtet. Daraus resultieren ebenfalls Vorteile

154 Vgl. o. V., Wir liefern nicht an Hinz und Kunz, a.a.O., S. 15.

155 Vgl. Belz, Chr., Konstruktives Marketing in gesättigten und schrumpfenden Märkten, a.a.O., S. $151 \mathrm{f}$.

156 Zum Begriff des Lebenszyklus vgl. Meffert, $\mathrm{H}$., Interpretation und Aussagewert des Lebenszykluskonzeptes, in: Neuere Ansätze zur Marketingtheorie, Festschrift zum 80. Geburtstag von O. Schnutenhaus, Hrsg.: Hammann, P. u.a., Berlin 1973, S. $85 \mathrm{ff}$;; Höft, U., Lebenszykluskonzepte, Grundlagen für das strategische Marketing- und Technologiemanagement, Berlin 1992, S. $15 \mathrm{ff}$. 
für solche Reifenhersteller, die aufgrund ihrer Tätigkeit als Erstausrüster bereits über entsprechend intensive Kontakte verfügen.

Es ist festzuhalten, daß die Erstausrüstung einen hohen Einfluß auf das Ersatzmarktgeschäft nimmt. Dabei steht jedoch weniger der ursächlich auf die Ausrüstung neuer Fahrzeuge zurückzuführende Marktanteil bei deren Wiederbereifung im Vordergrund. Vielmehr scheint die grundsätzliche Bedeutung der Erstausrüstung für die objektive sowie die vom Handel und Endabnehmer wahrgenommene Qualität der Reifen der entscheidende Grund für den Erfolg dieser Marken im Ersatzgeschäft zu sein.

2. Markenpolitische Ziele und Grundsatzstrategien der Reifenhersteller als Ausgangspunkt der Planung von Markendurchsetzungsstrategien

Markenpolitische Ziele und Grundsatzstrategien der Reifenhersteller bilden den Ausgangspunkt der Planung von Markendurchsetzungsstrategien, indem sie zum einen anzustrebende Vorzugsstände und zum anderen strategische Rahmenbedingungen festlegen. Daher sind zunächst aus der Menge möglicher markenpolitischer Ziele die für eine modellgestützte Planung auf dem Reifenmarkt relevanten Ziele herauszuarbeiten. Des weiteren sind die Grundsatzstrategien der Markierung und Positionierung von Reifenherstellern festzustellen und deren Einfluß auf Entscheidungen über die Markendurchsetzungsstrategie zu kennzeichnen.

\subsection{Markenpolitische Ziele als anzustrebende Vorzugszustände}

\subsection{Systematisierung des Zielsystems}

Die Systematisierung einer Menge von Zielen erfolgt in der Regel nach dem Kriterium der "Mittel-Zweck"-Beziehung. Im Mittelpunkt steht die Frage, inwieweit ein Ziel "Mittelcharakter" zur Verwirklichung der übrigen Elemente der Zielmenge aufweist. Die Identifikation solcher Beziehungen ermöglicht 
eine hierarchische Ordnung von Zielen, bei der zwischen Ober-, Zwischen- und Unterzielen unterschieden werden kann (vgl. Abbildung 16). ${ }^{157}$

\begin{tabular}{|c|c|}
\hline Oberziel & Nebenziele \\
\hline - Markenwert & $\begin{array}{l}\text { - Effizienz } \\
\text { - Flexibilität } \\
\text { - Liquidität }\end{array}$ \\
\hline \multicolumn{2}{|c|}{ Zwischenziele } \\
\hline $\begin{array}{l}\text { - Gewinn } \\
\text { - Rentabilität }\end{array}$ & $\begin{array}{l}\text { - Umsatz } \\
\text { - Marktanteil }\end{array}$ \\
\hline \multicolumn{2}{|c|}{ Unterziele } \\
\hline $\begin{array}{l}\text { - Nachfragestärke } \\
\text { - Präferenzgrad } \\
\text { - Präferenzintensität } \\
\text { - Akzeptanzgrad }\end{array}$ & $\begin{array}{l}\text { - Angebotsstärke } \\
\text { - Distributionsgrad } \\
\text { - POS-Stärke }\end{array}$ \\
\hline
\end{tabular}

Abb. 16: Hierarchisch strukturiertes Zielsystem für die Planung von Markendurchsetzungsstrategien

Als markenpolitisches Oberziel wird in jüngerer Zeit verstärkt das Ziel des Markenwertes diskutiert. Allerdings besteht in der Literatur bislang keine einheitliche Vorstellung über die inhaltliche Abgrenzung dieses Begriffes. Das breite Spektrum reicht von rein finanzwirtschaftlichen über ausschließlich käuferverhaltenstheoretische Ansätze bis hin zu umfassenden Kriteriensystemen zur Beschreibung des Markenwertes. ${ }^{158}$ So finden sich beispielsweise folgende Definitionen, nach denen als Markenwert

157 Vgl. Heinen, E., Grundlagen betriebswirtschaftlicher Entscheidungen, a.a.O., S. 102 ff.; Kupsch, P., Unternehmungsziele, Stuttgart, New York 1979, S. 33 ff.

158 Die erheblichen Unterschiede in der Abgrenzung des Markenwertkonzeptes lassen sich daraus erklären, daß zunächst die bilanzielle Bewertung der Marke im Vorder- 
- "der Barwert aller zukünftigen Einzahlungsüberschüsse, die der Eigentümer aus der Marke erwirtschaftet" (finanzwirtschaftliche Abgrenzung), ${ }^{159}$

- "die Akkumulation aller Marketingmaßnahmen und deren subjektive Wahrnehmung, Bewertung und Speicherung durch die Konsumenten" (käuferverhaltenstheoretische Abgrenzung) ${ }^{160}$ oder

- "die Gesamtheit aller positiven und negativen Vorstellungen, die im Konsumenten ganz oder teilweise aktiviert werden, wenn er das Markenzeichen wahrnimmt, und die sich in ökonomischen Daten des Markenwettbewerbs spiegeln" (Abgrenzung durch umfassendes Kriteriensystem $)^{161}$

grund stand und erst in jüngerer Zeit der Markenwert auch als Steuerungsinstrument für die Markenführung diskutiert wird. Vgl. Hammann, P., von der Gathen, A., Bilanzierung des Markenwertes und kapitalmarktorientierte Markenbewertungsverfahren, in: Markenartikel, H. 5, 1994, S. 204 ff.; Franzen, O., Trommsdorf, V., Riedel, F., Ansätze der Markenbewertung und Markenbilanz, in: Markenartikel, H. 8, 1994, S. 372 f.; Seif, F. T., Bilanzwert der Marke, in: Markenartikel, H. 8, 1994, S. $388 \mathrm{f}$.

159 Vgl. Kaas, K.-P., Langfristige Werbewirkung und Brand Equity, in: Werbeforschung \& Praxis, H. 3, 1990, S. 48. Eine ähnliche Definition wird auch von Simon und Sullivan zugrunde gelegt. Vgl. Keller, K. L., Conceptualizing, Measuring and Managing Customer-Based Brand Equity, in: Journal of Marketing, January 1993, S. 1.

$160 \mathrm{Vgl}$. Esch, F.-R., Markenwert und Markensteuerung, Eine verhaltenswissenschaftliche Perspektive, in: Thexis, H. 5/6, 1993, S. 58; Bekmeier, S., Markenwert und Markenstärke - Markenevaluierung aus konsumentenorientierter Perspektive, in: Markenartikel, H. 8, 1994, S. 383 ff. Siehe zu anderen verhaltenswissenschaftlichen Untersuchungen des Markenwertes auch Andresen, T., Innere Markenbilder: MAX wie er wurde, was er ist, in: Planung und Analyse, H. 1, 1991, S. 31 ff.; Keller, K. L., Conceptualizing, Measuring, and Managing Customer-Based Brand Equity, a.a.O, S. $1 \mathrm{ff}$.

161 Dieser Definition des Markenwertes von Schulz und Brandmeyer liegt eine Markenbilanz mit 19 Teilkriterien zugrunde, die den Markenwert umfassend abbilden sollen. Vgl. Schulz, R., Brandmeyer, K., Die Marken-Bilanz, Ein neues Instrument für die Bewertung und Steuerung von Marken stellt sich vor, in: Marketing Journal, H. 4, 1989, S. $360 \mathrm{ff}$. Ähnlich kombiniert das Markenwertkonzept INTERBRAND den Gewinn einer Marke mit ihrer Marktstärke, die sich $u$. a. in Kriterien wie der Stellung im Markt, der Stabilität im Sinne von Langlebigkeit oder dem langfristigen Trend im Hinblick auf die Bedeutung der Marke für den Kunden ausdrückt. Vgl. 0 . V., Marken - How much in Dollar?, in: asw, H. 8, 1989, S. 51 ff. Der wesentliche Nachteil dieser Markenwertansätze besteht darin, daß die einzelnen Ziele als unabhängige Kriterien in ein Punktbewertungsmodell einfließen, ohne daß deren Wirkungszusammenhänge berücksichtigt werden. Zu weiteren Kritikpunkten vgl. 0 . V., Wieviel ist der Markenwert wert?, in: asw, H. 3, 1990, S. 26 sowie Brandmeyer, K., 
definiert wird. Hier soll der finanzwirtschaftlichen Interpretation des Markenwertes als Oberziel der Markenpolitik der Vorzug gegeben werden, da sich in dem so definierten Ziel letztlich das erwerbswirtschaftliche Interesse des Unternehmens an der Marke ausdrückt. 162 Somit wird für die vorliegende Arbeit der Markenwert als Barwert aller Ein- und Auszahlungen definiert, die im Zusammenhang mit der Erstellung und dem Verkauf von Produkten und Leistungen dieser Marke anfallen.

Auf einer zwischengelagerten Ebene finden sich wirtschaftliche Erfolgsziele wie Gewinn, Umsatz und Rentabilität. ${ }^{163}$ Der zentrale Unterschied zwischen diesen Größen und dem Markenwert ist in der Länge des betrachteten Zeitraums zu sehen. Während der Markenwert alle zukünftigen Zahlungen berücksichtigt, sind Gewinn, Umsatz und Rentabilität periodenbezogen definiert. Sie beschreiben somit den monetären Markenerfolg nur für ein relativ eng begrenztes Zeitintervall. Zur Kennzeichnung der Marktstellung dient auf dieser Zielebene der Marktanteil. Er repräsentiert den relativen Markterfolg einer Marke im Vergleich zu den Wettbewerbern. ${ }^{164}$

Den Unterzielen kommt schließlich die Aufgabe zu, eine Verbindung zwischen der Festlegung von Zielen und den sich anschließenden Entscheidungen über die zu wählenden Handlungsalternativen zu schaffen. Durch die Unterziele wird bereits die Breite des weiteren Entscheidungskanals eingegrenzt, so daß Ziel- und Strategieentscheidung zu einem eng verknüpf-

Schulz, R., Die Markenbilanz im Kreuzverhör, in: Markenartikel, H. 5, 1990, S. $236 \mathrm{f}$.

162 Zu empirischen Untersuchungen von Unternehmenszielen vgl. Fritz, W. U.a., Unternehmensziele in Industrie und Handel, a.a.O., S. $375 \mathrm{ff}$; Meffert, H., Kirchgeorg, M., Umweltschutz als Unternehmensziel, in: Marketing-Schnittstellen, Hrsg.: Specht, G., Silberer, G., Engelhardt, W. H., Stuttgart 1989, S. 179 ff.

163 Diese Ziele werden auch als wirtschaftliche Formalziele bezeichnet. Vgl. Schierenbeck, H., Grundzüge der Betriebswirtschaftslehre, 11. Aufl., München, Wien 1993, S. 62 .

164 Allerdings sind mengen- und wertmäßige Operationalisierungsansätze dieses Zieles dazu unterschiedlich geeignet. Bei einer mengenmäßigen Betrachtung ist streng genommen nur ein Erfolgsvergleich mit Marken desselben Preisniveaus zulässig. Dagegen berücksichtigt die wertmäßige Erfassung des Marktanteils implizit den Zusammenhang zwischen Preishöhe und abgesetzter Menge. Vgl. zur Bedeutung des Marktanteils auch Tolle, E., Steffenhagen, $H_{\text {., Kategorien des Markenerfolges }}$ und einschlägige Meßmethoden, in: Markenartikel, H. 8, 1994, S. $378 \mathrm{ff}$. 
ten, interdependenten Prozeß werden. Darüber hinaus ermöglicht die Vorgabe spezifischer, auf die Art der Strategie bezogener Ziele eine Überprüfung der Zielerreichung. So ist für eine push-orientierte Markendurchsetzungsstrategie das Ausmaß der unmittelbar angestrebten Reaktion des Handels vorzugeben, während bei einer pull-orientierten Markendurchsetzungsstrategie die gewünschte Reaktion des Endabnehmers als direktes Ziel zu formulieren ist. In diesem Sinne werden im folgenden die Zielkonstrukte der Angebots- und Nachfragestärke eingeführt. Diese sind als Konstrukte zur vereinfachten, prägnanten Beschreibung eines mehrdimensionalen Phänomens zu verstehen, bei denen verschiedene, das Angebotsverhalten des Handels bzw. Nachfrageverhalten des Endabnehmers charakterisierende, operationale (Teil-)Ziele gebündelt werden.

Neben der hierarchischen Anordnung von Zielen lassen sich Haupt- und Nebenziele unterscheiden. ${ }^{165}$ Letztere können auch als Nebenbedingungen verstanden werden, die es bei der Erreichung der Hauptziele einzuhalten gilt. So stellen der effiziente Einsatz von Ressourcen, ${ }^{166}$ die Flexibilität im Sinne der Wahrung ausreichender Dispositionsspielräume ${ }^{167}$ sowie die Erhaltung der Liquidität des Unternehmens zu berücksichtigende Nebenziele dar.

\subsection{Ziele der Nachfragestärke}

Ziele der Nachfragestärke beschreiben das angestrebte Nachfrageverhalten der Endabnehmer gegenüber der Marke. Hierbei lassen sich psychographische und kaufverhaltensbezogene Ziele voneinander unterscheiden. Mit psychographischen Zielen sollen die das Kaufverhalten des Konsumenten bestimmenden inneren Vorgänge abgebildet und als anzustrebende Vor-

$165 \mathrm{Vgl}$. Heinen, E., Grundlagen betriebswirtschaftlicher Entscheidungen, a.a.O., S. $107 \mathrm{ff}$.

166 Zu einer Übersicht von Unternehmensressourcen vgl. Kreilkamp, E., Strategisches Management und Marketing, a.a.O., S. $187 \mathrm{ff}$.

167 Zu einer ausführlichen Diskussion des Themenbereichs der Flexibilität vgl. Meffert, H., Zum Problem der betriebswirtschaftlichen Flexibilität, in: ZfB, 1969, S. 779 ff.; Meffert, H., Größere Flexibilität als Unternehmungskonzept, in: ZfbF, H. 2, 1985, S. $121 \mathrm{ff}$. 
zugszustände operationalisiert werden. Am häufigsten werden in der Literatur in diesem Zusammenhang die Ziele der Markenbekanntheit, der Markenaktualität, der Einstellung bzw. des Markenimage, der Markenkaufabsicht, der Markenzufriedenheit sowie der Markenpräferenz genannt (vgl. Abbildung 17). ${ }^{168}$

\begin{tabular}{|l|l|}
\hline Psychographische Ziele & Kaufverhaltensbezogene Ziele \\
\hline - Markenbekanntheit & - Anzahl der Käufer einer Marke \\
- Markenaktualität & - Kaufhäufigkeit der Marke \\
- Einstellung / Markenimage & - Menge pro Kauf der Marke \\
- Markenkaufabsicht & - Wiederkaufrate \\
- Markenzufriedenheit & - Markentreue \\
- Markenpräferenz & \\
\hline
\end{tabular}

Abb. 17: Markenpolitische Ziele zur Beschreibung der Nachfragestärke einer Marke

Als Markenbekanntheit wird die aktive oder passive Bewußtseinspräsenz eines Markennamens beim Konsumenten bezeichnet. Im allgemeinen wird der aktiven Bekanntheit zumindest für Low-Involvement-Käufe ${ }^{169}$ eine gewisse prognostische Relevanz für das Kaufverhalten zugestanden, während der passiven Bekanntheit in dieser Hinsicht keine Geltung beigemessen wird. ${ }^{170}$ Etwas weiter gefaßt und mit vermutlich größerem Einfluß auf die Kaufentscheidung als die Markenbekanntheit ist das Ziel der Markenaktualität zu verstehen. Hierbei geht es darum, eine Wahrnehmung der Marke als

168 Vgl. Tolle, E., Steffenhagen, H., Kategorien des Markenerfolges und einschlägige Meßmethoden, a.a.O., S. $378 \mathrm{ff}$.

169 Unter Involvement ist das innere Engagement zu verstehen, mit dem sich der Konsument einem Gegenstand oder einer Aktivität zuwendet. Der Begriff des LowInvolvement drückt ein entsprechend geringes inneres Engagement beim Kauf aus. Vgl. Kroeber-Riel, W., Konsumentenverhalten, a.a.O., S. 375.

170 Von aktiver Bekanntheit wird gesprochen, wenn der Konsument bei entsprechenden internen oder externen Stimuli von sich aus an den Markennamen denkt. Dagegen setzt die passive Bekanntheit nur voraus, daß der Konsument sich bei Wahrnehmung des Markennamens an diesen erinnert. Vgl. Steffenhagen, H., Markenbekanntheit als Werbeziel, Theorie und Operationalisierung, in: ZfB, H. 10, 1976, S. $715 \mathrm{ff}$. 
beachtenswerte, aktuelle Kaufalternative zu erreichen.171 Die höchste Bedeutung zur Erklärung des Kaufverhaltens wird in der Literatur jedoch dem Konstrukt der Einstellung bzw. des Image ${ }^{172}$ zugesprochen, da in diesem eine relative dauerhafte Prädisposition gegenüber der Marke zum Ausdruck kommt. ${ }^{173}$ Die Bildung von Einstellungen beruht nach gängiger Literaturmeinung auf der differenzierten Bewertung von Markeneigenschaften. ${ }^{174}$ Die Erfassung der Einstellung in der gedanklich antizipierten Kaufsituation führt zur Kaufabsicht. Da der Konsument hierbei sowohl sein verfügbares Budget als auch den entstehenden Beschaffungsaufwand berücksichtigt, prognostiziert die Kaufabsicht das zu erwartende Verhalten genauer als die unabhängig von den Kaufbedingungen ermittelte Einstellung. ${ }^{175}$

Mit der Markenzufriedenheit wird die Übereinstimmung zwischen der vom Endabnehmer erwarteten und der wahrgenommenen Leistung beschrieben. Im Gegensatz zur Einstellung setzt die Markenzufriedenheit damit Erfahrungen des Konsumenten mit der Marke voraus und wird in der Regel als zeit-

171 Vgl. Kroeber-Riel, W., Strategie und Technik der Werbung, a.a.O., S. 34 f.

172 In der Literatur besteht keine eineitliche Meinung darüber, inwieweit die Konstrukte der Einstellung und des Image übereinstimmen. So sieht Kroeber-Riel beide Begriffe weitgehend identisch, wogegen Mazanec sich für eine explizite Trennung ausspricht. In der vorliegenden Arbeit werden beide Begriffe synonym verwendet. Vgl. Kroeber-Riel, W., Konsumentenverhalten, a.a.O., S. 190; Mazanec, J., Strukturmodelle des Konsumverhaltens, Wien 1978, S. 59 ff.; Trommsdorff, V., Konsumentenverhalten, a.a.O., S. 131 ff.; Hätty, H., Markentransfer, Heidelberg 1989, S. 69 ff.; Herzig, O. A., Markenbilder/Markenwelten - Neue Wege in der Imageforschung, Wien 1991, S. $8 \mathrm{ff}$.

173 Unter dem Begriff der Einstellung ist die innere Bereitschaft eines Individuums zu verstehen, auf bestimmte Stimuli der Umwelt konsistent positiv oder negativ zu reagieren. Objekte der Einstellungen können Dinge, Personen oder Themen sein. Vgl. Meffert, H., Marketingforschung und Käuferverhalten, 2. Aufl., Wiesbaden 1992, S. 55.

174 Dies kommt insbesondere in Modellen zur mehrdimensionalen Messung von Einstellungen zum Ausdruck. Vgl. zu einer ausführlichen Diskussion von Einstellungsmodellen Trommsdorff, V., Die Messung von Produktimages für das Marketing, Grundlagen und Operationalisierung, Köln u.a. 1975, S. 48 ff.

175 Vgl. Freitag, D. E., Methodische Probleme bei der Realisation eines Einstellungsmodells, in: Jahrbuch der Absatz- und Verbrauchsforschung, H. 2, 1973, S. 157 ff.; Dehlhees, K. H., Systemorientierte Darstellung des Kaufprozesses als Entscheidungshilfe des Marketing, in: Jahrbuch der Absatz- und Verbrauchsforschung, H. 2, 1973, S. 285 ff.; Weinberg, P., Das Entscheidungsverhalten von Konsumenten, a.a.O., S. $27 \mathrm{f}$. 
lich weniger stabil betrachtet. Allerdings stellt die Zufriedenheit eine wichtige Voraussetzung für die Wiederkaufentscheidung dar. ${ }^{176}$ Die aus Sicht des Endabnehmers wahrgenommene Vorzugswürdigkeit einer Marke kommt in der Markenpräferenz zum Ausdruck. Häufig werden Präferenzen als relativierte Einstellungen interpretiert, bei denen durch Vergleich der ermittelten Einstellungswerte auf die Kaufwahrscheinlichkeit der Marke geschlossen wird. 177

Zu den kaufverhaltensbezogenen Zielen werden in der Literatur die den Marktanteil bestimmenden Einzelgrößen (Anzahl der Käufer, Kaufhäufigkeit, Menge pro Kauf), die Wiederkaufrate sowie die Markentreue gezählt. 178

Besonders betont wird bei den kaufverhaltensbezogenen Zielen die Bedeutung der Markentreue. Gerade mit diesem Ziel sind jedoch erhebliche Abgrenzungsprobleme verbunden. Allein der Wiederholungskauf einer Marke ist kein ausreichender Maßstab zur Beurteilung der Markentreue. Es kann sich um rein zufällige Wiederholungen des Kaufes oder $z$. B. um eine Entscheidung für das jeweils preiswerteste Produkt handeln, ohne daß eine

176 Vgl. Bruhn, M., Marketing und Konsumentenzufriedenheit, in: WISU, H. 6, 1985, S. $300 \mathrm{ff}$.

177 In der Präferenztheorie herrscht keine Einigkeit darüber, inwieweit der Preis als Determinante der Präferenz anzusehen ist. So nimmt Gierl in seinem Untersuchungsdesign den Preis als Einflußgröße der Präferenzbildung auf, während Böcker diesen explizit aus der Präferenzdefinition ausschließt. Es steht zu vermuten, daß der Preis primär mit der Zielsetzung ausgeschlossen wird, Unterschiede in den Preisbereitschaften aus der Präferenz heraus erklären zu können. Im Hinblick auf die prognostische Relevanz erscheint es jedoch wenig sinnvoll, die Vorzugswürdigkeit einer Marke unter Vernachlässigung eines kaufentscheidenden Kriteriums zu definieren. In der vorliegenden Arbeit wird daher der Preis als eine präfenzbildende Markeneigenschaft verstanden. Vgl. Böcker, F., Präferenzpolitik, in: Vahlens Großes Marketinglexikon, Hrsg.: Diller, H., München 1992, S. 883 ff.; Böcker, F., Präferenzforschung als Mittel marktorientierter Unternehmensfühung, in: ZfbF, H. 7/8, 1986, S. 543 ff.; Gierl, H., Von der Präferenz zum Kauf eines Markenartikels, in: Markenartikel, H. 10, 1987, S. $459 \mathrm{ff}$.

178 Die mengenmäßige (wertmäßige) Wiederkaufsrate ergibt sich aus der Relation der Absatzmenge (des Umsatzes) einer Marke bei den Wiederholungskäufern dieser Marke zur Absatzmenge (Umsatz), die (der) aus den gesamten Käufen der Endabnehmer in der Produktart resultiert. Vgl. Steffenhagen, Marketing, a.a.O., S. 71; Tolle, E., Steffenhagen, $H$., Kategorien des Markenerfolges und einschlägige Meßmethoden, a.a.O., S. $378 \mathrm{ff}$. 
innere Bindung des Endabnehmers an die Marke besteht. ${ }^{179}$ Speziell im Reifenmarkt erweitert sich diese Problemstellung um einen zusätzlichen Aspekt. Da die am Fahrzeug montierte Reifenmarke nicht notwendigerweise auf eine frühere Wahlentscheidung des gegenwärtigen Fahrzeugnutzers zurückzuführen ist, stimmen die Bereifung des Fahrzeuges und die Markenpräferenzen des Endabnehmers nicht notwendigerweise überein. Hier wäre zu hinterfragen, ob der ("Wieder"-)Kauf der montierten Marke oder der stets gleiche Kauf einer bestimmten Marke als Markentreue zu verstehen ist.

In der vorliegenden Arbeit soll aus den genannten Alternativen markenpolitischer Ziele die Präferenz als zentrale Zielgröße zur Beschreibung der Nachfragestärke gewählt werden.180 Die Entscheidung für dieses Ziel erklärt sich aus drei Gründen: Erstens kann dieses Ziel, differenziert nach seinem Ausmaß, sowohl im Hinblick auf die Erst- als auch die Wiederkäufer einer Marke definiert werden. Damit entfällt die Notwendigkeit, für diese beiden Gruppen inhaltlich unterschiedliche Ziele festzulegen. ${ }^{181}$ Zweitens läßt sich die Präferenz gut im Hinblick auf das angestrebte Verhalten von Käuferaggregaten operationalisieren. Sie ist beispielsweise als Prozentsatz der

179 Das Ziel der Markentreue läßt sich demnach anhand vielfältiger Dimensionen charakterisieren, so z. B. danach, ob es sich um bewußte oder unbewußte, handlungsoder einstellungsmäßige sowie um eine Mehr- oder Einmarkentreue handelt. Nolte kommt im Ergebnis seiner Diskussion der Markentreue zu folgender Definition: Markentreue ist "die von einer Entscheidungseinheit aufgrund eines psychologischevaluativen Entscheidungsprozesses und der daraus resultierenden Markeneinstellung über einen Zeitraum hin vorgenommene Konzentrierung der Käufe auf eine oder einige wenige der in einer Produktkategorie angebotenen Marken". Vgl. Nolte, H., Die Markentreue im Konsumgüterbereich, a.a.O., S. 109. Vgl. auch Jacoby, J., Brand Loyalty, A Conceptual Definition, in: Proceedings of the 79th American Psychological Association Convention 1971, S. 655 f.

180 Grundsätzlich erfüllen alle der hier aufgeführten markenpolitischen Ziele die bei einer solchen Auswahl zu stellenden Anforderungen der praxeologischen und theoretischen Relevanz. Hierfür spricht allein die Breite des Schrifttums, das sich sowohl aus praktischer als auch aus theoretischer Perspektive mit diesen Zielen auseinandersetzt. Auch können alle Ziele zur Beschreibung der Nachfragestärke herangezogen werden, da sie unmittelbar kaufverhaltensbezogen sind oder zumindest die dem Kaufverhalten zugrundeliegende Prädisposition kennzeichnen. $\mathrm{Zu}$ den Kriterien der praxeologischen und theoretischen Relevanz vgl. Mayer, H., Werbewirkung und Kaufverhalten unter ökonomischen und psychologischen Aspekten, Stuttgart 1990, S. $49 \mathrm{ff}$.

181 So läßt sich beispielsweise das Ziel der Markentreue nur für Käufer definieren, die mindestens einmal zuvor die gleiche Reifenmarke (direkt oder indirekt mit dem Fahrzeug) erworben haben. 
Käufer darzustellen, die eine bestimmte Reifenmarke vorziehen. ${ }^{182}$ Drittens kann die Präferenz geeignet derart operationalisiert werden, daß nicht nur die Anzahl der eine Marke präferierenden Konsumenten, sondern auch deren interne Bindung an die Marke zum Ausdruck kommt.

Entsprechend soll das Ziel der Präferenz hier wie folgt operationalisiert werden. ${ }^{183}$ Als Präferenzgrad einer Marke wird der prozentuale Anteil an Reifenkäufern bezeichnet, die sich in einer "fair-choice"-Situation für die betreffende Marke entscheiden würden. 184 Die Präferenzintensität einer Marke kommt in dem Ausmaß zum Ausdruck, in dem der Endabnehmer Such- und Wegeaufwand akzeptiert und in seiner Kaufentscheidung am Point of Sale beeinflußbar ist. ${ }^{185}$ Die Präferenzintensität kann demnach als prozentualer Anteil an Reifenkäufern mit "fair-choice"-Präferenz für eine Marke beschrieben werden, die bereit sind,

182 Der im Rahmen der Modellbildung zugrunde gelegte benutzerorientiert-subjektive Ansatz setzt voraus, daß Käuferaggregate betrachtet werden. Mit einer Einschätzung des Verhaltens einzelner Individuen wäre der Entscheidungsträger allein von der Anzahl der für eine ausreichend große Stichprobe zu beschreibenden Käufer überfordert (Ausnahmen ergeben sich lediglich für den Fall, daß die Anzahl der zu berücksichtigenden Marktteilnehmer sehr gering ist). Dementsprechend sind auch die markenpolitischen Ziele als Vorzugszustände des Käuferaggregates festzulegen. Wenig geeignet sind in diesem Zusammenhang alle Ziele, die graduelle Ausprägungen bestimmter Sachverhalte bei Individuen beschreiben. Dies gilt beispielsweise für die Markeneinstellung, die in aggregierter Form lediglich eine Aussage über den angestrebten durchschnittlichen Einstellungswert in der Käuferschaft zuläßt. Zu Aggregationsvorschriften der Einstellung vgl. auch Steffenhagen, $H_{\text {., }}$ Wirkungen absatzpolitischer Instrumente, a.a.O., S. 128 f. sowie die dort zitierte Literatur.

183 Zur Unterscheidung des Präferenzgrades bei verschiedenen Käufergruppen vgl. Kap. C. 3.12 dieser Arbeit.

184 Dies entspricht der "unmodified preference" zur Kennzeichnung der Breite der Nachfragestärke. Vgl. Kap. A. 2 dieser Arbeit. Vgl. auch Kapferer, J.-N., Die Marke, a.a.O., S. 308 f.

185 Die Präferenzstärke wird hier im Hinblick auf die Berücksichtigung des Beschaffungsaufwandes ähnlich dem Konstrukt der Kaufabsicht beschrieben. Dieses Konstrukt ist jedoch bereits stark am intendierten Verhalten eines konkret anstehenden Kaufs orientiert, während die Präferenzstärke eher auf das grundsätzlich zu erwartende Kaufverhalten abzielt. 
- für den Kauf gewisse Anstrengungen im Hinblick auf den Such- und Wegeaufwand auf sich zu nehmen. Die Wahl der Einkaufsstätte erfolgt dabei grundsätzlich nach dem Kriterium der Verfügbarkeit einer Marke. Endabnehmer, die sich in dieser Weise verhalten, können als "markenfixierte" Reifenkäufer beschrieben werden;

- die Marke zu erwerben, falls hierdurch kein wesentlicher Such- und Wegeaufwand entsteht und keine andere, ebenfalls als akzeptabel betrachtete Marke in erheblich stärkerem Maße am Point of Sale herausgestellt wird. Entsprechend wird die Verfügbarkeit der Marke bei der Einkaufsstättenwahl berücksichtigt, wenn hiermit kein wesentlicher Zusatzaufwand verbunden ist. In diesem Fall kann von "markenorientierten" Reifenkäufern gesprochen werden;

- die Marke nur dann zu erwerben, wenn sie in der beliebig gewählten Einkaufsstätte vorhanden ist und keine andere akzeptable Marke am Point of Sale stärker gefördert wird. Bei der Auswahl der Einkaufsstätte stellt die Verfügbarkeit der Marke kein Entscheidungskriterium dar. Zu dieser Gruppe zählende Endabnehmer sollen als "markenbewußte" Reifenkäufer bezeichnet werden.

Die größtmögliche Präferenzintensität ist somit erreicht, wenn alle Endabnehmer mit "fair-choice"-Präferenz für eine Marke weder durch den Beschaffungsaufwand noch durch situative Einflüsse am Point of Sale von ihrer ursprünglichen Kaufabsicht abweichen, d. h. der Gruppe der markenfixierten Reifenkäufer zuzurechnen sind. Die geringste Präferenzintensität ist gegeben, wenn es sich ausschließlich um markenbewußte Käufer handelt.

Das Ziel der Präferenz beschränkt sich bei der Kennzeichnung des Kaufverhaltens gegenüber einer Marke auf diejenigen Endabnehmer, von denen die betreffende Marke vorgezogen wird. Darüber hinaus ist das Verhalten gegenüber dieser Marke jedoch auch bei indifferenten Käufern oder solchen mit anderweitiger Präferenz zu beschreiben. Für den Reifenmarkt gilt allgemein, daß der Händler einen nicht markenfixierten Kunden zum Kauf jeder Reifenmarke bewegen kann, die nach Kriterien wie Bekanntheit, Image und preislicher Positionierung von dem Kunden grundsätzlich akzeptiert wird. Damit bietet es sich an, als zweites Ziel der Nachfragestärke die Akzeptanz einer Marke zu definieren, d. h. die generelle Bereitschaft des 
Endabnehmers, eine bestimmte Marke zu kaufen. Unter dem Akzeptanzgrad einer Marke soll im folgenden der prozentuale Anteil an indifferenten Reifenkäufern oder solchen mit anderweitiger Präferenz verstanden werden, die eine solche Bereitschaft gegenüber der betrachteten Marke besitzen.

\subsection{Ziele der Angebotsstärke}

Der angestrebte, im Handel auf den Endabnehmer wirkende Angebotsdruck $^{186}$ einer Marke läßt sich mit Hilfe der Ziele des Distributionsgrades sowie der POS-Stärke bestimmen.

Der Distributionsgrad stellt ein Maß für die Erhältlichkeit187 einer Marke dar, das sowohl in numerischer als auch gewichteter Form operationalisiert werden kann. ${ }^{188}$ Welche der beiden Operationalisierungsalternativen geeigneter ist, um die zu erreichende Angebotsstärke zu beschreiben, ist in Abhängigkeit von der Ausprägung des Präferenzziels zu entscheiden. Für Marken mit einer ausgeprägten Präferenzintensität ist der anzustrebende Distributionsgrad in numerischer Form vorzugeben. Da der Endabnehmer seine Einkaufsstätte schwerpunktmäßig nach der Marke wählt, die er zu kaufen beabsichtigt, ist eine Aussage über die notwendige Anzahl an Verkaufsstellen aussagefähiger als der damit abgedeckte Distributionsanteıi am Gesamtmarktumsatz. Hier ist nicht die Größe der einzelnen Einkaufsstätten entscheidend, sondern allein die Frage nach der Anzahl an Verkaufsstellen, die erforderlich ist, damit der vom Reifenkäufer akzeptierte Such- und Wegeaufwand eingehalten wird. Anders stellt sich die Situation bei einer geringen Präferenzintensität der Marke dar. In diesem Fall entspricht die

186 Die Begriffe Angebotsstärke und Angebotsdruck bringen den gleichen Sachverhalt zum Ausdruck und werden daher synonym verwendet.

187 Aus Vereinfachungsgründen soll zur Kennzeichnung der Verfügbarkeit das Problem der Nichtvorrätigkeit von Marken in Einkaufsstätten, die diese grundsätzlich führen, vernachlässigt werden.

188 Der numerische Distributionsgrad mißt ausschließlich die Zahl der die Marke führenden Absatzstellen in bezug auf die Gesamtzahl dieser Stellen, während der gewichtete Distributionsgrad deren Bedeutung, z. B. gemessen am Umsatz, berücksichtigt. Ebenfalls geeignet, allerdings weniger im Rahmen der Markenführung gebräuchlich, ist das Maß der Distributionsdichte, deren Bezugsgröße nicht die Absatzstellen, sondern eine nicht absatzmittlerbezogene Einheit wie z. B. die Fläche eines Gebietes ist. Vgl. Ahlert, D., Distributionspolitik, a.a.O., S. $199 \mathrm{ff}$. 
Umsatzbedeutung einer Einkaufsstätte, in der die Marke geführt wird, dem Anteil an Reifenkäufern, für die die betreffende Marke verfügbar ist. Hier wird die Angebotsstärke geeigneter durch das Ausmaß des zu erreichenden gewichteten Distributionsgrades beschrieben.

Mit dem Ziel der POS-Stärke wird die zu erreichende Förderung der Marke im Vergleich zu Konkurrenzprodukten innerhalb der Einkaufsstätte wiedergegeben. ${ }^{189}$ Mit Bezug auf den in der Literatur in diesem Zusammenhang vielfach verwendeten Begriff des Regalplatzes ist die POS-Stärke als Ausdruck für das Ausmaß der im Handel für die Marke erbrachten, endabnehmergerichteten Leistungen zu verstehen. ${ }^{190}$ Bei diesen Leistungen kann es sich um die physische oder verbale Präsentation der Marke sowie um ihre kommunikative Herausstellung durch Displays, markenbezogene Werbung des Handels etc. handeln. ${ }^{191} \mathrm{Im}$ Reifenmarkt kommt die Förderung einer Marke vornehmlich durch das Empfehlungsverhalten des Händlers zum Ausdruck. In diesem Sinne kann die POS-Stärke einer Marke als der Anteil operationalisiert werden, zu dem der Handel diese gegenüber den Reifenkäufern empfiehlt. Bezugsgröße ist dabei die Gesamtheit aller Empfehlungen der Händler, von denen die betreffende Marke im Sortiment geführt wird.

189 Dies entspricht der "in-store-attractiveness" zur Kennzeichnung der Tiefe der Angebotsstärke. Vgl. Kap. A. 2 dieser Arbeit.

190 Vgl. Cairns, J. P., Suppliers, Retailers and Shelf Space, a.a.O., S. 34 ff. In der Literatur besteht allerdings keine einheitliche Meinung darüber, welche Aktivitäten des Handels genau zum Regalplatz zu zählen sind. Hansen nimmt hier eine Erweiterung von der "Unterbringung der Produkte auf händlerischen Verkaufsflächen" zur "Präsentation des Laden-Werbematerials" vor. Vgl. Hansen, P., Die handelsgerichtete Absatzpolitik der Hersteller im Wettbewerb um den Regalplatz, a.a.O., S. 93 f. sowie S. $186 \mathrm{ff}$. Zur POS-Stärke als Ausdruck endabnehmergerichteter Handelsaktivitäten vgl. auch Steffenhagen, H., Keller, D., Herstellerkonditionen und Handelsleistung, in: Markenartikel, H. 9, 1990, S. 398 ff.

191 Vgl. auch Hansen, U., Absatz- und Beschaffungsmarketing des Einzelhandels, a.a.O., S. $299 \mathrm{ff}$. 


\subsection{Grundsatzstrategien der Reifenhersteller als strategische Rah- menbedingungen für Markendurchsetzungsstrategien}

\subsection{Grundsatzstrategien der Markierung}

Auf dem Reifenmarkt war ursprünglich der Name des Herstellers identisch mit dem Markennamen des Reifens. Aufgrund von Unternehmensakquisitionen der letzten Jahre sind mittlerweile jedoch einige Hersteller im Besitz verschiedener Marken, die parallel geführt werden. Dabei sind unter einer Marke z. T. verschiedene Reifenarten und -technologien zusammengefaßt (z. B. "Standardreifen", runderneuerte Reifen, Niederquerschnittsreifen), die durchaus auch selbständige Marken bilden könnten. Die Reifenhersteller verfolgen demnach entweder eine Dachmarken- oder eine MehrmarkenFamilienmarkenstrategie (vgl. Abbildung 18). ${ }^{192}$ Bei letzterer ist es den Reifenherstellern mit wenigen Ausnahmen nicht gelungen, die eigenen Marken unterschiedlich zu positionieren. Hier bestehen substitutive Beziehungen zwischen allen oder zumindest einem Teil der Marken des jeweiligen Portfolios. ${ }^{193}$ Allerdings wird seitens der Reifenfachhändler zumindest bei einem Reifenhersteller auch eine komplementäre Beziehung zwischen den geführten Marken gesehen. ${ }^{194}$

Die Dachmarken- und Familienmarkenstrategien bieten gegenüber einer Produktmarkenstrategie erhebliche Synergievorteile für die Markendurchsetzung. Mit Blick auf den Handel erhöht sich dessen Aufnahmebereitschaft für ein neues Produkt, sofern bereits positive Erfahrungen mit anderen Produkten derselben Marke vorliegen. ${ }^{195}$ Damit ist der angestrebte Distribu-

192 Eine Kombination aus Dachmarken- und Mehrmarkenstrategie ist theoretisch nicht möglich und wird deshalb von vornherein ausgeschlossen. Die Punkte innerhalb der Abbildung verdeutlichen, welche Strategien von den Reifenherstellern verfolgt werden.

193 Unter einem Markenportfolio ist die Gesamtheit der von einem Unternehmen gleichzeitig geführten Marken zu verstehen. Vgl. auch Laforet, S., Saunders, J., Managing Brand Portfolios, How the leaders do it, in: Journal of Advertising Research, Sept./Oct. 1994, S. $64 \mathrm{ff}$.

194 Quelle: Unveröffentlichte Protokolle von Expertengesprächen mit Reifenfachhändlern.

195 Vgl. Meffert, H., Strategien zur Profilierung von Marken, a.a.O., S. 139 ff.; Titze, A., Marken-Macht, in: Manager Magazin, H. 5, 1991, S. $206 \mathrm{ff}$. 
tionsgrad einfacher zu erreichen als bei Produktmarken. Zudem ist der Handel aufgrund des Vertrauensvorsprunges eher bereit, der Marke eine hohe POS-Stärke einzuräumen, auch wenn im Gegenzug hierzu diejenige anderer Marken verringert werden muß. Gerade auf dem Reifenmarkt steht der Handel der Vielzahl bereits existierender Marken kritisch gegenüber. ${ }^{196}$ Die Einführung eines neuen Reifens unter einer eigenständigen Marke würde unmittelbar auf den Widerstand der Reifenhändler stoßen, der nur mit großem Aufwand zur überwinden wäre. Demgegenüber zeigt die hohe Erneuerungsrate der Reifenherstellersortimente, daß die Sortimentsausweitung oder -änderung innerhalb der gleichen Marke mit wesentlich geringeren Aufnahmebarrieren verbunden ist.

\begin{tabular}{|c|c|c|c|}
\hline \multirow{3}{*}{$\begin{array}{l}\text { Anzahl der } \\
\text { Produkte } \\
\text { pro Marke }\end{array}$} & \multirow{3}{*}{$\begin{array}{c}\text { Anzahl der } \\
\text { Marken } \\
\text { pro Markt }\end{array}$} & eine Marke & mehrere Marken \\
\hline & & \multirow[t]{2}{*}{$\begin{array}{l}\text { Einmarken- } \\
\text { strategie }\end{array}$} & $\begin{array}{l}\text { Mehrmarken- } \\
\text { strategie }\end{array}$ \\
\hline & & & $\begin{array}{l}\text { komplemen-substitutive } \\
\text { täre Marken Marken }\end{array}$ \\
\hline ein Produkt & $\begin{array}{l}\text { Produkt- } \\
\text { marken- } \\
\text { strategie }\end{array}$ & & \\
\hline $\begin{array}{l}\text { mehrere } \\
\text { Produkte }\end{array}$ & $\begin{array}{l}\text { Familien- } \\
\text { marken- } \\
\text { strategie }\end{array}$ & & \\
\hline $\begin{array}{l}\text { alle Produkte } \\
\text { des Unter- } \\
\text { nehmens }\end{array}$ & $\begin{array}{l}\text { Dach- } \\
\text { marken- } \\
\text { strategie }\end{array}$ & & \\
\hline
\end{tabular}

Abb. 18: Markierungsstrategien im Reifenmarkt

196 Quelle: Unveröffentlichte Protokolle von Expertengesprächen mit Reifenfachhändlern. 
Die Vorteile im Hinblick auf die Beeinflussung des Endabnehmers beruhen darauf, daß die Kommunikationsaktivitäten gleichzeitig allen Produkten, die unter einer Marke geführt werden, zugute kommen. Des weiteren besteht eine höhere Werbeeffizienz bei Einführung eines neuen Produktes, da Bekanntheit und Image der Marke schon aufgebaut sind und die Ressourcen darauf konzentriert werden können, das Interesse des Nachfragers an dem neuen Produkt zu wecken. ${ }^{197}$ Der überwiegend wenig sachkundige Reifenkäufer ist bereits bei der gegenwärtigen Anzahl von Reifenmarken nicht in der Lage, eine fundierte Beurteilung deren unterschiedlicher Leistungen vorzunehmen, die als Basis stabiler Präferenzen dienen könnte. Viel weniger noch ist er dazu fähig, zwischen einer Vielzahl von Produktmarken zu differenzieren. Die Markierungsstrategie der Dachmarke oder zumindest der Familienmarken ist damit im Reifenmarkt als notwendige Voraussetzung zu betrachten, eine pull-orientierte Markendurchsetzungsstrategie realisieren zu können.

Wird vom Reifenhersteller eine Mehrmarkenstrategie verfolgt, bei der die Marken in substitutiver Beziehung zueinander stehen, so ist zu beachten, daß hiermit die Verhandlungsposition des Handels verbessert wird. Dieser sieht in den Marken alternative Möglichkeiten zur Befriedigung der gleichen Kundenbedürfnisse. Die auf die einzelne Marke bezogenen Hineinverkaufsmaßnahmen können vom Handel gegeneinander ausgespielt werden und die Ausweitungen von Distributionsgrad und POS-Stärke einer Marke zu Lasten anderer Marken des Herstellers gehen. So wird auch seitens der Reifenhändler den Herstellern bescheinigt, daß ihre Marken z. T. in einem unternehmensinternen Wettbewerb stehen, der "gut für die Konditionen ist". 198

Eine Mehrmarkenstrategie mit Marken, die in einem komplementären Verhältnis zueinander stehen, kann die Erreichung einer hohen Angebotsstärke erleichtern. Die Marken stellen keine Substitute dar, sondern ergänzen sich innerhalb des Sortimentes. Der Reifenhändler kann an dieser Konstellation insofern ein gewisses Interesse besitzen, als daß sich seine

197 Vgl. Smith, D. C., Brand extensions and advertising efficiency, what can and cannot be expected, in: Journal of Advertising Research, Nov./Dec. 1989, S. $11 \mathrm{ff}$.

198 Quelle: Unveröffentlichte Protokolle von Expertengesprächen mit Reifenfachhändlern. 
Transaktionskosten ${ }^{199}$ verringern. Je geringer die Anzahl der Reifenhersteller, mit denen er in direktem Kontakt stehen muß, um ein vollständiges Sortiment anbieten zu können, um so weniger Zeit- und Koordinationsaufwand ist für Vertretergespräche, Bestellungen, Rechnungsabwicklung etc. erforderlich. Im Reifenmarkt ist allerdings zu beobachten, daß die Reifenhersteller ihre Marken z. T. auch unternehmensorganisatorisch trennen, beispielsweise in Form verschiedener Außendienstorganisationen. In einem solchen Fall ergeben sich keine Vorteile für den Handel und entsprechend keinerlei Erleichterung bei der Markendurchsetzung. 200

\subsection{Grundsatzstrategien der Positionierung}

Abbildung 19 zeigt in Profildarstellung das vom Endabnehmer wahrgenommene Image ausgewählter Reifenhersteller bzw. -marken und verdeutlicht damit verschiedene Positionierungen, die von Reifenmarken eingenommen werden. ${ }^{201}$ Die beste Beurteilung hinsichtlich der Grunddimensionen Qualität, Innovation und Programmbreite erhält in dem Beispiel deutlich die Marke A. Sie wird von ihrer Positionierung auf allen drei Dimensionen als besonders stark gesehen. Bezüglich des emotionalen Zusatznutzens ergibt sich ein widersprüchliches Bild. Obwohl jeweils $36 \%$ der Befragten in der Marke einen Reifen für Autofans und sportliche Fahrer sehen, ist nahezu die gleiche Zahl der Meinung, es handele sich vor allem um Reifen für nor-

199 Zum Begriff der Transaktionskosten vgl. Kaas, K.-P., Marketing und neue Institutionenlehre, Arbeitspapier Nr. 1 aus dem Forschungsprojekt Marketing und ökonomische Theorie der Universität Frankfurt, Frankfurt/Main 1993, S. 1 ff.

Quelle: Unveröffentlichte Protokolle von Expertengesprächen mit Reifenfachhändlern.

201 Die Ergebnisse entstammen einer unveröffentlichten Studie eines Reifenherstellers. Befragt wurden Autofahrer, die das Unternehmen bzw. die Marke kennen $(n=800)$. Aufgrund des Informationsstandes der Endabnehmer kann davon ausgegangen werden, daß die Begriffe des Reifenherstellers und der -marke von diesen als Synonyme verstanden werden. Zur Interpretation sind sowohl der Verlauf des Profils an sich als auch die Ausprägungen der Eigenschaften im Vergleich der Marken untereinander heranzuziehen. Eine geringere Eigenschaftsausprägung kann zum einen darauf hinweisen, daß die betreffende Leistung tatsächlich als schlechter wahrgenommen wird. Zum anderen kann es sich um einen Indikator dafür handeln, daß die befragten Personen, obwohl sie angeben, die Marke zu kennen, nicht ausreichend über deren Leistung informiert sind, um eine entsprechende Beurteilung vornehmen zu können. 


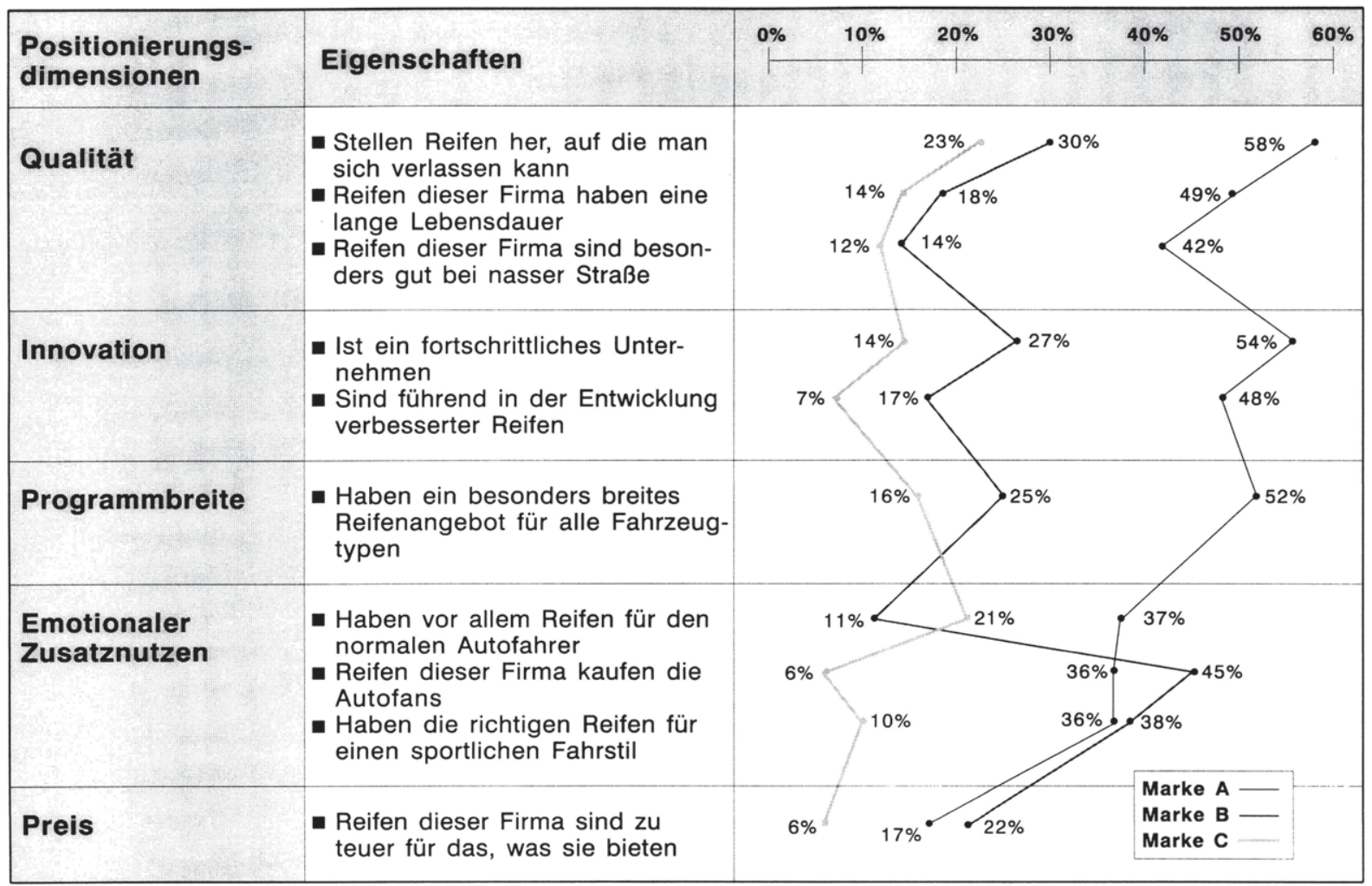

Abb. 19: Ausprägungen verschiedener Positionierungen im Reifenmarkt (Quelle: Unveroffentlichte Studie eines Reifenherstellers) 
male Fahrer. Im direkten Markenvergleich z. B. mit der Marke C sind zwar alle drei Werte hoch ausgeprägt, doch scheint keine eindeutige Vorstellung darüber zu bestehen, ob ein Zusatznutzen in Form der Assoziation mit dem Autofan und der Sportlichkeit vorliegt. Wesentlich prägnanter ist in dieser Hinsicht die Positionierung der Marke B. Dort liegt eine klare Betonung der Dimensionen des emotionalen Zusatznutzens vor, bei dem die Marke als sportlicher Reifen für den Autofan positioniert ist. Demgegenüber stellt die Marke $\mathbf{C}$ aus Sicht des Endabnehmers einen preiswerten Reifen für den Normalfahrer dar. Neben einem günstigen Preis-Leistungsverhältnis verfügt diese Marke über keine besonders hervorzuhebenden Eigenschaften.

Aus diesen verschiedenen Positionierungsstrategien heraus ergeben sich unterschiedliche Rahmenbedingungen für die Strategie der Markendurchsetzung. So ist eine Einschätzung der Qualität von Reifen für den Endabnehmer äußerst schwierig. Die Qualitätseigenschaften eines Reifens lassen sich nicht durch reine Anschauung bestimmen und sind selbst bei längerer Verwendung nur schwer einschätzbar. Auch die Möglichkeiten der Vermittlung zusätzlicher Informationen durch das Produkt oder die Verpackung sind stark eingegrenzt.202 Der Aufbau eines ausgesprochenen Qualitätsimage verlangt im Reifenmarkt daher entweder eine pull-orientierte Markendurchsetzungsstrategie oder aber eine Push-Orientierung, die primär auf eine intensive Beratung des Endabnehmers am Point of Sale abzielt.

Gleiches gilt für die innovationsorientierte Positionierung, die von den Reifenkäufern noch wesentlich schwerer wahrzunehmen ist, da es sich im Reifenmarkt zumeist um kontinuierliche technologische Verbesserungen handelt. Versucht der Reifenhersteller, seine Innovationsorientierung durch die Einführung stark veränderter Technologien zu verdeutlichen, ist darüber hinaus der Unsicherheit über die Akzeptanz der Innovation am Markt zu begegnen. ${ }^{203} \mathrm{Da}$ sich die wenig sachkundigen Reifenkäufer bei ihrer Kaufentscheidung wesentlich an der Empfehlung des Händlers orientieren,

202 Vgl. hierzu Kap. B. 3.11 dieser Arbeit.

203 Zu Kaufwiderständen bei Innovationen vgl. Müller, N., Marketingstrategien in HighTech-Märkten, a.a.O., S. $75 \mathrm{ff}$. Vgl. auch Walters, M., Marktwiderstände und Marketingplanung, Wiesbaden 1984, S. 51 ff. u. S. $155 \mathrm{ff}$. 
spricht in diesem Fall vieles für eine stärkere Push-Orientierung zumindest in der Einführungsphase der Technologie.204

Die Programmbreite des Reifenangebotes als zentrales Positionierungsmerkmal eines Herstellers ist am Point of Sale eingeschränkt darstellbar. Aufgrund des umfangreichen Platzbedarfes von Reifen sowie der geringen Aussagefähigkeit des Reifenäußeren für den wenig interessierten Käufer werden vielfach nur ausgewählte Muster im Verkaufsraum ausgestellt. Auch im Verkaufsgespräch dürfte die Breite des Angebotes einer Marke nur bedingt zum Ausdruck kommen. Der Reifenhändler kann kein Interesse daran haben, den Endabnehmer durch die Vielfalt der Varianten zu verwirren. Vielmehr ist zu erwarten, daß er - den Anforderungen des Kunden entsprechend - zielgenau für jede Marke den geeigneten Reifentyp nennt. Eine Positionierung gegenüber dem Endabnehmer auf der Dimension der Programmbreite scheint somit eher eine pull-orientierte Markendurchsetzungsstrategie zu erfordern.

Auch ein emotionaler Zusatznutzen ist im Reifenmarkt am Point of Sale nur bedingt vermittelbar. Dem Verkauf von Reifen hängt das Image "schmutziger Hände" nach, das sich durchaus bei einer Vielzahl von Reifenfachhändlern in der Realität widerspiegelt.205 Von einer derartigen Einkaufsatmosphäre geht kaum ein emotionaler Nutzen auf die Marke über. Im Gegensatz zu vielen anderen Branchen steht im Reifenmarkt zudem der Begriff des Reifenfachhandels nicht sinngemäß für das Angebot höherwertiger Produkte. Im Sortiment des Reifenfachhandels finden sich vielmehr Marken aller Preislagen, so daß auch unter diesem Aspekt keine Imageübertragung stattfinden kann. Eine solche scheint lediglich für den Fall möglich, daß die Einkaufsstätte durch die Ausweitung des Sortimentes auf andere Serviceleistungen (z. B. Bremsendienst, Stoßdämpfereinbau, Achsenvermessung) und entsprechende Fahrzeugteile in der Lage ist, sich auf bestimmte Marken zu konzentrieren.206 Beispielsweise ist durch das Unter-

204 Vgl. hierzu Kap. B. 3.34 dieser Arbeit.

205 Vgl. o. V., Wir wollen Problemlöser des Autofahrers sein, Interview mit Gerd Grünenwald, Verkaufsdirektor Deutsche Goodyear $\mathrm{GmbH}$, in: Dynamik im Handel, H. 11,1989 , S. 24 f.

206 Zur Sortimentsausweitung im Reifenfachhandel vgl. auch BRV, Erfolgskonzepte 2000, a.a.O., S. $126 \mathrm{ff}$. 
nehmen Goodyear das Handelskonzept "Goodyear Reifen + Auto Service" entwickelt worden. Die angeschlossenen Fachhändler treten in einem "sauberen" und aktiven Erscheinungsbild als "Das Fitness-Center für $\mathrm{lhr}$ Auto" mit einem professionellen Serviceangebot "rund ums Auto" auf. Für den Reifenkauf wird eine Erlebnisatmosphäre mit einem "jungen, dynamischen" Image aufgebaut, das sich auf die Marke Goodyear überträgt.207 Gleichwohl ist zu berücksichtigen, daß ein selektiver Vertrieb, der sich auf wenige, besonders geeignete Verkaufsstellen beschränkt, zu einer Verringerung des maximal erreichbaren Distributionsgrades führt. Insofern erscheint bei der gegenwärtigen Situation des Handels im Reifenmarkt eine Positionierung von Reifenmarken auf der Dimension des emotionalen Zusatznutzens eher eine pull-orientierte Strategie zu verlangen.

\section{Einsatz des Push- und Pull-Instrumentariums als Ausdruck von Markendurchsetzungsstrategien}

\subsection{Darstellung des Push- und Pull-Instrumentariums im Reifenmarkt}

Marketinginstrumente beschreiben Aktivitätsarten und Entscheidungstatbestände zur Beeinflussung der Marktteilnehmer, in denen sich die zuvor getroffenen Entscheidungen über die Strategien der Markierung und Positionierung sowie der Markendurchsetzung konkretisieren. Zur Systematisierung der Instrumente werden diese zumeist den Bereichen Produkt, Distribution, Kommunikation und Kontrahierung zugeordnet.208 Mit Blick auf die Ausgestaltung der Markendurchsetzungsstrategie ist ferner die Ausrichtung des Instrumentes genauer zu beschreiben. So können einige Marketinginstrumente sowohl als Push-Instrument des Hineinverkaufs, als PushInstrument des Herausverkaufs sowie als Pull-Instrument eingesetzt werden. Abbildung 20 gibt einen Überblick über die im folgenden darzustellenden Instrumente.

207 Vgl. o. V., Das Fitness-Center für Ihr Auto, Goodyear Reifen + Auto Service, in: Dynamik im Handel, H. 11, 1989, S. 20 ff.

$208 \mathrm{Zu}$ einer vergleichbaren Vorgehensweise siehe Meffert, H., Marketing, a.a.O., S. $114 \mathrm{f}$. Zu anderen Formen der Systematisierung von Marketing-Instrumenten vgl. Ahlert, D., Distributionspolitik, a.a.O., S. 20. 


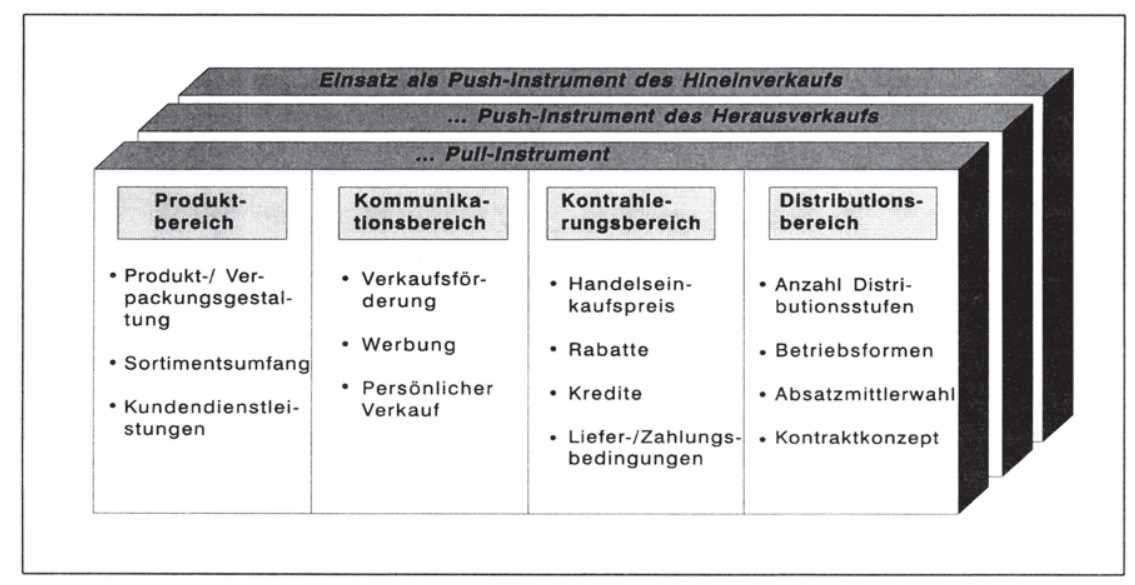

Abb. 20: Systematisierung des Push- und Pull-Instrumentariums

\subsection{Instrumente im Produktbereich}

Marketinginstrumente aus dem Produktbereich umfassen alle marktgerichteten Aktivitäten, die sich auf die Gestaltung des Leistungsprogramms des Unternehmens beziehen. ${ }^{209}$ Hierbei sind Entscheidungen über die Produktund Verpackungsgestaltung, den Sortimentsumfang sowie die Kundendienstleistungen zu treffen.

Bei der Produkt- und Verpackungsgestaltung steht zur Förderung des Hineinverkaufs im Reifenmarkt vor allem die Produktqualität im Sinne einer geringen Fehlerquote sowie die Handhabung des Reifens bei der Montage im Vordergrund. Einer Befragung von Reifenfachhändlern zufolge lassen sich hierbei wesentliche Unterschiede zwischen den Marken feststellen. ${ }^{210}$

209 Zu den Entscheidungstatbeständen des Produktbereiches vgl. Meffert, H., Marketing, a.a.O., S. 361 ff.; Kotler, P., Bliemel, F. W., Marketing-Management, Analyse, Planung, Umsetzung und Steuerung, 7. Aufl., Stuttgart 1992, S. 621 ff.; Meffert, H., Erfolgreiches Marketing in der Rezession, Strategien und Maßnahmen in engeren Märkten, Wien 1994, S. 92 ff.

210 Befragt wurden Reifenfachhändler. Die Ergebnisse stammen aus einer unveröffentlichten Studie eines Reifenherstellers ( $n=436$ ). 
Das Instrument der Verpackung entfällt im Reifenmarkt nahezu vollständig. ${ }^{211}$ Für den transportunempfindlichen und gut stapelbaren Reifen ergibt sich keine Notwendigkeit, durch eine Verpackung zum Schutz des Produktes oder zur Rationalisierung des Transport- und Lagerhaltungsaufwandes beizutragen. Eine zusätzliche Verpackung würde nur den Umfang und damit die erforderliche Lagerfläche des ohnehin voluminösen Produktes ausweiten.

Zur Förderung des Herausverkaufs sind die Produkteigenschaften grundsätzlich dahingehend zu gestalten, daß die Leistungsmerkmale möglichst auffällig und selbstkommunizierend sind und die Marke zudem vom Wettbewerber differenzieren. Hier stehen den Herstellern relativ wenig Möglichkeiten zur Verfügung. Form und Größe des Reifens sind technisch bedingt vorgegeben. Breit- und Niederquerschnittsreifen sind zwar gegenüber einem Standardreifen in ihrem Äußeren verändert, unterscheiden sich jedoch nicht von den entsprechenden Produkten der Wettbewerber. Eine Unterscheidung und Beurteilung individueller Reifenprofile ist für den Endabnehmer kaum möglich. Auch die Verpackung entfällt weitgehend als Informationsträger. Allenfalls finden sich schmale Verpackungsstreifen oder Aufkleber am Reifen angebracht.

In jüngster Zeit wird der Versuch unternommen, eine Differenzierung über die Reifenfarbe zu erreichen. In Anlehnung an die aus früheren Jahren bekannten Weißwandreifen erhalten die Reifen an der Seitenwand eine farbige Aufschrift. So wird gegenwärtig unter der Marke Semperit ein Reifen mit dem weiß-, mint- oder pink-farbenen Schriftzug "SEMPERIT TOPSPEED" angeboten. 212 Sofern nicht mehrere Wettbewerber entsprechende Reifen führen, kann die Produktgestaltung in dieser Form zu einem erfolgversprechenden Pull-Instrument werden, da die Farbigkeit des Reifens eine besonders einprägsame Eigenschaft darstellt.

211 Zum Begriff und den Funktionen der Verpackung vgl. Hansen, U., Leitherer, E., Produktpolitik, 2. Aufl., Stuttgart 1984, S. 93 ff.; Brockhoff, K., Produktpolitik, a.a.O., S. 163; Seidler, J., Bedeutung der Verpackungspolitik für die Markengestaltung, H. 6, 1994, S. 280.

212 Vgl. Continental AG (Hrsg.): Geschäftsbericht 1993, Hannover 1993, S. 16. 
Obgleich die Unterschiede bei Reifen für den Endabnehmer kaum sichtbar sind, ist der Sortimentsumfang bei Reifenherstellern sehr groß. Die Vielzahl an Varianten ist zum einen technisch bedingt, ergibt sich zum anderen aber auch durch den Versuch der Hersteller, ihre Produkte auf verschiedene Käufersegmente abzustimmen.213 So unterscheidet beispielsweise Michelin zwischen Reifen für den comfortbetonten ("Pilot cx"), den harmonisch ausgewogenen ("Pilot hx") und den sportlichen ("Pilot sx") Fahrstil.

Für den Hineinverkauf sind damit sowohl positive als auch negative Aspekte verbunden. Zur Rationalisierung des Einkaufs bevorzugt der Handel zwar insbesondere für seinen Sortimentskern solche Marken, mit deren Produktvarianten die Nachfrage möglichst umfassend abgedeckt werden kann. Die Produktvielfalt stößt jedoch auf den Widerstand des Handels, wenn aufgrund von Substitutionsbeziehungen zwischen den Varianten nur eine Zersplitterung, nicht aber eine Steigerung des Umsatzes erreicht wird.

Der Herausverkauf wird unterstützt, falls sich durch die Einführung von Produktvarianten die POS-Stärke der Marke erhöht, weil aufgrund beschränkter Lagerkapazitäten der Reifenhändler Wettbewerbsmarken in geringerem Umfang gelagert und damit auch angeboten werden. Zudem ist von jeder Produktvariante der Marke eine gewisse Mindestanzahl vorrätig zu halten, so daß es zu steigendem Lageraufwand kommt und hierdurch das eigene Interesse des Handels an einem schnellen Abverkauf wächst.

Eine Wirkung als Pull-Instrument kann im Zusammenhang mit der Sortimentsbreite nur bedingt angenommen werden. Das geringe Interesse am Thema Reifen seitens der Käufer sowie die unzureichende Wahrnehmbarkeit der verschiedenen Leistungseigenschaften sprechen eher dafür, daß eine zunehmende Variantenzahl keinen Einfluß auf die Nachfragestärke der Marke besitzt.

213 Technisch bedingte Variationen ergeben sich aufgrund unterschiedlicher Anforderungen an Höchstgeschwindigkeit und Tragfähigkeit des Reifens. 
Für Kundendienstleistungen ${ }^{214}$ ergeben sich umfassende Ansatzpunkte, diese im Sinne eines Push-Instrumentes dafür zu nutzen, die Zusammenarbeit von Hersteller und Handel auf eine langfristige, kooperative Basis zu stellen. Der einzelne Reifenfachhändler befindet sich selbst in einem intensiven Wettbewerb zu anderen Fachhändlern und den Absatzmittlern der weiteren Vertriebswege. Gleichzeitig fehlen inm aufgrund beschränkter finanzieller und personeller Ressourcen sowie einem vielfach ungenügenden Know-how die Möglichkeiten, sich seinen Kunden gegenüber als modernes, leistungsfähiges Unternehmen zu präsentieren. Hier können vom Hersteller unterstützende Beratungsleistungen, z. B. im Hinblick auf Fragen der Einkaufsstättengestaltung, der Personalführung, der EDV-Systeme etc., angeboten werden. ${ }^{215}$ So offeriert beispielsweise die Continental AG dem Handel ein umfassendes, modular aufgebautes Paket an Beratungsleistungen. Diese reichen von einer Stärken-Schwächen-Analyse für die einzelnen Bereiche über standortspezifische Marktforschungsuntersuchungen bis hin zur Entwicklung und Implementierung innerorganisatorischer Systeme und eines individuell erarbeiteten Marktauftritts des Händlers gegenüber seinen Kunden (vgl. Abbildung 21). ${ }^{216}$

Weitere Kundendienstleistungen bestehen in der Schulung des Händlerpersonals sowie einem guten Lieferservice. ${ }^{217}$ Eine hohe Lieferbereitschaft und -genauigkeit trägt besonders vor dem Hintergrund der großen Variantenviel-

214 Das Instrument des Kundendienstes umfaßt Nebenleistungen zur Absicherung der Funktionstüchtigkeit von Produkten, Zusatzleistungen zur Ergänzung des Produktes sowie sogenannte eigenständige Leistungen, die integraler Bestandteil des Produktnutzens sind. Vgl. Meffert, $\mathrm{H}$., Der Kundendienst als Marketinginstrument Einführung in die Problemkreise des Kundendienstmanagements, in: Kundendienstmanagement, Hrsg.: Meffert, H., Frankfurt/Main, Bern 1982, S. 2 f.; Bauche, K., Segmentierung von Kundendienstleistungen auf investiven Märkten, Dargestellt am Beispiel von Personal Computern, Frankfurt/Main 1994, S. 3 f.

215 Vgl. auch Irrgang, W., Strategien im vertikalen Marketing, Handelsorientierte Konzeptionen der Industrie, München 1989, S. 102.

216 Vgl. Simon, R. S., Marketing-Service als Mittel zur Differenzierung im stagnierenden Reifenmarkt, a.a.O., S. $29 \mathrm{ff}$.

217 Zur Einordnung des Lieferservice zum Instrument des Kundenservice vgl. Specht, G., Distributionsmanagement, 2. Aufl., Stuttgart u.a. 1992, S. 91. Zum Lieferservice vgl. auch Pfohl, H.-Chr., Zur Formulierung einer Lieferservice-Politik - Theoretische Aussagen zum Angebot von Sekundärleistungen als absatzpolitisches Instrument, in: ZfbF, H. 3, 1977, S. $239 \mathrm{ff}$. 
falt von Reifen dazu bei, die Lagerhaltungskosten beim Handel zu verringern und darüber hinaus dessen Risiko zu reduzieren, auftretenden Nachfrageschwankungen nicht entsprechen zu können. ${ }^{218}$

\begin{tabular}{|l|l|}
\hline Modul & Leistungen \\
\hline Unternehmensberatung & $\begin{array}{l}\text { Ermittlung eines Stärken-/Schwächenprofils und Ableitung konkreter } \\
\text { Empfehlungen für Maßnahmen in Bereichen wie Werbung / Kunden- } \\
\text { ansprache, Finanzierung / Controlling, Logistik, Führungsstrukur / } \\
\text { Organisation und Personalberatung }\end{array}$ \\
\hline Corporate Identity & $\begin{array}{l}\text { Individuelle Gestaltung des Gesamtauftritts des Unternehmens z.B. im } \\
\text { Hinblick auf Außenfassade der Einkaufsstätte, Firmenfahrzeuge und } \\
\text { Kleidung des Personals }\end{array}$ \\
\hline Ladengestaltung & $\begin{array}{l}\text { Angebot eines speziell auf die Bedürfnisse des Reifenfachhandels ab- } \\
\text { gestimmten, individuell anpaßbaren Ladeneinrichtungssystems }\end{array}$ \\
\hline Marktforschung & $\begin{array}{l}\text { Ermittlung des Imageprofils des Handelsunternehmens, regionale Ana- } \\
\text { lyse des Endabnehmerverhaltens sowie Standortanalyse für Neugrün- } \\
\text { dungen und Filialeröffnungen }\end{array}$ \\
\hline $\begin{array}{l}\text { Elektronische } \\
\text { Datenverarbeitung }\end{array}$ & $\begin{array}{l}\text { Analyse des EDV-technischen Anforderungsprofils und darauf abge- } \\
\text { stimmte Bewertung verfügbarer Alternativen sowie Unterstützung bei } \\
\text { der Einführung des empfohlenen EDV-Systems }\end{array}$ \\
\hline Schulungen & $\begin{array}{l}\text { Generelle und händlerspezifische Schulungen im Bereich Technik / } \\
\text { Montage und Verkauf }\end{array}$ \\
\hline $\begin{array}{l}\text { Werbung I } \\
\text { Absatzförderung }\end{array}$ & $\begin{array}{l}\text { Entwicklung und Durchführung von Werbe- und Verkaufsförderungs- } \\
\text { aktionen }\end{array}$ \\
\hline
\end{tabular}

Abb. 21: Handelsgerichtete Leistungen der Continental AG (Quelle: Simon, R. S., Marketing-Service als Mittel zur Differenzierung im stagnierenden Reifenmarkt, a.a.O., S. 29 ff.)

\subsection{Instrumente im Kommunikationsbereich}

Marketinginstrumente aus dem Kommunikationsbereich befassen sich mit der bewußten Gestaltung der absatzmarktgerichteten Informationen. Wichtige Entscheidungstatbestände bilden die Werbung, die Verkaufsförderung sowie der persönliche Verkauf. 219

218 Als Pull-Instrument werden Kundendienstleistungen im Reifenmark̄̄ nicht eingesetzt.

219 Darüber hinaus zählen Messen, Public Relations sowie das Sponsoring zu Entscheidungstatbeständen des Kommunikationsbereiches. Diese Instrumente werden im folgenden nicht weiter erläutert, da sie zumeist nicht marken-, sondern 
Als klassisches Push-Instrument des Kommunikationsbereiches wird häufig die Verkaufsförderung genannt.220 Allerdings ist festzustellen, daß Maßnahmen der Verkaufsförderung der Mehrzahl nach aus anderen Instrumentebereichen stammen. Beispiele sind Sondervarianten eines Produktes, unterstützende Werbematerialien am Point of Sale sowie Konditionen- und Preisveränderungen. Eigenständige Maßnahmen bilden die Ausnahme, wie etwa Preisausschreiben, Gewinnspiele etc. Der spezifische, verkaufsfördernde Charakter ergibt sich zumeist durch die zeitliche Befristung des Einsatzes und die sich umgehend einstellenden Reaktionen von Handel und Endabnehmer.221

Die Werbung tritt als Instrument des Hineinverkaufs in Form der handelsgerichteten Ansprache z. B. als Anzeigenwerbung in Fachzeitschriften 222 oder als Direktwerbung auf und dient primär dazu, den Handel über die Leistung und die Eigenschaften der Marke zu informieren. Der Einsatz von Werbung beim Herausverkauf kann entweder unmittelbar am Point of Sale oder aber in Form einer Gemeinschaftswerbung von Hersteller und Handel vorgenommen werden. Am Point of Sale erfolgt im Reifenhandel die Werbung primär über verkaufsunterstützendes Prospekt- und Anschauungsmaterial, im Inneren der Einkaufsstätte aufgestellte und an der Außenfassade angebrachte Displays sowie über spezielle Präsentationsregale für Reifen. Darüber hinaus bietet es sich an, videounterstützte Kommunikationsmittel einzusetzen, die die vergleichsweise besten Möglichkeiten zur

gesamtunternehmensbezogen eingesetzt werden. Vgl. Meffert, H., Integrierte Marktkommunikation, unveröffentlichtes Skript, Münster 1991, S. $81 \mathrm{ff}$.

220 Vgl. zur Verkaufsförderung Bautz, D., Der Promotion-Erfolg muß nicht kurzfristig sein, in: asw, H. 2, 1992, S. 116 ff.; Cristofolini, P., Markenpolitik und Verkaufsförderung, in: Markenartikel, H. 9, 1994, S. 426 ff. Zu Maßnahmen der Verkaufsförderung vgl. Döppner, H. W., Verkaufsförderung - eine Marketing-Funktion, Dargestellt am Beispiel der Konsumgüterindustrie, Berlin 1977, S. 43 ff.; Kellner, J., Promotions, Zielsetzungen, Techniken und Fallbeispiele, Landsberg a. L. 1982, S. $47 \mathrm{ff}$. Zur Wirksamkeit von Verkaufsförderungsaktionen vgl. Diller, H., Brielmaier, A., Die Wirksamkeit von Verkaufsförderungsaktionen, Ein Marktexperiment, Arbeitspapier Nr. 28 des Lehrstuhls für Marketing an der Universität Erlangen-Nürnberg, Nürnberg 1993, S. $14 \mathrm{ff}$.

221 Vgl. auch Diller, H., Das Zielsystem der Verkaufsförderung, in: WiSt, H. 10, 1984, S. $497 \mathrm{f}$.

222 Zu Fachzeitschriften vgl. Wehrle, F., Chancen und Risiken für Fachzeitschriften im europäischen Binnenmarkt, in: Europa 1992, Chancen und Risiken für das Marketing, Hrsg.: Bruhn, M., Wehrle, F., 2. Aufl., Münster-Hiltrup 1990, S. 217 ff. 
Veranschaulichung der Leistungsfähigkeit eines Reifens bieten. ${ }^{223}$ Bei der Gemeinschaftswerbung werden vom Hersteller in aller Regel sogenannte Werbekostenzuschüsse gewährt, um mit der eigenen Marke in der lokal orientierten Werbung des Händlers berücksichtigt zu werden. ${ }^{224}$

Für die pull-orientierte Markendurchsetzungsstrategie ist die endabnehmergerichtete Werbung das zentrale Marketinginstrument. Abbildung 22a zeigt die zeitliche Entwicklung des Werbeaufkommens auf dem Reifenmarkt. 225

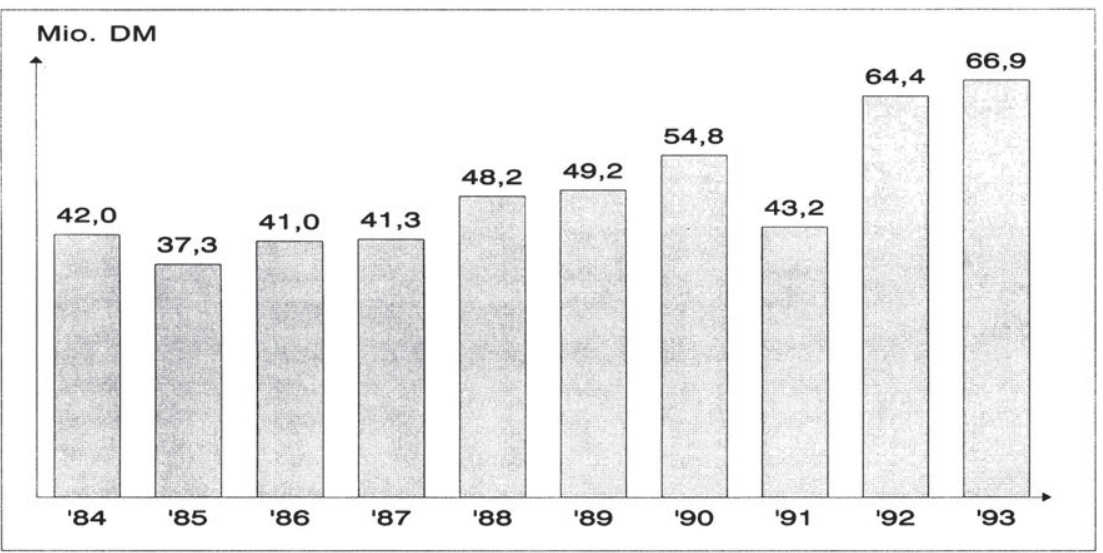

Abb. 22a: Entwicklung des Werbeaufkommens im deutschen Reifenmarkt (Quelle: Unveroffentlichte Studie eines Reifenherstellers; Daten der Nielsen Werbeforschung S+P GmbH)

223 Vgl. Schmitz, B., Die zukünftige Bedeutung von interaktiven Kommunikationssystemen am Point of Sale, in: Der Handel für die Märkte von Morgen, Perspektiven und Entwicklungen, Hrsg.: Gruber, H., Titze, A., Frankfurt/Main 1990, S. 172 ff.

224 Vgl. Irrgang, W., Strategien im vertikalen Marketing, a.a.O., S. 86. Zur kooperativen Werbung vgl. auch Schmalen, H., Kommunikationspolitik, Stuttgart u.a. 1985, S. $202 \mathrm{f}$.

225 Ab 1990 wird das gesamte Werbeaufkommen für die alten und neuen Bundesländer ausgewiesen. Da nur ein geringer Anteil der Werbung auf Fachzeitschriften entfällt (in 1993 ein Anteil von 4,1\%), handelt es sich primär um das endabnehmergerichtete Werbeaufkommen. Die in der Abbildung wiedergegebenen Informationen beruhen auf unveröffentlichten Unterlagen eines Reifenherstellers sowie auf Datenmaterial, das von der Nielsen Werbeforschung $S+P$ GmbH zur Verfügung gestellt wurde. 
Hier ist gerade in den Jahren 1992 und 1993 ein erheblicher Anstieg zu verzeichnen. Dies deutet darauf hin, daß die Hersteller nunmehr verstärkt versuchen, sich unmittelbar kommunikativ gegenüber den Endabnehmern zu profilieren und die Nachfragestärke ihrer Marken auszubauen. Dies gilt in besonderem Maße für die beiden bedeutendsten Marken Michelin und Conti, die allein $35 \%$ des Gesamtwerbeaufkommens in 1993 auf sich vereinen. Bei den für die Werbung ausgewählten Medien stehen eindeutig TV und Publikumszeitschriften im Vordergrund (vgl. Abbildung 22b).

Das Instrument des persönlichen Verkaufs 226 wird von den Reifenherstellern intensiv zur Einflußnahme auf den Handel genutzt. Die Außendienstmitarbeiter der Hersteller suchen die Reifenhändler in relativ kurzen Abständen auf. So erhalten die befragten Reifenfachhändler zwischen 3 und 10 Vertreterbesuche pro Woche. Die jeweilige Gesprächsdauer beträgt zwischen 15 Minuten und 1 Stunde, wobei nicht nur geschäftliche Themen (Neuproduktvorstellung, Konditionen, Herstelleraktionen), sondern auch private Angelegenheiten (Hobbies, Familie etc.) zur Sprache kommen. Dies unterstreicht die Tatsache, daß die Bedeutung des "menschlichen Kontaktes" und der persönlichen Beziehung von den Reifenhändlern überwiegend hoch eingeschätzt wird. Umbesetzungen im Außendienst und damit der Wegfall der gewachsenen Beziehungen können im Extremfall dazu führen, daß die Zusammenarbeit von Hersteller und Händler nachdrücklich beeinträchtigt wird. 227

\subsection{Instrumente im Kontrahierungsbereich}

Im Rahmen des Instrumenteeinsatzes im Kontrahierungsbereich sind Entscheidungen über die Preis- und Konditionengestaltung zu treffen. ${ }^{228}$

226 Zum Instrument des persönlichen Verkaufs vgl. Meffert, H., Marketing, a.a.O., S. $481 \mathrm{ff}$. Der persönliche Verkauf des Herstellers an den Endabnehmer ist aufgrund der unterstellten Mehrstufigkeit des Absatzes hier ausgeschlossen.

227 Quelle: Unveröffentlichte Protokolle von Expertengesprächen mit Reifenfachhändlern.

$228 \mathrm{Zu}$ den Entscheidungstatbeständen des Kontrahierungsbereiches vgl. Meffert, $H$., Marketing, a.a.O., S. 118 ff.; Kotler, P., Bliemel, F. W., Marketing-Management, a.a.O., S. $621 \mathrm{ff}$; Meffert, H., Erfolgreiches Marketing in der Rezession, Strategien und Maßnahmen in engeren Märkten, Wien 1994, S. $116 \mathrm{ff}$. 


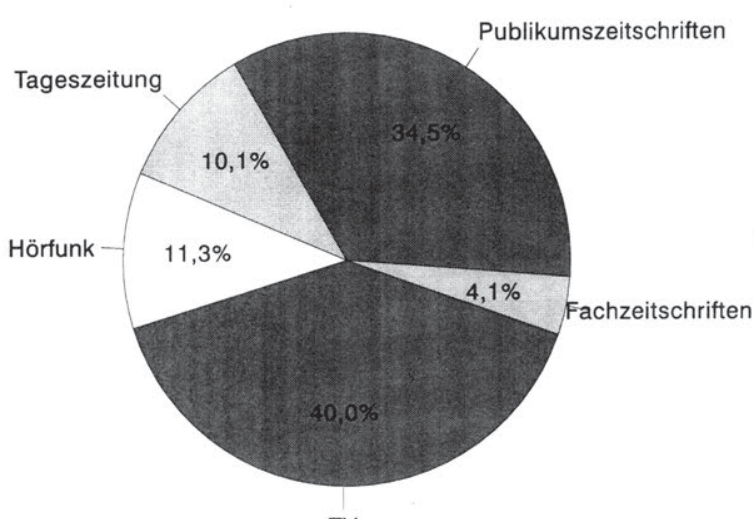

TV

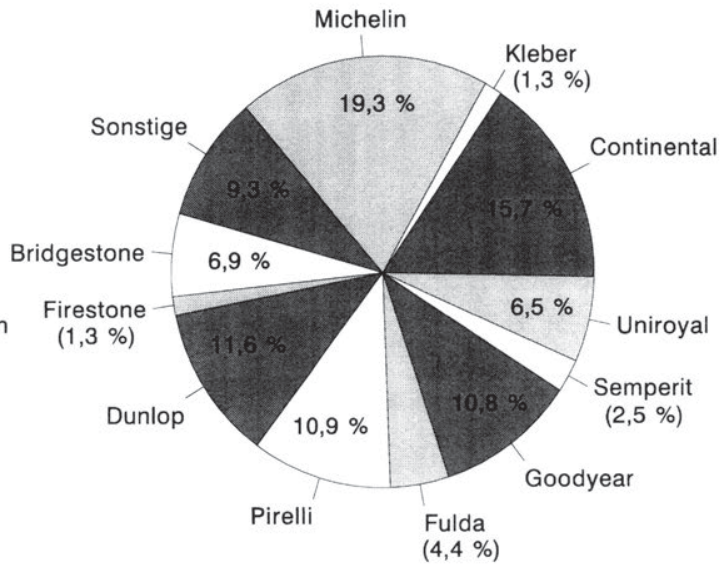

8

\footnotetext{
Abb. 22b: Aufteilung des Werbeaufkommens auf Medien und Reifenmarken in 1993
} 
Der Einkaufspreis des Handels kann wohl als das sensibelste Instrument im Hinblick auf den Hineinverkauf gelten. Bei einem homogenen und breit erhältlichen Gut wie dem des Reifens stellt der am Gesamtmarkt herausgebildete Endabnehmerpreis ein Datum dar, das von einem einzelnen Reifenhändler nur innerhalb eines engen Korridors nach oben überschritten werden kann. So kommt eine Untersuchung auf dem Reifenmarkt zu dem Ergebnis, daß die kritische Schwelle für die Akzeptanz beim Reifenkäufer ca. $10 \%-20 \%$ über dem als Mittelwert empfundenen Preis eines Reifens liegt. 229 Insofern determiniert der Einkaufspreis die für den Handel maximal erreichbare Spanne. ${ }^{230}$

Ein niedriger Handelseinkaufspreis trägt somit zu einer wirksamen Förderung des Hineinverkaufs bei. Gleichzeitig besteht jedoch für den Hersteller die Gefahr, daß sich bei Weitergabe des Preisvorteils durch den Handel an den Endabnehmer die angestrebte Positionierung der Marke nicht adäquat im Preis widerspiegelt.231 Gerade der auf dem Reifenfachhandel lastende Wettbewerbsdruck führt dazu, daß Reifenhändler vielfach mit bekannten Marken preisaggressiv werben, um die Preisgünstigkeit der Einkaufsstätte zu verdeutlichen. ${ }^{232}$

Für den Herausverkauf gilt es, bezüglich des Endabnehmerpreises zu berücksichtigen, daß am Point of Sale unmittelbare Vergleichsmöglichkeiten mit Wettbewerbsmarken bestehen. Diese sind um so bedeutender, je weniger gefestigt das Preiswürdigkeitsurteil beim Endabnehmer ist, d. h. der Wert einer Marke von diesem eingeschätzt werden kann und als Beurteilungsmaßstab des Angebotspreises gilt. Da die Einschätzung der Leistung eines Reifens schwierig ist, kann für den Reifenmarkt angenommen werden,

229 Vgl. hierzu auch Diller, H., Preisimage und Preiswerbung im Reifenhandel, a.a.O., S. $16 \mathrm{ff}$.

230 Zum Zusammenhang von Handelseinkaufspreis und Handelsgewinn vgl. auch Behrends, Chr., Category Management, Von der Vision zur Praxis, in: LZ, 3. Juni 1994, S. 59.

231 Vgl. Pepels, W., Die Beziehungen von Marketing-Interessen auf Hersteller- und Handelsstufe, a.a.O., S. 496.

232 Zur Preiswerbung vgl. Diller, H., Preisimage und Preiswerbung im Reifenhandel, a.a.O., S. $7 \mathrm{ff}$. 
daß der Preisbeurteilung zumeist ein Preisgünstigkeitsurteil zugrunde liegt, bei dem die Konkurrenzmarken zum Vergleich herangezogen werden. ${ }^{233}$

Als Pull-Instrument dient der Preis im Falle einer grundsätzlichen Positionierung der Marke auf niedrigem Preisniveau. Über die Tagespreiswerbung des Händlers hinaus wird hiermit durch den Hersteller ein dauerhaft gültiges Preisimage vermittelt, das den niedrigen Preis als zentrale Stärke der Marke beim Endabnehmer verankert.

Bei den Konditioneninstrumenten ${ }^{234}$ werden im Zusammenhang mit dem Hineinverkauf zumeist Listungsgebühren für die Aufnahme einer Marke in das Sortiment des Händlers entrichtet. Mengen- und zeitpunktabhängige Rabatte zielen darauf $a b$, die Bestellungen großer Mengen zu einem möglichst frühen Zeitpunkt zu erreichen. Auf diese Weise läßt sich ein wirksamer Mengendruck erzielen, da die zur Ausschöpfung der Konditionen eingegangenen Verpflichtungen entsprechende Anstrengungen des Handels beim Abverkauf erfordern. Allerdings wird von den Reifenhändlern die Unübersichtlichkeit der Konditionensysteme einiger Hersteller beanstandet. Die Vielzahl an Rabattstufen führt dazu, daß die Einstandskosten der auf dem Lager befindlichen Reifen kaum zu berechnen sind.235 Über die Konditionen hinaus erhöhen vorteilhafte Liefer- und Zahlungsbedingungen die finanzielle Liquidität und Flexibilität des Händlers und verringern dessen Kosten. Beispielsweise kann der Zahlungszeitraum derart bemessen werden, daß die Ware voraussichtlich bereits an den Endabnehmer weiterveräußert ist oder Kredite zur Neugestaltung der Ladeneinrichtung zur Verfügung gestellt werden.

233 Beim Preiswürdigkeitsurteil wird der Preis explizit im Verhältnis zur Qualität bewertet, während bei der Preisgünstigkeit die Preise ähnlicher, aber aufgrund der Beurteilungsunsicherheit des Endabnehmers nicht zwingend leistungmäßig identischer Konkurrenzmarken zugrunde gelegt werden. Beide Urteile können zu unterschiedlichen Ergebnissen führen, da die Marke bei der Preiswürdigkeit für sich bewertet wird, während die Einschätzung der Preisgünstigkeit stets im Wettbewerbsvergleich erfolgt. Vgl. Diller, H., Preispolitik, 2. Aufl., Stuttgart u.a. 1991, S. 96 ff.

$234 \mathrm{Zu}$ Konditioneninstrumenten vgl. Meffert, H., Marketing, a.a.O., S. 346 ff.; Steffenhagen, H., Keller, D., Herstellerkonditionen und Handelsleistung, a.a.O., S. $398 \mathrm{ff}$.

235 Quelle: Unveröffentlichte Protokolle von Expertengesprächen mit Reifenfachhändlern. 
Zur unmittelbaren Förderung des Herausverkaufs werden insbesondere Aktionsrabatte für konkrete Abverkaufsmaßnahmen des Handels zur Verfügung gestellt oder dessen Kosten für die werbliche Förderung der Marke in Teilen übernommen. ${ }^{236}$ Als Pull-Instrument wird das Konditioneninstrumentarium auf dem Reifenmarkt dagegen nicht eingesetzt. Anders als z. B. im Automobilsektor werden seitens der Hersteller keine Absatzkredite oder ähnliche Vergünstigungen direkt den Endabnehmern gegenüber eingeräumt.

\subsection{Instrumente im Distributionsbereich}

Entscheidungstatbestände aus dem Distributionsbereich beziehen sich auf den Weg eines Produktes vom Hersteller zum Endabnehmer. Hierzu gehört die Selektion der Absatzmittler mit Festlegung der Anzahl von Distributionsstufen und Wahl der Betriebsformen sowie die Entscheidung über das Kontraktkonzept. 237

Bezüglich der Anzahl von Distributionsstufen ist zwischen zwei Aspekten abzuwägen. ${ }^{238}$ Zum einen ist bei einer großen Zahl von Absatzmittlern die push-orientierte Markendurchsetzungsstrategie unter ökonomischen Kriterien vielfach nur dann sinnvoll realisierbar, wenn sich die Anstrengungen auf eine überschaubare Anzahl vorgelagerter Absatzmittler beschränken, die im Sinne einer Multiplikatorenwirkung Einfluß auf nachgelagerte Stufen nehmen. Zum anderen gilt jedoch, daß mit zunehmender Länge des Absatzkanals die Steuerbarkeit des letztlich auf den Endabnehmer zielenden Angebotes der Marke abnimmt. Im Reifenmarkt ist die Anzahl der eigenständigen Händler relativ hoch. Besonders das Instrument des persönlichen Verkaufs

236 Vgl. Irrgang, W., Strategien im vertikalen Marketing, a.a.O., S. 86 f.

237 Zum Distributionsbereich werden unter anderem auch die sogenannten Akquisitions- und Stimulierungskonzepte gezählt, die darüber Aufschluß geben, wie Absatzmittler zur Aufnahme der Marke und ihrer Förderung bewegt werden können. Vgl. Meffert, H., Marketing-Management, a.a.O., S. 173; Irrgang, W., Strategien im vertikalen Marketing, a.a.O., S. 79 ff. Da sich dahinter jedoch der Einsatz von Marketinginstrumenten anderer Bereiche verbirgt, werden diese beiden Konzepte hier nicht als eigenständige Entscheidungen des Distributionsbereiches verstanden.

238 Zur Anzahl der Distributionsstufen vgl. Meffert, H., Marketing, a.a.O., S. 118; Ahlert, D., Distributionspolitik, a.a.O., S. 153. 
wird sich daher auf größere Reifenhändler beschränken müssen, die in ihrer Funktion als Großhändler entsprechend auf die Vielzahl kleinerer Händler einwirken.

Bei der Betriebsform des Handels239 ist zunächst die Markenimageadäquate Auswahl zu beachten. Grundsätzlich ergeben sich hierbei im Reifenmarkt jedoch wenig Beschränkungen, da von den relevanten Vertriebswegen zumindest keine negativen Imageeffekte auf die Reifenmarke wirken. Von ihrem Anteil am Gesamtmarktumsatz stellen der Reifenfachhandel sowie der Automobilhandel die primären Betriebsformen dar, über die der Absatz des Reifenherstellers zu erfolgen hat. Ungeeignet ist der Reifenhandel allerdings im Hinblick auf die Beeinflussung des Bedarfsverhaltens. Da die Endabnehmer erst zum Reifenfachhändler kommen, wenn der Bedarf bereits erkannt wurde, ist eine Bedarfsstimulierung nicht möglich. Anders ist die Situation dagegen bei Automobilhäusern, an Tankstellen oder in KfzWerkstätten, die der Endabnehmer auch aus anderen Gründen als dem Reifenkauf aufsucht.

Bei der Auswahl geeigneter Absatzmittler ist vor allem zu berücksichtigen, daß umsatzstarke Händler i. d. R. eine Vielzahl von Marken führen, wodurch es innerhalb der Einkaufsstätte zum intensiven Markenwettbewerb kommt und eine hohe POS-Stärke nur schwer durchsetzbar ist. Konzentriert sich die Auswahl dagegen auf kleinere Absatzmittler mit nur wenigen Marken im Sortiment, ist zwar die Anzahl240 der einzugehenden Beziehungen

239 Als Handelsbetriebsformen können standardisierte Typen möglicher WarenDienstleistungskombinationen aufgefaßt werden. Als solche werden häufig Fachgeschäfte, Verbrauchermärkte, Warenhäuser etc. unterschieden. Vgl. Specht, G., Distributionsmanagement, a.a.O., S. 60 ff. Für eine ausführliche Diskussion der eng zusammenhängenden Begriffe Betriebsformen und Betriebstypen vgl. Wöllenstein, S., Betriebstypenprofilierung in vertraglichen Vertriebssystemen, Eine Analyse von Einflußfaktoren und Erfolgswirkungen auf der Grundlage eines Vertragshändlersystems im Automobilhandel, Diss., Münster 1994, S. $21 \mathrm{ff}$.

240 Dabei ist zu berücksichtigen, daß mit wachsender Zahl der Absatzmittler die Abwicklungs- und Logistikkosten steigen und es zunehmend schwieriger wird, einen einheitlichen Marktauftritt für die Marke zu gewährleisten. Vgl. Belz, Chr., Die Beschränkung der Absatzkanäle als Marketing-Strategie, in: Jahrbuch der Absatzund Verbrauchsforschung, H. 1, 1990, S. 27 ff. Für Beispiele zu Auswirkungen unkontrollierter Distributionskanäle und Maßnahmen zu deren Beschränkung vgl. o.V., Abspecken und sich wohlfühlen, in: asw, H. 11, 1990, S. $81 \mathrm{ff}$. 
größer, jedoch die POS-Stärke aufgrund des schwächeren Markenumfelds in der Einkaufsstätte um so ausgeprägter.

Mit dem Kontraktkonzept ist schließlich die Art der Verhaltensabstimmung im Absatzkanal anzusprechen, die von einer Lenkung durch reine Marktprozesse bis hin zu einer engen vertraglichen Bindung reicht. ${ }^{241}$ Bei steigendem Zentralisationsgrad 242 der Koordinationsform ist die POS-Stärke vom Hersteller zunehmend autonom vorzugeben, wie es im Rahmen der "controlled distribution" erfolgt. Ein hoher Bindungsgrad erlaubt die langfristige Absicherung einer push-orientierten Markendurchsetzungsstrategie, die besonders von Bedeutung ist, wenn sich der Erfolg nur langfristig einstellt. Ohne entsprechende Sicherungsmaßnahmen läuft der Hersteller andernfalls Gefahr, die anfänglich erreichte POS-Stärke zu verlieren oder sogar ausgelistet zu werden.243

\subsection{Aktivitätsniveau des Push- und Pull-Instrumentariums}

Über die inhaltliche Darstellung des Push- und Pull-Instrumentariums hinaus ergibt sich für die modellgestützte Planung die Frage, wie dessen Grad an Aktivität (Aktivitätsniveau) operationalisiert werden kann.

Steffenhagen schlägt im Zusammenhang mit der Bestimmung von Aktivitätsniveaus vor, jedes Marketinginstrument zunächst in heterogene, elementare Aktivitäten zu zerlegen, diese anhand der Dimensionen Quali-

$241 \mathrm{Zu}$ möglichen Formen solcher Vereinbarungen siehe ausführlich Ahlert, D., Vertikale Kooperationsstrategien im Vertrieb, in: ZfB, H. 1, 1982, S. 62 ff.

242 Nach Grossekettler lassen sich die Koordinationsformen zwischen Hersteller und Handel in einem zweidimensionalen Raum anhand der Dimensionen Zentralisationsgrad als Ausdruck für die Stärke der Weisungsbefugnis des Hersteller und Bindungsgrad als Maß für die vorab vertraglich festgelegten Entscheidungen einordnen. Vgl. Grossekettler, H., Die gesamtwirtschaftliche Problematik vertraglicher Vertriebssysteme, in: Vertragliche Vertriebssysteme zwischen Industrie und Handel, Hrsg.: Ahlert, D., Wiesbaden 1981, S. 255 ff.

243 So zeigen Olver und Farris auf, daß es vergleichsweise einfach ist, die Aufnahme neuer Produkte beim Handel zu erreichen, jedoch um so schwieriger, diese abzusichern. Die Begründung ist darin zu sehen, daß das jeweils schwächste Produkt im Sortiment vom Handel kurzerhand gegen die Neueinführung ausgetauscht wird. Olver, J. M., Farris, P. W., Push and Pull, A One-Two Punch for Packaged Products, a.a.O., S. 57. 
tät, räumliche-personelle Zielung und zeitliche Reichweite zu beschreiben und mit Hilfe der Größe des Kontaktpotentials zu operationalisieren.244 Erst nach der Erfassung der Niveaus aller elementaren Aktivitäten wird durch die anschließende instrumentespezifische Aggregation ein Aktivitätsniveau für das jeweilige Marketinginstrument ermittelt. ${ }^{245}$

In der Unternehmenspraxis erfolgt dagegen die Beschreibung von Aktivitätsniveaus der Marketinginstrumente zumeist anhand kostenmäßig quantifizierbarer Marketingbudgets. Aus Gründen der Vereinfachung, Meßproblemen bei der Erfassung von Niveaus elementarer Aktivitäten und dem Fehlen geeigneter Aggregationsvorschriften wird das Aktivitätsniveau des Instrumenteeinsatzes durch die Höhe der innerhalb eines definierten Zeitraums zur Verfügung stehenden finanziellen Mittel charakterisiert.

Diese Form der Operationalisierung scheint für die Planung von Markendurchsetzungsstrategien ausreichend. Hierbei sind nicht die Aktivitätsniveaus einzelner Instrumente festzulegen, sondern auf einem relativ hohen Aggregationsniveau ist über die grundsätzliche Abstimmung und Schwerpunktsetzung beim Instrumenteeinsatz zu entscheiden. Entsprechend soll für diese Arbeit das Aktivitätsniveau des Push- bzw. PullInstrumentariums durch den Umfang der für die entsprechenden Instrumente insgesamt verfügbaren finanziellen Mittel in einer Periode definiert werden (Push-Budget, Pull-Budget). ${ }^{246}$

244 Die Qualitätsdimension umfaßt verschiedenartige Aktivitätsmerkmale. Dabei stehen vor allem die inhaltliche und formale Ausgestaltung von Botschaften im Vordergrund (Innovativität einer Werbeidee, Originalität der Gestaltung einer Verpackung, Format einer Anzeige oder Dauer eines TV-Spots). In der räumlich-personellen Zielung zeigt sich die "Breitenwirkung" des Instrumenteeinsatzes, in dem primär die Anzahl der mit einer Aktivität potentiell beeinflußbaren Marktteilnehmer (Endabnehmer, Händler) zum Ausdruck kommt. Mit der zeitlichen Reichweite werden der Zeitpunkt des Beginns sowie die Dauer der Aktivität beschrieben. Das Kontaktpotential ist als Erwartungswert der Summe aller mit der Aktivität realisierbaren Kontakte zu den Marktteilnehmern zu verstehen. Vgl. hierzu Steffenhagen, H., Wirkungen absatzpolitischer Instrumente, a.a.O., S. $45 \mathrm{ff}$.

245 Diese Vorgehensweise wird von Steffenhagen vorgeschlagen, der davon ausgeht, daß jedes absatzpolitische Instrument aus einer Vielzahl heterogener, elementarer Aktivitäten besteht, von denen marktstimulierende Wirkungen ausgehen können. Vgl. Steffenhagen, H., Wirkungen absatzpolitischer Instrumente, a.a.O., S. 45 ff.

246 Zum Begriff des Budgets vgl. Diller, H., Budgetierungstechniken, in: Marketingplanung, Hrsg.: Diller, H., München 1980, S. 120; Nieschlag, R., Dichtl, E., 
Ausnahmen bilden Marketinginstrumente aus dem Kontrahierungsbereich. Generell sind diese Instrumente vergleichsweise einfach zu operationalisieren. Sie können direkt als monetäre Größen oder aber als Prozentwert einer solchen Größe beschrieben werden. Allerdings ist eine kostenmäßige Zuweisung zu den Budgets nicht möglich.247 So lassen sich beispielsweise Rabatte gegenüber dem Handel nicht als Kosten interpretieren. Ohne an dieser Stelle theoretische Aspekte des Kostenbegriffs zu vertiefen, ist festzustellen, daß derartige "Kosten" weder als wertmäßige Kosten, ${ }^{248}$ noch als Opportunitätskosten eingeordnet werden können. ${ }^{249}$

Zudem würde eine solche Vorgehensweise das Problem aufwerfen, daß die Budgethöhe nicht im vorhinein festzulegen ist, da die "Gesamtkosten" stückbezogener Preisnachlässe erst nach Ermittlung der erreichten Absatzmenge festgestellt werden können. Damit sind Marketinginstrumente aus dem Kontrahierungsbereich getrennt zu operationalisieren und erst anschließend mit dem Push- bzw. Pull-Budget zu verknüpfen. 250

Hörschgen, H., a.a.O., S. 855 ff.; Lehmann, F. O., Strategische Budgetierung, Entwurf und Fundierung eines Instruments des strategischen Controllings, Frankfurt/Main u. a. 1993, S. 47.

247 Im Gegensatz zu dieser Meinung führt Barzen Kostenbudgets für kontrahierungspolitische Instrumente ein, ohne sich aber mit den damit zusammenhängenden Problemstellungen auseinanderzusetzen. Vgl. Barzen, D., Marketing-Budgetierung, Frankfurt/Main u. a. 1990, S. $58 \mathrm{ff}$.

248 Im Sinne des wertmäßigen Begriffs sind Kosten definiert als "... bewerteter Verbrauch von Gütern und Dienstleistungen für die Herstellung und den Absatz von betrieblichen Leistungen und die Aufrechterhaltung der dafür erforderlichen Kapazitäten". Vgl. Wöhe, G., Einführung in die allgemeine Betriebswirtschaftslehre, 15. Aufl., München 1984, S. 1133.

249 Opportunitätskosten sind solche des entgangenen Gewinns. Vgl. Wöhe, G., Einführung in die allgemeine Betriebswirtschaftslehre, a.a.O., S. 701. Rabatte lassen sich insbesondere deshalb nicht als Opportunitätskosten bezeichnen, da es in Frage steht, ob ohne den entsprechenden Preisnachlaß überhaupt ein Gewinn erzielt werden könnte (z. B. Gefahr der Auslistung), oder aber dieser nicht geringer ausfallen würde (z. B. weil die Förderung der Marke durch den Handel entfällt). 


\subsection{Wirkungsbeziehungen und Interdependenzen des Push- und Pull- Instrumentariums}

Die Analyse der Beziehungen zwischen dem Push- und Pull-Instrumentarium und den markenpolitischen Zielen stellt die Grundlage zur Strukturierung des Modells und zur Formulierung der Erklärungsgleichungen dar. Hierbei soll im folgenden zwischen direkten und indirekten Wirkungsbeziehungen sowie zeitlich-horizontalen und zeitlich-vertikalen Interdependenzen unterschieden werden.

Wirkungsbeziehungen beschreiben den Zusammenhang zwischen dem Aktivitätsniveau eines Marketinginstrumentes und der Ausprägung eines Zieles. Von einer direkten Wirkungsbeziehung soll gesprochen werden, wenn ein unmittelbarer Zusammenhang zwischen Instrument und Ziel besteht, während bei indirekten Beziehungen das Instrument über zwischengeschaltete Ziele auf das letztlich betrachtete Ziel wirkt. ${ }^{251}$ So zeigt Abbildung 23 beispielsweise eine direkte Wirkungsbeziehung des PushInstrumentariums auf die Angebotsstärke und eine indirekte Wirkung auf die Nachfragestärke und den Marktanteil.

Interdependenzen sind jeweils zwischen zwei Instrumenten oder dem Instrumenteeinsatz verschiedener Perioden im Hinblick auf eine gemeinsame Zielwirkung definiert. Zeitlich horizontale Interdependenzen liegen vor, wenn innerhalb einer Periode der Zielbeitrag eines Marketinginstrumentes vom Aktivitätsniveau eines anderen Instrumentes abhängig ist. Dabei ist ein substitutiver Wirkungsverbund gegeben, wenn durch unterschiedliche Kombinationen der Aktivitätsniveaus die gleiche Gesamtwirkung erzielt werden kann. Ergänzen bzw. fördern sich zwei Instrumente in ihrer Wirkung, handelt es sich um einen komplementären Wirkungsverbund. Bei zeitlich vertikaler Interdependenz ist die Wirkung des Instrumentes abhängig vom Aktivitätsniveau des gleichen oder eines anderen Instrumentes in vor- oder nachgelagerten Perioden. ${ }^{252}$

251 Vgl. ähnlich auch Steffenhagen, H., Wirkungen absatzpolitischer Instrumente, a.a.O., S. 190.

252 Vgl. Herlitz, P., Zur optimalen Kombination des absatzpolitischen Instrumentariums, Diss., Berlin 1968, S. 5; Adam, D., Kurzlehrbuch Planung, 2. Aufl., Wiesbaden 1983, S. 62 ff.; Schweiger, G., Die Absatzfunktion als Grundlage der Optimierung des Marketing-Mix, in: der Markt, Nr. 50, 1974, S. 29 ff. Zu einer ausführlichen Dis- 


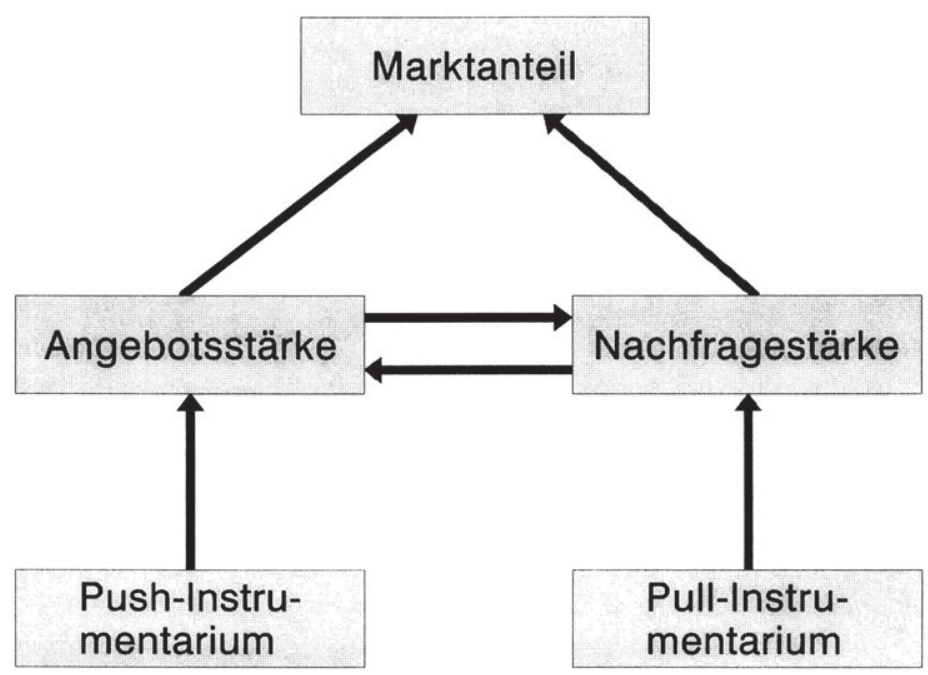

Abb. 23: Schematische Darstellung der Wirkungsbeziehungen des Pushund Pull-Instrumentariums

\subsection{Wirkungsbeziehungen des Push-Instrumentariums}

Vom Push-Instrumentarium geht eine direkte Wirkung auf die Angebotsstärke der Marke aus. Mit zunehmendem Aktivitätsniveau des Instrumentariums werden dem Handel verstärkt Anreize gegeben, die Marke in das Sortiment aufzunehmen und zu fördern. Besonders zu beachten ist hierbei ein bestimmtes Mindestniveau bzw. ein Schwellenwert der Aktivitäten.

Zur besseren Veranschaulichung sei in diesem Zusammenhang zunächst das Verhalten eines einzelnen Händlers betrachtet. Im allgemeinen ist davon auszugehen, daß der Reifenhändler eine neue Marke nur gegen Entrichtung einer Listungsgebühr in sein Sortiment aufnimmt. Darüber hinaus wird der Händler versuchen, weitere "Gebühren" z. B. in Form von zusätzMarketing-Mix-Interaktion, Theorie, empirische Befunde, strategische Implikationen, in: ZfbF, H. 2, 1992, S. $87 \mathrm{ff}$. 
lichen Jahresboni dafür zu verlangen, die Marke nicht auszulisten.253 Aus Herstellersicht ist damit ein Mindestniveau des Push-Instrumentariums erforderlich, das ausschließlich dazu dient, die Listung der Marke zu erreichen bzw. abzusichern.

Mit der Aufnahme der Marke in das Sortiment richtet sich ein gewisser Teil der Verkaufsanstrengungen des Händlers auf deren Absatz. So wird er Reifen und Werbematerialien dieser Marke zu Präsentationszwecken im Verkaufsraum ausstellen und die Marke im Verkaufsgespräch zu einem bestimmten Anteil gegenüber dem Endabnehmer empfehlen. Das Ausmaß dieser "freiwilligen" Förderung ist von dem Vorteil abhängig, den der Händler sich von der Marke verspricht. Handelt es sich um eine leistungsstarke Marke mit hoher Nachfragestärke, deren Angebot sich positiv auf den Umsatz und das Image des Händlers auswirkt, wird er aus Eigeninteresse bestrebt sein, der Marke eine exponierte Stellung im Sortiment einzuräumen. Schwächere Marken dagegen, die eher der Vervollständigung des Sortimentes dienen, erhalten nur ein notwendiges Mindestmaß an POSStärke. Um eine Förderung der Marke zu erreichen, die über den freiwillig vom Händler eingeräumten Umfang hinausgeht, ist seitens des Herstellers das Aktivitätsniveau des Push-Instrumentariums weiter zu erhöhen, beispielsweise in Form zusätzlicher Konditionen oder ergänzender Serviceleistungen (vgl. Abbildung 24). ${ }^{254}$

Im Hinblick auf das Gesamtbudget des Push-Instrumentariums erscheint es damit sinnvoll, zwischen einem Akquisitions- und einem Förderungsbudget zu unterscheiden. Die Höhe des Akquisitionsbudgets bestimmt sich durch die Anzahl der Händler und das jeweils für die Listung notwendige Mindestniveau des Push-Instrumentariums. Eine Veränderung des Budgets führt dementsprechend zu einer Erhöhung bzw. Verringerung des Distributionsgrades. Die Höhe des Förderungsbudgets ergibt sich aus den gesamten Aktivitätsniveaus der Push-Instrumente, die erforderlich sind, um die gewünschte POS-Stärke der Marke in den Einkaufsstätten zu erreichen.

253 Vgl. Irrgang, W., Strategien im vertikalen Marketing, a.a.O., S. 85.

254 Für den Zusammenhang zwischen dem Aktivitätsniveau eines Marketinginstruments und der Marktteilnehmerreaktion wird im allgemeinen ein s-förmiger oder konkaver Verlauf der Wirkungsfunktion unterstellt. Vgl. Nieschlag, R., Dichtl, E., Hörschgen, H., a.a.O., S. 513 f.; Steffenhagen, H., Marketing, a.a.O., S. 193 f. 


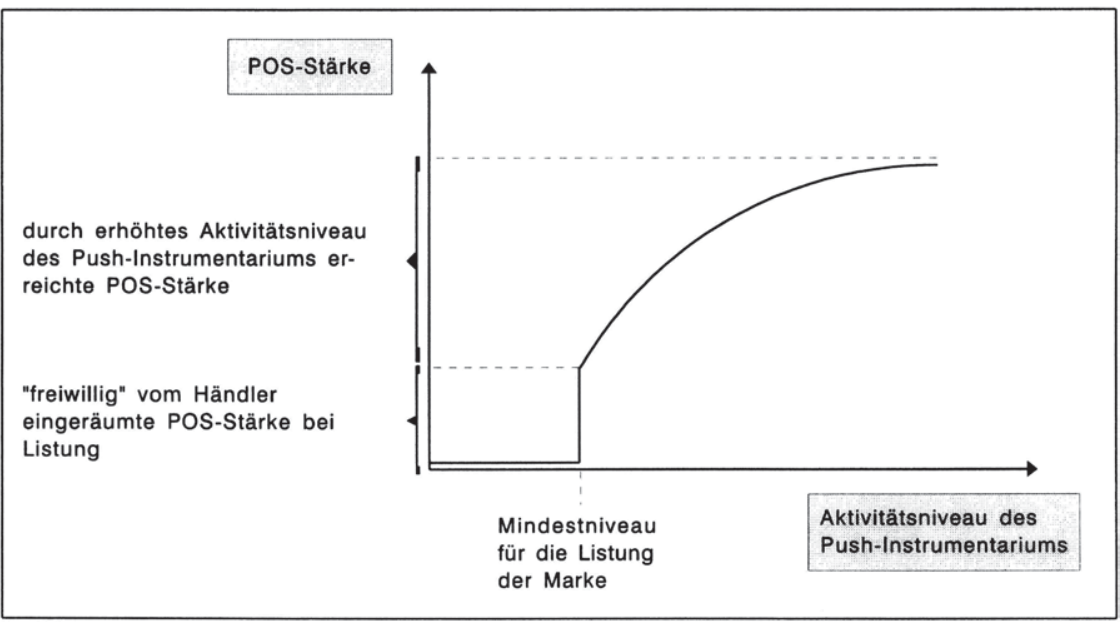

Abb. 24: Zusammenhang von POS-Stärke und Aktivitätsniveau des PushInstrumentariums

Akquisitions- und Förderungsbudget verfolgen nicht nur unterschiedliche Zielsetzungen, sondern richten sich in Teilen auch auf verschiedene Händlergruppen. Während eine Ausweitung des Akquisitionsbudgets stets dazu dient, neue Absatzmittler zu gewinnen, kann sich eine Erhöhung des Förderungsbudgets auch ausschließlich auf solche Händler beziehen, die bereits dem Distributionssystem des Herstellers angehören. Beide Budgets sind allerdings über die Anzahl der insgesamt zu berücksichtigenden Absatzmittler miteinander verbunden. Je höher das Akquisitionsbudget und damit der erzielte Distributionsgrad, um so größer ist das erforderliche Förderungsbudget zur Aufrechterhaltung einer bestimmten POS-Stärke. So werden mit der Akquisition neuer Händler zusätzliche Mittel erforderlich, um nicht nur die Aufnahme der Marke, sondern ebenfalls das gewünschte Ausmaß an Förderung in den jeweiligen Einkaufsstätten zu erreichen.

Eine indirekte Wirkung geht vom Push-Instrumentarium über die Angebots- auf die Nachfragestärke aus. Ein hoher Distributionsgrad und eine ausgeprägte POS-Stärke tragen dazu bei, daß der Käufer über die Existenz der Marke und die Eigenschaften der hierunter geführten Produkte informiert 
wird.255 Gerade die im Reifenmarkt vorherrschende Bedienungsform der Fremdbedienung ist hierzu besonders befähigt. Der Händler kann im Rahmen des Verkaufsgespräches jeden Käufer, für den die Marke grundsätzlich geeignet ist, auf diese Alternative aufmerksam machen, die Vorteile herausstellen und eine Empfehlung aussprechen. Bekanntheit und Image der Marke werden hierüber gefördert und damit die Basis zur Bildung von Präferenzen geschaffen.

\subsection{Wirkungsbeziehungen des Pull-Instrumentariums}

Das Pull-Instrumentarium wirkt unmittelbar auf die Nachfragestärke der Marke. Durch eine direkte Ansprache des Endabnehmers werden Akzeptanz und Präferenz für die Marke geschaffen. Letztere veranlaßt den Reifenkäufer dazu, die Einkaufsstätte bereits mit einer bestimmten Kaufabsicht zu betreten und die Marke beim Handel gezielt nachzufragen.

Auch hier ist ein Mindestniveau der Instrumenteaktivitäten notwendig, um überhaupt eine Wirkung zu erreichen. Durch die Informationsüberflutung des Endabnehmers wird erst beim Überschreiten eines Schwellenwertes des Aktivitätsniveaus dessen Aufmerksamkeit geweckt und damit die Voraussetzung zur weiteren Aufnahme von Informationen über die Marke erfüllt. ${ }^{256}$ Vergegenwärtigt man sich, daß z. B. die Betrachtungszeit einer ganzseitigen Anzeige in Zeitschriften durchschnittlich ca. 2 Sekunden beträgt, gleichzeitig die Kosten für eine solche Anzeige bei bekannten, auflagenstarken Publikumszeitschriften aber durchaus zwischen 60.000 DM und 100.000 DM liegen, so wird schnell die Größenordnung der notwendigen Mindestbudgets deutlich.257 Darüber hinaus nimmt die erreichte Wirkung eines kommunikativen Kontaktes in der sich daran anschließenden Zeit kontinuierlich ab. Die im Rahmen des Pull-Budgets dominierenden Kommunikationsausgaben

255 Vgl. Heeler, R. M., On the Awareness Effects of Mere Distribution, in: Marketing Science, H. 3, 1986, S. 273.

256 Zu einer Übersicht über Prozeßmodelle der Kommunikationswirkung vgl. Mayer, $\mathrm{H}$., Werbewirkung und Kaufverhalten, a.a.O., S. $60 \mathrm{ff}$.

257 Vgl. Kroeber-Riel, W., Strategie und Technik der Werbung, a.a.O., S. 143. Zu den Kosten unterschiedlicher Medien vgl. Mediaanalyse '94, Hrsg.: Axel Springer Verlag, Hamburg 1994. 
sind somit nicht einmalig zu tätigen, sondern auf einem gewissen Niveau aufrechtzuerhalten oder zumindest periodisch zu wiederholen. 258

Indirekte Wirkungen des Pull-Instrumentariums gehen über die Nachfrageauf die Angebotsstärke aus. Aus Sicht des Handels ist eine Marke unter Umsatzkriterien um so attraktiver, je größer der Präferenz- und auch der Akzeptanzgrad ist. Mit zunehmendem Präferenzgrad steigt die Anzahl der Käufer, die eine Marke von vornherein präferieren, und deren Bedürfnissen der Handel mit der Aufnahme dieser Marke in das Sortiment unmittelbar entsprechen kann. Ein hoher Akzeptanzgrad trägt dazu bei, daß mit dem Angebot der Marke auch solche Kunden zufriedengestellt werden können, die entweder indifferent sind oder ursprünglich eine andere Marke bevorzugen. Für den Handel ergibt sich daraus der Vorteil, die Sortimentstiefe verringern zu können, da den Kunden mit der betreffenden Marke eine akzeptierte Alternative geboten wird. ${ }^{259}$

Besteht neben einem hohen Präferenzgrad auch eine hohe Präferenzintensität, kommt der Händler nicht umhin, die Marke zu führen. Andernfalls geht er das Risiko ein, markenfixierte und -orientierte Endabnehmer als Kunden zu verlieren. Allein die Tatsache, daß die Marke aus diesem Grunde vom Handel in das Sortiment aufgenommen wird, bedeutet jedoch nicht, daß ihr ebenfalls eine hohe POS-Stärke eingeräumt wird. Wie das Beispiel der "controlled distribution" im Reifenmarkt zeigt, werden nicht-herstellereigene Marken vornehmlich zur Präsentation eines tiefen Sortimentes geführt, ohne deren Abverkauf jedoch zu forcieren. Zudem werden Reifenhersteller stark nachgefragter Marken im allgemeinen dem Handel gegenüber zu weniger Konditionenzugeständnissen bereit sein. Für den Reifenhändler ist es daher attraktiver, den Kunden im Verkaufsgespräch zum Kauf einer weniger nachfragestarken Marke zu bewegen. Ein solches Verhalten

258 Zur zeitlichen Entwicklung der Kommunikationswirkung vgl. Little, J. D. C., Aggregate Advertising Models: The State of the Art, in: Operations Research, No. 4, S. $631 \mathrm{ff}$.; Schmalen, H., Kommunikationspolitik, a.a.O., S. $56 \mathrm{ff}$.

259 Vgl. Müller-Hagedorn, L., Heidel, B., Die Sortimentstiefe als absatzpolitisches Instrument, in: ZfbF, H. 1, 1986, S. 39 ff.; Gierl, H., Planung der Sortimentstiefe im Handel, in: der Markt, H. 1, 1992, S. $20 \mathrm{ff}$. 
wird konkret auch von den Reifenfachhändlern bestätigt. 260 Hier greifen wiederum die hohen Einflußmöglichkeiten der Fremdbedienung im Reifenhandel.

\subsection{Zeitlich-horizontale Interdependenzen zwischen Push- und Pull- Instrumentarium}

Zeitlich-horizontale Interdependenzen führen dazu, daß der gleiche Marktanteil über verschiedene Kombinationen der Aktivitätsniveaus des Pushund Pull-Instrumentariums erreicht werden kann. Graphisch läßt sich dieser Zusammenhang über ein Reaktionsgebirge abbilden (vgl. Abbildung 25). ${ }^{261}$

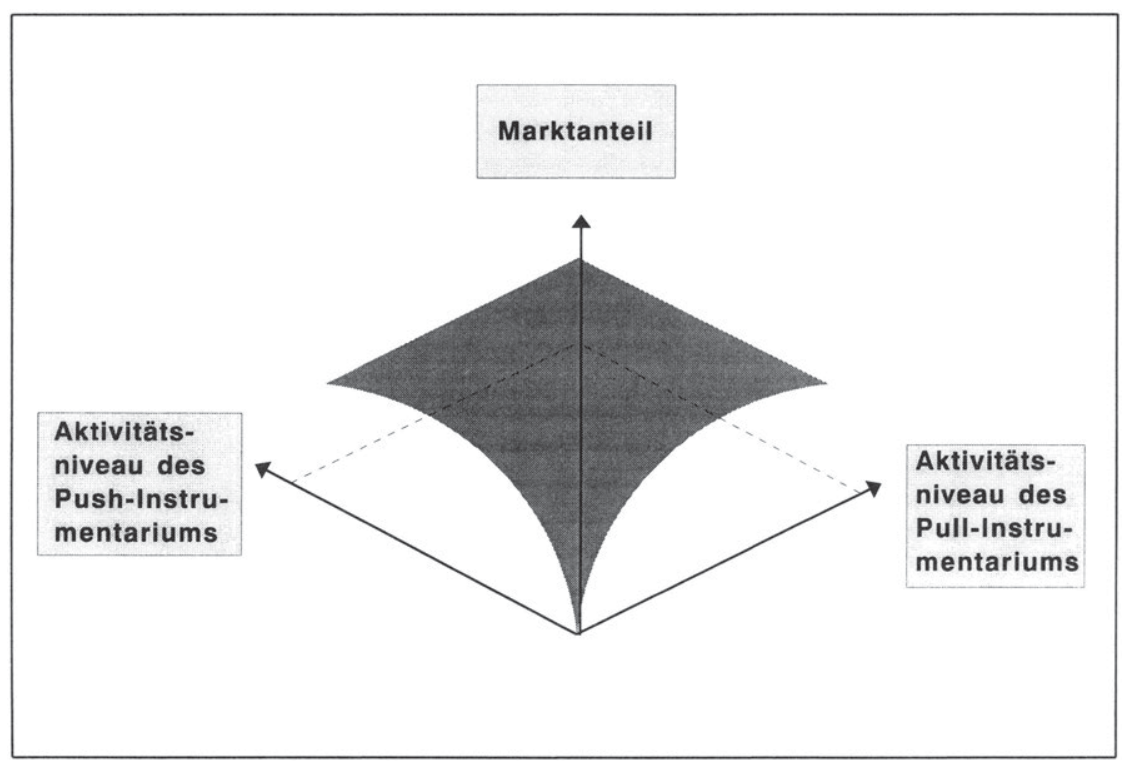

Abb. 25: Zeitlich-horizontale Interdependenzen zwischen Push- und PullInstrumentarium im Hinblick auf das Marktanteilsziel

260 Quelle: Unveröffentlichte Protokolle von Expertengesprächen mit Reifenfachhändlern.

261 Die Mindestniveaus der beiden Instrumentarien wurden aus Übersichtlichkeitsgründen nicht in die Abbildung aufgenommen. Zum Reaktionsgebirge vgl. auch Steffenhagen, H., Wirkungen absatzpolitischer Instrumente, a.a.O., S. $192 \mathrm{ff}$. 
Verantwortlich für die Interdependenzen sind die direkten und indirekten, von den Instrumentarien auf die Angebots- und Nachfragestärke ausgehenden Wirkungsbeziehungen. Um diese Beziehungen inhaltlich erklären zu können, ist eine differenzierte Untersuchung der sich über die Teilziele der Angebots- und Nachfragestärke ergebenden Zusammenhänge notwendig. Hierbei steht die Betrachtung der in Abbildung 26 aufgeführten Beziehungen im Vordergrund. 262

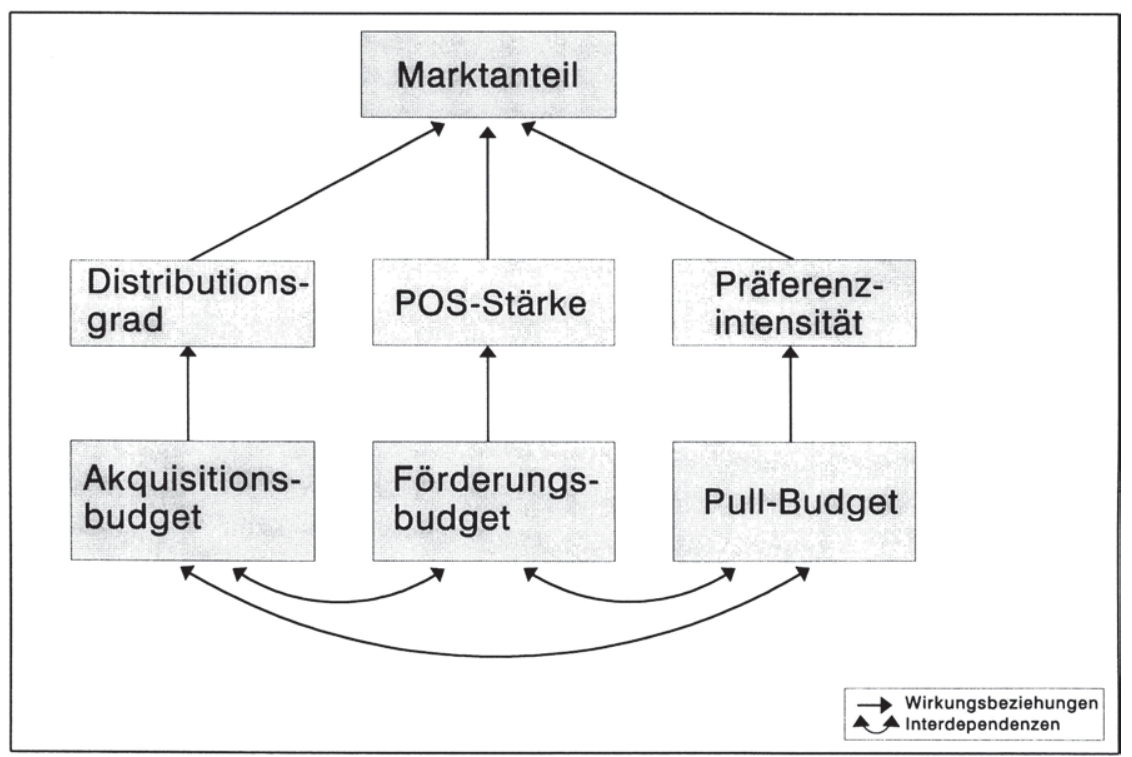

Abb. 26: Vereinfachte Darstellung der zeitlich-horizontalen Interdependenzen des Push- und Pull-Instrumentariums

Die über den Distributionsgrad und die Präferenzintensität entstehenden Interdependenzen beruhen auf dem unterschiedlichen Beschaffungsaufwand, den markenfixierte, -orientierte und -bewußte Endabnehmer zu tragen bereit sind. Je höher die Präferenzintensität, desto geringer ist der erforderliche Distributionsgrad, um einen bestimmten Marktanteil zu erreichen. So

262 Aus Gründen der Übersichtlichkeit wurden hier die indirekten Wirkungsbeziehungen des Push- und Pull-Instrumentariums auf die Ziele des Distributionsgrades, der POS-Stärke und der Präferenzintensität nicht eingetragen. 
ist bei markenfixierten Endabnehmern bereits ein relativ niedriger Distributionsgrad ausreichend, um die Präferenz für eine Marke in einen tatsächlichen Kauf zu überführen, da in dieser Gruppe ein relativ hoher Beschaffungsaufwand beim Reifenkauf akzeptiert wird. Demgegenüber wird es bei den markenbewußten Endabnehmern nur zu einem Kauf entsprechend der Präferenz kommen, wenn die Marke in der vom Kunden beliebig gewählten Einkaufsstätte auch verfügbar ist.

Ähnliches gilt für Interdependenzen, die sich aus dem Zusammenhang von POS-Stärke und Präferenzintensität ableiten lassen.263 Bei markenfixierten Endabnehmern genügt ein Mindestmaß an POS-Stärke, um die Präferenzen in einen Kauf zu überführen. Hier ist ein Aktivitätsniveau des PushInstrumentariums ausreichend, mit dem sichergestellt wird, daß präferenzbeeinträchtigende Eindrücke vermieden werden. Dies bedeutet für den Reifenmarkt vor allem, daß der Händler sich gegenüber dem Käufer nicht negativ über die Marke äußert. Zudem ist eine imageschädigende Präsentation der Reifen (z. B. verstaubte/verschmutzte Ware, unordentlicher Aufbau, mangelnde optische Heraushebung einer hochpreisigen Marke gegenüber Billigprodukten etc.) zu verhindern. Im Hinblick auf die Gruppe der markenbewußten Endabnehmer ist dagegen ein Aktivitätsniveau erforderlich, mit dem zumindest eine den Wettbewerbern gleichwertige POS-Stärke erreicht wird. Andernfalls ist aufgrund der hohen Beeinflußbarkeit der Endabnehmer anzunehmen, daß diese von ihrer "fair-choice"-Präferenz abweichen werden. Hierzu kann bereits die besonders auffällige und interessante Präsentation der Reifen einer anderen Marke im Verkaufsraum ausreichen.

Schließlich sind Interdependenzen zu berücksichtigen, die sich zwischen dem zur Akquisition und dem zur Förderung eingesetzten Push-Instrumentarium aufgrund des Zusammenhangs von POS-Stärke und Distributionsgrad ergeben. Der Hersteller kann sich mit seinen Aktivitäten auf eine relativ geringe Anzahl von Händlern beschränken, um durch den konzentrierten Einsatz des Förderungsbudgets eine hohe POS-Stärke in den entsprechenden Einkaufsstätten zu erreichen. Gerade für den Fall, daß der Reifenhersteller mehrere, verschieden positionierte Marken führt und vor dem Hinter-

263 Eine fehlende Berücksichtigung der Präferenzintensität mag eine Erklärung dafür sein, daß z. T. sehr unterschiedliche Einschätzungen hinsichtlich der Wirksamkeit einer Verkaufsflächenvariation bestehen. Vgl. Curhan, R. C., The Relationship Between Shelf Space and Unit Sales in Supermarkets, a.a.O., S. $406 \mathrm{ff}$. 
grund der Beeinflußbarkeit des Reifenkäufers kann hiermit ein sehr hoher Anteil am gesamten Abverkauf des Händlers erzielt werden. Wird das PushInstrumentarium dagegen primär auf die Erreichung eines hohen Distributionsgrades ausgerichtet und auf die gezielte Beeinflussung der POS-Stärke weitgehend verzichtet, setzt sich der gleiche Marktanteil aus kleineren Absatzanteilen an einer größeren Zahl von Händlern zusammen.

Zwischen den Aktivitätsniveaus des Push-Instrumentariums der Akquisition und der Förderung sowie des Pull-Instrumentariums ist somit in jedem Fall ein substitutiver Wirkungsverbund gegeben. Aufgrund der indirekten Wirkungen, die zuvor im Rahmen der Wirkungsbeziehungen identifiziert wurden, ist ferner davon auszugehen, daß die Interdependenzen auch komplementären Charakter besitzen, $d$. $h$. die Instrumentarien sich gegenseitig in inrer Wirkung fördern. So erhöht die mit dem Pull-Instrumentarium herbeigeführte Nachfragestärke der Marke die Aufnahmebereitschaft des Handels. Damit steigt der bei konstantem Aktivitätsniveau des Push-Instrumentariums erreichbare Distributionsgrad und führt dadurch letztlich zu einem größeren Marktanteil. Umgekehrt werden mit der durch das Push-Instrumentarium erzielten Angebotsstärke Bekanntheit und Image der Marke gefördert. Damit reduziert sich die Wahrnehmungsschwelle und die Aufnahmebereitschaft des Endabnehmers gegenüber dem Pull-Instrumentarium, 264 so daß trotz konstantem Aktivitätsniveau eine steigende Wirkung auf die Nachfragestärke erreicht wird und sich somit wiederum ein höherer Marktanteil einstellt.

\subsection{Zeitlich-vertikale Interdependenzen zwischen Push- und Pull- Instrumentarium}

Zeitlich-vertikale Interdependenzen sind primär bei der Einführung neuer Marken oder aber neuer Produkte unter einer bestehenden Marke von Bedeutung. So stellte sich beispielsweise die Situation bei dem Versuch von

264 Vgl. hierzu auch Kroeber-Riel, W., Strategie und Technik der Werbung, a.a.O., S. 102 u. S. $131 \mathrm{f}$. 
Michelin, das TRX-Reifensystem als echte Innovation am Markt einzuführen, wie folgt dar:265

- Die Fahrzeuge waren durch den Automobilhersteller noch nicht mit den für TRX-Reifen geeigneten Felgen ausgerüstet, so daß für den Endabnehmer eine zusätzliche Investition beim Kauf dieses neuen Reifentyps notwendig wurde.

- Die vom Hersteller versprochenen Eigenschaften des Reifens waren vor dem Kauf nur durch Informationen Dritter zu verifizieren und konnten auch während der Verwendungsphase nur bedingt selber beurteilt werden.

- Weder durch die Erstausrüstung neuer Fahrzeuge, noch durch das Angebot im Handel konnte für den Endabnehmer der Eindruck einer breiten Marktakzeptanz entstehen. Insofern war aus Konsumentensicht der zukünftige Erfolg der Technologie ungewiß und die Investition in neue Felgen als finanzielles Risiko einzuschätzen.

- Für den Handel erforderte die Aufnahme des TRX-Systems in das Sortiment die Anschaffung neuer Montagegeräte und Schulungen des Personals. Darüber hinaus erhöhte sich durch das parallele Angebot des nicht mit dem bisherigen Standard kompatiblen TRX-Systems der Lageraufwand, und es verringert sich die Lagerflexibilität.

Idealerweise wären in einer solchen Ausgangssituation gleichzeitig die Automobilhersteller dahingehend zu beeinflussen, ihre Erstausrüstung auf die neue Technologie umzustellen, durch ein hohes Aktivitätsniveau des Push-Instrumentariums eine ausgeprägte Angebotsstärke zu erreichen und durch den intensiven Einsatz des Pull-Instrumentariums eine hohe Nachfragestärke anzustreben. ${ }^{266}$ Ressourcenbeschränkungen grenzen jedoch die Gesamthöhe des für die Push- und Pull-Instrumente zur Verfügung stehen-

265 Vgl. Belz, Chr., Konstruktives Marketing in gesättigten und schrumpfenden Märkten, a.a.O., S. 198 ff. sowie Kap. B. 1.2 dieser Arbeit.

266 Die Durchsetzung einer Marke bzw. eines neuen Produktes über die Erstausrüstung soll hier nicht weiter betrachtet werden. Die in Kap. B. 1.5 dieser Arbeit aufgezeigten Zusammenhänge zwischen Erstausrüstungs- und Ersatzgeschäft gelten hier analog. Darüber hinaus ist auf die Langfristigkeit einer Durchsetzungsstrategie über die Erstausrüstung hinzuweisen. Bei dem aufgezeigten, vereinfachten Beispiel würden erst nach 3 Jahren und zu diesem Zeitpunkt erst 1,25 \% der Fahrzeuge von Reifenkäufern mit Felgen des TRX-Systems ausgerüstet sein. 
den Budgets ein, d. h. Aktivitätsniveau und zeitliche Entwicklung des Instrumenteeinsatzes sind auch unter Ressourcenaspekten aufeinander abzustimmen.

Die Untersuchung des Informationsverhaltens der Endabnehmer hat deutlich gezeigt,267 daß die Herstellerkommunikation nur in geringem Maße als kaufentscheidende Informationsquelle genutzt wird. Insofern sind zu Beginn der Einführung mit dem Pull-Instrumentarium allenfalls Interesse und Akzeptanz, jedoch kaum klare Präferenzen bei den Endabnehmern zu erreichen. Der Reifenkäufer wird sich vielmehr beim Händler über die neue Marke bzw. das neue Produkt informieren und erst auf dieser Basis eine Präferenz herausbilden. Mit einer pull-orientierten Markendurchsetzungsstrategie zu Beginn der Einführung begibt sich damit der Reifenhersteller in die Gefahr einer "Zeitfalle". Sobald Endabnehmer sich für die neue Marke bzw. das Produkt zu interessieren beginnen, muß diese(s) bereits im Handel verfügbar sein. Andernfalls besteht das Risiko eines schnell abflachenden Interesses, womit ein Teil der getätigten Investitionen in Pull-Instrumente seine potentielle Wirkung einbüßt.

Ein hohes Aktivitätsniveau des Push-Instrumentariums führt demgegenüber schnell zu einer hohen Angebotsstärke im Handel. Durch starke Anreize, wie z. B. finanzielle Unterstützungen bei der Beschaffung der Montagesysteme oder attraktive Preiskonditionen, wird der Reifenhändler nicht nur zur Aufnahme der Marke in sein Sortiment bewegt, sondern dient in seiner Funktion als Marktgestalter zur Schaffung von Präferenzen bei den Käufern. Präferenzfördernd wirkt neben der Beratung des Reifenkäufers durch den einzelnen Händler das generelle Engagement des Handels für die neue Marke bzw. das Produkt, da hiermit Unsicherheiten des Käufers abgebaut werden.

Je größer die vom Endabnehmer wahrgenommene Akzeptanz seitens des Handels für die neue Marke bzw. das Produkt ist, um so eher wird er davon ausgehen, einen guten (funktionales Risiko) und sicheren (gesundheitliches Risiko) Reifen zu erwerben. Zudem verringert sich die Unsicherheit im Hinblick auf die zukünftige Marktentwicklung des Reifens. So sind die zusätzlichen Ausgaben für neue Felgen beim TRX-System nicht nachträglich in 
Frage zu stellen (psychologisches Risiko), weil das System wenig erfolgreich sein könnte, und es steht nicht zu befürchten, daß beim nächsten Reifenkauf keine Reifen für diese speziellen Felgen mehr angeboten werden (finanzielles Risiko). ${ }^{268}$

Im Reifenmarkt scheint somit in der Einführungsphase einer neuen Marke bzw. eines Produktes die push-orientierte Markendurchsetzungsstrategie erfolgversprechender. Sind der angestrebte Distributionsgrad und die gewünschte POS-Stärke erreicht und hiermit auch eine bestimmte Nachfragestärke aufgebaut, kann im Zeitablauf eine Verlagerung der Aktivitäten zu einer stärkeren Pull-Orientierung erfolgen. Aufgrund der zwischenzeitlich entstandenen Nachfrage erhöht sich die Aufnahmebereitschaft und die "freiwillig" eingeräumte POS-Stärke seitens des Handels. Damit werden bislang durch das Push-Instrumentarium gebundene Ressourcen frei und können zu einer Erhöhung des Aktivitätsniveaus des Pull-Instrumentariums eingesetzt werden. Hierdurch steigt die Nachfragestärke, wodurch wiederum das Push-Instrumentarium zurückgenommen werden kann (vgl. Abbildung 27). Grenzen der Verlagerung des Instrumenteschwerpunktes von der Pushzur Pull-Orientierung sind erreicht, wenn eine weitere Budgetveränderung zu einer Verschlechterung des jeweils betrachteten, hierarchisch obersten Zieles führt bzw. bei konstanter Zielerreichung keine Verbesserung der untergeordneten Ziele mehr möglich ist. 


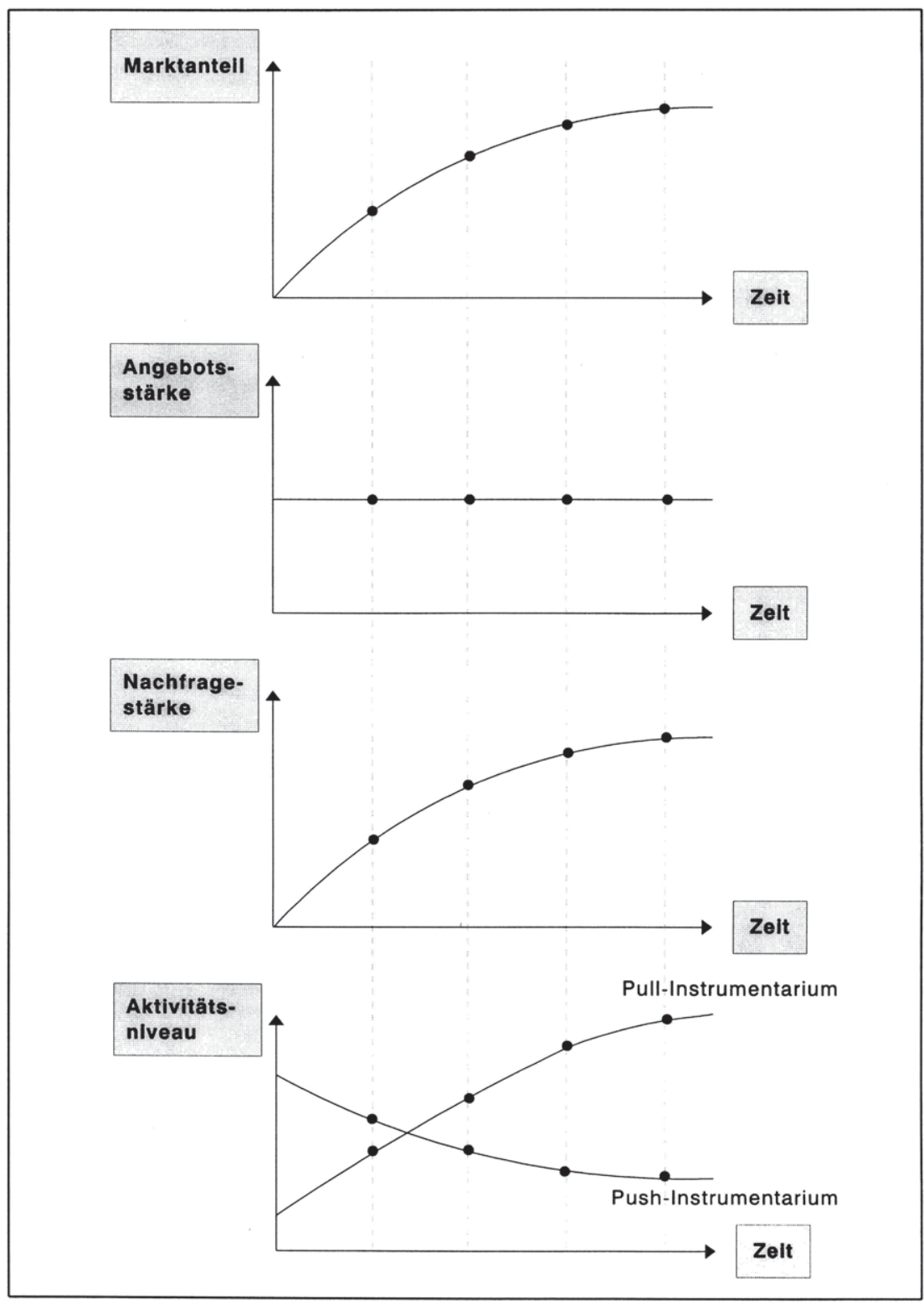

Abb. 27: Idealtypischer Verlauf einer Marken- bzw. Produkteinführung mit Wechsel von einer Push- zur Pull-Orientierung 


\section{Modellgestützte Planung von Markendurchsetzungsstrategien}

\section{Grundlagen der Gestaltung eines Modells zur Planung von Mar- kendurchsetzungsstrategien}

Auf Basis der bislang vorgenommenen inhaltlichen Analyse des Planungsgegenstandes ist im weiteren ein eigenes entscheidungsunterstützendes Modell für die Planung von Markendurchsetzungsstrategien im Reifenmarkt zu entwickeln. Ausgangspunkte der Modellentwicklung sind die Beschreibung der zu beachtenden Strukturdefekte sowie die Festlegung grundlegender Anforderungen an das Modell. Vor diesem Hintergrund sind verschiedene Ansätze der Modellgestaltung zu diskutieren und Implikationen für die Entwicklung des eigenen Modells abzuleiten.

\subsection{Strukturdefekte und Anforderungen an das Modell als Ausgangs- punkt der Entwicklung}

Praxisbezogene Planungsprobleme sind in ihrer Mehrzahl durch sogenannte Strukturdefekte geprägt. Häufig wird deshalb auch von Planung in schlechtstrukturierten Entscheidungssituationen gesprochen. Abhängig davon, auf welche Merkmale sich die Mängel beziehen, können folgende Defekte voneinander unterschieden werden:267

267 Die Begriffe Defekte und Mängel werden hier synonym verwendet. Zu den einzelnen Defekten vgl. ausführlich Adam, D., Zur Problematik der Planung in schlechtstrukturierten Entscheidungssituationen, in: Neue Aspekte der betrieblichen Planung, Schriften zur Unternehmensführung, Hrsg.: Jacob, H., Band 28, Wiesbaden 1980, S. 47 ff.; Adam D., Planungsüberlegungen in wirkungsdefekten Problemsituationen, in: WISU, 1980, H. 8, S. 382 ff.; Adam, D., Planungsüberlegungen in bewertungs- und zielsetzungsdefekten Problemsituationen, in: WISU, 1980, H. 3, S. 127 ff. u. $173 \mathrm{ff}$; Berens, W., Beurteilung von Heuristiken, Neuorientierung und Vertiefung am Beispiel logistischer Probleme, Wiesbaden 1992, S. 16 ff. In der Literatur werden im allgemeinen nur Zielsetzungs-, Bewertungs-, Wirkungs- und Lösungsdefekte voneinander unterschieden. Aufgrund ihrer besonderen Bedeutung für die Modellbildung werden in der vorliegenden Arbeit zusätzlich Meß- und Prognosedefekte betrachtet. 
- Zielsetzungsdefekte,

- Bewertungs-, Meß- und Prognosedefekte,

- Wirkungsdefekte sowie

- Lösungsdefekte.

Zielsetzungsdefekte liegen vor, wenn keine eindeutige, operationale und eindimensionale Zielfunktion gegeben ist. Im Rahmen der Planung von Markendurchsetzungsstrategien fehlt zunächst die Eindeutigkeit der Ziele. So zeigt allein die Diskussion um die Definition des Markenwertes, die Frage der prognostischen Relevanz von Markenbekanntheit und Markeneinstellung sowie die Einführung neuer Konstrukte wie der "Markenidentität", daß in der Literatur keine einheitliche Meinung darüber existiert, welche markenpolitischen Ziele primär zu verfolgen sind.

Für die modellgestützte Planung ist diese defekte Ausgangssituation in eine nicht defekte, für das Modell rechenbare Planungssituation zu überführen. ${ }^{268}$ Hierzu wurde bereits bei der inhaltlichen Analyse des Planungsgegenstandes eine Auswahl aus der Menge von Zielen getroffen, die für die Planung von Markendurchsetzungsstrategien von Bedeutung sind. ${ }^{269}$ Da keine objektiv richtige Wahl der Zielinhalte möglich ist, repräsentieren die festgelegten Ziele zwar nur das subjektive Problemverständnis des für die Auswahl verantwortlichen Entscheidungsträgers, definieren jedoch eine eindeutige und in dieser Hinsicht damit nicht mehr defekte Planungssituation für das Modell.

Ein weiterer Zielsetzungsdefekt besteht in der fehlenden Operationalität der Mehrzahl markenpolitischer Ziele. Beispielsweise umfaßt das Ziel der POS-Stärke eine Vielzahl quantitativer (Anzahl der Empfehlungen, Fläche des Regalplatzes etc.) und qualitativer (Überzeugungskraft, mit der eine Marke empfohlen wird, räumliche Anordnung der Regalfläche innerhalb der Einkaufsstätte etc.) Aspekte. Je nach Wahl dieser Merkmale und dem Vorgehen bei der Aggregation der Teilwerte zu einem Gesamtwert der POS-

268 Vgl. hierzu auch Adam, D., Planung und Entscheidung, Modelle-Ziele-Methoden, 3. Aufl., Wiesbaden 1993, S. 15.

Vgl. Kap. B. 2.1 dieser Arbeit. 
Stärke ergeben sich unterschiedliche Zielinhalte und Ausprägungen. Auch hier wurde bereits durch eine subjektiv festgelegte Operationalisierung der markenpolitischen Ziele eine nicht defekte Planungssituation für das Modell geschaffen.

Der Zieldefekt einer fehlenden Eindimensionalität der Zielfunktion liegt vor, wenn gleichzeitig verfolgte Ziele in konfliktärer Beziehung zueinander stehen. 270 Eine solche Situation ist bei der Markendurchsetzungsstrategie beispielsweise für die Ziele Marktanteil und Gewinn denkbar. Wird mit Hilfe zusätzlicher Produktvarianten der Marktanteil der Marke erhöht, kann sich durch die hiermit entstehenden Komplexitätskosten in der Beschaffung, der Produktion, der Logistik etc. gleichzeitig deren Gewinn verringern. ${ }^{271}$

Im Hinblick auf die Entwicklung eines Erklärungsmodells ist dieser Defekt jedoch von untergeordneter Bedeutung, da zunächst nur die Werte der Zielvariablen ausgewiesen werden. Insofern verlagert sich die Problematik konfliktärer Zielbeziehungen auf den Bereich außerhalb des Modells. Die Entscheidung über die zu wählende Handlungsalternative wird hier erst nach der Modellberechnung vom Entscheidungsträger getroffen. Ausnahmen bestehen, wenn die Ausprägungen der verschiedenen Ziele bereits innerhalb des Modells z. B. über Nutzenfunktionen bewertet und zu einem Gesamtzielwert aggregiert werden sollen.272 In diesem Fall sind entsprechende Bewertungs- und Aggregationsvorschriften in das Modell aufzunehmen.

Bei Bewertungs-, Meß- und Prognosedefekten lassen sich für die Variablen keine eindeutigen Wertansätze finden, die Ausprägungen der Variablen nicht präzise messen oder deren Entwicklung nicht genau prognostizieren.

270 Ein Zielkonflikt entsteht, wenn die Erreichung eines Zieles die Erfüllung des anderen Zieles behindert oder ausschließt. Vgl. Heinen, E., Grundlagen betriebswirtschaftlicher Entscheidungen, a.a.O., S. 95 ff.; Bamberg, G., Coenenberg, A. G., Betriebswirtschaftliche Entscheidungslehre, 6. Aufl., München 1991, S. 46.

$271 \mathrm{Zu}$ Komplexitätskosten vgl. Fischer, T. M., Variantenvielfalt und Komplexität als betriebliche Bestimmungsfaktoren, in: Kostenrechnungspraxis, H. 1, 1993, S. 27 ff.; Becker, W., Komplexitätskosten, in: Kostenrechnungspraxis, H. 3, 1992, S. 171 ff.; Lingenau, V., Kostenwirkungen der Variantenvielfalt, in: Kostenrechnungspraxis, H. 5,1994, S. $307 \mathrm{ff}$.

Vgl. hierzu Kap. C. 3.32 dieser Arbeit. 
Bewertungsdefekte bestehen im Rahmen der Planung von Markendurchsetzungsstrategien vor allem bei der Quantifizierung von Aktivitätsniveaus des Marketinginstrumentariums. Sowohl im Push- als auch im Pull-Instrumentarium sind verschiedene Marketinginstrumente zusammengefaßt, die sich in der Art ihrer Aktivitäten z. T. erheblich voneinander unterscheiden. Daher ist es nur unter stark vereinfachenden Annahmen möglich, deren Einzelniveaus zu einem Gesamtaktivitätsniveau zu aggregieren. Um zu einer nicht defekten Planungssituation zu gelangen, wurden bereits bei der inhaltlichen Analyse die Aktivitätsniveaus als aggregierte Budgets definiert, die den insgesamt zur Verfügung stehenden Umfang der finanziellen Mittel für den Einsatz des Push- und Pull-Instrumentariums beschreiben. ${ }^{273}$

Defekte der Messung bestehen insbesondere mit Blick auf die Erhebung des Marktteilnehmerverhaltens. So sind über rein technische Probleme der Messung (Validität des Meßinstrumentes, Antwortverhalten der Befragten, Interviewereinfluß etc.) hinaus ${ }^{274}$ Untersuchungen des Präferenzverhaltens der Endabnehmer oder des Empfehlungsverhaltens des Handels zunächst ausschließlich für die betrachtete Stichprobe und den Zeitpunkt ihrer Erhebung gültig. Nur unter bestimmten Annahmen (Repräsentativität der Stichprobe, ${ }^{275}$ Zeitstabilität des Verhaltens) kann davon ausgegangen werden, daß die ermittelten Werte auch für die Grundgesamtheit und zumindest für einen Teil des Planungszeitraumes gelten. Prognosedefekte bestehen nahezu in jedem Fall, in dem Veränderungen der Planungssituation während des betrachteten Zeitraums möglich sind. So sind das zukünftige Verhalten von Handel und Endabnehmer oder die Entwicklung von Marktvolumina und Technologien nur mit Unsicherheit prognostizierbar. 276

273 Vgl. Kap. A. 2. dieser Arbeit.

$274 \mathrm{Zu}$ Problemen der Messung vgl. auch Meffert, H., Marketingforschung und Käuferverhalten, a.a.O., S. $202 \mathrm{ff}$.

275 Zur Repräsentativität von Stichproben vgl. Bleymüller, J., Stichprobenverfahren von Wirtschaftswissenschaftlern, Manuskript, Münster 1989, S. 2 ff.

276 Auf die Implikationen der Meß- und Prognosedefekte sowie der im folgenden noch zu beschreibenden Wirkungsdefekte für die modellgestützte Planung ist im Kap. C. 1.3 dieser Arbeit einzugehen. 
Wirkungsdefekte können auf zwei Ursachen beruhen. Zum einen kann Unkenntnis über die erfolgversprechenden Handlungsalternativen bestehen. Unzureichende Kenntnisse der Besonderheiten des Marktteilnehmerverhaltens beim Reifenkauf können z. B. dazu führen, daß die Anforderungen von Handel und Reifenkäufer an die Herstellerleistungen unbekannt sind oder falsch wahrgenommen werden. Zudem kann es zu Fehleinschätzungen hinsichtlich der zu wählenden Marketinginstrumente und der grundsätzlich bestehenden Beeinflussungsmöglichkeiten, z. B. im Hinblick auf das Bedarfsverhalten des Reifenkäufers oder der von inm gewählten Informationsquellen, kommen.

Einer solchen Form von Wirkungsdefekten wurde durch die inhaltliche Analyse der auf dem Reifenmarkt zu berücksichtigenden situativen Faktoren entgegengewirkt. Mit der strukturierten und systematischen Untersuchung des Marktes wurden hier die für die Modellentwicklung erforderlichen Informationsvoraussetzungen geschaffen. Andererseits ist eine zweite Art von Wirkungsdefekten gegeben, wenn der Zusammenhang zwischen dem Aktivitätsniveau des Marketinginstrumentariums und den Ausprägungen der Ziele nicht ermittelbar ist. Von ihrer Wirkung schwer kontrollierbare Einflüsse wie Aktivitäten der Wettbewerber, die Kombination direkter und indirekter Instrumentewirkungen sowie periodenübergreifende Wirkungen in Form von Wirkungsverzögerungen und Verharrungseffekten tragen dazu bei, daß der Wirkungszusammenhang nur bedingt bestimmt werden kann. 277

Schließlich bestehen Lösungsdefekte, wenn keine effiziente Lösungsmethode existiert. Hierbei ist der Begriff der "Lösung" abhängig von der Art des Modells zu interpretieren. Bei Entscheidungsmodellen wird von einer Lösung gesprochen, wenn eine im Hinblick auf die Erwartungsvariablen gute oder optimale Kombination der Entscheidungsvariablen gefunden ist. Im Zusammenhang mit Erklärungsmodellen ist unter einer Lösung die Berechnung der Erwartungsvariablen bei einer bestimmten Konstellation der Entscheidungsvariablen zu verstehen. Die Komplexität des Zusammenwirkens

277 Vgl. Diller, H., Ausstrahlungseffekte, in: Betriebswirtschaftliche Gesetze, Effekte und Prinzipien, Hrsg.: Schanz, G., München 1979, S. 99 ff.; Vgl. ausführlich Simon, H., Goodwill und Marketingstrategie, Wiesbaden 1985. 
von Marketinginstrumenteeinsatz des Herstellers, den Verhaltensweisen von Handel und Endabnehmer sowie der periodenübergreifend wirkenden Reifenausstattung des Fahrzeugs tragen im Reifenmarkt zu einer lösungsdefekten Situation bei.

Es kann somit festgehalten werden, daß im Hinblick auf die Ziel- und Bewertungsdefekte bereits eine geeignete Situation für die modellgestützte Planung vorliegt. Demgegenüber bleiben die Fragen nach der Handhabung von Meß- und Prognosedefekten, der Unsicherheit bei der Festlegung von Wirkungszusammenhängen und der zu wählenden Lösungsmethode noch zu beantworten.

Neben der Analyse bestehender Defekte sind als Ausgangspunkt der Modellentwicklung Gestaltungsanforderungen zu formulieren. Über die bereits getroffene grundsätzliche Entscheidung für einen benutzerorientiertsubjektiven Ansatz hinaus sind hierbei leistungsorientierte, benutzerorientierte sowie design- und kostenbezogene Anforderungen festzulegen (vgl. Abbildung 28). ${ }^{278}$

Zu den leistungsbezogenen Anforderungen zählen die Vollständigkeit, Validierbarkeit, Berechenbarkeit und prognostische Relevanz des Modells. Die Forderung nach Vollständigkeit bezieht sich auf die möglichst umfassende Berücksichtigung aller für das Planungsproblem relevanten Entscheidungstatbestände, situativen Faktoren, Wirkungsbeziehungen und Zielgrößen. Diese sind entweder explizit als Modellvariablen oder -beziehungen zu formulieren oder implizit bei der Festlegung von Variablenwerten und Funktionsparametern zu beachten.

278 Siehe hierzu auch Meffert, der im gleichen Sinne eine Unterscheidung von management-, objekt- und designsystem-bezogenen Kriterien vornimmt. Vgl. Meffert, $H$., Computergestützte Marketing-Informationssysteme, a.a.O., S. 55. Zu den einzelnen Kriterien vgl. Little, J. D. C., Modelle und Manager, a.a.O., S. $207 \mathrm{ff}$;; Breitung, A., Informationssysteme, Ein methodologischer Beitrag von Datenverarbeitungsprozessen in Marketing-Informationssystemen, Diss., Münster 1974, S. 227 ff.; Meffert, H., Steffenhagen, H., Marketing-Prognosemodelle, a.a.O., S. 55 ff.; Voß, W.-D., Modellgestützte Markenpolitik, a.a.O., S. $29 \mathrm{ff}$. 


\begin{tabular}{|c|c|c|}
\hline $\begin{array}{l}\text { Leistungsbe- } \\
\text { zogene Anfor- } \\
\text { derungen }\end{array}$ & $\begin{array}{l}\text { Benutzerorien- } \\
\text { tierte Anforde- } \\
\text { rungen }\end{array}$ & $\begin{array}{l}\text { Design- und } \\
\text { kostenbezogene } \\
\text { Anforderungen }\end{array}$ \\
\hline $\begin{array}{l}\text { - Vollständigkeit } \\
\text { - Validierbarkeit } \\
\text { - Prognostische } \\
\text { Relevanz }\end{array}$ & $\begin{array}{l}\text { - Einfachheit } \\
\text { - Benutzungs- } \\
\text { sicherheit } \\
\text { - Kontrollierbar- } \\
\text { keit } \\
\text { - Dialogfähig- } \\
\text { keit }\end{array}$ & $\begin{array}{l}\text { - Wirtschaft- } \\
\text { lichkeit } \\
\text { - Anpassungs- } \\
\text { fähigkeit } \\
\text { - Integrations- } \\
\text { fähigkeit }\end{array}$ \\
\hline
\end{tabular}

Abb. 28: Anforderungen an die Modellgestaltung

Das Kriterium der Validierbarkeit richtet sich grundsätzlich auf die Übereinstimmung der im Modell abgebildeten und empirisch beobachtbaren Beziehungen der Modellvariablen. Hier besteht die Notwendigkeit, die für das Modell benötigten Daten zu erheben und die Parameter der zugrunde gelegten Beziehungen schätzen zu können. ${ }^{279}$ Diese Anforderung relativiert sich allerdings dahingehend, daß beim benutzerorientiert-subjektiven Ansatz zunächst das Ziel im Vordergrund steht, das interne Modell des Entscheidungsträgers abzubilden. Bei diesem Ansatz ist die Validierbarkeit derart zu interpretieren, daß der Entscheidungsträger in der Lage sein muß, für die von ihm angenommenen Beziehungen subjektive Schätzungen abgeben zu können.

Die Forderung der prognostischen Relevanz zielt darauf $a b$, daß eine Übereinstimmung der vom Modell ermittelten und in der Zukunft tatsächlich eintretenden Ausprägungen der Erwartungsvariablen bestehen muß. Wenngleich das interne Modell des Entscheidungsträgers nicht notwendigerweise mit der realen Situation übereinstimmt, so hat das Modell im

279 Diese Aspekte werden auch unter dem Begriff der Validierbarkeit eines Modells subsumiert. Vgl. Meffert, H., Steffenhagen, H., Marketing-Prognosemodelle, a.a.O., S. 56; Steffenhagen, H., Wirkungen absatzpolitischer Instrumente, a.a.O., S. $22 \mathrm{ff}$. 
Praxiseinsatz dennoch zu zeigen, daß die berechneten Ergebnisse von Relevanz für die Entscheidung sind. Dies setzt zumindest voraus, daß die Ergebnisse die für die Realsituation tatsächlich beste Handlungsalternative aufzeigen.

Benutzerorientierte Anforderungen beziehen sich auf Kriterien der Einfachheit, Benutzungssicherheit, Dialogfähigkeit und Kontrollierbarkeit. Mit dem Prinzip der Einfachheit soll die Transparenz und Verständlichkeit des Modells sichergestellt werden. Details werden nur insoweit abgebildet, wie der Entscheidungsträger sie tatsächlich zur Entscheidungsfindung heranzieht. Die Benutzungssicherheit erleichtert den Umgang mit dem Modell, indem von vornherein nur solche Angaben zugelassen werden, die zu sinnvollen Ergebnissen führen. Das Modellverhalten muß zudem vom Entscheidungsträger kontrollierbar, d. h. die Berechnungen innerhalb des Modells nachvollziehbar sein. Die Dialogfähigkeit verlangt die Möglichkeit der interaktiven Arbeit des Entscheidungsträgers mit dem Modell, die sowohl aufgrund des benutzerorientiert-subjektiven Ansatzes notwendig wird als auch dem Gedanken des Experimentierens mit dem Modell unter verschiedenen Annahmen der Planungssituation entspricht.

Design- und kostenbezogene Anforderungen berücksichtigen schließlich die Wirtschaftlichkeit des Modells sowie die notwendige Integrations- und Anpassungsfähigkeit. Mit dem Kriterium der Wirtschaftlichkeit280 wird gefordert, daß die Kosten der Modellentwicklung und -implementierung sowie der Datenbeschaffung in einem angemessenen Verhältnis zur Erhöhung von Geschwindigkeit und Verbesserung der Qualität der Planung stehen müssen. Die Anforderung der Anpassungsfähigkeit richtet sich auf die Notwendigkeit, neue Erkenntnisse in dem Modell berücksichtigen zu können. Dies gilt sowohl für die verwendeten Daten als auch für die Modellstruktur. Abschließend ist die Forderung nach Integrationsfähigkeit zu stellen, die eine Einbindung des Modells in bereits bestehende Planungssysteme erlaubt.

280 Zur Forderung der Wirtschaftlichkeit von Modellen vgl. Naert, P. A., Leeflang, P. S., Building implementable marketing models, Boston 1978, S. $353 \mathrm{ff}$. 


\subsection{Darstellung von Ansätzen zur Gestaltung von Modellen}

Ansätze der Modellgestaltung lassen sich nach mehreren Dimensionen systematisieren. Dabei sind vor allem der Aggregationsgrad der Marktteilnehmerreaktion, dessen Abstraktionsgrad, die Berücksichtigung von Unsicherheit und dynamischen Entwicklungen, die Vollständigkeit des Modells sowie die verwendete Lösungsmethode zur Kennzeichnung heranzuziehen (vgl. Abbildung 29).

\begin{tabular}{|c|c|c|}
\hline Dimension & \multicolumn{2}{|c|}{ Ausprägungen } \\
\hline Abstraktionsgrad & detailanalytisch & $\hookrightarrow \longrightarrow$ globalanalytisch \\
\hline Aggregationsgrad & Mikromodell & $\hookrightarrow$ Makromodell \\
\hline Zeitbezug & statisch & $\hookrightarrow \rightarrow$ dynamisch \\
\hline Modellumfang & Totalmodell & $\triangleleft \longrightarrow$ Partialmodell \\
\hline Unsicherheit & deterministisch & $\triangleleft \rightarrow$ stochastisch \\
\hline Lösungstechnik & algorithmisch & $\hookrightarrow$ simulativ \\
\hline
\end{tabular}

Abb. 29: Dimensionen zur Systematisierung von Modelltypen

Bezüglich des Aggregationsgrades der untersuchten Marktteilnehmerreaktion wird im allgemeinen zwischen Mikro- und Makromodellen differenziert. ${ }^{281}$ Mikromodelle stellen den einzelnen Marktteilnehmer in den Mittelpunkt der Betrachtung und ermitteln das Reaktionsverhalten jedes Indivi-

281 Vgl. Meffert, H., Steffenhagen, H., Marketing-Prognosemodelle, a.a.O., S. 37 ff.; Kulla, B., Ergebnisse oder Erkenntnisse - liefern makroanalytische Simulationsmodelle etwas Brauchbares?, in: Biethahn, J., Schmidt, B., Simulation als betriebliche Entscheidungshilfe, Methoden, Werkzeuge, Anwendungen, Berlin u. a. 1987, S. $5 \mathrm{f}$. 
duums. Wenngleich hierdurch eine hohe Trennschärfe bei der Analyse von Wirkungen verschiedener Einflußfaktoren auf das Verhalten erreicht werden kann, sprechen in aller Regel der Aufwand einer auf das Individuum bezogenen Datenbeschaffung, die hohe Modellkomplexität sowie Probleme der Schätzung von Parametern der Erklärungsgleichungen gegen einen solchen Modellansatz. Darüber hinaus sind Mikromodelle in aller Regel nicht mit einem benutzerorientiert-subjektiven Vorgehen vereinbar, da sich die Einschätzungen des Entscheidungsträgers hinsichtlich des Verhaltens im allgemeinen nur auf Aggregate von Marktteilnehmern beziehen können. Einer solchen Anforderung werden demgegenüber Makromodelle gerecht, da innen eine aggregierte Marktteilnehmerbetrachtung zugrunde liegt. Je nach Aggregationsgrad wird dabei das Reaktionsverhalten segmentspezifisch aufgeschlüsselt oder aber undifferenziert für alle, jeweils zu einer Gruppe gehörenden Marktteilnehmer (Endabnehmer, Handel etc.) erfaßt. Allerdings bleibt zu beachten, daß Makromodelle in den meisten Fällen mit Aggregationsfehlern behaftet sind, da sie nicht nur Unterschiede im individuellen Verhalten, sondern auch Art und Umfang der reaktionsauslösenden Stimuli nivellieren. 282

Bei der Klassifizierung von Modellen hinsichtlich ihres Abstraktionsgrades sind detail- und globalanalytische Ansätze voneinander zu differenzieren. ${ }^{283}$ Kennzeichnend für detailanalytische Ansätze ist die Berücksichtigung sogenannter intervenierender Variablen wie z. B. Markenbekanntheit und Einstellung, die das nicht beobachtbare Marktteilnehmerverhalten explizit abbilden sollen. Generell ist diesbezüglich festzustellen, daß die Analyse interner Prozesse der Marktteilnehmer und deren Beschreibung in Form hypothetischer Konstrukte wichtige Erkenntnisse für die Gestaltung des Marketinginstrumentariums liefern. Da bis heute jedoch keine überzeugenden, eindeutig bestätigten Beziehungen zwischen dem Instrumenteeinsatz, derartigen Konstrukten und schließlich dem beobachtbaren Verhalten er-

282 Vgl. Steffenhagen, H., Wirkungen absatzpolitischer Instrumente, a.a.O., S. 34 f.; Lilien, G. L., Kotler, P., Marketing Decision Making, A Model-Building Approach, New York 1987, S. 89 f.

283 Vgl. hierzu und im folgenden Meffert, H., Steffenhagen, H., Marketing-Prognosemodelle, a.a.O., S. 38 f.; Naert, P. A., Leeflang, P. S. H., Building implementable marketing models, a.a.O., S. $44 \mathrm{ff}$. 
mittelt werden konnten, sind detailanalytische Modelle als Instrumente der Entscheidungsunterstützung kritisch zu betrachten. Zwar mögen solche Modelle subjektiv den Anschein einer höheren Glaubwürdigkeit vermitteln, jedoch gilt es zu berücksichtigen, daß jede intervenierende Variable die Anzahl der mit Unsicherheit behafteten Erklärungsgleichungen erhöht und damit keinesfalls zwingend zur Verbesserung der Realitätstreue des abzubildenden Zusammenhangs von Entscheidungs- und Erwartungsvariablen beiträgt. ${ }^{284}$

Auf der anderen Seite ist ein völliger Verzicht auf die Abbildung interner Prozesse und die alleinige Fokussierung auf beobachtbares Verhalten, wie es bei globalanalytischen Modellen geschieht, mit dem Nachteil behaftet, daß für die Entscheidungen wichtige Verhaltensaspekte vernachlässigt werden. So bleibt beispielsweise die Präferenz markenorientierter und -bewußter Endabnehmer unberücksichtigt, wenn aufgrund einer unzureichenden Distribution kein Kauf zustande kommt und damit die ursprüngliche Intention im Verhalten nicht offensichtlich wird. Ein möglicher Kompromiß besteht darin, sich bei der Modellbildung auf solche intervenierenden Variablen zu beschränken, die einen sehr engen Bezug zum beobachtbaren Verhalten aufweisen. ${ }^{285}$

Die Frage der Berücksichtigung der Unsicherheit führt zur Unterscheidung von deterministischen und stochastischen Modellen. Beim deterministischen Ansatz wird unterstellt, daß weder die Modellvariablen, noch deren Beziehungen zueinander vom Zufall oder nicht erklärbaren Faktoren abhängig sind, sondern eindeutig festliegen. Unabhängige Variablen gehen jeweils mit einem bestimmten Wert in das Modell ein und führen als Bestandteil funktionaler Beziehungen zu genau einem Wert der entsprechenden abhängigen Variablen. ${ }^{286}$ In einem solchen Modellansatz wird

284

285

286

Vgl. Steffenhagen, H., Wirkungen absatzpolitischer Instrumente, a.a.O., S. 19 f.

Hierzu zählen beispielsweise die Ziele der Präferenz und Akzeptanz, wie sie in dieser Arbeit Verwendung finden. Vgl. Kap. B. 2.12.

Vgl. Topritzhofer, E., Absatzwirtschaftliche Modelle des Kaufentscheidungsprozesses, a.a.O., S. 33 ff.; Biethahn, J., Simulation - eine Methode zur Findung betriebswirtschaftlicher Entscheidungen?, a.a.O., S. 82; Ritzerfeld, U., MarketingMix-Strategien in Investitionsgütermärkten, a.a.O., S. 111. 
jedoch weder das Unsicherheitsproblem bei der Messung und Prognose der Kontextvariabeln erfaßt, noch geht die Unsicherheit bezüglich des Wirkungszusammenhangs von Entscheidungs- und Erwartungsvariablen in das Modell ein. ${ }^{287}$ Werden derartige Unsicherheiten bereits im Rahmen der Modellstruktur berücksichtigt, kann von stochastischen Modellen gesprochen werden. Der wesentliche Nachteil solcher Ansätze besteht in einem erhöhten Modellentwicklungs- und -berechnungsaufwand. So sind funktionale Beziehungen, die einem Zufallsprozeß unterliegen, schwieriger abzubilden als deterministische Zusammenhänge. Zudem führt die Verknüpfung von Variablen, die gleichzeitig mehrere Werte annehmen können, zu einer Vielzahl zu berechnender Kombinationsmöglichkeiten.

Des weiteren lassen sich statische und dynamische Modelle voneinander unterscheiden. Als statisch können Modellansätze bezeichnet werden, die entweder von einer einperiodigen Betrachtung ausgehen oder deren Variablenwerte und Beziehungszusammenhänge über die Zeit konstant bleiben. Dagegen unterliegen dynamische Modelle Veränderungen im Zeitablauf, die sich aus Veränderungen in den situativen Faktoren oder zeitübergreifenden Kopplungsbeziehungen ergeben können. ${ }^{288}$ Veränderte situative Faktoren resultieren beispielsweise aus der nicht vom Hersteller beeinflußbaren Entwicklung des Marktvolumens oder einem sich über die Zeit autonom verändernden Kaufverhalten der Endabnehmer. Kopplungsbeziehungen ergeben sich z. B. in Form von Verharrungseffekten bei der Präferenz für eine bestimmte Marke, da das erreichte Niveau am Ende einer Periode die Ausgangsbasis für die Folgeperiode darstellt. Kopplungsbeziehungen können auch über mehrere Perioden hinweg von Bedeutung sein, wie etwa beim Ersatzkauf, bei dem bereits länger zurückliegende Ereignisse die aktuelle Kaufentscheidung des Endabnehmers beeinflussen. Wenngleich dynamische Ansätze die Modellkomplexität steigern und zudem das Problem der Prognose zukünftiger Entwicklungen besteht, erhöht sich im Vergleich zu

287 Zur Unsicherheit vgl. Adam, D., Planung und Entscheidung, a.a.O., S. 14 und S. $194 \mathrm{ff}$.

288 Vgl. Rütschi, K., Marketing-Modelle, a.a.O., S. 6; Adam, D., Planung und Entscheidung, a.a.O., S. $72 \mathrm{ff}$. 
statischen Ansätzen gerade bei marktbezogenen Entscheidungen ohne Zweifel die Realitätsnähe des Modells.

Der Umfang eines Modells wird zumeist durch dessen Kennzeichnung als Total- oder Partialmodell zum Ausdruck gebracht. Eine Totalbetrachtung liegt vor, wenn sämtliche entscheidungsrelevanten Aspekte in das Modell einbezogen werden, während bei einer Partialbetrachtung nur jeweils ein bestimmter Teil der Problemstellung abgebildet wird. ${ }^{289}$ Um der Komplexität eines Totalmodells zu entgehen, gleichzeitig aber nicht die Nachteile eines aus dem Gesamtzusammenhang gelösten Partialmodells akzeptieren zu müssen, bietet sich ein Lösungsansatz an, bei dem die einzelnen Modellteile in ihrer Gesamtheit die Aspekte der Problemstellung möglichst abdecken, jedoch einen unterschiedlichen Detaillierungsgrad aufweisen. Die im Mittelpunkt des Interesses stehenden Beziehungen werden möglichst detailliert abgebildet, während solche zur Einordnung in den Gesamtzusammenhang nur in grobem Umfang berücksichtigt werden.

Schließlich sind Modelle hinsichtlich ihrer Lösungstechnik als algorithmische oder simulative Modelle zu kennzeichnen.290 Bei Entscheidungsmodellen wird mit einer algorithmischen Lösungstechnik ein genau festgelegter Rechenweg vorgegeben, um eine optimale oder möglichst gute Kombination der Entscheidungsvariablen im Hinblick auf die Ausprägungen der Erwartungsvariablen zu erreichen. Existieren solche Algorithmen nicht oder sind aufgrund der Komplexität des Modells zu aufwendig, kann auf die Lösungstechnik der Simulation zurückgegriffen werden. Dabei werden die Entscheidungsvariablen so lange variiert, bis die gewünschte Ausprägung der Erwartungsvariablen erreicht ist. Im Zusammenhang mit Erklärungsmodellen ist die algorithmische Lösungstechnik als festgelegter Weg zur Berechnung der Erwartungsvariablen zu verstehen. Enthält das Modell stochastische Variablen, die nicht auf numerischem oder analytischem Weg miteinander verknüpft werden können, wird die sogenannte Risikosimulation als

289 Vgl. Rütschi, K., Marketing-Modelle, a.a.O., S. 8.

290 Vgl. Meffert, H., Computergestützte Marketing-Informationssysteme, a.a.O., S. 58. 
Lösungsmethode herangezogen. ${ }^{291}$ Hierbei werden die Wahrscheinlichkeitsverteilungen der stochastischen Variablen simuliert und aus der Kombination möglicher Werte die Erwartungsvariablen berechnet.

In diesem Zusammenhang ist die Simulation als Lösungstechnik von dem Begriff der Simulation im Sinne eines Marktexperimentes abzugrenzen. Letztere ist als Instrument zur Untersuchung des Verhaltens von Modellen zu verstehen, bei dem die Werte der Entscheidungs- oder Kontextvariablen variiert werden, um die daraus resultierenden Veränderungen der Erwartungsvariablen beobachten zu können. 292

\subsection{Implikationen für die eigene Modellentwicklung}

Vor dem Hintergrund der aufgeführten Strukturdefekte und Gestaltungsanforderungen sowie der grundlegenden Ansätze der Modellgestaltung ist das Modell wie folgt zu konzipieren:

- Die Entscheidung für ein benutzerorientiert-subjektives Vorgehen verlangt den Ansatz eines Makromodells, bei dem die Verhaltensreaktionen vom Entscheidungsträger jeweils mit Bezug auf ein Marktteilnehmeraggregat geschätzt werden.

- Um die Wirkungsdefekte nicht durch die Einführung zusätzlicher Erklärungsgleichungen zu erhöhen, wird auf die Verwendung intervenierender Variablen verzichtet. Es kommen lediglich solche Variablen zum Einsatz, die das zu erwartende Verhalten von Handel und Endabnehmer direkt beschreiben. Insofern handelt es sich um ein globalanalytisches Modell mit verhaltensbezogenen Variablen.

Vgl. hierzu auch Kap. C. 3.31 dieser Arbeit.

292 Vgl. Biethahn, J., Optimierung und Simulation, Wiesbaden 1978, S. 5 f.; Voß, W.D., Modellgestützte Markenpolitik, a.a.O., S. 59; Herrmann, H.-J., Modellgestützte Planung im Unternehmen, a.a.O., S. $71 \mathrm{ff}$. 
- Die aufgrund von Meß-, Prognose- und Wirkungsdefekten bestehenden Unsicherheiten über die Ausprägungen von Kontextvariablen und die Beziehungen zwischen Entscheidungs- und Erwartungsvariablen sollen explizit in dem Modell berücksichtigt werden. Dazu sind stochastische Variablen und Wirkungsbeziehungen einzuführen.

- Die periodenübergreifenden Wirkungen des Marketinginstrumenteeinsatzes sowie die Bedeutung der vorhergegangenen Bereifung des Fahrzeuges für die aktuelle Kaufentscheidung macht einen dynamischen Modellansatz notwendig.

- Um der benutzerorientierten Anforderung der Einfachheit zu genügen, orientiert sich der Detaillierungsgrad der Modellbestandteile an deren Relevanz für die Fragestellung der Planung von Markendurchsetzungsstrategien. Während das Verhalten von Handel und Endabnehmer einer differenzierten Abbildung bedarf, sind Aspekte wie z. B. die Marktvolumensentwicklung nur auf aggregiertem Niveau zu beschreiben.

- Die Verwendung von stochastischen Variablen und Wirkungsbeziehungen erfordert den Einsatz der Risikosimulation als Lösungsmethode. Darüber hinaus soll das Modell zur Durchführung von Sensitivitätsanalysen geeignet sein, um die Umgebung der Ausgangsniveaus der Marketinginstrumentarien auf Verbesserungsmöglichkeiten hin untersuchen zu können.

\section{Darstellung und Würdigung ausgewählter Modelle der Literatur}

Im Anschluß an die Diskussion grundlegender Ansätze zur Gestaltung von Modellen stellt sich die Frage, in welcher konkreten Form eine geeignete modellhafte Abbildung des Planungsproblems erreicht werden kann. Hierzu sind zunächst Modelle der Literatur dahingehend zu untersuchen, wie Entscheidungstatbestände, situative Faktoren und Ziele sowie deren Beziehungen in Form von Variablen und mathematischen Funktionen beschrieben werden. 
Im folgenden werden dazu beispielhaft drei Modelle vorgestellt, deren Ansätze und Einsatzbereiche sich jeweils auf thematische Teilbereiche des hier zu entwickelnden Modells beziehen. Das zunächst darzustellende CIMDeveloppement-Reifenmarktmodell wurde speziell für den (französischen) Reifenmarkt entwickelt. Wenngleich als detailanalytisches Mikromodell formuliert, lassen sich hieraus Erkenntnisse über die Modellierung branchenspezifischer Besonderheiten des Reifenmarktes gewinnen. Als zweites ist das Modell von Farris, Olver und de Kluyver vorzustellen, das sich spezifisch mit der Problemstellung des Zusammenwirkens von Angebotsund Nachfragestärke im Rahmen der Planung von Markendurchsetzungsstrategien auseinandersetzt. Schließlich ist das von Little entwickelte BRANDAID-Modell zu kennzeichnen, dessen Konzeption auf dem benutzerorientiert-subjektiven Ansatz der Modellbildung basiert, wie er auch hier für die Modellentwicklung zugrunde gelegt wird.

\subsection{CIM-Devellopement-Reifenmarktmodel|293}

Ausgangsbasis des Reifenmodells des französischen Beratungsunternehmens CIM Devellopement ist die in Abbildung 30 wiedergegebene allgemeine Struktur eines Totalmodells zur Bestimmung und Beurteilung alternativer Marketing-Aktivitäten. ${ }^{294}$ Die einzelnen Teilmodelle repräsentieren die Aktionen und Reaktionen einer Institution oder einer Gruppe von Individuen.

293 Vgl. Hansen, H. R., Thabor, A., Marketing-Modelle, Anwendungsmöglichkeiten und Entwicklung computergestützter Modelle im Marketing, Berlin, New York 1973, S. $139 \mathrm{ff}$.

294 Das CIM-Developpement-Reifenmarktmodell zählt zu den sogenannten "largescale"-Simulationsmodellen, bei denen es sich um komplexe, detailanalytische Mikromodelle handelt, die zur Ermittlung der Modellergebnisse auf die Methode der Simulation zurückgreifen. Als die drei grundlegenden "large-scale"-Simulationsmodelle werden im allgemeinen die Modelle von Amstutz, Lavington und KlengerKrauter angesehen. Vgl. Amstutz, A. E., Computer Simulation of Competitive Market Response, Cambridge (Mass.), London 1976; Lavington, M. R., A Practical Simulation Model for Consumer Marketing, in: Operational Research Quarterly 1970, S. 25 ff.; Klenger, F., Krautter, F., Simulation des Käuferverhaltens, Teil I: Werbewirkung und Käuferverhalten, Teil II: Analyse eines Kaufprozesses, Teil III: Computermodell des Käuferverhaltens, Wiebaden 1972. Eine ausführliche Würdi-

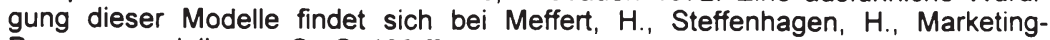
Prognosemodelle, a.a.O., S. $193 \mathrm{ff}$. 


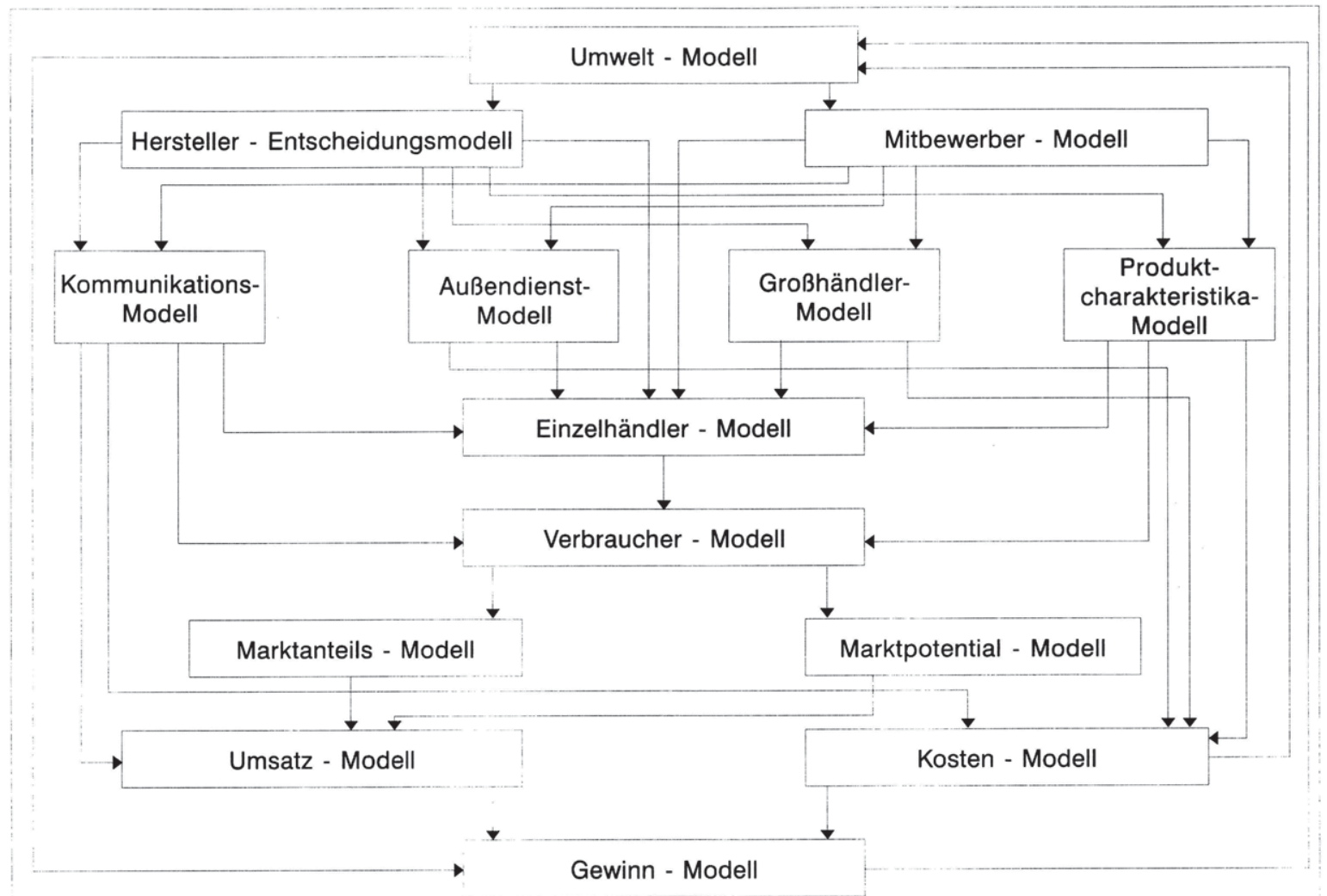

Abb. 30: Struktur des CIM-DEVELOPPEMENT-Modells (Quelle: Hansen, H. R., Thabor, A., Marketing-Modelle, a.a.O., S. 139) 
Dabei wird das Entscheidungs- und Reaktionsverhalten jeweils innerhalb dieser Teilmodelle durch Aggregation des Indvidualverhaltens ermittelt. Wechselwirkungen zwischen den Teilmodellen werden berücksichtigt, indem die Ergebnisse eines Teilmodells gleichzeitig als Eingangsdaten in andere Teilmodelle eingehen.

Dieses allgemeine Modell wurde für einen Reifenhersteller speziell für den französischen Markt konkretisiert, ${ }^{295}$ wobei die möglichst genaue Abbildung des Marken- und Einkaufsstättenwahlverhaltens des Endabnehmers (Reifenbenutzer-Modell), des Beschaffungs- und Endabnehmerbeeinflussungsverhaltens des Handels (Absatzmittlermodell) sowie der Wirkung der Kommunikationsanstrengungen des Herstellers (Kommunikationsmodell) im Vordergrund stehen. ${ }^{296}$ Abbildung 31 zeigt die wesentlichen Beziehungen auf, die es im folgenden näher zu kennzeichnen gilt.

Das Reifenbenutzermodell unterscheidet zur Ermittlung des tatsächlichen Absatzes die freie, spontane und modulierte Nachfrage des Endabnehmers nach einer bestimmten Reifenmarke.

Die freie Nachfrage nach einer Marke ergibt sich aus der Aggregation der freien Neigungen der individuellen Endabnehmer. Diese entsprechen jeweils der Wahrscheinlichkeit $q_{i k}$, daß ein Reifenbenutzer $k$ die Marke i kauft, wenn er in der Kaufentscheidung nicht durch die vorhandene Reifenausstattung seines Fahrzeuges beeinflußt wird, die Marke verfügbar ist und keine Einflußnahme des Händlers erfolgt. Die freie Neigung wird auf Basis eines Einstellungsmodells berechnet, indem zunächst die wahrgenommenen Idealund Realausprägungen von Reifeneigenschaften in Form eines "Präferenzindikators" $\left(B_{i k}\right)$ erfaßt werden. Dieser wird aus der Summe der Eigenschaftsbewertungen, $d . h$. der Differenzen zwischen den Idealvorstellungen

295 Das Modell wurde darüber hinaus an verschiedene weitere Branchen (Bankenmarkt, Verlagswesen, Pharmazeutika etc.) angepaßt. Vgl. Hansen, H. R., Thabor, A., Marketing-Modelle, a.a.O., S. 143. Für eine ausführliche Darstellung der Konkretisierung für den Bankenmarkt siehe Thabor, A., Ein Marketing-Mix-Simulationssystem - angewandt beim Bankenmarkt, in: Computergestützte Marketingplanung, Hrsg.: Hansen, H. R., München 1974, S. 587 ff.

$296 \mathrm{Zu}$ der folgenden Darstellung vgl. Hansen, H. R., Thabor, A., Marketing-Modelle, a.a.O., S. $171 \mathrm{ff}$. 


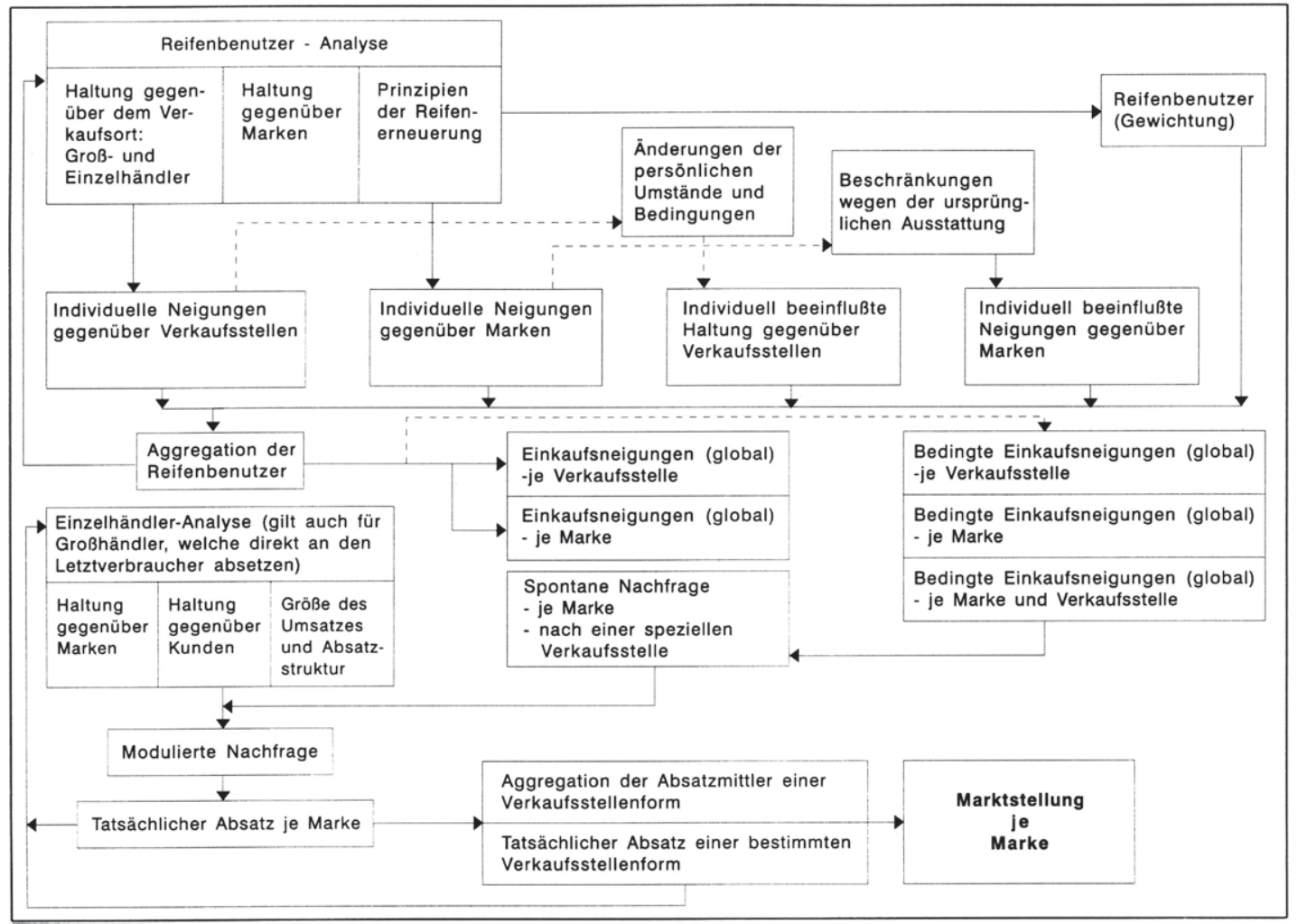

Abb. 31: Grundlegende Beziehungen des im CIM-DEVELOPPEMENT abgebildeten Reifenmarktes (Quelle: Hansen, H. R., Thabor, A., Marketing-Modelle, a.a.O., S. 188) 
des Endabnehmers und seiner Einschätzungen der jeweiligen Marke bezüglich der von ihm frei assoziierten Reifeneigenschaften gebildet.297 Zudem wird eine Untergrenze $\left(F_{k}\right)$ eingeführt, in der das geforderte Mindestmaß der Annäherung von Ideal- und Realausprägungen zum Ausdruck kommt. Für die Menge aller Reifenmarken, deren sich der Endabnehmer bewußt ist $\left(I_{k}\right)$ und deren Einstellungswerte die jeweilige Mindestanforderung $\left(B_{i k}-F_{k}>0\right)$ erfüllen $\left(I_{k}^{\prime}\right)$, gilt:298

$q_{i k}=\frac{B_{i k}-F_{k}}{\sum_{i d_{k}^{\prime}}\left(B_{i k}-F_{k}\right)}$

mit

$F_{k}=\max _{i \phi_{k}}\left(B_{i k}\right)-g \cdot \sigma_{k}$,

wobei der Parameter $\mathrm{g}$ ein nicht weiter beschriebener Gewichtungsfaktor ist und es sich bei $\sigma_{k}$ um die Standardabweichung der Eigenschaftsbewertungen handelt.

Die spontane Nachfrage nach einer Reifenmarke resultiert aus der Aggregation der bedingten Neigungen der Endabnehmer. Dem liegt jeweils die Wahrscheinlichkeit $p_{i k v}$ zugrunde, daß ein Reifenbenutzer $k$ die Marke $i$ kauft, falls er im Besitz der Automarke $v$ ist. In die Berechnung gehen dabei ein:

- die Wahrscheinlichkeit $\alpha_{k}$, daß sich k für denselben Reifen entscheidet, der sich bereits am Fahrzeug befindet, 299

297 Zusätzlich erfolgt eine Normierung des Präferenzindikators mit Bezug auf die Anzahl der vom individuellen Endabnehmer frei assoziierten Eigenschaften.

298 In allen anderen Fällen gilt $q_{i k}=0$.

299 Dabei wurde $\alpha_{k}$ als gewichteter Durchschnitt der Wahrscheinlichkeiten berechnet, mit der nicht auf eine andere Reifenmarke übergegangen wird, wenn 1, 2 oder 4 Reifen gewechselt werden. 
- die Wahrscheinlichkeit $a_{i k v}$, daß Reifenmarke $i$ am Fahrzeug von $k$ der Automarke $v$ montiert ist sowie

- die freie Neigung $q_{i k}$ für den Fall, daß sich k nicht für die aktuell montierte Reifenmarke entscheidet.

Die bedingte Neigung läßt sich damit darstellen als:

$p_{i k v}=\alpha_{k} \cdot a_{i k v}+\left(1-\alpha_{k}\right) \cdot q_{i k}$

Ebenso wie bei der Neigung gegenüber der Marke i läßt sich für jeden Reifenbenutzer seine Neigung $p_{j k c}^{\prime}$ für eine Verkaufsstelle j (Garage, Tankstelle, Großhändler, Warenhaus, Autohändler) ${ }^{300}$ unter Berücksichtigung der Umstände des Reifenwechsels c (z. B. Urlaubsreise) ermitteln. Dies führt schließlich zu der spontanen Nachfrage $q_{i j}$ nach der Marke $i$ in der Verkaufsstelle j, wobei w als Gewicht zur Berücksichtigung des "Kaufpotentials" des Reifenbenutzers dient:

$q_{i j}=\frac{\sum_{k} w_{k} \cdot p_{i k} \cdot p_{j k}^{\prime}}{\sum_{k} w_{k}}$

Die modulierte Nachfrage ergibt sich aus der spontanen Nachfrage, die um die Nichtverfügbarkeit bestimmter Reifenmarken in der Kaufsituation bereinigt ist. Sei

$T_{j 1}=\left(D_{1 j l}, \ldots, D_{n j l}\right)^{\prime}$

mit $D_{i j l}=\frac{q_{i j l}}{\sum_{i} q_{i j l}}$

die spontane Nachfrage in der Verkaufsstelle j des Einzelhändlers I nach den Marken $1, \ldots, n$, dann berechnet sich die modulierte Nachfrage $M_{j l}$ aus:

$M_{j 1}=T_{j 1}+k_{1} \cdot W_{1} \cdot U_{1}$

300 Der Begriff der Verkaufsstelle wird in dem Modell synonym zum Begriff der Betriebsform verwendet. 
Der Vektor $k_{1}$ beschreibt Vorratsprobleme des Einzelhändlers, und die Matrix $W_{1}$ gibt den Anteil der Marke i an, der bei Nichtverfügbarkeit auf die Marke i' übergeht. $U_{1}$ stellt einen $(n, 1)-V e k t o r$ dar, mit $U_{i l}=0$, falls die Marke i vorhanden ist und $U_{i 1}=1$, falls dies nicht der Fall sein sollte. Der schließlich durch den Vektor $V_{1}$ beschriebene Absatz der Marken beim Einzelhändler I berücksichtigt neben der modulierten Nachfrage die Verkaufsanstrengungen des Händlers in Form eines Förderungskoeffizienten-Vektors $F_{1}$ :

$V_{1}=M_{1}+F_{1}$

Die Ermittlung der Verkaufsanstrengungen selbst ist Gegenstand des Absatzmittlermodells. ${ }^{301}$ Dazu werden von jedem Einzelhändler insgesamt 11 Reifeneigenschaften der Wichtigkeit nach geordnet und die Reifenmarken entsprechend dieser Merkmale beurteilt. Sei $C_{\mathrm{IL}}$ der vom Einzelhändler I bewertete Rang des Kriteriums $L$, und $K_{\text {ill }}$ der Rang der Marke i im Hinblick auf $L$, dann ergibt sich der Präferenzindikator $H_{i l}$ des Einzelhändlers wie folgt:

$H_{\mathrm{il}}=\sum_{\mathrm{L}=1}^{11} \mathrm{G}_{\mathrm{IL}} \cdot \mathbf{N}_{\mathrm{iL}}$

mit

$G_{\mathrm{LL}}=6-C_{\mathrm{IL}}$ falls $C_{\mathrm{IL}} \leq 6$ sonst $G_{\mathrm{LL}}=0$

$N_{\text {ill }}=6-K_{\text {ill }}$ falls $K_{\text {ill }} \leq 6$ sonst $K_{\text {ill }}=0$

Von den 11 abgefragten Reifeneigenschaften gehen somit jeweils nur die 5 wichtigsten und von den Marken nur die bezüglich jedes Kriteriums 5 besten in die Bewertung ein. Damit wird unterstellt, daß der Einzelhändler nur eine beschränkte Zahl von Eigenschaften bzw. Marken zur Beurteilung heranzieht. Die Förderungskoeffizienten werden schließlich aufgrund der relativen Präferenz des Einzelhändlers für die einzelnen Marken ermittelt.

301 Das Absatzmittlermodell wurde sowohl für die Einzel- als auch für die Großhandelsebene entwickelt. Aufgrund deren sehr ähnlicher Struktur ist es hier jedoch ausreichend, das Einzelhandelsmodell zu beschreiben. 
Die Wirkung der Werbung auf die Neigung des Endabnehmers wird durch das Kommunikationsmodell erfaßt. Differenziert nach den Medien Fernsehen, Rundfunk, Zeitschriften und Zeitungen werden zunächst auf Basis der Mediennutzungsgewohnheiten der Reifenbenutzer die Wahrscheinlichkeiten ermittelt, mit denen es zu einem Werbekontakt kommt, dessen Botschaftsinhalte auch wahrgenommen werden. Zudem wird empirisch festgestellt, mit welcher Wahrscheinlichkeit die Werbung zu einer "Steigerung des Bewußtseins"302 des Endabnehmers bezüglich der Reifenmarken führt und welcher Einfluß durch die Assimilation ${ }^{303}$ der Werbebotschaft auf die Wahrnehmung der Eigenschaften dieser Marken ausgeht. Dazu wird die Annahme zugrunde gelegt, daß die Assimilationswahrscheinlichkeit direkt von den Einstellungen des Reifenbenutzers $k$ gegenüber der in der Werbung hervorgehobenen Markeneigenschaft $s$ abhängt. Die entsprechende Modellformulierung lautet:

$$
A_{k}=\frac{\sum_{s} r_{s} \cdot\left|n_{s k}\right|}{\sum_{s} r_{s}}
$$

Die Variable $r_{s}$ beschreibt den relativen Rang der Markeneigenschaften auf einer Kriterienskala und $n_{\text {sk }}$ das Einflußgewicht der Eigenschaft bei der Einstellung des Reifenbenutzers gegenüber dem Idealreifen. Kommt es zur Assimilation, so wird unterstellt, daß spätestens von diesem Zeitpunkt an die in der Werbebotschaft betonten Eigenschaften vom Endabnehmer mit der Reifenmarke assoziiert werden, sofern diese nicht mit dessen bisherigen Vorstellungen von der Marke unvereinbar sind. Daraus kann schließlich eine funktionale Beziehung zwischen Werbebotschaft und Einstellungsveränderung abgeleitet werden. Die Einstellung dient wiederum als Eingangsgröße für das Reifenbenutzermodell zur Berechnung der freien Nachfrage.

302 Der Begriff des Bewußtseins wird in dem Modell im Sinne der Markenbekanntheit verwendet.

303 Mit dem Begriff Assimilation wird in dem Modell das Verstehen und Erinnern einer Werbebotschaft bezeichnet. 


\subsection{BRANDAID-Model|304}

Das vornehmlich im Hinblick auf die Unterstützung von Marketing-Mix-Entscheidungen bei verpackten Konsumgütern entwickelte, jedoch in seinen Einsatzbereichen sehr flexible Modell BRANDAID basiert auf dem in Abbildung 32 dargestellten Marktsystem und umfaßt ein Grundmodell zur Umsatz- und Gewinnberechnung sowie Teilmodelle zur Werbung, Verkaufsförderung, Preispolitik und Vertretereinsatzplanung.

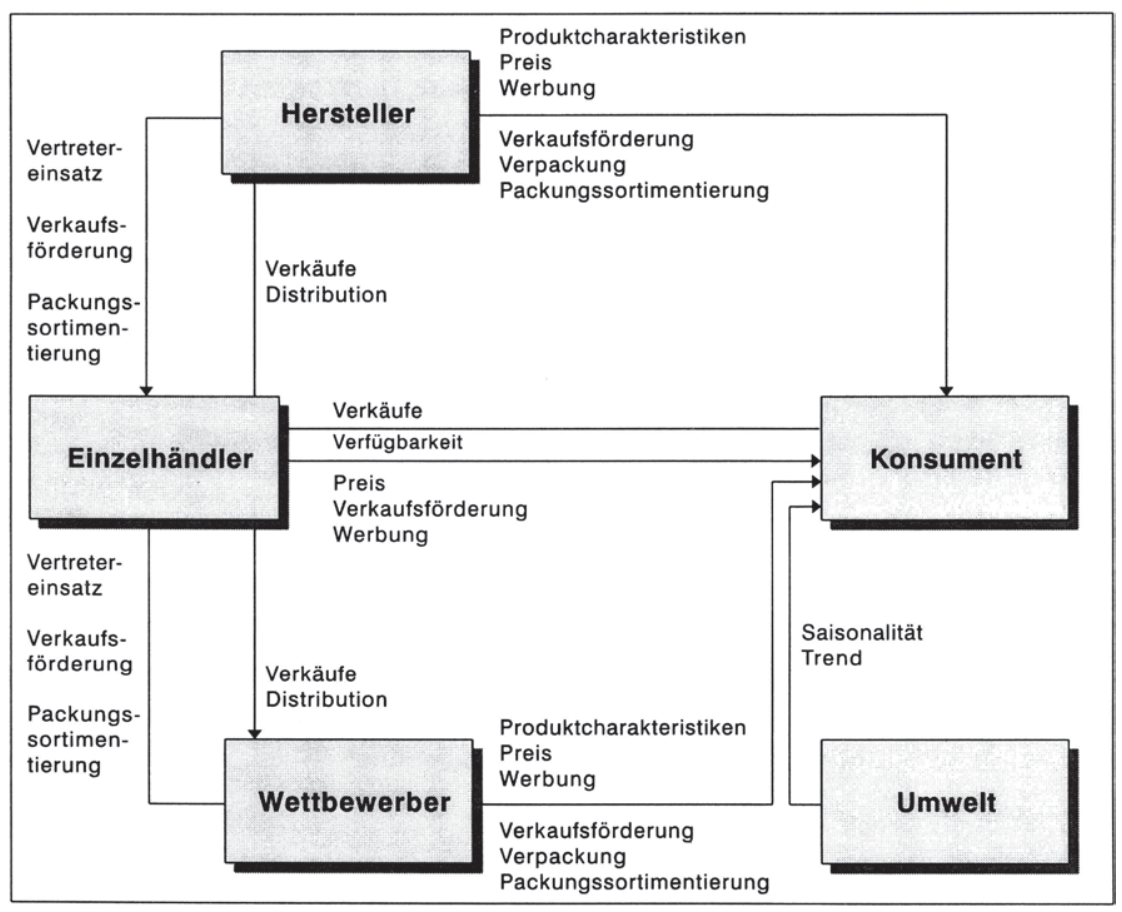

Abb. 32: Struktur des BRANDAID-Modells

(Quelle: Little, J. D. C., Ein On-Line-Marketing-Mix-Modell, a.a.O., S. 695)

304 Vgl. Little, J. D. C., Ein On-Line-Marketing-Mix-Modell, in: Computergestützte Marketingplanung, Hrsg.: Hansen, H. R., München 1974, S. 651 ff. sowie Meffert, H., Computergestützte Marketing-Informationssysteme, a.a.O., S. $117 \mathrm{ff}$. 
Das Umsatz- und Gewinnmodell geht davon aus, daß sich der Gewinnbeitrag $p$ in Periode $t$ (Geldeinheiten pro Kunde und Periode) des Produktes ergibt, indem der Bruttostückgewinn $g_{t}$ (Geldeinheit pro Verkaufseinheit) mit dem Absatz der Produktgruppe $S_{t}$ (verkaufte Einheiten pro Kunde und Periode) und dem Marktanteil des konkreten Produktes $m_{t}$ multipliziert wird und davon die Kosten $\mathrm{c}_{\text {it }}$ der Marketingaktivitäten i (in Geldeinheiten pro Kunde und Periode) abgezogen werden. Der aufsummierte Gesamtgewinn P innerhalb eines Planungszeitraums $T_{1}$ bis $T_{2}$ berechnet sich dann aus der Länge des Zeitraums $\Delta$ sowie der Anzahl der in den Perioden vorhandenen Kunden $\mathrm{N}_{\mathrm{t}}$ :

$p=\sum_{t=T_{1}}^{T_{2}} N_{t} \cdot\left(g_{t} \cdot m_{t} \cdot S_{t}-\sum_{i} c_{i t}\right) \cdot \Delta$

Den auf diesem Grundmodell aufbauenden Teilmodellen zur Planung der Marketingaktivitäten ist gemein, daß die Berechnungen auf Basis von Bezugsbedingungen erfolgen. BRANDAID unterstellt, daß der Entscheidungsträger bei der Planung für ein eingeführtes Produkt gedanklich von der bisher erreichten Marktstellung ausgeht. Dementsprechend werden Bezugsbedingungen definiert, die auf den Marktanteilen, Umsätzen und Marketingaktivitäten der Vergangenheit basieren. Veränderungen gegenüber diesen Bezugswerten werden in Form von Indizes zum Ausdruck gebracht. Die Gesamtwirkung aller Veränderungen ermittelt sich aus deren multiplikativer Verknüpfung:

$m_{t}=m_{0} \cdot \prod_{i e_{m}} e_{m, i t}$

$m_{0} \quad$ : Bezugsmarktanteil

$e_{m, j t}$ : Index der Auswirkungen von Marketingaktivität i auf den Marktanteil in Periode $t$

$I_{m} \quad$ : Menge der Einflüsse auf den Marktanteil

Dabei kann der Index sowohl eine feste Größe darstellen als auch in veränderlicher Form durch eine Reaktionsfunktion spezifiziert sein. So wird beim Werbemodell angenommen, daß es ein bestimmtes Maß an Erhaltungs- 
werbung gibt, das den ursprünglichen Marktanteil stabilisiert. Veränderungen dieser Erhaltungswerbung erhöhen bzw. verringern den Marktanteil. Als allgemeine Reaktionsfunktion zwischen Werbung und Marktanteil wird dabei folgende Beziehung gewählt:

$e_{t}=\alpha \cdot e_{t-1}+(1-\alpha) \cdot r\left(a_{t}\right)$

$e_{\mathrm{t}} \quad$ : Einfluß der Werbung auf den Marktanteil (Index) im Zeitraum $\mathrm{t}$

$\alpha \quad$ : Periodenübergreifende Wirkung der Werbung

$r\left(a_{t}\right)$ : Einfluß der Werbung auf den langfristigen Marktanteil (Index)

$a_{t} \quad$ : Werbequote in Periode $t$ (Index)

Die in dieser Funktion enthaltene Werbequote $a_{t}$ ermittelt sich aus den Wirkungen des für die Werbemaßnahme $j$ herangezogenen Werbemediums $\left(h_{j t}\right.$, Kontakte pro Geldeinheit) und des Werbemittels $\left(k_{\mathrm{jt}}\right)$ sowie aus den hierfür aufgewendeten Werbeausgaben $\mathrm{x}_{\mathrm{j} t}$. Unterschiede in den Werbemaßnahmen kommen in einem Gewicht $w_{j t}$ zum Ausdruck. Die Bezugsgrößen der Werte werden jeweils durch den Index 0 gekennzeichnet. Damit läßt sich $a_{t}$ darstellen als:

$a_{t}=\frac{\sum_{j} h_{j t} \cdot k_{j t} \cdot w_{j t} \cdot x_{j t}}{\sum_{j} h_{0 j} \cdot k_{0 j} \cdot w_{0 j} \cdot x_{0 j}}$

Optional verwendet Little anstelle der Werbequote die in t tatsächlich wirksame Werbung $\hat{a}_{t}$, die nach seinem Verständnis neben den aktuellen Werbeausgaben auch die Wirkung früherer Werbemaßnahmen im Ausmaß des Erinnerungsfaktors $\beta$ einbezieht:

$\hat{a}_{t}=\beta \cdot \hat{a}_{t-1}+(1-\beta) \cdot a_{t}$

Im Rahmen des Verkaufsförderungsmodells wird der Einfluß einer zeitlich begrenzten Preisreduzierung abgebildet, die dem Handel gewährt wird und diesen zu einer verstärkten Förderung des Produktes bewegen soll. Dabei wird berücksichtigt, daß sich die Preissenkung nur auf einen Teil $n_{t}$ des angebotenen Sortimentes bezieht, so daß der Zuwachs dieses Anteils r mit- 
unter im Ausmaß b zu Lasten des übrigen Sortimentes geht. ${ }^{305}$ In Abhängigkeit von der Verkaufsförderungsintensität $a_{t}{ }^{306}$ gilt somit für die Nettoerhöhung r't des Marktanteils:

$r_{t}^{\prime}=n_{t} \cdot r\left(a_{t}\right) \cdot(1-b)$

Bezüglich der Wirkung der Verkaufsförderungsmaßnahme wird zudem unterstellt, daß dem zunächst erreichten Maximum des entsprechenden Marktanteilsindex ein Minimum folgt, das sich aus vorangegangenen Vorratskäufen von Handel und Endabnehmer erklärt. Anschließend sind positive, jedoch mit der Zeit abnehmende Folgeeffekte zu erwarten, da Endabnehmer, die zu dem geförderten Produkt gewechselt sind, dieses auch in absehbarer Zukunft weiterhin kaufen. Wird mit $q_{\tau}$ der (exponentiell abnehmende) Marktanteilsgewinn in Periode $\tau$ als Anteil des ursprünglichen Marktanteilsgewinns durch die Verkaufsförderungsmaßnahme bezeichnet und mit $e_{0}$ die Auswirkung auf den Bezugsmarktanteil ohne diese Maßnahme, so läßt sich die Wirkung auf den Marktanteil $e_{t}$ (Index) in Periode $t$ wie folgt darstellen:

$$
\begin{aligned}
& e_{t}=e_{0} \prod_{\tau>0}^{t}\left(1+q_{t} \cdot r_{t-\tau}^{\prime}\right) \\
& q_{t}=q_{2} \cdot\left(q_{3} / q_{2}\right)^{\tau-2} \quad \text { für } t \geq 2
\end{aligned}
$$

Während im Verkaufsförderungsmodell die Wirkung einer kurzfristigen Preissenkung beschrieben wird, erfaßt das Preismodell die Entwicklung des Marktanteils in Abhängigkeit des vom Hersteller festgelegten Großhandelspreises $x_{t}$. Es wird unterstellt, daß die Händler diesen in Form eines Standardaufschlages an den Endabnehmer weitergeben, wobei neben der kontinuierlichen Wirkung $r$ zusätzlich die psychologische Wirkung $\psi$ des

305 Während Little an anderer Stelle stets das einzelne Produkt oder Erzeugnis als Bezugsobjekt wählt, wechselt er im Verkaufsförderungsmodell ohne weitere Begründung zum Begriff des Sortimentes.

306 Die Verkaufsförderungsintensität kann sowohl in bezug auf den Kunden (Geldeinheit pro Kunde) als auch in bezug auf die Verkaufseinheiten (Geldeinheit je verkaufte Einheit) definiert werden. Analog zum Vorgehen im Werbemodell werden dabei auch hier die getätigten Ausgaben mit Wirkungsfaktoren multipliziert, die Little als "Reichweiteneffizienz" und "Konsumenteneffektivität" bezeichnet. 
Über- bzw. Unterschreitens von Preisschwellen berücksichtigt wird. 307 Sei $x_{0}$ die Bezugsgröße des Preises, dann ergibt sich der Marktanteil $e_{t}$ (Index) aus

$e_{t}=\psi_{t}\left(x_{t}\right) \cdot r\left(x_{t} / x_{0}\right)$

Die Struktur des Vertretermodells stimmt weitgehend mit derjenigen des Werbemodells überein. Der "effektive" Vertretereinsatz $\hat{a}_{t}$ ergibt sich aus der aktuellen Höhe des Vertretereinsatzes $x_{t}$ (Geldeinheiten pro Kunde und Jahr), dessen Reichweiteneffizienz $h_{t}$ (Besuche pro Geldeinheit) und der Wirksamkeit pro Besuch $k_{t}$ sowie aus den noch mit $\beta$ wirksamen Vertreterbesuchen früherer Perioden:

$$
\begin{aligned}
& a_{t}=\frac{h_{t} \cdot k_{t} \cdot x_{t}}{h_{0} \cdot k_{0} \cdot x_{0}} \\
& \hat{a}=\beta \cdot \hat{a}_{t-1}+(1-\beta) \cdot a_{t}
\end{aligned}
$$

Die Wirkung des Vertretereinsatzes auf den Marktanteil $e_{t}$ (Index) resultiert schließlich aus der Produkttreue des Einzelhandels $\alpha$ sowie der langfristigen Reaktion des Marktanteils auf den Vertretereinsatz r:

$$
e_{t}=\alpha \cdot e_{t-1}+(1-\alpha) \cdot r\left(\hat{a}_{t}\right)
$$

Neben den Wirkungen der Marketingaktivitäten können in dem Gesamtmodell weitere Einflüsse wie Beschränkungen der Produktionskapazität, saisonale Schwankungen oder Trendentwicklungen berücksichtigt werden. Letztere werden beispielsweise wie folgt formuliert:

$$
e_{t}=e_{0} \prod_{\tau=1}^{t}\left(1+r_{\tau}\right)
$$

Dabei stellt $e_{0}$ den Ausgangswert dar, während $r_{\tau}$ die Wachstumsrate pro Periode beschreibt.

$307 \mathrm{Zu}$ den theoretischen Grundlagen der Wirkung von Preisschwellen vgl. auch Kaas, K.-P., Hay, Ch., Preisschwellen bei Konsumgütern - eine theoretische und empirische Analyse, in: ZfbF, 1982, S. $333 \mathrm{ff}$. 
Die Berücksichtigung des Verhaltens von Wettbewerbern erfolgt nach zwei von Little als Prinzipien der Symmetrie und Modularität bezeichneten Grundsätzen. Symmetrie bedeutet, daß die Teilmodelle zur Abbildung der eigenen Aktivitäten ebenfalls zur Beschreibung von Aktivitäten der Wettbewerber verwendet werden. Allerdings wird hierbei nicht notwendigerweise der gleiche Detailliertheitsgrad zugrunde gelegt. Mit dem Prinzip der Modularität wird ausgesagt, daß die Wettbewerbsaktivität i nicht etwa als Bezugsgröße zur Relativierung der Stärke eigener Aktivitäten erfaßt wird, ${ }^{308}$ sondern deren Wirkung jeweils auf einen eigenständigen Index, wie z. B. auf den Konkurrenzmarktanteil $\hat{e}_{m, i t}$ (Index), abgebildet wird. Die Wirkung auf den eigenen Marktanteil $e_{m, i t}$ (Index) wird anschließend mit Hilfe einer Umwandlungskonstante $\gamma_{i}$ berechnet:

$\mathrm{e}_{\mathrm{m}, \mathrm{t}}=1+\gamma_{\mathrm{i}} \cdot\left(1-\hat{\mathrm{e}}_{\mathrm{m}, \mathrm{t}}\right)$

Um schließlich in dem Modell Einzelhandelsaktivitäten erfassen zu können, wird $d_{i t}$ als Leistungsmaßstab für die Einzelhändlermaßnahme $i$ definiert und $r_{m, i}$ als die davon ausgehende Reaktion auf den Marktanteil $e_{m, i t}$ (Index). Damit gilt:

$e_{m, i t}=r_{m, i}\left(d_{i t}\right)$

Als ein Beispiel für den Leistungsmaßstab $d_{t}$ stellt Little die Verfügbarkeit im Sinne des Distributionsgrades eines Produktes heraus, deren Ausmaß durch die Wirkung $e_{\mathrm{d}, \mathrm{t}}$ der Marketingaktivität $j$ des Herstellers beeinflußt wird. Sei $d_{0}$ der Bezugswert von $d_{t}$ und $I_{d}$ die Menge aller Marketingaktivitäten, die Einfluß auf die Verfügbarkeit $d_{t}$ nehmen, dann läßt sich diese Beziehung wie folgt abbilden:

$d_{t}=d_{0} \cdot \prod_{j d_{d}} e_{d, j t}$

308 Eine solche Vorgehensweise würde beispielsweise im Werbemodell dazu führen, daß - stark vereinfacht - eine Modellformulierung der Art "eigene Werbung/(eigene Werbung + Konkurrenzwerbung)" zu wählen wäre. 
Der in dem Modell wohl interessanteste Einflußfaktor auf die Verfügbarkeit ist der Umsatz $v_{t}$ des Produktes, wiederum ausgedrückt als Index im Verhältnis zum Bezugsumsatz. Der Einfluß $e_{d, t}$ des Umsatzes auf die Verfügbarkeit berechnet sich nach:

$e_{\mathrm{d}, t}=\alpha \cdot e_{\mathrm{d} t-1}+(1-\alpha) \cdot r_{\mathrm{d}}\left(v_{t}\right)$,

wobei $\alpha$ den periodenübergreifenden Effekt repräsentiert und $r_{d}$ die langfristige Wirkung der Verfügbarkeit in bezug auf den Umsatz darstellt.

\subsection{Modell von Farris, Olver und De Kluyver ${ }^{309}$}

Farris, Olver und De Kluyver gehen in ihrem Modell, wie in Abbildung 33 dargestellt, von einem vergleichsweise einfachen Gesamtzusammenhang aus. Im Vordergrund steht die Analyse der Beziehungen zwischen Marktanteil und Distribution bei häufig gekauften Konsumgütern unter besonderer Berücksichtigung der Präferenzstärke der Marke.

Ausgangspunkt des Modells ist die Unterscheidung zwischen dem sogenannten uncompromised und compromised demand. Der aus dem uncom-

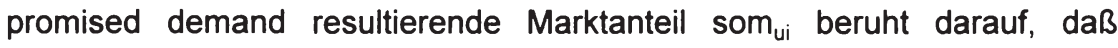
Endabnehmer diejenige Marke erwerben, die bereits vorher von innen präferiert wurde. Demgegenüber setzt sich der auf dem compromised demand basierende Marktanteil som $_{\mathrm{ci}}$ aus solchen Käufen der Marke i zusammen, bei denen die vom Endabnehmer ursprünglich präferierten Alternativen nicht

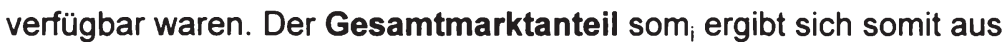

$\operatorname{som}_{\mathrm{i}}=\operatorname{som}_{\mathrm{ui}}+$ som $_{\mathrm{ci}}$

309 Vgl. Farris, P., Olver, J., De Kluyver, C., The Relationship between Distribution and Market Share, a.a.O., S. $107 \mathrm{ff}$.; vgl. auch Olver, J., Farris, P., Push and Pull, a.a.O., S. 56; Hughes, D. A., Commentary to Farris, P., Olver, J., De Kluyver, C., The Relationship between Distribution and Market Share, in: Marketing Science, No. 2, Spring 1989, S. 129 f.; Farris, P., Olver, J., De Kluyver, C., Reply to the Commentaries to Farris, P., Olver, J., De Kluyver, C., The Relationship between Distribution and Market Share, in: Marketing Science, No. 2, Spring 1989, S. $131 \mathrm{f}$. 


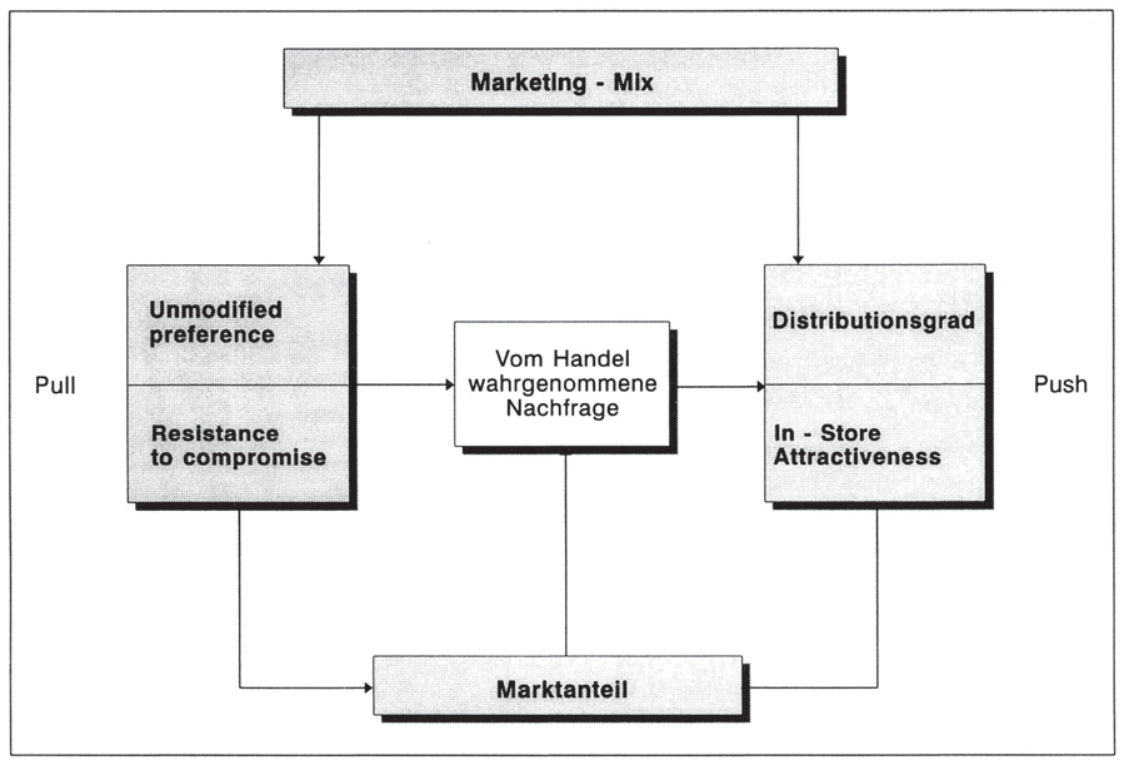

Abb. 33: Struktur des Modells von Farris, Olver und De Kluyver

In die Berechnung des Marktanteils des uncompromised demand gehen die unmodified preference $p_{i}$, die in-store-attractiveness $\beta_{i}$ sowie die effektive Verfügbarkeit PCVA ${ }_{i}$ der Marke i ein: ${ }^{310}$

$\operatorname{som}_{u i}=\beta_{i} \cdot p_{i} \cdot P C V A_{i}$

Unter der Voraussetzung, daß die Marke verfügbar ist, ermittelt sich deren Absatz somit aus den vom Handelseinfluß bereinigten Präferenzen der Endabnehmer sowie der relativen Präsentationsstärke der Marke am Point of Sale. Die effektive Verfügbarkeit einer Marke bestimmt sich dabei sowohl aus dem gewichteten Distributionsgrad $\mathrm{PCV}_{\mathrm{i}}$ als auch aus dem Beschaffungsverhalten der Endabnehmer. Sie steigt mit deren Bereitschaft, die der

310 Für eine differenzierte Berücksichtigung verschiedener Einflußfaktoren $S_{i k}$ der instore-attractiveness einer Marke $\mathrm{i}$ wird von den Autoren folgende Modellformulierung vorgeschlagen: $\beta_{i}=S_{i 1}^{r_{11}} \cdot S_{i 2}^{\gamma_{i 2}} \ldots . S_{i n}^{\gamma_{i n}}$, wobei $\gamma$ die Elastizität des jeweiligen Faktors ausdrückt. Zur unmodified preference sowie in-store-attractiveness vgl. auch Kap. A. 2. dieser Arbeit. 
Präferenz entsprechende Kaufabsicht auch für den Fall beizubehalten, daß die Marke nicht unmittelbar in der Einkaufsstätte vorhanden ist ("resistance to compromise", $\alpha_{\mathrm{i}}$ ):

$\mathrm{PCVA}_{\mathrm{i}}=1-\left(1-\mathrm{PCV}_{\mathrm{i}}\right)^{\alpha_{\mathrm{i}}}$

Wesentlich komplexer gestaltet sich die Formulierung des auf dem compromised demand beruhenden Marktanteils. ${ }^{311}$ Sei mit i die Marke gekennzeichnet, deren Marktanteil es zu ermitteln gilt, dann ist zunächst für alle Marken $L \neq i$ derjenige Teil der Nachfrage $c d a_{L}$ zu berechnen, der aufgrund von Verfügbarkeitsproblemen nicht befriedigt werden kann und damit zur Verteilung ansteht:

$c d a_{L}=\beta_{L} \cdot P_{L} \cdot\left(1-P C V_{L}\right)^{\alpha_{L}}$

$\mathrm{Da}$ in dem Modell zudem verschiedene Einkaufsstättenklassen ${ }^{312} \mathrm{~s}$ unterschieden werden, ist für jede Marke $L$ festzustellen, welchen Anteil die entsprechenden Einkaufsstätten an der nicht erfüllten Nachfrage besitzen $\left(\mathrm{Ssc}_{\mathrm{LS}}\right)$. Hierzu wird mit $\mathrm{PC}_{\mathrm{s}}$ der Absatzanteil einer Einkaufsstättenklasse am Gesamtabsatz der betreffenden Produktart bezeichnet und mit $d_{L s}$ eine Variable eingeführt, die den Wert 1 annimmt, falls die Marke $L$ in $s$ geführt wird und den Wert 0 erhält, wenn dies nicht der Fall ist:

$\operatorname{ssc}_{\mathrm{Ls}}=\frac{P C_{\mathrm{s}} \cdot\left(1-d_{L s}\right)}{1-P C V_{L}}$

Schließlich ist der Anteil $\mathrm{scd}_{\text {is }} \mathrm{zu}$ bestimmen, zu dem die nicht befriedigte Nachfrage in der Einkaufsstättenklasse s der Marke i zugute kommt. Hierzu werden die Eigenschaften dieser Marke (unmodified preference, in-storeattractiveness) in das Verhältnis zu denjenigen der verfügbaren Konkurrenzprodukte gesetzt:

311 Zur besseren Verständlichkeit wird im folgenden die Gesamtformel zunächst in Teilformeln zerlegt.

312 Als solche werden von den Autoren im Rahmen eines Beispiels aufgeführt: large stores, medium stores, small stores, convenience stores. 
$\operatorname{scd}_{\text {is }}=\frac{\beta_{\text {is }} \cdot P_{\text {is }} \cdot d_{\text {is }}}{\sum_{g \neq L} \beta_{g s} \cdot P_{g s} \cdot d_{g s}}$

Führt man die einzelnen Modellbestandteile zusammen, stellt sich der aus dem compromised demand resultierende Marktanteil wie folgt dar:

$\operatorname{som}_{\mathrm{ci}}=\sum_{\mathrm{L \neq i}}\left(\mathrm{cda}_{\mathrm{L}} \cdot \sum_{\mathrm{s}=1}^{\mathrm{S}} \mathrm{ssc}_{\mathrm{LS}} \cdot \mathrm{scd}_{\mathrm{is}}\right)$

Über diese generelle Modellformulierung hinaus werden zwei vereinfachende Alternativen vorgestellt, die jeweils von unterschiedlichen Annahmen bezüglich der Verfügbarkeit der Marken ausgehen. Bei der sogenannten "store-class-dependent stocking rule" wird unterstellt, daß allen Händlern die gleichen Anreize zur Führung der Marke im Sortiment geboten werden und diese zudem anhand der gleichen Kriterien über die Aufnahme einer Marke entscheiden. Dies führt dazu, daß die für den Handel attraktivste Marke in allen Einkaufsstätten geführt wird, die zweit-attraktivste Marke in allen Einkaufsstätten mit mindestens zwei Marken usw. Die sogenannte "store-class-independent stocking rule" unterstellt dagegen, daß die Wahrscheinlichkeit, daß eine Marke in einer bestimmten Einkaufsstätte verfügbar ist, ihrem gewichteten Distributionsgrad entspricht.

\subsection{Würdigung der Modelle und Implikationen für die eigene Modell- entwicklung}

Betrachtet man das in der Literatur übliche Vorgehen bei der Würdigung von Modellen, so ist festzustellen, daß häufig starke Redundanzen der Kritikpunkte auftreten. Da Modelle stets unter bestimmten Annahmen konzipiert und notwendigerweise z. T. stark vereinfachend sind, bieten sich in nahezu jedem Fall Ansatzpunkte für die folgende Kritik:

- Bestimmte Marketinginstrumente und situative Faktoren werden nicht berücksichtigt, 
- periodenübergreifende Wirkungen der Marketinginstrumente werden nicht erfaßt,

- Wechselwirkungen zwischen den Marketinginstrumenten kommen nicht oder nur ungenügend zum Ausdruck,

- die verwendeten multiplikativen oder additiven Verknüpfungen von Variablen entsprechen nicht den tatsächlichen Wirkungszusammenhängen,

- die angenommenen Beziehungen zwischen Instrumenteeinsatz und Marktteilnehmerverhalten sind willkürlich,

- der Zusammenhang von marktbezogenen Absatz- und unternehmensinternen Produktionsbedingungen wird vernachlässigt,

- Auswirkungen des Marketinginstrumenteeinsatzes auf die Kostensituation werden nicht adäquat abgebildet,

- die Existenz von Wettbewerbern, deren aktuelles und zukünftiges Verhalten sowie deren mögliche Reaktion auf eigene Entscheidungen gehen nicht in das Modell ein,

- bestimmte Beziehungen zwischen den Marktteilnehmern sind nicht in dem Modell enthalten,

- Veränderungen der Variablenwerte und der funktionalen Zusammenhänge während des Planungszeitraumes werden nicht beachtet und

- Teile des Modells sind nicht differenziert genug abgebildet.

Werden hiermit Vereinfachungen kritisiert, so gibt es darüber hinaus eine Klasse von Kritikpunkten, die stets bei komplexen Modellen angebracht werden:

- Eine korrekte Parameterschätzung der Wirkungsfunktionen ist aufgrund der Vielzahl simultan zu berücksichtigender Modellgleichungen und Interdependenzen nicht möglich,

- die zugrundeliegenden, differenzierten Hypothesen über das Konsumentenverhalten lassen sich nicht empirisch absichern,

- die verwendeten Größen lassen sich nur schwer operationalisieren und kaum mit hinreichender Genauigkeit messen und

- der umfassende Datenbedarf ist mit erheblichem Zeitaufwand verbunden und führt zu hohen Kosten. 
Für die Mehrzahl der insgesamt genannten Kritikpunkte lassen sich ohne weiteres auch Belege in den drei beschriebenen Modellen finden. Auf eine solche Form der Würdigung dieser Modelle soll jedoch verzichtet werden, da sich hieraus allenfalls Ansatzpunkte zu deren Verbesserung herausarbeiten lassen. Der Erkenntnisgewinn für die Entwicklung eines neuen Modells wäre jedoch vergleichsweise gering, da jedes Modell anderen Annahmen und Einschränkungen unterliegt. Vielmehr sollen hier diejenigen Aspekte der Modelle diskutiert werden, die bei der Entwicklung eines eigenen Ansatzes übernommen werden können oder zumindest Anregungen für die Modellierung geben können. Zu diesen Aspekten zählen die Frage der generellen Struktur des Modells, die Art der Verknüpfung von Variablen, die Form der Berücksichtigung des Verhaltens von Handel und Endabnehmer sowie die Art und der Umfang der Einbeziehung von Wettbewerbern.

Im Hinblick auf die generelle Struktur des Modells findet sich beim CIMDeveloppement-Modell eine Untergliederung des Gesamtmodells in einzelne Module. Damit erhöht sich die Transparenz der Modellstruktur, und es besteht eine hohe Flexibilität im Hinblick auf spätere Erweiterungen. Zudem wird durch dieses Vorgehen dem Gestaltungsprinzip der Einfachheit entsprochen, da die einzelnen Teilprogramme entsprechend ihrer Bedeutung für den Entscheidungsträger unterschiedlich differenziert ausgearbeitet werden können.

Das BRANDAID-Modell geht einen Schritt weiter, indem nicht inhaltlich zusammengehörige Bereiche modularisiert werden, sondern nahezu jede Einflußgröße als eigenständiges Modul betrachtet wird. Die entsprechenden Variablen können beliebig detailliert aufgenommen oder aber vollständig vernachlässigt werden, ohne daß andere Modellvariablen hiervon beeinflußt werden. Eine solche Struktur führt zwangsläufig zu der Kritik, daß sich die Wirkungsfunktionen verschiedener, auf eine gemeinsame Ergebnisvariable ausgerichteter Einflußgrößen nicht unabhängig voneinander schätzen lassen. Auch die anschließende multiplikative Verknüpfung der daraus fol- 
genden Indizes ist sicherlich nicht unproblematisch, muß allerdings aufgrund notwendiger Vereinfachungen akzeptiert werden. ${ }^{313}$

Einer direkten Übertragung auf das hier zu entwickelnde Modell steht jedoch ein anderer, benutzerorientierter Grund entgegen. Während beim BRANDAID-Modell die Wirkungen von Entscheidungsvariablen jeweils auf eine Erwartungsvariable abgebildet werden, soll hier das Endabnehmer- und Händlerverhalten differenzierter betrachtet werden. Eine Kombination von z. B. 3 Einflußgrößen, die auf 4 das Verhalten beschreibende Variablen wirken, würde jedoch bereits zu 3x4=12 Funktionen führen, die vom Entscheidungsträger $\mathrm{zu}$ bestimmen wären. Prinzipiell erscheint der Ansatz des BRANDAID-Modells zur Verknüpfung von Variablen jedoch aufgrund der gewonnenen Transparenz und Flexibilität des Modells durchaus sinnvoll. Daher soll er hier in abgewandelter Form Verwendung finden. Wie später noch zu zeigen ist, wird dabei nicht unmittelbar die Wirkung der Entscheidungsvariablen auf die Erwartungsvariablen abgebildet, sondern ein zweistufiges Vorgehen gewählt. ${ }^{314}$

Das Verhalten der Marktteilnehmer wird in den drei Modellen entsprechend ihrer global- bzw. detailanalytischen Konzeptionen mit unterschiedlicher Tiefe beschrieben. Betrachtet man die umfangreichen Annahmen des CIM-Devellopement-Modells, die allein zur Berechnung der Präferenzen von Endabnehmer und Händler vorauszusetzen sind, ${ }^{315}$ so zeigt sich hier sehr deutlich die grundsätzliche Problematik detailanalytischer Ansätze. Interessant ist bei diesem Modell allerdings die Unterscheidung zwischen der freien, spontanen und modulierten Nachfrage sowie dem tatsächlichen

313 Vgl. zu einer ausführlichen Kritik Meffert, H., Computergestützte Marketing-Informationssysteme, a.a.O., S. $138 \mathrm{ff}$.

314 Vgl. Kap. C. 3.11 dieser Arbeit.

315 Implizit werden u. a. folgende Annahmen bezüglich des Bewertungs- und Entscheidungsverhaltens des Endabnehmers getroffen: Additive Verknüpfung der Eigenschaftsbewertung; Mindestanforderung an Einstellungswert geht in die freie Neigung explizit ein; Mindestanforderung selbst wird mit Hilfe der Standardabweichung der Eigenschaftsbewertungen ermittelt. Für das Handelsverhalten wird $u$. a. unterstellt: Ausschließlich 5 Kriterien werden in der Bewertung berücksichtigt; Präferenz setzt sich additiv aus miteinander multipliziertem Rang der Bewertungen und Bedeutung von Markeneigenschaften zusammen. 
Absatz einer Marke. Eine solche Differenzierung verdeutlicht unterschiedliche Gründe für den Kauf bzw. Nichtkauf und zeigt damit zentrale Ansatzpunkte für Möglichkeiten und Hindernisse der Markendurchsetzung auf. Zu bemängeln ist jedoch, daß bei der spontanen Nachfrage für die aktuell am Fahrzeug montierte Marke die zugrundeliegenden Ursachen nicht zum Ausdruck kommen. Es kann sich sowohl um einen quasi automatischen Wiederkauf handeln als auch um eine Entscheidung, die auf Basis der freien Neigung getroffen wird. Da sich hieraus verschiedenartige Implikationen für die Markendurchsetzung ergeben, ist eine derartige Unterscheidung in dem zu entwickelnden Modell auf jeden Fall zu treffen.

Ungeeignet als Beispiel für die Modellierung des Endabnehmerverhaltens ist der BRANDAID-Ansatz. Dort wird nicht das Verhalten des Endabnehmers, sondern unmittelbar der Zusammenhang von Instrumenteeinsatz und Marktanteil abgebildet. Implizit kommt der Verhaltensaspekt zwar in den Indizes zum Ausdruck, mit denen der Ausgangsmarktanteil multipliziert wird. Für den Entscheidungsträger wird jedoch keine anschauliche Trennung zwischen der Beeinflussung bestimmter Verhaltensweisen des Käufers und dem daraus resultierenden Marktanteil vorgenommen.

Gut gelungen ist dies dagegen im Modell von Farris, Olver und De Kluyver, deren Marktanteilsberechnung auf Basis einer aggregierten, globalen Betrachtung des Endabnehmerverhaltens erfolgt. Die Bedeutung der Präferenz für eine Marke sowie der Einfluß inrer Verfügbarkeit und ihrer Förderung im Handel auf den Kauf wird deutlich herausgestellt. Intelligent gelöst wurde zudem die Problemstellung, festzustellen, ob eine präferierte Marke für den Endabnehmer in der Einkaufsstätte vorhanden ist. Die auf Basis des Distributionsgrades unter Einbeziehung des Endabnehmerverhaltens berechnete effektive Verfügbarkeit entspricht gerade der Wahrscheinlichkeit, daß der Endabnehmer die Marke tatsächlich antrifft.

Neben der eigenen sind Marken der Wettbewerber in dem Modell zu erfassen. Sowohl das CIM-Devellopement-Modell als auch das Modell von Farris, Olver und De Kluyver erfassen jeden Wettbewerber einzeln. Gegen 
ein solches Vorgehen sprechen vier wesentliche Gründe. ${ }^{316}$ Erstens stehen Informationen über die Konkurrenzprodukte in aller Regel nicht in derselben Ausführlichkeit zur Verfügung wie für die eigene Marke. Selbst wenn diese prinzipiell beschafft werden können, erhöhen sich hierdurch die Kosten und der Zeitaufwand in z. T. nicht unerheblichem Maße. Zweitens steigt die Anzahl der zu schätzenden Wirkungsbeziehungen. Jede Marke besitzt eine andere Vorgeschichte und eine andere aktuelle Stellung bei den Endabnehmern und beim Handel. Damit ist zu erwarten, daß kein einheitlicher Zusammenhang von Instrumenteeinsatz und Verhaltenswirkung gilt, sondern die Wirkungsfunktionen vielmehr individuell für die verschiedenen Marken zu bestimmen sind. ${ }^{317}$ Drittens besteht die Problematik, das zukünftige Verhalten der einzelnen Wettbewerber prognostizieren zu müssen. Diese ohnehin schwierige Aufgabe gewinnt vor allem an Komplexität, wenn Reaktionsmuster der verschiedenen Konkurrenten auf die eigenen Maßnahmen explizit im Modell erfaßt werden sollen. ${ }^{318}$ Viertens ist damit schließlich der Aspekt der unterschiedlichen Markenpositionierungen zu berücksichtigen. Längst nicht alle Reifenmarken konkurrieren miteinander bzw. stehen mit gleicher Intensität im Wettbewerb. Die differenzierte Betrachtung konkurrierender Marken erfordert somit eine genaue Kenntnis der Positionierung und der daraus resultierenden gegenseitigen Abhängigkeiten. ${ }^{319}$

316 Vgl. hierzu auch Little, J. D. C., Ein On-Line-Marketing-Mix-Modell, a.a.O., S. 674 f.

317 Dies mag auch eine Erklärung dafür sein, daß Untersuchungen zur Werbewirkung bis heute kaum überzeugende Ergebnisse liefern. Zur Beurteilung des aktuellen Standes der Werbewirkungsforschung vgl. Mayer, $H$., Werbewirkung und Kaufverhalten unter ökonomischen und psychologischen Aspekten, a.a.O., S. $196 \mathrm{ff}$.

$318 \mathrm{Zu}$ einem Beispiel für die explizite Berücksichtigung von Inderdependenzen zwischen den Wettbewerbern bei Marketingentscheidungen vgl. Kotler, $P$., Competitive Strategies for New Product Marketing over the Life Cycle, in: Management Science, Dec. 1965, S. B-104 ff. sowie die Darstellung bei Meffert, H. und Mitarbeiter, Die Anwendung mathematischer Modelle im Marketing, Wiesbaden, o.J., S. $10 \mathrm{ff}$. Eine kritische Analyse sowie Erweiterungen des Modells finden sich bei Grob, H. L., Computergestützte Preispolitik, Preisstrategische Entscheidungsmodelle für Marketing-Informationssysteme, Wiesbaden 1975, S. $91 \mathrm{ff}$.

319 In bezug auf das Instrument des Preises wird diese Abhängigkeit häufig in Form der Kreuzpreiselastizität ausgedrückt. Sie gibt an, um wieviel Prozent sich der Absatz einer Marke $i$ ändert, wenn der Preis der Marke j um $1 \%$ verändert wird. Vgl. Diller, H., Preispolitik, a.a.O., S. 66 f. In ihrer Funktion zur Charakterisierung der Konkurrenzsituation wird die Elastizität auch als Triffinscher Koeffizient bezeichnet. Vgl. 
Im BRANDAID-Modell werden diese Probleme dadurch umgangen, daß die konkurrierenden Marken zu einer einzigen fiktiven Wettbewerbsmarke zusammengefaßt werden. Dieses durchaus übliche Vorgehen ist allerdings dann kritisch zu betrachten, wenn hiermit die Wirkung von Konkurrenzmaßnahmen auf den eigenen Absatz abgebildet werden soll. Hinterfragt man, was dort letztlich als Aktivität der Konkurrenz und deren Wirkung beschrieben wird, so zeigen sich relativ schnell die Grenzen dieses Ansatzes. Dies wird besonders am Beispiel des Distributionsgrades deutlich. Der durch die fiktive Marke repräsentierte durchschnittliche Distributionsgrad subsumiert zum einen weit und gering distribuierte Marken und zeigt zum anderen keine Überschneidungen in der Distribution zur eigenen Marke auf. Damit läßt sich weder eine Aussage darüber treffen, welche Wirkungen vom Distributionsgrad der Konkurrenzmarken auf das Endabnehmerverhalten ausgehen, in welchem Umfang die Käufer ihre präferierte Marke am Point of Sale vorfinden, noch in welchem Konkurrenzumfeld sich die eigene Marke dort befindet. Eine Erhöhung des durchschnittlichen Distributionsgrades sagt zudem nichts darüber aus, ob alle darunter subsumierten Konkurrenten ihr Verhalten gleichmäßig verändert haben oder beispielsweise zwei bedeutende Wettbewerber stark offensiv agieren. Wechselseitige Reaktionen und Wirkungen zwischen dem eigenen und dem Verhalten des fiktiven Wettbewerbers repräsentieren somit kaum die tatsächlichen Marktverhältnisse. Eine fiktive Marke kann somit allenfalls gewisse Niveaugrößen repräsentieren, um Ausprägungen der Variablen für die eigene Marke nicht in absoluten, sondern in relativen Werten in die Modellberechnung eingehen zu lassen.

Vor diesem Hintergrund soll hier auf die explizite Abbildung des Verhaltens von Wettbewerbern verzichtet werden. Die konkurrierenden Marken werden allein aus Darstellungsgründen zu einer fiktiven Marke zusammengefaßt. Diese repräsentiert lediglich den nicht von der eigenen Marke erreichten Anteil der jeweiligen Erwartungsvariablen. Beispielsweise berechnet sich der Marktanteil dieser fiktiven Konkurrenzmarke aus:

Marktanteil fiktive Konkurrenzmarke $=100 \%$ - Marktanteil eigene Marke 
Aus diesem Vorgehen ist allerdings nicht die Schlußfolgerung zu ziehen, daß der Wettbewerb nicht beachtet würde. Schätzungen des Entscheidungsträgers über die Wirkungen eigener Maßnahmen erfolgen stets unter impliziter Berücksichtigung erwarteter Konkurrenzaktionen und -reaktionen. Insofern soll von den Fähigkeiten des Entscheidungsträgers Gebrauch gemacht werden, aus Erfahrungen heraus die relevanten Wettbewerber zu identifizieren, deren Verhalten einzuschätzen und hierdurch Aussagen darüber zu treffen, welche Wirkungen mit eigenen Maßnahmen voraussichtlich verbunden sein werden.

\section{Entwicklung eines Reifenmarktsimulationsmodells zur Planung von Markendurchsetzungsstrategien}

Für das im folgenden zu entwickelnde eigene Modell zur Planung von Markendurchsetzungsstrategien sind zunächst die Modellstruktur sowie die einzelnen Teilmodelle darzustellen. Anschließend ist das Vorgehen zur Ermittlung von Werten der Eingangsvariablen auf Basis subjektiver Schätzungen des Entscheidungsträgers zu beschreiben und die Art der Bestimmung von Parametern der Erklärungsgleichungen zu erläutern. Ferner ist zur Berechnung der Modellergebnisse auf die Lösungsmethode der Risikosimulation näher einzugehen.

Da diese mehrdimensionale Ergebnisse für die Erwartungsvariablen liefert, sind darüber hinaus Möglichkeiten zu charakterisieren, um eine Entscheidung bei verschiedenen, sich nicht dominierenden Handlungsalternativen herbeizuführen. Darüber hinaus lassen sich mit Hilfe der Sensitivitätsanalyse Wege aufzeigen, um eine Verbesserung oder sogar Optimierung des Budgeteinsatzes zu erreichen. Neben der konzeptionellen Entwicklung des Modells wird schließlich dessen computergestützte Implementierung vorgestellt. 


\subsection{Kennzeichnung der Struktur des Gesamtmodells und Darstellung der Teilmodelle}

Die Struktur des hier zu entwickelnden Modells ist in Abbildung 34 dargestellt.

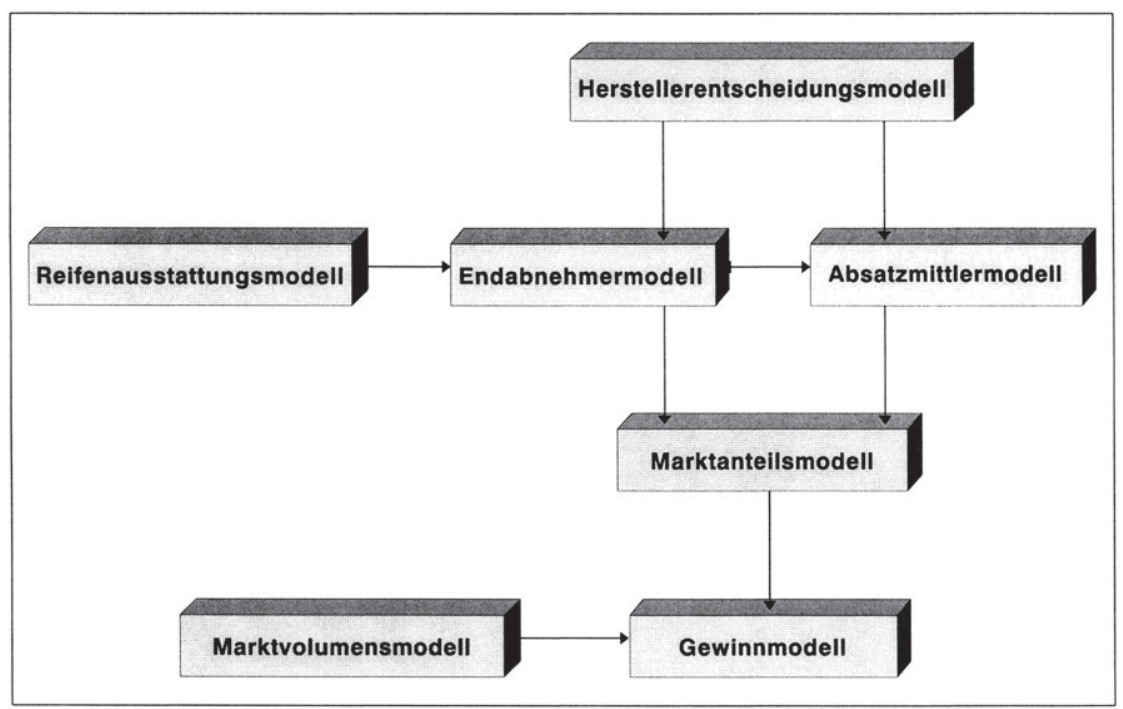

Abb. 34: Struktur des Reifenmarktsimulationsmodells

Ausgehend von den Entscheidungen des Herstellers über die Tatbestände der Markendurchsetzungsstrategie wird das Verhalten der Endabnehmer und des Handels ermittelt. Da die aktuelle Reifenausstattung einen bedeutenden Einfluß auf das Endabnehmerverhalten besitzt, wird dazu in einem eigenen Teilmodell festgestellt, welche Reifenmarke zum Kaufzeitpunkt am Fahrzeug montiert ist. Der Marktanteil ergibt sich aus der Zusammenführung des Endabnehmer- und Absatzmittlerverhaltens und geht schließlich zusammen mit dem Marktvolumen in das Gewinnmodell ein. Hier werden die entsprechenden Erlöse und entstehenden Kosten gegenübergestellt. 320

320 Es sei unterstellt, daß Erlöse und Kosten jeweils mit ihrem Auftreten zahlungswirksam werden. Damit lassen sich erstens diese Begriffe synonym zu den investitionstheoretischen Begriffen der Ein- und Auszahlungen verwenden, und zweitens 
Abbildung 35 zeigt die grundlegende Struktur der Variablenbeziehungen des Modells auf. Als Entscheidungsvariablen stehen das Pull-Budget, das Akquisitions- und Förderungsbudget sowie die Handelsspanne zur Verfü-gung. Darüber hinaus kann der Anteil an der Erstausrüstung vorgegeben werden. Die Entscheidungsvariablen sind über Erklärungsgleichungen mit Erwartungsvariablen zur Beschreibung der Marktteilnehmerreaktion verbunden. Diese entsprechen den Unterzielen der Nachfrage- und Angebotsstärke. Hierzu zählen der Präferenz- und der Akzeptanzgrad bei den unterschiedlichen Käufergruppen sowie der Distributionsgrad und das Empfehlungsverhalten des Handels. Als Zwischen- und Oberziele beschreibende Erwartungsvariablen gehen der Markenwert, die Periodengewinne sowie die Marktanteile der Perioden in das Modell ein.

Bezüglich der Kontextvariablen sind zwei Arten von Variablen zu unterscheiden. Kontextvariablen mit direktem Einfluß auf die Erklärungsgleichungen beeinflussen unmittelbar die Wirkungsbeziehungen zwischen den Entscheidungs- und den Erwartungsvariablen. Dazu gehören beispielsweise die aufgeführten Machtbeziehungen zwischen Hersteller und Handel oder das Rollenverständnis des Handels. Diese situativen Faktoren werden in dem Modell nicht explizit als Variablen aufgenommen, sondern im Sinne des benutzerorientiert-subjektiven Ansatzes vom Entscheidungsträger bei der Schätzung von Funktionsparametern der Erklärungsgleichungen berücksichtigt. Darüber hinaus existieren weitere situative Faktoren wie das Marktvolumen oder die Fahrleistung der Reifenkäufer. Diese nehmen zwar keinen direkten Einfluß auf die funktionalen Beziehungen zwischen den Entscheidungsvariablen und den Erwartungsvariablen zur Beschreibung der Marktteilnehmerreaktion, sind jedoch als Elemente des situativen Kontextes mit den Erwartungsvariablen verknüpft. Insofern gehen diese Faktoren als Kontextvariablen in das Modell ein.

Das Modell simuliert die Marktentwicklung innerhalb eines prinzipiell frei wählbaren, in Perioden gleicher Länge aufzuteilenden Planungszeitraumes. Im folgenden sei eine Periodenlänge von einem Jahr und ein Planungszeit

kann eine noch zu definierende Verteilung des Zahlungsstroms während der Periode angenommen werden. Vgl. zur Abgrenzung dieser Begriffe Schierenbeck, $H$., Grundzüge der Betriebswirtschaftslehre, a.a.O., S. $465 \mathrm{ff}$. 


\begin{tabular}{|c|c|c|c|}
\hline & \multirow{2}{*}{\begin{tabular}{|l|}
$\begin{array}{l}\text { Kontextvariablen mit } \\
\text { direktem Einfluß auf Er- } \\
\text { klärungsgleichungen }\end{array}$ \\
\\
Implizite Berücksichti- \\
gung im Rahmen der \\
Parameterschätzung \\
\end{tabular}} & \multicolumn{2}{|c|}{$\begin{array}{l}\text { Kontextvariablen ohne direkten Einfluß auf Erklä- } \\
\text { rungsgleichungen }\end{array}$} \\
\hline & & \multicolumn{2}{|c|}{$\begin{array}{l}\text { - Anteile der Reifenkäufer mit Präferenz für eine } \\
\text { Marke (abhängig/ unabhängig von montierter } \\
\text { Reifenmarke) und Indifferenz } \\
\text { - Marktvolumen/ -wachstum } \\
\text { - Fahrleistungen Reifenkäufer } \\
\text { - Reifenlebensdauer }\end{array}$} \\
\hline $\begin{array}{l}\text { Entscheidungs- } \\
\text { variablen }\end{array}$ & $\begin{array}{l}\text { Erklärungsglei- } \\
\text { chungen }\end{array}$ & $\begin{array}{l}\text { Erwartungsvariablen zur } \\
\text { Beschreibung der Markt- } \\
\text { teilnehmerreaktion (Un- } \\
\text { terziele) }\end{array}$ & \begin{tabular}{|l|} 
Erwartungsvariablen \\
zur Beschreibung \\
der Zwischen- und \\
Oberziele \\
\end{tabular} \\
\hline $\begin{array}{l}\text { - Akquisitionsbudget } \\
\text { - Förderungsbudget } \\
\text { - Handelsspanne } \\
\text { - Erstausrüstungsanteil }\end{array}$ & $\begin{array}{l}\text { Wirkungsbeziehun- } \\
\text { gen mit subjektiv ge- } \\
\text { schätzten Funktions- } \\
\text { parametern }\end{array}$ & $\begin{array}{l}\text { - Präferenzgrad für betrachte- } \\
\text { te Marke bei markenfixier- } \\
\text { ten/ -orientierten/ -be- } \\
\text { wußten Reifenkäufern } \\
\text { - Akzeptanzgrad für betrach- } \\
\text { tete Marke bei Käufern } \\
\text { mit anderer Präferenz und } \\
\text { bei indifferenten Käufern } \\
\text { - Distributionsgrad } \\
\text { - Empfehlungsverhalten ggü. } \\
\text { Reifenkäufern mit Präfe- } \\
\text { renz für betrachtete Marke } \\
\text { /andere Marke/ Indifferenz }\end{array}$ & $\begin{array}{l}\text { - Markenwert } \\
\text { - Periodengewinne } \\
\text { - Marktanteile der } \\
\text { Perioden }\end{array}$ \\
\hline
\end{tabular}

Abb. 35: Grundstruktur der Variablenbeziehungen des Modells 
raum von sieben Jahren angenommen. Der Grundgedanke der durchzuführenden Simulation besteht darin, daß ausgehend von der zum Startzeitpunkt $\mathrm{t}=0$ bestehenden Situation jeweils zum Ende einer Periode, d. $\mathrm{h}$. zu den Zeitpunkten $t=1, \ldots, 7$, das Verhalten von Reifenkäufern und Händlern beobachtet und der Marktanteil berechnet wird.

Ferner gelten zu diesen Zeitpunkten bestimmte Handelsspannen, Herstellungskosten und Endabnehmerpreise. Die Werte dieser zeitpunktbezogenen Größen beziehen sich jeweils auf den Beginn und das Ende einer Periode. Über deren Entwicklung während der Periode sind an gegebener Stelle geeignete Annahmen zu treffen. Als periodenbezogene Größen gehen die Budgets sowie das Marktvolumen in das Modell ein. Deren Werte beziehen sich jeweils auf eine gesamte Periode.

Diese explizite Unterscheidung von Perioden und Zeitpunkten wird häufig bei den in der Literatur beschriebenen Modellen vernachlässigt. Dies ist für den Fall zulässig, daß die Perioden relativ kurz sind. Bei der hier gewählten Periodenlänge von einem Jahr ist jedoch davon auszugehen, daß es innerhalb dieses Zeitraumes zu Veränderungen kommen kann, so daß das zu einem bestimmten Zeitpunkt beobachtete Verhalten keine Gültigkeit für die gesamte Periode besitzt. Gleiches gilt für periodenübergreifende Kaufverhaltenszusammenhänge. ${ }^{321}$ Daher wird im folgenden stets zwischen einem Zeitpunkt $t$ und der dazugehörigen Periode $n$ differenziert. Für den Zusammenhang zwischen $t$ und $n$ gilt, daß die betreffende Periode eingeschlossen wird durch die Zeitpunkte $\mathrm{t}-1$ und $\mathrm{t}$.

\subsection{Herstellerentscheidungsmodell}

In diesem ersten Teilmodell sind die vom Hersteller kontrollierbaren Entscheidungsvariablen der Markendurchsetzungsstrategie festzulegen. Zur unmittelbaren Einflußnahme auf den Endabnehmer steht zunächst das in Periode $\mathrm{n}$ für die Marke i wirksame Pull-Budget $\mathrm{pu}_{\text {in }}$ zur Verfügung.

321 Auf die Formulierung periodenübergreifender Kaufverhaltenszusammenhänge wird in Kap. C. 3.15 ausführlich eingegangen. 
Dieses Budget wird als Index definiert, der sich auf eine in Geldeinheiten ausgedrückte Basis puba ${ }_{n}$ bezieht. Zur Berücksichtigung von Kostensteigerungen oder -senkungen beim Einsatz der Marketinginstrumente im Verlauf des Planungszeitraumes wird für jede Periode eine prozentuale Veränderung $\Delta \mathrm{puba}_{\mathrm{n}}$ dieser Basis angenommen. Ausgehend von der Budgetbasis puba $_{0}$ zum Startzeitpunkt des Planungszeitraumes berechnet sich damit die jeweils für die Periode $n$ gültige Basis aus:

puba $_{n}=$ puba $_{0} \cdot \prod_{i=1}^{n}\left(1+\Delta\right.$ puba $\left._{1}\right)$

Die Einflußnahme auf den Handel erfolgt über Push-Budgets. Wie zuvor herausgearbeitet, ist hierbei zwischen dem Akquisitions- $\left(p a_{\text {in }}\right)$ und dem Förderungsbudget $\left(\mathrm{pf}_{\text {in }}\right)$ zu differenzieren. ${ }^{322}$ Auch diese Budgets werden als Indizes definiert, die sich jeweils auf eine in Geldeinheiten ausgedrückte Budgetbasis paban $_{n}$ bzw. pfban beziehen. Dabei sind wiederum entsprechende Veränderungsraten $\Delta \mathrm{paba}_{n} \mathrm{bzw} \Delta \mathrm{pfba}_{\mathrm{n}}$ zu berücksichtigen.

Um das nicht kostenmäßig bewertbare kontrahierungspolitische Instrumentarium dem Push-Budget zurechnen zu können, werden zunächst alle Maßnahmen der Kontrahierungspolitik in der potentiellen Handelsspanne $h_{i t}$ zusammengefaßt. Diese berechnet sich als Differenz zwischen dem vom Handel potentiell realisierbaren Endabnehmerpreis und dem tatsächlichen Handelseinkaufspreis, d. h. dem vom Handel zu zahlenden Preis unter Berücksichtigung aller kontrahierungspolitischen Vergünstigungen. Im weiteren liegt diesem Vorgehen der Gedanke zugrunde, daß die Handelsspanne Einfluß auf die Effizienz des Push-Budgets nimmt, d. h. die Wirkung einer Einheit (DM) des Push-Budgets steigt bzw. sinkt bei Änderung der Handelsspanne.

Dies führt zum Begriff der effektiv wirksamen Push-Budgets $p a_{\text {eff,in }}$ bzw. $\mathrm{pf}_{\text {eff,in }}$, die sich aus den bisher definierten (monetären) Akquisitions- und Förderungsbudgets sowie entsprechender Effizienzindizes $w_{a h, \text { in }}$ bzw. $w_{f n, i n}$ berechnen: 
$p a_{\text {eff }, \text { in }}=p a_{\text {in }} \cdot w_{\text {an,in }}$

$\mathrm{pf}_{\text {eff,in }}=p f_{\text {in }} \cdot w_{\text {fh,in }}$

Die Effizienzindizes stehen ihrerseits in funktionaler Abhängigkeit von der gewährten potentiellen Handelsspanne: 323

$w_{\text {ah,in }}=\frac{f_{1}\left(h_{i t-1}\right)+f_{1}\left(h_{i t}\right)}{2}$

$w_{\text {fh, in }}=\frac{f_{2}\left(h_{i t-1}\right)+f_{2}\left(h_{i t}\right)}{2}$

Hierbei zeigt sich das Problem, daß monetäre Budgets in Geldeinheiten pro Periode definiert sind, während die Handelsspanne eine zeitpunktbezogene Größe ist, die sich während der Periode verändern kann. Es sei angenommen, daß die mit der Handelsspanne während der Periode erreichte Effizienz des Push-Budgets sich als Durchschnitt der Wirkungen zu Beginn und zum Ende der Periode ergibt.

\subsection{Endabnehmermodell}

Ausgangspunkt des Endabnehmermodells ist die Strukturierung des Reifenkaufprozesses in der Einkaufsstätte. In einer ersten Grobbetrachtung (vgl. Abbildung 36) läßt sich das Verhalten der Reifenkäufer zunächst danach differenzieren, ob beim Betreten der Einkaufsstätte bereits eine bestimmte Präferenz vorliegt oder aber noch keine konkrete Vorstellung über die zu erwerbende Marke besteht. Findet sich im ersten Fall die verlangte Marke in der Einkaufsstätte und nimmt der Händler keinen weiteren Einfluß auf die Entscheidung des Endabnehmers, kommt es zur Umsetzung des Kaufvorhabens. Empfiehlt der Händler dagegen eine andere Marke, schließt sich der Endabnehmer dessen Vorschlag möglicherweise an und weicht damit von seiner ursprünglichen Präferenz ab. Ist die verlangte Marke nicht in der Einkaufsstätte vorhanden oder fragt der Reifenkäufer keine bestimmte Marke nach, werden die vom Händler geführten Reifen

323 Mit $f(\bullet)$ sei allgemein eine funktionale Abhängigkeit beschrieben, die es später noch näher zu spezifizieren gilt. Vgl. Kap. C. 3.22. 
angeboten bzw. empfohlen. Der Endabnehmer kann entweder eine der vorgeschlagenen Alternativen akzeptieren oder aber die Einkaufsstätte verlassen, um einen weiteren Händler aufzusuchen.

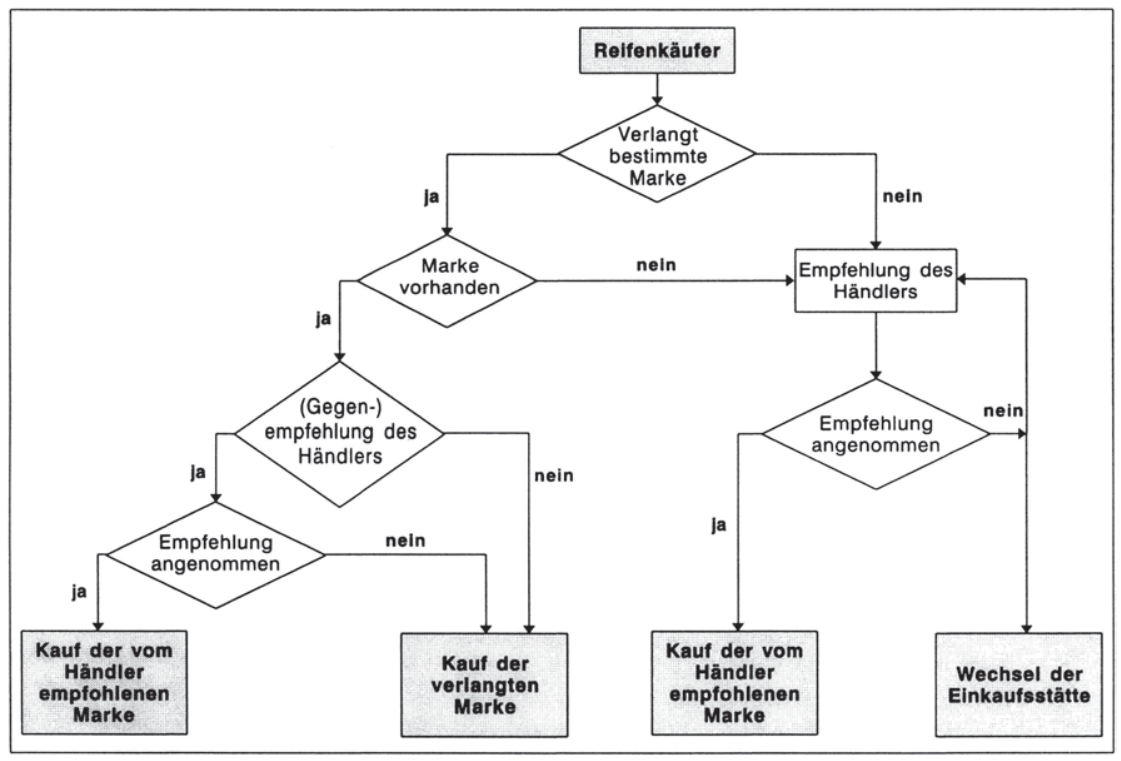

Abb. 36: Prozeß des Reifenkaufs innerhalb der Einkaufsstätte (in Anlehnung an Hansen, H. R., Thabor, A., Marketing Modelle, a.a.O., S. 185)

Aus dieser Grobbetrachtung heraus ist zunächst die Situation genauer zu beschreiben, in der ein bestimmter Reifen verlangt wird, der Reifenkäufer also eine Präferenz für eine Marke besitzt. In den vorherigen Ausführungen wurde bereits auf die besonders hohe Bedeutung des am Fahrzeug montierten Reifens für die Kaufentscheidung des Endabnehmers hingewiesen. Es scheint durchaus gerechtfertigt, davon auszugehen, daß zum Zeitpunkt der Kaufentscheidung ein Teil der Reifenkäufer pam ${ }_{t}$ stets die Marke nachfragt, die sich aktuell am Fahrzeug befindet. Damit gilt für die (Teil-) Wahrscheinlichkeit ppam $_{\text {it, }}$ daß Marke i zum Zeitpunkt t verlangt wird:

$\operatorname{ppam}_{\mathrm{it}}=\operatorname{pam}_{\mathrm{t}} \cdot \mathrm{mr}_{\mathrm{it}}$, 
wobei mit $\mathrm{mr}_{\mathrm{it}}$ die Wahrscheinlichkeit beschrieben wird, daß die Reifenmarke i zu diesem Zeitpunkt am Fahrzeug montiert ist. 324

Andere Reifenkäufer bilden ihre Präferenzen aufgrund einer Vielzahl von Informationen und Einflußfaktoren, die sich aus Marketingaktivitäten des Herstellers, eigenen Erfahrungen, Empfehlungen aus dem sozialen Umfeld usw. ergeben. Um den Unterschied zu Endabnehmern hervorzuheben, deren Präferenz sich stets an der jeweils montierten Marke orientiert, werden diese Personen als Käufer bezeichnet, die sich unabhängig von der aktuell am Fahrzeug befindlichen Reifenausstattung für eine bestimmte Marke entscheiden. Es sei pumt deren Anteil an der Gesamt-

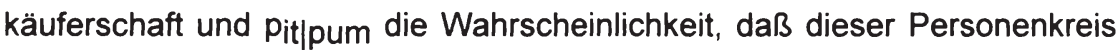
die Marke i präferiert. Bezogen auf die Gesamtheit der Reifenkäufer ergibt sich damit die (Teil-)Wahrscheinlichkeit ppum $_{i t}$, daß die Marke i zum Zeitpunkt t nachgefragt wird, aus:

ppum $_{\text {it }}=$ pum $_{\mathrm{t}} \cdot \mathrm{p}_{\text {itpum }}$

Dabei soll angenommen werden, daß sich $p_{\text {itpum }}$ aus dem zum vorhergegangenen Zeitpunkt $\mathrm{t}-1$ gültigen Wert sowie dem in Periode $n$ effektiv wirksamen Pull-Budget $\mathrm{pu}_{\text {in }}$ ergibt. Es gilt:

$p_{\text {itpum }}=f\left(p_{\text {it }-1 \mid \text { pum }}, p u_{\text {in }}\right)$

Da Präferenzen für eine Marke unterschiedlich stark ausgeprägt sein können, ist auch hier zwischen markenfixierten, -orientierten und -bewußten Endabnehmern zu differenzieren. Zudem ist zu berücksichtigen, daß die Präferenzstärke durchaus davon abhängig sein kann, ob lediglich der gerade montierte Reifen nachgefragt wird oder der Präferenz weitergehende Überlegungen und Entscheidungsprozesse zugrunde liegen. Damit sind $\mathrm{mf}_{\mathrm{t} \mid \mathrm{pam}, \mathrm{i}}, \mathrm{mo}_{\mathrm{t|pam}, \mathrm{i}}$ und $\mathrm{mb}_{\mathrm{t|pam}, \mathrm{i}}$ als Anteile markenfixierter, -orientierter und bewußter Endabnehmer an der Gruppe von Reifenkäufern zu definieren, die zum Zeitpunkt $t$ aufgrund des montierten Reifens die Marke i verlangen.

324 Zur Vereinfachung wird dabei unterstellt, daß alle Reifen am Fahrzeug der gleichen Marke angehören. 
Mit $\mathrm{mf}_{t \mid p u m, i}, \mathrm{mo}_{\mathrm{t} \mid \text { pum,i }}$ und $\mathrm{mb}_{\mathrm{t} \mid \mathrm{pum}, \mathrm{i}}$ werden analog hierzu die Präferenzstärken bei jenen Endabnehmern beschrieben, die unabhängig vom montierten Reifen eine Präferenz für Marke i besitzen. Der jeweilige Anteil ergibt sich wiederum aus dem entsprechenden Wert in $\mathrm{t}-1$ sowie dem in Periode $\mathrm{n}$ effektiv wirksamen Pull-Budget. Dabei ist es ausreichend, die Funktionen für jeweils zwei der drei Typen aufzustellen, da sich die jeweiligen Anteile der fixierten, orientierten und bewußten Reifenkäufer stets auf den Wert 1 summieren:

$$
\begin{aligned}
& m f_{\text {t|pam,i }}=f_{1}\left(m f_{t-1 \mid p a m, i}, p u_{i n}\right) \\
& m o_{\text {tppam,i }}=f_{2}\left(m o_{t-1 \mid p a m, i}, p u_{i n}\right) \\
& m b_{t \mid p a m, i}=1-m f_{t \mid p a m, i}-m o_{t \mid p a m, i} \\
& m f_{t \mid p u m, i}=f_{1}\left(m f_{t-1 \mid p u m, i}, p u_{i n}\right) \\
& m o_{t \mid p u m, i}=f_{2}\left(m o_{t-1 \mid p u m, i}, p u_{i n}\right) \\
& m b_{t \mid p u m, i}=1-m f_{t \mid p u m, i}-m o_{t \mid p u m, i}
\end{aligned}
$$

Bislang wurden lediglich Endabnehmer einbezogen, die in der Einkaufsstätte nach der Marke i verlangen. Darüber hinaus sind jedoch auch Reifenkäufer zu berücksichtigen, die zwar eine Konkurrenzmarke präferieren, deren ursprüngliche Kaufabsicht aber durchaus am Point of Sale noch beeinflußbar ist. Wie zuvor diskutiert, werden die konkurrierenden Marken in aggregierter Form betrachtet und zu einer fiktiven Konkurrenzmarke $\mathrm{k}$ zusammengefaßt. Dann berechnet sich der Anteil an Reifenkäufern, die die Marke $k$ abhängig ppam pt bzw. unabhängig ppum $_{k t}$ von der montierten Marke verlangen, aus:

$$
\begin{aligned}
& \operatorname{ppam}_{k t}=\operatorname{pam}_{\mathrm{t}} \cdot\left(1-\mathrm{mr}_{\mathrm{it}}\right) \\
& \operatorname{ppum}_{\mathrm{kt}}=\operatorname{pum}_{\mathrm{t}} \cdot\left(1-\mathrm{p}_{\mathrm{itpum}}\right)
\end{aligned}
$$

Von diesen sind jeweils alle nicht markenfixierten Käufer (1-mf $f_{t \mid p a m, k}$ bzw.1$\mathrm{mf}_{\mathrm{t} \mid p u m, \mathrm{k}}$ ) grundsätzlich dazu bereit, Empfehlungen des Händlers für einen anderen Reifen anzunehmen. Voraussetzung ist, daß es sich bei der vorgeschlagenen Alternative um eine Marke handelt, die Akzeptanz beim Reifenkäufer besitzt. 
Der Akzeptanzgrad der Marke i bei Endabnehmern mit Präferenz für die Konkurrenzmarke sei ausgedrückt durch $a_{i t \mid p a m, k}$ bzw. $a_{i t \mid p u m, k}$.

Ein großer Teil der Reifenkäufer ind $\mathrm{t}_{\mathrm{t}}$ betritt die Einkaufsstätte, ohne eine bestimmte Reifenmarke zu verlangen. Da durchaus angenommen werden kann, daß sich eine bestehende Präferenz für eine konkrete Marke auch in einer entsprechenden Nachfrage beim Händler artikulieren würde, ist hier davon auszugehen, daß diese Endabnehmer zunächst indifferent sind. Sie werden in der Einkaufsstätte nach weiteren Informationen suchen, um schließlich eine Entscheidung treffen zu können. Aufgrund des besonderen Stellenwertes der persönlichen Beratung wird hierbei letztlich die Empfehlung des Händlers ausschlaggebend sein, soweit dieser eine für den Reifenkäufer akzeptable Marke vorschlägt. Der Akzeptanzgrad von Marke i zum Zeitpunkt $t$ bei indifferenten Reifenkäufern sei mit $a_{i t \mid i n d}$ bezeichnet.

Ebenso wie die Präferenz ist auch die Akzeptanz für eine Marke durch das Pull-Budget des Herstellers beeinflußbar. In der bereits bekannten Form ergeben sich folgende Zusammenhänge:

$$
\begin{aligned}
& a p_{\text {itpam }, k}=f_{1}\left(a p_{\text {it-1pam, }, k}, p u_{\text {in }}\right) \\
& a p_{\text {itpum }, k}=f_{2}\left(a p_{\text {it-1|pum, }, k}, p u_{\text {in }}\right) \\
& a i_{\text {it ind }}=f_{3}\left(a i_{i t-1 \mid \text { ind }}, p u_{\text {in }}\right)
\end{aligned}
$$

Aus der bislang vorgenommenen Systematisierung des Verhaltens von Reifenkäufern lassen sich nunmehr fünf für die weiteren Teilmodelle relevante Käufergruppen ableiten, deren Anteile an der Gesamtkäuferschaft sich zum Zeitpunkt t wie folgt berechnen (vgl. Abbildung 37):

- Anteil markenfixierter Käufer $\mathrm{mf}_{\mathrm{it}}$ mit Präferenz für Marke i: $\mathrm{mf}_{\mathrm{it}}=\mathrm{ppam}_{\mathrm{it}} \cdot \mathrm{mf}_{\mathrm{upam}, \mathrm{i}}+\mathrm{ppum}_{\mathrm{it}} \cdot \mathrm{mf}_{\mathrm{upum}, \mathrm{i}}$

- Anteil markenorientierter Käufer mo it mit Präferenz für Marke i: $\mathrm{mo}_{\mathrm{it}}=\mathrm{ppam}_{\mathrm{it}} \cdot \mathrm{mo}_{\mathrm{tpam}, \mathrm{i}}+\mathrm{ppum}_{\mathrm{it}} \cdot \mathrm{mo}_{\mathrm{tpum}, \mathrm{i}}$

- Anteil markenbewußter Käufer $m b_{i t}$ mit Präferenz für Marke i: $m b_{i t}=$ ppam $_{i t} \cdot m b_{t p a m, i}+$ ppum $_{i t} \cdot \mathrm{mb}_{\mathrm{tppum}, \mathrm{i}}$ 


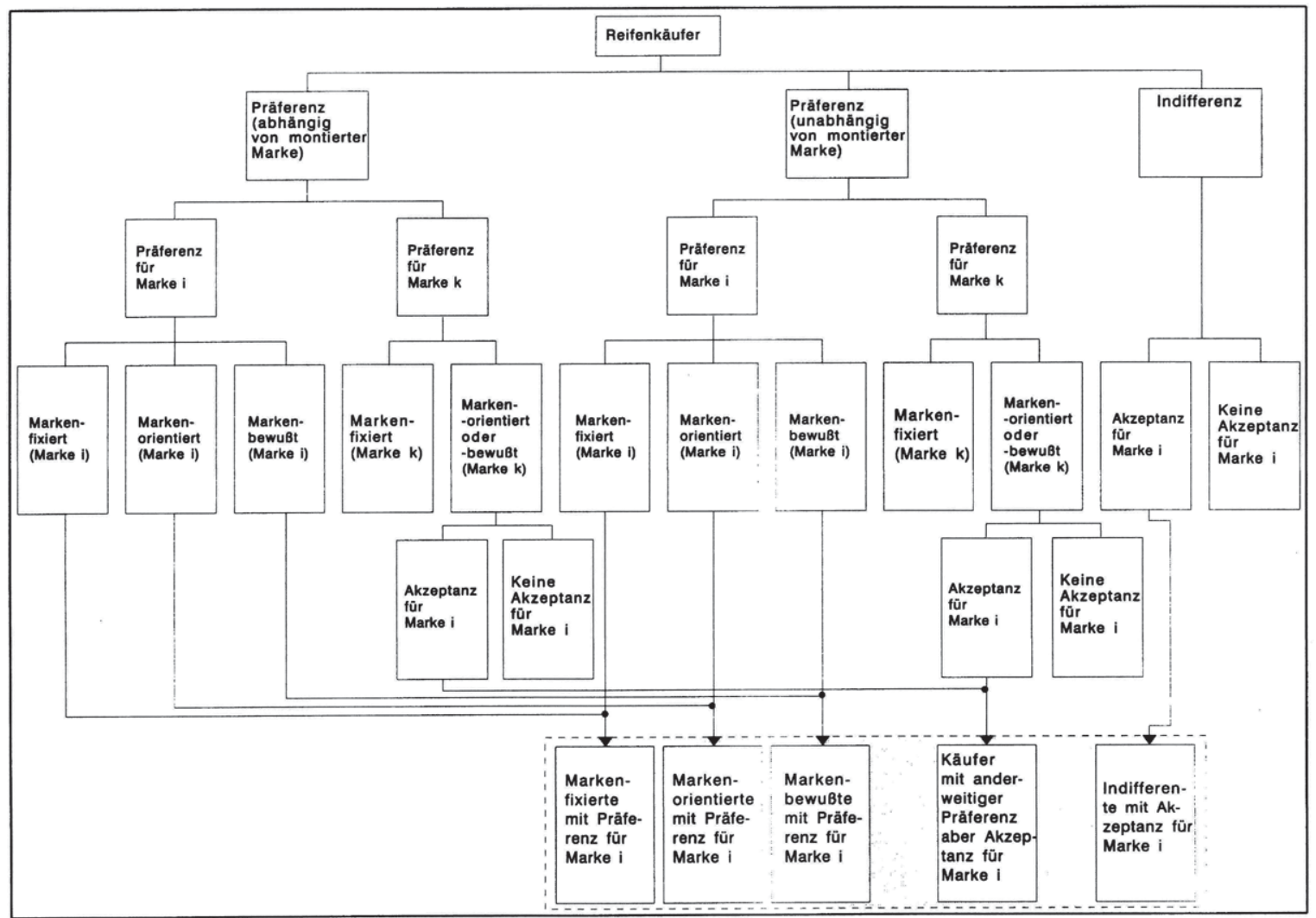


- Anteil der Käufer $a p_{i t}$, die zwar die Konkurrenzmarke präferieren, eine Empfehlung des Händlers für Marke i aber akzeptieren würden:

$\mathrm{ap}_{\mathrm{it}}=\operatorname{ppam}_{\mathrm{kt}} \cdot\left(1-\mathrm{mf}_{\mathrm{tpam}, \mathrm{k}}\right) \cdot \mathrm{ap}_{\mathrm{itpam}, \mathrm{k}}+\mathrm{ppum}_{\mathrm{kt}} \cdot\left(1-\mathrm{mf}_{\mathrm{t} \text { pum, }, \mathrm{k}}\right) \cdot \mathrm{ap}_{\mathrm{itpum}, \mathrm{k}}$

- Anteil der indifferenten Käufer $\mathrm{ai}_{\mathrm{it}}$, die eine Empfehlung des Händlers für Marke i akzeptieren würden:

$a i_{i t}=$ ind $_{t} \cdot a i_{\text {ittind }}$

\subsection{Absatzmittlermodell}

Im Verhaltensmodell der Absatzmittler sind der Distributionsgrad und die POS-Stärke der Marke in Abhängigkeit von den Push-Budgets des Herstellers zu ermitteln.

Der Distributionsgrad für Marke i zum Zeitpunkt $t$ wird als Funktion des effektiv wirksamen Akquisitionsbudgets in der entsprechenden Periode $n$ abgebildet:

$d g_{i t}=f\left(p a_{\text {eff,in }}\right)$

Je größer die Anzahl der Reifenkäufer, die die Marke i präferieren oder zumindest als Alternative akzeptieren, um so geringer sind die zu deren Sortimentsaufnahme erforderlichen Ausgaben pro Händler. Die Effizienz des Budgets steigt, d. h. bei gleichem Budget wird ein höherer Distributionsgrad erreicht. Um diesen Zusammenhang im Modell berücksichtigen zu können, werden wiederum Effizienzindizes $w_{a p, i n} b z w . w_{a a, i n}$ gebildet. Diese sind funktional abhängig von Präferenz und Akzeptanz der Marke: ${ }^{325}$

$$
\begin{aligned}
& w_{a p, i n}=\frac{f_{1}\left(m f_{i t-1}+m o_{i t-1}+m b_{i t-1}\right)+f_{1}\left(m f_{i t}+m o_{i t}+m b_{i t}\right)}{2} \\
& w_{a a, i n}=\frac{f_{2}\left(a p_{i t-1}+a i_{i t-1}\right)+f_{2}\left(a p_{i t}+a i_{i t}\right)}{2}
\end{aligned}
$$

325 Vereinfachend wird dabei unterstellt, daß $w_{a p, i}$ und $w_{a a, i}$ unabhängig voneinander sind. 
Damit verändert sich das effektiv wirksame Akquisitionsbudget und ist nunmehr definiert als: ${ }^{326}$

$p a_{\text {eff in }}=p a_{\text {in }} \cdot f_{1}\left(w_{\text {ap,in }}\right) \cdot f_{2}\left(w_{a a, i n}\right) \cdot f_{3}\left(w_{\text {ah,in }}\right)$

Die Förderung einer Marke im Reifenmarkt kommt vornehmlich durch das Empfehlungsverhalten des Handels zum Ausdruck. Mit Blick auf die Marke i sind dabei drei Situationen zu unterscheiden, die sich aus dem Nachfrageverhalten der Endabnehmer ergeben. Betritt der Reifenkäufer die Einkaufsstätte bereits mit Präferenz für Marke i, so verhält sich der Händler im günstigen Fall neutral oder bestätigt diesen sogar in seiner Kaufabsicht. Im ungünstigen Fall kommt es dagegen zu einer Gegenempfehlung, d. h. der Händler rät dem Endabnehmer zu einem Konkurrenzprodukt. Diese beiden Möglichkeiten werden in der Variable $e_{i j i}$ zusammengefaßt. Diese beschreibt die Wahrscheinlichkeit, daß der Händler keine Gegenempfehlung ausspricht, wenn der Reifenkäufer die Marke i präferiert.

Fragt der Endabnehmer dagegen die Konkurrenzmarke $\mathrm{k}$ nach, kehren sich günstiges und ungünstiges Verhalten des Händlers gerade ins Gegenteil. Um trotz der bekundeten Präferenz des Reifenkäufers einen Kauf der Marke i zu erreichen, muß der Händler diese aktiv anbieten und mit Nachdruck empfehlen. Die Wahrscheinlichkeit hierzu sei durch $e_{i \mid k}$ ausgedrückt. Bei indifferenten Reifenkäufern tritt schließlich die Situation ein, daß der Händler auf jeden Fall eine Empfehlung abgibt und sei es indirekt durch die Auswahl der im Verkaufsgespräch angebotenen Marken. Die Wahrscheinlichkeit, daß es sich hierbei um die Marke i handelt, wird durch $\mathrm{e}_{i \text { ind }}$ wiedergegeben.

Die drei Situationen unterscheiden sich in dem Engagement, das der Händler jeweils im Verkaufsgespräch für Marke i aufbringen muß. Dies reicht von der Unterlassung einer Gegenempfehlung über die vorteilhafte Darstellung der Marke bei indifferenten Reifenkäufern bis hin zur Überzeugung von Endabnehmern mit ursprünglich anderer Präferenz. Entsprechend unterschiedlich wird das Ausmaß der Leistungen sein, die der Hersteller im Rah-

326 Die Variable $w_{\text {ah,in }}$ wurde bereits an anderer Stelle als Effizienzindex der potentiellen Handelsspanne definiert. Vgl. Kap. C. 3.11 dieser Arbeit. 
men der Push-Budgets zu erbringen hat, um den Händler zu einem vorteilhaften Empfehlungsverhalten zu bewegen. Aus diesem Grunde wird für jede Situation eine eigene funktionale Beziehung zwischen dem für diese Zwecke eingesetzten effektiv wirksamen Förderungsbudget und den korrespondierenden Verhaltenswahrscheinlichkeiten definiert:

$$
\begin{aligned}
& e_{\text {itti }}=f_{1}\left(p f_{\text {eff,in }}\right) \\
& e_{\text {itk }}=f_{2}\left(p f_{\text {eff,in }}\right) \\
& e_{\text {ittind }}=f_{3}\left(p f_{\text {eff,in }}\right)
\end{aligned}
$$

Dabei gilt auch hier, daß sich das effektiv wirksame Budget aus dem monetären Förderungsbudget und der effizienzbeeinflussenden Wirkung von Präferenz und Akzeptanz der Endabnehmer gegenüber der Marke ergibt. Die entsprechenden Effizienzindizes des Förderungsbudgets $\left(w_{\mathrm{fp}, \text { in }}\right.$ bzw. $\mathrm{w}_{\mathrm{fa}, \text { in }}$ ) sind damit wie folgt $\mathrm{zu}$ beschreiben: 327

$$
\begin{aligned}
& w_{f p, i n}=\frac{f_{1}\left(m f_{i t-1}+m o_{i t-1}+m b_{i t-1}\right)+f_{1}\left(m f_{i t}+m o_{i t}+m b_{i t}\right)}{2} \\
& w_{f a, i n}=\frac{f_{2}\left(a p_{i t-1}+a i_{i t-1}\right)+f_{2}\left(a p_{i t}+a i_{i t}\right)}{2}
\end{aligned}
$$

Ferner geht der bereits zuvor definierte Effizienzindex $w_{f h}$, in in die Berechnung des effektiven Förderungsbudgets ein. Dieser beeinflußt die Effizienz des Budgets in Abhängigkeit von der potentiellen Handelsspanne.

Darüber hinaus gilt es zu beachten, daß die Wirkung des Förderungsbudgets abhängig ist vom Distributionsgrad. Wie bereits erläutert, führt ein über die Erhöhung des Akquisitionsbudgets zunehmender Distributionsgrad dazu, daß sich die zur Verfügung stehenden finanziellen Förderungsmittel auf eine größere Zahl von Händlern verteilen und somit nicht mehr die ursprünglich geplante Wirkung erzielen. Die Wirkungsabschätzung des Förderungsbudgets muß daher stets auf Grundlage eines Bezugswertes des Akquisitionsbudgets erfolgen. Veränderungen des Akquisitionsbudgets führen im glei-

327 Der auf der Handelsspanne beruhende Effizienzindex $w_{\mathrm{fh}}$, in wurde bereits zuvor definiert. Vgl. Kap. C. 2.22 dieser Arbeit. 
chen Verhältnis, wie sie den Distributionsgrad beeinflussen, zu einer Veränderung des effektiv wirksamen Förderungsbudgets. Damit gilt:

$$
p f_{\text {eff, },}=p f_{\text {in }} \cdot f_{1}\left(w_{\text {ap,it }}\right) \cdot f_{2}\left(w_{\text {aa,it }}\right) \cdot f_{3}\left(w_{\text {an,it }}\right) \cdot\left(\frac{d g_{\text {e,it }}}{d g_{\text {it }}}\right)
$$

Mit dge, it wird der Distributionsgrad bezeichnet, der sich bei einer bestimmten, als Bezugswert gewählten Höhe des Akquisitionsbudgets einstellen würde. Dieser wird ins Verhältnis gesetzt zu dem Distributionsgrad $\mathrm{dg}_{\mathrm{it}}$, der sich aufgrund der tatsächlichen Höhe des Akquisitionsbudgets ergibt.

\subsection{Marktanteilsmodell}

Führt man die Ergebnisse des Endabnehmer- und Absatzmittlermodells in einem Marktanteilsmodell zusammen, so ist dort das Zusammenwirken von Käufer- und Händlerverhalten abzubilden und zu einem Marktanteilswert zu verdichten. Dazu ist zunächst die effektive Verfügbarkeit der Marke zu ermitteln, die sowohl durch das Präferenzverhalten der Reifenkäufer als auch den Distributionsgrad bestimmt wird. Die effektive Verfügbarkeit einer Reifenmarke beschreibt die Wahrscheinlichkeit, mit der ein Reifenkäufer diese Marke in der von ihm gewählten Einkaufsstätte vorfindet. Markenfixierte Endabnehmer sind dadurch gekennzeichnet, daß sie sich bei der Wahl der Einkaufsstätte nach der von ihnen präferierten Marke richten. Gleiches gilt, wenn auch in geringerem Ausmaß, für markenorientierte Käufer. Die effektive Verfügbarkeit der präferierten Marke $v_{\text {ittmf,i }} b z w$. $v_{\text {ittmo,i }}$ ist somit bei beiden Gruppen größer als der Distributionsgrad dg $g_{i t}$. Hier gilt:

$v_{\text {ittmf,i }}=f_{1}\left(d g_{i t}\right)$ bzw. $\quad v_{i t \mid m o, i}=f_{2}\left(d g_{i t}\right)$

Demgegenüber entspricht bei markenbewußten Käufern die effektive Verfügbarkeit $v_{i t \mid m b, i}$ gerade dem Distributionsgrad. Das gleiche gilt für Endabnehmer mit Präferenz für eine andere Marke $\left(v_{\text {it|ap,i }}\right)$ und indifferente Reifenkäufer $\left(v_{i t \mid a i, i}\right)$ :

$v_{\text {ittmb,i }}=v_{\text {ittap,i }}=v_{\text {ittai,i }}=d g_{\text {it }}$ 
Bezüglich des Distributionsmaßes ist bei markenfixierten und -orientierten Reifenkäufern strenggenommen der numerische Distributionsgrad zu wählen. Da Endabnehmer gezielt nach der betreffenden Marke suchen, ist unter der Annahme einer räumlich gleichmäßigen Verteilung der Einkaufsstätten allein deren Anzahl von Bedeutung. Für alle weiteren Käufergruppen ist der gewichtete Distributionsgrad zugrunde zu legen. Die Differenzierung zwischen den beiden Distributionsmaßen ist dabei um so bedeutender, je mehr der Absatz schwerpunktmäßig über umsatzstarke oder -schwache Einkaufsstätten erfolgt. Hier sei vereinfachend angenommen, daß die Struktur des für Marke i gewählten Distributionssystems bezüglich der Umsatzbedeutung der Einkaufsstätten mit deren Grundgesamtheit übereinstimmt. In dieser Situation entsprechen sich die beiden Distributionsmaße, so daß auf eine Unterscheidung verzichtet werden kann.

Neben der Verfügbarkeit ist die Frage zu klären, wie Reifenkäufer sich gegenüber Empfehlungen des Händlers verhalten. Bei markenfixierten Endabnehmern besteht hierüber kein Zweifel. Soweit die Marke verfügbar ist, wird die bestehende Präferenz auf jeden Fall in einen Kauf umgesetzt. Demgegenüber soll für markenorientierte und -bewußte Endabnehmer unterstellt werden, daß diese sich einer Gegenempfehlung des Händlers für eine akzeptable Alternative anschließen. Eine solche Annahme scheint durchaus realistisch, wenn ergänzend gefordert wird, daß nur ernsthafte Verkaufsbemühungen des Händlers als Empfehlung bezeichnet werden. Zudem ist davon auszugehen, daß sich aufgrund der Austauschbarkeit der Marken jeweils mindestens eine als akzeptabel betrachtete Alternative im Sortiment findet. Gegenempfehlungen bei ursprünglicher Präferenz für Marke i führen damit aus Herstellersicht mit Bestimmtheit zum Verlust des Endabnehmers. Des weiteren wird auch für indifferente Käufer unterstellt, daß die Händlerempfehlung angenommen wird, sofern es sich um eine akzeptierte Marke handelt. Damit läßt sich der Marktanteil $m_{i}$ der Marke i wie folgt berechnen: 


\begin{tabular}{|c|c|c|c|c|}
\hline $\begin{array}{l}\text { Kăufer mit Práferenz oder } \\
\text { Akzeptanz fur Marke } i\end{array}$ & $\begin{array}{l}\text { Anteil an } \\
\text { Gesamt- } \\
\text { käuferschaft }\end{array}$ & $\begin{array}{l}\text { Effektive } \\
\text { Verfügbar- } \\
\text { keit der } \\
\text { Marke i }\end{array}$ & $\begin{array}{l}\text { Empfehlung } \\
\text { für Marke i } \\
\text { falls vorhan- } \\
\text { den }\end{array}$ & $\begin{array}{l}\text { Wahrscheinlichkeit für } \\
\text { den Kauf von Marke i }\end{array}$ \\
\hline Markenfixiert (Marke i) & $\mathrm{mf}_{\mathrm{it}}$ & $v_{i t \mid m f, i}$ & - & (1) $\mathrm{mf}_{\mathrm{it}} \mathrm{v}_{\mathrm{it} \mid \mathrm{mf}, \mathrm{i}}$ \\
\hline Markenorientiert (Marke i) & $\mathrm{mo}_{\text {it }}$ & $\mathrm{v}_{\mathrm{it} \mid \mathrm{mo}, \mathrm{i}}$ & $e_{i t \mid i}$ & (2) $\mathrm{mo}_{\text {it }} \cdot \mathrm{v}_{\mathrm{it} \mid \mathrm{mo}, \mathrm{i}} \mathrm{e}_{\mathrm{it} \mid \mathrm{i}}$ \\
\hline Markenbewußt (Marke i) & $m b_{\text {it }}$ & $d_{i t}$ & $e_{i t \mid i}$ & (3) $m b_{\text {it }} \cdot v_{i t \mid m b, i} \cdot e_{i t \mid j}$ \\
\hline Bewußt/Orientiert (Marke k) & $a p_{\text {it }}$ & $d_{i t}$ & $e_{i t \mid k}$ & (4) $a p_{i t} \cdot d_{i t} \cdot e_{i t \mid k}$ \\
\hline Indifferent & $a a_{i t}$ & $d_{i t}$ & $\mathrm{e}_{\text {itlind }}$ & $(5) a p_{i t} \cdot d_{i t} \cdot e_{i t \mid i n d}$ \\
\hline Marktanteil $\mathbf{m}_{\text {it }}$ & & & & $(1)+(2)+(3)+(4)+(5)$ \\
\hline
\end{tabular}

Abb. 38: Berechnung des Marktanteils

\subsection{Reifenausstattungsmodell}

Im Teilmodell zur Reifenausstattung sind die Anteile der zum Kaufzeitpunkt t am Fahrzeug montierten Marken festzustellen. Dabei sind periodenübergreifende Zusammenhänge zu berücksichtigen, die in der Literatur häufig wie folgt abgebildet werden:

$x(t)=\alpha \cdot x(t-1)$

Mit $x(t)$ wird in diesem speziellen Fall der Marktanteil einer bestimmten Marke beschrieben. Dieser Beziehung liegt in aller Regel die Annahme zugrunde, daß ein Teil der Käufer, die diese Marke in $t-1$ kaufen, sich auch in $t$ für dieselbe Marke entscheiden wird. Damit wird jedoch gleichermaßen eine homogene Käuferschaft unterstellt, bei der die Zeitdistanz zwischen zwei Käufen für alle Personen genau t-(t-1) beträgt.

Diese Annahme ist etwas weniger streng, wenn t nicht als Zeitpunkt, sondern als Periode interpretiert wird. In diesem Fall ist die Forderung ausreichend, daß die Personen mindestens einen Kauf pro Periode tätigen. Diese Annahme kann mit zunehmender Periodenlänge in steigendem Maße ein- 
gehalten werden. Wird das Intervall der Periode allerdings zu groß gewählt, lassen sich gerade die im Mittelpunkt des Interesses stehenden KaufWiederkaufbeziehungen bzw. Markenwechselwahrscheinlichkeiten nicht mehr analysieren. ${ }^{328}$

Derartige Prämissen scheinen durchaus für Produkte akzeptabel, bei denen es in vergleichsweise kurzen Abständen zu Ersatzkäufen kommt und deren Endabnehmer ein zumindest ähnliches Verbrauchsverhalten zeigen. Klassischerweise handelt es sich dabei um Güter des täglichen Bedarfs. Für den Reifenmarkt sind solche Annahmen hingegen aus zwei Gründen nicht zulässig. Zum einen beträgt der Zeitabstand zwischen zwei Reifenkäufen in aller Regel mehrere Jahre, so daß selbst eine Periodenlänge von 12 Monaten nicht ausreicht, um das Wiederkaufverhalten von einer zur nächsten Periode zu erfassen. Zum anderen ist die Käuferschaft im Hinblick auf ihre Fahrleistung sehr heterogen, wodurch sich stark unterschiedliche Reifennutzungsdauern und daraus resultierende Wiederbeschaffungszeiträume ergeben.

Daher erfolgt in dem hier zu entwickelnden Teilmodell die Ermittlung der zum Kaufzeitpunkt $t$ am Fahrzeug befindlichen Marke wie folgt: Zunächst sei mit der Zufallsvariable fl die jährliche Fahrleistung der Reifenkäufer (in $\mathrm{km}$ ) ausgedrückt und mit $\mathrm{rl}$ die technisch bedingte Reifenlebensdauer (in $\mathrm{km}$ ). Dann berechnet sich der Zeitpunkt $t_{w}$ des letzten Reifenwechsels aus:

$t_{w}=t-\frac{f l}{r l}$

Der Zeitpunkt liege innerhalb der ganzzahligen Periodengrenzen $t_{u}$ und $t_{0}: 329$

$t_{u} \leq t_{w} \leq t_{0}$

328 Besonders deutlich kommen diese Annahmen bei Markenwahlmodellen, wie $z$. B. sogenannten Markoffketten und linearen Lernmodellen, zum Ausdruck. Bei genauerer Betrachtung zeigt sich jedoch, daß es sich um eine grundlegende Problematik handelt, von der nahezu alle periodenübergreifenden Modelle des skizzierten Typs betroffen sind. Vgl. auch Meffert, H., Steffenhagen, H., Marketing-Prognosemodelle, a.a.O., S. $103 \mathrm{ff}$.

329 Dabei gilt $t_{0}=t_{u}+1$. 
Unterstellt man einen über die Periode kontinuierlichen Verlauf der Marktanteilsentwicklung, so ergibt sich die Wahrscheinlichkeit $m_{i t_{w}}$ daß bei dem Reifenwechsel zum Zeitpunkt $t_{w}$ die Marke $i$ auf das Fahrzeug montiert wurde, wie folgt (vgl. Abbildung 42):

$$
\begin{aligned}
& m_{i t_{w}}=m_{i t_{u}}+\frac{t_{w}-t_{u}}{t_{o}-t_{u}} \cdot\left(m_{i t_{o}}-m_{i t_{u}}\right) \\
& m_{\text {itu }_{u}}=\frac{n f_{t_{u o}}}{g f_{t_{u o}}} \cdot m_{e, i_{u}}+\left(1-\frac{n f_{t_{u o}}}{g f_{t_{u o}}}\right) \cdot m_{n, i t_{u}}
\end{aligned}
$$

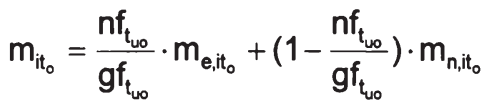

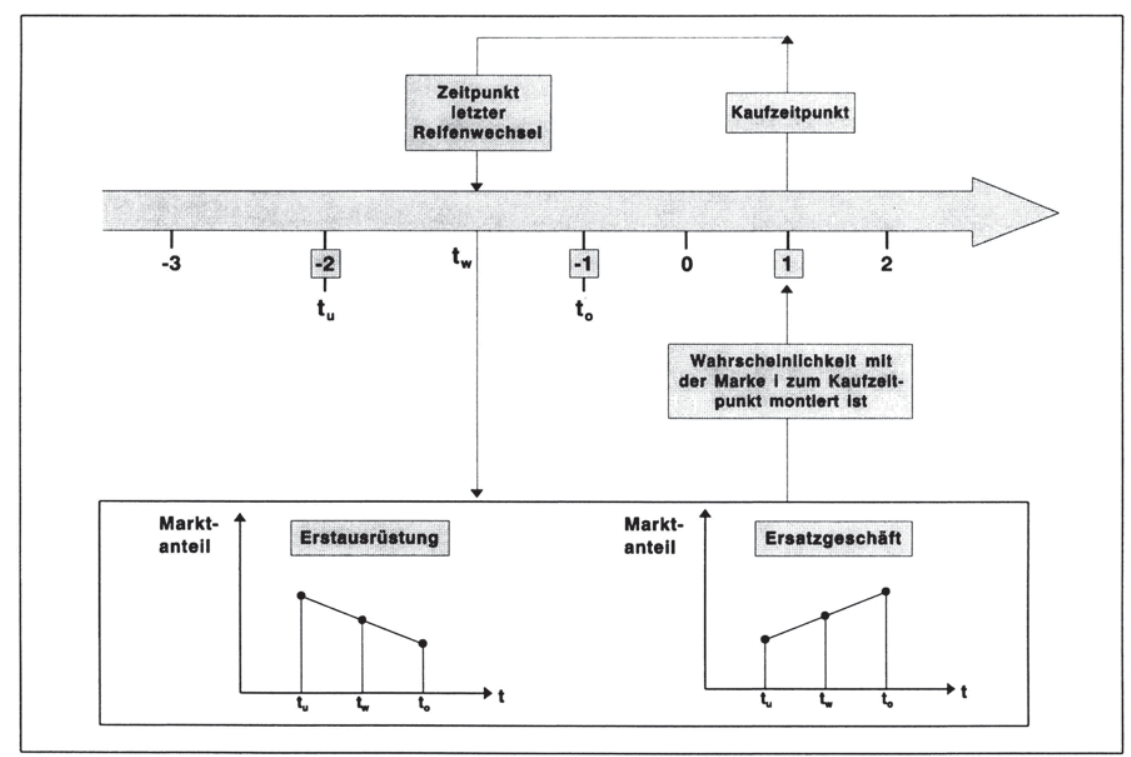

Abb. 39: Ermittlung der zum Kaufzeitpunkt montierten Reifenmarke

Die Variablen $m_{i t_{u}}$ und $m_{i t_{0}}$ werden aus den Marktanteilen im Ersatzgeschäft $\left(m_{e, i t}\right)$ und der Ausrüstung von Neufahrzeugen $\left(m_{n, i t}\right)$ berechnet, wie sie jeweils zu den durch die Periodengrenzen beschriebenen Zeitpunkten bestanden. Da sich die Gesamtvolumina des Ersatz- und Erstausrüstungsmarktes voneinander unterscheiden, sind die jeweiligen Marktanteile ent- 
sprechend zu gewichten. Hierzu wird die Anzahl der Neufahrzeuge $\mathrm{nf}_{\mathrm{t}_{\mathrm{uo}}}$ in Periode $\mathrm{t}_{\mathrm{uo}}{ }^{330}$ in das Verhältnis $\mathrm{zu}$ dem Gesamtfahrzeugbestand dieser Periode $\mathrm{gf}_{\mathrm{t}}$ gesetzt.

\subsection{Marktvolumensmodell}

Mit Hilfe dieses Teilmodells ist das Marktvolumen der einzelnen Perioden zu ermitteln, um im Zusammenhang mit den entsprechenden Marktanteilen den Umsatz der Marke bestimmen zu können.

Das Volumen eines Marktes kann grundsätzlich sowohl in Umsatz- als auch in Absatzgrößen ausgedrückt werden. ${ }^{331}$ Von diesen beiden Möglichkeiten ist hier die Beschreibung durch die Absatzmenge zu wählen, da sich der berechnete Marktanteil ebenfalls auf die Mengengröße bezieht. ${ }^{332}$ Unter dem Marktvolumen $\mathrm{mv}_{\mathrm{n}}$ wird somit im folgenden die Gesamtzahl der auf dem Markt innerhalb der Periode $\mathrm{n}$ abgesetzten Reifen verstanden. Wird mit $\mathrm{mv}_{1}$ das Marktvolumen in der ersten Periode des Planungszeitraums und mit $\Delta m v_{n}$ dessen prozentuale Veränderung in Periode $n$ bezeichnet, so ergibt sich:

$$
m v_{n}=m v_{1} \cdot \prod_{l=2}^{n}\left(1+\Delta m v_{1}\right)
$$

Aus Gründen der Modellvereinfachung wird auf eine eigenständige Prognose der Marktveränderungen verzichtet. Sollte eine solche gewünscht werden, bieten sich mit der Trend- und Indikatorprognose zwei grundle-

330 Mit $t_{u o}$ wird die Periode bezeichnet, deren Grenzen durch die Zeitpunkte $t_{u}$ und $t_{0}$ bestimmt sind.

Vgl. Meffert, H., Marketing, a.a.O., S. $216 \mathrm{f}$.

332 Bei der Marktanteilsberechnung wird zwar zunächst nicht die Kaufmenge ermittelt, sondern lediglich die Wahrscheinlichkeit, mit der eine bestimmte Marke in der Kaufsituation gewählt wird. Unter der Annahme, daß die Anzahl der beim Kauf erworbenen Reifen unabhängig von deren Marke ist, entspricht die berechnete Kaufwahrscheinlichkeit jedoch gerade auch deren Anteil an der Absatzmenge. 
gende Möglichkeiten der Erweiterung an. ${ }^{333}$ Bei der Trendprognose erfolgt eine Verknüpfung des Marktvolumens mit der Zeit, wobei der aus Vergangenheitswerten ermittelte Entwicklungstrend für die Zukunft fortgeschrieben wird.

Die Indikatorprognose geht demgegenüber davon aus, daß wesentliche Einflußfaktoren existieren, anhand derer die weitere Entwicklung der zu prognostizierenden Größe bestimmt werden kann. Ein solcher Ansatz bietet sich besonders für den Reifenmarkt an, da dort die Einflußfaktoren relativ gut identifizierbar sind und ein enger Zusammenhang zum Marktvolumen unterstellt werden kann. So ist es offensichtlich, daß für den Bedarf an Reifen der PKW-Bestand, 334 die Fahrleistung der Endabnehmer pro Jahr sowie die Reifenlebensdauer entscheidend sind.

\subsection{Gewinnmodell}

Zielsetzung dieses Teilmodells ist die Berechnung der anfallenden Kosten und erzielten Erlöse sowie der daraus resultierenden Gewinne. Hierzu sind zunächst mit $k_{h o}$ die stückbezogenen Herstellungskosten für den Startzeitpunkt des Planungszeitraums anzugeben. Die weitere Kostenentwicklung wird jeweils durch deren prozentuale Veränderung $\Delta \mathrm{k}_{\mathrm{hn}}$ pro Periode beschrieben.

333 Zu Trend- und Indikatorprognose vgl. Lo, L., Prognoseinformationen für kundendienstpolitische Entscheidungen - dargestellt an Beispielen des Fotomarktes, Diss., Münster 1979, S. 112 ff. u. S. 130 ff.; Meffert, H., Steffenhagen, H., MarketingPrognosemodelle, a.a.O., S. $63 \mathrm{ff}$ u. S. $78 \mathrm{ff}$.; Hüttner, M., Markt und Absatzprognose, Stuttgart u. a. 1982, S. 105 ff.; Lewandowsky, R., Mittelfristige Prognoseund Marketingsysteme, Bd. 2, Berlin 1980, S. $73 \mathrm{ff}$.

334 Informationen über die Entwicklung des PKW-Bestands sowie der Fahrleistung lassen sich beispielsweise der jährlich erstellten Prognose der Deutschen Shell AG zum Fahrzeugmarkt entnehmen. Vgl. Deutsche Shell Aktiengesellschaft (Hrsg.): Motorisierung in Deutschland, a.a.O., S. $1 \mathrm{ff}$. Eine ausführliche Diskussion der Absatzprognose im Automobilmarkt findet sich bei Kellner, M., Absatzprognose im Automobilmarkt, München 1987. 
Damit lassen sich die Herstellungskosten $k_{h t}$ zu den verschiedenen Planungszeitpunkten wie folgt berechnen: 335

$k_{h t}=k_{h o} \cdot \prod_{n=1}^{t}\left(1+\Delta k_{n n}\right)$

Häufig findet sich in Modellen zur Ermittlung zukünftiger Herstellungskosten das sog. Erfahrungskurvenkonzept ${ }^{336}$ wieder. Dessen grundlegende Annahme besteht darin, daß mit jeder Verdoppelung der im Zeitablauf kumulierten Produktionsmenge die inflationsbereinigten Stückkosten um einen bestimmten Prozentsatz (Lernrate) sinken. ${ }^{337}$ Neben der grundsätzlichen Kritik, der sich die Erfahrungskurve ausgesetzt sieht,, ${ }^{338}$ sprechen vor allem zwei Argumente gegen die Einbeziehung dieses Konzeptes in das hier zu entwickelnde Modell. Die beiden Argumente gehen dabei von gegensätzlichen Thesen aus.

Wie bereits erläutert, können sich hinter einer Marke verschiedene Produkte, Produktvarianten und auch -technologien verbergen. Unterstellt man einerseits, daß mit der Einführung jedes neuen Produktes bzw. jeder neuen Variante oder Technologie eine neue Erfahrungskurve beginnt, so würde deren Berücksichtigung in dem Modell eine wesentlich differenziertere Betrachtung erfordern, als sie auf der Markenebene möglich ist. Andererseits könnte man davon ausgehen, daß sich die Erfahrungskurve aufgrund der starken Ähnlichkeit zwischen den verschiedenen Produkten, Varianten und Technologien kontinuierlich fortsetzt, so daß die Betrachtungsebene der

$335 \mathrm{Zu}$ Herstellungskosten vgl. Schierenbeck, H., Grundzüge der Betriebswirtschaftslehre, a.a.O., S. $517 \mathrm{ff}$.

336 Vgl. zum Erfahrungskurvenkonzept ausführlich Henderson, B. D., Die Erfahrungskurve in der Unternehmensstrategie, Frankfurt, New York 1974.

337 Das Erfahrungskurvenkonzept läßt sich formal abbilden durch $k_{t}=k_{0} \cdot\left(x_{t} / x_{0}\right)^{\delta}$. Dabei entspricht $k$ den Stückkosten zum Zeitpunkt 0 bzw. $t$ und $x$ der bis zu diesen Zeitpunkten kumulierten Produktionsmenge. Mit $\delta$ wird die Stückkostenelastizität bezeichnet, die sich wie folgt aus der Lernrate $\alpha$ berechnet: $\delta=\ln (1-\alpha) / \ln 2$. Vgl. Simon, H., Preismanagement, a.a.O., S. 75.

338 Vgl. hierzu insbesondere die kritische Diskussion bei Kreilkamp, E., Strategisches Management und Marketing, a.a.O., S. $349 \mathrm{ff}$. 
Marke durchaus adäquat wäre. In diesem Fall ist allerdings auf die sehr hohe kumulierte Ausbringungsmenge hinzuweisen, die in der Vergangenheit bereits produziert wurde. Während Unternehmen in jungen Märkten relativ schnell eine Verdoppelung der Ausbringungsmenge erreichen, so daß der beschriebene Zusammenhang erhebliche Auswirkungen auf die Kosten nehmen kann, trifft dies bei traditionsreichen Reifenherstellern in einem weitgehend stagnierenden Markt - selbst bei einem Planungszeitraum von mehreren Jahren - wohl nur sehr bedingt zu.

Wenngleich das Erfahrungskurvenkonzept für den hier vorliegenden Fall somit ungeeignet erscheint, gehen dennoch unbestreitbar Effekte von einer Steigerung der Ausbringungsmenge auf die Kosten aus. In einer Erweiterung des Modells könnte diese beispielsweise für die variablen Kosten in der Form

$k_{h t}=k_{h t-1} \cdot \Delta k_{h n} \cdot f\left(x_{n}-x_{n-1}\right)$

Eingang finden. Mit der Funktion $f$ wird der im Zeitpunkt $t$ gegenüber dem Zeitpunkt t-1 erreichte Kostensenkungseffekt ausgedrückt, der auf einer Erhöhung der Absatzmenge ${ }^{339} \mathrm{x}$ in der $\mathrm{t}$ vorangehenden Periode $\mathrm{n}$ gegenüber $n-1$ beruht. 340 Die Variable $\Delta k_{h n}$ beschreibt in diesem Fall die Veränderungsrate, die sich ohne Absatzmengenerhöhung einstellen würde.

Als weitere Kostengröße sind die Budgets für Push- und Pull-Aktivitäten zu berücksichtigen. Die Gesamtkosten $k_{b, n}$ berechnen sich aus der Summe des jeweils auf die entsprechende Budgetbasis bezogenen Akquisitions-, Förderungs- und Pull-Budgets:

$$
k_{b, n}=p a_{b b, n} \cdot p a_{n}+p f_{b b, n} \cdot p f_{n}+p u_{b b, n} \cdot p u_{n}
$$

339 Die Absatzmenge sei dabei gleich der produzierten Ausbringungsmenge.

$340 \mathrm{Zu}$ unterschiedlichen Funktionszusammenhängen zwischen Kosten und Ausbringungsmenge vgl. Kistner, K.-P., Produktions- und Kostentheorie, Würzburg, Wien 1981, S. $109 \mathrm{ff}$. 
Die Ermittlung der stückbezogenen Erlöse erfolgt auf Basis des zum Zeitpunkt $\mathrm{t}$ gültigen Endabnehmerpreises $\mathrm{ep}_{\mathrm{it}}$ sowie der potentiellen Handelsspanne $h_{i t}{ }^{341}$ Veränderungen des Endabnehmerpreises während des Planungszeitraums, die z. B. auf einer Erhöhung des Marktpreisniveaus beruhen, werden wiederum in folgender Form abgebildet:

$\mathrm{ep}_{\mathrm{it}}=\mathrm{ep}_{\mathrm{i0}} \cdot \prod_{\tau=1}^{\mathrm{t}}\left(1+\Delta \mathrm{ep}_{\mathrm{i \tau}}\right)$

Mit $\Delta e p_{i t}$ sei die prozentuale Veränderung gegenüber dem Endabnehmerpreis zum Zeitpunkt $\mathrm{t}-1$ bezeichnet und mit $\mathrm{ep}_{\mathrm{i} 0}$ der Endabnehmerpreis zum Beginn des Planungszeitraums.

Der Periodengewinn $g_{\text {in }}$ für die Marke ergibt sich schließlich aus den Marktanteilen, Endabnehmerpreisen, Kosten und Handelsspannen zu Beginn und zum Ende der Periode sowie den Push- und Pull-Budgets.

$$
g_{\text {in }}=m v_{\text {in }} \cdot \frac{m_{i t-1} \cdot e p_{i t-1} \cdot\left(1-h_{i t-1}\right)+m_{i t} \cdot e p_{i t} \cdot\left(1-h_{i t}\right)}{2}-k_{b, n}
$$

Unter der Annahme, daß Erlöse und Kosten jeweils mit ihrem Auftreten zahlungswirksam werden, läßt sich der Markenwert als Kapitalwert der Periodengewinne ermitteln. 342 Vielfach wird dabei unterstellt, daß die entsprechenden Ein- und Auszahlungen jeweils zu Beginn oder Ende der Periode anfallen. Bei einer Periodenlänge von einem Jahr scheint eine solche Annahme jedoch unrealistisch.

341 Hierbei ist die Annahme zu treffen, daß die potentielle Handelsspanne vom Handel auch realisiert wird.

342 Vgl. zur Kapitalwertmethode Kruschwitz, L., Investitionsrechnung, 3. Aufl., Berlin, New York 1987, S. 66 ff. Zur Problematik der richtigen Wahl des Kalkulationszinsfußes siehe darüber hinaus Grob, H.-L., Periodenspezifische Mischzinsfüße als theoretisch richtige Kalkulationszinsfüße, in: ZfB, H. 3, 1982, S. $381 \mathrm{ff}$. 
Hier sei angenommen, daß der Zahlungsstrom sich gleichmäßig über die Periode verteilt. Für diesen Fall ergibt sich der Markenwert $c_{i}$ beim Kalkulationszinsfuß $z$ aus:

$c_{i}=\sum_{n=1}^{N} \int_{t-1}^{t} p g_{n} \cdot e^{-\delta \tau} d \tau=\sum_{n=1}^{N} \operatorname{pg}_{n} \cdot \frac{1}{\delta} \cdot\left(e^{-\delta \cdot(t-1)}-e^{-\delta \cdot t}\right)$

mit $\delta=\ln (1+z)$

\subsection{Eingangsvariablen und Erklärungsgleichungen}

Nachdem die Variablen des Modells definiert sowie dessen grundlegende Strukturen aufgezeigt sind, ist im folgenden auf die Bestimmung der Variablenwerte sowie die Konkretisierung der Erklärungsgleichungen einzugehen.

\subsection{Ermittlung von Werten der Eingangsvariablen}

Für alle Variablen, die nicht innerhalb des Modells berechnet werden, sind vom Entscheidungsträger Werte vorzugeben. Eine der zentralen Anforderungen an das hier zu entwickelnde Modell besteht darin, die Unsicherheit bezüglich der Werte dieser Eingangsvariablen explizit zu berücksichtigen. An die Stelle deterministischer Variablen mit als sicher angenommenen Werten treten daher stochastische Variablen, deren Werte einer Wahrscheinlichkeitsverteilung unterliegen. ${ }^{342}$

In diesem Zusammenhang sind zwei verschiedene Ansätze des Wahrscheinlichkeitsbegriffes zu unterscheiden. Nach dem subjektivistischen Verständnis ist die Wahrscheinlichkeit des Eintritts eines bestimmten Ereignisses als "Überzeugungsgrad" der die Eintrittsmöglichkeit beurteilenden Person zu interpretieren. Demgegenüber definiert der objektivistische

342 Vgl. Köhler, R., Uebele, H., Risikoanalysen bei der Evaluierung absatzorientierter Projekte, in: WiSt, H. 3, 1983, S. 122; Bamberg, G., Baur, F., Statistik, 3. Aufl., München 1984, S. $93 \mathrm{ff}$. 
Ansatz die Wahrscheinlichkeit als Grenzwert einer relativen Häufigkeit bei einer unendlichen Anzahl von Fällen. ${ }^{343}$ Da die gesamte Entwicklung des Modells vor dem Hintergrund des benutzerorientiert-subjektiven Ansatzes der Modellbildung erfolgt, ist hier konsequenterweise auch ein subjektivistischer Wahrscheinlichkeitsbegriff zugrunde zu legen. Dies schließt jedoch keinesfalls aus, daß in Fällen objektiv ermittelter Wahrscheinlichkeiten diese vom Entscheidungsträger als Informationsgrundlage für die eigene Schätzung genutzt werden.

Für die folgenden Ausführungen ist es ferner von Bedeutung, zwischen zwei Interpretationen der Wahrscheinlichkeitsverteilung zu differenzieren. Im Sinne der relativen Häufigkeit beschreibt eine Verteilung die Anzahl der Realisation unterschiedlicher Ausprägungen der Zufallsvariablen. ${ }^{344}$ Dies trifft beispielsweise für die jährliche Fahrleistung der Reifenkäufer zu, deren Häufigkeitsverteilung im Rahmen einer Befragung erhoben wurde (vgl. Abbildung 40). ${ }^{345}$

Die Fahrleistungen der einzelnen Personen in der Stichprobe und bei Repräsentationsschluß346 auch in der Grundgesamtheit sind tatsächlich unterschiedlich. Demgegenüber kann z. B. der Anteil an Personen mit Präferenz für eine bestimmte Marke zu einem Zeitpunkt nur einen Wert annehmen. Wird der Entscheidungsträger in diesem Zusammenhang nach seiner Einschätzung bezüglich der Eintrittswahrscheinlichkeit unterschiedlicher Werte befragt, entspricht dies nicht dem Sinn einer Häufigkeitsverteilung,

343 Vgl. Gottwald, R., Entscheidung unter Unsicherheit, Informationsdefizite und unklare Präferenzen, Wiesbaden 1990, S. 68; Eisenführ, F., Weber, M., Rationales Entscheiden, Berlin u. a., S. $148 \mathrm{ff}$.

344 Zum Begriff der Wahrscheinlichkeitsverteilung vgl. Bleymüller, J., Gehlert, G., Gülicher, H., Statistik für Wirtschaftswissenschaftler, München 1979, S. 39.

345 Die Ergebnisse entstammen einer internen Studie eines Reifenherstellers ( $n=1005)$.

346 Beim Repräsentationsschluß wird von der Verteilung der Variablenwerte in einer Stichprobe auf deren Verteilung in der Grundgesamtheit geschlossen. Vgl. hierzu Hammann, P., Erichson, B., Marktforschung, Stuttgart, New York 1978, S. 31; Bleymüller, J., Gehlert, G., Gülicher, H., Statistik für Wirtschaftswissenschaftler, a.a.O., S. 85 . 
sondern läßt sich vielmehr als "Überzeugungs-" oder "Glaubwürdigkeitsverteilung"347 interpretieren. ${ }^{348}$

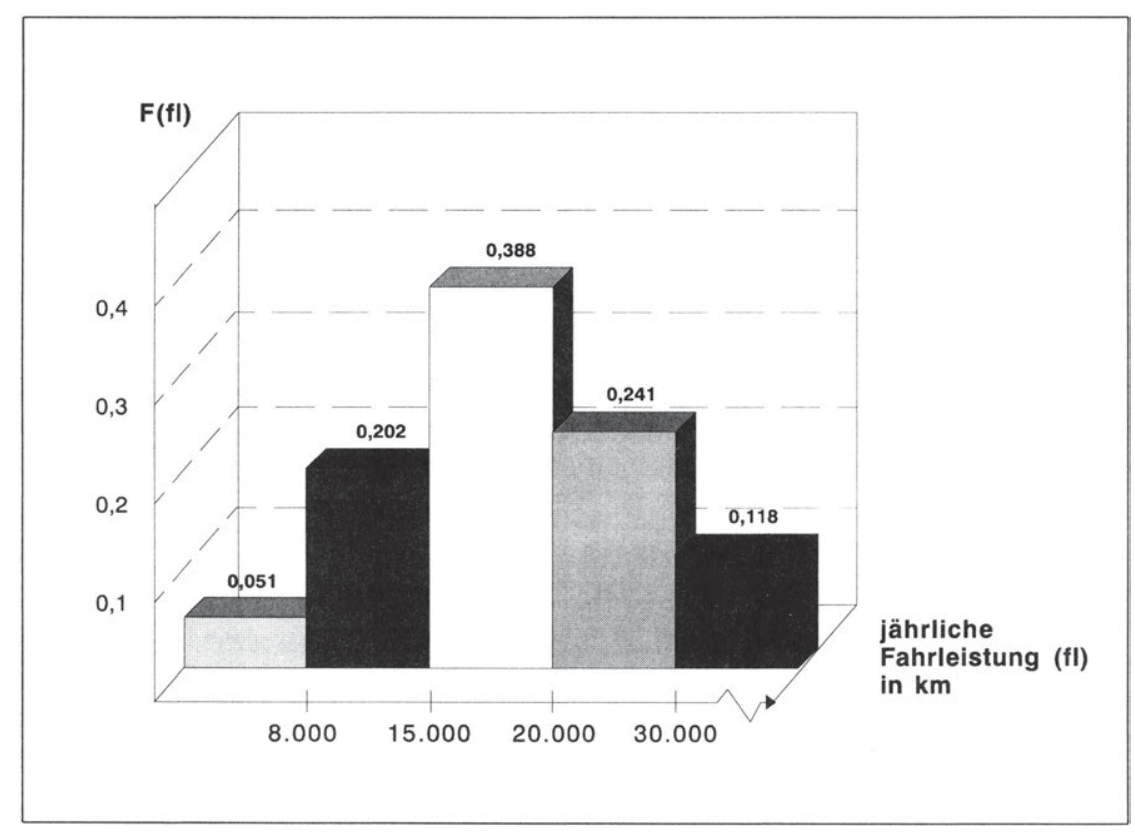

Abb. 40: Stichprobenverteilung der jährlichen Fahrleistung

Zur Bestimmung der Wahrscheinlichkeitsverteilung durch subjektive Schätzungen des Entscheidungsträgers ist festzulegen, ob ein "freier" Funktionsverlauf erhoben oder aber für die Funktionsform eine theoreti-

347 Vgl. auch Allen, D. H., Credibility Forecasts and their Application to the Economic Assessment of Novel Research and Developement Project, in: Operations Research Quarterly, No. 1, 1968, S. 25-42 sowie die Ausführungen von Schindel, V., Risikoanalyse, Darstellung und Bewertung von Risikorechnung am Beispiel von Investitionsentscheidungen, München 1977, S. 119 f.

348 Dem entspricht letztlich auch der Grundgedanke sog. Zugehörigkeitsfunktionen, die im Rahmen der Fuzzy-Theorie zur Abbildung unscharfer Zahlen verwendet werden. Zur Fuzzy-Theorie vgl. Dubois, D., Prade, H., Fuzzy sets and systems, Theory and applications, New York u. a. 1980; Zimmermann, H.-J., Fuzzy set theory and its applications, Boston u. a. 1985. 
sche Verteilung zugrunde gelegt werden soll. ${ }^{349}$ Ersteres Vorgehen führt zu dem Ergebnis, daß Punkt- oder Intervallschätzungen für die Eintrittswahrscheinlichkeiten verschiedener Ausprägungen vorliegen. So können z. B. mit der Fraktil-Methode die Wahrscheinlichkeiten durch Abfrage des Überbzw. Unterschreitens bestimmter Grenzen festgestellt werden. Dies kann entweder durch eine direkte ("Wie hoch ist die Wahrscheinlichkeit, daß mindestens $40 \%$ der Reifenkäufer eine bestimmte Präferenz besitzen?") oder indirekte Frage ("Ist es wahrscheinlicher, daß mindestens $40 \%$ der Reifenkäufer eine bestimmte Präferenz besitzen oder aber beim Münzwurf Zahl zu erhalten?)"350 geschehen. Ein wesentlicher Vorteil bei der Erhebung freier Funktionsverläufe ist darin zu sehen, daß der Befragte das Spektrum möglicher Werte sehr differenziert beurteilen kann. Daraus resultiert allerdings gleichermaßen der Nachteil, daß mit zunehmender Anzahl der abgefragten Funktionspunkte oder -intervalle der Aufwand für den Entscheidungsträger wächst. Die Bedeutung dieses Aspektes steigt insbesondere mit der im Modell enthaltenen Zahl stochastischer Variablen.

Legt man der Schätzung eine theoretische Verteilung zugrunde, reduziert sich der Umfang notwendiger Angaben. So sind z. B. bei der Normalverteilung nur Erwartungswert und Varianz zu erheben, um den Funktionsverlauf der Verteilung bestimmen zu können. Es besteht jedoch das Problem, daß sich die zu schätzenden Parameter häufig dem plastischen Vorstellungsvermögen entziehen. Selbst wenn deren inhaltliche Beschreibung dem Entscheidungsträger deutlich ist, gestaltet sich die Zuweisung eines konkreten Wertes, also z. B. der Varianzausprägung, als recht schwierig. ${ }^{351}$

349 Für eine Systematisierung und Diskussion von Methoden zur Erhebung subjektiver Wahrscheinlichkeiten vgl. Schütt, K.-P., Wahrscheinlichkeitsschätzungen im Computer-Dialog, Theorie, Methoden und eine experimentelle Studie zur Schätzung von subjektiven Wahrscheinlichkeiten, Stuttgart 1981, S. 92 ff. Vgl. auch Pfohl, H.-Ch., Messung subjektiver Wahrscheinlichkeiten, in: Materialien zur Betriebs- und Volkswirtschaft, Bd. 2, Wirtschaftliche Meßprobleme, Köln 1977, S. 23 ff.

350 Die zum Vergleich herangezogene Wahrscheinlichkeit wird jeweils solange variiert, bis der Entscheidungsträger indifferent zwischen den vorgeschlagenen Alternativen ist. Vgl. Schindel, V., Risikoanalyse, a.a.O., S. 120. 
Entsprechend den Gestaltungsanforderungen des benutzerorientiert-subjektiven Ansatzes der Modellbildung soll hier eine theoretische Verteilung gewählt werden, die zum einen die gedanklichen Vorstellungen des Entscheidungsträgers von der Realität möglichst gut repräsentiert und zum anderen anhand einfacher, verständlicher Angaben zu berechnen ist. ${ }^{352}$ Diesen Anforderungen scheint die Trapezverteilung gerecht zu werden (vgl. Abbildung 41). Es ist durchaus plausibel zu unterstellen, daß der Entscheidungsträger zwar nicht in der Lage ist, einen einzigen Wert als den wahrscheinlichsten zu identifizieren, wohl aber ein gewisses Intervall ange\left. ben kann ( $x{ } w_{\min } \ldots x e r w_{\max }\right)$, innerhalb dessen er den tatsächlichen Wert mit größter Wahrscheinlichkeit erwartet.

$f(x)$

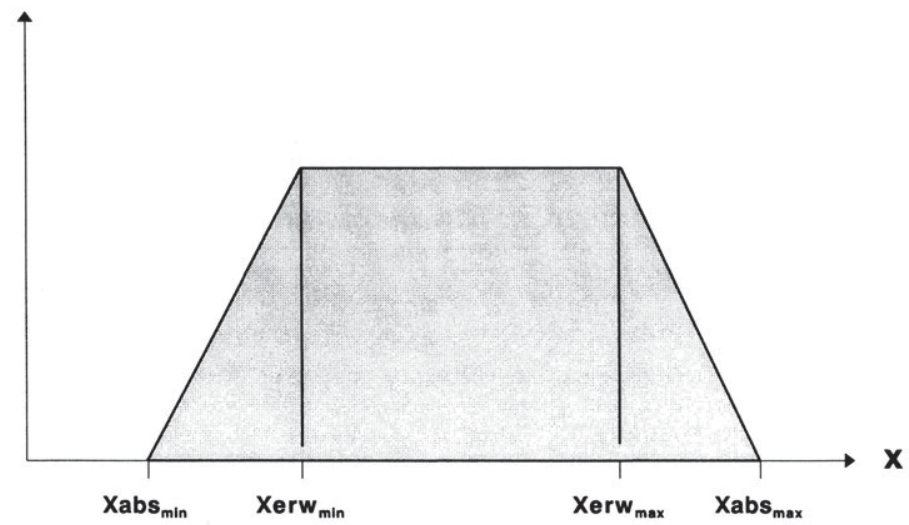

Abb. 41: Trapezverteilung der Modellvariablen

Darüber hinaus wird der Entscheidungsträger Vorstellungen über den absolut kleinsten $\left(x a b s_{\min }\right)$ bzw. größten ( $x_{a b s_{\max }}$ ) Wert besitzen, den die Variable

352 Ausführliche Beschreibungen der verschiedenen theoretischen Verteilungen finden sich in nahezu jedem statistischen Lehrbuch. Vgl. beispielsweise Stange, K., Angewandte Statistik, Zweiter Teil, Mehrdimensionale Probleme, Berlin u. a. 1971; Hartung, J., Elpelt, B., Klösener, K.-H., Lehr- und Handbuch der angewandten Statistik, 9. Aufl., München, Wien 1993. 
annehmen kann. ${ }^{353}$ Diese vier Angaben sind ausreichend, um die benötigte Verteilungsfunktion $F(x)$ der Wahrscheinlichkeiten zu berechnen. ${ }^{354}$ Mit Ausnahme der Variablen zur Beschreibung der jährlichen Fahrleistung wird diese Verteilung allen stochastischen Variablen des Modells zugrunde gelegt. 355

\subsection{Bestimmung von Parametern der Erklärungsgleichungen}

Das Modell enthält eine Reihe von Wirkungsbeziehungen zwischen den Variablen, deren genauer funktionaler Zusammenhang festzulegen ist. Auch hier kann wieder zwischen objektiven und subjektiven Ansätzen unterschieden werden. Bei ersteren handelt es sich um ökonometrische Wirkungsprognosen, die mit Hilfe mathematisch-statistischer Verfahren die Beziehungen zwischen den empirisch ermittelten Ausprägungen der Variablen analysieren. ${ }^{356}$ Wie bei der Ermittlung von Wahrscheinlichkeitsverteilungen sollen die Ergebnisse objektiver Ansätze als Informationsgrundlage verstanden werden. Die endgültige Schätzung der Wirkungsfunktion erfolgt auf Basis der subjektiven Angaben des Entscheidungsträgers.

353 Zu dieser Einschätzung vgl. auch Wolf, J., Lineare Fuzzy-Modelle zur Unterstützung der Investitionsentscheidung, Modellierung und Lösung von Investitionsproblemen mittels der Theorie unscharfer Mengen, Frankurt/Main u.a. 1988, S. 94 f. sowie die dort angegebene Literatur.

354 Zum Begriff der Verteilungsfunktion vgl. Bleymüller, J., Gehlert, G., Gülicher, H., Statistik für Wirtschaftswissenschaftler, a.a.O., S. 40 ff. Zur Funktion der Trapezverteilung vgl. die Darstellung im Anhang dieser Arbeit.

355 Das gilt auch für die Variable zur Beschreibung der Reifennutzungsdauer. Obwohl vermutet werden kann, daß mit einer Normalverteilung noch eine größere Abbildungsgenauigkeit erreichbar ist, wurde aus Gründen der Vereinfachung auf die Einführung einer weiteren Verteilungsform verzichtet.

356 Üblicherweise handelt es sich bei diesen Verfahren um Ansätze der Regressionsoder mitunter der Conjoint-Analyse. In neueren Forschungen wird zudem versucht, entsprechende Beziehungen durch den Einsatz sog. neuronaler Netze zu ermitteln. Vgl. Simon, H., Kucher, E., Die Bestimmung empirischer Preisabsatzfunktionen, Methoden, Befunde, Erfahrungen, in: ZfB, H. 2, S. 171 ff.; Gedenk, K., Skiera, B., Marketing-Planung auf der Basis von Reaktionsfunktionen, Funktionsschätzung und Optimierung, in: WiSt, H. 5, 1994, S. 258 ff.; Gaul, W., Decker, R., Neuronale Netze in der Kaufverhaltensforschung, in: asw, H. 7, 1994, S. $66 \mathrm{ff}$. 
Als der zentrale Ansatz zur Ermittlung subjektiv geschätzter Funktionen wird in der Literatur auch heute noch ein von Little bereits früh unterbreiteter Vorschlag genannt. ${ }^{357} \mathrm{Um}$ die Wirkung des Marketinginstrumenteeinsatzes auch ohne mathematisch-statistische Methoden bestimmen zu können, wurde von ihm das sogenannte ADBUDG-Modell eingeführt. Dessen Grundlagen werden im folgenden kurz dargelegt, um anschließend eine Weiterentwicklung vorzunehmen. 358

Das ADBUDG-Modell basiert auf zwei verschiedenen Teilfunktionen. Die Responsefunktion beschreibt zunächst den Zusammenhang zwischen dem Aktivitätsniveau eines Marketinginstrumentes ${ }^{359} x_{t}$ und dem hiermit erreichbaren Marktanteil $\mathrm{ma}_{\text {resp }, \mathrm{t}}$ innerhalb einer Periode. Der Funktionsverlauf wird durch die Angaben des Entscheidungsträgers zu vier Fragestellungen ermittelt:

1. Wie hoch muß das Aktivitätsniveau sein, um den derzeitigen Marktanteil halten zu können (Erhaltungsniveau eb)?

2. Welcher Marktanteil $\left(\mathrm{ma}_{\mathrm{min}}\right)$ wird am Ende der Planungsperiode voraussichtlich bei einem Aktivitätsniveau von 0 erreicht ?

3. Welcher Marktanteil $\left(\mathrm{ma}_{1,5 \cdot \mathrm{eb}}\right)$ wird am Ende der Planungsperiode voraussichtlich erreicht, wenn das Erhaltungsniveau um $50 \%$ erhöht wird?

4. Welcher Marktanteil $\left(\mathrm{ma}_{\max }\right)$ wird am Ende der Planungsperiode voraussichtlich erreicht, wenn das Aktivitätsniveau unbegrenzt ausgedehnt wird?

Das Modell geht dabei von einer konkaven oder s-förmigen Funktionsform aus. Ober- und Untergrenze der Funktionswerte ergeben sich aus den Fragen zum erzielbaren Marktanteil bei unbegrenztem Aktivitätsniveau und dem

357 Vgl. Little, J. D. C., Models and Managers, a.a.O., S. B-466 ff.; Barzen, D., Marketing-Budgetierung, a.a.O., S. 250 ff.; Lilien, G. L., Kotler, P., Moorthy, K. S., Marketing Models, a.a.O., S. $697 \mathrm{ff}$. sowie die dort angegebene Literatur.

$358 \mathrm{Zu}$ der folgenden Darstellung vgl. Little, J. D. C., Models and Managers, a.a.O., S. B-466 ff.

359 Little wählt als Marketinginstrument die Werbung und drückt dessen Aktivitätsniveau in Form des Werbebudgets aus. 
Marktanteilsminimum bei einem Aktivitätsniveau von 0 (vgl. Abbildung 42). Der Verlauf zwischen den Grenzen wird durch die Parameter $a$ und $b$ der Funktion bestimmt, die sich wie folgt berechnen: ${ }^{360}$

$$
m a_{r e s p, t}=m a_{\min }+\frac{\left(m a_{\max }-m a_{\min }\right) \cdot x_{t}^{a}}{b+x_{t}^{a}}
$$

mit

$$
\begin{aligned}
& a=\frac{1}{\ln (1,5)} \cdot \ln \left(\frac{m a_{\text {max }}-m a_{e b}}{m a_{e b}-m a_{\text {min }}} \cdot \frac{m a_{1,5 \cdot e b}-m a_{\text {min }}}{m a_{\text {max }}-m a_{1,5 \cdot e b}}\right) \\
& b=e b^{a} \cdot \frac{m a_{\text {max }}-m a_{e b}}{m a_{e b}-m a_{\text {min }}}
\end{aligned}
$$

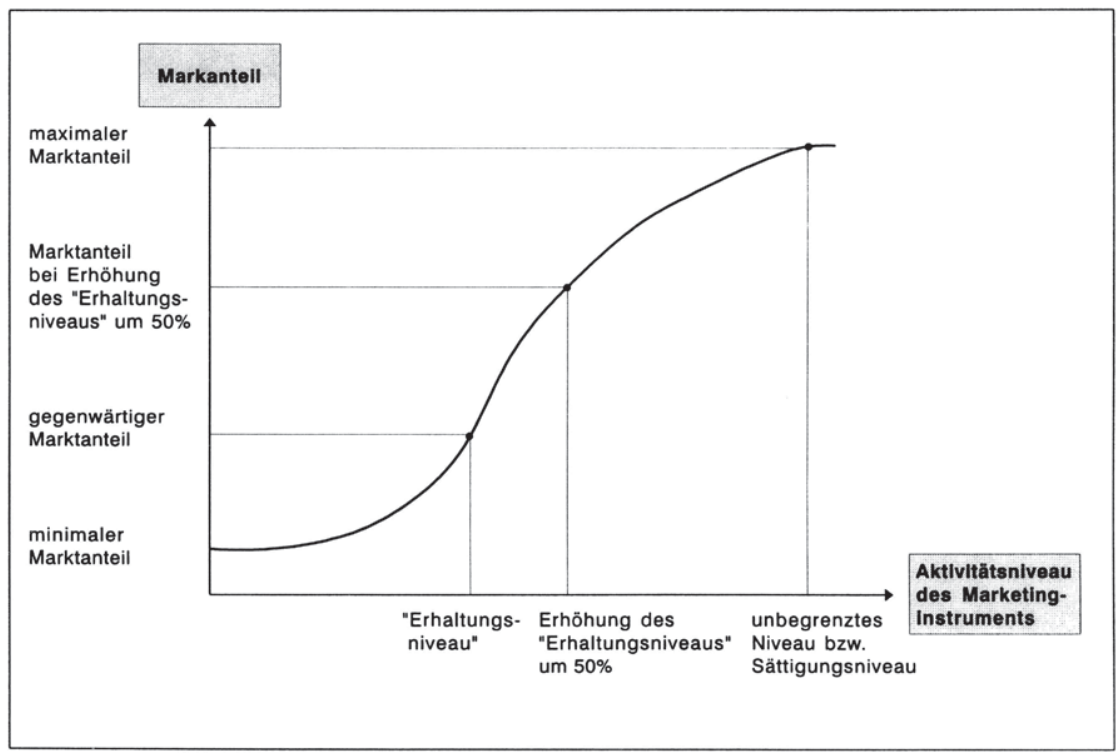

Abb. 42: Responsefunktion des ADBUDG-Modells

360 Ein s-förmiger Verlauf ergibt sich für a>1. Vgl. Lilien, G. L., Marketing Mix Analysis with Lotus 1-2-3, Redwood City 1986, S. 24. Zur Berechnung der Parameter vgl. Lilien, G. L., Kotler, P., Marketing Decision Making, a.a.O., S. 132. 
Die zweite Funktion des Modells ist die sogenannte Verfallskurve. Während mit Hilfe der Responsefunktion die Wirkung des Instrumenteeinsatzes innerhalb einer Periode abgebildet wird, verdeutlicht die Verfallskurve, daß die einmal erreichte Wirkung von einer zur nächsten Periode stetig abnimmt. Hierdurch wird das statische Modell zu einem einfachen dynamischen Ansatz erweitert. Dabei wird unterstellt, daß der Verfall mit gleichgroßen relativen Abnahmeraten erfolgt, die sich auf den Bereich zwischen dem aktuellen und dem sich langfristig einstellenden Marktanteilsminimum bei einem Aktivitätsniveau von 0 ( $\left.\mathrm{ma}_{\text {min,fif }}\right)$ beziehen (vgl. Abbildung 43).

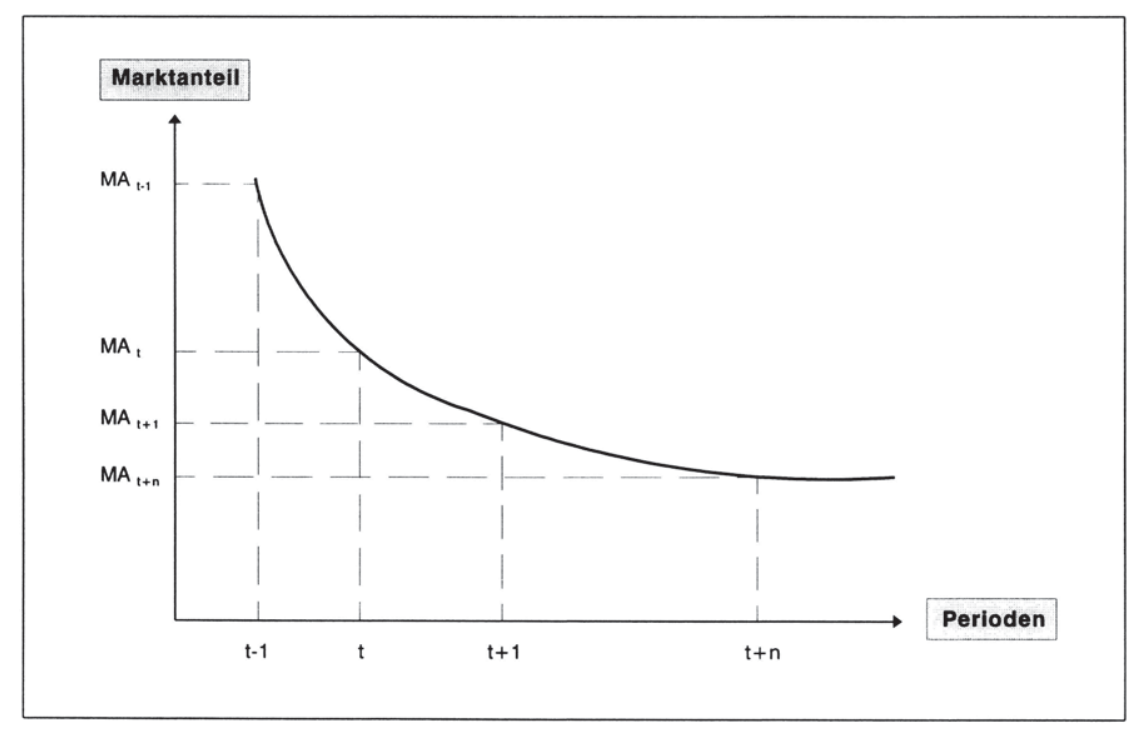

Abb. 43: Verfallsfunktion des ADBUDG-Modells

Letzteres ist zusätzlich $\mathrm{zu}$ den bisherigen Informationen vom Entscheidungsträger zu erfragen. Der grundsätzlich degressiv fallende Funktionsverlauf berechnet sich aus: ${ }^{361}$

361 Die Variable bv repräsentiert das "Beharrungsvermögen" des Marktanteils, d. h. den prozentualen Anteil, der auch ohne Werbung jeweils von einer zur nächsten Periode verbleibt. 
$m a_{\text {verf,t }}=m a_{\min , f f}+b v \cdot\left(m a_{t-1}-m a_{\min }\right)$

mit

bv $=\frac{m a_{\min }-m a_{\min , f f}}{m a_{e b}-m a_{\min , f f}}$

Führt man beide Teilfunktionen zusammen, ergibt sich der Marktanteil schließlich wie folgt:

$$
m a_{t}=\underbrace{m a_{\text {min,f }}+b v \cdot\left(m a_{t-1}-m a_{\text {min }}\right)}_{\text {Periodenminimum }\left(m a_{\text {min. } .1}\right)}+\underbrace{\frac{\left(m a_{\max }-m a_{\min }\right) \cdot x_{t}^{a}}{b+x_{t}^{a}}}_{\text {Wirkung des aktuellen Instrumenteeinsatzes }}
$$

Um diese Funktionen für die Entwicklung eines eigenen Modells nutzen zu können, sind zwei zentrale Erweiterungen notwendig:

1. Es muß die Möglichkeit geschaffen werden, auch solche Variablen als unabhängige Größen der Funktion zu verwenden, die in ihrem Wertebereich begrenzt sind.

2. Die Unsicherheit bezüglich der bestehenden Wirkungszusammenhänge muß explizit in der Beziehung berücksichtigt werden können.

Im Hinblick auf den Wertebereich der unabhängigen Variablen wurde bislang unterstellt, daß dieser unbegrenzt ist. So wird im ADBUDG-Modell die obere Grenze des Marktanteils (abhängige Variable) unter der Prämisse eines beliebig hohen Aktivitätsniveaus des Marketinginstrumentes (unabhängige Variable) festgelegt. Dieses Vorgehen ist jedoch nur möglich, wenn zumindest keine theoretischen Beschränkungen wie $z$. B. beim Werbebudget existieren. Wählt man als unabhängige Variablen dagegen relative Größen, wie z. B. den share of voice oder den Distributionsgrad, so ist deren gültiger Bereich auf die Werte zwischen 0 und 1 (bzw. $100 \%$ ) begrenzt. Spätestens für den Wert 1 muß die abhängige Variable ihren Maximalwert erreicht haben. Für diesen Fall wird eine veränderte Responsefunktion gewählt, die wie folgt formuliert ist: 
$m a^{\prime}$ resp.t $=m a_{\min }+\frac{\left(m a_{\max }-m a_{\min }\right) \cdot x_{t}^{\prime a}}{b+x_{t}^{\prime a}}$

mit

$x_{t}^{\prime}=\frac{x_{t}}{1-x_{t}}$

$a=\frac{1}{\ln (a b / e b)} \cdot \ln \left(\frac{m a_{\text {max }}-m a_{e b}}{m a_{e b}-m a_{\text {min }}} \cdot \frac{m a_{e b}-m a_{\text {min }}}{m a_{\text {max }}-m a_{e b}}\right)$

$\mathrm{b}=e b^{\mathrm{a}} \cdot \frac{\mathrm{ma} \mathrm{a}_{\max }-m a_{\mathrm{eb}}}{m a_{\mathrm{eb}}-m a_{\min }}$

Die erste Veränderung bezüglich des Wertebereiches gegenüber der Ausgangsfunktion besteht darin, daß die Responsefunktion nicht mehr unmittelbar auf die Variable $x_{t}$ angewendet wird, sondern diese zunächst eine Transformation erfährt. Hierbei strebt der transformierte Wert $x_{t}^{\prime}$ mit zunehmender Annäherung von $x_{t}$ an die Wertebereichsgrenze von 1 gegen unendlich. Damit wird funktionsintern gerade wieder der Fall einer nach oben unbeschränkten unabhängigen Variablen hergestellt.

Zur Modifizierung der Funktion ist in diesem Zusammenhang jedoch noch eine weitere Veränderung vorzunehmen, die sich auf die abgefragten Datenpunkte bezieht. So wurde zur Ermittlung der Funktion nach dem Wert des Marktanteils bei 50 \%iger Erhöhung des zur Aufrechterhaltung des Marktanteils notwendigen Aktivitätsniveaus des Marketinginstrumentes gefragt. Eine solche Angabe ist allerdings nur dann möglich, wenn durch die Erhöhung die Wertebereichsgrenze nicht überschritten wird. So läßt sich ein Distributionsgrad von $80 \%$ nicht nochmals um die Hälfte steigern. Aus diesem Grunde wird in der modifizierten Funktion keine feste Vorgabe für den Datenpunkt gegeben, sondern es kann innerhalb der Grenzen eb $<a b<1$ hierfür ein beliebiger Wert ab gewählt werden. Bei der Datenerhebung ändern sich somit die dritte und vierte Frage an den Entscheidungsträger im folgenden Sinne: 
Welcher Marktanteil wird am Ende der Planungsperiode erreicht, wenn

1. ...

2. ...

3. das Aktivitätsniveau auf den Wert ab ausgeweitet wird?

4. das Aktivitätsniveau sein Maximum annimmt?

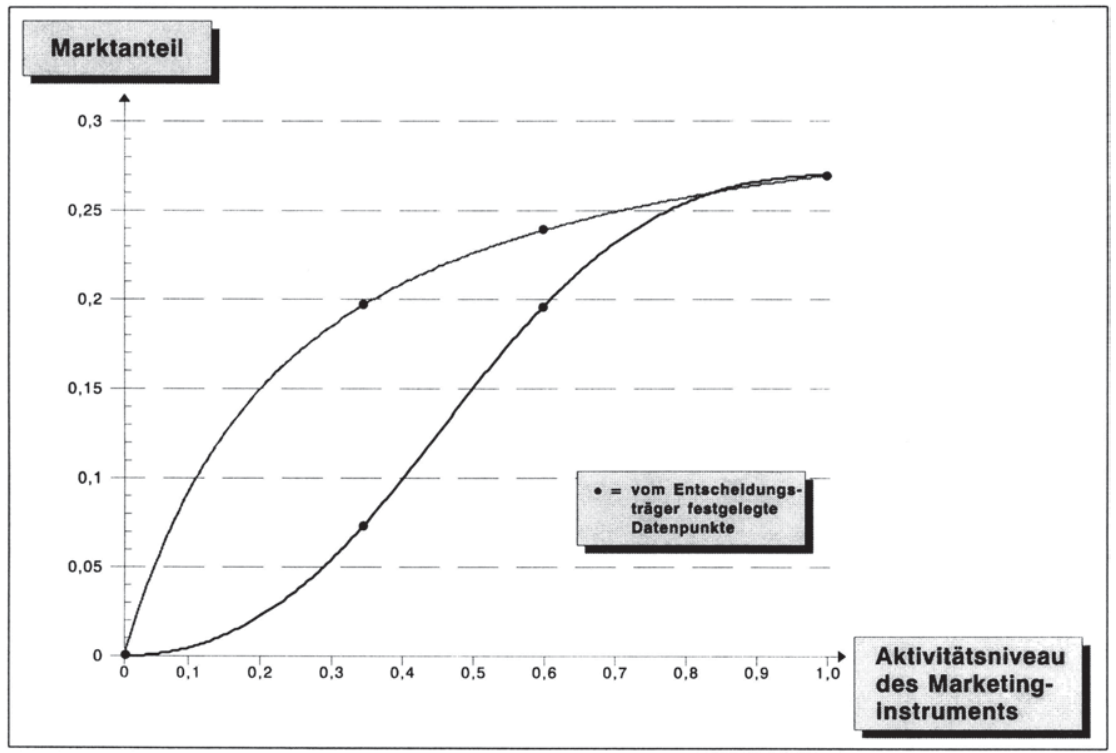

Abb. 44: Konkaver und s-förmiger Funktionsverlauf der modifizierten ADBUDG-Funktion

Wie Abbildung 44 zeigt, lassen sich mit der modifizierten Version der Funktion ebenfalls konkave und s-förmige Funktionsverläufe schätzen. Allerdings muß eingeräumt werden, daß die Funktion weniger robust gegenüber nicht geeigneten Angaben ist als die Ausgangsfunktion des ADBUDG-Modells. Während diese durch den nach oben nicht beschränkten Wertebereich der unabhängigen Variablen den "Freiraum" besitzt, um auf jeden Fall einen konkaven oder s-förmigen Verlauf zu konstruieren, "erzwingt" die modifizierte Funktion einen Verlauf mit vier genau definierten Punkten innerhalb eines bestimmten Bereiches. Es kann insbesondere dazu kommen, daß bei Annäherung an die Bereichsgrenze der unabhängigen 
Variablen ein exponentieller Anstieg stattfindet, um den oberen Wert der abhängigen Variablen noch zu erreichen. Dies bedeutet jedoch letztlich, daß die vier angegebenen Punkte nicht auf einer Funktion der gewünschten Form liegen können. Daher empfiehlt es sich, die geschätzte Funktion stets anhand ihrer graphischen Darstellung auf einen sinnvollen Verlauf zu überprüfen.

Als zweite wesentliche Änderung wurde die explizite Berücksichtigung von Unsicherheit in den Wirkungsbeziehungen genannt. Dem liegt die Annahme zugrunde, daß der Entscheidungsträger in aller Regel nicht in der Lage sein wird, die Wirkung eines bestimmten Wertes der unabhängigen Variablen auf das Ergebnis der abhängigen Variablen exakt angeben zu können. Dementsprechend sind dessen Angaben, z. B. bezüglich des Marktanteils bei einem bestimmten Aktivitätsniveau des Marketinginstrumentes, nur als Ausdruck der Beziehung zu interpretieren, der er die höchste Wahrscheinlichkeit zumißt. In dieser geschätzten Beziehung zeigt sich jedoch weder die Breite des Spektrums möglicher Marktanteilsergebnisse noch der Überzeugungsgrad des Entscheidungsträgers, mit dem er den Eintritt des entsprechenden Ergebnisses erwartet.

Daher sollen im folgenden statt deterministischer Funktionen stochastische Beziehungen Verwendung finden. Ebenso wie bei den Eingangswerten wird auch hier angenommen, daß der Entscheidungsträger durchaus eine Vorstellung darüber besitzt, in welchem Bereich sich das wahrscheinlichste Ergebnis befindet. Zudem wird unterstellt, daß er Unter- und Obergrenzen angeben kann, die in jedem Fall von dem Ergebnis eingehalten werden. Damit läßt sich wiederum die Trapezfunktion als Wahrscheinlichkeitsverteilung wählen. Diese repräsentiert nunmehr für jeden Wert der unabhängigen Variablen die entsprechenden Ausprägungen der abhängigen Variablen. Graphisch läßt sich der Zusammenhang in einer dreidimensionalen Abbildung darstellen (vgl. Abbildung 45).

Bei der deterministischen Responsefunktion war für die vier ausgewählten Aktivitätsniveaus jeweils genau ein Marktanteilsergebnis anzugeben. Nunmehr ist für jedes dieser vier Aktivitätsniveaus bzw. Budgets die angenommene Wahrscheinlichkeitsverteilung zu erheben. 
Dies führt zu folgendem Frageschema für subjektiv geschätzte stochastische Wirkungsbeziehungen:

"Angenommen es wäre ein Budget in Höhe von ... verfügbar. In welchem Bereich würde der erzielbare Marktanteil voraussichtlich am Ende der Periode liegen? Welcher Wert würde auf jeden Fall erreicht, und welcher wäre als absolute Obergrenze zu betrachten?"

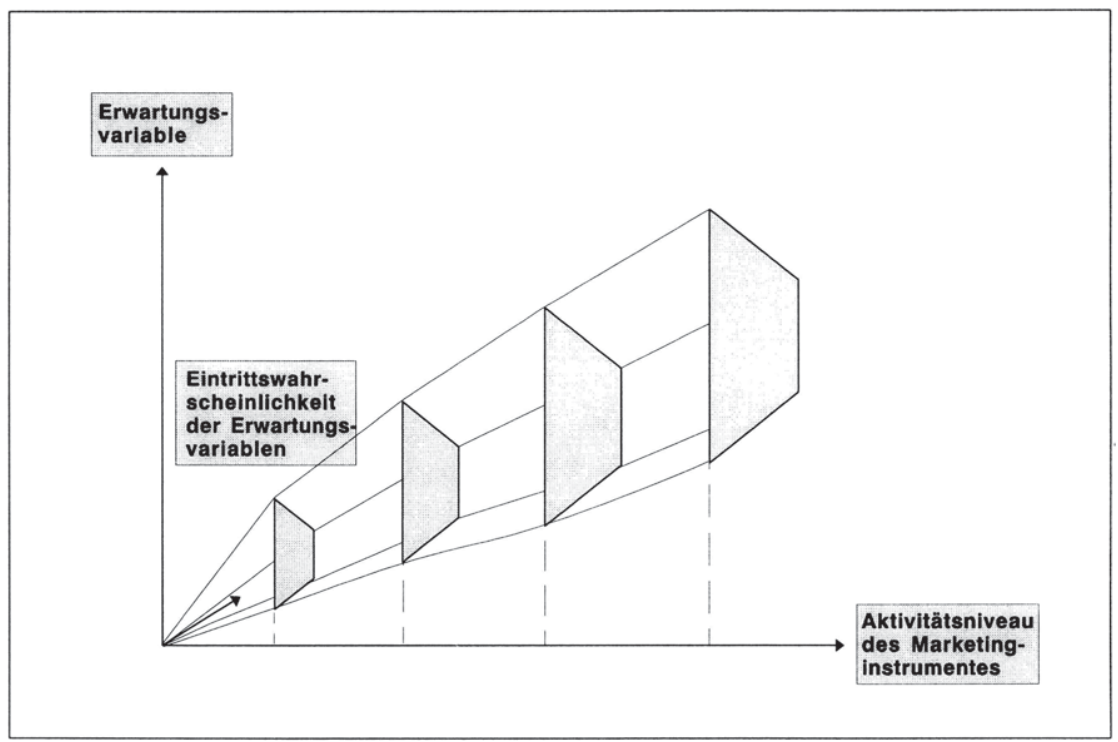

Abb. 45: Zusammenhang von Aktivitätsniveau des Marketinginstrumentes und der Erwartungsvariablen unter Berücksichtigung des unsicheren Wirkungszusammenhanges

Diese Formulierung ist unmittelbar auf die Fragestellungen 2-4 der Responsefunktion anwendbar. ${ }^{362}$ Problematisch ist dagegen die Übertragbarkeit auf die 1. Frage. Dort wurde nicht nach dem erreichbaren Marktanteil bei einem bestimmten Aktivitätsniveau gefragt, sondern gerade umgekehrt der Marktanteil vorgegeben. Ein solches Vorgehen ist mit Einführung der 
stochastischen Beziehung strenggenommen nicht mehr möglich, da sich die Unsicherheit dann gerade in verschiedenen Aktivitätsniveaus ausdrücken müßte. Die Frage ist folglich dahingehend zu modifizieren, daß der Entscheidungsträger ein Ausgangsniveau angeben soll, von dem er meint, daß der sich einstellende Marktanteil innerhalb eines Intervalls um den Marktanteilswert des vorangegangenen Zeitpunktes liegt.

An dieser Stelle sei zudem daran erinnert, daß die Wirkungsabschätzung des Förderungsbudgets stets auf der Grundlage eines Bezugspunktes des Akquisitionsbudgets erfolgen muß. ${ }^{363}$ Es bietet sich an, gerade dieses Ausgangsbudget als Bezugspunkt zu wählen und ihm darüber hinaus den (Index-)Wert 1 zu geben. Ferner gilt es zu beachten, daß die Art der Fragestellung für Beziehungen zu modifizieren ist, wie sie z. B. zwischen Distributionsgrad und effektiver Markenverfügbarkeit bestehen. Hier ist nicht die Wirkung eines bestimmten Aktivitätsniveaus bis zum Ende der Periode anzugeben, sondern ein zu jedem Zeitpunkt innerhalb der Periode gültiger Zusammenhang. Die ansonsten inhaltlich unveränderten Fragen sind dahingehend anzupassen, daß der Zeitraumbezug entfällt.

Sind die Angaben (absmin, erwmin, erwmax, absmax) zu den Wahrscheinlichkeitsverteilungen für die vier ausgewählten Aktivitätsniveaus $\left(b_{1}, . . b_{4}\right)^{364}$ ermittelt, stellt sich die Aufgabe, diese miteinander zu verbinden. Letztlich müssen daraus für jedes zulässige Aktivitätsniveau $x$ die erforderlichen Werte ( $x_{\text {absmin }}, x_{\text {erwmin }}, x_{\text {erwmax }}, x_{\text {absmax }}$ ) berechnet werden können, um daraus die jeweilige Verteilung abzuleiten. Ein zunächst gangbarer Weg scheint darin zu bestehen, jeweils die sich entsprechenden Werte der Wahrscheinlichkeitsverteilungen der vier Aktivitätsniveaus miteinander zu verknüpfen. Dies würde zu folgenden Funktionen führen:

Vgl. Kap. C. 3.13.

364 Im folgenden werden dabei stets folgende Aktivitätsniveaus der unabhängigen Variablen gewählt: $b w_{1}=0 ; \quad b w_{2}=A u s g a n g s w e r t$ der Planung; $b w_{3}=$ beliebig in den Grenzen $b_{2}<b w_{3}<b w_{4}$; $b w_{4}=1$ falls Variable eine relative Größe beschreibt, sonst $\mathrm{bw}_{4}=$ unbegrenztes Niveau. In der modifizierten Responsefunktion treten dementsprechend die Variablen $b_{w_{2}}$ und $b_{w_{3}}$ an die Stelle der Variablen eb und $a b$. 


$$
\begin{aligned}
& x_{a b s m i n}=f_{1}\left(x, b_{1, a b s m i n}, b_{2, a b s m i n}, b_{3, a b s m i n}, b w_{4, a b s m i n}\right) \\
& X_{\text {erwmin }}=f_{2}\left(x, b w_{1, e r w m i n}, b w_{2, e r w m i n}, b w_{3, e r w m i n}, b w_{4, e r w m i n}\right) \\
& X_{\text {erwmax }}=f_{3}\left(x, b w_{1, e r w m a x}, b_{2, e r w m a x}, b_{3, e r w m a x}, b w_{4, e r w m a x}\right) \\
& X_{\text {absmax }}=f_{4}\left(x, b w_{1, a b s m a x}, b_{2, a b s \max }, b_{3, a b s m a x}, b_{4, a b s m a x}\right)
\end{aligned}
$$

Es zeigt sich jedoch relativ schnell, daß ein solches Vorgehen nicht zu dem gewünschten Ergebnis führt. Jede der Funktionen stellt für sich eine eigenständige Responsefunktion dar, die unabhängig von den anderen berechnet wird. Aufgrund der daraus resultierenden, unterschiedlichen konkaven oder s-förmigen Funktionsverläufe kann es dazu kommen, daß sich die Graphen schneiden und damit für die Wahrscheinlichkeitsverteilungen keine sinnvollen Angaben mehr liefern.

Hier soll daher eine andere Vorgehensweise gewählt werden. Dazu ist zunächst die Responsefunktion zu berechnen, die auf den Erwartungs-

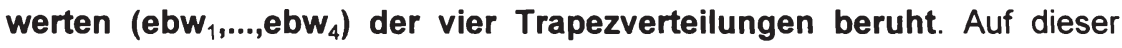
Funktion läßt sich dann für jedes Aktivitätsniveau $x$ dessen erwarteter Wert (ex) der gesuchten Verteilung ablesen. Den sich daran anschließenden Berechnungen liegt der Kerngedanke zugrunde, daß sich die Distanz zwischen diesem Erwartungswert und den gesuchten Angaben ( $x_{\text {absmin }}, x_{\text {enwmin }}$, $\left.x_{\text {erwmax }}, x_{\text {absmax }}\right)$ aus denjenigen Distanzen ergibt, die den beiden umliegenden Werten entsprechen. Exemplarisch von der Situation ausgehend, daß $b_{2}$ und $b w_{3}$ die für $x$ umliegenden Aktivitätsniveaus sind, $d$. $h$. es gilt

$\mathrm{bw}_{1}<\mathrm{bw}_{2}<\mathrm{x}<\mathrm{bw}_{3}<\mathrm{bw}_{4}$,

ergeben sich die zu ermittelnden Werte aus:

$$
\begin{aligned}
& X_{a b s \min }=g \cdot\left(e b w_{2}-b w_{2, a b s m i n}\right)+(1-g) \cdot\left(e b w_{3}-b w_{3, a b s m i n}\right) \\
& X_{\text {enwmin }}=g \cdot\left(e b w_{2}-b w_{2, e r w m i n}\right)+(1-g) \cdot\left(e b w_{3}-b w_{3, \text { erwmin }}\right) \\
& X_{e n w m a x}=g \cdot\left(e b w_{2}-b w_{2, e r w m a x}\right)+(1-g) \cdot\left(e b w_{3}-b w_{3, \text { erwmax }}\right) \\
& X_{a b s m a x}=g \cdot\left(e b w_{2}-b w_{2, a b s \max }\right)+(1-g) \cdot\left(e b w_{3}-b w_{3, a b s \max }\right)
\end{aligned}
$$


Der Parameter g stellt einen Gewichtungsfaktor dar, der die Stärke angibt, mit der die umliegenden Aktivitätsniveaus in die Berechnung eingehen (vgl. Abbildung 46):

$\mathrm{g}=\frac{\mathrm{ebw}_{3}-\mathrm{ex}}{\mathrm{ebw_{3 } - e b w _ { 2 }}}$

In der beschriebenen Weise läßt sich nunmehr vollständig die stochastische Beziehung zwischen einer abhängigen und einer unabhängigen Variablen bestimmen. Diese gilt bislang allerdings nur für die Responsefunktion. Sollen auch periodenübergreifende Wirkungen einbezogen werden, so ist zu beachten, daß sich von einer Periode zur nächsten das Periodenminimum des hier betrachteten Marktanteils ändert. Während die Form des Funktionsverlaufes gleich bleibt, kommt es dabei zu einer konstanten Niveauveränderung aller Funktionswerte. ${ }^{365}$ Diese ist jedoch erst zum Abschluß der Berechnung von $\mathrm{x}_{\mathrm{absmin}}, \ldots, \mathrm{x}_{\mathrm{absmax}} \mathrm{zu}$ berücksichtigen, da sich die ursprünglichen Angaben des Entscheidungsträgers auf das Minimum der aktuellen Periode beziehen. Die Werte von $\mathrm{x}_{\mathrm{absmin}}, \ldots, \mathrm{X}_{\mathrm{absmax}}$ sind somit wie folgt zu korrigieren:

$$
\begin{aligned}
& x_{a_{b s m i n}}=x_{a b s m i n}-m a_{\text {min }}+m a_{\text {min,t }} \\
& x_{\text {erwmin }}=x_{\text {erwmin }}-m a_{\text {min }}+m a_{\text {min, } t} \\
& x_{\text {erwmax }}=x_{\text {erwmax }}-m a_{\text {min }}+m a_{\text {min,t }} \\
& x_{\text {absmax }}=x_{\text {absmax }}-m a_{\text {min }}+m a_{\text {min, } t}
\end{aligned}
$$

\subsection{Berechnung und Interpretation der Modellergebnisse}

Die Verwendung stochastischer Variablen und Beziehungen innerhalb des Modells erfordert den Einsatz von Methoden, die in der Lage sind, aus den zugrunde gelegten Wahrscheinlichkeitsverteilungen die entsprechenden

365 Durch diese Konstruktion des ADBUDG-Modells kann es unter ungünstigen Bedingungen dazu kommen, daß der obere Wert der abhängigen Variablen überschritten wird. Da sich die Wirkung des Budgets stets auf die konstante Differenz ma $a_{\max }$ $\mathrm{ma}_{\min }$ bezieht, kann dieser Fall eintreten, wenn das Minimum der aktuellen Periode größer ist als das mit ma $\mathrm{m}_{\min }$ beschriebene Minimum. 


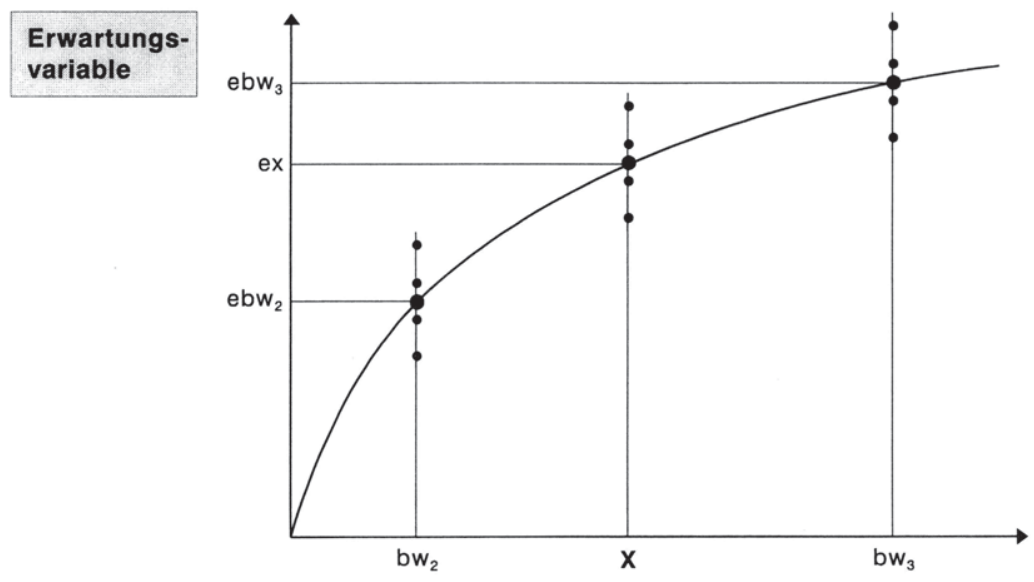

Vom Entscheidungstrāger erhobene Angaben zur Wahrscheinlichkeitsverteilung der Erwartungsvariablen bei Aktivitätsniveau $\mathrm{bw}_{2}, \mathrm{bw}_{3}$

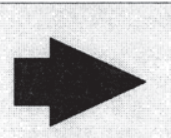

Absolutes Maximum $\rightarrow$ Erwartetes Maximum Erwartetes Minimum Absolutes Minimum
Aktivitätsniveau des Marketinginstrumentes

Abb. 46: Ermittlung der stochastischen Beziehung zwischen dem Aktivitätsniveau des Marketinginstrumentes und der Erwartungsvariablen bei trapezförmiger Wahrscheinlichkeitsverteilung 
Verteilungen der Erwartungsvariablen zu berechnen. Zudem sind Regeln anzugeben, wie die nunmehr durch zwei Dimensionen beschriebenen Ergebnisse interpretiert und bewertet werden können.

\subsection{Berechnung der Modellergebnisse durch Risikosimulation}

Methoden zur Berücksichtigung stochastischer Eingangsvariablen werden in der Literatur unter dem Begriff der Risikoanalyse zusammengefaßt. $366 \mathrm{Da}$ bei lassen sich grundsätzlich drei verschiedene Ansätze voneinander unterscheiden: 367

- Verfahren der Vollenumeration berechnen das Ergebnis für jede denkbare Kombination der Eingangsvariablen. Dies setzt jedoch voraus, daß die Werte diskreter Natur sind und die Gesamtzahl an Variablen und Werten möglichst gering ist. 368

- Analytische Verfahren verknüpfen die stochastischen Variablen mit Hilfe von Regeln der Wahrscheinlichkeitstheorie. Sie unterliegen damit weitgehenden Einschränkungen, die sich insbesondere auf die verwendeten Verteilungsfunktionen beziehen. ${ }^{369}$

366 Vgl. Hildenbrand, K., Systemorientierte Risikoanalyse in der Investitionsplanung, Berlin 1988, S. 33. Der Begriff der Riskoanalyse geht nach allgemeiner Literaturmeinung auf eine Veröffentlichung von Herz zurück. Vgl. Herz, D. B., Risk Analysis in Capital Investment, in: Harvard Business Review, H. 1, 1964, S. 95 ff. Zu einer übersetzten und überarbeiteten Fassung vgl. auch Herz, D. B., Risiko-Analyse bei Kapitalanlagen, in: Investitionsplanung, Hrsg.: Lüder, K., München 1977, S. 157 ff.

367 Vgl. Schindel, V., Risikoanalyse, a.a.O., S 30 ff.; Köhler, R., Uebele, H., Risikoanalysen bei der Evaluierung absatzorientierter Projekte, a.a.O., S. 121.

368 Zu einem solchen Beispiel vgl. Müller-Merbach, H., Risikoanalyse, in: Management Enzyklopädie, Bd. 8, München 1975, S. 2845 ff.

369 Vgl. zu solchen Ansätzen bspw. Hillier, F. S., The Derivation of Probabilistic Information for the Evaluation of Risky Investments, in: Management Science, 1963, S. $443 \mathrm{ff}$; ; Wagle, B., A Statistical Analysis of Risk in Capital Investment Projects, in: Operational Research Quarterly, H. 1, 1969, S. 13 ff. Zu einer ausführlichen Kritik der Ansätze siehe Wagener, F., Die partielle Risikoanalyse als Instrument der integrierten Unternehmensplanung, München 1978, S. $156 \mathrm{ff}$. 
- Mit den Verfahren der repräsentativen Stichprobe werden schließlich Zufallsprozesse nachgeahmt, die den zugrundeliegenden Wahrscheinlichkeitsverteilungen folgen. Bekanntgeworden sind solche Stichprobenverfahren insbesondere unter dem Begriff der Risikosimulation oder Monte-Carlo-Simulation. Diese soll auch hier zur Berechnung der Erwartungsvariablen verwendet werden, da sie wesentlich flexibler einsetzbar ist als analytische Verfahren und Vollenumerationsverfahren.

Die Vorgehensweise der Monte-Carlo-Simulation wird allgemein wie folgt dargestellt: 370

1. Zum Zeitpunkt $t$ wird zunächst für jede stochastische Eingangsvariable zufällig ein Wert aus dem zulässigen Wertebereich ermittelt. Dazu wird eine $[0,1]$-verteilte Zufallsvariable $z$ an der Verteilungsfunktion $F(x)$ der entsprechenden Variablen $x$ gespiegelt. Mathematisch ergibt sich $x$ somit aus der Umkehrung der Verteilungsfunktion: $x=\mathrm{F}^{-1}(\mathrm{z}) \cdot{ }^{371}$

2. Die Werte der Eingangsvariablen werden in das Modell eingesetzt und die Enwartungsvariablen berechnet.

3. Die ersten beiden Schritte werden für alle Planungszeitpunkte wiederholt.

4. Nachdem der gesamte Planungszeitraum einmal durchlaufen wurde, werden aggregierte Erwartungsvariablen, wie z. B. der Kapitalwert der Periodenergebnisse, berechnet.

5. Die Schritte 1.-4. werden für eine vorgegebene Anzahl an Simulationsläufen wiederholt.

370 Vgl. Wagener, F., Die partielle Risikoanalyse als Instrument der integrierten Unternehmensplanung, a.a.O., S. 188 ff.; Köhler, R., Uebele, H., Straplag AG, Teil II, Risikoanalytische Evaluierung von Preisstrategien bei einer Neuprodukteinführung, in: WiSt, H. 4, 1983, S. 212.

371 Zur Verteilungsfunktion und deren Umkehrung für die Trapezverteilung vgl. die Darstellung im Anhang dieser Arbeit. Zu einer umfassenden Übersicht von Techniken zur Generierung von Zufallswerten aus Wahrscheinlichkeitsverteilungen vgl. Rubinstein, R. Y., Simulation and the Monte Carlo Method, New York u. a. 1981, S. 38 ff.; Bratley, P., Fox, B. L., Schrage, L. E., A Guide to Simulation, 2. Ed., New York u. a. 1987, S. $124 \mathrm{ff}$. 
Abweichend von dieser Vorgehensweise sind hier zwei grundlegende Ergänzungen notwendig, die sich aus der Art der verwendeten Wahrscheinlichkeitsverteilungen sowie der Berücksichtigung stochastischer Zusammenhänge ergeben.

Wie bereits erläutert, 372 sind die Verteilungen der Fahrleistungen sowie der Reifenlebensdauer als Häufigkeitsverteilung zu verstehen, während die Verteilungen anderer Variablen des Modells als Glaubwürdigkeitsverteilungen zu interpretieren sind. Damit ist der erste Schritt im Rahmen der MonteCarlo-Simulation in zwei Stufen aufzuspalten. Zunächst ist eine "Untersimulation" für all diejenigen Berechnungen durchzuführen, die auf Werte von Häufigkeitsverteilungen Bezug nehmen. Dies trifft hier für die Berechnung des Anteils an Reifenkäufern zu, die zum Kaufzeitpunkt die betrachtete Marke i am Fahrzeug montiert haben. Dieser Anteil ergab sich aus der jährlichen Fahrleistung, der Reifenlebensdauer sowie den Marktanteilen im Ersatz- und Erstausrüstungsgeschäft zu dem daraus ermittelten Zeitpunkt des letzten Reifenwechsels. Für eine vorgegebene Zahl von Simulationsläufen ist diese Rechnung mit zufällig gezogenen Werten zu wiederholen, um daraus den erwarteten Anteil der Marke i zu erhalten. ${ }^{373}$ Im Rahmen der eigentlichen "Hauptsimulation" sind daran anschließend die Werte aller stochastischen Eingangsvariablen zu ermitteln, denen Glaubwürdigkeitsverteilungen zugrunde liegen.

Da stochastische Beziehungen Verwendung finden, lassen sich aus den schließlich vorliegenden Variablenwerten nicht unmittelbar die Erwartungsvariablen berechnen, sondern es sind zuvor zwei weitere Teilschritte vorzunehmen. Zum einen ist für jede Beziehung die gültige Wahrscheinlichkeitsverteilung zu bestimmen, zum anderen ist aus dieser Verteilung jeweils ein

372 Vgl. Kap. C. 2.21.

373 Eine analytische Berechnung des Erwartungswertes des Anteils an Reifenkäufern, bei dem zum Kaufzeitpunkt die betrachtete Marke i am Fahrzeug montiert ist, schließt sich aufgrund der Komplexität der notwendigen periodenübergreifenden Rückrechnung nahezu aus. Zudem ist diese Berechnung für jede Periode des Planungszeitraumes zu wiederholen, da sich jeweils die Zusammensetzung der einzubeziehenden Perioden ändert, und die Marktanteile zurückliegender Perioden Z. T. erst während des Simulationslaufes ermittelt werden. 
Zufallswert zu ermitteln. Letzterer stellt das Ergebnis der abhängigen Variablen in der entsprechenden Beziehung dar.

Insgesamt ergibt sich für die Monte-Carlo-Simulation damit der in Abbildung 47 dargestellte Ablauf von Simulationsschritten. Dabei ist besonders zu beachten, daß jeder einzelne Simulationslauf über die gesamte Planungsperiode erfolgt, und nicht etwa die festgelegte Anzahl von Simulationsläufen zuerst für einen Zeitpunkt und dann für den jeweils darauffolgenden durchgeführt wird. Eine solche Reihenfolge würde dazu führen, daß bei periodenübergreifenden Beziehungen stets nur der Erwartungswert übergeben würde und damit ein Teil der tatsächlichen Varianz unbeachtet bliebe.

Hinsichtlich der Anzahl notwendiger Simulationsläufe gelten die Regeln der induktiven Statistik zur Bestimmung des Umfangs von Stichproben. Grundlage ist die Festlegung des sogenannten Konfidenzintervalls, innerhalb dessen Grenzen sich der tatsächliche Wert der Erwartungsvariablen mit einer vorgegebenen Vertrauenswahrscheinlichkeit befinden soll. ${ }^{374}$ Die Ermittlung des hierfür notwendigen Stichprobenumfangs setzt allerdings voraus, daß die Standardabweichung der Stichprobe bekannt ist oder zumindest geschätzt werden kann. Diese Information liegt aber gerade erst nach Durchführung der Simulation vor, so daß die Anzahl der Simulationsläufe, d. h. des Stichprobenumfangs, im vorhinein nicht bestimmbar ist. Allenfalls kann ein sukzessives Vorgehen gewählt werden, bei dem erst eine willkürliche Zahl von Simulationsläufen durchgeführt wird, um auf Basis der gefundenen Standardabweichung die erforderliche Anzahl von Läufen zu berechnen. Ist diese höher als die willkürliche Vorgabe, werden zusätzliche Simulationsläufe durchgeführt. Dadurch kann sich jedoch wiederum die Standardabweichung ändern, so daß der Stichprobenumfang erneut zu berechnen ist und mit der tatsächlichen Anzahl von Simulationsläufen verglichen werden muß. Der Vorgang wird erst beendet, wenn die erforderliche Zahl von Simulationsläufen erreicht ist. ${ }^{375}$

374 Vgl. zur Berechnung des Stichprobenumfangs Bleymüller, J., Gehlert, G., Gülicher, H., Statistik für Wirtschaftswissenschaftler, a.a.O., S $89 \mathrm{f}$.

375 Vgl. Hildenbrand, K., Systemorientierte Risikoanalyse in der Investitionsplanung, a.a.O., S. 179. 


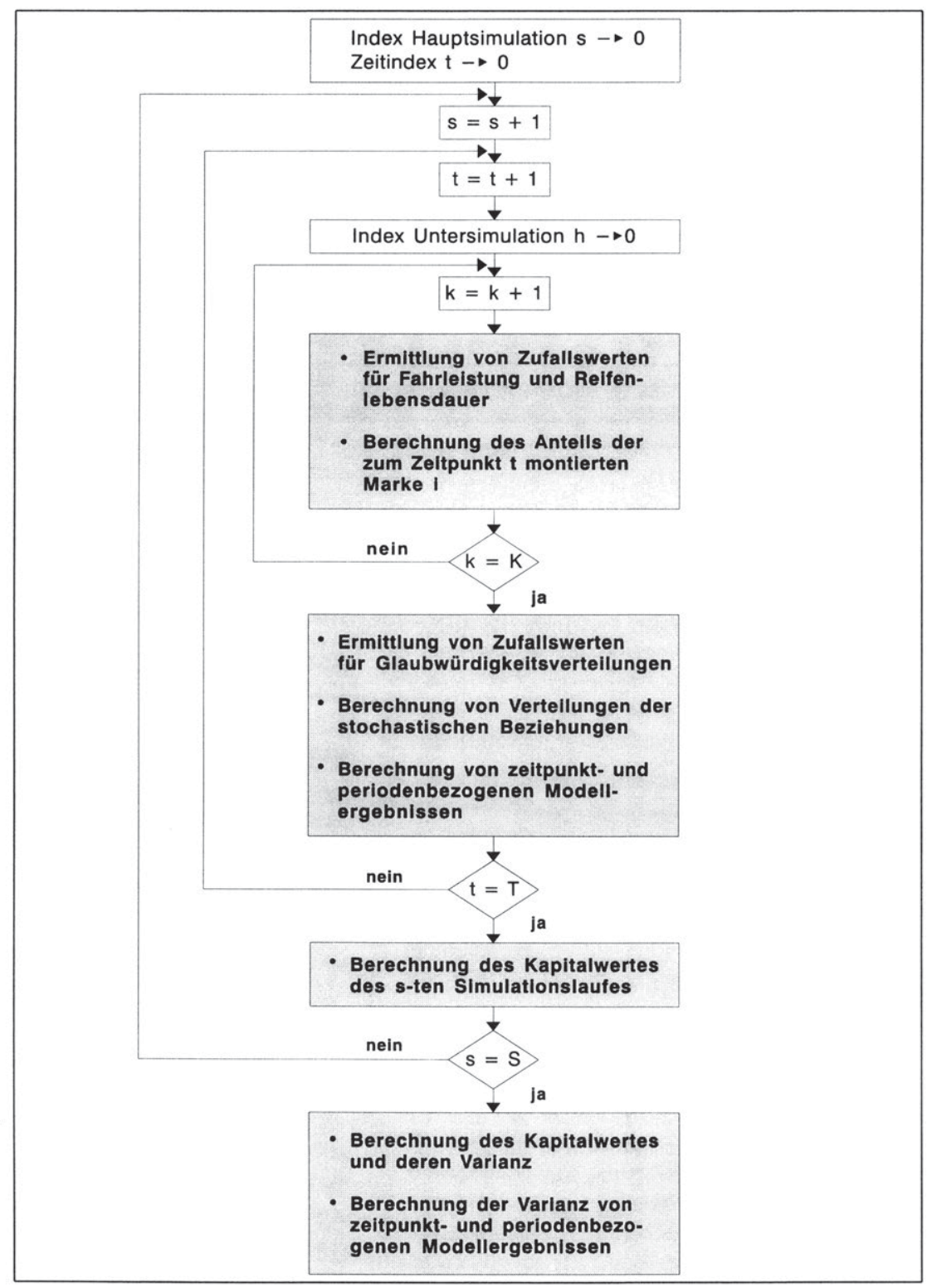

Abb. 47: Ablauf der Monte-Carlo-Simulation

(In Anlehnung an Schindel, V., Risikoanalyse, a.a.O., S. 44) 
Für das Modell wurde auf eine solche halbautomatische Berechnung des Stichprobenumfangs verzichtet, da sie die Handhabung und Verständlichkeit der Modellberechnung für den Entscheidungsträger eher erschwert als vereinfacht. Die erreichten Vertrauenswahrscheinlichkeiten der Erwartungswerte für die Gewinn- und Marktanteilsvariablen werden erst nach vollständiger Durchführung der Simulation ausgewiesen. Der Entscheidungsträger kann selbst darüber befinden, ob er diese für das von inm vorgegebene Konfidenzintervall als ausreichend erachtet oder die Simulation mit einer erhöhten Zahl von Durchläufen erneut beginnt.

Um die Vertrauenswahrscheinlichkeiten von stochastischen Variablen berechnen zu können, werden zumeist Annahmen über deren Verteilung getroffen. Aufgrund ihrer besonderen Eigenschaften geht man dabei häufig von einer Normalverteilung aus. ${ }^{376}$ Die vielfältigen Verknüpfungen innerhalb des Modells lassen jedoch keine sichere Aussage darüber zu, ob es sich in diesem Fall tatsächlich um normalverteilte Erwartungsvariablen handelt. Daher wird hier ein Ansatz auf Basis der sogenannten TschebyschewUngleichung gewählt, bei dem lediglich die Annahmen zu treffen sind, daß Erwartungswert und Standardabweichung endlich sind und letztere darüber hinaus bekannt ist. Die Vertrauenswahrscheinlichkeit 1- $\alpha$, daß sich der tatsächliche Erwartungswert einer Zufallsvariable innerhalb eines Konfidenzintervalls $[\bar{x}-\varepsilon, \bar{x}+\varepsilon]$ bei einer Anzahl von I Simulationsläufen befindet, ergibt sich dann aus: 377

$$
1-\alpha=1-\frac{\sigma^{2}}{1 \cdot \varepsilon^{2}}
$$

376 Zu diesen Eigenschaften zählen zum einen die Möglichkeiten, andere Verteilungen gut zu approximieren. Zum anderen wird aufgrund des zentralen Grenzwertsatzes der Statistik häufig angenommen, daß eine Zufallsvariable normalverteilt ist, wenn sie sich additiv aus vielen unabhängigen Zufallseinflüssen zusammensetzt, von denen jeder nur einen geringfügigen Einfluß besitzt. Vgl. Bamberg, G., Baur, F., Statistik, 3. Aufl., München 1984, S. 108 ff.; Autorenkollektiv Freie Universität Berlin u. a., Grundausbildung in Statistik für Wirtschaftswissenschaftler, Osnabrück, o. J., S. $317 \mathrm{f}$. $\bar{x}$ ist das ermittelte Stichprobenmittel, $\varepsilon$ eine vom Entscheidungsträger vorgegebene Abweichung.

377 Die Vertrauenswahrscheinlichkeit wird aufgrund der nur geringen Information über die Verteilung geringer ausfallen, $d . h$. vorsichtiger berechnet als z. B. bei Annahme einer Normalverteilung. Vgl. zu diesem Ansatz Autorenkollektiv Freie Universität Berlin u. a., a.a.O., S. $401 \mathrm{ff}$. 
Die Vertrauenswahrscheinlichkeit darf nicht falsch interpretiert werden. Sie stellt lediglich eine Information zur Übereinstimmung von Ergebnissen der Stichprobe und der im Modell dargestellten Grundgesamtheit dar, sagt aber nichts über die Validität des Modells oder mögliche Prämissenverletzungen aus. Die zentrale Prämisse bei der Monte-Carlo-Simulation besteht darin, daß die Wahrscheinlichkeitsverteilungen der stochastischen Variablen unabhängig voneinander sind, oder aber Abhängigkeiten explizit in der Modellformulierung und bei der Datenschätzung berücksichtigt werden. ${ }^{378}$ Gleiches gilt für die stochastischen Beziehungen. Obwohl eine solche Forderung nur in Ausnahmefällen vollständig erfüllt werden kann, läßt sich doch zeigen, daß ihr in diesem Modell weitgehend entsprochen wird.

Ein grundlegendes Prinzip zur Vermeidung von Abhängigkeiten besteht in der Schaffung "bedingter Variablen", die von vornherein nur für eine bestimmte Situation definiert sind. Beispielsweise ist die Frage, welche Marke vom Reifenkäufer präferiert wird, davon abhängig, ob dieser seine Präferenz anhand der montierten Reifenmarke oder losgelöst hiervon bildet. Daher wurden im Endabnehmermodell anstelle einer Variablen "Präferenz für Marke i" zwei solche Variablen definiert, die sich zum einen auf die Situation "Präferenz abhängig von montierter Marke" und zum anderen auf die Situation "Präferenz unabhängig von montierter Marke" beziehen. Analog hierzu wurde mit weiteren Variablen zur Beschreibung des Endabnehmer- wie auch des Absatzmittlerverhaltens verfahren.

Grundsätzlich abhängig voneinander sind diejenigen Variablen, deren Einzelwerte sich zu einem bestimmten Gesamtwert addieren. Betrachtet man z. B. die Wahrscheinlichkeiten für "Präferenz abhängig von montierter Marke", "Präferenz unabhängig von montierter Marke" und "Indifferenz", so müssen diese in der Summe den Wert 1 ergeben. Um die Abhängigkeit erfassen zu können, wurde in dieser Situation derart verfahren, daß vom

378 In der Literatur findet sich in diesem Zusammenhang zumeist die Forderung, daß die Wahrscheinlichkeit für die Realisation einer Eingangsgröße von denjenigen aller anderen unabhängig ist. Hierbei ist allerdings zu beachten, daß sich mit den Wahrscheinlichkeiten gleichzeitig auch der Wertebereich der Eingangsgrößen verändern kann, falls die Eintrittswahrscheinlichkeiten $p$ der einzelnen Werte zwischen $p=0$ und $p>0$ wechseln. Vgl. auch Kruschwitz, L., Bemerkungen zur Risikoanalyse aus theoretischer Sicht, in: ZfB, H. 7, 1980, S. 805. 
Entscheidungsträger nur Angaben zu den Verteilungen von zwei der drei Variablen verlangt werden. Während der Modellberechnung ergibt sich der Wert der dritten Variablen jeweils aus der Differenz von 1 und den Zufallswerten der beiden ersten Variablen.

Explizit berücksichtigt wird darüber hinaus die Abhängigkeit von Variablen, zwischen denen eine Wirkungsbeziehung angenommen wird. Über die stochastische Beziehung wird dabei für jeden Wert der abhängigen Variablen eine eigene Verteilung der unabhängigen Variablen ermittelt. Da es sich jeweils nur um einseitige und nicht um wechselseitige Wirkungen handelt, ist die gewählte Vorgehensweise ausreichend, um das Abhängigkeitsverhältnis in angemessener Form abzubilden. ${ }^{379}$

Die Grenzen eines Modells werden sicherlich erreicht, wenn der Einfluß einer Variablen auf den stochastischen Zusammenhang zweier anderer Variablen abzubilden ist. Obwohl theoretisch durchaus möglich, erhöhten sich die Komplexität des Modells und der Schätzaufwand für die anzugebenden Daten derart, daß dem Prinzip des benutzerorientiert-subjektiven Ansatzes nicht mehr gefolgt würde. Ein solcher Fall liegt beispielsweise für den Einfluß des Präferenzgrades auf die Beziehung von Push-Stärke und Distributionsgrad vor. Es ist anzunehmen, daß der Handel Veränderungen der Push-Stärke gegenüber unempfindlicher reagiert, wenn es sich um eine stark nachgefragte Marke handelt, als wenn eine weniger wichtige Marke betroffen wäre. Dies bedeutet modelltheoretisch, daß die abgebildete Elastizität der Beziehung zwischen Push-Stärke und Distributionsgrad entsprechend dem Präferenzgrad zu verändern wäre.

Um diesen Zusammenhang zumindest im Ansatz abzubilden, wurde für das Modell in dieser Arbeit der beschriebene Weg über die Einführung von Effizienzindizes gewählt. Damit wird zwar keine direkte Veränderung der Elastizitäten erreicht, aber immerhin eine fiktive Anpassung der jeweils

379 Ein weiterer Ansatz zur Bestimmung bedingter Verteilungen findet sich bei Heider, der von Beta-verteilten stochastischen Variablen ausgeht. Der Zusammenhang wird über jeweils zwei Funktionen abgebildet, mittels derer die Parameter Erwartungswert und Standardabweichung der abhängigen Variablen durch den Wert der unabhängigen Variablen gesteuert werden. Vgl. Heider, M., Simulationsmodell zur Risikoanalyse für Investitionsplanungen, Diss., Bonn 1969, S. 20 ff. 
unabhängigen Variablen vorgenommen. Berücksichtigt man darüber hinaus, daß die Schätzungen der Daten vor dem Hintergrund einer bestimmten Marke erfolgen und in der Realität zumeist keine grundlegenden, sondern nur inkrementale Veränderungen in der Markenführung stattfinden, so scheint die gewählte Vorgehensweise durchaus angemessen. Ändert sich die Ausgangssituation über die Zeit zu stark, kann auf Grundlage der Ergebnisse der ersten Perioden manuell eine veränderte Schätzung der Funktionen vorgenommen werden und die Simulation somit sukzessive für die weiteren Perioden fortgeschrieben werden.

Obgleich insgesamt versucht wurde, die für eine Monte-Carlo-Simulation unzulässigen Abhängigkeiten in dem Modell zu vermeiden oder aber explizit zu erfassen, konnten nicht alle theoretisch möglichen Beziehungen berücksichtigt werden. Dies trifft z. B. für eine potentielle Abhängigkeit des Kaufverhaltens von der jährlichen Fahrleistung oder für die Abhängigkeit der Lebensdauer eines Reifens von seiner Marke zu. Hier war jeweils abzuwägen zwischen der Abbildungsgenauigkeit und Prämissentreue auf der einen und einer erhöhten Modellkomplexität und einem steigenden Schätzaufwand auf der anderen Seite.

\subsection{Interpretation der Modellergebnisse und Entscheidung für eine Handlungsalternative}

Mit Hilfe der Monte-Carlo-Simulation lassen sich sogenannte Risikoprofile ermitteln. Als solche werden komplementäre Verteilungsfunktionen $\left(F^{*}=1-F\right)$ der jeweiligen Erwartungsvariablen bezeichnet, wie sie in Abbildung $48 \mathrm{im}$ Hinblick auf die Ergebnisgröße des Markenwertes beispielhaft für zwei Strategien der Markendurchsetzung dargestellt werden. ${ }^{380}$

380 Da es sich im streng-analytischen Sinne nicht um eine Verteilungsfunktion, sondern um eine kumulierte Häufigkeitsverteilung der Ergebniswerte handelt, wird in der Literatur z. T. auch von einer Quasi-Verteilungsfunktion gesprochen. Vgl. Köhler, R., Uebele, H., Risikoanalysen bei der Evaluierung absatzorientierter Projekte, a.a.O., S. 121. 


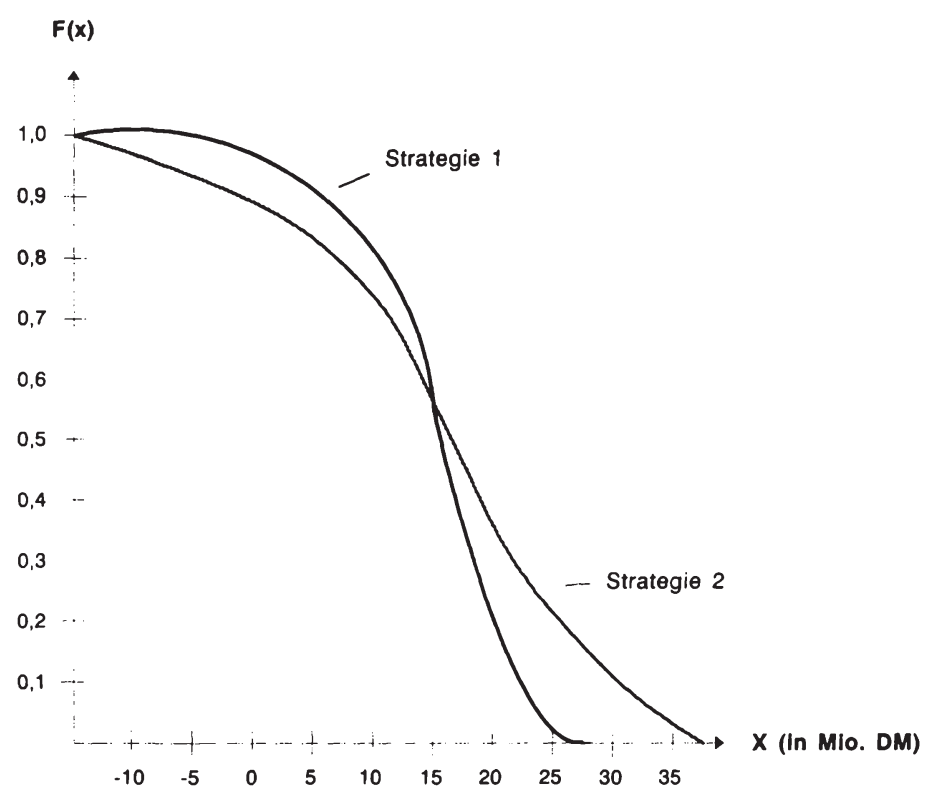

Abb. 48: Risikoprofile zweier Strategien der Markendurchsetzung

Der Verlauf der Graphen gibt an, mit welcher Mindestwahrscheinlichkeit jeweils der entsprechende Markenwert erreicht wird. Ist zwischen mehreren Alternativen zu wählen, empfiehlt es sich, die Strategien zunächst auf gegenseitige Dominanz zu prüfen. ${ }^{381}$ In dem abgebildeten Beispiel dominiert allerdings keine der beiden Alternativen. Dies läßt sich graphisch daran erkennen, daß sich die Profile schneiden. Für die weitergehende Analyse wird in der Literatur vorgeschlagen, den Risikoprofilen gewisse Mindestanforderungen gegenüberzustellen. Hierbei bietet es sich an, Mindest- bzw. Höchstwahrscheinlichkeiten für eine bestimmte Verlusthöhe, für das Erreichen des positiven Bereiches sowie für einen bestimmten gewünschten Markenwert anzugeben.

381 Hierbei handelt es sich um die sog. stochastische Dominanz, die wie folgt definiert ist: Strategie a dominiert Strategie b, wenn für jedes mögliche Ergebnis gilt, daß die Wahrscheinlichkeit, dieses zu erreichen, bei a nicht kleiner und für mindestens ein Ergebnis größer ist als bei b. Vgl. Gottwald, R., Entscheidung unter Unsicherheit, Informationsdefizite und unklare Präferenzen, Wiesbaden 1990, S. 162. 
Über das Risikoprofil hinaus liefert die Monte-Carlo-Simulation in aller Regel Angaben zum Erwartungswert und zur Standardabweichung. Damit ist die Voraussetzung geschaffen, die Regeln der klassischen Entscheidungstheorie bei Risiko anwenden zu können. ${ }^{382}$ Hierbei werden Erwartungswert und Standardabweichung nach dem $\mu-\sigma$-Prinzip zu einem Nutzenwert $u$ verdichtet, dessen Höhe die relative Bewertung der jeweiligen Alternative beschreibt. Mögliche Nutzenfunktionen, mit denen in der Literatur gearbeitet wird, sind z. B.:383

$u=\mu-\mathbf{a} \cdot \sigma$

$u=\mu-\mathbf{a} \cdot \sigma^{2}$

Durch den Parameter a kommt die Risikoeinstellung des Entscheidungsträgers zum Ausdruck. Verhält sich dieser risikoavers $(a>0)$, so wird er bei gleichem Erwartungswert stets die Alternative mit der geringsten Standardabweichung vorziehen. Demgegenüber führt ein risikofreudiges Verhalten $(a<0)$ in der gleichen Situation zur Wahl der Alternative mit der höchsten Standardabweichung. Ohne Relevanz für die Entscheidung ist die Standardabweichung bei einem risikoneutralen Verhalten $(a=0) .384$

Die zweite Möglichkeit zur Auswahl einer Strategie aus mehreren Alternativen beruht auf dem Konzept des Risikonutzens. Mittels einer vom Entscheidungsträger zu bestimmenden Risikonutzenfunktion werden jedem möglichen Ergebniswert eg $g_{j}$ einer Alternative Nutzenwerte $u\left(\mathrm{eg}_{\mathrm{j}}\right)$ zugeordnet,

382 In der klassischen Entscheidungstheorie wird beim Vorliegen von Unsicherheit zwischen den Situationen des Risikos und der Ungewißheit unterschieden. Während in der Risikosituation die subjektive oder objektive Wahrscheinlichkeit des Eintritts eines bestimmten Ereignisses bekannt ist, bestehen in der Ungewißheitssituation ausschließlich Informationen darüber, welche Ereignisse eintreten können. Vgl. Bamberg, G., Coenenberg, A. G., Betriebswirtschaftliche Entscheidungslehre, a.a.O., S. 17.

383 Vgl. Adam, D., Planung und Entscheidung, a.a.O., S. 221; Bitz, M., Entscheidungstheorie, München 1981, S. 102.

384 Vgl. Bamberg, G., Coenenberg, A. G., Betriebswirtschaftliche Entscheidungslehre, a.a.O., S. 89 . 
mit der Eintrittswahrscheinlichkeit des Ergebnisses $w_{j}$ gewichtet und $z u$ einem Gesamtnutzenwert $u$ addiert: 385

$u=\sum_{j} w_{j} \cdot n\left(e g_{j}\right)$

Für die zugrundeliegende Nutzenfunktion wird angenommen, daß sie sowohl die Höhen- als auch die Risikopräferenz des Entscheidungsträgers abbildet und daher dessen grundlegende Risikoeinstellung am Verlauf der Funktion erkennbar ist. ${ }^{386}$

Im Rahmen des hier zu entwickelnden Modells wurde auf die explizite Berechnung von Nutzenwerten verzichtet. Vielmehr beschränkten sich die Angaben zu den Ergebnissen auf wertfreie, statistische Kennziffern. Wie dargestellt, lassen sich Nutzenwerte bei vorliegender Nutzenfunktion jedoch sehr einfach berechnen, so daß bei Bedarf eine entsprechende Erweiterung des Modells ohne größeren Aufwand vorgenommen werden kann.

\subsection{Sensitivitätsanalyse und Optimierung}

Unter dem Begriff der Sensitivitätsanalyse wird im allgemeinen ein Vorgehen verstanden, bei dem der Zusammenhang zwischen Eingangs- und Erwartungsvariablen durch Variation der Eingangsgröße näher untersucht wird. Die Analyse bezieht sich jeweils auf eine Ausgangslösung, d. h. eine Kombination von Werten der Eingangsvariablen, die zu einem bestimmten Ergebnis führt. Grundsätzlich lassen sich zwei Typen der Sensitivitätsanalyse voneinander unterscheiden: 387

385 Vgl. Adam, D., Planung und Entscheidung, a.a.O., S. 213.

386 Diese Annahme ist in der Literatur nicht unumstritten und wird z. T. sehr kontrovers diskutiert. Vgl. hierzu sowie zu den Begriffen der Höhen- und Risikopräferenz Bamberg, G., Coenenberg, A. G., Betriebswirtschaftliche Entscheidungslehre, a.a.O., S. 92 ff. sowie die dort angegebene Literatur. Vgl. auch Gottwald, R., Entscheidung unter Unsicherheit, a.a.O., S. $300 \mathrm{ff}$.

387 Vgl. Schirmeister, R., Modell und Entscheidung, Möglichkeiten und Grenzen der Anwendung von Modellen zur Alternativenberechnung im Entscheidungsprozeß der 
- Einerseits wird die Stabilität einer gefundenen Lösung analysiert. Dazu wird nach Grenzen gesucht, innerhalb derer die Werte der Eingangsgrößen variieren können, ohne daß das Ergebnis einen vorgegebenen Bereich verläßt. Dieses Vorgehen wird in der Literatur auch als Verfahren der kritischen Werte bezeichnet.

- Andererseits wird die Wirkung von Veränderungen der Eingangsgrößen auf die Erwartungsvariablen untersucht. Abhängig davon, welche Variablen variiert werden, lassen sich zum einen Erkenntnisse darüber gewinnen, wie empfindlich das Ergebnis auf eine geänderte Datensituation reagiert, zum anderen lassen sich potentielle Verbesserungen der Ausgangslösung finden. Handelt es sich bei den zu verändernden Eingangsvariablen um Kontextvariablen, so werden damit alternative Umweltsituationen simuliert. Betreffen die Veränderungen dagegen Entscheidungsvariablen des Unternehmens, wird durch die Sensitivitätsanalyse nach verbesserten Lösungen gesucht.

Da das hier zu entwickelnde Modell von vornherein auf die Durchführung von Marktexperimenten angelegt wurde, sind Sensitivitätsanalysen ohne weiteres realisierbar. In beiden Formen der Analyse sind lediglich die gewünschten Eingangsgrößen schrittweise zu verändern, wobei sich nur die Abbruchkriterien voneinander unterscheiden. Entsprechend der Zielsetzung wird der Variationsvorgang beendet, wenn

- ein kritischer Wert der Erwartungsvariablen erreicht ist,

- ein zuvor festgelegtes Werteintervall der zu verändernden Eingangsvariablen durchlaufen ist oder

- sich keine weitere Ergebnisverbesserung erzielen läßt.

Diese Beschreibung der Sensitivitätsanalyse bezieht sich zunächst auf den Fall, daß die Werte deterministischer Variablen verändert werden. Bei stochastischen Eingangsvariablen ist zu differenzieren, ob eine Sensitivitätsanalyse in bezug auf mögliche Werteausprägungen oder im Hinblick auf die Verteilung selbst gewünscht ist. 
Der erste Fall wird quasi automatisch im Rahmen der Monte-Carlo-Simulation berücksichtigt. Indem entsprechend der vorgegebenen Wahrscheinlichkeitsverteilung die Variable mit verschiedenen Werten in die Modellberechnung eingeht, wird nicht nur bereits dem Gedanken der Sensitivitätsanalyse entsprochen, sondern es werden darüber hinaus noch Informationen über die jeweiligen Eintrittswahrscheinlichkeiten der Ergebnisse geliefert. Zum anderen ist es durchaus denkbar, die Wahrscheinlichkeitsverteilung selbst zum Gegenstand der Analyse zu machen. Aufgrund deren Mehrdimensionalität sind in diesem Fall jedoch Angaben dazu erforderlich, in welcher Weise die Verteilungen variieren sollen. So reicht das Spektrum der Möglichkeiten von einer Verschiebung der Verteilung auf der Wertedimension bis hin zu einer Veränderung des Funktionsverlaufs.

Auch hinsichtlich des Abbruchkriteriums sowie der Ergebnisdarstellung erhöht sich die Komplexität der Sensitivitätsanalyse, wenn stochastische Variablen in dem Modell enthalten sind. Selbst wenn die eigentliche Variation an deterministischen Größen vorgenommen wird, sind die Veränderungen der Ergebnisse mehrdimensional und betreffen sowohl deren Höhe als auch deren Eintrittswahrscheinlichkeit. Zur Komplexitätsreduzierung bieten sich grundsätzlich zwei Wege an, die beide darauf abzielen, jeweils eine eindimensionale Erwartungsvariable zu schaffen. Zum einen besteht die Möglichkeit, Höhe und Wahrscheinlichkeiten der Ergebnisse in Form von Nutzenfunktionen zu aggregieren. Zum anderen kann vereinfachend zunächst die Dimension des Risikos vernachlässigt und jeweils nur der Erwartungswert zur Analyse herangezogen werden.

Für ein entscheidungsunterstützendes Modell, das den Forderungen der zugrunde gelegten Gestaltungsprinzipien entsprechen soll, ist eine möglichst einfache, flexible und gut kommunizierbare Form der Sensitivitätsanalyse notwendig. Daher beschränkt sich das hier zu entwickelnde Modell darauf, deterministische Variablen einer solchen Analyse zu unterziehen und bei den Erwartungsvariablen nur den Erwartungswert zu berücksichtigen.

Insbesondere geht es darum, die Wirkung der Variation der Entscheidungsvariablen (Förderungs-, Akquisitions- und Pull-Budget sowie Handelsspanne) im Hinblick auf die Veränderung der Erwartungsvariablen 
(Markenwert, Periodengewinne, Marktanteile sowie die Variablen zur Beschreibung des Marktteilnehmerverhaltens) zu untersuchen. Neben einer Darstellung der Sensitivität von Ergebnissen in unmittelbarer Umgebung der Ausgangswerte dieser Variablen läßt sich damit auch schrittweise eine Ergebnisverbesserung herbeiführen, um ein zumindest lokales Optimum zu erreichen.

Die einfachste Vorgehensweise zur Ermittlung eines lokalen Optimums wird als Einzelschritt- oder Einzelfaktormethode bezeichnet, die sehr anschaulich in der Literatur mit der Wanderung eines Bergsteigers bei Nebel verglichen wird. 388 Demnach behält der Bergsteiger eine eingeschlagene Richtung solange bei, wie der Weg ansteigend ist. Erst wenn dieser sich neigt, ändert der Bergsteiger seine Richtung und folgt dem neuen Weg wiederum solange, wie er sich aufwärts bewegt. Dieses Vorgehen wird beibehalten, bis schließlich in keine Richtung mehr ein Anstieg festzustellen und damit ein Optimum gefunden ist. Übertragen auf das hier vorliegende Problem bedeutet dies, daß zunächst drei der vier zur Verfügung stehenden Entscheidungsvariablen konstant gehalten werden und die vierte Variable solange variiert wird, bis keine Ergebnisverbesserung mehr möglich ist. Auf diese Art werden nacheinander alle Entscheidungsvariablen verändert. Dieser Vorgang wird solange wiederholt, bis letztlich keine Ånderung mehr zu einer Verbesserung des Ergebnisses führt. ${ }^{389}$

Der wesentliche Vorteil dieses Verfahrens ist in seiner Einfachheit und hohen Durchschaubarkeit für den Entscheidungsträger zu sehen. Der zentrale Nachteil besteht darin, daß nicht überprüft werden kann, ob ein lokales oder globales Optimum vorliegt. Hierzu ist es notwendig, von möglichst unterschiedlichen Ausgangslösungen aus den Optimierungsprozeß zu wie-

388 Vgl. Schmidt, J. W., Taylor, R. E., Simulation and Analysis of Industrial Systems, Homewood, Georgetown 1970, S. 529 f.

389 Vgl. Biethahn, J., Optimierung und Simulation, a.a.O., S. 48 f.; Lilien, G. L., Marketing Mix Analysis with Lotus 1-2-3, a.a.O., S. 30. 
derholen, wodurch entweder weitere lokale Optima ermittelt werden oder das bereits gefundene Ergebnis als globales Optimum bestätigt wird. 390

\subsection{Computergestützte Implementierung des Modells}

Das bisher in seiner theoretischen Konzeption vorgestellte Modell wurde im Rahmen dieser Arbeit als computergestütztes Simulationsprogramm implementiert. Dabei bestand zunächst das Problem einer adäquaten Auswahl der zu verwendenden Programmiersprache.

Zur Implementierung von Simulationsmodellen wurde in der Vergangenheit eine Reihe spezifischer Simulationssprachen entwickelt, wie z. B. GPSS, SIMULA, SIMSCRIPT, DYNAMO oder DYMOSYS. 391 Grundsätzlich bietet der Einsatz solcher problemorientierter Sprachen den Vorteil einer kürzeren Entwicklungszeit bei der Umsetzung von Modellabläufen in Programmbefehle. Auch nachträgliche Änderungen lassen sich in aller Regel einfacher einführen, da die Anzahl der notwendigen Befehle vergleichsweise gering und die Struktur des Programms recht transparent ist. Dies gilt zumeist jedoch nur unter der Bedingung, daß die Konzeption des Modells der spezifischen Problemstellung der jeweiligen Sprache entspricht. Bei darüber hinausgehenden Anforderungen erweisen sich solche Sprachen eher als inflexibel. Dies gilt insbesondere auch für eine benutzerfreundliche Gestaltung der Kommunikation zwischen Modell und Entscheidungsträger. ${ }^{392}$

390 Vgl. Biethahn, J., Optimierung und Simulation, a.a.O., S. 51. Über dieses einfache Verfahren hinaus ist in der Literatur eine Vielzahl von komplexeren Methoden zur Zieloptimierung bei Simulationsmodellen entwickelt worden. Vgl. ebenda, S. 53 ff.; Dörnhöfer, K., Lösung von Optimierungsproblemen durch Simulation, in: Biethahn, J., Schmidt, B., Simulation als betriebliche Entscheidungshilfe, Methoden, Werkzeuge, Anwendungen, Berlin u. a. 1987, S. $64 \mathrm{ff}$.

391 Vgl. Mertens, P., Simulation, 2. Aufl., Stuttgart 1982, S. 39 ff.; Bratley, P., Fox, B. L., Schrage, L. E., A Guide to Simulation, a.a.O., S. 228 ff.; Pidd, M., Computer Simulation in Management Science, 2. Ed., Chichester 1988, S. $150 \mathrm{ff}$.

392 Zu dieser Einschätzung vgl. auch Hildenbrand, K., Systemorientierte Risikoanalyse in der Investitionsplanung, a.a.O., S. 124 ff.; Bratley, P., Fox, B. L., Schrage, L. E., A Guide to Simulation, a.a.O., S. 228 f.; Ritzerfeld, U., Marketing-Mix-Strategien in Investitionsgütermärkten, a.a.O., S. 124 f. 
Da im Rahmen dieser Arbeit weniger die Entwicklungsgeschwindigkeit als vielmehr eine hohe Flexibilität bei der Implementierung und eine benutzerfreundliche Gestaltung im Vordergrund standen, wurde auf den Einsatz einer spezifischen Simulationssprache verzichtet und statt dessen auf eine Universalsprache zurückgegriffen. Verwendung fand hierbei die Programmiersprache TURBO PASCAL 6.0.

Die für den Entscheidungsträger sichtbare Struktur des Programms gliedert sich in die Bereiche der Dateneingabe sowie der Simulationsdurchführung (vgl. Abbildung A1). ${ }^{393}$ Für die Dateneingabe werden verschiedene Bildschirmmasken bereitgestellt, in die die erforderlichen Angaben übersichtlich eingetragen werden können. Da jedoch für nahezu jede Variable vier Werte anzugeben sind und bei Funktionen sogar bis zu zwanzig Einträge notwendig sind, wurden hierbei gegenüber dem beschriebenen Modell bestimmte Vereinfachungen vorgenommen, um den Gesamtumfang der Dateneingabe auf ein überschaubares Ausmaß zu beschränken. Diese Vereinfachungen beziehen sich in erster Linie darauf, daß bestimmte Ausgangswerte und Veränderungsraten über die Perioden konstant gehalten werden.

Zur Berechnung der zum Kaufzeitpunkt montierten Reifenmarke waren Angaben zu den Marktanteilen im Ersatzmarkt- und Erstausrüstungsgeschäft sowie zum Verhältnis von Neufahrzeug- zum Gesamtfahrzeugbestand, sowohl für vergangene als auch zukünftige Zeitpunkte, notwendig. Rückwirkend werden diese Daten von dem Modell für die Zeitpunkte $t=-5$ bis $t=0$ berücksichtigt. Sollte der letzte Reifenwechsel länger zurückliegen, werden die Angaben zu $t=-5$ zugrunde gelegt. Für die Perioden des Planungszeitraums wird sowohl der Erstausrüstungsmarktanteil wie auch das den Fahrzeugbestand betreffende Verhältnis als konstant angenommen (vgl. Abbildung A18). Gleiches gilt für die jährliche Fahrleistung sowie die Reifenlebensdauer (vgl. Abbildung A19). Das Marktvolumen (vgl. Abbildung A17) sowie die Basisangaben zu den Budgets (vgl. Abbildung A16) werden jeweils für die erste Periode angegeben und unterliegen im weiteren einer

393 Die einzelnen Bildschirmmasken des Programms sind im Anhang der Arbeit abgebildet. 
konstanten Veränderungsrate. Die Angaben zu Herstellungskosten und Endabnehmerpreisen beziehen sich auf den Beginn der ersten Periode und verändern sich ebenfalls über die Zeit mit einer konstanten Rate. Die Basisangabe zur Handelsspanne besitzt für den gesamten Planungszeitraum Gültigkeit.

Bezüglich des Verhaltens der Endabnehmer ist vom Entscheidungsträger zunächst anzugeben, zu welchem Anteil diese - abhängig bzw. unabhängig von ihrer Reifenausstattung - eine bestimmte Marke präferieren (vgl. Abbildung A5). Wie an anderer Stelle erläutert, wird der entsprechende Prozentsatz indifferenter Personen auf Basis dieser Informationen jeweils während der Simulation berechnet. Bei den darauffolgenden Angaben sind die Wirkungen des Pull-Budgets im Hinblick auf das Endabnehmerverhalten gegenüber der betrachteten Marke i festzulegen (vgl. Abbildungen A6, A8 A9). Dem Entscheidungsträger wird dabei die Möglichkeit gegeben, die von inm geschätzten Zusammenhänge unmittelbar auch graphisch zu visualisieren. Hierzu wird die stochastische Beziehung durch vier Graphen abgebildet, die jeweils den Verlauf der den Trapezverteilungen zugrundeliegenden Werte zeigen (vgl. Abbildung A7). Auf die Darstellung der dritten Dimension, d. h. der Wahrscheinlichkeitsausprägungen, wurde aus Gründen der Übersichtlichkeit verzichtet. 394

Die effektive Verfügbarkeit einer Marke ist unter den verschiedenen Annahmen des Präferenzverhaltens in Abhängigkeit vom Distributionsgrad zu schätzen (vgl. Abbildung A10). Im Gegensatz zu den vorherigen Beziehungen, die auf dem Pull-Budget aufbauen, sind hierbei keine periodenübergreifenden Zusammenhänge zu berücksichtigen, so daß ausschließlich die vier für die Responsefunktion notwendigen Angaben erforderlich sind. Darüber hinaus liegt hier die Situation einer vom Wertebereich beschränkten, unabhängigen Variablen vor. Als höchster Wert wird dementsprechend vom Programm ein Distributionsgrad von $100 \%$ vorgegeben.

394 Die graphische Darstellung kann für jeden vom Entscheidungsträger gewünschten Wertebereich der unabhängigen Variablen, in diesem Fall also des Pull-Budgets, erfolgen. 
Zur Ermittlung des Distributionsgrades selbst sowie des Förderungsverhaltens des Handels sind im nächsten Schritt die Wirkungen der Push-Budgets zu bestimmen (vgl. Abbildungen A11 und A12). Die hierbei festgelegten Zusammenhänge bilden letztlich die Beziehung zwischen den effektiv wirksamen Push-Budgets und den entsprechenden Verhaltensvariablen des Handels ab. Um die Anschaulichkeit für den Entscheidungsträger jedoch zu erhöhen, empfiehlt es sich, dessen Schätzungen zuerst allein im Hinblick auf das monetäre Budget zu erfragen. Dies setzt allerdings voraus, daß der Entscheidungsträger eine bestimmte Situation bezüglich Handelsspanne, Präferenz und Akzeptanz zugrunde legt, bei deren Eintreffen die effektiv wirksamen und die monetären Push-Budgets übereinstimmen. Die Effizienz einer Budget-Mark muß also in dieser Situation genau den Wert 1 annehmen. Um die Beziehungen zwischen Handelsspanne, Akzeptanz und Präferenz auf der einen Seite sowie der Budgeteffizienz auf der anderen Seite angeben zu können, stehen weitere Eingabemöglichkeiten zur Verfügung (vgl. Abbildungen A13, A14 und A15).

Sind schließlich alle notwendigen Daten erfaßt und die Wirkungsbeziehungen ermittelt, können die gewünschten Simulationsexperimente durchgeführt werden. Das entsprechende Teilprogramm zur Simulationssteuerung bietet hierzu die Möglichkeit, das Pull-Budget, die Push-Budgets der Akquisition und Förderung sowie die Handelsspanne beliebig zu variieren und miteinander zu kombinieren (vgl. Abbildung A2). Die Anzahl der Simulationsläufe innerhalb eines Experimentes ist vom Entscheidungsträger frei wählbar und kann getrennt für Haupt- und Untersimulation festgelegt werden. Für die Zielgrößen des Marktanteils sowie des Gewinns lassen sich Konfidenzintervalle bestimmen. Diese werden als relative Abweichung von den in der Stichprobe berechneten Mittelwerten der Variablen ausgedrückt. Die Simulation stellt daraufhin als Ergebnisse die Erwartungswerte, Standardabweichungen und Vertrauenswahrscheinlichkeiten der Marktanteile, der Periodengewinne sowie des Kapitalwertes zur Verfügung. Zusätzlich werden die Erwartungswerte der in Abbildung A3 dargestellten Erwartungsvariablen ausgewiesen.

Für die Marktanteile und den Markenwert lassen sich ferner die Wahrscheinlichkeitsverteilungen und die Risikoprofile graphisch darstellen. Vor- 
aussetzung ist allerdings, daß der Entscheidungsträger vor Simulationsbeginn bereits den Bereich angeben kann, in dem sich die Ergebnisse befinden. Der Grund hierfür liegt darin, daß die Häufigkeitsverteilung bereits während des Simulationslaufes erstellt wird. Die Anzahl der dabei zugrunde gelegten Intervalle ist vom Programm vorgegeben, die Bereichsgrenzen sind jedoch vor dem Simulationslauf nicht automatisch feststellbar. Obgleich hiermit eine gewisse Einschränkung der Benutzerfreundlichkeit des Programms einhergeht, wurde dieser Weg aus Gründen der programmtechnischen Vereinfachung gewählt. In der praktischen Anwendung wird der Entscheidungsträger zunächst einen Probedurchgang mit einer geringen Anzahl von Simulationsläufen durchführen, um so den Ergebnisbereich abschätzen zu können, bevor er das eigentliche Simulationsexperiment durchführt.

Für Sensitivitätsanalysen steht ein eigenes Teilprogramm zur Verfügung (vgl. Abbildung A4). Auch hier sind wiederum das Pull-Budget, die PushBudgets sowie die Handelsspanne frei wählbar. Jeweils eine dieser Größen kann in einem vom Entscheidungsträger vorzugebenden Intervall variiert werden. Neben den Intervallgrenzen ist dabei die Anzahl der Simulationsschritte anzugeben. Das Programm berechnet daraus die Schrittweite, mit der die betrachtete Größe innerhalb des gewünschten Bereiches verändert wird. Ausgehend von der unteren Grenze wird anschließend sukzessive für jeden der daraus resultierenden Werte des Intervalls eine eigenständige Simulation durchgeführt.

Die Ergebnisse der Sensitivitätsanalyse lassen sich graphisch darstellen. Hierzu werden die Erwartungswerte einer auszuwählenden Erwartungsvariablen in einem Koordinatensystem über das Intervall der variierten Größe abgetragen. Der Entscheidungsträger kann zwischen mehreren Erwartungsvariablen wählen. Als einzige Einschränkung ist zu beachten, daß die Ergebnisse jeweils nur für eine vor Beginn der Sensitivitätsanalyse festzulegende Periode 395 ausgewiesen werden können. Diese Verein-

395 Eine Ausnahme bildet der Markenwert, der sich stets auf den Beginn des Planungszeitraumes bezieht. 
fachung wurde ebenfalls aus programmtechnischen Gründen vorgenommen, um die Anzahl der zwischenzuspeichernden Werte zu begrenzen.

\section{Planung von Markendurchsetzungsstrategien mit dem Reifen- marktsimulationsmodell anhand eines fiktiven Beispiels}

Nachdem das Reifenmarktsimulationsmodell sowohl in seiner Konzeption als auch der computergestützten Implementierung vorgestellt ist, soll dessen Einsatz bei der Entscheidungsunterstützung nun anhand eines Beispiels verdeutlicht werden. Die Daten dieses Beispiels sind zu einem Teil den Marktforschungsstudien eines Reifenherstellers entnommen. Die Studien entstanden allerdings zu verschiedenen Zeitpunkten und vor jeweils unterschiedlichem Befragungshintergrund, so daß deren Ergebnisse nur bedingt kompatibel sind. Eine Situation, die in der Praxis der Markenführung nicht untypisch sein dürfte. Da sich überdies nicht alle benötigten Informationen aus den Studien ergaben und auf eine weitergehende empirische Erhebung verzichtet werden sollte, erhebt das vorliegende Beispiel keinen Anspruch darauf, die Realität einer bestimmten Marke abzubilden. Es wird vielmehr eine fiktive Marke betrachtet, deren Werte aber durchaus realistische Ausprägungen besitzen.

\subsection{Datensituation des Beispiels}

Bezüglich des Präferenzverhaltens der Endabnehmer (vgl. Abbildung A5) wird angenommen, daß 30-45\% der Käufer bereits mit einem bestimmten Kaufwunsch die Einkaufsstätte betreten und sich hierbei an der aktuell am Fahrzeug befindlichen Marke orientieren. Von denjenigen, die dabei eine Konkurrenzmarke präferieren, sind 7-10 \% zur Gruppe der Markenfixierten zu zählen. Zwischen $12 \%$ und $18 \%$ der Reifenkäufer besitzen eine bestimmte Präferenz, die unabhängig von der zum Kaufzeitpunkt montierten Reifenmarke ist. Deren Bindung an die gewünschte Marke dürfte im Durchschnitt höher sein als bei Personen, die sich nach der Reifenausstattung richten. Dementsprechend werden von diesen Käufern, soweit sie eine Konkurrenzmarke präferieren, $11-14 \%$ als markenfixiert eingeschätzt. 
Von den Endabnehmern, die die Marke i präferieren, da diese am Fahrzeug montiert ist, werden in der Ausgangssituation 5-8\% als markenfixiert und $19-25 \%$ als markenorientiert eingeschätzt (vgl. Abbildung A6). Diese Anteile lassen sich durch Pull-Maßnahmen beeinflussen. Innerhalb der Periode gelten 4-7 \% bzw. 14-20\% bei vollkommener Streichung des Pull-Budgets als Untergrenze und 7-10\% bzw. 28-30\% bei unbeschränkter Budgetausdehnung als Obergrenze. Als langfristige Untergrenze werden 3-6 \% bzw. 9$15 \%$ angenommen. Betrachtet man jeweils die absoluten Veränderungen, so reagiert der Anteil markenfixierter Käufer sehr unempfindlich gegenüber Pull-Maßnahmen, während sich der Anteil der Markenorientierten deutlich beeinflussen läßt.

Sehr viel stärker ist die Wirkung einer Variation des Pull-Budgets bei Personen, die sich unabhängig von der Reifenausstattung entscheiden (vgl. Abbildung A8). Diese Käufergruppe ist generell den Pull-Aktivitäten des Herstellers gegenüber aufgeschlossen. Damit läßt sich nicht nur unmittelbar Einfluß auf den Anteil der Käufer nehmen, die hiervon Marke i präferieren, sondern auch auf die Intensität, mit der sie die Kaufabsicht verfolgen. Bei der Marke i handelt es sich um einen Reifen, der bereits eine relativ hohe Nachfragestärke zeigt. Er wird in der Ausgangssituation von 16-19\% der sich unabhängig von der Reifenausstattung entscheidenden Käufer präferiert, die sich überdies zu 20-23\% markenfixiert und zu 42-48 \% markenorientiert verhalten. Dementsprechend ist das Steigerungspotential weitaus niedriger als die mögliche Verschlechterung der Präferenzwerte bei Absenkung des Pull-Budgets.

Gleiches gilt für die Akzeptanz, die seitens der Endabnehmer der Marke i entgegengebracht wird (vgl. Abbildung A9). Auch hier liegt ein hohes Ausgangsniveau vor. Zwischen $70 \%$ und $80 \%$ aller Käufer, die nicht die Marke i präferieren, würden sie dennoch akzeptieren, wenn vom Händler ein entsprechender Vorschlag unterbreitet würde. Die höchste Akzeptanz wird mit 77-80 \% für indifferente Käufer angenommen, da sie sich am stärksten an der Empfehlung des Händlers orientieren. Die geringste Akzeptanz wird mit 70-76\% den Käufern zugeschrieben, die unabhängig von der Reifenausstattung bereits eine Präferenz für eine andere Marke besitzen. Dem liegt der Gedanke zugrunde, daß sich diese Käufer in ihrem Kaufent- 
scheidungsproze $ß$ intensiver mit den verschiedenen Alternativen auseinandersetzen und daher eher in der Lage sein werden, eine Marke als für sich ungeeignet $\mathrm{zu}$ beurteilen. ${ }^{396}$ Für diese soll damit aber auch die höchste Sensitivität bezüglich Änderungen des Pull-Budgets angenommen werden, da sie wahrscheinlich aufmerksamer als andere Käufer die Herstelleraktivitäten verfolgen und sich in ihrer Kaufentscheidung daran orientieren.

Hinsichtlich der effektiven Verfügbarkeit wird unterstellt, daß bei einem Distributionsgrad von $54 \%$ durchaus $90-96 \%$ der markenfixierten Käufer die Marke i tatsächlich auch in der Einkaufsstätte antreffen (vgl. Abbildung A10). Geht man davon aus, daß sich die Distribution einigermaßen gleichmäßig über die Fläche verteilt, so scheint bei der hohen Zahl an Einkaufsstätten die Annahme realistisch, daß nahezu jeder Käufer, der eine bestimmte Marke sucht, diese letztlich auch findet. Auch für markenorientierte Käufer wird daher bei diesem Distributionsgrad mit 82-88 \% von einer hohen effektiven Verfügbarkeit ausgegangen. Die Werte für die Mindestund Höchstgrenzen der Verfügbarkeit in Abhängigkeit vom Distributionsgrad sind von der Natur der Beziehung her mit $0 \%$ bzw. $100 \%$ vorgegeben.

Für das Absatzmittlermodell sind die Wirkungen des Push-Budgets auf den Distributionsgrad und das Empfehlungsverhalten anzugeben. In der Ausgangssituation beträgt der Distributionsgrad zwischen $52 \%$ und $58 \%$ (vgl. Abbildung A11). Aufgrund der beschriebenen Konkurrenzsituation ist davon auszugehen, daß dieser am Ende der Periode, selbst bei hohen Anstrengungen, nicht höher als 57-63 \% sein wird. Demgegenüber würde der Handel auf eine Verringerung des Akquisitionsbudgets empfindlicher reagieren. Allerdings verbleibt selbst bei einer vollständigen Streichung der Marke noch ein Distributionsgrad von 40-46 \% bzw. langfristig von $24-30 \%$. Dies spricht für die Attraktivität oder die Bedeutung der Marke. Diese würde von einem Teil der Händler auch ohne direkte Anreize des Herstellers geführt.

396 Bei weitgehend unbekannten Marken kann allerdings auch eine genau umgekehrte Situation vorliegen. Während eine solche Marke evtl. als "Geheimtip" von kenntnisreichen Käufern akzeptiert würde, hätten unbedarfte Reifenkäufer Bedenken aufgrund des geringen Bekanntheitsgrades. 
Die Beziehung zwischen dem Förderungsbudget und dem Empfehlungsverhalten des Handels zeigt allerdings, daß es weniger die Attraktivität ist, die den Handel zu seinem Verhalten motiviert (vgl. Abbildung A12). Da der Umfang, in dem die Marke i dem Käufer empfohlen wird, stark von den fördernden Leistungen des Herstellers abhängt, scheinen vielmehr der hohe Präferenz- und Akzeptanzgrad die Händler dazu zu bewegen, die Marke i als durchaus wichtigen Bestandteil des Sortimentes zu verstehen. So sinkt die Empfehlungsrate gegenüber den Indifferenten bei Händlern, die die Marke i führen, von 28-34\% in der Ausgangssituation, kurzfristig auf 20-26 $\%$ und langfristig sogar auf $7-13 \%$, wenn keine entsprechende Förderung seitens des Herstellers mehr gegeben ist. Besonders deutlich zeigt sich die Wirkung einer Streichung des Förderungsbudgets auch für die Situation, daß der Reifenkäufer die Marke $i$ ursprünglich präferiert. Selbst wenn der Händler diese Marke führt, wird er langfristig lediglich in 60-66 \% der Fälle keine Gegenempfehlung für einen anderen, aus seiner Sicht lukrativeren Reifen aussprechen.

Das effektiv wirksame Push-Budget setzte sich aus dem monetären Budget, der Handelsspanne, der Präferenz des Endabnehmers sowie dessen Akzeptanz gegenüber der Marke zusammen. Von der Handelsspanne geht bei einem Wert von $35 \%$ weder eine positive, noch eine negative Wirkung auf die Effizienz des monetären Push-Budgets aus, d. $h$. monetäres und effektiv wirksames Budget entsprechen sich gerade (vgl. Abbildung A13). Bei einer Verringerung der Handelsspanne sinkt diese Effizienz stark, bis sie schließlich den Wert 0 annimmt. Nun wäre anzunehmen, daß sich bei einer wesentlichen Erhöhung der Handelsspanne, im Extremfall bis zu 100 \%, die Effizienz des monetären Push-Budgets um ein Vielfaches steigern müßte, da sich die Attraktivität der Marke für den Handel erheblich erhöht. Eine solch extreme Ausweitung der Handelsspanne ist bei gegebenem Endabnehmerpreis jedoch allein aufgrund der eigenen Kosten- und Gewinnanteile des Herstellers nicht realisierbar. Auch würde die vermeintliche Wirkung ausbleiben, da seitens der Konkurrenz in jedem Fall Gegenmaßnahmen zu erwarten wären. Daß diese Aspekte bei der Schätzung der maximal erreichbaren Effizienzwerte zu berücksichtigen sind, erklärt deren möglicherweise zunächst gering scheinende Ausprägung von 1,25-1,40 für die zumindest theoretisch mögliche Handelsspanne von $100 \%$. 
Die gleiche Argumentation gilt für die Effizienzwirkung der Präferenz (vgl. Abbildung A14). Auch hier ist ein Präferenzgrad von $100 \%$ nur theoretisch erreichbar, in nicht-monopolistischen Märkten jedoch vollkommen unrealistisch. Die Effizienzwerte wurden hierfür mit 1,25-1,40 bzw. 1,30-1,45 derart gewählt, daß der daraus resultierende Beziehungsverlauf in den sinnvollen Wertebereichen der Präferenz die vermutete Wirkung adäquat repräsentiert. Schließlich ist in diesem Zusammenhang die Effizienzwirkung der Akzeptanz von Marke i zu schätzen. Vom Ausgangswert aus betrachtet läßt sich mit einer Steigerung der Akzeptanz wiederum nur eine vergleichsweise geringe Effizienzerhöhung erreichen, während ein Absinken der Akzeptanz mit erheblichen Effizienzverlusten verbunden wäre (vgl. Abbildung A15). Der Handel scheint die bestehende Akzeptanz für Marke i somit als weitgehend ausreichend zu betrachten.

Bei den Budgetdaten wird für das Akquisitionsbudget eine Basis von 12 Mio. DM, für das Förderungsbudget von 15 Mio. DM und für das PullBudget von 11 Mio. DM in der ersten Periode zugrunde gelegt. Die jährliche Steigerungsrate liegt zwischen $1 \%$ und $3 \%$. Die Basis für die Handelsspanne beträgt $35 \%$. Fixe Herstellkosten fallen in der ersten Periode in Höhe von 20,5-22,0 Mio. DM an. Die variablen Herstellkosten betragen zum Startzeitpunkt der Planungsperiode 34,5-36,0 DM und der Endabnehmerpreis zum gleichen Zeitpunkt 106-112 DM. Für diese Werte gilt ebenfalls eine Steigerungsrate von 1-3\%. Als Kalkulationszinssatz werden $10 \%$ angesetzt (vgl. Abbildung A16).

Für das Marktvolumen wird von einem Absatz von 30 bis 33 Mio. Reifen für die erste Periode ausgegangen. Die jährliche Steigerung wird auf einen Wert zwischen $2 \%$ und $5 \%$ geschätzt (vgl. Abbildung A17). Hinsichtlich der Marktanteile vergangener Perioden könnte die Ansicht vertreten werden, daß auf stochastische Variablen zu verzichten ist, da die Werte in aller Regel vorliegen. Da aber auch hier Unsicherheiten bestehen können, die sich beispielsweise aus Fehlern in der Messung des Marktanteils ergeben, soll diese wenigstens in kleinen Schwankungsintervallen berücksichtigt werden (vgl. Abbildung A18). Insgesamt war der Marktanteil im Ersatzgeschäft in den vergangenen Perioden relativ stabil und lag zu den Zeitpunkten $t=0$ und $t=-1$ bei ca. $12,9-13,3 \%$. Der Marktanteil in der Erstausrüstung ist 
dagegen kontinuierlich gesunken. Für die Planungsperiode soll angenommen werden, daß sich der Anteil auf einem Niveau von 15,6-15,9\% stabilisiert. Für die Reifenlebensdauer wird eine Laufleistung von 38.000-48.000 $\mathrm{km}$ unterstellt, die jährliche Fahrleistung wird im Bereich von 0 bis 60.000 $\mathrm{km}$ angenommen. Da für letztere keine Trapezverteilung gilt, sind die Wahrscheinlichkeiten jeweils für die gebildeten Intervalle anzugeben. Die Mehrzahl der Reifen (38\%) wird demnach zwischen 15.000 und 20.000 km pro Jahr gefahren (vgl. Abbildung A19).

\subsection{Ausgangssimulation und Optimierung}

Die Simulation wird im ersten Schritt für eine Ausgangssituation durchgeführt, bei der alle Budgetindizes sowie der Index für die Handelsspanne auf den Wert 1 gesetzt werden. Die Wirkungsbeziehungen waren derart zu schätzen, daß in dieser Situation in etwa der Marktanteil der Vorperiode gehalten wird. Mit einem berechneten Marktanteil von 12,8 \% für den Zeitpunkt $t=1$ wird dieser Zielsetzung sehr gut entsprochen. Auch über den Planungszeitraum hinweg ist der Marktanteil für diese Ausgangssituation stabil. Es ist damit ein Gleichgewicht gefunden, das die ebenfalls recht stabile Situation der vergangenen Jahre kontinuierlich fortschreibt (vgl. Abbildung A2).

Die Periodengewinne steigen demgegenüber von Jahr zu Jahr. Hierin spiegeln sich allerdings die Steigerungsraten für die Budgetausgaben, für die Kosten und Endabnehmerpreise sowie für das Marktvolumen wider. Der erwartete Markenwert für die sieben Perioden beträgt mit einer $98 \%$ igen Vertrauenswahrscheinlichkeit 519,79 \pm 5,19 Mio. DM.

Über die Detailergebnisse läßt sich aussagen, daß diese sich ebenfalls stabil über die Planungsperioden hinweg verhalten. Etwa $8 \%$ der Käufer besitzen eine Präferenz für Marke i. Hiervon verhalten sich $12 \%$ markenfixiert, $30 \%$ markenorientiert und $58 \%$ markenbewußt. Eine Präferenz für andere Marken besitzen $45 \%$ der Käufer. Der Anteil indifferenter Reifenkäufer beträgt $47 \%$, wobei von diesen immerhin $78 \%$ auch die Marke i akzeptierten sofern sie vom Händler vorgeschlagen würden. Der erreichte 
Distributionsgrad beträgt $58 \%$, wodurch eine Verfügbarkeit von $94 \%$ für markenfixierte und $81 \%$ für markenorientierte Käufer hergestellt wird. In 90 $\%$ der Fälle spricht der Händler, der die Marke i im Sortiment führt, keine Gegenempfehlung aus, wenn der Kunde diese bereits beim Betreten der Einkaufsstätte präferiert. Er empfiehlt sie explizit in $7 \%$ der Fälle, in denen der Endabnehmer eine Präferenz für eine andere Marke besitzt und in $31 \%$ der Fälle, in denen es sich um indifferente Käufer handelt.

Auf dieser Ausgangssituation basierend wurde mit Hilfe der Sensitivitätsanalyse versucht, eine Ergebnisverbesserung zu erzielen. Als zu optimierende Zielgröße wurde dabei der Markenwert gewählt. Die einzelnen Simulationsläufe sind in den Abbildungen A20 bis A22 dargestellt. Ein weiterer Simulationslauf brachte keine weitere Verbesserung des Markenwertes.

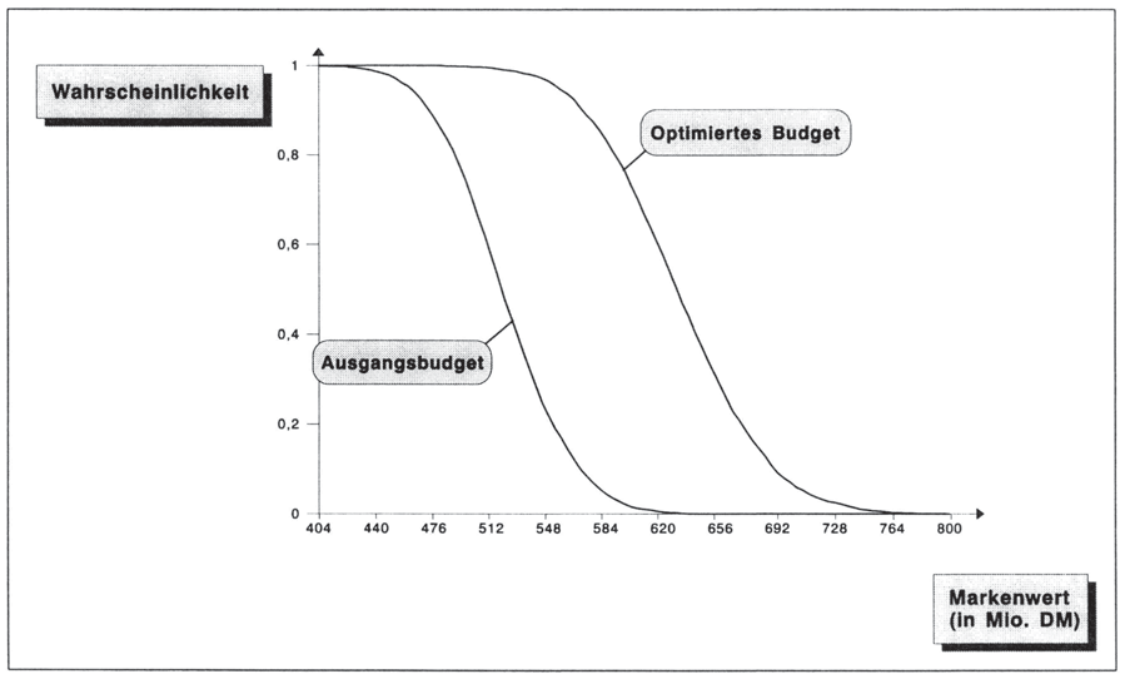

Abb. 49: Risikoprofile des Markenwertes bei Ausgangs- und optimiertem Budget

Mit den derart ermittelten Werten wurde daraufhin eine erneute Simulation durchgeführt. Es zeigt sich, daß nunmehr zum Zeitpunkt $t=1$ ein Marktanteil von $14,1 \%$ erreicht wird. Diesem Ergebnis zufolge führen die veränderten Budgets zu einer Marktanteilssteigerung um ca. $10 \%$ innerhalb der ersten Periode. Der zu optimierende Kapitalwert konnte ebenfalls erhöht werden. 


\section{Zusammenfassung und Ausblick}

\section{Zusammenfassende Würdigung der modellgestützten Planung von Markendurchsetzungsstrategien}

Angesichts der zunehmenden Komplexität markenpolitischer Entscheidungen und der gleichzeitigen Forderung, die Effizienz und Effektivität in der Markenpolitik zu steigern, war es die generelle Zielsetzung der vorliegenden Arbeit, einen Beitrag zur modellgestützten Planung von Strategien der Markendurchsetzung zu leisten. Dabei sollten als Grundlage die inhaltiche Analyse des Planungsgegenstandes sowie darauf aufbauend die Entwicklung eines eigenen Modells im Vordergrund stehen. Als situativer Kontext wurde der Untersuchung der Privatkundenbereich des deutschen Reifenmarktes zugrunde gelegt.

Im Rahmen der zunächst vorgenommenen Strukturierung des Planungsprozesses in der Markenpolitik wurden Strategien der Markendurchsetzung als Instrumentalstrategien charakterisiert, die der Durchsetzung markenpolitischer Grundsatzstrategien der Markierung und Positionierung dienen. Als Ausprägungsformen von Markendurchsetzungsstrategien konnten die Push- und die Pull-Orientierung identifiziert werden, die eine handelsbzw. endabnehmergerichtete Schwerpunktsetzung des Instrumenteeinsatzes bei der Markendurchsetzung beschreiben.

Zur Darstellung des Reifenmarktes als beispielhaft gewähltem situativen Kontext für die Planung von Markendurchsetzungsstrategien waren im Anschluß daran einzelne Teilmärkte voneinander abzugrenzen, die grundlegende Marktstruktur aufzuzeigen sowie die Wettbewerbssituation und die Marktentwicklung zu kennzeichnen. Im Mittelpunkt stand daraufhin die Untersuchung der für die Entscheidung über die Art der Markendurchsetzung relevanten Faktoren des Verhaltens von Endabnehmern und Handel.

Bezüglich des Endabnehmerverhaltens wurden hierzu das Bedarfsverhalten, das Einkaufsstättenwahlverhalten sowie die Form der genutzten Informationsquellen und des damit zusammenhängenden Markenwahlverhaltens analysiert. Hinsichtlich des Bedarfsverhaltens konnte gezeigt werden, daß die grundsätzlich für die Wahl der Markendurchsetzungsstrategie wichtige Frage, wie der Bedarf der Endabnehmer stimuliert werden kann, für den 
Reifenmarkt von untergeordneter Bedeutung ist. Das fehlende Interesse der Käufer an dem Produkt "Reifen" führt dazu, daß der Zeitpunkt der Wahrnehmung des Bedarfs technisch bedingt ist und weitgehend außerhalb des Einflußbereiches des Reifenherstellers liegt. Für die Einkaufsstättenwahl wurde deutlich, daß die Mehrzahl der Reifenkäufer sich bei ihrer Entscheidung an handelsspezifischen Leistungen orientiert und zudem eine hohe Bindung an den Händler des Vertrauens zeigt. Dennoch macht auch ein nicht unerheblicher Teil der Käufer die Verfügbarkeit einer bestimmten Reifenmarke zur Voraussetzung für die Wahl der Einkaufsstätte. Mit Blick auf die genutzten Informationsquellen und das daraus resultierende Markenwahlverhalten ließ sich feststellen, daß der eigenen positiven Erfahrung mit der Reifenmarke die höchste Bedeutung für die Kaufentscheidung zukommt. Ebenfalls als sehr wichtig wird die Empfehlung des Händlers für die Reifenmarke gesehen. Die endabnehmergerichtete Werbung des Reifenherstellers nimmt dagegen aus Sicht der befragten Reifenkäufer keinen wesentlichen Einfluß auf die Kaufentscheidung.

Für das Verhalten des Handels konnte dargestellt werden, daß sich der Reifenfachhandel eher in der Rolle des Marktgestalters als in der des Marktvermittlers sieht. Die Machtbeziehungen zwischen Hersteller und Handel zeigen eine relativ schwache Position der Reifenhersteller, die sich im wesentlichen aus der hohen Austauschbarkeit der Reifenmarken ergibt. Warenpräsentation und Bedienungsform sind im Reifenhandel durch die Fremdbedienung geprägt. Dies ermöglicht dem Händler Präferenz und Akzeptanz der verschiedenen Marken unmittelbar im Verkaufsgespräch zu prüfen und erhöht zudem seine Einflußmöglichkeiten auf die Kaufentscheidung des Endabnehmers.

Aufgrund der hohen Bedeutung, die seitens der Reifenhersteller dem Erstausrüstungsgeschäft zugemessen wird, war des weiteren der Zusammenhang zwischen Erstausrüstungs- und Ersatzmarkt kritisch zu überprüfen. Hierbei stellte sich heraus, daß weniger der durch einen "automatischen" Wiederkauf erreichte Marktanteil, als vielmehr die Auswirkungen auf die objektive und von seiten des Handels und der Endabnehmer wahrgenommenen Qualität der Reifen den Stellenwert der Erstausrüstung aus Sicht des Herstellers begründen. 
Die Festlegung markenpolitischer Ziele stellte den Ausgangspunkt der Planung von Markendurchsetzungsstrategien dar. Hier waren geeignete Zielgrößen auszuwählen und ein hierarchisch geordnetes Zielsystem aufzubauen. Als markenpolitisches Oberziel wurde der finanzwirtschaftlich definierte Markenwert herangezogen, da hierin letztlich das erwerbswirtschaftliche Interesse des Unternehmens an der Marke zum Ausdruck kommt und insofern eine direkte Anbindung markenpolitischer Ziele an übergeordnete Unternehmensziele erfolgt.

Spezifisch auf die Strategien der Markendurchsetzung abgestellt, wurden im Sinne von Unterzielen die Zielkonstrukte der Angebots- und Nachfragestärke eingeführt. Damit wurde zum einen eine Verknüpfung von Ziel- und Strategieentscheidungen erreicht. So ist die Angebotsstärke einer Marke als unmittelbares Ziel der push-orientierten Markendurchsetzungsstrategie zu verstehen, während die Erhöhung der Nachfragestärke das direkte Ziel der pull-orientierten Markendurchsetzung ist. Zum anderen konnte durch die Zusammenfassung verschiedener Merkmale der Handels- und Endabnehmerreaktionen unter diesen Konstrukten die inhaltliche Diskussion der Wirkungen push- und pull-orientierter Strategien der Markendurchsetzung besser veranschaulicht werden. Zur Präzisierung der Angebots- und Nachfragestärke wurden der Präferenz- und Akzeptanzgrad, die Präferenzintensität, der Distributionsgrad sowie die POS-Stärke als deren Teilziele definiert. In diesem Zusammenhang wurden drei Käufergruppen von Endabnehmern mit Präferenz für eine bestimmte Marke unterschieden. Abhängig vom Ausmaß des Beschaffungsaufwandes, den die Endabnehmer für den Kauf der Marke zu tragen bereit sind, und der Beeinflußbarkeit ihrer Präferenz am Point of Sale wurden die Gruppen der markenfixierten, -orientierten und -bewußten Reifenkäufer voneinander unterschieden.

Während markenpolitische Ziele anzustrebende Vorzugszustände definieren, bilden Grundsatzstrategien der Markierung und Positionierung strategische Rahmenbedingungen für die Markendurchsetzungsstrategie. Hier konnte aufgezeigt werden, daß die von den Reifenherstellern verfolgten Dachmarken- und Markenfamilienstrategien erhebliche Synergievorteile gegenüber Produktmarkenstrategien bieten. Allerdings ist bei der Mehrmarkenstrategie zu beachten, daß Reifenhersteller durch substitutive Beziehungen der eigenen Marken die relative Machtposition des Handels stärken und damit die Markendurchsetzung erschweren. Demgegenüber bieten komple- 
mentär positionierte Marken eines Herstellers dem Handel zusätzliche Anreize zu deren Aufnahme in das Sortiment, so daß sich die Markendurchsetzung erleichtert. Für den Zusammenhang von Positionierungs- und Markendurchsetzungsstrategien wurde herausgestellt, daß die Qualitäts- und Innovationsorientierung in aller Regel nicht durch das Produkt selbst kommunizierbar sind, sondern entweder durch die endabnehmergerichtete Kommunikation des Herstellers oder die intensive Beratung des Endabnehmers am Point of Sale dargestellt werden müssen. Zudem wurde deutlich, daß ein emotionaler Zusatznutzen gegenwärtig im Reifenhandel nur bedingt vermittelbar ist.

Die Markendurchsetzungsstrategien werden schließlich durch den Einsatz der Marketinginstrumente realisiert. Systematisiert nach Produkt-, Kommunikations-, Kontrahierungs- und Distributionsbereich wurde deren Einsatz als Push-Instrumente des Hinein- und Herausverkaufs sowie als PullInstrument für den Reifenmarkt dargestellt. Über die Beschreibung der Instrumenteinhalte hinaus war die Frage einer geeigneten Operationalisierung der Aktivitätsniveaus zu beantworten. Da bei Markendurchsetzungsstrategien der Instrumenteeinsatz auf einem sehr hohen Aggregationsniveau geplant wird, war es ausreichend, die Aktivitätsniveaus des Push- bzw. PullInstrumentariums durch den Umfang der für die jeweiligen Instrumente insgesamt zur Verfügung stehenden finanziellen Mittel in einer Periode zu beschreiben.

Für die Analyse der Beziehungen zwischen dem Push- und Pull-Instrumentarium und den markenpolitischen Zielen wurde zwischen direkten und indirekten Wirkungsbeziehungen sowie zeitlich-horizontalen und zeitlich-vertikalen Interdependenzen differenziert. Bei den direkten Wirkungsbeziehungen war vor allem die Existenz bestimmter Schwellenwerte für das Aktivitätsniveau des Push-Instrumentariums herauszuarbeiten. Deren Einhaltung ist die Voraussetzung für die Aufnahme einer Marke in das Sortiment durch den Händler. Bezüglich des Gesamtbudgets des Push-Instrumentariums konnte in diesem Sinne zwischen einem Akquisitions- und einem Förderungsbudget unterschieden werden.

Als indirekte Wirkungen waren solche zu identifizieren, die vom PushInstrumentarium über die Angebots- auf die Nachfragestärke ausgehen bzw. vom Pull-Instrumentarium über die Nachfrage- auf die Angebotsstärke. Auf 
deren Grundlage konnten anschließend zeitlich-horizontale Interdependenzen untersucht werden, die dazu führen, daß derselbe Marktanteil mit verschiedenen Kombinationen von Aktivitätsniveaus des Push- und PullInstrumentariums erreicht wird. Hier war insbesondere herauszustellen, daß bei einem vorgegebenen Marktanteil die erforderliche Höhe des Distributionsgrades und der POS-Stärke mit abnehmender Präferenzintensität der Marke steigt. Für die primär bei der Einführung neuer Marken oder neuer Produkte unter einer Marke bedeutsamen zeitlich-vertikalen Interdependenzen konnte die Problematik der sogenannten "Zeitfalle" aufgezeigt werden, bei der das durch eine pull-orientierte Markendurchsetzung erreichte anfängliche Interesse des Käufers schnell abflacht, wenn die Marke bzw. das Produkt nicht unmittelbar im Handel verfügbar ist. Zudem wurde festgestellt, daß sich durch eine hohe Angebotsstärke die vom Endabnehmer wahrgenommenen Kaufrisiken einer neuen Marke bzw. eines neuen Produktes verringern.

Im Anschluß an diese inhaltliche Analyse des Planungsgegenstandes war dann ein eigenes entscheidungsunterstützendes Modell für die Planung von Markendurchsetzungsstrategien im Reifenmarkt zu entwickeln. Als Ausgangspunkt wurden hierfür zunächst die zu beachtenden Strukturdefekte diskutiert und dabei herausgearbeitet, daß die Handhabung von Meß-, Prognose- und Wirkungsdefekten ein zentrales Problem bei der Entwicklung des Modells darstellt. Neben der Untersuchung bestehender Defekte waren Gestaltungsanforderungen an das Modell zu formulieren. Als leistungsbezogene Anforderungen wurden die Vollständigkeit, Validierbarkeit und prognostische Relevanz aufgeführt. Mit Blick auf die Benutzerorientierung waren Einfachheit, Benutzungssicherheit, Kontrollierbarkeit und Dialogfähigkeit des Modells zu fordern. Ferner wurden als design- und kostenbezogene Anforderungen die Wirtschaftlichkeit, Anpassungsfähigkeit und Integrationsfähigkeit des Modells genannt.

Vor dem Hintergrund der Strukturdefekte und formulierten Anforderungen waren im darauffolgenden grundlegende Ansätze zur Gestaltung von Modellen zu diskutieren und Implikationen für die eigene Modellentwicklung abzuleiten. Bereits an früherer Stelle war die Entscheidung getroffen worden, einen benutzerorientiert-subjektiven Ansatz der Modellbildung zu wählen, bei dem das mathematische Modell die internen Vorstellungen des Entscheidungsträgers von der Realität widerspiegelt und die Funktion eines 
"Intelligenzverstärkers" einnimmt. Dieser Ansatz verlangt im Hinblick auf den Aggregationsgrad die Entwicklung eines Makromodells. Um die Wirkungsdefekte nicht durch zusätzliche Erklärungsgleichungen zu erhöhen, wurde auf die Einführung intervenierender Variablen verzichtet und statt dessen ein globalanalytischer Ansatz mit verhaltensbezogenen Variablen gewählt. Zur expliziten Berücksichtigung von Meß-, Prognose- und Wirkungsdefekten wurde festgelegt, stochastische Variablen und Wirkungsbeziehungen in dem Modell zu verwenden. Da mit den Marketinginstrumenten und der Reifenausstattung des Fahrzeuges periodenübergreifende Effekte verbunden sind, war ferner ein dynamischer Modellansatz notwendig. Schließlich wurde ein differenziertes Vorgehen beim Detaillierungsgrad der Modellteile festgelegt und als Lösungstechnik aufgrund der stochastischen Elemente des Modells die Risikosimulation eingesetzt.

Über die grundlegenden Ansätze zur Gestaltung von Modellen hinaus stellte sich die Frage, in welcher konkreten Form eine geeignete modellhafte Abbildung des Planungsproblems zu erreichen ist. Um hierzu Ansatzpunkte und Anregungen für die eigene Modellentwicklung zu gewinnen, wurden drei Modelle der Literatur dahingehend untersucht, wie die Entscheidungstatbestände, situative Faktoren und Ziele als Variablen und deren Beziehungen als mathematische Funktionen abgebildet werden. Aufgrund ihrer verschiedenen thematischen Schwerpunkte wurden dazu das reifenmarktspezifische Modell der CIM-Devellopement, das auf die Abstimmung von Angebots- und Nachfragestärke ausgerichtete Modell von Farris, Olver und de Kluyver sowie das nach dem benutzerorientiert-subjektiven Ansatz von Little entwickelte Modell BRANDAID ausgewählt.

Angesichts der im Rahmen der Modellanalyse gewonnenen Erkenntnisse wurde eine modulare Modellstruktur mit einer Form der Variablenverknüpfung gewählt, wie sie sich in ähnlicher Form auch im BRANDAID-Modell findet. Ansatzpunkte für die Modellformulierung des Endabnehmerverhaltens lieferten das CIM-Devellopement-Modell mit der Unterscheidung von freier, spontaner und modulierter Nachfrage sowie das Modell von Farris, Olver und de Kluyver mit der Einführung der effektiven Verfügbarkeit. Bezüglich der Berücksichtigung von Marken der Wettbewerber konnte herausgearbeitet werden, daß sowohl die Abbildung jedes einzelnen Wettbewerbers als auch die Aggregation der Wettbewerbsmarken zu einer fiktiven Marke mit erheblichen Problemen behaftet ist. Aus diesem Grund wurde entschieden, 
auf eine explizite Abbildung des Verhaltens von Wettbewerbern zu verzichten und dieses statt dessen implizit in den Schätzungen des Entscheidungsträgers über die Wirkungen eigener Maßnahmen zu berücksichtigen. Allein aus Darstellungsgründen wurden die Konkurrenzmarken für die Modellentwicklung zu einer fiktiven Marke zusammengefaßt, die jedoch nur im Sinne einer Residualvariablen den von der eigenen Marke nicht erreichten Anteil der jeweiligen Erwartungsvariablen beschreibt.

Vor dem Hintergrund der Gestaltungsanforderungen sowie der gewonnenen Erkenntnisse über spezifische Möglichkeiten der konkreten Modellierung konnte die Entwicklung des eigenen Reifenmarktsimulationsmodells erfolgen. In einem ersten Teilmodell, dem Herstellerentscheidungsmodell, waren die vom Hersteller kontrollierbaren Entscheidungsvariablen der Markendurchsetzungsstrategie abzubilden. Hierzu wurden Variablen für das Pull-Budget sowie das Akquisitions- und Förderungsbudget definiert. Darüber hinaus wurden alle handelsgerichteten kontrahierungspolitischen Maßnahmen in der Handelsspanne zusammengefaßt, um hieraus anschließend das effektiv wirksame Push-Budget zu berechnen.

Ausgangspunkt des Endabnehmermodells war die Strukturierung des Reifenkaufprozesses in der Einkaufsstätte. Hier galt es zu berücksichtigen, daß sich Endabnehmer beim Reifenkauf sowohl an der bereits bestehenden Reifenausstattung des Fahrzeuges orientieren als auch unabhängig davon Präferenzen bilden. Die Präferenzen können dabei ebenso für die betrachtete Marke als auch für eine fiktive Konkurrenzmarke bestehen und entsprechend der Unterscheidung von markenfixierten, -orientierten und -bewußten Käufern unterschiedlich stark ausgeprägt sein. Ferner waren Reifenkäufer zu beachten, die beim Betreten der Einkaufsstätte indifferent im Hinblick auf die Markenwahlentscheidung sind, und es war der Fall zu erfassen, daß Käufer zwar keine Präferenz für die betrachtete Marke besitzen, diese aber als Kaufalternative akzeptieren würden. Hieraus konnten schließlich fünf Käufergruppen gebildet werden, deren Präferenz und Akzeptanz für die Marke durch das Pull-Budget des Herstellers sowie die zum Kaufzeitpunkt am Fahrzeug montierte Marke bestimmt werden.

Im Absatzmittlermodell waren der Distributionsgrad und die POS-Stärke der Marke in Abhängigkeit von den Push-Budgets des Herstellers abzubilden. Dabei war zu beachten, daß sich mit zunehmender Akzeptanz und 
Präferenz der Reifenkäufer für die Marke die Bereitschaft des Handels zu deren Aufnahme und Förderung erhöht. Um diesen Zusammenhang zu beschreiben, wurden zusätzliche Effizienzindizes eingeführt. Diese beeinflussen das bereits im Herstellerentscheidungsmodell berechnete effektiv wirksame Push-Budget im Hinblick auf die Relation von Aktivitätsniveau und erreichtem Distributionsgrad bzw. erreichter POS-Stärke. Ferner war zu berücksichtigen, daß die Wirkung des Förderungsbudgets abhängig ist vom Distributionsgrad. Eine Korrektur des effektiv wirksamen Förderungsbudgets wurde in dem Maße vorgenommen, in dem eine Veränderung des Akquisitionsbudgets den Distributionsgrad beeinflußt.

Im Marktanteilsmodell erfolgte die Zusammenführung der ermittelten Ergebnisse des Endabnehmer- und Absatzmittlermodells. Dazu waren zunächst die effektive Verfügbarkeit einer Marke zu modellieren und Annahmen über das Verhalten des Reifenkäufers gegenüber Empfehlungen des Händlers zu treffen. Anschließend konnte der Marktanteil auf Basis der berechneten Teilwahrscheinlichkeiten für Präferenz und Akzeptanz gegenüber einer Marke, der effektiven Verfügbarkeit sowie des Empfehlungsverhaltens des Händlers bestimmt werden.

Das Teilmodell zur Reifenausstattung wurde entwickelt, um den zum Kaufzeitpunkt bestehenden Anteil der Marke an der Reifenausstattung der Fahrzeuge festzustellen. Hier konnte gezeigt werden, daß die vielfach übliche Form der Abbildung periodenübergreifender Beziehungen nur unter bestimmten Annahmen zulässig ist. Daher wurde hier auf Basis der Lebensdauer von Reifen und der Fahrleistungen der Reifenkäufer eine Rückrechnung vom Kaufzeitpunkt auf den genauen Zeitpunkt des letzten Reifenwechsels vorgenommen. Die Marktanteile einer Marke, wie sie zu diesem Zeitpunkt im Erstausrüstungs- und Ersatzgeschäft bestanden haben, sowie das Verhältnis von Neufahrzeug- zum Gesamtfahrzeugbestand bestimmen dann den Anteil der Marke an den montierten Reifen zum Kaufzeitpunkt.

Unter Berücksichtigung des im Marktvolumensmodell ermittelten Absatzvolumens, der fixen und variablen Herstellungskosten sowie der Handelsspanne konnten schließlich im Gewinnmodell die Periodengewinne und der Markenwert berechnet werden. In diesem Zusammenhang war auch auf das Konzept der Erfahrungskurve einzugehen, das sich in der Literatur häufig zur Prognose der Entwicklung von Herstellungskosten findet. Es konnte 
gezeigt werden, daß dieses Konzept für den vorliegenden Fall einer markenbezogenen Betrachtung des Absatzes, hohen kumulierten Produktionsmengen der Reifenhersteller in der Vergangenheit und einem weitgehend stagnierenden Markt nicht geeignet ist.

Nachdem die Variablen und deren grundlegenden Beziehungen mit der Entwicklung der Teilmodelle definiert waren, mußten die Ausprägungen der Eingangsvariablen ermittelt sowie die Parameter der Erklärungsgleichungen bestimmt werden. Zur Ermittlung von Werten der Eingangsvariablen war es zunächst erforderlich, zwischen dem subjektivistischen und dem objektivistischen Wahrscheinlichkeitsbegriff zu differenzieren. Ersterer wurde in Konsequenz des benutzerorientiert-subjektiven Ansatzes der Modellgestaltung für diese Arbeit gewählt. Darüber hinaus war bei der Interpretation der Wahrscheinlichkeitsverteilungen zwischen der Häufigkeitsverteilung und der Glaubwürdigkeitsverteilung zu unterscheiden. Ferner standen zur Bestimmung der Wahrscheinlichkeitsverteilungen sowohl die Möglichkeit des freien Funktionsverlaufes als auch die Verwendung theoretischer Verteilungen zur Wahl. Aufgrund der geringeren Komplexität wurde entschieden, bis auf eine Ausnahme alle Variablen durch eine theoretische Verteilung zu beschreiben. Als hierfür geeignete Wahrscheinlichkeitsverteilung konnte die Trapezverteilung identifiziert werden, die sich aus vier, für den Entscheidungsträger sehr anschaulichen Datenpunkten ermitteln läßt.

Für die Bestimmung von Parametern der Erklärungsgleichungen wurde auf den von Little im Rahmen des ADBUDG-Modell verwendeten Ansatzes der subjektiven Funktionsschätzung zurückgegriffen. Das aus einer Response- und einer Verfallsfunktion bestehende Modell mit s-förmigem oder konkavem Funktionsverlauf erfuhr dabei zwei zentrale Erweiterungen. Die erste Erweiterung bezog sich darauf, auch solche Variablen als unabhängige Größen der Funktion zuzulassen, die in ihrem Wertebereich begrenzt sind. Bei der zweiten Erweiterung handelte es sich um die explizite Berücksichtigung der Unsicherheit in den Wirkungsbeziehungen. Die deterministische Funktion wurde damit zu einer stochastischen Beziehung weiterentwickelt, die auf der bereits bekannten Trapezverteilung aufbaut.

Zur Berechnung der Modellergebnisse wurde die Risikosimulation herangezogen, die in der Literatur auch als Monte-Carlo-Simulation bezeichnet wird. Aufbauend auf den grundlegenden Ablaufschritten der Risikosimulation 
waren hier wiederum zwei Erweiterungen vorzunehmen. Diese wurden aufgrund der Art der verwendeten Wahrscheinlichkeitsverteilungen sowie der Berücksichtigung stochastischer Wirkungszusammenhänge notwendig. Da in dem Modell neben den Glaubwürdigkeitsverteilungen auch eine Häufigkeitsverteilung Verwendung fand, war die Risikosimulation in eine Unterund eine Hauptsimulation aufzuspalten. Während in der Untersimulation zunächst die Werte derjenigen Variablen berechnet werden, denen Häufigkeitsverteilungen zugrunde liegen, werden im Rahmen der eigentlichen Hauptsimulation die Werte aller Variablen ermittelt, die auf Glaubwürdigkeitsverteilungen beruhen. Da das Modell darüber hinaus stochastische Beziehungen enthält, waren zwei weitere Teilschritte zur Berechnung der Werte von Erwartungsvariablen einzuführen. Erstens war für jede stochastische Beziehung die für den Wert der unabhängigen Variable gültige Wahrscheinlichkeitsverteilung zu berechnen und zweitens aus dieser Verteilung ein Zufallswert zu ermitteln, der das Ergebnis der Erwartungsvariablen darstellt.

Im Hinblick auf die notwendige Anzahl von Simulationsläufen konnte auf die Regeln der induktiven Statistik zur Bestimmung des Stichprobenumfangs zurückgegriffen werden. Da sich der notwendige Stichprobenumfang bei gegebenem Konfidenzintervall und festgelegter Vertrauenswahrscheinlichkeit jedoch erst festlegen läßt, wenn die Standardabweichung der Stichprobe bekannt ist, wurde auf deren automatische bzw. halbautomatische Berechnung durch das Modell verzichtet. Statt dessen wird die Vertrauenswahrscheinlichkeit für das vorgegebene Konfidenzintervall ausgewiesen, so daß der Entscheidungsträger selbst darüber befinden kann, ob er die erreichte Vertrauenswahrscheinlichkeit als ausreichend betrachtet oder aber die Zahl der Simulationsläufe erhöht. Die Berechnung der Vertrauenswahrscheinlichkeit wurde auf Basis der sogenannten Tschebyschew-Ungleichung vorgenommen, die relativ geringe Anforderungen an die Annahmen bezüglich der zugrundeliegenden Wahrscheinlichkeitsverteilungen stellt.

Die mit der Risikosimulation berechneten Erwartungsvariablen sind durch die zwei Dimensionen der Höhe und der Eintrittswahrscheinlichkeit der Werte beschrieben. Zur Interpretation der Ergebnisse und der Entscheidung für eine der Alternativen lassen sich diese zum einen als Risikoprofile darstellen. Hier wurde empfohlen, die Alternativen zunächst auf gegenseitige Dominanz zu prüfen und zudem Mindestanforderungen im Hinblick 
auf Eintrittswahrscheinlichkeiten und Höhe der Ergebniswerte zu formulieren. Zum anderen wurden Möglichkeiten aufgezeigt, die Regeln der klassischen Entscheidungstheorie sowie des Risikonutzens zur Bewertung und Auswahl heranzuziehen. Die schließlich gewählte Handlungsalternative stellt eine Ausgangslösung für die Planung von Markendurchsetzungsstrategien dar. Um die Stabilität der Lösung zu prüfen, alternative Umweltsituationen zu analysieren und Verbesserungen dieser Lösungen zu erreichen, war die Sensitivitätsanalyse zu erläutern. In diesem Zusammenhang wurden verschiedene Möglichkeiten des Einsatzes der Sensitivitätsanalyse aufgezeigt und dabei insbesondere das Vorgehen der Einzelschrittmethode dargestellt. Mit dieser Methode konnte gezeigt werden, wie eine schrittweise Verbesserung der Ausgangslösung bis hin zu einem (zumindest lokalen) Optimum erreichbar ist.

Zusätzlich zur theoretischen Konzeption wurde das Modell im Rahmen dieser Arbeit auch als computergestütztes Simulationsprogramm implementiert. Um eine hohe Flexibilität bei der Implementierung und eine benutzerfreundliche Gestaltung des Programms zu erreichen, wurde dabei zur Programmierung anstelle einer spezifischen Simulationssprache eine Universalsprache (PASCAL 6.0) verwendet. Für die Dateneingabe stellt das Programm dem Entscheidungsträger verschiedene Bildschirmmasken bereit, in die die erforderlichen Angaben übersichtlich eingetragen werden können. Um die Anzahl der notwendigen Eintragungen auf ein überschaubares Maß zu reduzieren und das Simulationsprogramm für den Entscheidungsträger handhabbar zu gestalten, waren dabei Vereinfachungen bezüglich der Datenanforderungen des Modells vorzunehmen.

Zur Simulationssteuerung erlaubt das Programm, die Entscheidungsvariablen des Pull-Budgets, des Akquisitions- und Förderungsbudgets sowie der Handelsspanne beliebig zu variieren und miteinander zu kombinieren. Neben einer tabellarischen Ausgabe der Ergebnisse lassen sich für den Markenwert sowie die Marktanteile der Perioden das Risikoprofil und die Dichtefunktion graphisch darstellen. Im Rahmen der Sensitivitätsanalyse bietet das Simulationsprogramm die Möglichkeit, die einzelnen Budgets sowie die Handelsspanne innerhalb anzugebender Bereiche frei zu variieren. Der daraus resultierende Funktionsverlauf der Erwartungswerte kann für alle Erwartungsvariablen graphisch dargestellt werden. 
Schließlich war in einem letzten Schritt der Einsatz des Programms anhand eines fiktiven Beispiels zu veranschaulichen. Auf Grundlage einer beschriebenen Datensituation wurde dazu zuerst eine Ausgangssimulation mit den gegenwärtig bestehenden Budgets durchgeführt. Hierbei zeigte sich eine stabile Situation der Art, daß in den Folgeperioden nahezu keine Marktanteilsveränderungen stattfanden. Anschließend wurde versucht, mit Hilfe der Sensitivitätsanalyse zu einer Verbesserung der Ausgangslösung zu gelangen. Im Ergebnis konnten hiermit ein Marktanteilszuwachs um ca. $10 \%$ und eine Verbesserung des Markenwertes um etwa $20 \%$ erreicht werden.

\section{Implikationen für die Unternehmenspraxis}

Die Analyse des Planungsgegenstandes von Markendurchsetzungsstrategien hat gezeigt, daß Entscheidungsträger in realen Planungssituationen der Markenpolitik eine Vielzahl externer und interner Einflußfaktoren berücksichtigen müssen, die zudem durch verschiedene Wechselwirkungen gekennzeichnet sind. Die hohe Komplexität des Planungsprozesses dürfte daher im Rahmen intuitiver, nicht durch geeignete Planungsinstrumente unterstützte Entscheidungsprozesse zumeist nur zu suboptimalen Lösungen hinsichtlich der Durchsetzung übergeordneter markenpolitischer Entscheidungen führen. In diesem Sinne hat die vorliegende Arbeit aufgezeigt, daß Planungsabläufe durch Entscheidungsunterstützungsmodelle sinnvoll ergänzt werden können. Vor diesem Hintergrund ergeben sich für die Unternehmenspraxis beim Einsatz derartiger Modelle im Rahmen der markenpolitischen Planung folgende Implikationen:

- Zunächst bietet sich die Nutzung von branchenspezifisch konzipierten, computergestützten Simulationsmodellen an, um auf diesem Wege die Ergebnisse komplexer Entscheidungsprozesse im Rahmen der Markenführung einerseits zu validieren und andererseits die Prozesse aus Sicht der Entscheidungsträger transparenter zu gestalten. Die Simulationsergebnisse sind dabei unmittelbar für Entscheidungen über die Verteilung von Marketingbudgets auf handels- und endverbraucherorientierte Instrumente geeignet und dienen zur besseren Abstimmung von Angebots- und Nachfragestärke als den zentralen Zielgrößen der Markendurchsetzung. 
- Eng damit verbunden ist die Erfüllung einer Intelligenzverstärkerfunktion durch die explizite Erfassung und den Realitätsabgleich (Validierung) der internen, vom Entscheider implizit im Entscheidungsprozeß zugrunde gelegten Vorstellungen über die Struktur des relevanten Marktes und das Verhalten der Marktteilnehmer.

- Bei längerfristiger Nutzung von Simulationsmodellen läßt sich durch unternehmensspezifische Längsschnittanalysen eine bessere Kalibrierung der Modellparameter und Wirkungsbeziehungen erreichen. Im Ergebnis kann dies zum Aufbau eines unternehmensindividuell ausgerichteten Entscheidungsunterstützungssystems führen, das durch Know-how-Vorteile letztlich sogar in Wettbewerbsvorteile mündet und zur nachhaltigen Erhöhung der Effizienz von wiederkehrenden markenpolitischen Entscheidungen beitragen kann.

\section{Implikationen für die weitere Forschung}

Modelltheoretische Arbeiten haben eine lange Tradition in der Fundierung betriebswirtschaftlicher Entscheidungen. Dies gilt auch und insbesondere für den Bereich der modellgestützten Marketingplanung. Dennoch haben sich modellgestützte Ansätze in der Unternehmenspraxis bislang noch nicht auf breiter Front durchgesetzt. Es war daher ein wesentliches Anliegen der vorliegenden Arbeit, durch ihre branchenspezifische Ausrichtung die Einsatzfähigkeit von Entscheidungsunterstützungsmodellen in der Markenpolitik nachzuweisen und damit ihre praktische Relevanz zu dokumentieren.

Angesichts eines notwendigerweise eng gewählten Problem- und Branchenfokusses und gleichzeitig vielfältigen Einsatzmöglichkeiten und Ausgestaltungsformen von Entscheidungsunterstützungsmodellen in der Unternehmenspraxis ergeben sich für die weiterführende wissenschaftliche Forschung folgende Implikationen bzw. Aufgaben:

- Die Konzeption des Reifenmarktsimulationsmodells hat aufgezeigt, daß die Einsatzfähigkeit eines Modells wesentlich von der Erfassung der Marktstruktur und des Marktteilnehmerverhaltens eines spezifischen Branchenkontextes abhängt. Die Entwicklung branchenübergreifend einsetzbarer Modelle erscheint daher nicht sinnvoll realisierbar. Statt dessen 
ist die Konzeption und Bereitstellung weiterer branchenspezifischer Simulationsmodelle zur Entscheidungsunterstützung im Bereich der Markenpolitik anzuraten.

- Im vorliegenden Simulationsmodell wurde ein Teil der Kontextfaktoren lediglich implizit über die Parameterschätzungen des Entscheidungsträgers berücksichtigt. Änderungen der Kontextfaktoren und ihre Auswirkungen auf die Erwartungsvariablen werden daher im Rahmen der Modellanwendung nicht hinreichend transparent abgebildet. Vor diesem Hintergrund erscheint die explizite Einbeziehung von Kontextfaktoren in die Modellspezifikation wünschenswert.

- Über die subjektive Schätzung des Entscheidungsträgers hinaus kann eine bessere Kalibrierung der den Modellen zugrundeliegenden Parameter und Wirkungsfunktionen durch die Einbeziehung von Marktforschungsdaten erreicht werden. Zu denken wäre hier beispielsweise an die Integration von Marktforschungsdaten zum Informations- und Kaufverhalten von Konsumenten.

- Um die Modellkomplexität gering zu halten, bietet sich eine Reduzierung der Modellstruktur auf der Basis vorgelagerter Erfolgsfaktoren-Studien an. Auf diesem Wege könnte z. B. die Auswahl der in ein Simulationsmodell einzubeziehenden Marketinginstrumente auf diejenigen beschränkt werden, die einen besonders hohen Erfolgseinfluß aufweisen.

- Schließlich empfiehlt sich die Entwicklung von Entscheidungsunterstützungsmodellen auch für den Bereich übergeordneter Markierungsstrategien (z. B. Einmarken- vs. Mehrmarkenstrategien). Eine Verknüpfung dieser Modelle mit Simulationsmodellen auf der Markendurchsetzungsebene führt im Ergebnis zu hierarchisch strukturierten Modellen zur integrierten Planung markenpolitischer Entscheidungen. 


\section{Anhang}

Michael Szeliga - 978-3-631-75081-0

Downloaded from PubFactory at 01/11/2019 08:32:20AM

via free access 


\section{Verzeichnis des Anhangs}

Abb. A1: Hauptmenü des Reifenmarktsimulationsmodells

Abb. A2: Eingabemaske zur Steuerung der Simulation und Ergebnisse der Ausgangssimulation

Abb. A3: Datenmaske zur Ausgabe der Detailergebnisse und Ergebnisse der Ausgangssimulation

Abb. A4: Eingabemaske zur Steuerung der Sensitivitätsanalyse 246

Abb. A5: Daten zum Präferenzverhalten der Endabnehmer 247

Abb. A6: Daten zur Wirkung des Pull-Budgets auf die Präferenz der Endabnehmer (Präferenz für Marke i abhängig von montierter Marke)

Abb. A7: Zweidimensionale Darstellung des geschätzten stochastischen Zusammenhanges zwischen Pull-Budget (Index) und Präferenz 248

Abb. A8: Daten zur Wirkung des Pull-Budgets auf die Präferenz der Endabnehmer (Präferenz für Marke i unabhängig von montierter Marke)

Abb. A9: Daten zur Wirkung des Pull-Budgets auf die Akzeptanz der Endabnehmer

Abb. A10: Daten zum Zusammenhang von Distributionsgrad und effektiver Verfügbarkeit.

Abb. A11: Daten zur Wirkung des Push-Budgets auf den Distributionsgrad..... 
Abb. A12: Daten zur Wirkung des Push-Budgets auf die Förderung der Marke im Handel.

Abb. A13: Daten zum Zusammenhang von Handelsspanne und dem effektiv wirksamen Push-Budget.

Abb. A14: Daten zum Zusammenhang von Präferenz und dem effektiv wirksamen Push-Budget.

Abb. A15: Daten zum Zusammenhang von Akzeptanz und dem effektiv wirksamen Push-Budget. 252

Abb. A16: Angaben zu Budgets, Handelsspanne, Kosten und Endabnehmerpreis. 252

Abb. A17: Daten zur Entwicklung des Marktvolumens 253

Abb. A18: Daten zu Marktanteilen aus den Vorperioden sowie Anteil der Erstausrüstung im Planungszeitraum

Abb. A19: Daten zur Reifenlebensdauer und der Fahrleistung pro Jahr

Abb. A20: Sensitivitätsanalyse zur Verbesserung der Kombination von Aktivitätsniveaus des Push- und Pull-Instrumentariums (Durchgang 1)..... 255

Abb. A21: Sensitivitätsanalyse zur Verbesserung der Kombination von Aktivitätsniveaus des Push- und Pull-Instrumentariums (Durchgang 2) 256

Abb. A22: Sensitivitätsanalyse zur Verbesserung der Kombination von Aktivitätsniveaus des Push- und Pull-Instrumentariums (Durchgang 3). 257

Abb. A23: Ergebnisse der Simulation und Optimierung der Budgets ..... 258 
Abb. A24: Detailergebnisse der Simulation nach Optimierung der Budgets..

Trapezvert.: Berechnung der Trapezverteilung 259 


\begin{tabular}{|l|}
\hline \multicolumn{1}{|c|}{ Reifenmarktsimulation } \\
\hline Simulationssteuerung \\
Sensitivitätsanalyse \\
Präferenzuerhalten der Endabnehmer (1) \\
Präferenzuerhalten der Endabnehmer (2) \\
Präferenzuerhalten der Endabnehmer (3) \\
Akzeptanzuerhalten der Endabnehmer \\
Uerfugbarkeit der Marke \\
Distributionsgrad \\
Förderung der Marke im Handel \\
Effektiv wirksames Push-Budget (1) \\
Effektiv wirksames Push-Budget (2) \\
Effektiv wirksames Push-Budget (3) \\
Budget-, Preis und Kostendaten \\
Entwicklung des Marktvolumens \\
Marktanteile der Vorperioden \\
Reifenlebensdauer und Fahrleistung \\
Daten Speichern \\
Daten Laden \\
Ende
\end{tabular}

Abb. A1: Hauptmenü des Reifenmarktsimulationsmodells

\begin{tabular}{|c|c|c|c|c|c|c|c|c|}
\hline \multicolumn{9}{|c|}{ Simulation } \\
\hline \multicolumn{2}{|l|}{ Budget } & Bas is & Si mu 1. & & Simulation & K-Int. & Min. & $\operatorname{Max}$ \\
\hline \multirow{2}{*}{\multicolumn{2}{|c|}{$\begin{array}{l}\text { Push-Budget (Akqui.) } \\
\text { Push-Budget (Förder.) } \\
\text { Handelsspanne }\end{array}$}} & \multirow{2}{*}{$\begin{array}{r}12.00 \\
15.00 \\
0.35\end{array}$} & \multirow{2}{*}{$\begin{array}{l}1.00 \\
1.00 \\
1.00\end{array}$} & & $\begin{array}{l}\text { Marktante il } \\
\text { Markenwert }\end{array}$ & $\begin{array}{l}0.010 \\
0.010\end{array}$ & \begin{tabular}{l|l}
0 & 0.000 \\
0 & 400.0
\end{tabular} & $\begin{array}{l}0.080 \\
880.0\end{array}$ \\
\hline & & & & & \multirow{2}{*}{$\begin{array}{l}S-\text { Haupt lauf } \\
S-\text { Unter lauf }\end{array}$} & & & зяดด \\
\hline \multicolumn{2}{|l|}{ Pull-Budget } & 11.00 & 1.00 & & & & & 28 \\
\hline Zum Zeitpunkt & MW & 1 & 2 & & 4 & 5 & 6 & $?$ \\
\hline $\begin{array}{l}\text { Marktanteil } \\
\text { - Erwartungs. } \\
\text { - Standardab. } \\
\text { - Vertrauens. }\end{array}$ & & $\begin{array}{r}0.1282 \\
0.8058 \\
0.99\end{array}$ & $\begin{array}{r}0.1294 \\
0.0057 \\
0.99\end{array}$ & $\begin{array}{r}0.1290 \\
0.0858 \\
0.99\end{array}$ & $\begin{array}{r}0.1286 \\
0.0059 \\
0.99\end{array}$ & $\begin{array}{r}0.1286 \\
0.0868 \\
0.99\end{array}$ & $\begin{array}{r}0.1285 \\
0.8061 \\
0.99\end{array}$ & $\begin{array}{r}0.1284 \\
0.0062 \\
0.99\end{array}$ \\
\hline $\begin{array}{l}\text { MW/Gewinn } \\
\text { - Erwartungs. } \\
\text { - Standardab. } \\
\text { - Vertrauens. }\end{array}$ & $\begin{array}{r}519.79 \\
36.84 \\
0.98\end{array}$ & $\begin{array}{r}87.24 \\
5.79 \\
0.99\end{array}$ & $\begin{array}{r}93.60 \\
7.86 \\
0.98\end{array}$ & $\begin{array}{r}181.45 \\
8.54 \\
0.98\end{array}$ & $\begin{array}{r}188.78 \\
9.39 \\
0.98\end{array}$ & $\begin{array}{r}116.82 \\
10.19 \\
0.97\end{array}$ & $\begin{array}{r}125.62 \\
11.96 \\
0.97\end{array}$ & $\begin{array}{r}134.78 \\
12.84 \\
0.97\end{array}$ \\
\hline
\end{tabular}

Abb. A2: Eingabemaske zur Steuerung der Simulation und Ergebnisse der Ausgangssituation 


\begin{tabular}{|c|c|c|c|c|c|c|c|}
\hline \multicolumn{8}{|c|}{ Detailergebnisse } \\
\hline Zuw Zeitpunkt & 1 & 2 & 3 & 4 & 5 & 6 & 7 \\
\hline $\begin{array}{l}\text { Präferenz fuir Marke } \\
\text { davon Markenf ixierte } \\
\text { Markenorientierte } \\
\text { Markenbewuste }\end{array}$ & $\begin{array}{l}0.0752 \\
0.1174 \\
0.3007 \\
0.5819\end{array}$ & $\begin{array}{l}0.0766 \\
0.1163 \\
0.2992 \\
0.5845\end{array}$ & $\begin{array}{l}0.0760 \\
0.1170 \\
0.2996 \\
0.5834\end{array}$ & $\begin{array}{l}0.0755 \\
0.1173 \\
0.3001 \\
0.5826\end{array}$ & $\begin{array}{l}0.0754 \\
0.1174 \\
0.3003 \\
0.5823\end{array}$ & $\begin{array}{l}0.0752 \\
0.1174 \\
0.3005 \\
0.5821\end{array}$ & $\begin{array}{l}0.0752 \\
0.1174 \\
0.3003 \\
0.5823\end{array}$ \\
\hline $\begin{array}{l}\text { Präferenz andere Marke } \\
\text { davon Akzeptanz Marke }\end{array}$ & $\begin{array}{l}0.4503 \\
0.6801\end{array}$ & $\begin{array}{l}0.4473 \\
0.6798\end{array}$ & $\begin{array}{l}0.4487 \\
0.6801\end{array}$ & $\begin{array}{l}0.4497 \\
0.6797\end{array}$ & $\begin{array}{l}0.4496 \\
0.6802\end{array}$ & $\begin{array}{l}0.4489 \\
0.6798\end{array}$ & $\begin{array}{l}0.4496 \\
0.6802\end{array}$ \\
\hline $\begin{array}{l}\text { Ind ifferente } \\
\text { davon Akzeptanz Marke }\end{array}$ & $\begin{array}{l}0.4745 \\
0.7848\end{array}$ & $\begin{array}{l}0.4761 \\
0.7849\end{array}$ & $\begin{array}{l}0.4753 \\
0.7849\end{array}$ & $\begin{array}{l}0.4748 \\
0.7849\end{array}$ & $\begin{array}{l}0.4750 \\
0.7848\end{array}$ & $\begin{array}{l}0.4759 \\
0.7849\end{array}$ & $\begin{array}{l}0.4751 \\
0.7849\end{array}$ \\
\hline $\begin{array}{l}\text { Distributionsgrad } \\
\text { Uerfugbar flir Markenfix. } \\
\text { Markenorien. }\end{array}$ & $\begin{array}{l}0.5838 \\
0.9413 \\
0.8069\end{array}$ & $\begin{array}{l}0.5842 \\
0.9417 \\
0.8069\end{array}$ & $\begin{array}{l}\text { D. } 5844 \\
\text { D. } 9416 \\
0.8074\end{array}$ & $\begin{array}{l}0.5844 \\
0.9418 \\
0.8077\end{array}$ & $\begin{array}{l}0.5842 \\
0.9413 \\
0.8075\end{array}$ & $\begin{array}{l}0.5842 \\
0.9414 \\
0.8071\end{array}$ & $\begin{array}{l}0.5838 \\
0.9414 \\
0.8069\end{array}$ \\
\hline $\begin{array}{l}\text { Keine Gegenempfehlung } \\
\text { Empfehlung bei anderer } P \text {. } \\
\text { Emf pehlung bei Indifferenz }\end{array}$ & $\begin{array}{l}0.8976 \\
0.0708 \\
0.3125\end{array}$ & $\begin{array}{l}0.8975 \\
0.0715 \\
0.3127\end{array}$ & $\begin{array}{l}0.8977 \\
0.0716 \\
0.3123\end{array}$ & $\begin{array}{l}0.8974 \\
0.0717 \\
0.3123\end{array}$ & $\begin{array}{l}0.8973 \\
0.0716 \\
0.3125\end{array}$ & $\begin{array}{l}0.8974 \\
0.0713 \\
0.3125\end{array}$ & $\begin{array}{l}0.8975 \\
0.0711 \\
0.3127\end{array}$ \\
\hline
\end{tabular}

Abb. A3: Datenmaske zur Ausgabe der Detailergebnisse und Ergebnisse der Ausgangssituation

\begin{tabular}{|c|c|c|c|}
\hline \multicolumn{4}{|c|}{ Sensitiuitätsanalyse } \\
\hline Budgets & Basis & Simul. & Ergebnisvariablen \\
\hline $\begin{array}{l}\text { A:Push-Budget (Akqui.) } \\
\text { B:Push-Budget (Förde.) } \\
\text { C:Handelsspanne } \\
\text { D:Pull-Budget }\end{array}$ & $\begin{array}{r}12.00 \\
15.08 \\
0.35 \\
11.08\end{array}$ & $\begin{array}{l}1.00 \\
1.00 \\
1.00 \\
1.00\end{array}$ & \multirow{5}{*}{$\begin{array}{l}\text { B:Markenwert } \\
\text { C:Gewinn } \\
\text { D:Marktanteil } \\
\text { F:Präferenz für Marke } \\
\text { G:davon Markenf ixierte } \\
\text { H: } \quad \text { Markenorientierte } \\
\text { I: Markenbewupte } \\
\text { J:Präferenz andere Marke } \\
\text { K:davon Akzeptanz Marke } \\
\text { L: Indifferente } \\
\text { M:davon Akzeptanz Marke } \\
\text { N:Distributionsgrad } \\
\text { O: Uerfügbar für Markenfix. } \\
\text { P:Uerfügbar für Markenorien. } \\
\text { Q: Keine Gegenempfehlung } \\
\text { R:Empfehlung bei anderer P. } \\
\text { T:Empfehlung bei Indifferenz }\end{array}$} \\
\hline \multicolumn{2}{|l|}{ Simulationsdaten } & & \\
\hline \multicolumn{2}{|c|}{$\begin{array}{l}\text { Zu variierendes Budget } \\
\text { Periode der Ergebnisuariablen }\end{array}$} & $\begin{array}{l}a \\
1\end{array}$ & \\
\hline \multicolumn{2}{|l|}{$\begin{array}{r}\text { Budgetbereich von } \\
\text { bis }\end{array}$} & $\begin{array}{l}1.00 \\
2.00\end{array}$ & \\
\hline \multicolumn{2}{|l|}{$\begin{array}{l}\text { Simulationsschritte } \\
\text { Simulationshaupt lauf } \\
\text { Simulationsunterlauf }\end{array}$} & $\begin{array}{r}20 \\
100 \\
20\end{array}$ & \\
\hline \multicolumn{2}{|c|}{ S=Simulation } & & $E=$ Ende \\
\hline
\end{tabular}

Abb. A4: Eingabemaske zur Steuerung der Sensitivitätsanalyse 


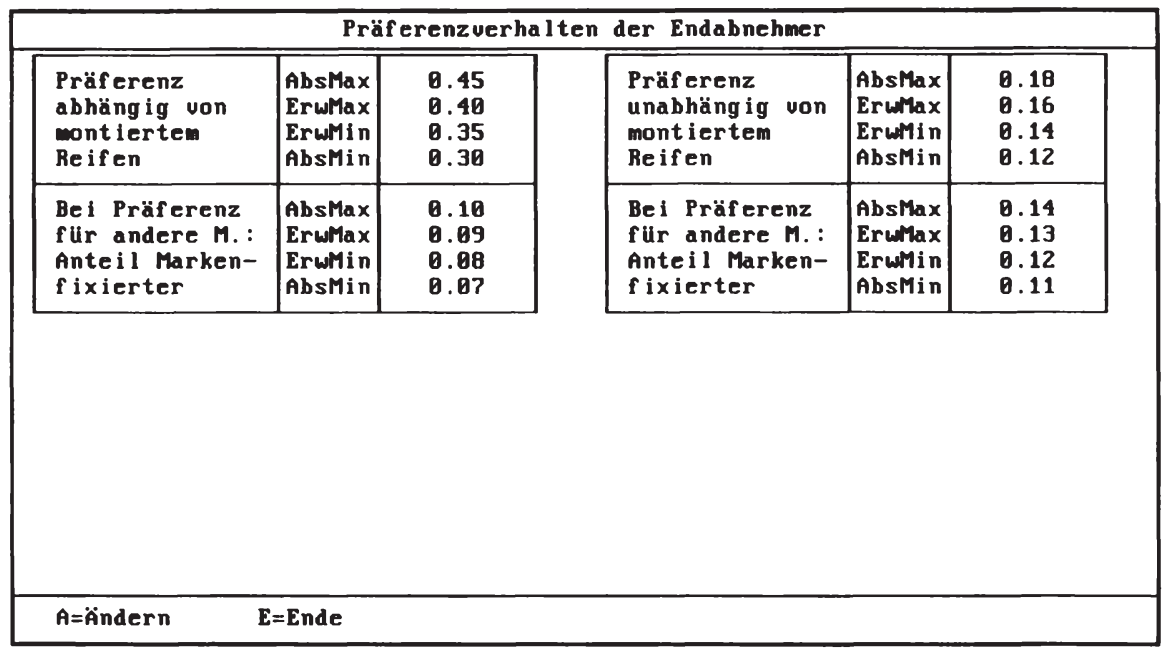

Abb. A5: Daten zum Präferenzverhalten der Endabnehmer

\begin{tabular}{|c|c|c|c|c|c|c|}
\hline \multicolumn{7}{|c|}{ Wirkung des Pull-Budgets auf Präferenz der Endabnehmer } \\
\hline & & Min langf $r$ & Min kurzfr & Ausgang & Auswe i tung & Maxi mum \\
\hline \multicolumn{7}{|l|}{$\begin{array}{l}\text { Präferenz für } \\
\text { Marke i abhäng. } \\
\text { von montierter } \\
\text { Marke }\end{array}$} \\
\hline \multirow{2}{*}{$\begin{array}{l}\text { Anteil } \\
\text { Marken- } \\
\text { fixierter }\end{array}$} & Budget & 0 & 0 & 1.00 & 1.50 & Unbegrenzt \\
\hline & $\begin{array}{l}\text { AbsMax } \\
\text { ErwMax } \\
\text { ErwMin } \\
\text { AbsMin }\end{array}$ & $\begin{array}{l}0.06 \\
0.05 \\
0.04 \\
0.03\end{array}$ & $\begin{array}{l}0.87 \\
0.06 \\
0.05 \\
0.84\end{array}$ & $\begin{array}{l}0.08 \\
0.07 \\
0.06 \\
0.05\end{array}$ & $\begin{array}{l}0.09 \\
0.08 \\
0.07 \\
0.06\end{array}$ & $\begin{array}{l}0.10 \\
0.89 \\
0.08 \\
0.07\end{array}$ \\
\hline \multirow{2}{*}{$\begin{array}{l}\text { Anteil } \\
\text { Marken- } \\
\text { orientierter }\end{array}$} & Budget & $\mathbf{0}$ & B & 1.00 & 1.50 & Unbegrenzt \\
\hline & $\begin{array}{l}\text { AbsMax } \\
\text { ErwMax } \\
\text { ErwMin } \\
\text { AbsMin }\end{array}$ & $\begin{array}{l}0.15 \\
0.13 \\
0.11 \\
0.09\end{array}$ & $\begin{array}{l}0.20 \\
0.18 \\
0.16 \\
0.14\end{array}$ & $\begin{array}{l}0.25 \\
0.23 \\
0.21 \\
0.19\end{array}$ & $\begin{array}{l}0.28 \\
0.26 \\
0.24 \\
0.22\end{array}$ & $\begin{array}{l}0.34 \\
0.32 \\
0.38 \\
0.28\end{array}$ \\
\hline \multicolumn{7}{|c|}{ A, B=Grafik } \\
\hline
\end{tabular}

Abb. A6: Daten zur Wirkung des Pull-Budgets auf die Präferenz der Endabnehmer (Präferenz für Marke i abhängig von montierter Marke) 


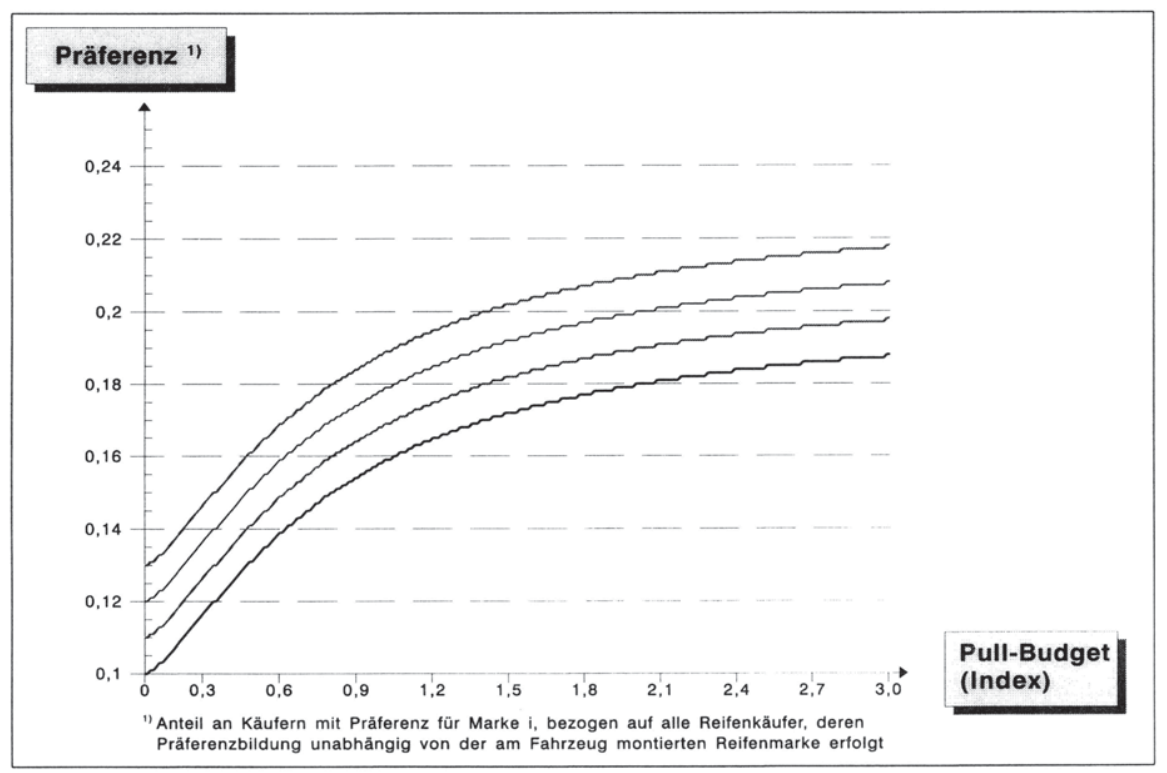

Abb. A7: Zweidimensionale Darstellung des geschätzten stochastischen Zusammenhanges zwischen Pull-Budget (Index) und Präferenz

\begin{tabular}{|c|c|c|c|c|c|c|}
\hline \multicolumn{7}{|c|}{ ts auf Präferenz der Endabnehmer } \\
\hline & & Thin langfr & Fin kurzfr & Ausgang & Ausweitung & Taximum \\
\hline \multirow[b]{2}{*}{$\begin{array}{l}\text { Präferenz fïr } \\
\text { Marke i unabh. } \\
\text { von montierter } \\
\text { Marke } \\
\qquad 1 / A\end{array}$} & Budget & 0 & 0 & 1.00 & 2.00 & Unbegrenzt \\
\hline & $\begin{array}{l}\text { AbsMax } \\
\text { ErwMax } \\
\text { ErwMin } \\
\text { AbsMin }\end{array}$ & $\begin{array}{l}0.05 \\
0.04 \\
0.03 \\
0.02\end{array}$ & $\begin{array}{l}0.13 \\
0.12 \\
0.11 \\
0.10\end{array}$ & $\begin{array}{l}0.19 \\
0.18 \\
0.17 \\
0.16\end{array}$ & $\begin{array}{l}0.21 \\
0.20 \\
0.19 \\
0.18\end{array}$ & $\begin{array}{l}0.23 \\
0.22 \\
0.21 \\
0.20\end{array}$ \\
\hline \multirow{2}{*}{$\begin{array}{l}\text { Anteil } \\
\text { Marken- } \\
\text { fixierter }\end{array}$} & Budget & 0 & 0 & 1.00 & 2.00 & Unbegrenzt \\
\hline & $\begin{array}{l}\text { AbsMax } \\
\text { ErwMax } \\
\text { ErwMin } \\
\text { AbsMin }\end{array}$ & $\begin{array}{l}0.08 \\
0.06 \\
0.04 \\
0.02\end{array}$ & $\begin{array}{l}0.18 \\
0.17 \\
0.16 \\
0.15\end{array}$ & $\begin{array}{l}0.23 \\
0.22 \\
0.21 \\
0.20\end{array}$ & $\begin{array}{l}0.24 \\
0.23 \\
0.22 \\
0.21\end{array}$ & $\begin{array}{l}0.25 \\
0.24 \\
0.23 \\
0.22\end{array}$ \\
\hline \multirow[b]{2}{*}{$\begin{array}{l}\text { Anteil } \\
\text { Marken- } \\
\text { orientierter }\end{array}$} & Budget & 0 & $\theta$ & 1.00 & 2.00 & Unbegrenzt \\
\hline & $\begin{array}{l}\text { AbsMax } \\
\text { ErwMax } \\
\text { ErwMin } \\
\text { AbsMin }\end{array}$ & $\begin{array}{l}0.18 \\
0.16 \\
0.14 \\
0.12\end{array}$ & $\begin{array}{l}0.36 \\
0.34 \\
0.32 \\
0.30\end{array}$ & $\begin{array}{l}0.48 \\
0.46 \\
0.44 \\
0.42\end{array}$ & $\begin{array}{l}0.50 \\
0.48 \\
0.46 \\
0.44 \\
\end{array}$ & $\begin{array}{l}0.54 \\
0.52 \\
0.50 \\
0.48\end{array}$ \\
\hline $1,2,3=$ Daten & $A, B, C=C$ & f ik & Grafik & & & \\
\hline
\end{tabular}

Abb. A8: Daten zur Wirkung des Pull-Budgets auf die Präferenz der Endabnehmer (Präferenz für Marke i unabhängig von montierter Marke) 


\begin{tabular}{|c|c|c|c|c|c|c|}
\hline \multicolumn{2}{|c|}{ Wirkung des } & \multicolumn{4}{|c|}{ Pull-Budgets auf Akzeptanz der Endabnehmer } & \multirow[b]{2}{*}{ Maximum } \\
\hline & & Min langfr & Min kurzfr & Ausgang & Auswe itung & \\
\hline \multirow{2}{*}{$\begin{array}{l}\text { Akzeptanz von } \\
\text { Marke i, bei } \\
\text { vom montierten } \\
\text { Reifen abhäng. } \\
\text { Präferenz fur } \\
\text { andere } M . \quad 1 / A\end{array}$} & Budget & $\theta$ & 0 & 1.00 & 1.58 & Unbegrenzt \\
\hline & $\begin{array}{l}\text { AbsMax } \\
\text { ErwMax } \\
\text { ErwMin } \\
\text { AbsMin }\end{array}$ & $\begin{array}{l}0.66 \\
0.64 \\
0.62 \\
0.68\end{array}$ & $\begin{array}{l}0.74 \\
0.72 \\
0.70 \\
0.68\end{array}$ & $\begin{array}{l}0.79 \\
0.77 \\
0.75 \\
0.73\end{array}$ & $\begin{array}{l}0.80 \\
0.78 \\
0.76 \\
0.74\end{array}$ & $\begin{array}{l}0.82 \\
8.88 \\
8.78 \\
8.76\end{array}$ \\
\hline \multirow{2}{*}{$\begin{array}{l}\text { Akzeptanz won } \\
\text { Marke i, bei } \\
\text { vom montierten } \\
\text { Reifen unabh. } \\
\text { Präferenz fuir } \\
\text { andere } M . \quad 2 / B\end{array}$} & Budget & D & D & 1.00 & 1.50 & Unbegrenzt \\
\hline & $\begin{array}{l}\text { AbsMax } \\
\text { ErwMax } \\
\text { ErwMin } \\
\text { AbsMin }\end{array}$ & $\begin{array}{l}0.60 \\
0.58 \\
0.56 \\
0.54\end{array}$ & $\begin{array}{l}0.70 \\
0.68 \\
0.66 \\
0.64\end{array}$ & $\begin{array}{l}0.76 \\
0.74 \\
0.72 \\
0.78\end{array}$ & $\begin{array}{l}0.78 \\
0.76 \\
0.74 \\
0.73\end{array}$ & $\begin{array}{l}8.81 \\
0.79 \\
0.77 \\
0.75\end{array}$ \\
\hline \multirow{2}{*}{$\begin{array}{l}\text { Akzeptanz von } \\
\text { Marke i bei } \\
\text { Ind ifferenz }\end{array}$} & Budget & 0 & 0 & 1.60 & 1.50 & Unbegrenzt \\
\hline & $\begin{array}{l}\text { AbsMax } \\
\text { ErwMax } \\
\text { ErwMin } \\
\text { AbsMin }\end{array}$ & $\begin{array}{l}0.68 \\
0.64 \\
0.62 \\
0.60 \\
\end{array}$ & $\begin{array}{l}0.76 \\
0.74 \\
0.72 \\
0.70 \\
\end{array}$ & $\begin{array}{l}0.80 \\
0.79 \\
0.78 \\
0.77 \\
\end{array}$ & $\begin{array}{l}0.82 \\
0.79 \\
0.77 \\
0.75 \\
\end{array}$ & $\begin{array}{l}0.83 \\
0.81 \\
0.79 \\
0.77 \\
\end{array}$ \\
\hline $1, \overline{2}$, & $A, \bar{B}, \mathbf{C}=\mathrm{C}$ & & Grafik & & & \\
\hline
\end{tabular}

Abb. A9: Daten zur Wirkung des Pull-Budgets auf die Akzeptanz der Enddabnehmer

\begin{tabular}{|c|c|c|c|c|c|}
\hline \multicolumn{6}{|c|}{ Zusammenhang von Distributionsgrad und effektiver Verfügbarkeit } \\
\hline \multirow[b]{3}{*}{$\begin{array}{l}\text { Effektive } \\
\text { Uerf uigbarke it } \\
\text { bei Marken- } \\
\text { fixierten }\end{array}$} & & Minimum & Ausgang & Auswe it tung & Maximum \\
\hline & Distr. & 0 & 0.54 & 0.70 & 1 \\
\hline & $\begin{array}{l}\text { AbsMax } \\
\text { ErwMax } \\
\text { ErwMin } \\
\text { AbsMin }\end{array}$ & $\begin{array}{l}0.00 \\
0.00 \\
0.00 \\
0.00\end{array}$ & $\begin{array}{l}0.96 \\
0.94 \\
0.92 \\
0.90\end{array}$ & $\begin{array}{l}0.98 \\
0.97 \\
0.96 \\
0.95\end{array}$ & $\begin{array}{l}1.00 \\
1.00 \\
1.00 \\
1.00\end{array}$ \\
\hline \multirow[b]{2}{*}{$\begin{array}{l}\text { Effektive } \\
\text { Uerfügbarkeit } \\
\text { bei Marken- } \\
\text { orientierten } \\
\qquad 2 / B\end{array}$} & Distr. & $\mathbf{B}$ & 8.54 & 8.70 & 1 \\
\hline & $\begin{array}{l}\text { AbsMax } \\
\text { Erwhlax } \\
\text { Erwhin } \\
\text { AbsMin }\end{array}$ & $\begin{array}{l}0.00 \\
0.00 \\
0.00 \\
0.00\end{array}$ & $\begin{array}{l}0.82 \\
0.80 \\
0.78 \\
0.76\end{array}$ & $\begin{array}{l}0.88 \\
0.86 \\
0.84 \\
0.82\end{array}$ & $\begin{array}{l}1.08 \\
1.00 \\
1.08 \\
1.00\end{array}$ \\
\hline $1,2=$ Daten & $A, B=$ Graf ik & G=Graf i & ten & \multicolumn{2}{|c|}{$E=$ Ende } \\
\hline
\end{tabular}

Abb. A10: Daten zum Zusammenhang von Distributionsgrad und effektiver Verfügbarkeit 


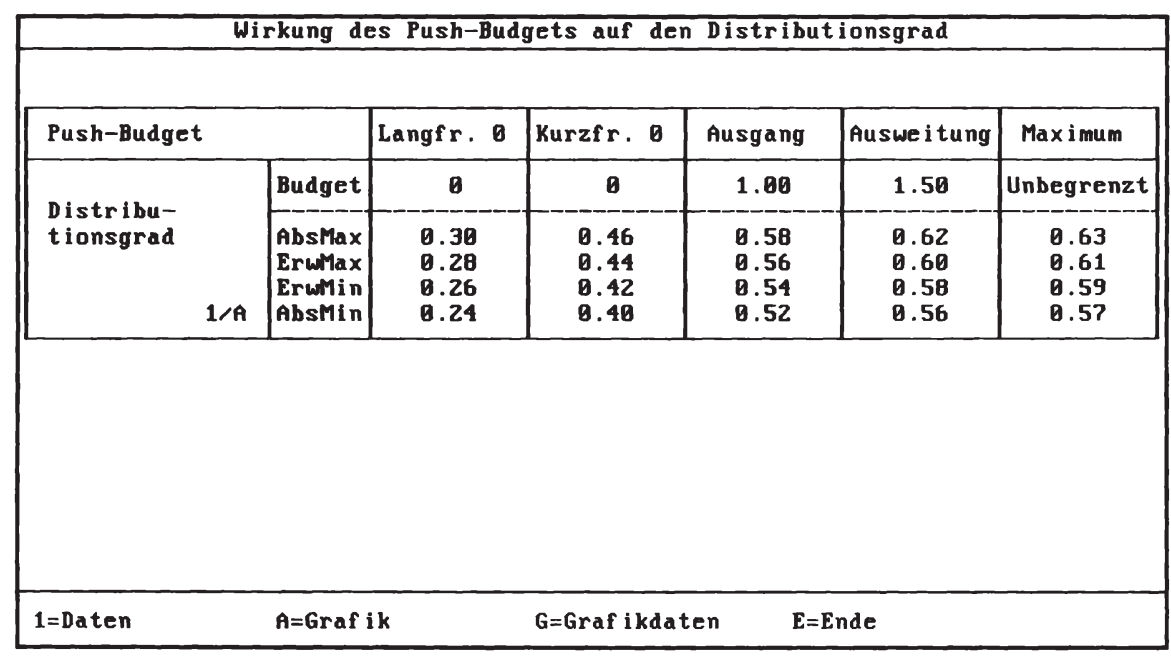

Abb. A11: Daten zur Wirkung des Push-Budgets auf den Distributionsgrad

\begin{tabular}{|c|c|c|c|c|c|c|}
\hline \multicolumn{7}{|c|}{ Wirkung des Push-Budgets auf die Förderung im Handel } \\
\hline & & Min langfr & Min kurzfr & Ausgang & Auswe i tung & Maximum \\
\hline \multirow[b]{2}{*}{$\begin{array}{l}\text { Empfehlung fir } \\
\text { Marke i bei } \\
\text { Ind ifferenz }\end{array}$} & Budget & 0 & $\mathbf{0}$ & 1.00 & 3.00 & Unbegrenzt \\
\hline & $\begin{array}{l}\text { AbsMax } \\
\text { ErwMax } \\
\text { ErwMin } \\
\text { AbsMin }\end{array}$ & $\begin{array}{l}0.13 \\
0.11 \\
0.09 \\
0.07\end{array}$ & $\begin{array}{l}0.26 \\
0.24 \\
0.22 \\
0.20\end{array}$ & $\begin{array}{l}0.34 \\
0.32 \\
0.36 \\
0.28\end{array}$ & $\begin{array}{l}0.35 \\
0.33 \\
0.31 \\
0.29\end{array}$ & $\begin{array}{l}0.36 \\
0.34 \\
0.32 \\
0.31\end{array}$ \\
\hline \multirow[b]{2}{*}{$\begin{array}{l}\text { Empfehlung fur } \\
\text { Marke i bei } \\
\text { Präferenz für } \\
\text { andere Marke } \\
\text { 2/B }\end{array}$} & Budget & 0 & 0 & 1.00 & 3.00 & Unbegrenzt \\
\hline & $\begin{array}{l}\text { AbsMax } \\
\text { ErwMax } \\
\text { ErwMin } \\
\text { AbsMin }\end{array}$ & $\begin{array}{l}0.00 \\
0.00 \\
0.00 \\
0.00\end{array}$ & $\begin{array}{l}0.04 \\
0.03 \\
0.02 \\
0.01\end{array}$ & $\begin{array}{l}0.10 \\
0.08 \\
0.06 \\
0.04\end{array}$ & $\begin{array}{l}0.11 \\
0.09 \\
0.07 \\
0.05\end{array}$ & $\begin{array}{l}0.12 \\
0.10 \\
0.08 \\
0.06\end{array}$ \\
\hline \multirow[b]{2}{*}{$\begin{array}{l}\text { Keine Gegen- } \\
\text { empfehlung bei } \\
\text { Präferenz für } \\
\text { Marke i } \\
\quad 3 / \mathrm{C}\end{array}$} & Budget & B & B & 1.00 & 3.00 & Unbegrenzt \\
\hline & $\begin{array}{l}\text { AbsMax } \\
\text { ErwMax } \\
\text { Erwilin } \\
\text { AbsMin }\end{array}$ & $\begin{array}{l}0.66 \\
0.64 \\
0.62 \\
0.60\end{array}$ & $\begin{array}{l}0.82 \\
0.80 \\
0.78 \\
0.76\end{array}$ & $\begin{array}{l}0.92 \\
0.90 \\
0.88 \\
0.86\end{array}$ & $\begin{array}{l}0.93 \\
0.92 \\
0.91 \\
0.90\end{array}$ & $\begin{array}{l}0.94 \\
0.93 \\
0.92 \\
0.91\end{array}$ \\
\hline $1,2,3=$ Daten & \multicolumn{2}{|c|}{$A, B, C=$ Graf ik } & \multicolumn{4}{|c|}{$E=$ Ende } \\
\hline
\end{tabular}

Abb. A12: Daten zur Wirkung des Push-Budgets auf die Förderung der Marke im Handel 


\begin{tabular}{|c|c|c|c|c|c|}
\hline \multicolumn{6}{|c|}{ Zusammenhang von Handelsspanne und effektiv wirksamen Push-Budget } \\
\hline \multirow[b]{3}{*}{$\begin{array}{l}\text { Effektiu } \\
\text { wirskames } \\
\text { Push-Budget } \\
\text { Akquisition }\end{array}$} & & Minimum & Ausgang & Auswe it ung & Maximum \\
\hline & Spanne & 0 & 0.35 & 0.40 & 1 \\
\hline & $\begin{array}{l}\text { AbsMax } \\
\text { ErwMax } \\
\text { ErwMin } \\
\text { AbsMin }\end{array}$ & $\begin{array}{l}0.00 \\
0.00 \\
0.00 \\
0.00\end{array}$ & $\begin{array}{l}1.10 \\
1.05 \\
1.90 \\
0.95\end{array}$ & $\begin{array}{l}1.25 \\
1.20 \\
1.15 \\
1.10\end{array}$ & $\begin{array}{l}1.46 \\
1.35 \\
1.38 \\
1.25\end{array}$ \\
\hline \multirow[b]{2}{*}{$\begin{array}{l}\text { Effektiv } \\
\text { wirksames } \\
\text { Push-Budget } \\
\text { Förderung } \\
\qquad \text { 2/B }\end{array}$} & Spanne & 0 & 0.35 & 0.40 & 1 \\
\hline & $\begin{array}{l}\text { AbsMax } \\
\text { ErwMax } \\
\text { ErwMin } \\
\text { AbsMin }\end{array}$ & $\begin{array}{l}0.00 \\
0.00 \\
0.08 \\
0.08\end{array}$ & $\begin{array}{l}1.10 \\
1.05 \\
1.00 \\
0.95\end{array}$ & $\begin{array}{l}1.25 \\
1.20 \\
1.15 \\
1.10\end{array}$ & $\begin{array}{l}1.40 \\
1.35 \\
1.30 \\
1.25\end{array}$ \\
\hline $1,2=$ Daten & $A, B=$ Graf ik & $G=\operatorname{Graf} i$ & \multicolumn{2}{|c|}{$E=$ Ende } & \\
\hline
\end{tabular}

Abb. A13: Daten zum Zusammenhang von Handelsspanne und dem effektiv wirksamen Push-Budget

\begin{tabular}{|c|c|c|c|c|c|}
\hline \multicolumn{6}{|c|}{ Zusamenhang von Präferenz und } \\
\hline \multirow[b]{3}{*}{$\begin{array}{l}\text { Effektiu } \\
\text { wirksawes } \\
\text { Push-Budget } \\
\text { Akquisition }\end{array}$} & & Minimum & fusgang & Auswe i tung & Maximum \\
\hline & Präf. & $\boldsymbol{\theta}$ & 0.88 & 8. 38 & 1 \\
\hline & $\begin{array}{l}\text { AbsMax } \\
\text { ErwMax } \\
\text { ErwMin } \\
\text { AbsMin }\end{array}$ & $\begin{array}{l}0.24 \\
0.22 \\
0.20 \\
0.18\end{array}$ & $\begin{array}{l}1.10 \\
1.85 \\
1.08 \\
0.95\end{array}$ & $\begin{array}{l}1.20 \\
1.15 \\
1.10 \\
1.05\end{array}$ & $\begin{array}{l}1.40 \\
1.35 \\
1.38 \\
1.25\end{array}$ \\
\hline \multirow[b]{2}{*}{$\begin{array}{l}\text { Effektiu } \\
\text { wirksames } \\
\text { Push-Budget } \\
\text { Förderung } \\
\qquad 2 / B\end{array}$} & Präf. & 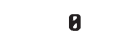 & 0.88 & 0.30 & 1 \\
\hline & $\begin{array}{l}\text { AbsMax } \\
\text { Erwhax } \\
\text { ErwMin } \\
\text { AbsMin }\end{array}$ & $\begin{array}{l}\text { B. } 34 \\
\text { 0.32 } \\
\text { B.38 } \\
\text { 0.28 }\end{array}$ & $\begin{array}{l}1.18 \\
1.85 \\
1.88 \\
0.95\end{array}$ & $\begin{array}{l}1.15 \\
1.18 \\
1.05 \\
1.08\end{array}$ & $\begin{array}{l}1.45 \\
1.48 \\
1.35 \\
1.38\end{array}$ \\
\hline $1,2=$ Daten & $A, B=$ Graf ik & \multicolumn{2}{|c|}{$G=$ Grafikdaten } & $=$ Ende & \\
\hline
\end{tabular}

Abb. A14: Daten zum Zusammenhang von Präferenz und dem effektiv wirksamen Push-Budget 


\begin{tabular}{|c|c|c|c|c|c|}
\hline \multicolumn{6}{|c|}{ Zusammenhang uon Akzeptanz und effektiv wirksamen Push-Budget } \\
\hline \multirow{3}{*}{$\begin{array}{l}\text { Effektiv } \\
\text { wirksames } \\
\text { Push-Budget } \\
\text { Akquisition }\end{array}$} & & Minimum & Ausgang & Auswe itung & Maximum \\
\hline & Akzept & 0 & 0.60 & 0.85 & 1 \\
\hline & $\begin{array}{l}\text { AbsMax } \\
\text { ErwMax } \\
\text { ErwMin } \\
\text { AbsMin }\end{array}$ & $\begin{array}{l}0.00 \\
0.00 \\
0.00 \\
0.00\end{array}$ & $\begin{array}{l}1.10 \\
1.05 \\
1.00 \\
0.95\end{array}$ & $\begin{array}{l}1.20 \\
1.15 \\
1.10 \\
1.05\end{array}$ & $\begin{array}{l}1.25 \\
1.20 \\
1.15 \\
1.10\end{array}$ \\
\hline \multirow[b]{2}{*}{$\begin{array}{l}\text { Effektiu } \\
\text { wirksames } \\
\text { Push-Budget } \\
\text { Förderung }\end{array}$} & Akzept & $\theta$ & 0.60 & 0.85 & 1 \\
\hline & $\begin{array}{l}\text { AbsMax } \\
\text { ErwMax } \\
\text { ErwMin } \\
\text { AbsMin }\end{array}$ & $\begin{array}{l}0.00 \\
0.00 \\
0.00 \\
0.00\end{array}$ & $\begin{array}{l}1.10 \\
1.05 \\
1.00 \\
0.95\end{array}$ & $\begin{array}{l}1.15 \\
1.10 \\
1.05 \\
1.00\end{array}$ & $\begin{array}{l}1.20 \\
1.15 \\
1.10 \\
1.05\end{array}$ \\
\hline $1,2=$ Daten & $A, B=\operatorname{Graf} i k$ & $\mathbf{G}=$ Graf $\mathbf{i}$ & aten & \multicolumn{2}{|l|}{$E=$ Ende } \\
\hline
\end{tabular}

Abb. A15: Daten zum Zusammenhang von Akzeptanz und dem effektiv wirksamen Push-Budget

\begin{tabular}{|c|c|c|c|c|c|c|c|}
\hline \multicolumn{8}{|c|}{ Budget-, Preis- und Kostendaten } \\
\hline \multicolumn{2}{|l|}{ Budget } & \multirow{2}{*}{$\frac{\text { Basis }}{12.00}$} & \multirow{2}{*}{\begin{tabular}{|l} 
Steig \\
0.03 \\
0.02 \\
0.02 \\
0.01
\end{tabular}} & \multirow[b]{2}{*}{$\begin{array}{l}\text { Fixe } \\
\text { Herste 11- } \\
\text { Kosten } \\
\text { (Mio. DM) }\end{array}$} & \multirow[b]{2}{*}{$\begin{array}{l}\text { AbsMax } \\
\text { ErwMax } \\
\text { ErwMin } \\
\text { AbsMin }\end{array}$} & \multirow{2}{*}{$\begin{array}{l}\text { Basis } \\
22.00 \\
21.50 \\
21.00 \\
20.50\end{array}$} & \multirow{2}{*}{\begin{tabular}{|c} 
Steig \\
0.03 \\
0.02 \\
0.02 \\
0.01
\end{tabular}} \\
\hline $\begin{array}{l}\text { Push-Budget } \\
\text { Akquisition } \\
\text { (Mio. DM) }\end{array}$ & $\begin{array}{l}\text { AbsMax } \\
\text { ErwMax } \\
\text { ErwMin } \\
\text { AbsMin }\end{array}$ & & & & & & \\
\hline $\begin{array}{l}\text { Push-Budget } \\
\text { Förderung } \\
\text { (Mio. DM) }\end{array}$ & $\begin{array}{l}\text { AbsMax } \\
\text { ErwMax } \\
\text { ErwMin } \\
\text { AbsMin }\end{array}$ & 15.00 & $\begin{array}{l}0.03 \\
0.02 \\
0.02 \\
0.01\end{array}$ & $\begin{array}{l}\text { Uariable } \\
\text { Herstel l- } \\
\text { kosten (DM) }\end{array}$ & $\begin{array}{l}\text { AbsMax } \\
\text { ErwMax } \\
\text { ErwMin } \\
\text { AbsMin }\end{array}$ & $\begin{array}{l}36.00 \\
35.50 \\
35.80 \\
34.50\end{array}$ & $\begin{array}{l}0.03 \\
0.02 \\
0.02 \\
0.01\end{array}$ \\
\hline $\begin{array}{l}\text { Pull-Budget } \\
\text { (Mio. DM) }\end{array}$ & $\begin{array}{l}\text { AbsMax } \\
\text { ErwMax } \\
\text { ErwMin } \\
\text { AbsMin }\end{array}$ & 11.00 & $\begin{array}{l}0.03 \\
0.02 \\
0.02 \\
0.01\end{array}$ & $\begin{array}{l}\text { Endabnehmer- } \\
\text { preis (DM) }\end{array}$ & $\begin{array}{l}\text { AbsMax } \\
\text { ErwMax } \\
\text { ErwMin } \\
\text { AbsMin }\end{array}$ & $\begin{array}{l}112.00 \\
110.00 \\
108.00 \\
106.00\end{array}$ & $\begin{array}{l}0.03 \\
0.02 \\
0.02 \\
0.01\end{array}$ \\
\hline Handelssp. & & & 0.35 & Kalk-Zins & & & 0.10 \\
\hline$A=$ Ändern & $\mathbf{E}=\mathbf{E}$ & & & & & & \\
\hline
\end{tabular}

Abb. A16: Angaben zu Budgets, Handelsspanne, Kosten und Endabnehmerpreis 


\begin{tabular}{|c|c|c|c|c|}
\hline \multicolumn{5}{|c|}{ Entwicklung des Marktuolumens } \\
\hline & \multicolumn{2}{|l|}{ In Periode } & 1 & Steigerung \\
\hline & $\begin{array}{l}\text { Markt volumen } \\
\text { (Mio. Stück) }\end{array}$ & $\begin{array}{l}\text { AbsMax } \\
\text { ErwMax } \\
\text { ErwMin } \\
\text { AbsMin }\end{array}$ & $\begin{array}{l}33.00 \\
32.60 \\
31.00 \\
30.08\end{array}$ & $\begin{array}{l}0.05 \\
0.04 \\
0.03 \\
0.02\end{array}$ \\
\hline$A=$ Ändern & $E=$ Ende & & & \\
\hline
\end{tabular}

Abb. A17: Daten zur Entwicklung des Marktvolumens

Marktanteile der Uorperioden

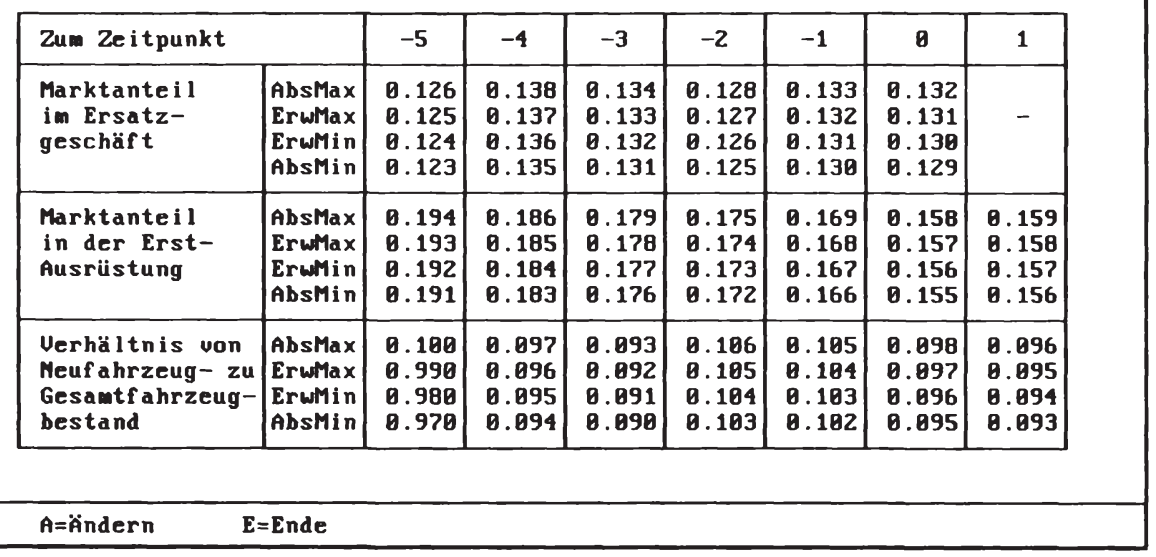

Abb. A18: Daten zu Marktanteilen aus den Vorperioden sowie Anteil an der Erstausrüstung im Planungszeitraum 


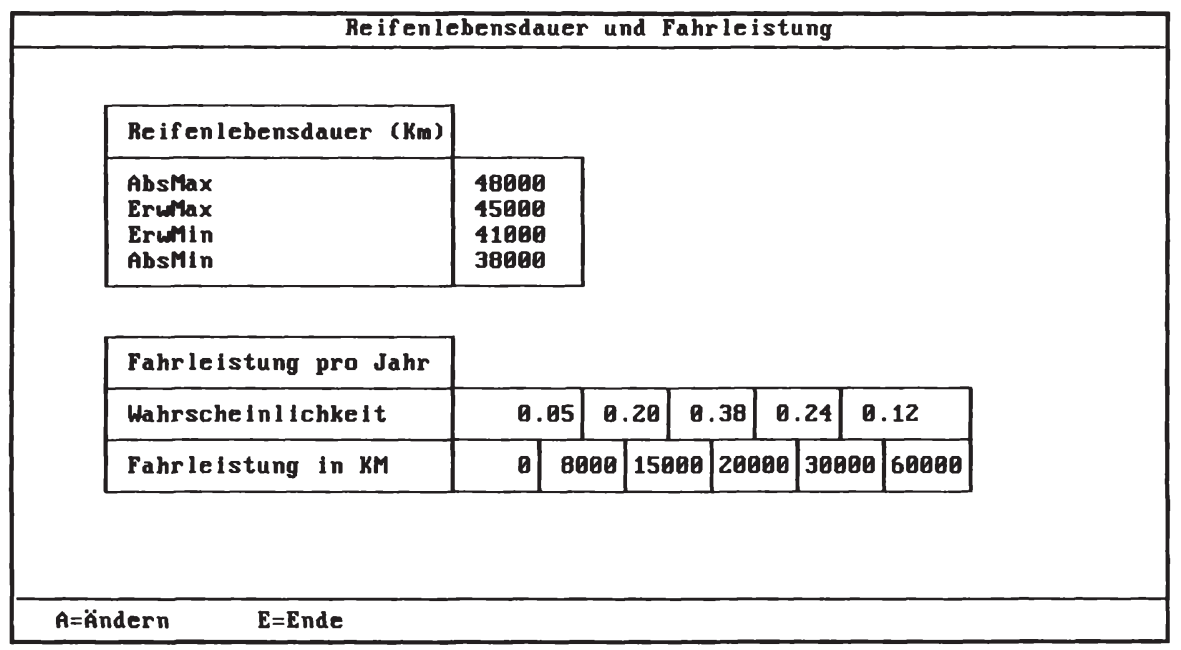

Abb. A19: Daten zur Reifenlebensdauer und der Fahrleistung pro Jahr 


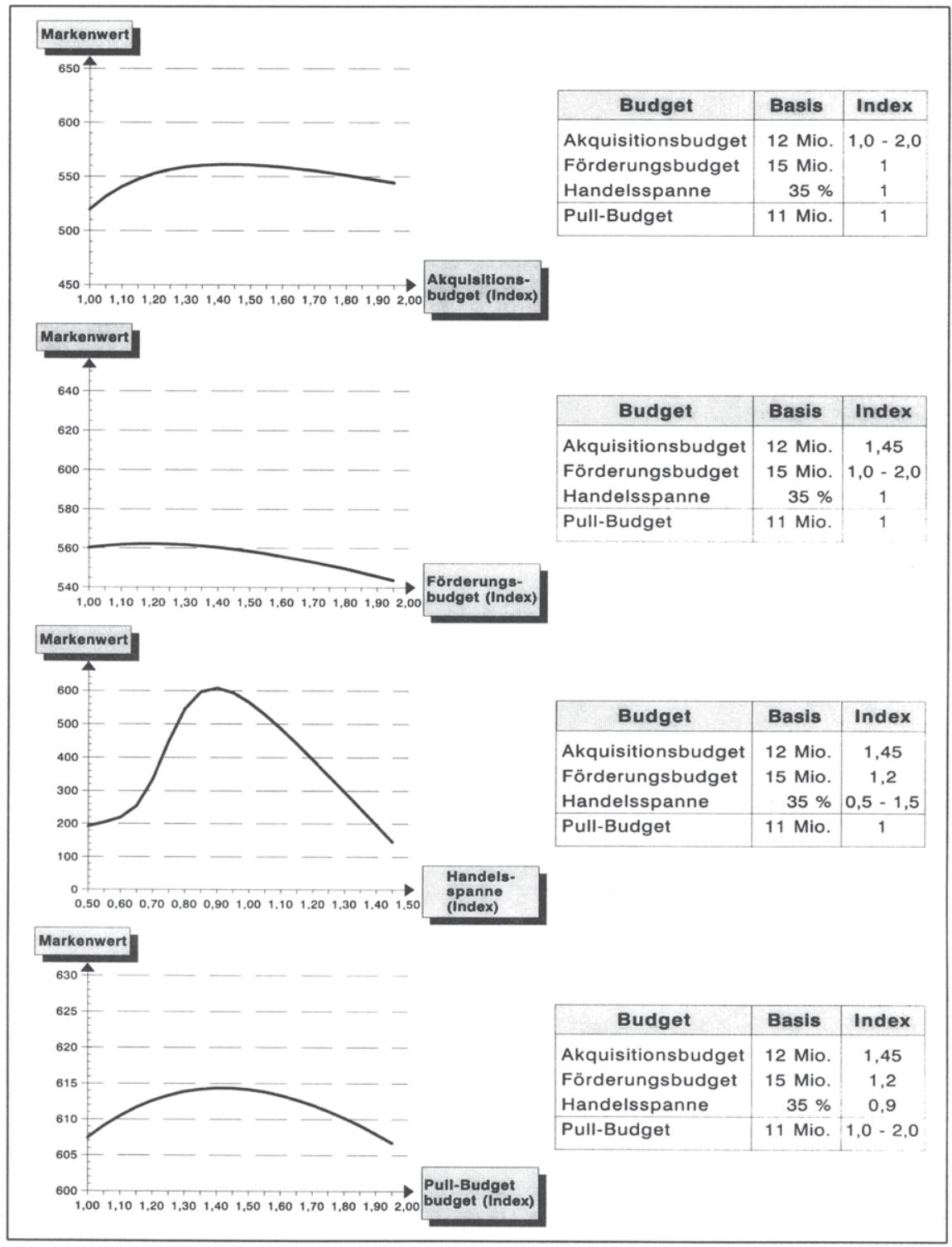

Abb. A20: Sensitivitätsanalyse zur Verbesserung der Kombination von Aktivitätsniveaus des Push- und Pull-Instrumentariums (Durchgang 1) 


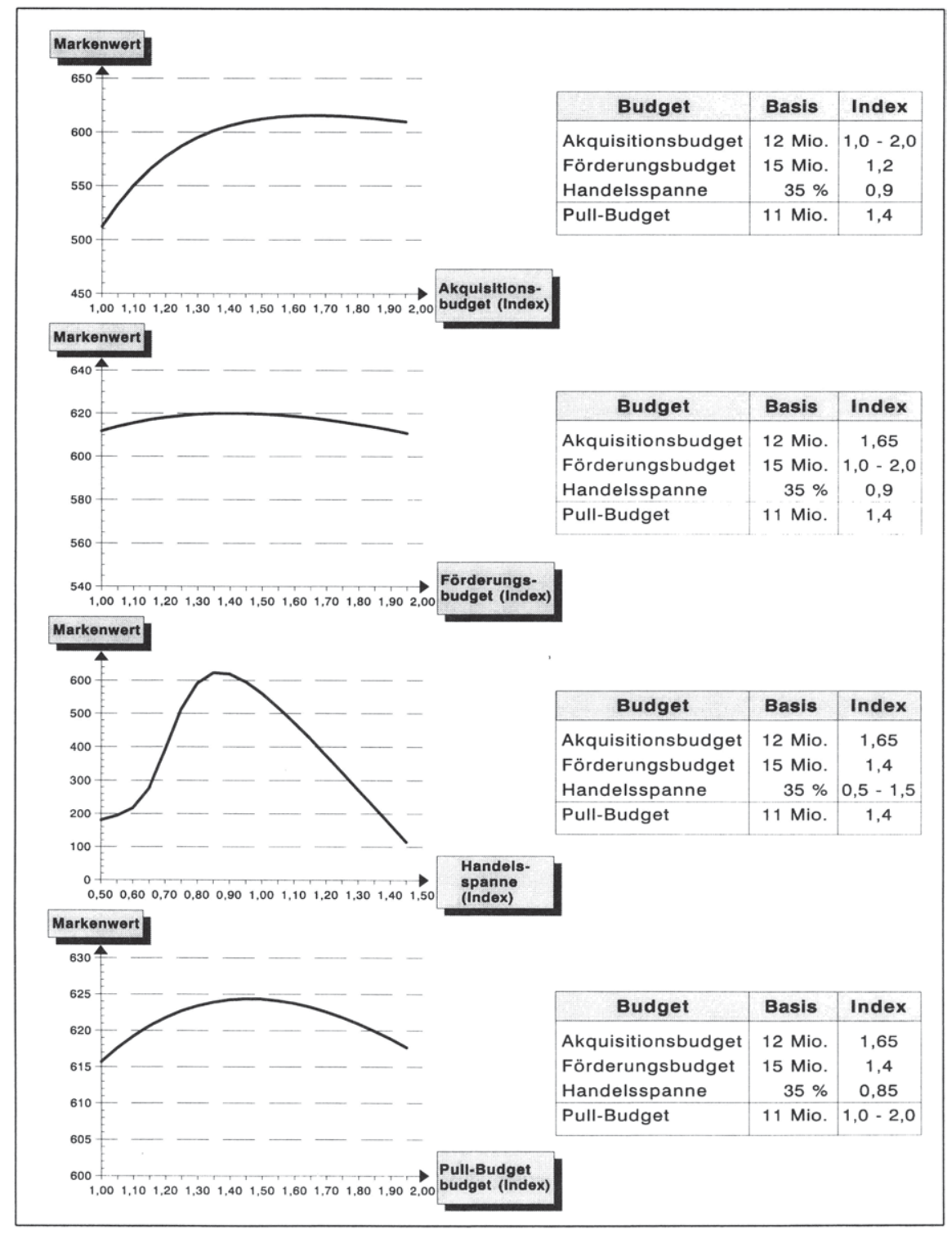

Abb. A21: Sensitivitätsanalyse zur Verbesserung der Kombination von Aktivitätsniveaus des Push- und Pull-Instrumentariums (Durchgang 2) 


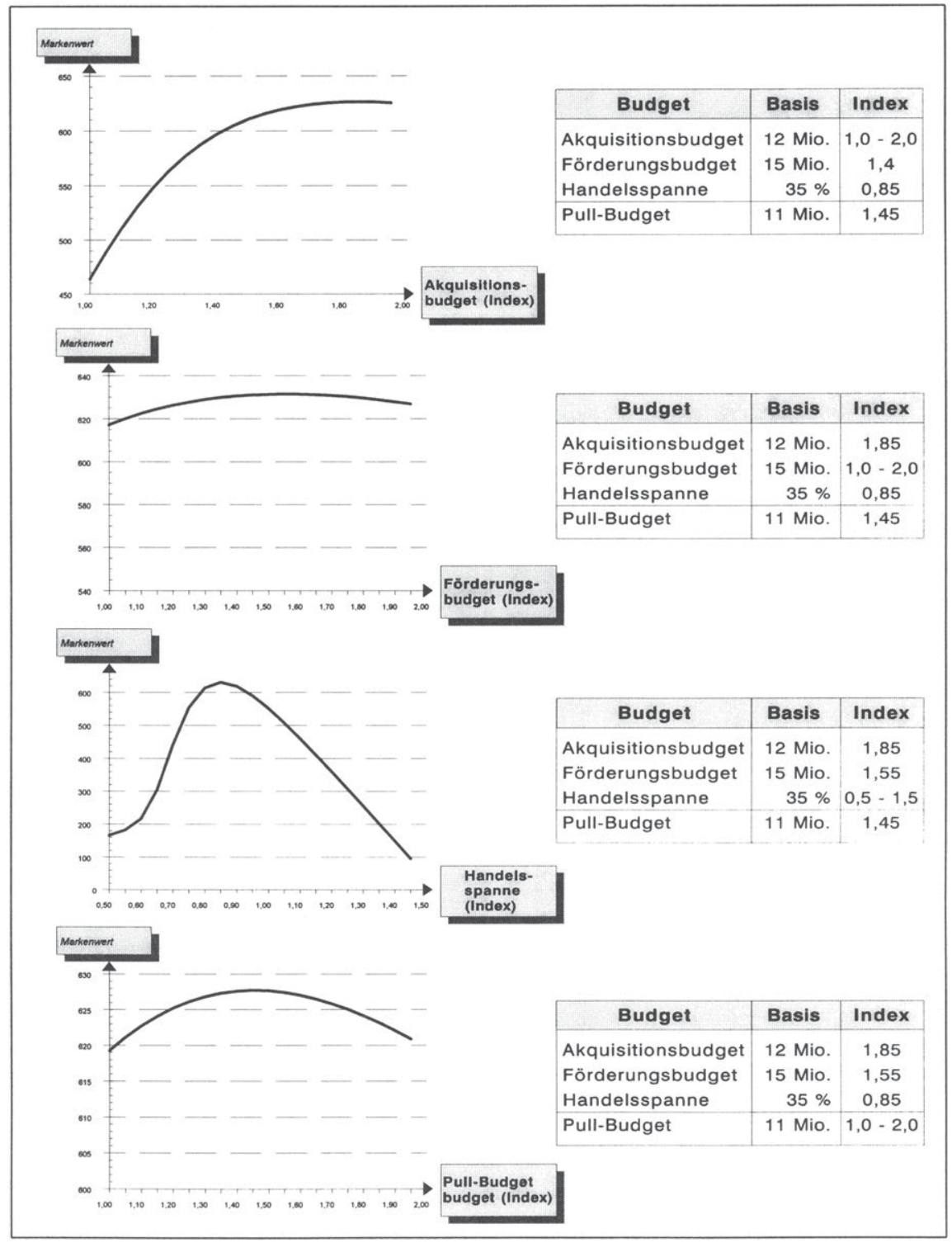

Abb. A22: Sensitivitätsanalyse zur Verbesserung der Kombination von Aktivitätsniveaus des Push- und Pull-Instrumentariums (Durchgang 3) 


\begin{tabular}{|c|c|c|c|c|c|c|c|c|}
\hline \multicolumn{9}{|c|}{ Simulation } \\
\hline \multicolumn{2}{|l|}{ Budget } & Basis & Simu 1 . & & Simulation & K-Int & t. Min. & $\operatorname{Max}$. \\
\hline \multicolumn{2}{|c|}{$\begin{array}{l}\text { Push-Budget (Akqui.) } \\
\text { Push-Budget (Förder.) } \\
\text { Handelsspanne }\end{array}$} & $\begin{array}{l}12.00 \\
15.00\end{array}$ & $\begin{array}{l}1.85 \\
1.55\end{array}$ & & $\begin{array}{l}\text { Marktante il } \\
\text { Markenwert }\end{array}$ & $\begin{array}{l}0.010 \\
0.010\end{array}$ & \begin{tabular}{c|c}
10 & 0.000 \\
10 & 400.0
\end{tabular} & $\begin{array}{l}0.000 \\
800.0\end{array}$ \\
\hline \multicolumn{2}{|l|}{ Pull-Budget } & 11.00 & 1.45 & & $\begin{array}{l}\text { S-Haupt lauf } \\
\text { S-Unter lauf }\end{array}$ & & & $\begin{array}{r}3000 \\
28\end{array}$ \\
\hline Zum Zeitpunkt & MW & 1 & 2 & 3 & 4 & 5 & 6 & 7 \\
\hline $\begin{array}{l}\text { Marktanteil } \\
\text { - Erwartungs. } \\
\text { - Standardab. } \\
\text { - Vertrauens. }\end{array}$ & & $\begin{array}{r}0.1410 \\
0.0061 \\
0.99\end{array}$ & $\begin{array}{r}0.1421 \\
0.0062 \\
0.99\end{array}$ & $\begin{array}{r}0.1426 \\
8.0063 \\
0.99\end{array}$ & $\begin{array}{r}0.1433 \\
0.0065 \\
0.99\end{array}$ & $\begin{array}{r}0.1437 \\
0.0065 \\
0.99\end{array}$ & $\begin{array}{r}0.1440 \\
0.0065 \\
0.99\end{array}$ & $\begin{array}{r}0.1440 \\
0.0065 \\
0.99\end{array}$ \\
\hline $\begin{array}{l}\text { MW/Gewinn } \\
\text { - Erwartungs. } \\
\text { - Standardab. } \\
\text { - Uertrauens. }\end{array}$ & $\begin{array}{r}629.80 \\
47.44 \\
0.98\end{array}$ & $\begin{array}{r}95.97 \\
7.02 \\
0.98\end{array}$ & $\begin{array}{r}112.38 \\
10.04 \\
0.97\end{array}$ & $\begin{array}{r}122.91 \\
10.96 \\
0.97\end{array}$ & $\begin{array}{r}133.80 \\
11.99 \\
0.97\end{array}$ & $\begin{array}{r}145.31 \\
13.83 \\
0.97\end{array}$ & $\begin{array}{r}157.22 \\
14.14 \\
8.97\end{array}$ & $\begin{array}{r}169.49 \\
15.34 \\
0.97\end{array}$ \\
\hline
\end{tabular}

Abb. A23: Ergebnisse der Simulation nach Optimierung der Budgets

\begin{tabular}{|c|c|c|c|c|c|c|c|}
\hline \multicolumn{8}{|c|}{ Detailergebnisse } \\
\hline Zum Zeitpunkt & 1 & 2 & 3 & 4 & 5 & 6 & $?$ \\
\hline $\begin{array}{l}\text { Präferenz für Marke } \\
\text { davon Markenf ixierte } \\
\text { Markenorientierte } \\
\text { Markenbewupte }\end{array}$ & $\begin{array}{l}0.0793 \\
0.1412 \\
0.3525 \\
0.5863\end{array}$ & $\begin{array}{l}\text { B. } 0868 \\
\text { B.1462 } \\
\text { B.3517 } \\
\text { B.5081 }\end{array}$ & $\begin{array}{l}0.0814 \\
0.1396 \\
0.3510 \\
0.5095\end{array}$ & $\begin{array}{l}0.0829 \\
0.1387 \\
0.3494 \\
0.5119\end{array}$ & $\begin{array}{l}0.0835 \\
0.1384 \\
0.3489 \\
0.5128\end{array}$ & $\begin{array}{l}0.0839 \\
0.1381 \\
0.3486 \\
0.5133\end{array}$ & $\begin{array}{l}0.0843 \\
0.1382 \\
0.3483 \\
0.5136\end{array}$ \\
\hline $\begin{array}{l}\text { Präferenz andere Marke } \\
\text { davon Akzeptanz Marke }\end{array}$ & $\begin{array}{l}0.4465 \\
0.7095\end{array}$ & $\begin{array}{l}0.4438 \\
0.7096\end{array}$ & $\begin{array}{l}0.4422 \\
0.7092\end{array}$ & $\begin{array}{l}0.4416 \\
0.7893\end{array}$ & $\begin{array}{l}0.4413 \\
0.7096\end{array}$ & $\begin{array}{l}0.4413 \\
0.7095\end{array}$ & $\begin{array}{l}0.4416 \\
0.7893\end{array}$ \\
\hline $\begin{array}{l}\text { Ind ifferente } \\
\text { davon Akzeptanz Marke }\end{array}$ & $\begin{array}{l}0.4742 \\
0.7799\end{array}$ & $\begin{array}{l}0.4754 \\
0.7798\end{array}$ & $\begin{array}{l}0.4764 \\
0.7795\end{array}$ & $\begin{array}{l}0.4755 \\
0.7793\end{array}$ & $\begin{array}{l}0.4752 \\
0.7796\end{array}$ & $\begin{array}{l}0.4748 \\
0.7798\end{array}$ & $\begin{array}{l}0.4741 \\
0.7799\end{array}$ \\
\hline $\begin{array}{l}\text { Distributionsgrad } \\
\text { Uerfigbar fur Markenfix. } \\
\quad \text { Markenorien. }\end{array}$ & $\begin{array}{l}0.6447 \\
0.9549 \\
0.8299\end{array}$ & $\begin{array}{l}0.6447 \\
0.9546 \\
0.8299\end{array}$ & $\begin{array}{l}0.6445 \\
0.9546 \\
0.8295\end{array}$ & $\begin{array}{l}0.6446 \\
0.9544 \\
0.8296\end{array}$ & $\begin{array}{l}0.6449 \\
0.9548 \\
0.8297\end{array}$ & $\begin{array}{l}0.6448 \\
0.9549 \\
0.8295\end{array}$ & $\begin{array}{l}0.6447 \\
0.9544 \\
0.8297\end{array}$ \\
\hline $\begin{array}{l}\text { Keine Gegenempfehlung } \\
\text { Empfehlung bei anderer P. } \\
\text { Emf pehlung bei Ind ifferenz }\end{array}$ & $\begin{array}{l}0.8807 \\
0.0683 \\
0.3066\end{array}$ & $\begin{array}{l}0.8809 \\
0.0685 \\
0.3068\end{array}$ & $\begin{array}{l}0.0812 \\
0.0688 \\
0.3068\end{array}$ & $\begin{array}{l}0.8815 \\
0.0688 \\
0.3064\end{array}$ & $\begin{array}{l}0.8812 \\
0.0682 \\
0.3067\end{array}$ & $\begin{array}{l}0.8811 \\
0.0683 \\
0.3070\end{array}$ & $\begin{array}{l}0.8807 \\
0.0681 \\
0.3068\end{array}$ \\
\hline
\end{tabular}

Abb. A24: Detailergebnisse der Simulation nach Optimierung der Budgets 


\section{Trapezverteilung:}

Zur Berechung der Verteilungsfunktion einer Trapezverteilung wird diese zunächst in zwei Dreiecks- und eine Rechtecksverteilung zerlegt. Deren jeweiliger Anteil an der Gesamtfläche ergibt sich aus:

$$
\begin{aligned}
& F_{1}=\frac{\text { xerw }_{\text {min }}-\text { xabs }_{\text {min }}}{\text { Xabs }_{\text {max }}-\text { Xabs }_{\text {min }}-\text { Xerw }_{\text {min }}+\text { Xerw }_{\text {max }}} \\
& F_{2}=\frac{\text { xerw }_{\text {max }}-\text { xerw }_{\text {min }}}{\text { Xabs }_{\text {max }}-\text { Xabs }_{\text {min }}-\text { Xerw }_{\text {min }}+\text { Xerw }_{\text {max }}} \\
& F_{3}=\frac{\text { xabs }_{\text {max }}-\text { xerw }_{\text {max }}}{\text { Xabs }_{\text {max }}-\text { Xabs }_{\text {min }}-\text { Xerw }_{\text {min }}+\text { Xerw }_{\text {max }}}
\end{aligned}
$$

Für den Bereich der linken Dreiecksverteilung ( $x_{a b s_{\min }} \leq x \leq x e r w_{\min }$ ) gilt:

$$
\begin{aligned}
& F(x)=\int_{\text {abs }_{\min }}^{x} \alpha \cdot\left(v-x_{a b s_{\text {min }}}\right) d v=\frac{\alpha}{2} \cdot\left(x-x_{a b s_{\text {min }}}\right)^{2} \\
& \alpha=\frac{2 \cdot F_{1}}{\left(x e r w_{\text {min }}-x a b s_{\min }\right)^{2}}
\end{aligned}
$$

Für den Bereich der Rechteckverteilung ( $x e r w_{\min }<x<x e r w_{\max }$ ) ergibt sich:

$$
F(x)=F_{1}+\int_{x w_{\min }}^{x} \frac{F_{2}}{x} d v=\frac{F_{2} \cdot\left(x-x e r w_{\text {max }}-x e r w_{\text {min }}\right)}{x e r w_{\text {max }}-x e r w_{\text {min }}}+F
$$

Im Bereich der rechten Dreiecksverteilung ( $x e r w_{\max } \leq x \leq$ xabs $_{\max }$ ) gilt:

$$
\begin{aligned}
F(x) & =F_{1}+F_{2}+\int_{\text {erw }_{\text {max }}}^{x} \beta \cdot\left(\text { xabs }_{\max }-v\right) d v \\
& =\beta \cdot\left(\text { xabs }_{\max } x-\frac{1}{2} x^{2}-x a b s_{\max } \cdot x e r w_{\max }+\frac{1}{2} x^{2} e r w_{\max }\right)+F_{1}+F_{2} \\
\beta & =\frac{2 \cdot F_{3}}{\left(\text { xabs }_{\max }-x e r w_{\max }\right)^{2}}
\end{aligned}
$$


Damit gilt für die Umkehrfunktion $\mathrm{F}^{-1}(\mathrm{x})$ :

$F^{-1}(x)= \begin{cases}x_{\text {abs }}+\sqrt{\frac{2 \cdot x}{\alpha}} & \text { falls (1) } \\ x e r w_{\text {min }}+\left(x e r w_{\text {max }}-x e r w_{\text {min }}\right) \cdot\left(\frac{x-F_{1}}{F_{2}}\right) & \text { falls (2) } \\ x_{\text {abs }} \text { max }-\sqrt{\left(x_{\text {abs }}-x e r w_{\text {max }}\right)^{2}+\frac{2 \cdot\left(F_{1}+F_{2}-x\right)}{\beta}} & \text { falls (3) }\end{cases}$

(1): $0 \leq x \leq F\left(x_{\text {min }}\right)$

(2): $F\left(x e r w_{\text {min }}\right) \leq x \leq F\left(x e r w_{\text {max }}\right)$

(3): $F\left(x e r w_{\max }\right) \leq x \leq 1$ 


\section{Literaturverzeichnis}

Aaker, D. A., Management des Markenwertes, Frankfurt/Main, New York 1992.

Achenbaum, A. A., Mitchel, F. K., Pulling away from push marketing, in: Harvard Business Review, No. 3, 1987, S. 38-40.

Adam, D., Zur Problematik der Planung in schlecht-strukturierten Entscheidungssituationen, in: Neue Aspekte der betrieblichen Planung, Schriften zur Unternehmensführung, Hrsg.: Jacob, H., Band 28, Wiesbaden 1980, S. 47-75.

Adam, D., Planungsüberlegungen in bewertungs- und zielsetzungsdefekten Problemsituationen, in: WISU, 1980, H. 3, S. 127-130 u. 173-180.

Adam D., Planungsüberlegungen in wirkungsdefekten Problemsituationen, in: WISU, 1980, H. 8, S. 382-386.

Adam, D., Kurzlehrbuch Planung, 2. Aufl., Wiesbaden 1983.

Adam, D., Planung und Entscheidung, Modelle-Ziele-Methoden, 3. Aufl., Wiesbaden 1993.

Ahlert, D., Vertikale Kooperationsstrategien im Vertrieb, in: ZfB, H. 1, 1982, S. 62-93.

Ahlert, D., Distributionspolitik, Das Management des Absatzkanals, Stuttgart, New York 1985.

Allen, D. H., Credibility Forecasts and their Application to the Economic Assessment of Novel Research and Developement Project, in: Operations Research Quarterly, No. 1, 1968, S. 25-42.

Amstutz, A. E., Computer Simulation of Competitive Market Response, Cambridge (Mass.), London 1976.

Andresen, T., Innere Markenbilder: MAX - wie er wurde, was er ist, in: Planung und Analyse, H. 1, 1991, S. 28-34.

Andritzky, K., Die Operationalisierbarkeit von Theorien zum Konsumentenverhalten, Berlin 1976.

Autorenkollektiv Freie Universität Berlin u. a., Grundausbildung in Statistik für Wirtschaftswissenschaftler, Osnabrück, O. J. 
Backhaus, K., Investitionsgütermarketing, 2. Aufl., München 1990.

Baetge, J., Betriebswirtschaftliche Systemtheorie, Opladen 1974.

Bamberg, G., Baur, F., Statistik, 3. Aufl., München 1984.

Bamberg, G., Coenenberg, A. G., Betriebswirtschaftliche Entscheidungslehre, 6. Aufl., München 1991.

Barth, K., Betriebswirtschaftslehre des Handels, Wiesbaden 1988.

Barzen, D., Marketing-Budgetierung, Frankfurt/Main u. a. 1990.

Batzer, E., Greipl, E., Die Bedeutung von Hersteller- und Handelsmarke für den Handel, in: Marke und Markenartikel als Instrumente des Wettbewerbs, Hrsg.: Dichtl, E., Eggers, W., München 1992, S. $185-204$.

Bauche, K., Segmentierung von Kundendienstleistungen auf investiven Märkten, Dargestellt am Beispiel von Personal Computern, Frankfurt/Main 1994.

Bäuerle, P., Zur Problematik der Konstruktion praktikabler Entscheidungsmodelle, in: ZfB, H. 2, 1989, S. 175-193.

Bautz, D., Der Promotion-Erfolg muß nicht kurzfristig sein, in: asw, H. 2, 1992, S. 116-121.

BBDO (Hrsg.): Der neue Realismus - Verbraucherverhalten in der Rezession, Düsseldorf 1994.

Becker, J., Marketing-Konzeption, Grundlagen des strategischen MarketingManagements, 5. Aufl., München 1993.

Becker, W., Komplexitätskosten, in: Kostenrechnungspraxis, H. 3, 1992, S. 71-173.

Behrends, Chr., Category Management, Von der Vision zur Praxis, in: LZ, 3. Juni 1994, S. 58-63.

Bekmeier, S., Markenwert und Markenstärke - Markenevaluierung aus konsumentenorientierter Perspektive, in: Markenartikel, H. 8, 1994, S. 383-387. 


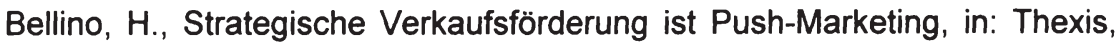
H. 2, 1993, S. 6-12.

Belz, Chr., Konstruktives Marketing in gesättigten und schrumpfenden Märkten, Band 2, Habil., St. Gallen 1987.

Belz, Chr., Die Beschränkung der Absatzkanäle als Marketing-Strategie, in: Jahrbuch der Absatz- und Verbrauchsforschung, H. 1, 1990, S. $7-40$.

Berekoven, L., Von der Markierung zur Marke, in: Marke und Markenartikel als Instrumente des Wettbewerbs, Hrsg.: Dichtl, E., Eggers, W., München 1992, S. 25-45.

Berens, W., Beurteilung von Heuristiken, Neuorientierung und Vertiefung am Beispiel logistischer Probleme, Wiesbaden 1992.

Berry, L. L., Zeithaml, V. A., Parasuraman, A., Five Imperatives for Improving Service Quality, in: Sloan Management Review, No. 4, 1990, S. 29-38.

Bidlingmaier, J., Geschäftsprinzipien und Betriebsformen des Einzelhandels, in: ZfbF, H. 12, 1963, S. 590-599.

Bielefeld, K. W., Marken II: Kampf um die Wertschöpfung, in: Harvardmanager, H. 3, 1992, S. 34-46.

Biethahn, J., Optimierung und Simulation, Wiesbaden 1978.

Biethahn, J., Simulation - eine Methode zur Findung betriebswirtschaftlicher Entscheidungen?, in: Biethahn, J., Schmidt, B., Simulation als betriebliche Entscheidungshilfe, Methoden, Werkzeuge, Anwendungen, Berlin u. a. 1987, S. 79-91.

Bitz, M., Entscheidungstheorie, München 1981.

Blex, W., Marchal, G., Risiken im Akquisitionsprozeß - Ein Überblick, in: BFuP, H. 2, 1990, S. 85-103.

Bleymüller, J., Gehlert, G., Gülicher, H., Statistik für Wirtschaftswissenschaftler, München 1979.

Bleymüller, J., Stichprobenverfahren von Wirtschaftswissenschaftlern, Manuskript, Münster 1989. 
Böcker, F., Präferenzforschung als Mittel marktorientierter Unternehmensführung, in: ZfbF, H. 7/8, 1986, S. 543-574.

Böcker, F., Präferenzpolitik, in: Vahlens Großes Marketinglexikon, Hrsg.: Diller, H., München 1992, S. 883-885.

Bolz, J., Wettbewerbsorientierte Standardisierung der internationalen Marktbearbeitung, Eine empirische Analyse in europäischen Schlüsselmärkten, Darmstadt 1992.

Bourgeois III, L. J., Strategy and Environment - A conceptual integration, in: Academy of Management Review, No. 1, S. 25-39.

Bratley, P., Fox, B. L., Schrage, L. E., A Guide to Simulation, 2. Ed., New York u. a. 1987.

Brandmeyer, K., Schulz, R., Die Markenbilanz im Kreuzverhör, in: Markenartikel, H. 5, 1990, S. 236 -239.

Breitung, A., Informationssysteme, Ein methodologischer Beitrag von Datenverarbeitungsprozessen in Marketing-Informationssystemen, Diss., Münster 1974.

Brockhoff, K., Produktpolitik, 3. Aufl., Stuttgart, Jena 1993.

Brombacher R., Entscheidungsunterstützungssysteme für das MarketingManagement, Gestaltungs- und Implementierungsansatz für die Konsumgüterindustrie, Berlin, Heidelberg 1988.

Bruhn, M., Marketing und Konsumentenzufriedenheit, in: WISU, H. 6, 1985 , S. 300-307.

Bundesverband Reifenhandel und Vulkaniseurhandwerk e.V. (Hrsg.): Erfolgskonzept 2000, Der deutsche Reifenfachhandel und sein Wettbewerbsumfeld - Chancen einer zukünftig profitablen Marktbehauptung, Köln 1990.

Bundesverband Reifenhandel und Vulkaniseurhandwerk e.V. (Hrsg.): "TÜVgeprüfter Reifen-Fachbetrieb - freiwillige Prüfung gemäß Anforderungsprofil des BRV und des TÜV Bayern/Sachsen", Köln 1994.

Bundesverband Reifenhandel und Vulkaniseurhandwerk e.V. (Hrsg.): Reifenersatzgeschäft Deutschland 1993/94 - Marktdaten. 
Cairns, J. P., Suppliers, Retailers and Shelf Space, in: Journal of Marketing, No. 3, 1962, S. 34-36.

Continental AG (Hrsg.): Geschäftsbericht 1993, Hannover 1993.

Cristofolini, P. M., Markenpolitik und Verkaufsförderung, in: Markenartikel, H. 9, 1994, S. 426-430.

Curhan, R. C., The Relationship Between Shelf Space and Unit Sales in Supermarkets, in: Journal of Marketing Research, November 1972, S. 406-412.

Dannenberg, J., Mikrocomputergestützte Instrumente der strategischen Unternehmensplanung, Wiesbaden 1990.

Day, G. S., Buyer Attitudes and Brand Choice Behavior, New York, London 1970.

Dehlhees, K. H., Systemorientierte Darstellung des Kaufprozesses als Entscheidungshilfe des Marketing, in: Jahrbuch der Absatz- und Verbrauchsforschung, H. 2, 1973, S. 285-297.

Deutsche Shell Aktiengesellschaft (Hrsg.): Motorisierung in Deutschland: Mehr Senioren fahren länger Auto, Shell Szenarien des PKWBestands und der Neuzulassungen bis zum Jahre 2010 mit einem Ausblick auf 2020, Hamburg 1993.

Dichtl, E., Müller, S., Herausforderungen für die Markenartikelindustrie an der Schwelle zu einem neuen Jahrzehnt, Ergebnisse einer DelphiBefragung, Arbeitspapier Nr. 94 des Instituts für Marketing der Universität Mannheim, Mannheim 1992.

Diller, H., Ausstrahlungseffekte, in: Betriebswirtschaftliche Gesetze, Effekte und Prinzipien, Hrsg.: Schanz, G., München 1979, S. 91-119.

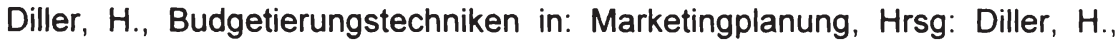
München 1980, S. 115-125.

Diller, H., Das Zielsystem der Verkaufsförderung, in: WiSt, H. 10, 1984, S. 494-499.

Diller, H., Preispolitik, 2. Aufl., Stuttgart u.a. 1991. 
Diller, $\mathrm{H}$., Preisimage und Preiswerbung im Reifenhandel, Arbeitspapier Nr. 18 des Lehrstuhls für Marketing an der Universität Erlangen-Nürnberg, Nürnberg 1993.

Diller, H., Brielmaier, A., Die Wirksamkeit von Verkaufsförderungsaktionen, Ein Marktexperiment, Arbeitspapier Nr. 28 des Lehrstuhls für Marketing an der Universität Erlangen-Nürnberg, Nürnberg 1993.

Dingeldey, K., Herstellermarketing im Wettbewerb um den Handel, Berlin 1975.

Domman, D., Erfolgreicher Persönlicher Verkaufen, in: Handbuch Marketing-Kommunikation, Hrsg.: Berndt, R., Hermanns, A., Wiesbaden 1993, S. 751-764.

Döppner, H. W., Verkaufsförderung - eine Marketing-Funktion, Dargestellt am Beispiel der Konsumgüterindustrie, Berlin 1977.

Dörnhöfer, K., Lösung von Optimierungsproblemen durch Simulation, in: Biethahn, J., Schmidt, B., Simulation als betriebliche Entscheidungshilfe, Methoden, Werkzeuge, Anwendungen, Berlin u. a. 1987 , S. 61-69.

Dubois, D., Prade, H., Fuzzy sets and systems, Theorie and applications, New York u. a. 1980.

Eisenführ, F., Weber, M., Rationales Entscheiden, Berlin u. a. 1993.

Engelhardt, W. H., Erscheinungsformen und absatzpolitische Probleme von Angebots- und Nachfrageverbunden, in: ZfbF, H. 1, 1976, S. 77-90.

Engelhardt, W. H., Mehrstufige Absatzstrategien, in: ZfbF-Kontaktstudium, 1976, S. 175-182.

Esch, F.-R., Markenwert und Markensteuerung, Eine verhaltenswissenschaftliche Perspektive, in: Thexis, H. 5/6, 1993, S. 56-65.

Farris, P., Olver, J., De Kluyver, C., Reply to the Commentaries to Farris, P., Olver, J., De Kluyver, C., The Relationship between Distribution and Market Share, in: Marketing Science, No. 2, Spring 1989, S. 131-132. 
Farris, P., Olver, J., De Kluyver, C., The Relationship between Distribution and Market Share, in: Marketing Science, No. 2, Spring 1989, S. 107-128.

Fischer, T. M., Variantenvielfalt und Komplexität als betriebliche Bestimmungsfaktoren, in: Kostenrechnungspraxis, H. 1, 1993, S. 27-31.

Florenz, P. J., Konzept des vertikalen Marketing, Entwicklung und Darstellung am Beispiel der deutschen Automobilwirtschaft, Diss., Köln 1991.

Franzen, O., Trommsdorff, V., Riedel, F., Ansätze der Markenbewertung und Markenbilanz, in: Markenartikel, H. 8, 1994, S. 372-376.

Freitag, D. E., Methodische Probleme bei der Realisation eines Einstellungsmodells, in: Jahrbuch der Absatz- und Verbrauchsforschung, H. 2, 1973, S. 157-193.

French, J. R. P., Raven, B., The Bases of Social Power, in: Studie in Social Power, Hrsg.: Cartwright, D., 3. Aufl., Ann Arbor 1959, S. 150-167.

Freter, M., Marktsegmentierung, Stuttgart 1983.

Fritz, W., Warentest und Konsumgütermarketing, Wiesbaden 1984.

Fritz, W. u.a., Unternehmensziele in Industrie und Handel, in: DBW, H. 4, 1985, S. $375-394$.

Funke, U. H., Mathematical Models in Marketing, A collection of Abstracts, Berlin u. a. 1976.

Gaul, W., Both, M., Computergestütztes Marketing, Berlin u. a. 1990.

Gaul, W., Decker, R., Neuronale Netze in der Kaufverhaltensforschung, in: asw, H. 7, 1994, S. 66-69.

GDI-Handelstrendletter 1/94, Gottlieb Duttweiler Institut, Rüschlikon 1994.

Gedenk, K., Skiera, B., Marketing-Planung auf der Basis von Reaktionsfunktionen, Funktionsschätzung und Optimierung, in: WiSt, H. 5 , 1994, S. 258-262.

Gierl, H., Von der Präferenz zum Kauf eines Markenartikels, in: Markenartikel, H. 10, 1987, S. 458-467. 
Gierl, H., Planung der Sortimentstiefe im Handel, in: der Markt, H. 1, 1992, S. 20-26.

Gordon, W., Handelsmarken, Das Äquivalent für Erfolg in den Neunzigern, in: Planung und Analyse, H. 3, 1994, S. 52-59.

Gottwald, R., Entscheidung unter Unsicherheit, Informationsdefizite und unklare Präferenzen, Wiesbaden 1990.

Grob, H. L., Computergestützte Preispolitik, Preisstrategische Entscheidungsmodelle für Marketing-Informationssysteme, Wiesbaden 1975.

Grob, H.-L., Periodenspezifische Mischzinsfüße als theoretisch richtige Kalkulationszinsfüße, in: ZfB, H. 4, 1982, S. 381-395.

Grossekettler, H., Die gesamtwirtschaftliche Problematik vertraglicher Vertriebssysteme, in: Vertragliche Vertriebssysteme zwischen Industrie und Handel, Hrsg.: Ahlert, D., Wiesbaden 1981, S. 255-263.

Größer, H., Der klassische Markenartikel - Versuch einer Wesensbestimmung, in: Markenartikel, H. 5, 1991, S. 200- 207.

Gümbel, R., Die Sortimentspolitik in den einzelnen Betrieben des Wareneinzelhandels, Köln, Opladen 1963.

Gümbel, R., Handel, Markt und Ökonomik, Wiesbaden 1985.

Gussek, F., Erfolg in der strategischen Markenführung, Wiesbaden 1992.

Haedrich, G., Informationssystem und Marketingplanung, in: Operationale Entscheidungshilfen für die Marketingplanung, Hrsg.: Haedrich, G., Berlin, New York 1977, S. 26-29.

Haedrich, G., Tomczak, T., Strategische Markenführung, Planung und Realisierung von Marketingstrategien für eingeführte Produkte, Bern, Stuttgart 1990.

Haedrich, G., Gussek, F., Tomczak, T., Instrumentelle Strategiemodelle als Komponenten im Marketingplanungsprozeß, in: DBW, H. 2, 1990, S. 205-222.

Hammann, P., Erichson, B., Marktforschung, Stuttgart, New York 1978. 
Hammann, P., von der Gathen, A., Bilanzierung des Markenwertes und kapitalmarkorientierte Markenbewertungsverfahren, in: Markenartikel, H. 5, 1994, S. 204-211.

Hansen, H. R., Thabor, A., Marketing-Modelle, Anwendungsmöglichkeiten und Entwicklung computergestützter Modelle im Marketing, Berlin, New York 1973.

Hansen, P., Die handelsgerichtete Absatzpolitik der Hersteller im Wettbewerb um den Regalplatz, Berlin 1972.

Hansen, U., Absatz- und Beschaffungsmarketing des Einzelhandels, Eine Aktionsanalyse, Göttingen 1990.

Hansen, U., Leitherer, E., Produktpolitik, 2. Aufl., Stuttgart 1984.

Hanssmann, F., Systemforschung am Beispiel der strategischen Unternehmensmodelle, in: WiSt, H. 8, S. 357-363.

Hartung, J., Elpelt, B., Klösener, K.-H., Lehr- und Handbuch der angewandten Statistik, 9. Aufl., München, Wien 1993.

Hätty, H., Markentransfer, Heidelberg 1989.

Heeler, R., M., On the Awareness Effects of Mere Distribution, in: Marketing Science, H. 3, 1986, S. 273.

Heider, M., Simulationsmodell zur Risikoanalyse für Investitionsplanungen, Diss., Bonn 1969.

Heinemann, M., Einkaufsstättenwahl und Firmentreue des Konsumenten, Wiesbaden 1976.

Heinen, E., Zum Wissenschaftsprogramm der entscheidungsorientierten Betriebswirtschaftslehre, in: ZfB, H. 4, 1969, 207-220.

Heinen, E., Grundlagen betriebswirtschaftlicher Entscheidungen, Das Zielsystem der Unternehmen, 3. Aufl., Wiesbaden 1976.

Heinen, E., Einführung in die Betriebswirtschaftslehre, 9. Aufl., Wiesbaden 1985.

Heinhold, M., Simultane Unternehmensplanungsmodelle - ein Irrweg?, in: DBW, H. 6, 1989, S. 689-708. 
Henderson, B. D., Die Erfahrungskurve in der Unternehmensstrategie, Frankfurt, New York 1974.

Herlitz, P., Zur optimalen Kombination des absatzpolitischen Instrumentariums, Diss., Berlin 1968.

Herrmann, H.-J., Modellgestützte Planung im Unternehmen, Entwicklung eines Rahmenkonzeptes, Wiesbaden 1992.

Herz, D. B., Risk Analysis in Capital Investment, in: Harvard Business Review, H. 1, 1964, S. 95-106.

Herz, D. B., Risiko-Analyse bei Kapitalanlagen, in: Investitionsplanung, Hrsg.: Lüder, K., München 1977, S. 157-175.

Herzig, O. A., Markenbilder/Markenwelten - Neue Wege in der Imageforschung, Wien 1991.

Hesse, H. W., Kommunikation und Diffusion von Produktinnovationen im Konsumgüterbereich, Berlin 1987.

Heß, G., Marktsignale und Wettbewerbsstrategie, Theoretische Fundierung und Fälle aus der Unternehmenspraxis, Stuttgart 1991.

Hildenbrand, K., Systemorientierte Risikoanalyse in der Investitionsplanung, Berlin 1988.

Hillier, F. S., The Derivation of Probabilistic Information for the Evoluation of Risky Investements, in: Management Science, 1963, S. 443-457.

Hinterhuber, H. H., Strategische Unternehmensführung, II. Strategisches Handeln, 5. Aufl., Berlin, New York 1992.

Höft, U., Lebenszykluskonzepte, Grundlagen für das strategische Marketingund Technologiemanagement, Berlin 1992, S. 15-46.

Höhl, J., Zweitmarken - Ein entscheidungsorientierter Ansatz aus der Sicht des Markenartikelherstellers bei Gütern des täglichen Bedarfs, Gelsenkirchen 1982.

Homburg, C., Modellgestützte Unternehmensplanung, Wiesbaden 1991.

Huber, W. R., Markenpolitische Strategien des Konsumgüterherstellers, Frankfurt/Main 1988. 
Hughes, D. A., Commentary to Farris, P., Olver, J., De Kluyver, C., The Relationship between Distribution and Market Share, in: Marketing Science, No. 2, Spring 1989, S. 129-130.

Hünerberg, R., Marketingsysteme und -modelle, Zur Entscheidungslehre im Marketing, Opladen 1974.

Hüttner, M., Markt und Absatzprognose, Stuttgart u. a. 1982.

Irrgang, W., Strategien im vertikalen Marketing, Handelsorientierte Konzeptionen der Industrie, München 1989.

Irrgang, W., Auf dem Weg zu mehr Partnerschaft? - Neue Chancen für das Verhältnis Hersteller/Handel, in: Marketingforschung und Management, H. 1, 1994, S. 3-6.

Jacoby, J., Brand Loyalty, A Conceptual Definition, in: Proceedings of the 79th American Psychological Association Convention 1971, S.655-656.

Kaas, K.-P., Langfristige Werbewirkung und Brand Equity, in: Werbeforschung \& Praxis, H. 3, 1990, S. 48-53.

Kaas, K.-P., Marketing und neue Institutionenlehre, Arbeitspapier Nr. 1 aus dem Forschungsprojekt Marketing und ökonomische Theorie der Universität Frankfurt, Frankfurt/Main 1993.

Kaas, K.-P., Hay, Ch., Preisschwellen bei Konsumgütern - eine theoretische und empirische Analyse, in: ZfbF, 1982, S. 333-346.

Kapferer, J.-N., Die Marke - Kapital des Unternehmens, Landsberg/Lech 1992.

Katz, R., Informationsquellen der Konsumenten, Eine Analyse der Divergenzen zwischen Beurteilung und Nutzung, Wiesbaden 1983.

Keller, K. L., Conceptualizing, Measuring and Managing Customer-Based Brand Equity, in: Journal of Marketing, January 1993, S. 1-22.

Kellner, J., Promotions, Zielsetzungen, Techniken und Fallbeispiele, Landsberg a. L. 1982.

Kellner, M., Absatzprognose im Automobilmarkt, München 1987. 
Kemmna, H., Das Key Account Management der 90er Jahre, in: Thexis, H. 1, 1990, S. 29-35.

Kieser, A., Kubicek, H., Organisation, 3. Aufl., Berlin, New York 1992.

Kirchgeorg, M., Ökologieorientiertes Unternehmensverhalten, Wiesbaden 1990.

Kistner, K.-P., Produktions- und Kostentheorie, Würzburg, Wien 1981.

Klenger, F., Krautter, F., Simulation des Käuferverhaltens, Teil I: Werbewirkung und Käuferverhalten, Teil II: Analyse eines Kaufprozesses, Teil III: Computermodell des Käuferverhaltens, Wiebaden 1972.

Knoblich, H., Die typologische Methode in der Betriebswirtschaftslehre, in: WiSt, H. 4, 1972, S. 141-147.

Koch, H., Integrierte Unternehmensplanung, Wiesbaden 1982.

Köhler, R., Beiträge zum Marketing-Management, 2. Aufl., München 1991.

Köhler, R. , Marke und Verbraucher - Zu den Grundlagen lebenslanger Bindung, in: Markenartikel, H. 7, 1993, S. 340-347.

Köhler, R., Uebele, H., Risikoanalysen bei der Evaluierung absatzorientierter Projekte, in: WiSt, H. 3, 1983, S. 119-127.

Köhler, R., Uebele, H., Straplag AG, Teil II, Risikoanalytische Evaluierung von Preisstrategien bei einer Neuprodukteinführung, in: WiSt, H. 4, 1983, S. 211-213.

Konert, F.-J., Vermittlung emotionaler Erlebniswerte - Eine Positionierungsund Profilierungsstrategie für Markenartikel auf gesättigten Märkten, in: Markenartikel, H. 12, 1986, S. 576-578.

Kotler, P., Competitive Strategies, for New Product Marketing over the Life Cycle, in: Management Science, Dec. 1965, S. B-104-B-119.

Kotler, P., Bliemel, F. W., Marketing-Management, Analyse, Planung, Umsetzung und Steuerung, 7. Aufl., Stuttgart 1992.

Kreilkamp, E., Strategisches Management und Marketing, Berlin, New York 1987. 
Kroeber-Riel, W., Marketingtheorie, Verhaltensorientierte Erklärungen von Marketingaktionen, Köln 1972.

Kroeber-Riel, W., Strategie und Technik der Werbung, Verhaltenswissenschaftliche Ansätze, 3. Aufl., Stuttgart u.a. 1991.

Kroeber-Riel, W., Konsumentenverhalten, 5. Aufl., München 1992.

Kruschwitz, L., Bemerkungen zur Risikoanalyse aus theoretischer Sicht, in: ZfB, H. 7, 1980, S. 800-808.

Kruschwitz, L., Investitionsrechnung, 3. Aufl., Berlin, New York 1987.

Kühn, R., Der Markenartikel, in: Marketing Journal, H. 5, 1983, S. 374-376.

Kulla, B., Ergebnisse oder Erkenntnisse - liefern makroanalytische Simulationsmodelle etwas Brauchbares?, in: Biethahn, J., Schmidt, B., Simulation als betriebliche Entscheidungshilfe, Methoden, Werkzeuge, Anwendungen, Berlin u. a. 1987, S. 3-23.

Kümpers, U. A., Marketingführerschaft, Eine Verhaltenswissenschaftliche Analyse des vertikalen Marketing, Diss., Münster 1976.

Kunkel, R., Vertikales Marketing im Herstellerbereich, Bestimmungsfaktoren und Gestaltungselemente stufenübergreifender Marketing-Konzeptionen, München 1977.

Kupsch, P., Unternehmungsziele, Stuttgart, New York 1979.

Laforet, S., Saunders, J., Managing Brand Portfolios, How the leaders do it, in: Journal of Advertising Research, Sept./Oct. 1994, S. 64-76.

Lavington, M. R., A Practical Simulation Model for Consumer Marketing, in: Operational Research Quarterly 1970, S. 25-45.

Lehmann, F. O., Strategische Budgetierung, Entwurf und Fundierung eines Instruments des strategischen Controllings, Frankfurt/Main u. a. 1993.

Leitherer, E., Die Entwicklung des Markenwesens - Von den Ursprüngen bis zum Beginn der fünfziger Jahre, Veröffentlichung der Gesellschaft zur Erforschung des Markenwesens e.V., Hrsg: Berekoven, L., München 1988. 
Lewandowsky, R., Mittelfristige Prognose- und Marketingsysteme, Bd. 2, Berlin 1980.

Lilien, G. L., Marketing Mix Analysis with Lotus 1-2-3, Redwood City 1986.

Lilien, G. L., Kotler, P., Marketing Decision Making, A Model-Building Approach, New York 1987.

Lilien, G. L., Kotler, P., Moorthy, K. S., Marketing Models, Englewood Cliffs 1992.

Lingenau, V., Kostenwirkungen der Variantenvielfalt, in: Kostenrechnungspraxis, H. 5, 1994, S. 307-315.

Little, J. D. C., Aggregate Advertising Models, The State of the Art, in: Operations Research, No. 4, 1971, S. 629-667.

Little, J. D. C., Ein On-Line-Marketing-Mix-Modell, in: Computergestützte Marketingplanung, Hrsg.: Hansen, H. R., München 1974, S. 651704.

Little, J. D. C., Models and Managers: The Concept of a Decision Calculus, in: Management Science, No. 8, 1970, S. 466-485. Deutsche Übersetzung: Modelle und Manager: Das Konzept eines Decision Calculus, in: Operationale Entscheidungshilfen für die Marketingplanung, Hrsg.: Haedrich, G., Berlin, New York 1977, S. 201-230.

Lo, L., Prognoseinformationen für Kundendienstpolitische Entscheidungen Dargestellt am Beispielen des Fotomarktes, Diss., Münster 1979.

Maas, R.-M., Absatzwege, Konzeptionen und Modelle, Wiesbaden 1980.

Mann, G., Entwicklungstendenzen des Markenartikel - Herstellerperspektive, in: Markenartikel, H. 10, 1994, S. 484-488.

Mayer, $\mathrm{H}$., Werbewirkung und Kaufverhalten unter ökonomischen und psychologischen Aspekten, Stuttgart 1990.

Mazanec, J., Strukturmodelle des Konsumverhaltens, Wien 1978.

Mediaanalyse '94, Hrsg.: Axel Springer Verlag, Hamburg 1994.

Meffert, H., Zum Problem der betriebswirtschaftlichen Flexibilität, in: ZfB, 1969, S. $779-800$. 
Meffert, H., Systemtheorie aus betriebswirtschaftlicher Sicht, in: Systemanalyse in den Wirtschafts- und Sozialwissenschaften, Hrsg.: Schenk, K. E., Berlin 1971, S. 174-206.

Meffert, H., Unternehmensziele, in: Jahrbuch des Marketing, Hrsg.: Schöttle, K. M., Essen 1971, S. 22-34.

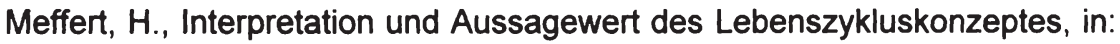
Neuere Ansätze zur Marketingtheorie, Festschrift zum 80. Geburtstag von O. Schnutenhaus, Hrsg.: Hammann, P. u.a., Berlin 1973 , S. $85-134$.

Meffert, H., Computergestützte Marketing-Informationssysteme und Marketing-Modelle, in: Computergestützte Marketingplanung, Hrsg.: Hansen, H. R., München 1974, S. 11-31.

Meffert, H., Computergestützte Marketing-Informationssysteme, Konzeptionen, Modellanwendungen, Entwicklungsstrategien, Wiesbaden 1975.

Meffert, H., Die Beurteilung und Nutzung von Informationsquellen beim Kauf von Konsumgütern, in: Konsumentenverhalten und Informationen, Hrsg.: Meffert, H., Steffenhagen, H., Freter, H., Wiesbaden 1979, S. 39-65.

Meffert, H., Produktmanagement: Funktionen und organisatorische Eingliederung, in: WISU, 1979, S. 68-72 u. 120-125.

Meffert, H., Der Kundendienst als Marketinginstrument - Einführung in die Problemkreise des Kundendienstmanagments, in: Kundendienstmanagement, Hrsg.: Meffert, H., Frankfurt/Main, Bern 1982.

Meffert, H., Markenpolitik im Wettbewerb (I), in: WISU, H. 9, 1983, S. 407410.

Meffert, H., Größere Flexibilität als Unternehmungskonzept, in: ZfbF, H. 2, 1985, S. 121-137.

Meffert, H. Marketing, Grundlagen der Absatzpolitik, 7. Aufl., Wiesbaden 1986.

Meffert, H., Perspektiven des Marketing im Spannungsfeld zwischen Standardisierung und Differenzierung, Thesen zu den Herausforderungen der 90er Jahre, in: Markenartikel, H. 10, 1986, S. 442-444. 
Meffert, H., Marketing und strategische Unternehmensführung - ein wettbewerbsorientierter Kontingenzansatz, in: Strategische Unternehmensplanung, Hrsg.: Hahn, D., Taylor, B., 4. Aufl., Heidelberg, Wien 1986, S. 660-684.

Meffert, H., Markenstrategien als Waffe im Wettbewerb, in: Handbuch strategische Führung, Hrsg.: Henzler, H. A., Wiesbaden 1988, S. 581610.

Meffert, H., Marketingstrategien in unterschiedlichen Marktsituationen, in: Handbuch des Marketing, Anforderungen an Marketingkonzeptionen aus Wissenschaft und Praxis, Hrsg.: Bruhn, M., München 1989 , S. 277-306.

Meffert, H., Marktanalyse, in: Handwörterbuch der Planung, Hrsg.: Szyperski, N., Stuttgart 1989, Sp. 1020-1030.

Meffert, H., Entwicklungslinien des Marketing - Akzente der marktorientierten Führung in den 90er Jahren, in: Jahrbuch des Marketing, Hrsg.: Schöttle, K. M., 5. Auflg., Essen 1990, S. 12-21.

Meffert, H., Herausforderungen an die Markenführung in den 90er Jahren, in: Markenartikel, H. 6, 1991, S. 264-265.

Meffert, H., Integrierte Marktkommunikation, unveröffentlichtes Skript, Münster 1991.

Meffert, H., Erfolgreiches Marketing in den neunziger Jahren, in: Marketing im Umbruch - Chancen und Gefahren für Unternehmer, Zürich 1991, S. 11-22.

Meffert, H., Marketingforschung und Käuferverhalten, 2. Aufl., Wiesbaden 1992.

Meffert, H., Strategien zur Profilierung von Marken, in: Marke und Markenartikel als Instrumente des Wettbewerbs, Hrsg.: Dichtl, E., Eggers, W., München 1992, S. 129-156.

Meffert, H., Erfolgreiches Marketing in der Rezession, Strategien und Maßnahmen in engeren Märkten, Wien 1994.

Meffert, H., Marketing-Management - Analyse, Strategie, Implementierung, Wiesbaden 1994. 
Meffert, H., Markenführung in der Bewährungsprobe, in: Markenartikel, H. 10, 1994, S. 478-481.

Meffert, H., Entscheidungsorientierter Ansatz der Markenpolitik, in: Handbuch Markenartikel, Hrsg.: Bruhn, M., Stuttgart 1994.

Meffert, H., Kirchgeorg, M., Umweltschutz als Unternehmensziel, in: Marketing-Schnittstellen, Hrsg.: Specht, G., Silberer, G., Engelhardt, W. H., Stuttgart 1989, S. 179-199.

Meffert, H., Steffenhagen, H., Marketing-Prognosemodelle, Stuttgart 1977.

Meffert, H., Steffenhagen, H., Freter, H., Entwicklung und Schwerpunkte der Käuferverhaltensforschung, in: Konsumentenverhalten und Informationen, Hrsg.: Meffert, H., Steffenhagen, H., Freter, H., Wiesbaden 1979 , S. 23-35.

Meffert, $H$. und Mitarbeiter, Die Anwendung mathematischer Modelle im Marketing, Wiesbaden, o.J.

Mei-Folter, A., Barber, F., Händler als Markengestalter, Aufbau vertikaler Handelsmarken als Zukunftschance, in: LZ, Nr. 41, 15. Oktober 1993, S. J 14.

Mellerowicz, K., Markenartikel - die ökonomischen Gesetze ihrer Preisbildung und Preisbindung, München, Wien 1963.

Merkle, H., Globale Trends - Globale Chance für die Werbung, in: Markenartikel, H. 7, 1994, S. 336-340.

Mertens, P., Simulation, 2. Aufl., Stuttgart 1982.

Michael, B. M., Die Marke ist tot, Es lebe die Marke!, in: Markenartikel, H. 1, 1994, S. 22-25.

Müller, G.-M., Dachmarkenstrategie, in: Markenartikel, H. 4, 1994, S. 142148.

Müller, N., Marketingstrategien in High-Tech-Märkten, Typologisierung, Ausgestaltungsformen und Einflußfaktoren auf der Grundlage strategischer Gruppen, Diss., Münster 1994.

Müller-Hagedorn, L., Heidel, B., Die Sortimentstiefe als absatzpolitisches Instrument, in: ZfbF, H. 1, 1986, S. 39-63. 
Müller-Merbach, H., Risikoanalyse, in: Management Enzyklopädie, Bd. 8, 1975 , S. 2845-2850.

Naert, P. A., Leeflang, P. S., Building implementable marketing models, Boston 1978.

Nieschlag, R., Dichtl, E., Hörschgen, H., Marketing, 15. Aufl., Berlin 1988.

Nolte, H., Die Markentreue im Konsumgüterbereich, Bochum 1976.

o. V. Unwucht im Ersatzgeschäft, in: Industriemagazin, H. 2, 1987, S. 142147.

o. V., Das Fitness-Center für Ihr Auto, Goodyear Reifen + Auto Service, in:

Dynamik im Handel, H. 11, 1989, S. 20-23.

o. V., Wir wollen Problemlöser des Autofahrers sein, Interview mit Gerd Grünenwald, Verkaufsdirektor Deutsche Goodyear $\mathrm{GmbH}$, in: Dynamik im Handel, H. 11, 1989, S. 24-25.

o. V., Marken - How much in Dollar?, in: asw, H. 8, 1989, S. 50-54.

o. V., Der gute Ruf entscheidet, in: asw, H. 9, 1990, S. 36.

o. V., Weltgrößter Reifenhersteller erwartet schwere Zeiten, in: Handelsblatt vom 27.6.90, S. 23.

o. V., Wieviel ist der Markenwert wert?, in: asw, H. 3, 1990, S. 26.

o. V., Wir liefern nicht an Hinz und Kunz, Interview mit Bridgestone Geschäftsführer Freitag, in: asw, H. 7, 1990, S. 15-18.

o. V., Gefahr im Marketing, in: w\&v, Nr. 27, 6. Juli 1990, S. 12.

o. V. Abspecken und sich wohlfühlen, in: asw, H. 11, 1990, S. 81-91.

o. V., Pull-Effekt à la Öko, in: asw, H. 11, 1991, S. 60-68.

o. V., Continental erwartet ein schwieriges Jahr, in: FAZ vom 27.2.93, S. 16.

o. V., Contintental ist der Reifenmarkt eingebrochen, in: FAZ vom 22.10.93, S. 22.

o. V., Der Wintereinbruch hilft dem Handel mehr als den Herstellern, in: FAZ vom 27.2.1993, S. 16. 
o. V., Reifenkonzern spürt die Wende zum Besseren, in: Handelsblatt vom 26./27.8.1994, S. 21.

o. V., Höherer Gewinn bei Continental, in: FAZ vom 14.3.1995, S. 19.

Oehme, W., Handelsmarketing, 2. Aufl., München 1992.

Olver, J. M., Farris, P. W., Push and Pull, A One-Two Punch for Packaged Products, in: Sloan Management Review, Fall 1989, S. 53-67.

Pepels, W., Die Beziehungen von Marketing-Interessen auf Hersteller- und Handelsstufe, in: Markenartikel, H. 10, 1988, S. 494-501.

Pepels, W., Positionierungsstrategien, in: Markenartikel, H. 4, 1994, S. 422425.

Pfohl, H.-Ch., Messung subjektiver Wahrscheinlichkeiten, in: Materialien zur Betriebs- und Volkswirtschaft, Bd. 2, Wirtschaftliche Meßprobleme, Köln 1977, S. 23-36.

Pfohl, H.-Ch., Zur Formulierung einer Lieferservice-Politik - Theoretische Aussagen zum Angebot von Sekundärleistungen als absatzpolitisches Instrument, in: ZfbF, H. 3, 1977, S. 239-255.

Pidd. M., Computer Simulation in Management Science, 2. Ed., Chichester 1988.

Porter, M. E., Wettbewerbsstrategie, Frankurt/Main 1992.

Reutterer, T., Analytische Modelle zur Unterstützung von Positionierungsentscheidungen - Versuch einer Bestandsaufnahme, in: der Markt, H. 2, 1994, S. 88-109.

Rieger, B., Die Kunst sich zwischen die Stühle zu plazieren. Plazieren Positionieren - Segmentieren, in: asw H. 10, 1984, S. 156-165.

Riesenbeck. H., Voss, W.-D., Marke oder Masse, in: Manager Magazin, H. 6, 1989, S. 223-233.

Ritzerfeld, U., Marketing-Mix-Strategien in Investitionsgütermärkten - Marktstrukturspezifische Strategieempfehlungen aufgrund einer Analyse von Lieferanten-Kunden-Beziehungen und einer Simulationsstudie, Diss., Aachen 1993. 
Rubinstein, R. Y., Simulation and the Monte Carlo Method, New York u. a. 1981.

Rudolph, M., Mehrstufiges Marketing für Einsatzstoffe, Anwendungsvoraussetzungen und Strategietypen, Frankfurt/Main u. a. 1989.

Rühli, E., Entscheidungsorientierter Ansatz und Allgemeine Betriebswirtschaftslehre, in: Die Betriebswirtschaftslehre im Spannungsfeld zwischen Generalisierung und Spezialisierung, Hrsg.: Kirsch, W.; Picot, A., Wiesbaden 1989, S. 99-117.

Rütschi, K., Marketing-Modelle, Systemanalyse, Formalisierung und Aufbau, Winterthur 1972.

Sander, M., Die Bestimmung und Steuerung des Wertes von Marken, Eine Analyse aus Sicht des Markeninhabers, Heidelberg 1994, S. 51.

Sasseen, J., Tyre Makers on the Rack, in: International Management, March 1993, S. 40-43.

Schierenbeck, H., Grundzüge der Betriebswirtschaftslehre, 11. Aufl., München, Wien 1993.

Schindel, V., Risikoanalyse, Darstellung und Bewertung von Risikorechnung am Beispiel von Investitionsentscheidungen, München 1977.

Schirmeister, R., Modell und Entscheidung, Möglichkeiten und Grenzen der Anwendung von Modellen zur Alternativenberechnung im Entscheidungsprozeß der Unternehmung, Stuttgart 1980.

Schmalen, H., Kommunikationspolitik, Stuttgart u.a. 1985.

Schmidt, J. W., Taylor, R. E. Simulation and Analysis of Industrial Systems, Homewood, Georgetown 1970.

Schmitz, B., Die zukünftige Bedeutung von interaktiven Kommunikationssystemen am Point of Sale, in: Der Handel für die Märkte von Morgen, Perspektiven und Entwicklungen, Hrsg.: Gruber, $H$., Titze, A., Frankfurt/Main 1990, S. 172-181.

Schulz, R., Brandmeyer, K., Die Marken-Bilanz im Kreuzverhör: ein Instrument zur Bestimmung und Steuerung von Markenwerten, in: Markenartikel, H. 7, 1989, S. 364-370. 
Schulz, R., Brandmeyer, K., Die Marken-Bilanz, Ein neues Instrument für die Bewertung und Steuerung von Marken stellt sich vor, in: Marketing Journal, H. 4, 1989, S. 360-363.

Schütt, K.-P., Wahrscheinlichkeitsschätzungen im Computer-Dialog, Theorie, Methoden und eine experimentelle Studie zur Schätzung von subjektiven Wahrscheinlichkeiten, Stuttgart 1981.

Schwärzler, A., Rentabel variieren, in: asw, H. 7, 1991, S. 64-66.

Schweiger, G., Die Absatzfunktion als Grundlage der Optimierung des Marketing-Mix, in: der Markt, H. 50, 1974, S. 29-38.

Seidler, J., Bedeutung der Verpackungspolitik für die Markengestaltung, H. 6,1994, S. 278-284.

Seif, F. T., Bilanzwert der Marke, in: Markenartikel, H. 8, 1994, S. 388-389.

Simon, H., Goodwill und Marketingstrategie, Wiesbaden 1985.

Simon, H., Scanner-Technologie, Revolution im Handelsmarketing?, in: Marktorientierte Unternehmensführung und Innovation, Hrsg.: Meffert, H., Münster 1985, S. 201-216.

Simon, H., Management strategischer Wettbewerbsvorteile, in: Wettbewerbsvorteile und Wettbewerbsfähigkeit, Hrsg.: Simon, H., Stuttgart 1988, S. 1-17.

Simon, H., Marketing-Mix-Interaktion, Theorie, empirische Befunde, strategische Implikationen, in: ZfbF, H. 2, 1992, S. 87-110.

Simon, H., Preismangagment, 2. Aufl., Wiesbaden 1993.

Simon, H., Kucher, E., Die Bestimmung empirischer Preisabsatzfunktionen. Methoden, Befunde, Erfahrungen, in: ZfB, H. 1, 1980, S. 171-183.

Simon, R. S., Marketing-Service als Mittel zur Differenzierung im stagnierenden Reifenmarkt, in: Thexis, H. 1, 1986, S. 28-32.

Smith, D. C., Brand extensions and advertising efficiency, what can and cannot be expected, in: Journal of Advertising Research, Nov./Dec. 1989, S. 11-20.

Specht, G., Distributionsmanagement, 2. Aufl., Stuttgart u.a. 1992. 
Stange, K., Angewandte Statistik, Zweiter Teil, Mehrdimensionale Probleme, Berlin u. a. 1971.

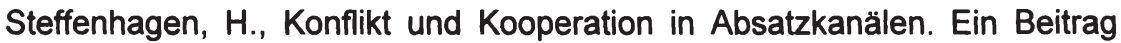
zur verhaltensorientierten Marketingtheorie, in: Schriftenreihe Unternehmensführung und Marketing, Bd. 5, Hrsg.: Meffert, H., Wiesbaden 1975.

Steffenhagen, H., Markenbekanntheit als Werbeziel, Theorie und Operationalisierung, in: ZfB, H. 10, 1976, S. 715-734.

Steffenhagen, H., Wirkungen absatzpolitischer Instrumente, Theorie und Messung der Marktreaktionen, Stuttgart 1978.

Steffenhagen, H., Der Strategiebegriff in der Marketingplanung, Arbeitspapier des Instituts für Marketing der Universität Münster, Nr. 29, Münster 1982.

Steffenhagen, H., Keller, D., Herstellerkonditionen und Handelsleistung, in: Markenartikel, H. 9, 1990, S. 398-403.

Steffenhagen, H., Marketing, Eine Einführung, 2. Aufl., Stuttgart u. a. 1991.

Steinmann, H., Schreyögg, G., Management, Stuttgart 1990.

Thabor, A., Ein Markting-Mix-Simulationssystem - angewandt beim Bankenmarkt, in: Computergestützte Marketingplanung, Hrsg.: Hansen, H. R., München 1974, S. 587-650.

Theisen, P., Optimierungsmodelle der Distributionspolitik, in: ZfB, H. 2, 1977, S. 65-88.

Thies, G., Vertikales Marketing, Marktstrategische Partnerschaft zwischen Industrie und Handel, Berlin, New York 1976.

Titze, A., Marken-Macht, in: Manager Magazin, H. 5, 1991, S. 206-215.

Tolle, E., Steffenhagen, H., Kategorien des Markenerfolges und einschlägige Meßmethoden, in: Markenartikel, H. 8, 1994, S. 378-382.

Tomczak, T., Gussek, F., Handelsorientierte Anreizsysteme der Konsumgüterindustrie, in: ZfB, H. 7, 1992, S. 783-806. 
Topritzhofer, E., Absatzwirtschaftliche Modelle des Kaufentscheidungsprozesses unter besonderer Berücksichtigung des Markenwahlaspektes, Wien 1974.

Träger, W., Push und Pull: Oder was soll der Hersteller beim Einzelhandel tun?, in: Marketing-Journal, H. 4, 1969, S. 262-263.

Triffin, R., Monopolistic Competition and General Equilibrium Theory, 7. Ed., Cambrigde 1962.

Trommsdorff, V., Die Messung von Produktimages für das Marketing, Grundlagen und Operationalisierung, Köln u.a. 1975.

Trommsdorff, V., Konsumentenverhalten, Stuttgart u. a. 1989.

Turban, E., Decision Support and Expert Systems, Managerial Perspectives, New York 1988.

Unger, F., Markenpolitik und -strategie angesichts geänderten Verbraucherverhaltens, in: VM International, H. 2, 1990, S. 4-10.

Voigt, K. I., Strategische Planung und Unsicherheit, Wiesbaden 1992.

Voß, W.-D., Modellgestützte Markenpolitik, Planung und Kontrolle markenpolitischer Entscheidungen auf der Grundlage computergestützter Informationssysteme, Wiesbaden 1983.

Voß, W.-D., Marketing in der Sackgasse? - Ansatzpunkte zur Weiterentwicklung des Marketing in der Konsumgüterindustrie, in: Marktorientierte Unternehmensführung im Umbruch, Effizienz und Flexibilität als Herausforderungen des Marketing, Hrsg.: Bruhn, M., Meffert, H., Wehrle, F., Stuttgart 1994, S. 115-131.

Wagener, F., Die partielle Risikoanalyse als Instrument der integrierten Unternehmensplanung, München 1978.

Wagle, B., A Statistical Analysis of Risk in Capital Investment Projects, in: Operational Research Quarterly, H. 1, 1969, S. 13-33.

Walters, M., Marktwiderstände und Marketingplanung, Wiesbaden 1984.

Wehrle, F., Chancen und Risiken für Fachzeitschriften im europäischen Binnenmarkt, Europa 1992, in: Chancen und Risiken für das Marketing, Hrsg.: Bruhn, M., Wehrle, F., 2. Aufl., Münster-Hiltrup 1990, S. 217-226. 
Weinberg, P., Das Entscheidungsverhalten von Konsumenten, Paderborn 1981.

Weinberg, P., Erlebnismarketing, München 1992.

Welge, M. K., Unternehmungsführung, Band 3, Controlling, Stuttgart 1983.

Westbrook, G., Der Preisabstand schwindet dahin, Trends auf dem europäischen Eigenmarkenmarkt, in: LZ, Nr. 40, 8. Oktober 1993, S. 158-159.

Wicke, L., Der Umweltschutz als Herausforderung und Chance für den Markenartikel, in: Marke und Markenartikel als Instrumente des Wettbewerbs, Hrsg.: Dichtl, E., Eggers, W., München 1992, S. 157184.

Windhorst, K-G., Wertewandel und Konsumentenverhalten, Münster 1985.

Wirtschaftsverband der deutschen Kautschukindustrie e. V. (Hrsg.), Kautschukindustrie: Probleme trotz leichter Konjunkturerholung, Pressenotiz vom 4.10.1994.

Wöhe, G., Einführung in die allgemeine Betriebswirtschaftslehre, 15. Aufl., München 1984.

Wolf, J., Lineare Fuzzy-Modelle zur Unterstützung der Investitionsentscheidung, Modellierung und Lösung von Investitionsproblemen mittels der Theorie unscharfer Mengen, Frankurt/Main u.a. 1988.

Wöllenstein, S., Betriebstypenprofilierung in vertraglichen Vertriebssystemen, Eine Analyse von Einflußfaktoren und Erfolgswirkungen auf der Grundlage eines Vertragshändlersystems im Automobilhandel, Diss., Münster 1994.

Zantov, R., Systemorientierte Marketinganalyse der Banken, Die Systemforschung als Grundlage generell anwendbarer Analysemodelle, dargestellt am Beispiel der Bankmarketinganalyse, Florenz 1978.

Zentes, J., Die Optimalkomplexion von Entscheidungsmodellen, Köln u.a. 1976.

Zentes, J., Trade-Marketing, Eine neue Dimension in den Hersteller-Händler-Beziehungen, in: Marketing ZFP, H. 4, 1989, S. 224-229.

Zimmermann, H.-J., Fuzzy set theory and its applications, Boston u. a. 1985. 


\section{SCHRIFTEN ZUM MARKETING}

Band 1 Friedrich Wehrle: Strategische Marketingplanung in Warenhăusern. Anwendung der Portfolio-Methode. 1981. 2. Auflage. 1984.

Band 2 Jürgen Althans: Die Übertragbarkeit von Werbekonzeptionen auf internationale Mărkte. Analyse und Exploration auf der Grundlage einer Befragung bei europaweit tătigen Werbeagenturen. 1982.

Band 3 Günter Kimmeskamp: Die Rollenbeurteilung von Handelsvertretungen. Eine empirische Untersuchung zur Einschätzung des Dienstleistungsangebotes durch Industrie und Handel. 1982.

Band 4 Manfred Bruhn: Konsumentenzufriedenheit und Beschwerden. Erklărungsansătze und Ergebnisse einer empirischen Untersuchung in ausgewählten Konsumbereichen. 1982.

Band 5 Heribert Meffert (Hrsg.): Kundendienst-Management. Entwicklungsstand und Entscheidungsprobleme der Kundendienstpolitik. 1982.

Band 6 Ralf Becker: Die Beurteilung von Handelsvertretern und Reisenden durch Hersteller und Kunden. Eine empirische Untersuchung zum Vergleich der Funktionen und Leistungen. 1982.

Band 7 Gerd Schnetkamp: Einstellungen und Involvement als Bestimmungsfaktoren des sozialen Verhaltens. Eine empirische Analyse am Beispiel der Organspendebereitschaft in der Bundesrepublik Deutschland. 1982.

Band 8 Stephan Bentz: Kennzahlensysteme zur Erfolgskontrolle des Verkaufs und der MarketingLogistik. Entwicklung und Anwendung in der Konsumgüterindustrie. 1983.

Band 9 Jan Honsel: Das Kaufverhalten im Antiquitätenmarkt. Eine empirische Analyse der Kaufmotive, ihrer Bestimmungsfaktoren und Verhaltenswirkungen. 1984.

\section{SCHRIFTEN ZU MARKETING UND MANAGEMENT}

Band 10 Matthias Krups: Marketing innovativer Dienstleistungen am Beispiel elektronischer Wirtschaftsinformationsdienste. 1985.

Band 11 Bernd Faehsler: Emotionale Grundhaltungen als Einflußfaktoren des Käuferverhaltens. Eine empirische Analyse der Beziehungen zwischen emotionalen Grundhaltungen und ausgewählten Konsumstrukturen. 1986.

Band 12 Ernst-Otto Thiesing: Strategische Marketingplanung in filialisierten Universalbanken. Integrierte Filial- und Kundengruppenstrategien auf der Grundlage erfolgsbeeinflussender Schlüsselfaktoren. 1986.

Band 13 Rainer Landwehr: Standardisierung der internationalen Werbeplanung. Eine Untersuchung der Prozeßstandardisierung am Beispiel der Werbebudgetierung im Automobilmarkt. 1988.

Band 14 Paul-Josef Patt: Strategische Erfolgsfaktoren im Einzelhandel. Eine empirische Analyse am Beispiel des Bekleidungsfachhandels. 1988. 2. Auflage. 1990.

Band 15 Elisabeth Tolle: Der Einfluß ablenkender Tätigkeiten auf die Werbewirkung. Bestimmungsfaktoren der Art und Höhe von Ablenkungseffekten bei Rundfunkspots. 1988.

Band 16 Hanns Ostmeier: Ökologieorientierte Produktinnovationen. Eine empirische Analyse unter besonderer Berücksichtigung ihrer Erfolgseinschătzung. 1990.

Band 17 Bernd Büker: Qualitătsbeurteilung investiver Dienstleistungen. Operationalisierungsansătze an einem empirischen Beispiel zentraler EDV-Dienste. 1991.

Band 18 Kerstin Ch. Monhemius: Umweltbewußtes Kaufverhalten von Konsumenten. Ein Beitrag zur Operationalisierung, Erklärung und Typologie des Verhaltens in der Kaufsituation. 1993. 
Band 19 Uwe Schürmann: Erfolgsfaktoren der Werbung im Produktlebenszyklus. Ein Beitrag zur Werbewirkungsforschung. 1993.

Band 20 Ralf Birkelbach: Qualitătsmanagement in Dienstleistungscentern. Konzeption und typenspezifische Ausgestaltung unter besonderer Berücksichtigung von Verkehrsflughäfen. 1993.

Band 21 Simone Frömbling. Zielgruppenmarketing im Fremdenverkehr von Regionen. Ein Beitrag zur Marktsegmentierung auf der Grundlage von Werten, Motiven und Einstellungen. 1993.

Band 22 Marcus Poggenpohl: Verbundanalyse im Einzelhandel auf der Grundlage von Kundenkarteninformationen. Eine empirische Untersuchung von Verbundbeziehungen zwischen Abteilungen. 1994.

Band 23 Kai Bauche: Segmentierung von Kundendienstleistungen auf investiven Mărkten. Dargestellt am Beispiel von Personal Computern. 1994.

Band 24 Ewald Werthmöller: Räumliche Identität als Aufgabenfeld des Städte- und Regionenmarketing. Ein Beitrag zur Fundierung des Placemarketing. 1995.

Band 25 Nicolaus Müller: Marketingstrategien in High-Tech-Märkten. Typologisierung, Ausgestaltungsformen und Einflußfaktoren auf der Grundlage strategischer Gruppen. 1995.

Band 26 Nicolaus Henke: Wettbewerbsvorteile durch Integration von Geschăftsaktivitäten. Ein zeitablaufbezogener wettbewerbsstrategischer Analyseansatz unter besonderer Berücksichtigung des Einsatzes von Kommunikations- und Informationssystemen (KIS). 1995.

Band 27 Kai Laakmann: Value-Added Services als Profilierungsinstrument im Wettbewerb. Analyse, Generierung und Bewertung. 1995.

Band 28 Stephan Wöllenstein: Betriebstypenprofilierung in vertraglichen Vertriebssystemen. Eine Analyse von Einflußfaktoren und Erfolgswirkungen auf der Grundlage eines Vertragshändlersystems im Automobilhandel. 1996.

Band 29 Michael Szeliga: Push und Pull in der Markenpolitik. Ein Beitrag zur modellgestützten Marketingplanung am Beispiel des Reifenmarktes. 1996. 